\title{
RATIONALITY PROBLEM FOR ALGEBRAIC TORI
}

\author{
AKINARI HOSHI AND AIICHI YAMASAKI
}

\begin{abstract}
We give the complete stably rational classification of algebraic tori of dimensions 4 and 5 over a field $k$. In particular, the stably rational classification of norm one tori whose Chevalley modules are of rank 4 and 5 is given. We show that there exist exactly 487 (resp. 7, resp. 216) stably rational (resp. not stably but retract rational, resp. not retract rational) algebraic tori of dimension 4, and there exist exactly 3051 (resp. 25, resp. 3003) stably rational (resp. not stably but retract rational, resp. not retract rational) algebraic tori of dimension 5. We make a procedure to compute a flabby resolution of a $G$-lattice effectively by using the computer algebra system GAP. Some algorithms may determine whether the flabby class of a $G$-lattice is invertible (resp. zero) or not. Using the algorithms, we determine all the flabby and coflabby $G$-lattices of rank up to 6 and verify that they are stably permutation. We also show that the Krull-Schmidt theorem for $G$-lattices holds when the rank $\leq 4$, and fails when the rank is 5. Indeed, there exist exactly 11 (resp. 131) $G$-lattices of rank 5 (resp. 6) which are decomposable into two different ranks. Moreover, when the rank is 6 , there exist exactly 18 G-lattices which are decomposable into the same ranks but the direct summands are not isomorphic. We confirm that $H^{1}(G, F)=0$ for any Bravais group $G$ of dimension $n \leq 6$ where $F$ is the flabby class of the corresponding $G$-lattice of rank $n$. In particular, $H^{1}(G, F)=0$ for any maximal finite subgroup $G \leq \operatorname{GL}(n, \mathbb{Z})$ where $n \leq 6$. As an application of the methods developed, some examples of not retract (stably) rational fields over $k$ are given.
\end{abstract}

\section{Contents}

1. Introduction

2. Preliminaries: Tate cohomology and flabby resolutions

3. CARAT ID of the $\mathbb{Z}$-classes in dimensions 5 and 6

4. Krull-Schmidt theorem fails for dimension 5

4.0. Classification of indecomposable maximal finite groups $G \leq \mathrm{GL}(n, \mathbb{Z})$ of dimension $n \leq 6$

4.1. Krull-Schmidt theorem (1)

4.2. Krull-Schmidt theorem (2)

4.3. Maximal finite groups $G \leq \mathrm{GL}(n, \mathbb{Z})$ of dimension $n \leq 6$

4.4. Bravais groups of dimension $n \leq 6$ and corresponding quadratic forms

5. GAP algorithms: the flabby class $\left[M_{G}\right]^{f l}$

5.0. Determination whether $M_{G}$ is flabby (coflabby)

5.1. Construction of the flabby class $\left[M_{G}\right]^{f l}$ of the $G$-lattice $M_{G}$

5.2. Determination whether $\left[M_{G}\right]^{f l}$ is invertible

5.3. Computation of $E$ with $\left[\left[M_{G}\right]^{f l}\right]^{f l}=[E]$

5.4. Possibility for $\left[M_{G}\right]^{f l}=0$

5.5. Verification of $\left[M_{G}\right]^{f l}=0$ : Method I

5.6. Verification of $\left[M_{G}\right]^{f l}=0$ : Method II

5.7. Verification of $\left[M_{G}\right]^{f l}=0$ : Method III

6. Flabby and coflabby $G$-lattices

7. $H^{1}\left(G,\left[M_{G}\right]^{f l}\right)=0$ for any Bravais group $G$ of dimension $n \leq 6$

8. Norm one tori

9. Tate cohomology: GAP computations

10. Proof of Theorem 1.27

11. Proof of Theorem 1.28

12. Proof of Theorem 12.3

13. Application of Theorem 12.3

2010 Mathematics Subject Classification. Primary 11E72, 12F20, 13A50, 14E08, 20C10, 20 G15.

Key words and phrases. Rationality problem, algebraic tori, stably rational, retract rational, flabby resolution, Krull-Schmidt theorem, Bravais group, Tate cohomology.

This work was partially supported by JSPS KAKENHI Grant Numbers 22740028, 24540019, 25400027. Some part of this work was done during the authors' visit to National Taiwan University, the National Center for Theoretic Sciences (Taipei Office), whose support is gratefully acknowledged. 
14. Tables for the stably rational classification of algebraic $k$-tori of dimension 5

\section{INTRODUCTION}

Let $k$ be a field and $K$ be a finitely generated field extension of $k$. A field $K$ is called rational over $k$ (or $k$-rational for short) if $K$ is purely transcendental over $k$, i.e. $K$ is isomorphic to $k\left(x_{1}, \ldots, x_{n}\right)$, the rational function field over $k$ with $n$ variables $x_{1}, \ldots, x_{n}$ for some integer $n$. $K$ is called stably $k$-rational if $K\left(y_{1}, \ldots, y_{m}\right)$ is $k$-rational for some algebraically independent elements $y_{1}, \ldots, y_{m}$ over $K$. When $k$ is an infinite field, $K$ is called retract $k$-rational if there is a $k$-algebra $R$ contained in $K$ such that (i) $K$ is the quotient field of $R$, and (ii) the identity map $1_{R}: R \rightarrow R$ factors through a localized polynomial ring over $k$, i.e. there is an element $f \in k\left[x_{1}, \ldots, x_{n}\right]$, which is the polynomial ring over $k$, and there are $k$-algebra homomorphisms $\varphi: R \rightarrow k\left[x_{1}, \ldots, x_{n}\right][1 / f]$ and $\psi: k\left[x_{1}, \ldots, x_{n}\right][1 / f] \rightarrow R$ satisfying $\psi \circ \varphi=1_{R}$ (cf. [Sal84a]). $K$ is called $k$-unirational if $k \subset K \subset k\left(x_{1}, \ldots, x_{n}\right)$ for some integer $n$. It is not difficult to see that " $k$-rational" $\Rightarrow$ "stably $k$-rational" $\Rightarrow$ "retract $k$-rational" $\Rightarrow$ " $k$-unirational".

Let $L$ be a finite Galois extension of $k$ and $G=\operatorname{Gal}(L / k)$ be the Galois group of the extension $L / k$. Let $M=\bigoplus_{1 \leq i \leq n} \mathbb{Z} \cdot u_{i}$ be a $G$-lattice with a $\mathbb{Z}$-basis $\left\{u_{1}, \ldots, u_{n}\right\}$, i.e. finitely generated $\mathbb{Z}[G]$-module which is $\mathbb{Z}$-free as an abelian group. Let $G$ act on the rational function field $L\left(x_{1}, \ldots, x_{n}\right)$ over $L$ with $n$ variables $x_{1}, \ldots, x_{n}$ by

$$
\sigma\left(x_{i}\right)=\prod_{j=1}^{n} x_{j}^{a_{i, j}}, \quad 1 \leq i \leq n
$$

for any $\sigma \in G$, when $\sigma\left(u_{i}\right)=\sum_{j=1}^{n} a_{i, j} u_{j}, a_{i, j} \in \mathbb{Z}$. The field $L\left(x_{1}, \ldots, x_{n}\right)$ with this action of $G$ will be denoted by $L(M)$. There is the duality between the category of $G$-lattices and the category of algebraic $k$-tori which split over $L$ (see $\operatorname{Vos} 98$, page 27, Example 6]). In fact, if $T$ is an algebraic $k$-torus, then the character group $X(T)=\operatorname{Hom}\left(T, \mathbb{G}_{m}\right)$ of $T$ may be regarded as a $G$-lattice. Conversely, for a given $G$-lattice $M$, there exists an algebraic $k$-torus $T$ which splits over $L$ such that $X(T)$ is isomorphic to $M$ as a $G$-lattice.

The invariant field $L(M)^{G}$ of $L(M)$ under the action of $G$ may be identified with the function field of the algebraic $k$-torus $T$. Note that the field $L(M)^{G}$ is always $k$-unirational (see [Vos98, page 40, Example 21]). Tori of dimension $n$ over $k$ correspond bijectively to the elements of the set $H^{1}(\mathcal{G}, \operatorname{GL}(n, \mathbb{Z}))$ where $\mathcal{G}=\operatorname{Gal}\left(k_{\mathrm{s}} / k\right)$ since $\operatorname{Aut}\left(\mathbb{G}_{m}^{n}\right)=\mathrm{GL}(n, \mathbb{Z})$. The $k$-torus $T$ of dimension $n$ is determined uniquely by the integral representation $h: \mathcal{G} \rightarrow \mathrm{GL}(n, \mathbb{Z})$ up to conjugacy, and the group $h(\mathcal{G})$ is a finite subgroup of $\operatorname{GL}(n, \mathbb{Z})$ (see $\operatorname{Vos} 98$, page 57, Section 4.9])).

Let $K / k$ be a separable field extension of degree $n$ and $L / k$ be the Galois closure of $K / k$. Let $G=$ Gal $(L / k)$ and $H=\operatorname{Gal}(L / K)$. The Galois group $G$ may be regarded as a transitive subgroup of the symmetric group $S_{n}$ of degree $n$. Let $R_{K / k}^{(1)}\left(\mathbb{G}_{m}\right)$ be the norm one torus of $K / k$, i.e. the kernel of the norm map $R_{K / k}\left(\mathbb{G}_{m}\right) \rightarrow \mathbb{G}_{m}$ where $R_{K / k}$ is the Weil restriction (see Vos98, page 37, Section 3.12]). The norm one torus $R_{K / k}^{(1)}\left(\mathbb{G}_{m}\right)$ has the Chevalley module $J_{G / H}$ as its character module and the field $L\left(J_{G / H}\right)^{G}$ as its function field where $J_{G / H}=$ $\left(I_{G / H}\right)^{\circ}=\operatorname{Hom}_{\mathbb{Z}}\left(I_{G / H}, \mathbb{Z}\right)$ is the dual lattice of $I_{G / H}=\operatorname{Ker} \varepsilon$ and $\varepsilon: \mathbb{Z}[G / H] \rightarrow \mathbb{Z}$ is the augmentation map (see $\left[\right.$ Vos98, Section 4.8]). We have the exact sequence $0 \rightarrow \mathbb{Z} \rightarrow \mathbb{Z}[G / H] \rightarrow J_{G / H} \rightarrow 0$ and rank $J_{G / H}=n-1$. Write $J_{G / H}=\oplus_{1 \leq i \leq n-1} \mathbb{Z} x_{i}$. Then the action of $G$ on $L\left(J_{G / H}\right)=L\left(x_{1}, \ldots, x_{n-1}\right)$ is nothing but (11).

The aim of this paper is to give the stably rational classification of algebraic $k$-tori of dimensions 4 and 5 (cf. Vos98, [Kun07] and the references therein). It is easy to see that all the 1-dimensional algebraic $k$-tori $T$, i.e. the trivial torus $\mathbb{G}_{m}$ and the norm one torus $R_{L / k}^{(1)}\left(\mathbb{G}_{m}\right)$ with $[L: k]=2$, are $k$-rational.

Theorem 1.1 (Voskresenskii [Vos67]). All the 2-dimensional algebraic $k$-tori $T$ are $k$-rational. In particular, for any finite subgroups $G \leq \mathrm{GL}(2, \mathbb{Z}), L\left(x_{1}, x_{2}\right)^{G}$ is $k$-rational.

There are 13 non-conjugate finite subgroups of $\mathrm{GL}(2, \mathbb{Z})$. By Theorem 1.1 , we see that for decomposable 3-dimensional $k$-tori $T=T_{1} \times T_{2}$ with $\operatorname{dim} T_{1}=1$ and $\operatorname{dim} T_{2}=2$, the function fields $L(T)=L(M)^{G}$ are $k$-rational where $M=M_{1} \oplus M_{2}$ with rank $M_{1}=1$ and rank $M_{2}=2$.

Let $S_{n}$ (resp. $A_{n}, D_{n}, C_{n}$ ) be the symmetric (resp. the alternating, the dihedral, the cyclic) group of degree $n$ of order $n$ ! (resp. $n ! / 2,2 n, n)$. For $2 \leq n \leq 4$, the GAP ID $(n, i, j, k)$ of a finite subgroup $G$ of $\operatorname{GL}(n, \mathbb{Z})$ stands for the $k$-th $\mathbb{Z}$-class of the $j$-th $\mathbb{Q}$-class of the $i$-th crystal system of dimension $n$ as in [BBNWZ78, Table 1$]$ and GAP. There are $73 \mathbb{Z}$-classes forming $32 \mathbb{Q}$-classes which are classified into 7 crystal systems in $\mathrm{GL}(3, \mathbb{Z})$.

The birational classification of 3 -dimensional $k$-tori is given by Kunyavskii Kun90. 
Theorem 1.2 (Kunyavskii [Kun90]). Let $L / k$ be a Galois extension and $G \simeq \operatorname{Gal}(L / k)$ be a finite subgroup of $\mathrm{GL}(3, \mathbb{Z})$ which acts on $L\left(x_{1}, x_{2}, x_{3}\right)$ via (1). Then $L\left(x_{1}, x_{2}, x_{3}\right)^{G}$ is not $k$-rational if and only if $G$ is conjugate to one of the 15 groups which are given as in Table 1 . Moreover, if $L\left(x_{1}, x_{2}, x_{3}\right)^{G}$ is not $k$-rational, then it is not retract $k$-rational.

Table 1: $L(M)^{G}$ not retract $k$-rational, rank $M=3, M$ : indecomposable (15 cases)

\begin{tabular}{|c|c|c|c|c|c|c|c|c|}
\hline${ }^{t} G$ in Kun90 & GAP ID & $G$ & ${ }^{t} G$ in Kun90 & GAP ID & $G$ & ${ }^{t} G$ in Kun90 & GAP ID & $G$ \\
\hline$U_{1}$ & $(3,3,1,3)$ & $C_{2}^{2}$ & $U_{6}$ & $(3,4,7,2)$ & $D_{4} \times C_{2}$ & $U_{11}$ & $(3,7,5,3)$ & $S_{4} \times C_{2}$ \\
\hline$U_{2}$ & $(3,3,3,3)$ & $C_{2}^{3}$ & $U_{7}$ & $(3,7,2,2)$ & $A_{4} \times C_{2}$ & $U_{12}$ & $(3,7,5,2)$ & $S_{4} \times C_{2}$ \\
\hline$U_{3}$ & $(3,4,4,2)$ & $D_{4}$ & $U_{8}$ & $(3,7,3,3)$ & $S_{4}$ & $W_{1}$ & $(3,4,3,2)$ & $C_{4} \times C_{2}$ \\
\hline$U_{4}$ & $(3,4,6,3)$ & $D_{4}$ & $U_{9}$ & $(3,7,3,2)$ & $S_{4}$ & $W_{2}$ & $(3,3,3,4)$ & $C_{2}^{3}$ \\
\hline$U_{5}$ & $(3,7,1,2)$ & $A_{4}$ & $U_{10}$ & $(3,7,4,2)$ & $S_{4}$ & $W_{3}$ & $(3,7,2,3)$ & $A_{4} \times C_{2}$ \\
\hline
\end{tabular}

For the last statement of Theorem 1.2, see [Kan12, page 25, the fifth paragraph].

If we adopt the action of $G$ as in (1), we should take the transpose ${ }^{t} G$ of the matrix group $G$ as in [Kun90. (cf. Theorem 13.4 in Section 13). We will give an alternative proof of Theorem 1.2 using the algorithms of this paper (see Example 5.3). For $n=4$, some birational invariants are computed by Popov Pop98.

Let $T=R_{K / k}^{(1)}\left(\mathbb{G}_{m}\right)$ be the norm one torus defined by $K / k$. The rationality problem for norm one tori is investigated by [EM74, CTS77, Hür84, CTS87, LeB95, CK00, LL00, [Flo] and End11].

Theorem 1.3 (Endo and Miyata [EM74, Theorem 1.5], Saltman [Sal84a, Theorem 3.14]). Let K/k be a finite Galois field extension and $G=\operatorname{Gal}(K / k)$. The following conditions are equivalent:

(i) $R_{K / k}^{(1)}\left(\mathbb{G}_{m}\right)$ is retract $k$-rational;

(ii) all the Sylow subgroups of $G$ are cyclic.

Theorem 1.4 (Endo and Miyata [EM74, Theorem 2.3], Colliot-Thélène and Sansuc [CTS77, Proposition 3]). Let $K / k$ be a finite Galois field extension and $G=\operatorname{Gal}(K / k)$. The following conditions are equivalent:

(i) $R_{K / k}^{(1)}\left(\mathbb{G}_{m}\right)$ is stably k-rational;

(ii) all the Sylow subgroups of $G$ are cyclic and $H^{4}(G, \mathbb{Z}) \simeq \widehat{H}^{0}(G, \mathbb{Z})$ where $\widehat{H}$ is the Tate cohomology (see Section 2);

(iii) $G=C_{m}$ or $G=C_{n} \times\left\langle\sigma, \tau \mid \sigma^{k}=\tau^{2^{d}}=1, \tau \sigma \tau^{-1}=\sigma^{-1}\right\rangle$ where $d \geq 1, k \geq 3, n, k$ : odd, and $\operatorname{gcd}\{n, k\}=1$;

(iv) $G=\left\langle s, t \mid s^{m}=t^{2^{d}}=1, t s t^{-1}=s^{r}, m: o d d, r^{2} \equiv 1(\bmod m)\right\rangle$.

Theorem 1.5 (Endo End11, Theorem 2.1]). Let $K / k$ be a finite non-Galois, separable field extension and $L / k$ be the Galois closure of $K / k$. Assume that the Galois group of $L / k$ is nilpotent. Then the norm one torus $R_{K / k}^{(1)}\left(\mathbb{G}_{m}\right)$ is not retract $k$-rational.

Theorem 1.6 (Endo End11, Theorem 3.1]). Let $K / k$ be a finite non-Galois, separable field extension and $L / k$ be the Galois closure of $K / k$. Let $G=\operatorname{Gal}(L / k)$ and $H=\operatorname{Gal}(L / K) \leq G$. Assume that all the Sylow subgroups of $G$ are cyclic. Then the norm one torus $R_{K / k}^{(1)}\left(\mathbb{G}_{m}\right)$ is retract $k$-rational, and the following conditions are equivalent:

(i) $R_{K / k}^{(1)}\left(\mathbb{G}_{m}\right)$ is stably $k$-rational;

(ii) $G=D_{n}$ with $n$ odd $(n \geq 3)$ or $G=C_{m} \times D_{n}$ where $m, n$ are odd, $m, n \geq 3, \operatorname{gcd}\{m, n\}=1$, and $H \leq D_{n}$ is of order 2 ;

(iii) $H=C_{2}$ and $G \simeq C_{r} \rtimes H, r \geq 3$ odd, where $H$ acts non-trivially on $C_{r}$.

Theorem 1.7 (Colliot-Thélène and Sansuc [CTS87, Proposition 9.1], LeB95, Theorem 3.1], CK00, Proposition 0.2], [LL00, Endo [End11, Theorem 4.1], see also [End11, Remark 4.2 and Theorem 4.3]). Let $K / k$ be a nonGalois separable field extension of degree $n$ and $L / k$ be the Galois closure of $K / k$. Assume that $\operatorname{Gal}(L / k)=S_{n}$, $n \geq 3$, and $\operatorname{Gal}(L / K)=S_{n-1}$ is the stabilizer of one of the letters in $S_{n}$.

(i) $R_{K / k}^{(1)}\left(\mathbb{G}_{m}\right)$ is retract $k$-rational if and only if $n$ is a prime;

(ii) $R_{K / k}^{(1)}\left(\mathbb{G}_{m}\right)$ is (stably) $k$-rational if and only if $n=3$.

Let $\left[R_{K / k}^{(1)}\left(\mathbb{G}_{m}\right)\right]^{(t)}$ be the product of $t$ copies of the norm one torus $R_{K / k}^{(1)}\left(\mathbb{G}_{m}\right)$.

Theorem 1.8 (Endo End11, Theorem 4.4]). Let $K / k$ be a non-Galois separable field extension of degree $n$ and $L / k$ be the Galois closure of $K / k$. Assume that $\operatorname{Gal}(L / k)=A_{n}, n \geq 4$, and $\operatorname{Gal}(L / K)=A_{n-1}$ is the stabilizer of one of the letters in $A_{n}$. 
(i) $R_{K / k}^{(1)}\left(\mathbb{G}_{m}\right)$ is retract $k$-rational if and only if $n$ is a prime.

(ii) For some positive integer $t,\left[R_{K / k}^{(1)}\left(\mathbb{G}_{m}\right)\right]^{(t)}$ is stably $k$-rational if and only if $n=5$.

The first main result of this paper is the stably rational classification of algebraic $k$-tori of dimension 4 . There are $710 \mathbb{Z}$-classes forming $227 \mathbb{Q}$-classes which are classified into 33 crystal systems in $\mathrm{GL}(4, \mathbb{Z})$.

Let $F_{20} \simeq C_{5} \rtimes C_{4}$ be the Frobenius group of order 20 .

Theorem 1.9 (Stably rational classification of algebraic $k$-tori of dimension 4$)$. Let L/ $k$ be a Galois extension and $G \simeq \operatorname{Gal}(L / k)$ be a finite subgroup of $\mathrm{GL}(4, \mathbb{Z})$ which acts on $L\left(x_{1}, x_{2}, x_{3}, x_{4}\right)$ via (1).

(i) $L\left(x_{1}, x_{2}, x_{3}, x_{4}\right)^{G}$ is stably $k$-rational if and only if $G$ is conjugate to one of the 487 groups which are not in Tables 2,3 and 4 .

(ii) $L\left(x_{1}, x_{2}, x_{3}, x_{4}\right)^{G}$ is not stably but retract $k$-rational if and only if $G$ is conjugate to one of the 7 groups which are given as in Table 2 .

(iii) $L\left(x_{1}, x_{2}, x_{3}, x_{4}\right)^{G}$ is not retract $k$-rational if and only if $G$ is conjugate to one of the 216 groups which are given as in Tables 3 and 4 .

Table 2: $L(M)^{G}$ not stably but retract $k$-rational, rank $M=4, M$ : indecomposable (7 cases)

\begin{tabular}{|c|c|c|c|c|c|c|c|}
\hline GAP ID & $G$ & GAP ID & $G$ & GAP ID & $G$ & GAP ID & $G$ \\
\hline$(4,31,1,3)$ & $F_{20}$ & $(4,31,2,2)$ & $C_{2} \times F_{20}$ & $(4,31,5,2)$ & $S_{5}$ & $(4,33,2,1)$ & $C_{3} \rtimes C_{8}$ \\
\hline$(4,31,1,4)$ & $F_{20}$ & $(4,31,4,2)$ & $S_{5}$ & $(4,31,7,2)$ & $C_{2} \times S_{5}$ & & \\
\hline
\end{tabular}

Table 3: $L(M)^{G}$ not retract $k$-rational, $M=M_{1} \oplus M_{2}$, rank $M_{1}=3$, rank $M_{2}=1, M_{2}$ : indecomposable (64 cases)

\begin{tabular}{|c|c|c|c|c|c|c|c|}
\hline GAP ID & GAP ID & GAP ID & GAP ID & GAP ID & GAP ID & GAP ID & GAP ID \\
\hline$(4,4,3,6)$ & $(4,6,2,4)$ & $(4,7,7,2)$ & $(4,13,1,3)$ & $(4,13,7,8)$ & $(4,24,3,5)$ & $(4,25,3,2)$ & $(4,25,7,4)$ \\
\hline$(4,4,4,4)$ & $(4,6,2,8)$ & $(4,12,2,4)$ & $(4,13,2,4)$ & $(4,13,8,3)$ & $(4,24,4,3)$ & $(4,25,3,4)$ & $(4,25,8,2)$ \\
\hline$(4,4,4,6)$ & $(4,6,2,9)$ & $(4,12,3,7)$ & $(4,13,3,4)$ & $(4,13,8,4)$ & $(4,24,4,5)$ & $(4,25,4,4)$ & $(4,25,8,4)$ \\
\hline$(4,5,1,9)$ & $(4,6,3,3)$ & $(4,12,4,6)$ & $(4,13,4,3)$ & $(4,13,9,3)$ & $(4,24,5,3)$ & $(4,25,5,2)$ & $(4,25,9,4)$ \\
\hline$(4,5,2,4)$ & $(4,6,3,6)$ & $(4,12,4,8)$ & $(4,13,5,3)$ & $(4,13,10,3)$ & $(4,24,5,5)$ & $(4,25,5,4)$ & $(4,25,10,2)$ \\
\hline$(4,5,2,7)$ & $(4,7,3,2)$ & $(4,12,4,9)$ & $(4,13,6,3)$ & $(4,24,1,5)$ & $(4,25,1,2)$ & $(4,25,6,2)$ & $(4,25,10,4)$ \\
\hline$(4,6,1,4)$ & $(4,7,4,3)$ & $(4,12,5,6)$ & $(4,13,7,6)$ & $(4,24,2,3)$ & $(4,25,1,4)$ & $(4,25,6,4)$ & $(4,25,11,2)$ \\
\hline$(4,6,1,8)$ & $(4,7,5,2)$ & $(4,12,5,7)$ & $(4,13,7,7)$ & $(4,24,2,5)$ & $(4,25,2,4)$ & $(4,25,7,2)$ & $(4,25,11,4)$ \\
\hline
\end{tabular}

Table 4: $L(M)^{G}$ not retract $k$-rational, rank $M=4, M$ : indecomposable (152 cases)

\begin{tabular}{|c|c|c|c|c|c|c|c|c|}
\hline GAP ID & GAP ID & GAP ID & GAP ID & GAP ID & GAP ID & GAP ID & GAP ID & GAP ID \\
\hline$(4,5,1,12)$ & $(4,12,4,12)$ & $(4,13,9,4)$ & $(4,19,3,2)$ & $(4,24,2,4)$ & $(4,25,9,5)$ & $(4,29,8,1)$ & $(4,32,12,2)$ & $(4,33,1,1)$ \\
\hline$(4,5,2,5)$ & $(4,12,5,8)$ & $(4,13,9,5)$ & $(4,19,4,3)$ & $(4,24,2,6)$ & $(4,25,10,3)$ & $(4,29,8,2)$ & $(4,32,13,3)$ & $(4,33,3,1)$ \\
\hline$(4,5,2,8)$ & $(4,12,5,9)$ & $(4,13,10,4)$ & $(4,19,4,4)$ & $(4,24,4,4)$ & $(4,25,10,5)$ & $(4,29,9,1)$ & $(4,32,13,4)$ & $(4,33,4,1)$ \\
\hline$(4,5,2,9)$ & $(4,12,5,10)$ & $(4,13,10,5)$ & $(4,19,5,2)$ & $(4,24,5,4)$ & $(4,25,11,3)$ & $(4,32,1,2)$ & $(4,32,14,3)$ & $(4,33,5,1)$ \\
\hline$(4,6,1,6)$ & $(4,12,5,11)$ & $(4,18,1,3)$ & $(4,19,6,2)$ & $(4,24,5,6)$ & $(4,25,11,5)$ & $(4,32,2,2)$ & $(4,32,14,4)$ & $(4,33,6,1)$ \\
\hline$(4,6,1,11)$ & $(4,13,1,5)$ & $(4,18,2,4)$ & $(4,22,1,1)$ & $(4,25,1,3)$ & $(4,29,1,1)$ & $(4,32,3,2)$ & $(4,32,15,2)$ & $(4,33,7,1)$ \\
\hline$(4,6,2,6)$ & $(4,13,2,5)$ & $(4,18,2,5)$ & $(4,22,2,1)$ & $(4,25,2,3)$ & $(4,29,1,2)$ & $(4,32,4,2)$ & $(4,32,16,2)$ & $(4,33,8,1)$ \\
\hline$(4,6,2,10)$ & $(4,13,3,5)$ & $(4,18,3,5)$ & $(4,22,3,1)$ & $(4,25,2,5)$ & $(4,29,2,1)$ & $(4,32,5,2)$ & $(4,32,16,3)$ & $(4,33,9,1)$ \\
\hline$(4,6,3,4)$ & $(4,13,5,4)$ & $(4,18,3,7)$ & $(4,22,5,1)$ & $(4,25,4,3)$ & $(4,29,3,2)$ & $(4,32,6,2)$ & $(4,32,18,2)$ & $(4,33,11,1)$ \\
\hline$(4,6,3,7)$ & $(4,13,5,5)$ & $(4,18,4,4)$ & $(4,22,5,2)$ & $(4,25,5,3)$ & $(4,29,3,3)$ & $(4,32,7,2)$ & $(4,32,18,3)$ & $(4,33,12,1)$ \\
\hline$(4,6,3,8)$ & $(4,13,6,5)$ & $(4,18,4,5)$ & $(4,22,6,1)$ & $(4,25,5,5)$ & $(4,29,4,1)$ & $(4,32,8,2)$ & $(4,32,19,2)$ & $(4,33,13,1)$ \\
\hline$(4,12,2,5)$ & $(4,13,7,9)$ & $(4,18,5,5)$ & $(4,22,7,1)$ & $(4,25,6,3)$ & $(4,29,4,2)$ & $(4,32,9,4)$ & $(4,32,19,3)$ & $(4,33,14,1)$ \\
\hline$(4,12,2,6)$ & $(4,13,7,10)$ & $(4,18,5,6)$ & $(4,22,8,1)$ & $(4,25,6,5)$ & $(4,29,5,1)$ & $(4,32,9,5)$ & $(4,32,20,2)$ & $(4,33,14,2)$ \\
\hline$(4,12,3,11)$ & $(4,13,7,11)$ & $(4,18,5,7)$ & $(4,22,9,1)$ & $(4,25,7,3)$ & $(4,29,6,1)$ & $(4,32,10,2)$ & $(4,32,20,3)$ & $(4,33,15,1)$ \\
\hline$(4,12,4,10)$ & $(4,13,8,5)$ & $(4,19,1,2)$ & $(4,22,10,1)$ & $(4,25,8,3)$ & $(4,29,7,1)$ & $(4,32,11,2)$ & $(4,32,21,2)$ & $(4,33,16,1)$ \\
\hline$(4,12,4,11)$ & $(4,13,8,6)$ & $(4,19,2,2)$ & $(4,22,11,1)$ & $(4,25,9,3)$ & $(4,29,7,2)$ & $(4,32,11,3)$ & $(4,32,21,3)$ & \\
\hline
\end{tabular}

More detailed information of the stably rational classification of algebraic $k$-tori of dimension 4 is given as in Table 7. In Table 7, \# on the second column stands for the number of $\mathbb{Z}$-classes in each $\mathbb{Q}$-classes, and the list $[s, r, u]$ stands for the number $s$ (resp. $r, u$ ) of $\mathbb{Z}$-classes whose invariant field $L(M)^{G}$ is stably $k$-rational (resp. not stably but retract $k$-rational, not retract $k$-rational) in each $\mathbb{Q}$-classes $(4, i, j)$. For example, [11,0,2] in the GAP ID $(4,5,1)$ in Table 7 means that the 1 st $\mathbb{Q}$-class of the 5 th crystal system of dimension 4 consists of 13 $\mathbb{Z}$-classes and $L(M)^{G}$ is $k$-stably rational for $11 \mathbb{Z}$-classes of them, and is not retract $k$-rational for $2 \mathbb{Z}$-classes.

Let $G(n, i)$ be the $i$-th group of order $n$ in GAP GAP. Let $d T m$ be the $m$-th transitive subgroup of $S_{d}$ (cf. BM83] and GAP]).

By Theorem 1.9, we have the following theorem.

Theorem 1.10. Let $K / k$ be a separable field extension of degree 5 and $L / k$ be the Galois closure of $K / k$. Assume that $G=\operatorname{Gal}(L / k)$ is a transitive subgroup of $S_{5}$ which acts on $L\left(x_{1}, x_{2}, x_{3}, x_{4}\right)$ via (1), and $H=\mathrm{Gal}(L / K)$ is 
the stabilizer of one of the letters in $G$. Then the stably rational classification of norm one tori $R_{K / k}^{(1)}\left(\mathbb{G}_{m}\right)$ is given as in Table 5.

Table 5:

\begin{tabular}{lllll} 
& & & $\begin{array}{l}\text { GAP ID of the } \\
G \text {-action on } J_{G / H}\end{array}$ & $\begin{array}{l}L\left(J_{G / H}\right)^{G} \\
=L\left(x_{1}, x_{2}, x_{3}, x_{4}\right)^{G}\end{array}$ \\
\hline $5 T 1$ & $C_{5}$ & $G(5,1)$ & $(4,27,1,1)$ & stably $k$-rational \\
$5 T 2$ & $D_{5}$ & $G(10,1)$ & $(4,27,3,2)$ & stably $k$-rational \\
$5 T 3$ & $F_{20}$ & $G(20,3)$ & $(4,31,1,3)$ & not stably but retract $k$-rational \\
$5 T 4$ & $A_{5}$ & $G(60,5)$ & $(4,31,3,2)$ & stably $k$-rational \\
$5 T 5$ & $S_{5}$ & $G(120,34)$ & $(4,31,4,2)$ & not stably but retract $k$-rational
\end{tabular}

Theorem 1.10 is already known except for the case of $A_{5}$ (see Theorems [1.3, 1.4, 1.6, 1.7 and 1.8). Stably $k$ rationality of $R_{K / k}^{(1)}\left(\mathbb{G}_{m}\right)$ for the case $A_{5}$ is asked by S. Endo in [End11, Remark 4.6]. By Theorems 1.8 and 1.10, we get:

Corollary 1.11. Let $K / k$ be a non-Galois separable field extension of degree $n$ and $L / k$ be the Galois closure of $K / k$. Assume that $\operatorname{Gal}(L / k)=A_{n}, n \geq 4$, and $\operatorname{Gal}(L / K)=A_{n-1}$ is the stabilizer of one of the letters in $A_{n}$. Then $R_{K / k}^{(1)}\left(\mathbb{G}_{m}\right)$ is stably $k$-rational if and only if $n=5$.

For $n=5$, the CARAT ID $(n, i, j)$ of a finite subgroup $G$ of $\operatorname{GL}(5, \mathbb{Z})$ stands for the $j$-th $\mathbb{Z}$-class of the $i$-th $\mathbb{Q}$-class in $\operatorname{GL}(5, \mathbb{Z})$ in the CARAT package [Carat of GAP1 (see Section 3 for the details of the CARAT ID of dimension $n \leq 6)$. There are $6079 \mathbb{Z}$-classes forming $955 \mathbb{Q}$-classes in $\operatorname{GL}(5, \mathbb{Z})$.

The second main result of this paper gives the stably rational classification of algebraic $k$-tori of dimension 5 . We will display Tables 11 to 15 of Theorem 1.12 in Section 14.

Theorem 1.12 (Stably rational classification of algebraic $k$-tori of dimension 5 ). Let L/ $k$ be a Galois extension and $G \simeq \operatorname{Gal}(L / k)$ be a finite subgroup of $\mathrm{GL}(5, \mathbb{Z})$ which acts on $L\left(x_{1}, x_{2}, x_{3}, x_{4}, x_{5}\right)$ via (1).

(i) $L\left(x_{1}, x_{2}, x_{3}, x_{4}, x_{5}\right)^{G}$ is stably $k$-rational if and only if $G$ is conjugate to one of the 3051 groups which are not in Tables 11, 12, 13, 14 and 15 .

(ii) $L\left(x_{1}, x_{2}, x_{3}, x_{4}, x_{5}\right)^{G}$ is not stably but retract $k$-rational if and only if $G$ is conjugate to one of the 25 groups which are given as in Table 11.

(iii) $L\left(x_{1}, x_{2}, x_{3}, x_{4}, x_{5}\right)^{G}$ is not retract $k$-rational if and only if $G$ is conjugate to one of the 3003 groups which are given as in Tables 12, 13, 14 and 15 .

Remark 1.13. For the 25 groups $G$ as in Theorem 1.12 (ii), the corresponding $G$-lattices $M$ are decomposable $M \simeq M_{1} \oplus M_{2}$ where $M_{1}$ is a $G / N$-lattice of rank $4, N=\left\{\sigma \in G \mid \sigma(v)=v\right.$ for any $\left.v \in M_{1}\right\}$ and $G / N$ is one of the 7 groups as in Theorem 1.9 (ii) (Table 2) (see Example 4.12). In particular, if $M$ is an indecomposable $G$-lattice of rank 5 , then $L(M)^{G}$ is either stably $k$-rational or not retract $k$-rational.

More detailed information of the stably rational classification of algebraic $k$-tori of dimension 5 is given as in Table 16 (see also the explanation of Table 7 above).

Theorem 1.14. Let $K / k$ be a separable field extension of degree 6 and $L / k$ be the Galois closure of $K / k$. Assume that $G=\operatorname{Gal}(L / k)$ is a transitive subgroup of $S_{6}$ which acts on $L\left(x_{1}, x_{2}, x_{3}, x_{4}, x_{5}\right)$ via (1), and $H=\mathrm{Gal}(L / K)$ is the stabilizer of one of the letters in $G$. Then the stably rational classification of norm one tori $R_{K / k}^{(1)}\left(\mathbb{G}_{m}\right)$ is given as in Table 6.

\footnotetext{
${ }^{1}$ The generators and some information about the groups $G \leq \mathrm{GL}(5, \mathbb{Z})$ for the CARAT ID $(5, i, j)$ are available at the secondnamed author's web page http://www.math.h.kyoto-u.ac.jp/〜yamasaki/Algorithm/
} 
Table 6:

\begin{tabular}{lllll} 
& & & CARAT ID of the & $L\left(J_{G / H}\right)^{G}$ \\
$G$ & & $G(n, i)$ & $G$-action on $J_{G / H}$ & $=L\left(x_{1}, x_{2}, x_{3}, x_{4}, x_{5}\right)^{G}$ \\
\hline $6 T 1$ & $C_{6}$ & $G(6,2)$ & $(5,461,4)$ & stably $k$-rational \\
$6 T 2$ & $S_{3}$ & $G(6,1)$ & $(5,173,4)$ & stably $k$-rational \\
$6 T 3$ & $D_{6}$ & $G(12,4)$ & $(5,391,4)$ & stably $k$-rational \\
$6 T 4$ & $A_{4}$ & $G(12,3)$ & $(5,580,2)$ & not retract $k$-rational \\
$6 T 5$ & $C_{3} \times S_{3}$ & $G(18,3)$ & $(5,823,4)$ & not retract $k$-rational \\
$6 T 6$ & $C_{2} \times A_{4}$ & $G(24,13)$ & $(5,606,2)$ & not retract $k$-rational \\
$6 T 7$ & $S_{4}$ & $G(24,12)$ & $(5,607,2)$ & not retract $k$-rational \\
$6 T 8$ & $S_{4}$ & $G(24,12)$ & $(5,608,2)$ & not retract $k$-rational \\
$6 T 9$ & $S_{3}^{2}$ & $G(36,10)$ & $(5,855,6)$ & not retract $k$-rational \\
$6 T 10$ & $C_{3}^{2} \rtimes C_{4}$ & $G(36,9)$ & $(5,853,5)$ & not retract $k$-rational \\
$6 T 11$ & $C_{2} \times S_{4}$ & $G(48,48)$ & $(5,623,2)$ & not retract $k$-rational \\
$6 T 12$ & $A_{5}$ & $G(60,5)$ & $(5,952,1)$ & not retract $k$-rational \\
$6 T 13$ & $S_{3}^{2} \rtimes C_{2}$ & $G(72,40)$ & $(5,892,4)$ & not retract $k$-rational \\
$6 T 14$ & $S_{5}$ & $G(120,34)$ & $(5,947,1)$ & not retract $k$-rational \\
$6 T 15$ & $A_{6}$ & $G(360,118)$ & $(5,951,1)$ & not retract $k$-rational \\
$6 T 16$ & $S_{6}$ & $G(720,763)$ & $(5,953,1)$ & not retract $k$-rational
\end{tabular}

In Theorems 1.9, 1.10, 1.12 and 1.14, we do not know whether the field $L(M)^{G}$ is $k$-rational when the field is stably $k$-rational except for few cases (see [Vos98, Chapter 2]). 
Table 7: stably rational classification of algebraic $k$-tori of dimension 4

\begin{tabular}{|c|c|c|c|c|c|c|c|c|c|}
\hline GAP ID & $\#$ & {$[s, r, u]$} & $G(n, i)$ & & GAP ID & \# & {$[s, r, u]$} & $G(n, i)$ & \\
\hline$(4,1,1)$ & 1 & {$[1,0,0]$} & $G(1,1)$ & $\{1\}$ & $(4,14,8)$ & 6 & {$[6,0,0]$} & $G(12,4)$ & $D_{6}$ \\
\hline$(4,1,2)$ & 1 & {$[1,0,0]$} & $G(2,1)$ & $C_{2}$ & $(4,14,9)$ & 6 & {$[6,0,0]$} & $G(12,4)$ & $D_{6}$ \\
\hline$(4,2,1)$ & 2 & {$[2,0,0]$} & $G(2,1)$ & $C_{2}$ & $(4,14,10)$ & 6 & {$[6,0,0]$} & $G(24,14)$ & $C_{2}^{2} \times S_{3}$ \\
\hline$(4,2,2)$ & 2 & {$[2,0,0]$} & $G(2,1)$ & $C_{2}$ & $(4,15,1)$ & 2 & {$[2,0,0]$} & $G(12,5)$ & $C_{6} \times C_{2}$ \\
\hline$(4,2,3)$ & 2 & {$[2,0,0]$} & $G(4,2)$ & $C_{2}^{2}$ & $(4,15,2)$ & 2 & {$[2,0,0]$} & $G(12,5)$ & $C_{6} \times C_{2}$ \\
\hline$(4,3,1)$ & 3 & {$[3,0,0]$} & $G(2,1)$ & $C_{2}$ & $(4,15,3)$ & 2 & {$[2,0,0]$} & $G(12,5)$ & $C_{6} \times C_{2}$ \\
\hline$(4,3,2)$ & 3 & {$[3,0,0]$} & $G(4,2)$ & $C_{2}^{2}$ & $(4,15,4)$ & 2 & {$[2,0,0]$} & $G(12,4)$ & $D_{6}$ \\
\hline$(4,4,1)$ & 6 & {$[6,0,0]$} & $G(4,2)$ & $C_{2}^{2}$ & $(4,15,5)$ & 4 & {$[4,0,0]$} & $G(12,4)$ & $D_{6}$ \\
\hline$(4,4,2)$ & 7 & {$[7,0,0]$} & $G(4,2)$ & $C_{2}^{2}$ & $(4,15,6)$ & 2 & {$[2,0,0]$} & $G(24,14)$ & $C_{2}^{2} \times S_{3}$ \\
\hline$(4,4,3)$ & 6 & {$[5,0,1]$} & $G(4,2)$ & $C_{2}^{2}$ & $(4,15,7)$ & 4 & {$[4,0,0]$} & $G(24,14)$ & $C_{2}^{2} \times S_{3}$ \\
\hline$(4,4,4)$ & 6 & {$[4,0,2]$} & $G(8,5)$ & $C_{2}^{3}$ & $(4,15,8)$ & 2 & {$[2,0,0]$} & $G(24,15)$ & $C_{6} \times C_{2}^{2}$ \\
\hline$(4,5,1)$ & 13 & {$[11,0,2]$} & $G(4,2)$ & $C_{2}^{2}$ & $(4,15,9)$ & 2 & {$[2,0,0]$} & $G(24,14)$ & $C_{2}^{2} \times S_{3}$ \\
\hline$(4,5,2)$ & 9 & {$[4,0,5]$} & $G(8,5)$ & $C_{2}^{3}$ & $(4,15,10)$ & 4 & {$[4,0,0]$} & $G(24,14)$ & $C_{2}^{2} \times S_{3}$ \\
\hline$(4,6,1)$ & 12 & {$[8,0,4]$} & $G(8,5)$ & $C_{2}^{3}$ & $(4,15,11)$ & 2 & {$[2,0,0]$} & $G(24,14)$ & $C_{2}^{2} \times S_{3}$ \\
\hline$(4,6,2)$ & 12 & {$[6,0,6]$} & $G(8,5)$ & $C_{2}^{3}$ & $(4,15,12)$ & 2 & {$[2,0,0]$} & $G(48,51)$ & $C_{2}^{3} \times S_{3}$ \\
\hline$(4,6,3)$ & 8 & {$[3,0,5]$} & $G(16,14)$ & $C_{2}^{4}$ & $(4,16,1)$ & 3 & {$[3,0,0]$} & $G(8,3)$ & $D_{4}$ \\
\hline$(4,7,1)$ & 2 & {$[2,0,0]$} & $G(4,1)$ & $C_{4}$ & $(4,17,1)$ & 3 & {$[3,0,0]$} & $G(6,1)$ & $S_{3}$ \\
\hline$(4,7,2)$ & 2 & {$[2,0,0]$} & $G(4,1)$ & $C_{4}$ & $(4,17,2)$ & 2 & {$[2,0,0]$} & $G(12,4)$ & $D_{6}$ \\
\hline$(4,7,3)$ & 2 & {$[1,0,1]$} & $G(8,2)$ & $C_{4} \times C_{2}$ & $(4,18,1)$ & 3 & {$[2,0,1]$} & $G(8,2)$ & $C_{4} \times C_{2}$ \\
\hline$(4,7,4)$ & 4 & {$[3,0,1]$} & $G(8,3)$ & $D_{4}$ & $(4,18,2)$ & 5 & {$[3,0,2]$} & $G(16,3)$ & $\left(C_{4} \times C_{2}\right) \rtimes C_{2}$ \\
\hline$(4,7,5)$ & 2 & {$[1,0,1]$} & $G(8,3)$ & $D_{4}$ & $(4,18,3)$ & 7 & {$[4,0,3]$} & $G(16,3)$ & $\left(C_{4} \times C_{2}\right) \rtimes C_{2}$ \\
\hline$(4,7,6)$ & 2 & {$[2,0,0]$} & $G(8,3)$ & $D_{4}$ & $(4,18,4)$ & 5 & {$[3,0,2]$} & $G(16,11)$ & $C_{2} \times D_{4}$ \\
\hline$(4,7,7)$ & 2 & {$[1,0,1]$} & $G(16,11)$ & $C_{2} \times D_{4}$ & $(4,18,5)$ & 7 & {$[4,0,3]$} & $G(32,27)$ & $C_{2}^{4} \rtimes C_{2}$ \\
\hline$(4,8,1)$ & 2 & {$[2,0,0]$} & $G(3,1)$ & $C_{3}$ & $(4,19,1)$ & 2 & {$[1,0,1]$} & $G(16,4)$ & $C_{4} \rtimes C_{4}$ \\
\hline$(4,8,2)$ & 2 & {$[2,0,0]$} & $G(6,2)$ & $C_{6}$ & $(4,19,2)$ & 2 & {$[1,0,1]$} & $G(16,2)$ & $C_{4}^{2}$ \\
\hline$(4,8,3)$ & 3 & {$[3,0,0]$} & $G(6,1)$ & $S_{3}$ & $(4,19,3)$ & 2 & {$[1,0,1]$} & $G(32,25)$ & $C_{4} \times D_{4}$ \\
\hline$(4,8,4)$ & 3 & {$[3,0,0]$} & $G(6,1)$ & $S_{3}$ & $(4,19,4)$ & 4 & {$[2,0,2]$} & $G(32,28)$ & $\left(C_{4} \times C_{2}^{2}\right) \rtimes C_{2}$ \\
\hline$(4,8,5)$ & 3 & {$[3,0,0]$} & $G(12,4)$ & $D_{6}$ & $(4,19,5)$ & 2 & {$[1,0,1]$} & $G(32,34)$ & $C_{4}^{2} \rtimes C_{2}$ \\
\hline$(4,9,1)$ & 1 & {$[1,0,0]$} & $G(6,2)$ & $C_{6}$ & $(4,19,6)$ & 2 & {$[1,0,1]$} & $G(64,226)$ & $D_{4}^{2}$ \\
\hline$(4,9,2)$ & 1 & {$[1,0,0]$} & $G(6,2)$ & $C_{6}$ & $(4,20,1)$ & 1 & {$[1,0,0]$} & $G(12,2)$ & $C_{12}$ \\
\hline$(4,9,3)$ & 1 & {$[1,0,0]$} & $G(12,5)$ & $C_{6} \times C_{2}$ & $(4,20,2)$ & 1 & {$[1,0,0]$} & $G(12,2)$ & $C_{12}$ \\
\hline$(4,9,4)$ & 1 & {$[1,0,0]$} & $G(12,4)$ & $D_{6}$ & $(4,20,3)$ & 2 & {$[2,0,0]$} & $G(12,1)$ & $C_{3} \rtimes C_{4}$ \\
\hline$(4,9,5)$ & 1 & {$[1,0,0]$} & $G(12,4)$ & $D_{6}$ & $(4,20,4)$ & 2 & {$[2,0,0]$} & $G(24,5)$ & $C_{4} \times S_{3}$ \\
\hline$(4,9,6)$ & 2 & {$[2,0,0]$} & $G(12,4)$ & $D_{6}$ & $(4,20,5)$ & 1 & {$[1,0,0]$} & $G(24,9)$ & $C_{12} \times C_{2}$ \\
\hline$(4,9,7)$ & 1 & {$[1,0,0]$} & $G(24,14)$ & $C_{2}^{2} \times S_{3}$ & $(4,20,6)$ & 1 & {$[1,0,0]$} & $G(24,10)$ & $C_{3} \times D_{4}$ \\
\hline$(4,10,1)$ & 1 & {$[1,0,0]$} & $G(4,1)$ & $C_{4}$ & $(4,20,7)$ & 2 & {$[2,0,0]$} & $G(24,6)$ & $D_{12}$ \\
\hline$(4,11,1)$ & 1 & {$[1,0,0]$} & $G(3,1)$ & $C_{3}$ & $(4,20,8)$ & 1 & {$[1,0,0]$} & $G(24,10)$ & $C_{3} \times D_{4}$ \\
\hline$(4,11,2)$ & 1 & {$[1,0,0]$} & $G(6,2)$ & $C_{6}$ & $(4,20,9)$ & 2 & {$[2,0,0]$} & $G(24,5)$ & $C_{4} \times S_{3}$ \\
\hline$(4,12,1)$ & 7 & {$[7,0,0]$} & $G(4,1)$ & $C_{4}$ & $(4,20,10)$ & 2 & {$[2,0,0]$} & $G(24,10)$ & $C_{3} \times D_{4}$ \\
\hline$(4,12,2)$ & 6 & {$[3,0,3]$} & $G(8,2)$ & $C_{4} \times C_{2}$ & $(4,20,11)$ & 2 & {$[2,0,0]$} & $G(24,6)$ & $D_{12}$ \\
\hline$(4,12,3)$ & 13 & {$[11,0,2]$} & $G(8,3)$ & $D_{4}$ & $(4,20,12)$ & 4 & {$[4,0,0]$} & $G(24,8)$ & $\left(C_{6} \times C_{2}\right) \rtimes C_{2}$ \\
\hline$(4,12,4)$ & 13 & {$[7,0,6]$} & $G(8,3)$ & $D_{4}$ & $(4,20,13)$ & 4 & {$[4,0,0]$} & $G(24,8)$ & $\left(C_{6} \times C_{2}\right) \rtimes C_{2}$ \\
\hline$(4,12,5)$ & 11 & {$[5,0,6]$} & $G(16,11)$ & $C_{2} \times D_{4}$ & $(4,20,14)$ & 1 & {$[1,0,0]$} & $G(24,7)$ & $C_{2} \times\left(C_{3} \rtimes C_{4}\right)$ \\
\hline$(4,13,1)$ & 6 & {$[4,0,2]$} & $G(8,2)$ & $C_{4} \times C_{2}$ & $(4,20,15)$ & 1 & {$[1,0,0]$} & $G(48,35)$ & $C_{2} \times C_{4} \times S_{3}$ \\
\hline$(4,13,2)$ & 6 & {$[4,0,2]$} & $G(8,2)$ & $C_{4} \times C_{2}$ & $(4,20,16)$ & 2 & {$[2,0,0]$} & $G(48,38)$ & $D_{4} \times S_{3}$ \\
\hline$(4,13,3)$ & 6 & {$[4,0,2]$} & $G(8,3)$ & $D_{4}$ & $(4,20,17)$ & 2 & {$[2,0,0]$} & $G(48,38)$ & $D_{4} \times S_{3}$ \\
\hline$(4,13,4)$ & 6 & {$[4,0,2]$} & $G(8,3)$ & $D_{4}$ & $(4,20,18)$ & 1 & {$[1,0,0]$} & $G(48,45)$ & $C_{6} \times D_{4}$ \\
\hline$(4,13,5)$ & 5 & {$[2,0,3]$} & $G(16,10)$ & $C_{4} \times C_{2}^{2}$ & $(4,20,19)$ & 1 & {$[1,0,0]$} & $G(48,36)$ & $C_{2} \times D_{12}$ \\
\hline$(4,13,6)$ & 6 & {$[4,0,2]$} & $G(16,11)$ & $C_{2} \times D_{4}$ & $(4,20,20)$ & 4 & {$[4,0,0]$} & $G(48,38)$ & $D_{4} \times S_{3}$ \\
\hline$(4,13,7)$ & 12 & {$[6,0,6]$} & $G(16,11)$ & $C_{2} \times D_{4}$ & $(4,20,21)$ & 2 & {$[2,0,0]$} & $G(48,43)$ & $C_{2} \times\left(\left(C_{6} \times C_{2}\right) \rtimes C_{2}\right)$ \\
\hline$(4,13,8)$ & 6 & {$[2,0,4]$} & $G(16,11)$ & $C_{2} \times D_{4}$ & $(4,20,22)$ & 1 & {$[1,0,0]$} & $G(96,209)$ & $C_{2} \times S_{3} \times D_{4}$ \\
\hline$(4,13,9)$ & 5 & {$[2,0,3]$} & $G(16,11)$ & $C_{2} \times D_{4}$ & $(4,21,1)$ & 2 & {$[2,0,0]$} & $G(6,2)$ & $C_{6}$ \\
\hline$(4,13,10)$ & 5 & {$[2,0,3]$} & $G(32,46)$ & $C_{2}^{2} \times D_{4}$ & $(4,21,2)$ & 2 & {$[2,0,0]$} & $G(12,5)$ & $C_{6} \times C_{2}$ \\
\hline$(4,14,1)$ & 4 & {$[4,0,0]$} & $G(6,2)$ & $C_{6}$ & $(4,21,3)$ & 4 & {$[4,0,0]$} & $G(12,4)$ & $D_{6}$ \\
\hline$(4,14,2)$ & 4 & {$[4,0,0]$} & $G(6,2)$ & $C_{6}$ & $(4,21,4)$ & 2 & {$[2,0,0]$} & $G(24,14)$ & $C_{2}^{2} \times S_{3}$ \\
\hline$(4,14,3)$ & 8 & {$[8,0,0]$} & $G(6,1)$ & $S_{3}$ & $(4,22,1)$ & 2 & {$[1,0,1]$} & $G(9,2)$ & $C_{3}^{2}$ \\
\hline$(4,14,4)$ & 4 & {$[4,0,0]$} & $G(12,5)$ & $C_{6} \times C_{2}$ & $(4,22,2)$ & 2 & {$[1,0,1]$} & $G(18,5)$ & $C_{6} \times C_{3}$ \\
\hline$(4,14,5)$ & 4 & {$[4,0,0]$} & $G(12,4)$ & $D_{6}$ & $(4,22,3)$ & 3 & {$[2,0,1]$} & $G(18,3)$ & $C_{3} \times S_{3}$ \\
\hline$(4,14,6)$ & 6 & {$[6,0,0]$} & $G(12,4)$ & $D_{6}$ & $(4,22,4)$ & 3 & {$[2,0,1]$} & $G(18,3)$ & $C_{3} \times S_{3}$ \\
\hline$(4,14,7)$ & 6 & {$[6,0,0]$} & $G(12,4)$ & $D_{6}$ & $(4,22,5)$ & 5 & {$[3,0,2]$} & $G(18,4)$ & $C_{3}^{2} \rtimes C_{2}$ \\
\hline
\end{tabular}


Table 7 (continued): stably rational classification of algebraic $k$-tori of dimension 4

\begin{tabular}{|c|c|c|c|c|c|c|c|c|c|}
\hline GAP ID & $\#$ & {$[s, r, u]$} & $G(n, i)$ & & GAP ID & $\#$ & {$[s, r, u]$} & $G(n, i)$ & \\
\hline$(4,22,6)$ & 3 & {$[2,0,1]$} & $G(36,12)$ & $C_{6} \times S_{3}$ & $(4,30,5)$ & 1 & {$[1,0,0]$} & $G(36,6)$ & $C_{3} \times\left(C_{3} \rtimes C_{4}\right)$ \\
\hline$(4,22,7)$ & 3 & {$[2,0,1]$} & $G(36,13)$ & $C_{2} \times\left(C_{3}^{2} \rtimes C_{2}\right)$ & $(4,30,6)$ & 1 & {$[1,0,0]$} & $G(48,38)$ & $D_{4} \times S_{3}$ \\
\hline$(4,22,8)$ & 4 & {$[3,0,1]$} & $G(36,10)$ & $S_{3}^{2}$ & $(4,30,7)$ & 1 & {$[1,0,0]$} & $G(72,30)$ & $C_{3} \times\left(\left(C_{6} \times C_{2}\right) \rtimes C_{2}\right)$ \\
\hline$(4,22,9)$ & 5 & {$[4,0,1]$} & $G(36,10)$ & $S_{3}^{2}$ & $(4,30,8)$ & 1 & {$[1,0,0]$} & $G(72,23)$ & $\left(C_{6} \times S_{3}\right) \rtimes C_{2}$ \\
\hline$(4,22,10)$ & 4 & {$[3,0,1]$} & $G(36,10)$ & $S_{3}^{2}$ & $(4,30,9)$ & 1 & {$[1,0,0]$} & $G(72,21)$ & $\left(C_{3} \times\left(C_{3} \rtimes C_{4}\right)\right) \rtimes C_{2}$ \\
\hline$(4,22,11)$ & 4 & {$[3,0,1]$} & $G(72,46)$ & $C_{2} \times S_{3}^{2}$ & $(4,30,10)$ & 1 & {$[1,0,0]$} & $G(144,154)$ & $\left(C_{2} \times S_{3}^{2}\right) \rtimes C_{2}$ \\
\hline$(4,23,1)$ & 1 & {$[1,0,0]$} & $G(18,5)$ & $C_{6} \times C_{3}$ & $(4,30,11)$ & 1 & {$[1,0,0]$} & $G(144,136)$ & $\left(C_{2} \times\left(C_{3}^{2} \rtimes C_{4}\right)\right) \rtimes C_{2}$ \\
\hline$(4,23,2)$ & 1 & {$[1,0,0]$} & $G(36,14)$ & $C_{6}^{2}$ & $(4,30,12)$ & 2 & {$[2,0,0]$} & $G(144,115)$ & $\left(C_{2} \times\left(C_{3}^{2} \rtimes C_{4}\right)\right) \rtimes C_{2}$ \\
\hline$(4,23,3)$ & 1 & {$[1,0,0]$} & $G(36,12)$ & $C_{6} \times S_{3}$ & $(4,30,13)$ & 1 & {$[1,0,0]$} & $G(288,889)$ & $\left(C_{2}^{2} \times S_{3}^{2}\right) \rtimes C_{2}$ \\
\hline$(4,23,4)$ & 1 & {$[1,0,0]$} & $G(36,12)$ & $C_{6} \times S_{3}$ & $(4,31,1)$ & 4 & {$[2,2,0]$} & $G(20,3)$ & $C_{5} \rtimes C_{4}$ \\
\hline$(4,23,5)$ & 2 & {$[2,0,0]$} & $G(36,13)$ & $C_{2} \times\left(C_{3}^{2} \rtimes C_{2}\right)$ & $(4,31,2)$ & 2 & {$[1,1,0]$} & $G(40,12)$ & $C_{2} \times\left(C_{5} \rtimes C_{4}\right)$ \\
\hline$(4,23,6)$ & 2 & {$[2,0,0]$} & $G(36,12)$ & $C_{6} \times S_{3}$ & $(4,31,3)$ & 2 & {$[2,0,0]$} & $G(60,5)$ & $A_{5}$ \\
\hline$(4,23,7)$ & 1 & {$[1,0,0]$} & $G(72,48)$ & $C_{2} \times C_{6} \times S_{3}$ & $(4,31,4)$ & 2 & {$[1,1,0]$} & $G(120,34)$ & $S_{5}$ \\
\hline$(4,23,8)$ & 1 & {$[1,0,0]$} & $G(72,49)$ & $C_{2}^{2} \times\left(C_{3}^{2} \rtimes C_{2}\right)$ & $(4,31,5)$ & 2 & {$[1,1,0]$} & $G(120,34)$ & $S_{5}$ \\
\hline$(4,23,9)$ & 2 & {$[2,0,0]$} & $G(72,46)$ & $C_{2} \times S_{3}^{2}$ & $(4,31,6)$ & 2 & {$[2,0,0]$} & $G(120,35)$ & $C_{2} \times A_{5}$ \\
\hline$(4,23,10)$ & 2 & {$[2,0,0]$} & $G(72,46)$ & $C_{2} \times S_{3}^{2}$ & $(4,31,7)$ & 2 & {$[1,1,0]$} & $G(240,189)$ & $C_{2} \times S_{5}$ \\
\hline$(4,23,11)$ & 1 & {$[1,0,0]$} & $G(144,192)$ & $C_{2}^{2} \times \stackrel{S_{3}^{2}}{2}$ & $(4,32,1)$ & 2 & {$[1,0,1]$} & $G(8,4)$ & $Q_{8}$ \\
\hline$(4,24,1)$ & 6 & {$[5,0,1]$} & $G(12,3)$ & $A_{4}$ & $(4,32,2)$ & 2 & {$[1,0,1]$} & $G(16,6)$ & $C_{8} \rtimes C_{2}$ \\
\hline$(4,24,2)$ & 6 & {$[2,0,4]$} & $G(24,13)$ & $C_{2} \times A_{4}$ & $(4,32,3)$ & 2 & {$[1,0,1]$} & $G(16,8)$ & $Q D_{8}$ \\
\hline$(4,24,3)$ & 6 & {$[5,0,1]$} & $G(24,12)$ & $S_{4}$ & $(4,32,4)$ & 2 & {$[1,0,1]$} & $G(16,13)$ & $\left(C_{4} \times C_{2}\right) \rtimes C_{2}$ \\
\hline$(4,24,4)$ & 6 & {$[3,0,3]$} & $G(24,12)$ & $S_{4}$ & $(4,32,5)$ & 3 & {$[1,0,2]$} & $G(24,3)$ & $\mathrm{SL}(2,3)$ \\
\hline$(4,24,5)$ & 6 & {$[2,0,4]$} & $G(48,48)$ & $C_{2} \times S_{4}$ & $(4,32,6)$ & 2 & {$[1,0,1]$} & $G(32,43)$ & $\left(C_{2} \times D_{4}\right) \rtimes C_{2}$ \\
\hline$(4,25,1)$ & 5 & {$[2,0,3]$} & $G(24,13)$ & $C_{2} \times A_{4}$ & $(4,32,7)$ & 2 & {$[1,0,1]$} & $G(32,7)$ & $\left(C_{8} \rtimes C_{2}\right) \rtimes C_{2}$ \\
\hline$(4,25,2)$ & 5 & {$[2,0,3]$} & $G(24,13)$ & $C_{2} \times A_{4}$ & $(4,32,8)$ & 2 & {$[1,0,1]$} & $G(32,11)$ & $\left(C_{4} \times C_{4}\right) \rtimes C_{2}$ \\
\hline$(4,25,3)$ & 5 & {$[2,0,3]$} & $G(24,12)$ & $S_{4}$ & $(4,32,9)$ & 5 & {$[3,0,2]$} & $G(32,6)$ & $\left(\left(C_{4} \times C_{2}\right) \rtimes C_{2}\right) \rtimes C_{2}$ \\
\hline$(4,25,4)$ & 5 & {$[3,0,2]$} & $G(24,12)$ & $S_{4}$ & $(4,32,10)$ & 2 & {$[1,0,1]$} & $G(32,49)$ & $\left(C_{2} \times D_{4}\right) \rtimes C_{2}$ \\
\hline$(4,25,5)$ & 5 & {$[1,0,4]$} & $G(48,49)$ & $C_{2}^{2} \times A_{4}$ & $(4,32,11)$ & 3 & {$[1,0,2]$} & $G(48,29)$ & $\operatorname{GL}(2,3)$ \\
\hline$(4,25,6)$ & 5 & {$[1,0,4]$} & $G(48,48)$ & $C_{2} \times S_{4}$ & $(4,32,12)$ & 2 & {$[1,0,1]$} & $G(64,134)$ & $\left(\left(C_{2} \times D_{4}\right) \rtimes C_{2}\right) \rtimes C_{2}$ \\
\hline$(4,25,7)$ & 5 & {$[2,0,3]$} & $G(48,48)$ & $C_{2} \times S_{4}$ & $(4,32,13)$ & 4 & {$[2,0,2]$} & $G(64,32)$ & $\left(\left(C_{8} \rtimes C_{2}\right) \rtimes C_{2}\right) \rtimes C_{2}$ \\
\hline$(4,25,8)$ & 5 & {$[2,0,3]$} & $G(48,48)$ & $C_{2} \times S_{4}$ & $(4,32,14)$ & 4 & {$[2,0,2]$} & $G(64,138)$ & \\
\hline$(4,25,9)$ & 5 & {$[2,0,3]$} & $G(48,48)$ & $C_{2} \times S_{4}$ & $(4,32,15)$ & 2 & {$[1,0,1]$} & $G(64,34)$ & \\
\hline$(4,25,10)$ & 5 & {$[1,0,4]$} & $G(48,48)$ & $C_{2} \times S_{4}$ & $(4,32,16)$ & 3 & {$[1,0,2]$} & $G(96,204)$ & $\left(\left(C_{2} \times D_{4}\right) \rtimes C_{2}\right) \rtimes C_{3}$ \\
\hline$(4,25,11)$ & 5 & {$[1,0,4]$} & $G(96,226)$ & $C_{2}^{2} \times S_{4}$ & $(4,32,17)$ & 2 & {$[1,0,1]$} & $G(128,928)$ & $D_{4}^{2} \rtimes C_{2}$ \\
\hline$(4,26,1)$ & 1 & {$[1,0,0]$} & $G(8,1)$ & $C_{8}$ & $(4,32,18)$ & 3 & {$[1,0,2]$} & $G(192,201)$ & \\
\hline$(4,26,2)$ & 1 & {$[1,0,0]$} & $G(16,7)$ & $D_{8}$ & $(4,32,19)$ & 3 & {$[1,0,2]$} & $G(192,1493)$ & \\
\hline$(4,27,1)$ & 1 & {$[1,0,0]$} & $G(5,1)$ & $C_{5}$ & $(4,32,20)$ & 3 & {$[1,0,2]$} & $G(192,1494)$ & \\
\hline$(4,27,2)$ & 1 & {$[1,0,0]$} & $G(10,2)$ & $C_{10}$ & $(4,32,21)$ & 3 & {$[1,0,2]$} & $G(384,5602)$ & \\
\hline$(4,27,3)$ & 2 & {$[2,0,0]$} & $G(10,1)$ & $D_{5}$ & $(4,33,1)$ & 1 & {$[0,0,1]$} & $G(24,11)$ & $C_{3} \times Q_{8}$ \\
\hline$(4,27,4)$ & 1 & {$[1,0,0]$} & $G(20,4)$ & $D_{10}$ & $(4,33,2)$ & 1 & {$[0,1,0]$} & $G(24,1)$ & $C_{3} \rtimes C_{8}$ \\
\hline$(4,28,1)$ & 1 & {$[1,0,0]$} & $G(12,2)$ & $C_{12}$ & $(4,33,3)$ & 1 & {$[0,0,1]$} & $G(24,3)$ & $\mathrm{SL}(2,3)$ \\
\hline$(4,28,2)$ & 1 & {$[1,0,0]$} & $G(24,6)$ & $D_{12}$ & $(4,33,4)$ & 1 & {$[0,0,1]$} & $G(48,17)$ & $\left(C_{3} \times Q_{8}\right) \rtimes C_{2}$ \\
\hline$(4,29,1)$ & 3 & {$[1,0,2]$} & $G(18,3)$ & $C_{3} \times S_{3}$ & $(4,33,5)$ & 1 & {$[0,0,1]$} & $G(48,33)$ & $\mathrm{SL}(2,3) \rtimes C_{2}$ \\
\hline$(4,29,2)$ & 2 & {$[1,0,1]$} & $G(36,12)$ & $C_{6} \times S_{3}$ & $(4,33,6)$ & 1 & {$[0,0,1]$} & $G(48,29)$ & $\mathrm{GL}(2,3)$ \\
\hline$(4,29,3)$ & 5 & {$[2,0,3]$} & $G(36,10)$ & $S_{3}^{2}$ & $(4,33,7)$ & 1 & {$[0,0,1]$} & $G(72,25)$ & $C_{3} \times \operatorname{SL}(2,3)$ \\
\hline$(4,29,4)$ & 4 & {$[2,0,2]$} & $G(36,9)$ & $C_{3}^{2} \rtimes C_{4}$ & $(4,33,8)$ & 1 & {$[0,0,1]$} & $G(96,201)$ & $\left(\mathrm{SL}(2,3) \rtimes C_{2}\right) \rtimes C_{2}$ \\
\hline$(4,29,5)$ & 2 & {$[1,0,1]$} & $G(72,46)$ & $C_{2} \times S_{3}^{2}$ & $(4,33,9)$ & 1 & {$[0,0,1]$} & $G(96,193)$ & $\mathrm{GL}(2,3) \rtimes C_{2}$ \\
\hline$(4,29,6)$ & 3 & {$[2,0,1]$} & $G(72,45)$ & $C_{2} \times\left(C_{3}^{2} \rtimes C_{4}\right)$ & $(4,33,10)$ & 1 & {$[0,0,1]$} & $G(96,67)$ & $\mathrm{SL}(2,3) \rtimes C_{4}$ \\
\hline$(4,29,7)$ & 4 & {$[2,0,2]$} & $G(72,40)$ & $S_{3}^{2} \rtimes C_{2}$ & $(4,33,11)$ & 1 & {$[0,0,1]$} & $G(144,125)$ & $\left(C_{3} \times \mathrm{SL}(2,3)\right) \rtimes C_{2}$ \\
\hline$(4,29,8)$ & 4 & {$[2,0,2]$} & $G(72,40)$ & $S_{3}^{2} \rtimes C_{2}$ & $(4,33,12)$ & 1 & {$[0,0,1]$} & $G(192,988)$ & $\left(\mathrm{GL}(2,3) \rtimes C_{2}\right) \rtimes C_{2}$ \\
\hline$(4,29,9)$ & 3 & {$[2,0,1]$} & $G(144,186)$ & $C_{2} \times\left(S_{3}^{2} \rtimes C_{2}\right)$ & $(4,33,13)$ & 1 & {$[0,0,1]$} & $G(288,860)$ & \\
\hline$(4,30,1)$ & 1 & {$[1,0,0]$} & $G(12,1)$ & $C_{3} \rtimes C_{4}$ & $(4,33,14)$ & 2 & {$[0,0,2]$} & $G(576,8277)$ & \\
\hline$(4,30,2)$ & 1 & {$[1,0,0]$} & $G(24,5)$ & $C_{4} \times S_{3}$ & $(4,33,15)$ & 1 & {$[0,0,1]$} & $G(576,8282)$ & \\
\hline$(4,30,3)$ & 1 & {$[1,0,0]$} & $G(24,10)$ & $C_{3} \times D_{4}$ & $(4,33,16)$ & 1 & {$[0,0,1]$} & $G(1152,1574$ & \\
\hline$(4,30,4)$ & 2 & {$[2,0,0]$} & $G(24,8)$ & $\left(C_{6} \times C_{2}\right) \rtimes C_{2}$ & & & & & \\
\hline
\end{tabular}


Let $M$ be a $G$-lattice. $M$ is called a permutation $G$-lattice if $M$ has a $\mathbb{Z}$-basis permuted by $G$, i.e. $M \simeq$ $\oplus_{1 \leq i \leq m} \mathbb{Z}\left[G / H_{i}\right]$ for some subgroups $H_{1}, \ldots, H_{m}$ of $G$. $M$ is called a stably permutation $G$-lattice if $M \oplus P \simeq P \prime$ for some permutation $G$-lattices $P$ and $P^{\prime} . M$ is called invertible if it is a direct summand of a permutation $G$-lattice, i.e. $P \simeq M \oplus M^{\prime}$ for some permutation $G$-lattice $P$ and a $G$-lattice $M^{\prime}$. $M$ is called coflabby if $H^{1}(H, M)=0$ for any subgroup $H$ of $G$. $M$ is called flabby if $\widehat{H}^{-1}(H, M)=0$ for any subgroup $H$ of $G$ where $\widehat{H}$ is the Tate cohomology (see Section 2 ).

Definition 1.15 (see [EM74, Section 1], [Vos98, Section 4.7]). Let $\mathcal{C}(G)$ be the category of all $G$-lattices. Let $\mathcal{S}(G)$ be the full subcategory of $\mathcal{C}(G)$ of all permutation $G$-lattices and $\mathcal{D}(G)$ be the full subcategory of $\mathcal{C}(G)$ of all invertible $G$-lattices. Let

$$
\mathcal{H}^{i}(G)=\left\{M \in \mathcal{C}(G) \mid \widehat{H}^{i}(H, M)=0 \text { for any } H \leq G\right\}(i= \pm 1)
$$

be the class of " $\widehat{H}^{i}$-vanish" $G$-lattices where $\widehat{H}^{i}$ is the Tate cohomology (see Section 2). Then one has the inclusions $\mathcal{S}(G) \subset \mathcal{D}(G) \subset \mathcal{H}^{i}(G) \subset \mathcal{C}(G)(i= \pm 1)$.

Definition 1.16 (The commutative monoid $\mathcal{C}(G) / \mathcal{S}(G)$ ). We say that two $G$-lattices $M_{1}$ and $M_{2}$ are similar if there exist permutation $G$-lattices $P_{1}$ and $P_{2}$ such that $M_{1} \oplus P_{1} \simeq M_{2} \oplus P_{2}$. We denote the similarity class of $M$ by $[M]$. The set of similarity classes $\mathcal{C}(G) / \mathcal{S}(G)$ becomes a commutative monoid (with respect to the sum $\left[M_{1}\right]+\left[M_{2}\right]:=\left[M_{1} \oplus M_{2}\right]$ and the zero $0=[P]$ where $\left.P \in \mathcal{S}(G)\right)$.

For a $G$-lattice $M$, there exists a short exact sequence of $G$-lattices $0 \rightarrow M \rightarrow P \rightarrow F \rightarrow 0$ where $P$ is permutation and $F$ is flabby which is called a flasque resolution of $M$ (see Theorem 2.12). The similarity class $[F] \in \mathcal{C}(G) / \mathcal{S}(G)$ of $F$ is determined uniquely and is called the flabby class of $M$. We denote the flabby class $[F]$ of $M$ by $[M]^{f l}$. We say that $[M]^{f l}$ is invertible if $[M]^{f l}=[E]$ for some invertible $G$-lattice $E$ (see Definition 2.13 .

The flabby class $[M]^{f l}$ plays crucial role in the rationality problem for $L(M)^{G}$ as follows (see Voskresenskii's fundamental book [Vos98, Section 4.6], see also e.g. Swan [Swa83, Kunyavskii Kun90, Section 2], Lemire, Popov and Reichstein [LPR06, Section 2], Kang [Kan12], Yamasaki [Yam12]):

Theorem 1.17 (Endo and Miyata, Voskresenskii, Saltman). Let L/k be a finite Galois extension with Galois group $G=\operatorname{Gal}(L / k)$ and $M$ and $M^{\prime}$ be $G$-lattices.

(i) (Endo and Miyata [EM73, Theorem 1.6]) $[M]^{f l}=0$ if and only if $L(M)^{G}$ is stably k-rational.

(ii) (Voskresenskii Vos74, Theorem 2]) $[M]^{f l}=\left[M^{\prime}\right]^{f l}$ if and only if $L(M)^{G}$ and $L\left(M^{\prime}\right)^{G}$ are stably $k$-isomorphic, i.e. there exist algebraically independent elements $x_{1}, \ldots, x_{m}$ over $L(M)^{G}$ and $y_{1}, \ldots, y_{n}$ over $L\left(M^{\prime}\right)^{G}$ such that $L(M)^{G}\left(x_{1}, \ldots, x_{m}\right) \simeq L\left(M^{\prime}\right)^{G}\left(y_{1}, \ldots, y_{n}\right)$.

(iii) (Saltman Sal84a, Theorem 3.14]) $[M]^{f l}$ is invertible if and only if $L(M)^{G}$ is retract $k$-rational.

Theorem 1.18 (Colliot-Thélène and Sansuc [CTS77, Corollaire 1]). Let $G$ be a finite group. The following conditions are equivalent:

(i) $\left[J_{G}\right]^{f l}$ is coflabby;

(ii) any Sylow subgroup of $G$ is cyclic or generalized quaternion $Q_{4 n}$ of order $4 n(n \geq 2)$;

(iii) any abelian subgroup of $G$ is cyclic;

(iv) $H^{3}(H, \mathbb{Z})=0$ for any subgroup $H$ of $G$.

Remark 1.19. (1) It is known that each of the conditions (i)-(iv) of Theorem 1.18 is equivalent to the condition that $G$ has periodic cohomology, i.e. there exists $q \neq 0$ and $u \in \widehat{H}^{q}(G, \mathbb{Z})$ such that the cup product map $u \cup-: \widehat{H}^{n}(G, \mathbb{Z}) \rightarrow \widehat{H}^{n+q}(G, \mathbb{Z})$ is an isomorphism for any $n \in \mathbb{Z}$ (see [CE56. Theorem 11.6]).

(2) $H^{3}(H, \mathbb{Z}) \simeq H^{1}\left(H,\left[J_{G}\right]^{f l}\right)$ for any subgroup $H$ of $G$ (see [Vos70, Theorem 7] and [CTS77, Proposition 1]).

Theorem 1.20 (Endo and Miyata [EM82, Theorem 2.1]). Let $G$ be a finite group. The following conditions are equivalent:

(i) $\mathcal{H}^{1}(G) \cap \mathcal{H}^{-1}(G)=\mathcal{D}(G)$, i.e. any flabby and coflabby $G$-lattice is invertible;

(ii) $\left[J_{G} \otimes_{\mathbb{Z}} J_{G}\right]^{f l}=\left[\left[J_{G}\right]^{f l}\right]^{f l}$ is invertible;

(iii) any $p$-Sylow subgroup of $G$ is cyclic for odd $p$ and cyclic or dihedral (including Klein's four group) for $p=2$.

Note that $\left[J_{G}\right]^{f l}=\left[J_{G} \otimes_{\mathbb{Z}} J_{G}\right]$ (see $[$ EM82, Section 2]). 
For $G$-lattice $M$, it is not difficult to see

$$
\begin{array}{ccc}
\text { permutation } \Rightarrow \text { stably permutation } & \Rightarrow \text { invertible } \Rightarrow \text { flabby and coflabby } \\
\Downarrow & \Downarrow \\
{[M]^{f l}=0} & \Rightarrow & {[M]^{f l} \text { is invertible. }}
\end{array}
$$

The above implications in each step cannot be reversed. Swan Swa60 gave an example of $Q_{8}$-lattice $M$ of rank 8 which is not permutation but stably permutation: $M \oplus \mathbb{Z} \simeq \mathbb{Z}\left[Q_{8}\right] \oplus \mathbb{Z}$. This also indicates that the direct sum cancellation fails (see also Theorem 4.6). Colliot-Thélène and Sansuc [CTS77, Remarque R1] and CTS77, Remarque R4] gave examples of $S_{3}$-lattice $M$ of rank 4 which is not permutation but stably permutation: $M \oplus \mathbb{Z} \simeq \mathbb{Z}\left[S_{3} /\langle\sigma\rangle\right] \oplus \mathbb{Z}\left[S_{3} /\langle\tau\rangle\right]$ where $S_{3}=\langle\sigma, \tau\rangle$ (see also Table 8 of Theorem[6.3) and of $F_{20}$-lattice $\left[J_{F_{20} / C_{4}}\right]^{f l}$ of the Chevalley module $J_{F_{20} / C_{4}}$ of rank 4 which is not stably permutation but invertible (see also Theorem 1.23 and Theorem 1.27(ii), (iv) and (v)). By Theorems 1.3, 1.17(ii) and 1.18, the flabby class $\left[J_{Q_{8}}\right]^{f l}$ of the Chevalley module $J_{Q_{8}}$ of rank 7 is not invertible but flabby and coflabby (we may take [ $\left.J_{Q_{8}}\right]^{f l}$ of rank 9, see Example 8.3). The inverse direction of the vertical implication holds if $M$ is coflabby (see Lemma 2.11).

By using the interpretation as in Theorem 1.17. Theorems 1.3, 1.4, 1.6, 1.7 and 1.8 may be obtained by the following theorems:

Theorem 1.21 (Endo and Miyata [EM74, Theorem 1.5]). Let $G$ be a finite group. The following conditions are equivalent:

(i) $\left[J_{G}\right]^{f l}$ is invertible;

(ii) all the Sylow subgroups of $G$ are cyclic;

(iii) $\mathcal{H}^{-1}(G)=\mathcal{H}^{1}(G)=\mathcal{D}(G)$, i.e. any flabby (resp. coflabby) G-lattice is invertible.

Theorem 1.22 (Endo and Miyata EM74, Theorem 2.3], see also [CTS77, Proposition 3]). Let G be a finite group. The following conditions are equivalent:

(i) $\left[J_{G}\right]^{f l}=0$;

(ii) $\left[J_{G}\right]^{\text {fl }}$ is of finite order in $\mathcal{C}(G) / \mathcal{S}(G)$;

(iii) all the Sylow subgroups of $G$ are cyclic and $H^{4}(G, \mathbb{Z}) \simeq \widehat{H}^{0}(G, \mathbb{Z})$;

(iv) $G=C_{m}$ or $G=C_{n} \times\left\langle\sigma, \tau \mid \sigma^{k}=\tau^{2^{d}}=1, \tau \sigma \tau^{-1}=\sigma^{-1}\right\rangle$ where $d \geq 1, k \geq 3, n, k$ : odd, and $\operatorname{gcd}\{n, k\}=1$;

(v) $G=\left\langle s, t \mid s^{m}=t^{2^{d}}=1, t s t^{-1}=s^{r}, m: o d d, r^{2} \equiv 1(\bmod m)\right\rangle$.

Theorem 1.23 (Endo End11, Theorem 3.1]). Let $G$ be a non-abelian group whose Sylow subgroups are all cyclic. Let $H$ be a non-normal subgroup of $G$ which contains no normal subgroup of $G$ except $\{1\}$. Then $\left[J_{G / H}\right]^{\text {fl }}$ is invertible, and the following conditions are equivalent:

(i) $\left[J_{G / H}\right]^{f l}=0$;

(ii) $\left[J_{G / H}\right]^{\text {fl }}$ is of finite order in $\mathcal{C}(G) / \mathcal{S}(G)$;

(iii) $G=D_{n}$ with $n$ odd $(n \geq 3)$ or $G=C_{m} \times D_{n}$ where $m, n$ are odd, $m, n \geq 3$, $\operatorname{gcd}\{m, n\}=1$, and $H \leq D_{n}$ is of order 2 ;

(iv) $H=C_{2}$ and $G \simeq C_{r} \rtimes H, r \geq 3$ odd, where $H$ acts non-trivially on $C_{r}$.

A partial result of Theorem 1.23 was given by Colliot-Thélène and Sansuc [CTS77, Remarque R4].

Theorem 1.24 (Colliot-Thélène and Sansuc [CTS87, Proposition 9.1], [LeB95, Theorem 3.1], [CK00, Proposition 0.2], [LL00], Endo [End11, Theorem 4.1], see also [End11, Remark 4.2 and Theorem 4.3]). Let $n \geq 2$ be an integer.

(i) $\left[J_{S_{n} / S_{n-1}}\right]^{f l}$ is invertible if and only if $n$ is a prime.

(ii) $\left[J_{S_{n} / S_{n-1}}\right]^{f l}=0$ if and only if $n=2,3$.

(iii) $\left[J_{S_{n} / S_{n-1}}\right]^{f l}$ is of finite order in $\mathcal{C}(G) / \mathcal{S}(G)$ if and only if $n=2,3$.

Theorem 1.25 (Endo End11, Theorem 4.5]). Let $n \geq 3$ be an integer.

(i) $\left[J_{A_{n} / A_{n-1}}\right]^{f l}$ is invertible if and only if $n$ is a prime.

(ii) $\left[J_{A_{n} / A_{n-1}}\right]^{f l}$ is of finite order in $\mathcal{C}(G) / \mathcal{S}(G)$ if and only if $n=3,5$.

Note that $\left[J_{A_{3} / A_{2}}\right]^{f l}=\left[J_{C_{3}}\right]^{f l}=0$. By Dre75, Corollary 3.3], $\left[J_{A_{5} / A_{4}}\right]^{f l}$ is of finite order in $\mathcal{C}(G) / \mathcal{S}(G)$. Indeed, we get $\left[J_{A_{5} / A_{4}}\right]^{f l}=0$ (see Theorems 1.9 and 1.10, Table 5, Corollary 1.11 and Theorem 1.27 (i) below).

Definition 1.26 (The $G$-lattice $M_{G}$ of a finite subgroup $G$ of $\operatorname{GL}(n, \mathbb{Z})$ ). Let $G$ be a finite subgroup of $\mathrm{GL}(n, \mathbb{Z})$. The $G$-lattice $M_{G}$ of rank $n$ is defined to be the $G$-lattice with a $\mathbb{Z}$-basis $\left\{u_{1}, \ldots, u_{n}\right\}$ on which $G$ acts by $\sigma\left(u_{i}\right)=\sum_{j=1}^{n} a_{i, j} u_{j}$ for any $\sigma=\left[a_{i, j}\right] \in G$. 
The stably rational classification of $k$-tori of dimensions 4 and 5 (Theorem 1.9 and Theorem 1.12 ) may be obtained by the following two theorems respectively.

Theorem 1.27. Let $G$ be a finite subgroup of $\mathrm{GL}(4, \mathbb{Z})$ and $M_{G}$ be the $G$-lattice of rank 4 as in Definition 1.26 . (i) $\left[M_{G}\right]^{f l}=0$ if and only if $G$ is conjugate to one of the 487 groups which are not in Tables 2,3 and 4 .

(ii) $\left[M_{G}\right]^{f l}$ is not zero but invertible if and only if $G$ is conjugate to one of the 7 groups which are given as in Table 2 .

(iii) $\left[M_{G}\right]^{f l}$ is not invertible if and only if $G$ is conjugate to one of the 216 groups which are given as in Tables 3 and 4 .

(iv) $\left[M_{G}\right]^{f l}=0$ if and only if $\left[M_{G}\right]^{f l}$ is of finite order in $\mathcal{C}(G) / \mathcal{S}(G)$.

(v) For the group $G \simeq S_{5}$ of the GAP ID $(4,31,5,2)$ in (ii), we have

$$
-\left[M_{G}\right]^{f l}=\left[J_{S_{5} / S_{4}}\right]^{f l} \neq 0
$$

(vi) For the group $G \simeq F_{20}$ of the $\operatorname{GAP} \operatorname{ID}(4,31,1,4)$ in (ii), we have

$$
-\left[M_{G}\right]^{f l}=\left[J_{F_{20} / C_{4}}\right]^{f l} \neq 0 .
$$

Theorem 1.28. Let $G$ be a finite subgroup of $\mathrm{GL}(5, \mathbb{Z})$ and $M_{G}$ be the G-lattice of rank 5 as in Definition 1.26 . (i) $\left[M_{G}\right]^{f l}=0$ if and only if $G$ is conjugate to one of the 3051 groups which are not in Tables $11,12,13,14$ and 15.

(ii) $\left[M_{G}\right]^{f l}$ is not zero but invertible if and only if $G$ is conjugate to one of the 25 groups which are given as in Table 11.

(iii) $\left[M_{G}\right]^{f l}$ is not invertible if and only if $G$ is conjugate to one of the 3003 groups which are given as in Tables $12,13,14$ and 15 .

(iv) $\left[M_{G}\right]^{f l}=0$ if and only if $\left[M_{G}\right]^{f l}$ is of finite order in $\mathcal{C}(G) / \mathcal{S}(G)$.

Remark 1.29. (i) By the interpretation as in Theorem 1.17 Theorem1.27(v), (vi) claims that the corresponding two tori $T$ and $T^{\prime}$ of dimension 4 are not stably $k$-rational and are not stably $k$-isomorphic but the torus $T \times T^{\prime}$ of dimension 8 is stably $k$-rational.

(ii) When $[M]^{f l}$ is invertible, the inverse element of $[M]^{f l}$ is given by $-[M]^{f l}=\left[[M]^{f l}\right]^{f l}$ (see Lemma 2.15$)$. Hence Theorem $1.27(\mathrm{v})$ also claims that $\left[\left[M_{G}\right]^{f l}\right]^{f l}=\left[J_{S_{5} / S_{4}}\right]^{f l}$ and $\left[\left[J_{S_{5} / S_{4}}\right]^{f l}\right]^{f l}=\left[M_{G}\right]^{f l}$.

We will give proofs of Theorem 1.27 and Theorem 1.28 in Section 10] and Section [1] respectively.

By using the algorithms in Section [5, we show the following theorem:

Theorem (Theorem 6.2 and Theorem 6.3). Let $G$ be a finite subgroup of $\mathrm{GL}(n, \mathbb{Z})$ and $M_{G}$ be the G-lattice of rank $n$ as in Definition 1.26 .

(i) When $n \leq 3, M_{G}$ is flabby and coflabby if and only if $M_{G}$ is permutation.

(ii) When $n=4, M_{G}$ is flabby and coflabby if and only if $M_{G}$ is permutation or the GAP ID of $G$ is one of $(4,14,2,2),(4,14,3,3),(4,14,3,4),(4,14,8,2)$.

(There are 11 conjugacy classes of subgroups of $S_{4}$ and hence 15 flabby and coflabby G-lattices of rank 4 in total.) (iii) When $n=5, M_{G}$ is flabby and coflabby if and only if $M_{G}$ is permutation or the CARAT ID of $G$ is one of $(5,218,4),(5,911,4),(5,918,4),(5,931,4)$.

(There are 19 conjugacy classes of subgroups of $S_{5}$ and hence 23 flabby and coflabby $G$-lattices of rank 5 in total.) (iv) When $n=6, M_{G}$ is flabby and coflabby if and only if $M_{G}$ is permutation or the CARAT ID of $G$ is one of the 50 triples

$\begin{array}{lllll}(6,159,14), & (6,161,14), & (6,161,28), & (6,197,14), & (6,226,14), \\ (6,226,40), & (6,231,39), & (6,238,27), & (6,246,21), & (6,366,27), \\ (6,1087,20), & (6,1090,18), & (6,1142,8), & (6,2043,4), & (6,2051,9), \\ (6,2068,6), & (6,2069,6), & (6,2069,12), & (6,2070,12), & (6,2079,14), \\ (6,2079,28), & (6,2088,18), & (6,2105,12), & (6,2154,26), & (6,2156,40), \\ (6,2156,80), & (6,2188,39), & (6,2968,4), & (6,2969,4), & (6,2969,8), \\ (6,2977,6), & (6,3068,7), & (6,3068,8), & (6,3071,7), & (6,3071,8), \\ (6,3073,7), & (6,3073,8), & (6,3073,15), & (6,3073,16), & (6,3076,7), \\ (6,3076,8), & (6,3091,11), & (6,3091,12), & (6,5210,14), & (6,5262,11), \\ (6,5321,6), & (6,5421,6), & (6,5475,6), & (6,5477,11), & (6,5487,11) .\end{array}$


(There are 56 conjugacy classes of subgroups of $S_{6}$ and hence 106 flabby and coflabby G-lattices of rank 6 in total.)

(v) When $n \leq 6, M$ is flabby and coflabby if and only if $M$ is stably permutation. Indeed, flabby and coflabby G-lattices $M$ which are not permutation in (ii), (iii), (iv) are stably permutation as in Table 8.

Definition 1.30 (Decomposition type). Let $G$ be a finite group and $M$ be a $G$-lattice. A $G$-lattice $M$ is said to be decomposable if there exist non-trivial $G$-lattices $U_{1}$ and $U_{2}$ such that $M \simeq U_{1} \oplus U_{2}$. A $G$-lattice is said to be indecomposable if it is not decomposable. When $M$ decomposes into indecomposable $G$-lattices $M \simeq U_{1} \oplus \cdots \oplus U_{r}$ of rank $n_{1}, \ldots, n_{r}$, we say that a decomposition type $\mathrm{DT}(M)$ of $M$ is $\left(n_{1}, \ldots, n_{r}\right)$.

For $n \leq 6$, the number of $G$-lattices $M_{G}$ of rank $n$ for a given decomposition type $\mathrm{DT}\left(M_{G}\right)$ is as follows:

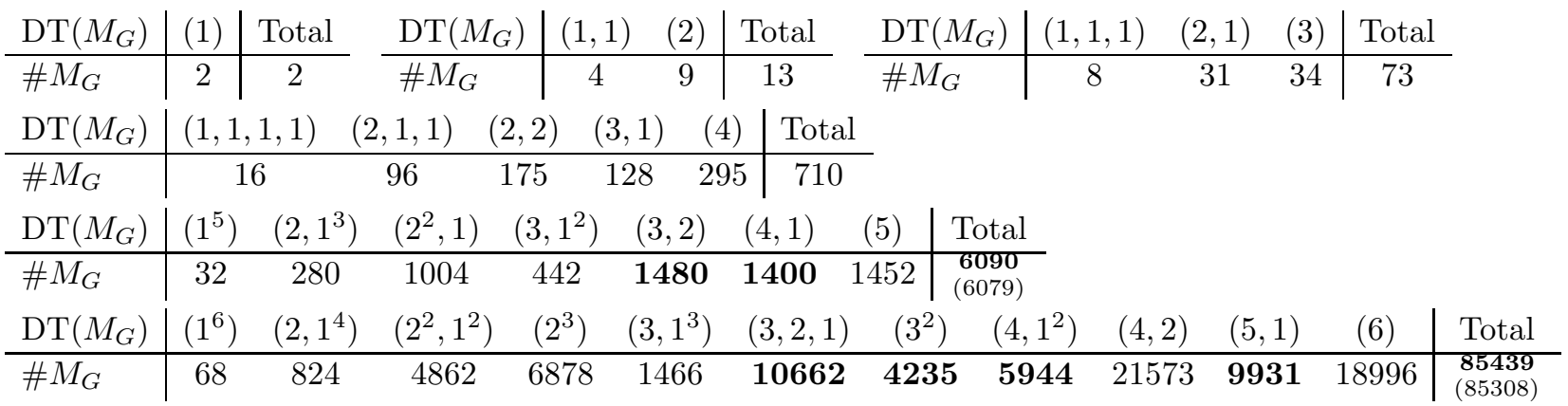

Let $G$ be a finite subgroup of $\mathrm{GL}(n, \mathbb{Z})$ and $M_{G}$ be the $G$-lattice of rank $n$ as in Definition 1.26 For $n \leq 4$, we will show that the Krull-Schmidt theorem holds for $M_{G}$, i.e. if $M_{G} \simeq M_{1} \oplus \cdots \oplus M_{l} \simeq N_{1} \oplus \cdots \oplus N_{m}$ for indecomposable $G$-lattices $M_{i}$ and $N_{j}$, then $l=m$ and, after a suitable renumbering of the $N_{j}, M_{i} \simeq N_{i}$ for any $1 \leq i \leq m$. However, it turns out that the Krull-Schmidt theorem fails for $M_{G}$ when the rank of $M_{G}$ is 5 . We split the Krull-Schmidt theorem for $M_{G}$ into the following two parts:

(KS1) If $M_{G} \simeq M_{1} \oplus \cdots \oplus M_{l} \simeq N_{1} \oplus \cdots \oplus N_{m}$ for indecomposable $G$-lattices $M_{i}$ and $N_{j}$, then $l=m$ and, after a suitable renumbering of the $N_{j}$, rank $M_{i}=\operatorname{rank} N_{i}$ for any $1 \leq i \leq m$;

(KS2) If $M_{G} \simeq M_{1} \oplus \cdots \oplus M_{m} \simeq N_{1} \oplus \cdots \oplus N_{m}$ for indecomposable $G$-lattices $M_{i}$ and $N_{i}$ with rank $M_{i}=$ rank $N_{i}$ for any $1 \leq i \leq m$, then after a suitable renumbering of the $N_{i}, M_{i} \simeq N_{i}$ for any $1 \leq i \leq m$.

Krull-Schmidt theorem holds for $M_{G}$ if and only if the conditions (KS1) and (KS2) hold for $M_{G}$.

Theorem (Theorem 4.6). Let $G$ be a finite subgroup of $\mathrm{GL}(n, \mathbb{Z})$ and $M_{G}$ be the G-lattice of rank $n$ as in Definition 1.26

(i) When $n \leq 4$, the Krull-Schmidt theorem holds for $M_{G}$, i.e. if $M_{G} \simeq M_{1} \oplus \cdots \oplus M_{l} \simeq N_{1} \oplus \cdots \oplus N_{m}$ for indecomposable $G$-lattices $M_{i}$ and $N_{j}$, then $l=m$ and, after a suitable renumbering of the $N_{j}, M_{i} \simeq N_{i}$ for any $1 \leq i \leq m$.

(ii) When $n=5$, (KS2) holds for $M_{G}$, and the Krull-Schmidt theorem fails for $M_{G}$ if and only if (KS1) fails for $M_{G}$ if and only if the CARAT ID of $G$ is one of the 11 triples

$(5,188,4),(5,189,4),(5,190,6),(5,191,6),(5,192,6),(5,193,4),(5,205,6),(5,218,8),(5,219,8),(5,220,4),(5,221,4)$.

For the exceptional 11 cases, the decomposition types of $M_{G}$ are $(3,2)$ and $(4,1)$ and $G$ is a subgroup of the group $C_{2} \times D_{6}$ of the CARAT ID $(5,205,6)$.

(iii) When $n=6,(\mathrm{KS} 1)$ fails for $M_{G}$ if and only if the CARAT ID of $G$ is one of the 131 triples

$(6,2013,8),(6,2018,4),(6,2023,6),(6,2024,6),(6,2025,6),(6,2026,6),(6,2033,6),(6,2042,8),(6,2043,8),(6,2044,4)$,

$(6,2045,4),(6,2048,5),(6,2049,8),(6,2050,8),(6,2051,8),(6,2052,8),(6,2058,5),(6,2059,5),(6,2067,5),(6,2068,5)$,

$(6,2069,5),(6,2069,11),(6,2070,9),(6,2071,9),(6,2072,10),(6,2072,11),(6,2076,24),(6,2076,25),(6,2077,24),(6,2077,25)$,

$(6,2078,24),(6,2078,25),(6,2079,24),(6,2079,25),(6,2087,15),(6,2088,15),(6,2089,17),(6,2089,18),(6,2094,9),(6,2102,24)$,

$(6,2102,25),(6,2105,9),(6,2106,9),(6,2107,10),(6,2107,11),(6,2108,15),(6,2109,15),(6,2110,17),(6,2110,18),(6,2111,15)$,

$(6,2139,9)$, 
$(6,40,4),(6,41,4),(6,44,6),(6,45,6),(6,47,4),(6,53,4),(6,54,4),(6,54,8),(6,55,4),(6,63,4)$,

$(6,64,6),(6,65,4),(6,66,6),(6,67,6),(6,75,4),(6,75,8),(6,76,8),(6,76,12),(6,77,8),(6,77,12)$,

$(6,78,4),(6,78,8),(6,79,6),(6,80,4),(6,81,8),(6,81,12),(6,90,4),(6,99,4),(6,108,4),(6,108,8)$,

$(6,109,8),(6,109,12),(6,110,4),(6,111,6),(6,112,8),(6,112,12),(6,113,4),(6,114,6),(6,115,6),(6,145,4)$,

$(6,2070,10),(6,2070,11),(6,2071,10),(6,2071,11),(6,2072,12),(6,2072,13),(6,2076,26),(6,2076,27),(6,2077,26),(6,2077,27)$,

$(6,2078,26),(6,2078,27),(6,2079,26),(6,2079,27),(6,2087,16),(6,2087,17),(6,2088,16),(6,2088,17),(6,2089,19),(6,2089,20)$,

$(6,2094,10),(6,2094,11),(6,2102,26),(6,2102,27),(6,2105,10),(6,2105,11),(6,2106,10),(6,2106,11),(6,2107,12),(6,2107,13)$,

$(6,2108,16),(6,2108,17),(6,2109,16),(6,2109,17),(6,2110,19),(6,2110,20),(6,2111,16),(6,2111,17),(6,2139,10),(6,2139,11)$.

For the former 51 cases (resp. the latter 80 cases), the decomposition types of $M_{G}$ are $(3,2,1)$ and $(4,1,1)$ (resp. $(3,3)$ and $(5,1))$ and $G$ is a subgroup of the group $C_{2}^{2} \times D_{6}$ of the CARAT ID $(6,2139,9)\left(\right.$ resp. $D_{6} \times D_{4}$ of the CARAT ID $(6,145,4))$.

(iv) When $n=6,(\mathrm{KS} 2)$ fails for $M_{G}$ if and only if the CARAT ID of $G$ is one of the 18 triples

$(6,2072,14),(6,2076,28),(6,2077,28),(6,2078,28),(6,2079,28),(6,2089,21),(6,2102,28),(6,2107,14),(6,2110,21),(6,2295,2)$,

$(6,3045,3),(6,3046,3),(6,3047,3),(6,3052,5),(6,3053,5),(6,3054,3),(6,3061,5),(6,3066,3)$.

For the former 10 cases, the decomposition type of $M_{G}$ is $(4,2)$ and $G$ is the group $D_{6}$ of the CARAT ID $(6,2295,2)$ or a subgroup of the 3 groups $C_{2} \times D_{6}$ of the CARAT ID $s(6,2102,28),(6,2107,14)$ and $(6,2110,21)$. For the latter 8 cases, the decomposition type of $M_{G}$ is $(5,1)$ and $G$ is a subgroup of the group $C_{2} \times S_{5}$ of the CARAT ID $(6,3054,3)$.

The following flow chart presents the structure of the GAP algorithms which will be given in Section 5 .

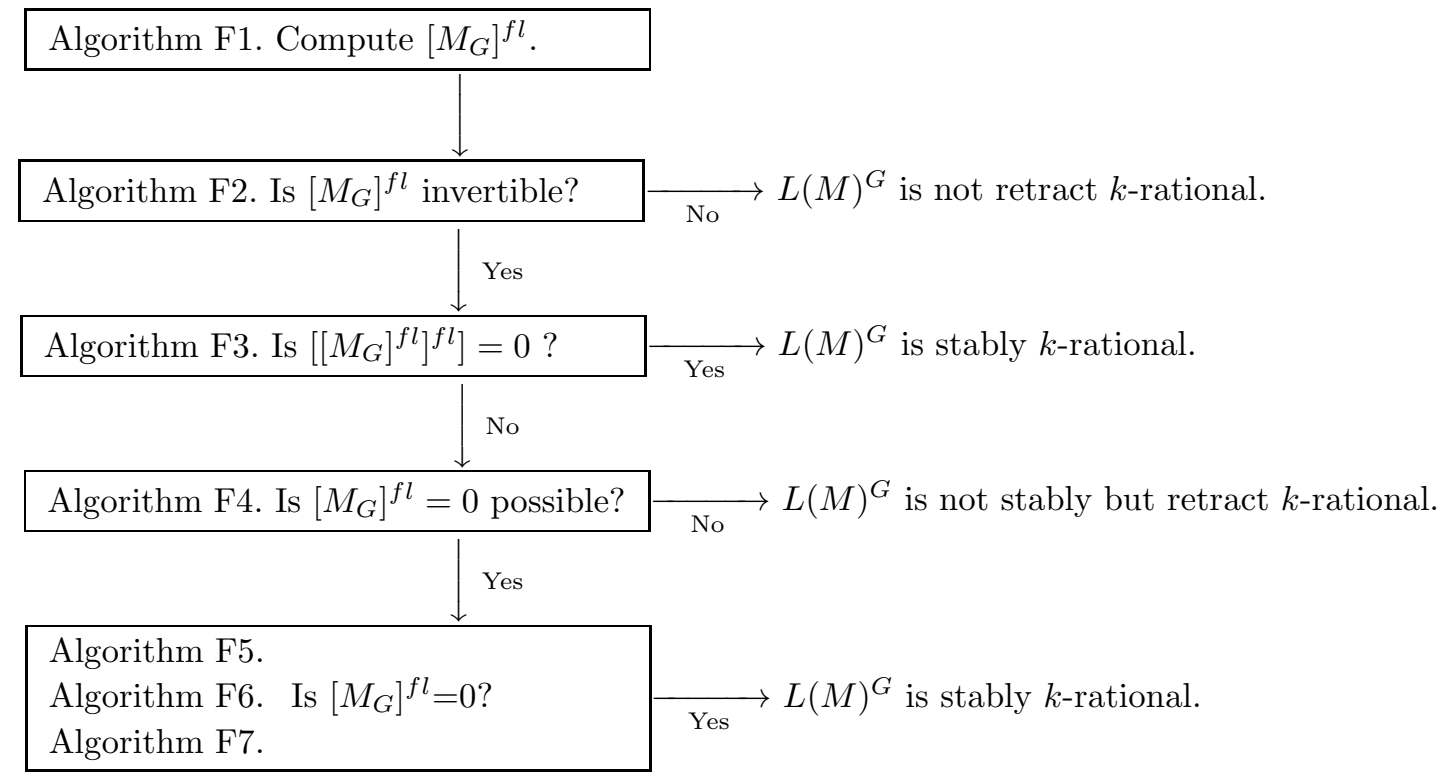

Using the algorithms as in Section [5 and Section 6, we may verify the following isomorphism which gives the smallest example exhibiting the failure of the Krull-Schmidt theorem for permutation $G$-lattices (see Section 4 and Dress's paper [Dre73, Proposition 9.6]):

Proposition (The Krull-Schmidt theorem fails for permutation $D_{6}$-lattices, see Proposition 6.7). Let $D_{6}$ be the dihedral group of order 12 and $\{1\}, C_{2}^{(1)}, C_{2}^{(2)}, C_{2}^{(3)}, C_{3}, C_{2}^{2}, C_{6}, S_{3}^{(1)}, S_{3}^{(2)}$ and $D_{6}$ be the conjugacy classes of subgroups of $D_{6}$. Then the following isomorphism of permutation $D_{6}$-lattices holds:

$$
\begin{aligned}
& \mathbb{Z}\left[D_{6}\right] \oplus \mathbb{Z}\left[D_{6} / C_{2}^{2}\right]^{\oplus 2} \oplus \mathbb{Z}\left[D_{6} / C_{6}\right] \oplus \mathbb{Z}\left[D_{6} / S_{3}^{(1)}\right] \oplus \mathbb{Z}\left[D_{6} / S_{3}^{(2)}\right] \\
\simeq & \mathbb{Z}\left[D_{6} / C_{2}^{(1)}\right] \oplus \mathbb{Z}\left[D_{6} / C_{2}^{(2)}\right] \oplus \mathbb{Z}\left[D_{6} / C_{2}^{(3)}\right] \oplus \mathbb{Z}\left[D_{6} / C_{3}\right] \oplus \mathbb{Z}^{\oplus 2} .
\end{aligned}
$$

By the classification of Bravais groups of dimension $n \leq 6$ as in Subsection 4.4 and the algorithms in Section 5 and Section 6] we will show the following theorem. 
Theorem (Theorem 7.1). If $G$ is a Bravais group of dimension $n \leq 6$, then $H^{1}\left(G,\left[M_{G}\right]^{\text {fl }}\right)=0$. In particular, if $G$ is a maximal finite subgroup $G \leq \mathrm{GL}(n, \mathbb{Z})$ where $n \leq 6$, then $H^{1}\left(G,\left[M_{G}\right]^{f l}\right)=0$.

As an application of the method of this paper, we give examples of not retract $k$-rational fields which are related to the rationality problem under the finite group action, e.g. Noether's problem (see [HKY11], YYam12], HKK14.).

Let $k$ be a field of char $k \neq 2$ and $k(x, y, z)$ be the rational function field over $k$ with variables $x, y, z$. We consider the $k$-involution (i.e. $k$-automorphism of order 2 )

$$
\sigma_{a, b, c, d}: x \mapsto-x, \quad y \mapsto \frac{-a x^{2}+b}{y}, \quad z \mapsto \frac{-c x^{2}+d}{z} \quad\left(a, b, c, d \in k^{\times}\right)
$$

on $k(x, y, z)$ and the rationality problem of $k(x, y, z)^{\left\langle\sigma_{a, b, c, d}\right\rangle}$ over $k$, namely whether the fixed field $k(x, y, z)^{\left\langle\sigma_{a, b, c, d}\right\rangle}$ is $k$-rational. We see that the fixed field $k(x, y, z)^{\left\langle\sigma_{a, b, c, d}\right\rangle}$ is $k$-isomorphic to $k(x, y, z)^{\left\langle\sigma_{\tau(a), \tau(b), \tau(c), \tau(d)}\right\rangle}$ for $\tau \in D_{4}$ where $D_{4}=\langle(a b d c),(a b)(c d)\rangle$ is the permutation group on the set $\{a, b, c, d\}$ which is isomorphic to the dihedral group of order 8 . Let $m=[k(\sqrt{a}, \sqrt{b}, \sqrt{c}, \sqrt{d}): k]$. Hence the rationality problem is determined by the following 22 cases:

(C1) $m=1$;

(C2) $m=2$, (1) $a, b, c \in k^{\times 2} ;(2) a, b, d \in k^{\times 2} ;(3) a, c, d \in k^{\times 2} ;$ (4) b,c,d $\in k^{\times 2}$;

(C3) $m=2$, (1) $a, b, c d \in k^{\times 2}$; (2) b, d, ac $\in k^{\times 2}$; (3) $d, c, a b \in k^{\times 2}$; (4) $c, a, b d \in k^{\times 2}$;

(C4) $m=2$, (1) $a, d, b c \in k^{\times 2}$; (2) b, c, ad $\in k^{\times 2}$;

(C5) $m=2$, (1) $a, b d, c d \in k^{\times 2}$; (2) b, cd, ac $\in k^{\times 2}$; (3) $d, a c, a b \in k^{\times 2}$; (4) $c, a b, b d \in k^{\times 2}$;

(C6) $m=2, a b, a c, a d \in k^{\times 2}$;

(C7) $m=4$, (1) $a, b \in k^{\times 2}$; (2) $b, d \in k^{\times 2}$; (3) $d, c \in k^{\times 2}$; (4) $c, a \in k^{\times 2}$;

(C8) $m=4$, (1) $a, d \in k^{\times 2}$; (2) b,c $\in k^{\times 2}$;

(C9) $m=4$, (1) $a, b c \in k^{\times 2}$; (2) b, ad $\in k^{\times 2}$; (3) $d, b c \in k^{\times 2}$; (4) $c, a d \in k^{\times 2}$;

(C10) $m=4$, (1) $a, b d \in k^{\times 2} ;(2) b, d c \in k^{\times 2} ;$ (3) $d, a c \in k^{\times 2}$; (4) $c, a b \in k^{\times 2}$;

(5) $a, c d \in k^{\times 2}$; (6) $b, a c \in k^{\times 2}$; (7) $d, a b \in k^{\times 2}$; (8) $c, b d \in k^{\times 2}$;

(C11) $m=4$, (1) $a, b c d \in k^{\times 2}$; (2) b, acd $\in k^{\times 2}$; (3) $d, a b c \in k^{\times 2}$; (4) $c, a b d \in k^{\times 2}$;

(C12) $m=4$, (1) $a b, c d \in k^{\times 2} ;(2) b d, a c \in k^{\times 2}$;

(C13) $m=4$, (1) $a b, a c \in k^{\times 2}$; (2) bd, ab $\in k^{\times 2}$; (3) $c d, b d \in k^{\times 2}$; (4) $a c, c d \in k^{\times 2}$;

(C14) $m=4, a d, b c \in k^{\times 2}$;

(C15) $m=4$, (1) ab, acd $\in k^{\times 2} ;$ (2) bd, abc $\in k^{\times 2} ;$ (3) $c d, a b d \in k^{\times 2}$; (4) ac, bcd $\in k^{\times 2}$;

(C16) $m=4$, (1) ad, abc $\in k^{\times 2} ;(2) b c, a b d \in k^{\times 2}$;

(C17) $m=8$, (1) $a \in k^{\times 2}$; (2) $b \in k^{\times 2}$; (3) $d \in k^{\times 2}$; (4) $c \in k^{\times 2}$;

(C18) $m=8$, (1) $a b \in k^{\times 2}$; (2) $a c \in k^{\times 2}$; (3) $b d \in k^{\times 2}$; (4) $c d \in k^{\times 2}$;

(C19) $m=8$, (1) $a d \in k^{\times 2} ;(2) b c \in k^{\times 2}$;

(C20) $m=8$, (1) $a b c \in k^{\times 2} ;$ (2) bcd $\in k^{\times 2}$; (3) $a b d \in k^{\times 2}$; (4) acd $\in k^{\times 2}$;

(C21) $m=8$, abcd $\in k^{\times 2}$;

(C22) $m=16$.

We see that if one of the conditions (C1), (C2), (C3), (C5), (C6), (C7), (C10), (C12), (C13) holds, then $k(x, y, z)^{\left\langle\sigma_{a, b, c, d}\right\rangle}$ is $k$-rational (see Lemma 12.5).

Theorem (Theorem 12.3). Let $k$ be a field of char $k \neq 2$ and $k(x, y, z)$ be the rational function field over $k$ with variables $x, y, z$. Let $\sigma_{a, b, c, d}$ be a $k$-involution on $k(x, y, z)$ defined by

$$
\sigma_{a, b, c, d}: x \mapsto-x, \quad y \mapsto \frac{-a x^{2}+b}{y}, \quad z \mapsto \frac{-c x^{2}+d}{z} \quad\left(a, b, c, d \in k^{\times}\right)
$$

and $m=[k(\sqrt{a}, \sqrt{b}, \sqrt{c}, \sqrt{d}): k]$.

(i) $k(x, y, z)^{\left\langle\sigma_{a, b, c, d}\right\rangle}=k\left(t_{1}, t_{2}, t_{3}, t_{4}\right)$ where $t_{1}, t_{2}, t_{3}, t_{4}$ satisfy the relation

$$
\left(t_{1}^{2}-a\right)\left(t_{4}^{2}-d\right)=\left(t_{2}^{2}-b\right)\left(t_{3}^{2}-c\right)
$$

(ii) $k(x, y, z)^{\left\langle\sigma_{a, b, c, d}\right\rangle}$ is $k$-isomorphic to $k(x, y, z)^{\left\langle\sigma_{\tau(a), \tau(b), \tau(c), \tau(d)}\right\rangle}$ for $\tau \in D_{4}$ where $D_{4}=\langle(a b d c),(a b)(c d)\rangle$ is the permutation group on the set $\{a, b, c, d\}$ which is isomorphic to the dihedral group of order 8 .

(iii) If one of the following conditions holds, then $k(x, y, z)^{\left\langle\sigma_{a, b, c, d}\right\rangle}$ is not retract $k$-rational: 
(C15) $m=4$, (1) $a b, a c d \in k^{\times 2}$; (2) $b d, a b c \in k^{\times 2}$; (3) $c d, a b d \in k^{\times 2}$; (4) $a c, b c d \in k^{\times 2}$;

(C16) $m=4$, (1) $a d, a b c \in k^{\times 2} ;(2) b c, a b d \in k^{\times 2}$;

(C18) $m=8$, (1) $a b \in k^{\times 2} ;(2) a c \in k^{\times 2} ;(3) b d \in k^{\times 2} ;(4) c d \in k^{\times 2}$;

(C19) $m=8$, (1) $a d \in k^{\times 2}$; (2) $b c \in k^{\times 2}$;

(C20) $m=8$, (1) $a b c \in k^{\times 2} ;(2) b c d \in k^{\times 2} ;$ (3) $a b d \in k^{\times 2} ;$ (4) $a c d \in k^{\times 2}$;

(C21) $m=8, a b c d \in k^{\times 2}$;

(C22) $m=16$.

We do not know whether the field $k(x, y, z)^{\left\langle\sigma_{a, b, c, d}\right\rangle}$ is $k$-rational for the cases (C4), (C8), (C9), (C11), (C14), (C17).

We organize this paper as follows. In Section 2, we recall known results and prepare some basic materials, e.g. Galois cohomology, Tate cohomology, flabby resolution of a $G$-lattice. We explain the relationship between these materials and the stably rational classification of algebraic $k$-tori. In Section 3 we explain how to access the GAP ID and the CARAT ID of a finite subgroup $G$ of $\mathrm{GL}(n, \mathbb{Z})(n \leq 6)$ of this paper. In Section 4 , we show that the Krull-Schmidt theorem for $G$-lattices holds when the rank $\leq 4$, and fails when the rank is 5 and 6 by using the GAP ID and the CARAT ID. The classification of maximal finite groups $G \leq \mathrm{GL}(n, \mathbb{Z})$ and Bravais groups of dimension $n$ where $n \leq 6$ will be also given in Subsections 4.3 and 4.4 respectively. In Section 5 , we give some algorithms to compute a flabby resolution of a $G$-lattice effectively in GAP. We also give some algorithms which may determine whether the flabby class of the $G$-lattice is invertible (resp. zero) or not. These algorithms enable us to classify the function fields of algebraic $k$-tori up to stably equivalence. In Section 6 , the classification of all the flabby and coflabby $G$-lattices of rank up to 6 will be given. In Section 7 , we confirm that $H^{1}\left(G,\left[M_{G}\right]^{f l}\right)=0$ for any Bravais group $G$ of dimension $n \leq 6$. In particular, we get that $H^{1}\left(G,\left[M_{G}\right]^{f l}\right)=0$ for maximal finite subgroups $G \leq \operatorname{GL}(n, \mathbb{Z})$ where $n \leq 6$. In Section 8 , we obtain the stably rational classification of norm one tori of dimensions 4 and 5. The same classification for dimensions 6 and 10 will also be given. In Section 9, we give some GAP algorithms for computing the Tate cohomologies. In Sections 10 and 11] we will prove Theorem 1.27 and Theorem 1.28 respectively by using the algorithms which are given in Sections 3, 4, 5 and 9. Using the algorithms in Section 5, we show Theorem 12.3 which provides some examples of not retract $k$-rational fields in Section 12, In Section 13, an application of Theorem 12.3 which is related to Noether's problem will be given. Tables 11 to 15 in Theorems 1.12 and 1.28 are located in Section 14. We also give detailed information of the stably rational classification of algebraic $k$-tori of dimension 5 as in Table 16 in Section 14

Acknowledgments. The authors would like to thank Ming-chang Kang for giving them useful and valuable comments. They also would like to thank Shizuo Endo for valuable comments and for fruitful discussions, in particular, about Section 4. The first-named author wishes to gratefully thank his teacher Yumiko Hironaka for continuous encouragement. The authors also would like to thank the referee who draws their attention to Bravais groups and gives them useful suggestions and comments. Subsections $4.3,4.4$ and Section 7 are added according to referee's valuable advice.

\section{Preliminaries: Tate cohomology and flabby Resolutions}

First we recall the definitions of Galois cohomology and Tate cohomology. See for details Cartan and Eilenberg [CE56, ChapterXII] and Brown [Bro82, Chapter VI].

Definition 2.1 ( $n$-cochains, coboundary homomorphisms). Let $G$ be a group and $M$ be an additive $G$-module. Let $n \geq 0$ be an integer and $C^{n}(G, M)$ be the additive group of all maps from $G^{n}$ to $M\left(G^{0}=1\right)$. The elements of $C^{n}(G, M)$ are called the $n$-cochains. The coboundary homomorphisms

$$
d^{n}: C^{n}(G, M) \rightarrow C^{n+1}(G, M)
$$

are defined as

$$
\begin{aligned}
\left(d^{n} \varphi\right)\left(g_{1}, \ldots, g_{n+1}\right)= & g_{1} \cdot \varphi\left(g_{2}, \ldots, g_{n+1}\right) \\
& +\sum_{i=1}^{n}(-1)^{i} \varphi\left(g_{1}, \ldots, g_{i-1}, g_{i} g_{i+1}, g_{i+2}, \ldots, g_{n+1}\right) \\
& +(-1)^{n+1} \varphi\left(g_{1}, \ldots, g_{n}\right) .
\end{aligned}
$$

Lemma 2.2. $d^{n+1} \circ d^{n}=0$ holds, and $(C(G, M), d)$ becomes a cochain complex.

Definition 2.3 (Group cohomology). Define the group of $n$-cocycles as

$$
Z^{n}(G, M)=\operatorname{Ker}\left(d^{n}\right)
$$


the group of $n$-coboundaries as

and the $n$-th cohomology group as

$$
\left\{\begin{array}{l}
B^{0}(G, M)=0, \\
B^{n}(G, M)=\operatorname{Im}\left(d^{n-1}\right) \quad(n \geq 1),
\end{array}\right.
$$

$$
H^{n}(G, M)=Z^{n}(G, M) / B^{n}(G, M) \quad(n \geq 0) .
$$

Definition 2.4 (Tate cohomology). Let $G$ be a finite group and $M$ be an additive $G$-module. Define the trace $\operatorname{map} T_{G}: M \rightarrow M$ as

$$
T_{G}(m)=\sum_{g \in G} g \cdot m,
$$

the group of 0 -cocycles and the group of $(-1)$-cocycles as

$$
\left\{\begin{array}{l}
\widehat{Z}^{0}(G, M)=M^{G}=H^{0}(G, M), \\
\widehat{Z}^{-1}(G, M)=\operatorname{Ker}\left(T_{G}\right),
\end{array}\right.
$$

the group of 0-coboundaries and the group of (-1)-coboundaries as

$$
\left\{\begin{array}{l}
\widehat{B}^{0}(G, M)=\operatorname{Im}\left(T_{G}\right) \\
\widehat{B}^{-1}(G, M)=\sum_{g \in G} \operatorname{Im}\left(g-\mathrm{id}_{M}\right)
\end{array}\right.
$$

and the n-th Tate cohomology group as

$$
\widehat{H}^{n}(G, M)= \begin{cases}H^{n}(G, M) & (n \geq 1), \\ \widehat{Z}^{0}(G, M) / \widehat{B}^{0}(G, M) & (n=0), \\ \widehat{Z}^{-1}(G, M) / \widehat{B}^{-1}(G, M) & (n=-1), \\ H_{-n-1}(G, M) & (n \leq-2)\end{cases}
$$

where $H_{i}$ is the $i$-th homology group.

Assume that $G$ is a finite group and $M$ is a $G$-lattice, i.e. finitely generated $\mathbb{Z}[G]$-module which is $\mathbb{Z}$-free as an abelian group. Both $\widehat{Z}^{n}(G, M)$ and $\widehat{B}^{n}(G, M)$ are free $\mathbb{Z}$-modules of finite rank, and it turns out that the groups $\widehat{H}^{n}(G, M)$ have exponent dividing $\# G$ and hence finite for any $n \in \mathbb{Z}$. We have that $\widehat{H}^{n}(G, \mathbb{Z}[G])=0$ for $n= \pm 1$ (see Lemma 2.11) and that $\widehat{H}^{0}(G, \mathbb{Z}[G])=0$.

We will give some GAP algorithms for computing the Tate cohomology $\widehat{H}^{n}\left(G, M_{G}\right)$ in Section 9 .

In order to construct a flabby resolution of $M$, we use the following long exact sequence:

Lemma 2.5 (A long exact sequence). If

$$
0 \rightarrow A \stackrel{f}{\rightarrow} B \stackrel{g}{\rightarrow} C \rightarrow 0
$$

is an exact sequence of $G$-lattices, then there exists a long exact sequence of abelian groups

$$
\cdots \rightarrow \widehat{H}^{k-1}(G, C) \stackrel{d^{*}}{\longrightarrow} \widehat{H}^{k}(G, A) \stackrel{f^{*}}{\longrightarrow} \widehat{H}^{k}(G, B) \stackrel{g^{*}}{\longrightarrow} \widehat{H}^{k}(G, C) \stackrel{d^{*}}{\rightarrow} \widehat{H}^{k+1}(G, A) \rightarrow \cdots
$$

where $f^{*}$ and $g^{*}$ are the maps in cohomology induced from the cochain maps $f$ and $g$, and $d^{*}$ is the connecting homomorphism obtained by using the snake lemma.

Definition 2.6 (Dual $G$-lattice). Let $M$ be a $G$-lattice. The $G$-lattice $M^{\circ}=\operatorname{Hom}_{\mathbb{Z}}(M, \mathbb{Z})$ with $G$-action

$$
(g \cdot f)(m)=f\left(g^{-1} \cdot m\right) \quad\left(f \in M^{\circ}, m \in M, g \in G\right)
$$

is called the dual G-lattice of $M$.

Note that $\left(M^{\circ}\right)^{\circ} \simeq M$ and if $A(g)$ is the matrix representation of the action of $g \in G$ on the $G$-lattice $M$ with a fixed $\mathbb{Z}$-basis, then the matrix representation of the action of $g$ on $M^{\circ}$ is given by ${ }^{t} A\left(g^{-1}\right)$. In particular, if $M$ is permutation $G$-lattice, then $M \simeq M^{\circ}$.

Lemma 2.7 (see Arn84, Theorem 2.2]). $H^{n}(G, M) \simeq \widehat{H}^{-n}\left(G, M^{\circ}\right)$.

Definition 2.8 (Dual homomorphism). For $f \in \operatorname{Hom}_{\mathbb{Z}}\left(M_{1}, M_{2}\right)$, we define the dual homomorphism $f^{\circ} \in$ $\operatorname{Hom}_{\mathbb{Z}}\left(M_{2}^{\circ}, M_{1}^{\circ}\right)$ by

$$
f^{\circ}(l)(m)=l(f(m)) \quad\left(l \in M_{2}^{\circ}, m \in M_{1}\right)
$$


Lemma 2.9. If $A \stackrel{f}{\rightarrow} B \stackrel{g}{\rightarrow} C$ is an exact sequence of $G$-lattices, then $C^{\circ} \stackrel{g^{\circ}}{\rightarrow} B^{\circ} \stackrel{f^{\circ}}{\rightarrow} A^{\circ}$ is also an exact sequence of G-lattices.

We recall some basic facts of the theory of flabby (flasque) G-lattices (see CTS77, Swa83, Vos98, Chapter 2], Lor05, Chapter 2], [Swa10]).

Definition 2.10 (Permutation, stably permutation, invertible, flabby and coflabby $G$-lattices). Let $G$ be a finite group and $M$ be a $G$-lattice (i.e. finitely generated $\mathbb{Z}[G]$-module which is $\mathbb{Z}$-free as an abelian group).

(i) $M$ is called a permutation $G$-lattice if $M$ has a $\mathbb{Z}$-basis permuted by $G$, i.e. $M \simeq \oplus_{1 \leq i \leq m} \mathbb{Z}\left[G / H_{i}\right]$ for some subgroups $H_{1}, \ldots, H_{m}$ of $G$.

(ii) $M$ is called a stably permutation $G$-lattice if $M \oplus P \simeq P^{\prime}$ for some permutation $G$-lattices $P$ and $P^{\prime}$.

(iii) $M$ is called invertible (or permutation projective) if it is a direct summand of a permutation $G$-lattice, i.e. $P \simeq M \oplus M^{\prime}$ for some permutation $G$-lattice $P$ and a $G$-lattice $M^{\prime}$.

(iv) $M$ is called flabby (or flasque) if $\widehat{H}^{-1}(H, M)=0$ for any subgroup $H$ of $G$ where $\widehat{H}$ is the Tate cohomology.

(v) $M$ is called coflabby (or coflasque) if $H^{1}(H, M)=0$ for any subgroup $H$ of $G$.

Lemma 2.11 ([Len74, Propositions 1.1 and 1.2], see also [Swa83, Section 8]). Let E be an invertible G-lattice. Then

(i) $E$ is flabby and coflabby.

(ii) If $C$ is a coflabby $G$-lattice, then any short exact sequence $0 \rightarrow C \rightarrow N \rightarrow E \rightarrow 0$ splits.

Let $\mathcal{C}(G)$ be the category of all $G$-lattices and $\mathcal{S}(G)$ be the full subcategory of $\mathcal{C}(G)$ of all permutation $G$ lattices. Recall that two $G$-lattices $M_{1}$ and $M_{2}$ are similar if there exist permutation $G$-lattices $P_{1}$ and $P_{2}$ such that $M_{1} \oplus P_{1} \simeq M_{2} \oplus P_{2}$. The similarity class of $M$ is denoted by $[M]$. Then the set of similarity classes $\mathcal{C}(G) / \mathcal{S}(G)$ becomes a commutative monoid (with respect to the sum $\left[M_{1}\right]+\left[M_{2}\right]:=\left[M_{1} \oplus M_{2}\right]$ and the zero $0=[P]$ where $P \in \mathcal{S}(G))$ (see Definition [1.16).

Theorem 2.12 (Endo and Miyata EM74, Lemma 1.1], Colliot-Thélène and Sansuc [CTS77, Lemma 3], see also [Swa83, Lemma 8.5], Lor05, Lemma 2.6.1]). For any G-lattice $M$, there is a short exact sequence of G-lattices $0 \rightarrow M \rightarrow P \rightarrow F \rightarrow 0$ where $P$ is permutation and $F$ is flabby.

Definition 2.13 (Flabby resolution). The exact sequence $0 \rightarrow M \rightarrow P \rightarrow F \rightarrow 0$ as in Theorem 2.12 is called a flabby resolution of the $G$-lattice $M . \rho_{G}(M)=[F] \in \mathcal{C}(G) / \mathcal{S}(G)$ is called the flabby class of $M$, denoted by $[M]^{f l}=[F]$. Note that $[M]^{f l}$ is well-defined: if $[M]=\left[M^{\prime}\right],[M]^{f l}=[F]$ and $\left[M^{\prime}\right]{ }^{f l}=\left[F^{\prime}\right]$ then $F \oplus P_{1} \simeq F^{\prime} \oplus P_{2}$ for some permutation $G$-lattices $P_{1}$ and $P_{2}$, and therefore $[F]=\left[F^{\prime}\right]$ (cf. [Swa83, Lemma 8.7]). We say that $[M]^{f l}$ is invertible if $[M]^{f l}=[E]$ for some invertible $G$-lattice $E$.

For $G$-lattice $M$, it is not difficult to see

$$
\begin{array}{ccc}
\text { permutation } \Rightarrow \text { stably permutation } & \Rightarrow \text { invertible } \Rightarrow \text { flabby and coflabby } \\
\Downarrow & \Downarrow \\
{[M]^{f l}=0} & \Rightarrow & {[M]^{f l} \text { is invertible }}
\end{array}
$$

(see Section 11). Let $L / k$ be a finite Galois extension with Galois group $G=\operatorname{Gal}(L / k)$ and $M$ be a $G$-lattice. The flabby class $[M]^{f l}$ plays crucial role in the rationality problem for $L(M)^{G}$. In particular, by Theorem 1.17 (Endo and Miyata [EM73, Voskresenskii Vos74 and Saltman Sal84a]), we have the following fundamentals:

(i) $[M]^{f l}=0$ if and only if $L(M)^{G}$ is stably $k$-rational;

(ii) $[M]^{f l}=\left[M^{\prime}\right]^{f l}$ if and only if $L(M)^{G}$ and $L\left(M^{\prime}\right)^{G}$ are stably $k$-isomorphic, i.e. there exist algebraically independent elements $x_{1}, \ldots, x_{m}$ over $L(M)^{G}$ and $y_{1}, \ldots, y_{n}$ over $L\left(M^{\prime}\right)^{G}$ such that $L(M)^{G}\left(x_{1}, \ldots, x_{m}\right) \simeq$ $L\left(M^{\prime}\right)^{G}\left(y_{1}, \ldots, y_{n}\right)$

(iii) $[M]^{f l}$ is invertible if and only if $L(M)^{G}$ is retract $k$-rational.

Let $G \simeq \operatorname{Gal}(L / k)$ be a finite group and $M \simeq M_{1} \oplus M_{2}$ be a decomposable $G$-lattice. Let $N_{i}=\{\sigma \in G \mid$ $\sigma(v)=v$ for any $v \in M_{i}$ \} be the kernel of the action of $G$ on $M_{i}(i=1,2)$. Then $L(M)^{G}$ is the function field of an algebraic torus $T$ and is $k$-isomorphic to the free composite of $L\left(M_{1}\right)^{G}$ and $L\left(M_{2}\right)^{G}$ over $k$ where $L\left(M_{i}\right)^{G}=\left(L^{N_{i}}\right)\left(M_{i}^{N_{i}}\right)^{G / N_{i}}$ is the function field of some torus $T_{i}(i=1,2)$ with $T=T_{1} \times T_{2}$ and $M_{i}$ may be regarded as a $G / N_{i}$-lattice. 
Lemma 2.14. Let $G$ be a finite group and $M \simeq M_{1} \oplus M_{2}$ be a decomposable G-lattice with the flabby class $\rho_{G}(M)=[M]^{f l}$. Let $N_{1}$ be a normal subgroup of $G$ which acts on $M_{1}$ trivially. The G-lattice $M_{1}$ may be regarded as a $G / N_{1}$-lattice with the flabby class $\rho_{G / N_{1}}\left(M_{1}\right)$ as $G / N_{1}$-lattice. Then

(i) $\rho_{G}(M)=\rho_{G}\left(M_{1}\right)+\rho_{G}\left(M_{2}\right)$.

(ii) $\rho_{G}\left(M_{1}\right)=0$ if and only if $\rho_{G / N_{1}}\left(M_{1}\right)=0$.

(iii) $\rho_{G}\left(M_{1}\right)$ is invertible if and only if $\rho_{G / N_{1}}\left(M_{1}\right)$ is invertible.

Proof. (i) Let $0 \rightarrow M_{i} \rightarrow P_{i} \rightarrow F_{i} \rightarrow 0$ be flabby resolutions of $M_{i}$ as $G$-lattices $(i=1,2)$. Then $0 \rightarrow M \rightarrow$ $P_{1} \oplus P_{2} \rightarrow F_{1} \oplus F_{2} \rightarrow 0$ is a flabby resolution of $M$. Hence $\rho_{G}(M)=\left[F_{1} \oplus F_{2}\right]=\rho_{G}\left(M_{1}\right)+\rho_{G}\left(M_{2}\right)$. (ii), (iii) See [CTS77, Lemme 2] and [Kan09, Lemma 4.1].

Lemma 2.15 (Swan [Swa10, Lemma 3.1]). Let $0 \rightarrow M_{1} \rightarrow M \rightarrow M_{2} \rightarrow 0$ be a short exact sequence of $G$ lattices with $M_{2}$ invertible. Then $\rho_{G}(M)=\rho_{G}\left(M_{1}\right)+\rho_{G}\left(M_{2}\right)$. In particular, if $\rho_{G}\left(M_{1}\right)$ is invertible, then $-\rho_{G}\left(M_{1}\right)=\rho_{G}\left(\rho_{G}\left(M_{1}\right)\right)$.

Proof. See [Swa10, Lemma 3.1].

Lemma 2.16. Let $G \simeq \operatorname{Gal}(L / k)$ be a finite group and $M \simeq M_{1} \oplus M_{2}$ be a decomposable $G$-lattice.

(i) $L(M)^{G}$ is retract $k$-rational if and only if both of $L\left(M_{i}\right)^{G}(i=1,2)$ are retract $k$-rational.

(ii) If $L\left(M_{1}\right)^{G}$ and $L\left(M_{2}\right)^{G}$ are stably k-rational, then $K(M)^{G}$ is stably k-rational.

(iii) When rank $M_{i} \leq 3(i=1,2), L(M)^{G}$ is stably $k$-rational if and only if both of $L\left(M_{i}\right)^{G}(i=1,2)$ are stably $k$-rational.

Proof. See, for example, [HKK14, Theorem 6.5].

Let $H$ be a subgroup of $G$. For a $G$-lattice $M$, it can be regarded as a $H$-lattice by restricting the action of $G$ to $H$. We write this $H$-lattice as $\left.M\right|_{H}$.

Lemma 2.17. Let $G$ be a finite subgroup of $\operatorname{GL}(n, \mathbb{Z})$ and $M_{G}$ be the corresponding $G$-lattice as in Definition 1.26. Let $H$ be a subgroup of $G$.

(i) If $\rho_{G}\left(M_{G}\right)=0$, then $\rho_{H}\left(M_{H}\right)=0$.

(ii) If $\rho_{G}\left(M_{G}\right)$ is invertible, then $\rho_{H}\left(M_{H}\right)$ is invertible.

Proof. Let $0 \rightarrow M_{G} \rightarrow P \rightarrow F \rightarrow 0$ be a flabby resolution of $M_{G}$ as a $G$-lattice. Then $\left.\left.0 \rightarrow M_{G}\right|_{H} \rightarrow P\right|_{H} \rightarrow$ $\left.F\right|_{H} \rightarrow 0$ is a flabby resolution of $\left.M_{G}\right|_{H}=M_{H}$ as a $H$-lattice, because $\left.P\right|_{H}$ is a permutation $H$-lattice and $\left.F\right|_{H}$ is a flabby $H$-lattice. This shows that $\rho_{H}\left(M_{H}\right)=\left[\left.F\right|_{H}\right]$ as a $H$-lattice. If $G$-lattice $F$ is stably permutation (resp. invertible), then $\left.F\right|_{H}$ is stably permutation (resp. invertible) as a $H$-lattice.

\section{CARAT ID OF THE $\mathbb{Z}$-CLASSES IN DIMENSIONS 5 AND 6}

In this section, we will explain how to access the GAP ID and the CARAT ID of a finite subgroup $G$ of $\operatorname{GL}(n, \mathbb{Z})(n \leq 6)$. We need the GAP ([GAP]) packages CrystCat and CARAT to do the computations below.

The CrystCat package of GAP provides a catalog of $\mathbb{Q}$-classes and $\mathbb{Z}$-classes (conjugacy classes) of finite subgroups $G$ of $\mathrm{GL}(n, \mathbb{Q})$ and $\mathrm{GL}(n, \mathbb{Z})(2 \leq n \leq 4)$. For $2 \leq n \leq 4$, the GAP ID $(n, i, j, k)$ of a finite subgroup $G$ of $\operatorname{GL}(n, \mathbb{Z})$ means that $G$ belongs to the $k$-th $\mathbb{Z}$-class of the $j$-th $\mathbb{Q}$-class of the $i$-th crystal system of dimension $n$ in GAP (see also BBNWZ78, Table 1]).

The CARAT2 (Carat) package of GAP provides all conjugacy classes of finite subgroups of GL $(n, \mathbb{Q})(n \leq 6)$ (see [PS00]). There exist exactly 2 (reps. 13, 73, 710, 6079, 85308) $\mathbb{Z}$-classes forming 2 (resp. 10, 32, 227, 955, $7103) \mathbb{Q}$-classes in dimension $n=1$ (resp. 2, 3, 4, 5, 6) 3 .

After unpacking the CARAT, we get the $\mathbb{Q}$-catalog file carat-2.1b1/tables/qcatalog.tar.gz. Unpacking this file, we get lists of $\mathbb{Q}$-classes of $\mathrm{GL}(n, \mathbb{Q})(n=1, \ldots, 6)$ in qcatalog/data1, ., qcatalog/data6. Generators of each group are in individual files under the folders qcatalog/dim1/,.., qcatalog/dim6/.

\footnotetext{
${ }^{2}$ CARAT works on Linux or Mac OS X, but not on Windows.

${ }^{3}$ In the old version of CARAT, the number of $\mathbb{Q}$-classes of $\mathrm{GL}(6, \mathbb{Q}), 7104$, was not correct because of the overlapping two same $\mathbb{Q}$-classes (cf. $[\overline{P S 00}$ ). The second-named author detected it and reported the correct number, 7103, to the CARAT group (see also Carat]). It also turned out that the correct number of $\mathbb{Z}$-classes of $\mathrm{GL}(6, \mathbb{Q})$ is 85308 (in the old version, 85311 was wrong). This has been fixed in the current version 2.1b1 of CARAT.
} 
The second-named author wrote the perl script crystlst.pl to collect these generators into a single file. Files cryst1.gap, ..., cryst6.gap are lists of representatives of $\mathbb{Q}$-classes of $\mathrm{GL}(1, \mathbb{Q}), \ldots, \mathrm{GL}(6, \mathbb{Q})$ respectively. These files are available from http://www.math.h.kyoto-u.ac.jp/ yamasaki/Algorithm/as GLnQ.zip.

Let $G$ be a finite subgroup of $\mathrm{GL}(n, \mathbb{Z})$. CARAT has a command ZClassRepsQClass $(G)$ to compute the complete $\mathbb{Z}$-class representatives of the $\mathbb{Q}$-class of $G$. We split the $\mathbb{Q}$-class of $G$ into $\mathbb{Z}$-classes by the command ZClassRepsQClass (G). For the $l$-th group $\widetilde{G}$ in the list of $\mathbb{Z}$-classes obtained by ZClassRepsQClass $(G)$ where $G$ is the $m$-th group $(\mathbb{Q}$-class) in qcatalog/datan, we say that the CARAT ID of $\widetilde{G}$ is $(n, m, l)$.

The second-named author wrote a GAP program to determine the $\mathbb{Q}$-class and the $\mathbb{Z}$-class of a group $G$. The files crystcat.gap and caratnumber.gap contain programs related to the GAP ID and the CARAT ID respectively. The file caratnumber.gap uses other files which are packed in the crystdat.zip. This zip file should be packed at the current directory.

All the files above are available from http://www.math.h.kyoto-u.ac.jp/ ${ }^{\sim}$ yamasaki/Algorithm/.

MatGroupZClass $(\mathrm{n}, \mathrm{i}, \mathrm{j}, \mathrm{k})$ (build-in function of GAP) returns the group $G \leq \mathrm{GL}(n, \mathbb{Z})$ of the GAP ID $(n, i, j, k)$ when $2 \leq n \leq 4$.

CaratMatGroupZClass $(n, i, j)$ returns the group $G \leq \operatorname{GL}(n, \mathbb{Z})$ of the CARAT ID $(n, i, j)$ when $1 \leq n \leq 6$. CrystCatZClass (G) returns the $\operatorname{GAP} \mathrm{ID}(n, i, j, k)$ of $G \leq \mathrm{GL}(n, \mathbb{Z})$ when $1 \leq n \leq 4$.

CaratZClass $(G)$ returns the CARAT ID $(n, i, j)$ of $G \leq \operatorname{GL}(n, \mathbb{Z})$ when $1 \leq n \leq 6$.

$\operatorname{NrQClasses}(\mathrm{n})$ returns the number of $\mathbb{Q}$-classes in dimension $n$ when $1 \leq n \leq 6$.

$\operatorname{NrZClasses}(\mathrm{n}, \mathrm{m})$ returns the number of $\mathbb{Z}$-classes in the $m$-th $\mathbb{Q}$-class in dimension $n$ when $1 \leq n \leq 6$.

CrystCat2Carat (1) returns the CARAT ID of the group $G$ of the GAP ID $l$.

Carat2CrystCat ( 1 ) returns the GAP ID of the group $G$ of the CARAT ID $l$.

Example 3.1 (Functions in crystcat.gap and caratnumber.gap). We give some examples of the functions in crystcat.gap and caratnumber.gap. Note that caratnumber.gap needs the CARAT package in GAP.

Let $C_{n}$ be the cyclic group of order $n$ and $J_{n}=J_{C_{n}}$ be the Chevalley module of rank $n-1$ which is the dual of $I_{n}=\operatorname{Ker} \varepsilon$ where $\varepsilon: \mathbb{Z}\left[C_{n}\right] \rightarrow \mathbb{Z}$ is the augmentation map (see Section 1). Then $K\left(J_{n}\right)^{C_{n}}$ is the function field of the norm one torus $R_{K / k}^{(1)}\left(\mathbb{G}_{m}\right)$ where $K$ is a cyclic Galois extension of $k$ of degree $n$.

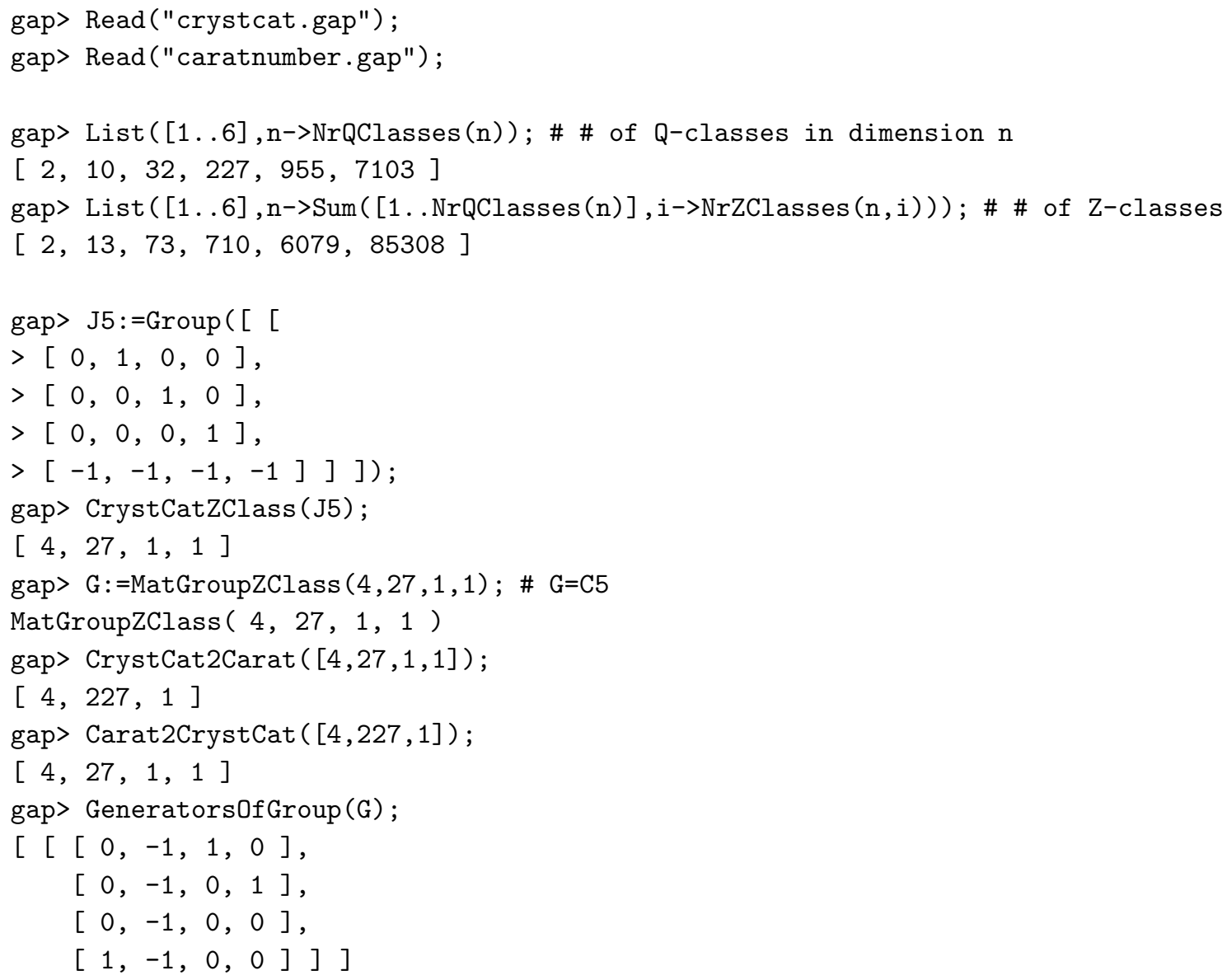




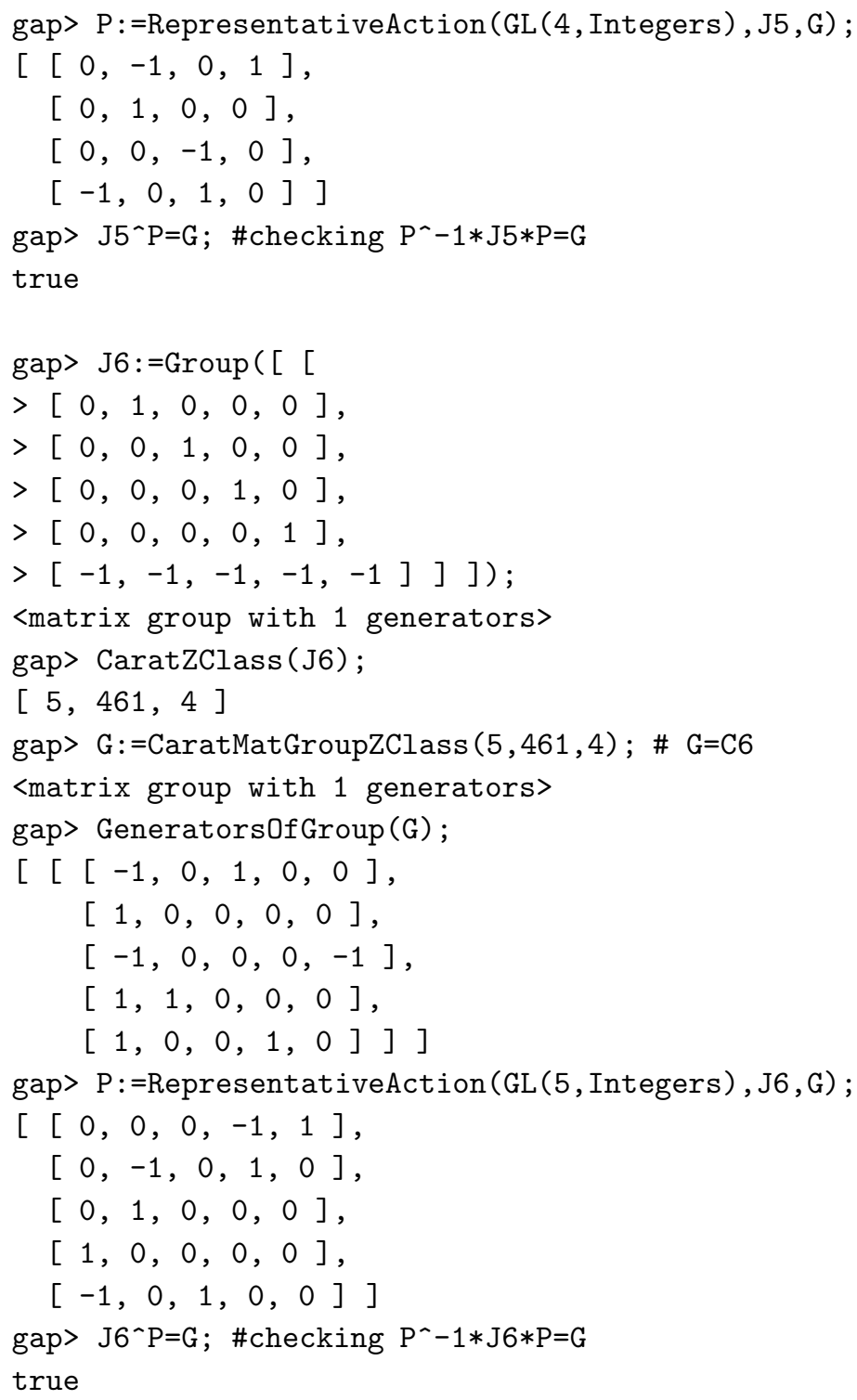

Some programs related to a flabby resolution are available from http://www.math.h.kyoto-u.ac.jp/ yamasaki/Algorithm/.

\section{KRULL-SCHMidT THEOREM FAILS FOR DIMENSION 5}

Let $G$ be a finite group and $\mathbb{Z}[G]$ be the integral group ring of $G$.

Definition 4.1 (Decomposable and reducible $G$-lattices). A $G$-lattice $M$ is said to be reducible if there exists a non-trivial $G$-invariant subspace of $M$. A $G$-lattice is said to be irreducible if it is not reducible. A $G$-lattice $M$ is said to be decomposable if there exist non-trivial $G$-lattices $U_{1}$ and $U_{2}$ such that $M \simeq U_{1} \oplus U_{2}$. A $G$-lattice is said to be indecomposable if it is not decomposable.

If a $G$-lattice $M$ is decomposable, then it is reducible. By Maschke's theorem, the converse holds for $\mathbb{Q}[G]$ modules, but not for $G$-lattices (i.e. finitely generated $\mathbb{Z}$-free $\mathbb{Z}[G]$-module).

We say that the direct sum cancellation holds for $G$-lattices if $M_{1} \oplus N \simeq M_{2} \oplus N$ implies $M_{1} \simeq M_{2}$ for $G$-lattices $M_{1}, M_{2}$ and $N$. We say that the Krull-Schmidt theorem holds for G-lattices if $M_{1} \oplus \cdots \oplus M_{l} \simeq N_{1} \oplus \cdots \oplus N_{m}$ for indecomposable $G$-lattices $M_{i}$ and $N_{j}$, then $l=m$ and, after a suitable renumbering of the $N_{j}, M_{i} \simeq N_{i}$ for any $1 \leq i \leq m$ (see Fac03]). Clearly, the Krull-Schmidt theorem holds for $G$-lattices implies that the direct sum cancellation holds for $G$-lattices.

By Krull-Schmidt-Azumaya theorem ([CR81, Theorem 6.12], see also [Azu50]), the Krull-Schmidt theorem holds for any $\mathbb{Z}_{p}[G]$-lattice where $\mathbb{Z}_{p}$ is the ring of $p$-adic integers. By [Jon65, Theorem 2] (see [CR81, Theorem $36.1]$ ), for $p$-group $G$ where $p$ is odd prime, the Krull-Schmidt theorem holds for $\mathbb{Z}_{(p)}[G]$-lattices where $\mathbb{Z}_{(p)}$ is 
the localization of $\mathbb{Z}$ at the prime ideal $(p)$. Note that the $G$-lattice $\mathbb{Z}[G / H]$ is indecomposable for any $H \leq G$ (see [CR87, Theorem 32.14]).

Endo and Hironaka [EH79, Theorem, page 161] (see [CR87, Theorem 50.29]) showed that if the direct sum cancellation holds for $G$-lattices, then $G$ is abelian, dihedral, $A_{4}, S_{4}$ or $A_{5}$ via the projective class group (see also Swa88, Corollary 1.3 and Section 7]). The question whether the Krull-Schmidt theorem holds for $G$-lattices is solved except for the case where $G$ is the dihedral group $D_{8}$ of order 16 (see also [Fac03, Theorem 9.1]).

Theorem 4.2 (Hindman, Klingler and Odenthal [HKO98, Theorem 1.6]). Let $G$ be a finite group which is not the dihedral group $D_{8}$ of order 16 . Then the Krull-Schmidt theorem holds for G-lattices if and only if one of the following conditions holds: (i) $G=C_{p}$ for prime $p \leq 19$; (ii) $G=C_{n}$ for $n=1,4,8$ or 9 ; (iii) $G=C_{2} \times C_{2}$; (iv) $G$ is the dihedral group $D_{4}$ of order 8 .

Two $G$-lattices $M, N$ are placed in the same genus $(M \approx N)$ if $M_{(p)} \simeq N_{(p)}$ for any prime ideal $(p)$. We say that the generalized Krull-Schmidt theorem holds for G-lattices if $M_{1} \oplus \cdots \oplus M_{l} \simeq N_{1} \oplus \cdots \oplus N_{m}$ for indecomposable $G$-lattices $M_{i}$ and $N_{j}$, then $l=m$ and, after a suitable renumbering of the $N_{j}, M_{i} \approx N_{i}$ for any $1 \leq i \leq m$. Clearly, the Krull-Schmidt theorem holds for $G$-lattices implies that the generalized Krull-Schmidt theorem holds for $G$-lattices. The following theorem was pointed out to the authors by S. Endo:

Theorem 4.3 (Dress [Dre70, Section 1]). Let $p$ be a prime number. The following conditions are equivalent:

(i) $G$ is a p-group;

(ii) the generalized Krull-Schmidt theorem holds for invertible G-lattices.

Proof. (This proof is due to S. Endo End12].) (i) $\Rightarrow$ (ii). If $p$ is odd prime, then the Krull-Schmidt theorem holds for $\mathbb{Z}_{(p)}[G]$-lattices. Assume that $G$ is a 2-group and $M$ is an invertible $G$-lattice. Since the Krull-Schmidt theorem holds for $\mathbb{Z}_{2}[G]$-lattices, $M_{2} \simeq \bigoplus \mathbb{Z}_{2}\left[G / H_{i}\right]$ where $M_{2}$ is the 2-adic completion of $M$ and $H_{i} \leq G$. It follows from Maranda's theorem CR81, Theorem 30.14] (see also [CR81, Proposition 30.17]) that $M_{(2)} \simeq \bigoplus \mathbb{Z}_{(2)}\left[G / H_{i}\right]$ where $M_{(2)}$ is the localization of $M$ at prime (2). The generalized Krull-Schmidt theorem holds for invertible $G$-lattices because $\mathbb{Z}_{(p)}[G / H]$ is indecomposable for any prime $p$ and any subgroup $H \leq G$.

(ii) $\Rightarrow$ (i). Assume that $G$ is not a $p$-group and $H \leq G$ is of order $p^{l} q^{m}$ where $p$ and $q$ are different primes and $l, m \geq 1$. Let $S y_{p}(H)$ be a $p$-Sylow subgroup of $H$. Then by [Dre70, Section 1] there exists $G$-lattice $M$ such that $\mathbb{Z}\left[H / S y_{p}(H)\right] \oplus \mathbb{Z}\left[H / S y_{q}(H)\right] \simeq M \oplus \mathbb{Z}$. By taking the tensor product $\mathbb{Z}[G] \otimes_{\mathbb{Z}[H]}$ of both sides, we get $\mathbb{Z}\left[G / S y_{p}(H)\right] \oplus \mathbb{Z}\left[G / S y_{q}(H)\right] \simeq \mathbb{Z}[G] \otimes_{\mathbb{Z}[H]} M \oplus \mathbb{Z}[G / H]$ as $G$-lattices. This contradicts that the generalized Krull-Schmidt theorem holds.

A $G$-set is a finite set with left $G$-action. The disjoint union $X \coprod X^{\prime}$ of $G$-sets $X$ and $X^{\prime}$ is also $G$-set. Two $G$-sets are isomorphic if there exists a bijection between them which preserves the action of $G$. A $G$-set $X$ may be written uniquely up to isomorphism as $X \simeq \bigsqcup_{H} a_{H}(X) G / H$ where $H$ runs through a set of representatives of conjugacy classes of subgroups of $G$ (see [CR87, Chapter 11], [Ben91, Chapter 5], [GW93, [Bou00] for related materials, e.g. Burnside ring). For $G$-sets $X$ and $X^{\prime}$, the direct sum of permutation $G$-lattices $\mathbb{Z}[X]$ and $\mathbb{Z}\left[X^{\prime}\right]$ is also permutation: $\mathbb{Z}[X] \oplus \mathbb{Z}\left[X^{\prime}\right] \simeq \mathbb{Z}\left[X \amalg X^{\prime}\right]$. A finite group $G$ is called cyclic $\bmod p$ (or $p$-hypoelementary) if the quotient group $G / O_{p}(G)$ is cyclic where $O_{p}(G)$ is the largest normal $p$-subgroup of $G$.

Theorem 4.4 (Dress [Dre73, Proposition 9.6]). Let $G$ be a finite group. The following conditions are equivalent:

(i) $G$ is cyclic mod $p$ for some $p$;

(ii) for any two $G$-sets $S, T, \mathbb{Z}[S] \simeq \mathbb{Z}[T]$ if and only if $S \simeq T$.

In particular, the Krull-Schmidt theorem holds for permutation G-lattices if and only if $G$ is cyclic mod $p$ for some $p$.

Using the algorithms in Sections [5 and 6, we will show the following proposition by constructing explicit isomorphism in Section 6 (see Example 6.8). We remark that the dihedral group $D_{6}$ of order 12 is the smallest group which is not cyclic mod $p$ for any $p$.

Proposition (The Krull-Schmidt theorem fails for permutation $D_{6}$-lattices, see Proposition 6.7). Let $D_{6}$ be the dihedral group of order 12 and $\{1\}, C_{2}^{(1)}, C_{2}^{(2)}, C_{2}^{(3)}, C_{3}, C_{2}^{2}, C_{6}, S_{3}^{(1)}, S_{3}^{(2)}$ and $D_{6}$ be the conjugacy classes of subgroups of $D_{6}$. Then the following isomorphism of permutation $D_{6}$-lattices holds:

$$
\begin{aligned}
& \mathbb{Z}\left[D_{6}\right] \oplus \mathbb{Z}\left[D_{6} / C_{2}^{2}\right]^{\oplus 2} \oplus \mathbb{Z}\left[D_{6} / C_{6}\right] \oplus \mathbb{Z}\left[D_{6} / S_{3}^{(1)}\right] \oplus \mathbb{Z}\left[D_{6} / S_{3}^{(2)}\right] \\
\simeq & \mathbb{Z}\left[D_{6} / C_{2}^{(1)}\right] \oplus \mathbb{Z}\left[D_{6} / C_{2}^{(2)}\right] \oplus \mathbb{Z}\left[D_{6} / C_{2}^{(3)}\right] \oplus \mathbb{Z}\left[D_{6} / C_{3}\right] \oplus \mathbb{Z}^{\oplus 2}
\end{aligned}
$$


Definition 4.5 (Decomposition type). Let $G$ be a finite group and $M$ be a $G$-lattice. When $M$ decomposes into indecomposable $G$-lattices $M \simeq U_{1} \oplus \cdots \oplus U_{r}$ of rank $n_{1}, \ldots, n_{r}$, we say that a decomposition type $\mathrm{DT}(M)$ of $M$ is $\left(n_{1}, \ldots, n_{r}\right)$. (This may not be unique.)

Let $G$ be a finite subgroup of $\operatorname{GL}(n, \mathbb{Z})$ and $M_{G}$ be the corresponding $G$-lattice of rank $n$ as in Definition 1.26 . The number of $G$-lattices $M_{G}$ for a given decomposition type $\mathrm{DT}\left(M_{G}\right)$ is as follows (see Example 4.9 below):

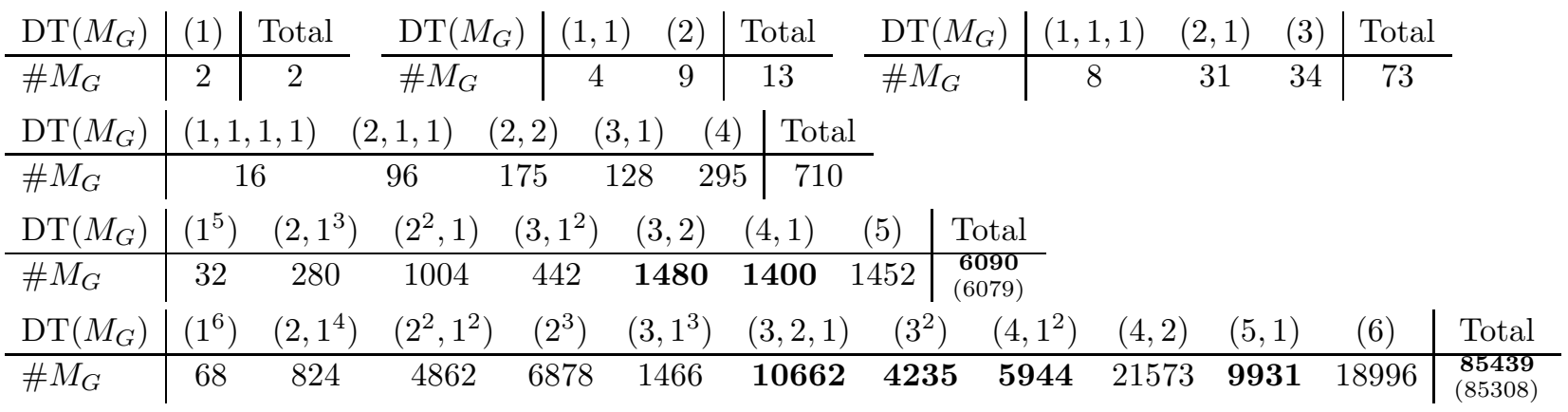

For $n \leq 4$, we see that the Krull-Schmidt theorem holds for $M_{G}$ of rank $n$, i.e. if $M_{G} \simeq M_{1} \oplus \cdots \oplus M_{l} \simeq$ $N_{1} \oplus \cdots \oplus N_{m}$ for indecomposable $G$-lattices $M_{i}$ and $N_{j}$, then $l=m$ and, after a suitable renumbering of the $N_{j}, M_{i} \simeq N_{i}$ for any $1 \leq i \leq m$. However, it turns out that the Krull-Schmidt theorem fails for $M_{G}$ when the rank $n$ of $M_{G}$ is 5 . We split the Krull-Schmidt theorem for $M_{G}$ into the following two parts:

(KS1) If $M_{G} \simeq M_{1} \oplus \cdots \oplus M_{l} \simeq N_{1} \oplus \cdots \oplus N_{m}$ for indecomposable $G$-lattices $M_{i}$ and $N_{j}$, then $l=m$ and, after a suitable renumbering of the $N_{j}$, rank $M_{i}=\operatorname{rank} N_{i}$ for any $1 \leq i \leq m$;

(KS2) If $M_{G} \simeq M_{1} \oplus \cdots \oplus M_{m} \simeq N_{1} \oplus \cdots \oplus N_{m}$ for indecomposable $G$-lattices $M_{i}$ and $N_{i}$ with rank $M_{i}=$ rank $N_{i}$ for any $1 \leq i \leq m$, then after a suitable renumbering of the $N_{i}, M_{i} \simeq N_{i}$ for any $1 \leq i \leq m$.

The Krull-Schmidt theorem holds for $M_{G}$ if and only if the conditions (KS1) and (KS2) hold for $M_{G}$.

Theorem 4.6. Let $G$ be a finite subgroup of $\mathrm{GL}(n, \mathbb{Z})$ and $M_{G}$ be the $G$-lattice as in Definition 1.26]. (i) When $n \leq 4$, the Krull-Schmidt theorem holds for $M_{G}$, i.e. if $M_{G} \simeq M_{1} \oplus \cdots \oplus M_{l} \simeq N_{1} \oplus \cdots \oplus N_{m}$ for indecomposable $G$-lattices $M_{i}$ and $N_{j}$, then $l=m$ and, after a suitable renumbering of the $N_{j}, M_{i} \simeq N_{i}$ for any $1 \leq i \leq m$.

(ii) When $n=5$, (KS2) holds for $M_{G}$, and the Krull-Schmidt theorem fails for $M_{G}$ if and only if (KS1) fails for $M_{G}$ if and only if the CARAT ID of $G$ is one of the 11 triples

$(5,188,4),(5,189,4),(5,190,6),(5,191,6),(5,192,6),(5,193,4),(5,205,6),(5,218,8),(5,219,8),(5,220,4),(5,221,4)$.

For the exceptional 11 cases, the decomposition types of $M_{G}$ are $(3,2)$ and $(4,1)$ and $G$ is a subgroup of the group $C_{2} \times D_{6}$ of the CARAT ID $(5,205,6)$.

(iii) When $n=6,(\mathrm{KS} 1)$ fails for $M_{G}$ if and only if the CARAT ID of $G$ is one of the 131 triples

$(6,2013,8),(6,2018,4),(6,2023,6),(6,2024,6),(6,2025,6),(6,2026,6),(6,2033,6),(6,2042,8),(6,2043,8),(6,2044,4)$,

$(6,2045,4),(6,2048,5),(6,2049,8),(6,2050,8),(6,2051,8),(6,2052,8),(6,2058,5),(6,2059,5),(6,2067,5),(6,2068,5)$,

$(6,2069,5),(6,2069,11),(6,2070,9),(6,2071,9),(6,2072,10),(6,2072,11),(6,2076,24),(6,2076,25),(6,2077,24),(6,2077,25)$,

$(6,2078,24),(6,2078,25),(6,2079,24),(6,2079,25),(6,2087,15),(6,2088,15),(6,2089,17),(6,2089,18),(6,2094,9),(6,2102,24)$,

$(6,2102,25),(6,2105,9),(6,2106,9),(6,2107,10),(6,2107,11),(6,2108,15),(6,2109,15),(6,2110,17),(6,2110,18),(6,2111,15)$,

$(6,2139,9)$,

$(6,40,4),(6,41,4),(6,44,6),(6,45,6),(6,47,4),(6,53,4),(6,54,4),(6,54,8),(6,55,4),(6,63,4)$,

$(6,64,6),(6,65,4),(6,66,6),(6,67,6),(6,75,4),(6,75,8),(6,76,8),(6,76,12),(6,77,8),(6,77,12)$,

$(6,78,4),(6,78,8),(6,79,6),(6,80,4),(6,81,8),(6,81,12),(6,90,4),(6,99,4),(6,108,4),(6,108,8)$,

$(6,109,8),(6,109,12),(6,110,4),(6,111,6),(6,112,8),(6,112,12),(6,113,4),(6,114,6),(6,115,6),(6,145,4)$,

$(6,2070,10),(6,2070,11),(6,2071,10),(6,2071,11),(6,2072,12),(6,2072,13),(6,2076,26),(6,2076,27),(6,2077,26),(6,2077,27)$,

$(6,2078,26),(6,2078,27),(6,2079,26),(6,2079,27),(6,2087,16),(6,2087,17),(6,2088,16),(6,2088,17),(6,2089,19),(6,2089,20)$,

$(6,2094,10),(6,2094,11),(6,2102,26),(6,2102,27),(6,2105,10),(6,2105,11),(6,2106,10),(6,2106,11),(6,2107,12),(6,2107,13)$,

$(6,2108,16),(6,2108,17),(6,2109,16),(6,2109,17),(6,2110,19),(6,2110,20),(6,2111,16),(6,2111,17),(6,2139,10),(6,2139,11)$. 
For the former 51 cases (resp. the latter 80 cases), the decomposition types of $M_{G}$ are $(3,2,1)$ and $(4,1,1)$ (resp. $(3,3)$ and $(5,1))$ and $G$ is a subgroup of the group $C_{2}^{2} \times D_{6}$ of the CARAT ID $(6,2139,9)$ (resp. $D_{6} \times D_{4}$ of the CARAT ID $(6,145,4))$.

(iv) When $n=6,(\mathrm{KS} 2)$ fails for $M_{G}$ if and only if the CARAT ID of $G$ is one of the 18 triples

$(6,2072,14),(6,2076,28),(6,2077,28),(6,2078,28),(6,2079,28),(6,2089,21),(6,2102,28),(6,2107,14),(6,2110,21),(6,2295,2)$,

$(6,3045,3),(6,3046,3),(6,3047,3),(6,3052,5),(6,3053,5),(6,3054,3),(6,3061,5),(6,3066,3)$.

For the former 10 cases, the decomposition type of $M_{G}$ is $(4,2)$ and $G$ is the group $D_{6}$ of the CARAT ID $(6,2295,2)$ or a subgroup of the 3 groups $C_{2} \times D_{6}$ of the CARAT IDs $(6,2102,28),(6,2107,14)$ and $(6,2110,21)$. For the latter 8 cases, the decomposition type of $M_{G}$ is $(5,1)$ and $G$ is a subgroup of the group $C_{2} \times S_{5}$ of the CARAT ID $(6,3054,3)$.

4.0. Classification of indecomposable maximal finite groups $G \leq \operatorname{GL}(n, \mathbb{Z})$ of dimension $n \leq 6$. Let $G \leq \mathrm{GL}(n, \mathbb{Z})$ be a finite matrix group. $G$ is called reducible (resp. irreducible, decomposable, indecomposable) if $M_{G}$ is reducible (resp. irreducible, decomposable, indecomposable) where $M_{G}$ is the corresponding $G$-lattice as in Definition 1.26, Let $\operatorname{Imf}(n, i, j) \leq \mathrm{GL}(n, \mathbb{Z})$ be the $j$-th $\mathbb{Z}$-class of the $i$-th $\mathbb{Q}$-class of the irreducible maximal finite group of dimension $n$ which corresponds to the build-in function $\operatorname{ImfMatrixGroup}(n, i, j)$ of GAP. For $n \leq 10$ and $n=p \leq 23$; prime, the irreducible maximal finite groups $\operatorname{Imf}(n, i, j)$ is determined by Plesken and Pohst [PP77] $(n \leq 7)$, PP80] $(n=8,9)$, Plesken [Ple85] $(n=p \leq 23$; prime) and Souvignier [Sou94] $(n=10)$.

For $n=2$, there exist exactly 2 irreducible maximal finite groups (Z्Z-classes) $\operatorname{Imf}(2,1,1) \simeq D_{4}$ and $\operatorname{Imf}(2,2,1) \simeq$ $D_{6}$ of order 8 and 12 of the GAP IDs $(2,3,2,1)$ and $(2,4,4,1)$.

For $n=3$, there exist exactly 3 irreducible maximal finite groups $\operatorname{Imf}(3,1,1) \simeq \operatorname{Imf}(3,1,2) \simeq \operatorname{Imf}(3,1,3) \simeq$ $C_{2} \times S_{4}$ of order 48 of the GAP IDs $(3,7,5,1),(3,7,5,2)$ and $(3,7,5,3)$.

For $n=4$, there exist exactly 6 irreducible maximal finite groups $\operatorname{Imf}(4,1,1), \operatorname{Imf}(4,2,1) \simeq D_{6}^{2} \rtimes C_{2}$, $\operatorname{Imf}(4,3,1) \simeq \operatorname{Imf}(4,3,2) \simeq C_{2} \times S_{5}, \operatorname{Imf}(4,4,1) \simeq C_{2}^{4} \rtimes S_{4}$ and $\operatorname{Imf}(4,5,1) \simeq C_{2} \times\left(S_{3}^{2} \rtimes C_{2}\right)$ of order 1152, $288,240,240,384$ and 144 of the GAP IDs $(4,33,16,1),(4,30,13,1),(4,31,7,1),(4,31,7,2),(4,32,21,1)$ and $(4,29,9,1)$ respectively. Note that the first one is isomorphic to the Wyle group $W\left(F_{4}\right)$ of type $F_{4}$ of order 1152 .

For $n=5$, there exist exactly 7 irreducible maximal finite groups $\operatorname{Imf}(5,1,1) \simeq \operatorname{Imf}(5,1,2) \simeq \operatorname{Imf}(5,1,3) \simeq$ $C_{2}^{5} \rtimes S_{5}$ of order 3840 of the CARAT IDs $(5,942,1),(5,942,2),(5,942,3)$ and $\operatorname{Imf}(5,2,1) \simeq \operatorname{Imf}(5,2,2) \simeq$ $\operatorname{Imf}(5,2,3) \simeq \operatorname{Imf}(5,2,4) \simeq C_{2} \times S_{6}$ of order 1440 of the CARAT IDs $(5,949,1),(5,949,4),(5,949,2),(5,949,3)$.

For $n=6$, there exist exactly 17 irreducible maximal finite groups $\operatorname{Imf}(6,1,1) \simeq \operatorname{Imf}(6,1,2) \simeq \operatorname{Imf}(6,1,3) \simeq$ $C_{2}^{6} \rtimes S_{6}, \operatorname{Imf}(6,2,1) \simeq D_{6}^{3} \rtimes S_{3}, \operatorname{Imf}(6,3,1) \simeq \operatorname{Imf}(6,3,2), \operatorname{Imf}(6,4,1) \simeq \operatorname{Imf}(6,4,2) \simeq C_{2} \times S_{7}, \operatorname{Imf}(6,5,1) \simeq$ $C_{2} \times \operatorname{PGL}\left(2, \mathbb{F}_{7}\right), \operatorname{Imf}(6,6,1) \simeq \operatorname{Imf}(6,6,2) \simeq \operatorname{Imf}(6,6,3) \simeq C_{2} \times S_{5}, \operatorname{Imf}(6,7,1) \simeq \operatorname{Imf}(6,7,2) \simeq\left(C_{2} \times S_{4}\right)^{2} \rtimes C_{2}$, $\operatorname{Imf}(6,8,1) \simeq\left(C_{2}^{5} \rtimes A_{6}\right) \rtimes C_{2}, \operatorname{Imf}(6,9,1) \simeq \operatorname{Imf}(6,9,2) \simeq D_{6} \times S_{4}$ of order 46080, 46080, 46080, 10368, 103680, 103680, 10080, 10080, 672, 240, 240, 240, 4608, 4608, 23040, 288 and 288 of the CARAT IDs $(6,2773,1)$, $(6,2773,3),(6,2773,2),(6,2803,1),(6,2804,2),(6,2804,1),(6,2932,1),(6,2932,2),(6,2945,1),(6,2952,1)$, $(6,2952,3),(6,2952,2),(6,2772,2),(6,2772,5),(6,2750,4),(6,2866,2)$ and $(6,2866,3)$ respectively.

Let $\operatorname{Indmf}(n, i, j) \leq \mathrm{GL}(n, \mathbb{Z})$ be the $j$-th $\mathbb{Z}$-class of the $i$-th $\mathbb{Q}$-class of the indecomposable maximal finite group of dimension $n$. We see that all the groups $\operatorname{Indmf}(n, i, j)$ coincide with $\operatorname{Imf}(n, i, j)$ for $n \leq 5$. However, this is not true for $n=6$. This phenomenon is suggested by [Ple78, Section V] (see also [PH84, Section V]). Indeed, it turns out that we need the one additional group $\operatorname{Indmf}(6,10,1) \simeq\left(C_{2} \times S_{4}\right)^{2}$ of order 2304 of the CARAT ID $(6,5517,4)$ in order to get all the indecomposable maximal finite groups. Namely, there exist exactly 18 indecomposable maximal finite groups of dimension 6 .

In summary, there exist exactly 2 (resp. $3,6,7,17)$ irreducible maximal finite groups $G \leq \mathrm{GL}(n, \mathbb{Z})$ (Z्Z-classes) and 2 (resp. 3, 6, 7, 18) indecomposable maximal finite groups $G \leq \mathrm{GL}(n, \mathbb{Z})$ (Z्Z-classes) of dimension 2 (resp. $3,4,5,6)$.

We will check this in the next subsection (see Example4.9 in Subsection 4.1 and Example 9.2). The algorithms given in this section are available from http://math.h.kyoto-u.ac.jp/ ^yamasaki/Algorithm/ as KS.gap.

$\operatorname{IndmfMatrixGroup}(n, i, j) \operatorname{returns} \operatorname{Indmf}(n, i, j)$ of dimension $n$ (this works only for $n \leq 6$ ).

IndmfNumberQClasses $(n)$ returns the number of $\mathbb{Q}$-classes of all the indecomposable maximal finite groups of dimension $n$ (this works only for $n \leq 6$ ).

Indmf NumberZClasses $(\mathrm{n}, \mathrm{i})$ returns the number of $\mathbb{Z}$-classes in the $i$-th $\mathbb{Q}$-class of the indecomposable maximal finite groups $\operatorname{Imf}(n, i, j)$ of dimension $n$ (this works only for $n \leq 6)$. 
AllImfMatrixGroups (n) returns all the irreducible maximal finite groups of dimension $n$.

AllIndmfMatrixGroups $(n)$ returns all the indecomposable maximal finite groups of dimension $n$ (this works only for $n \leq 6)$.

Algorithm Indmf (Constructing all the indecomposable maximal finite groups (Indmf) of dimension $n \leq 6$ ). (The following algorithm needs the CARAT package of GAP and caratnumber.gap).

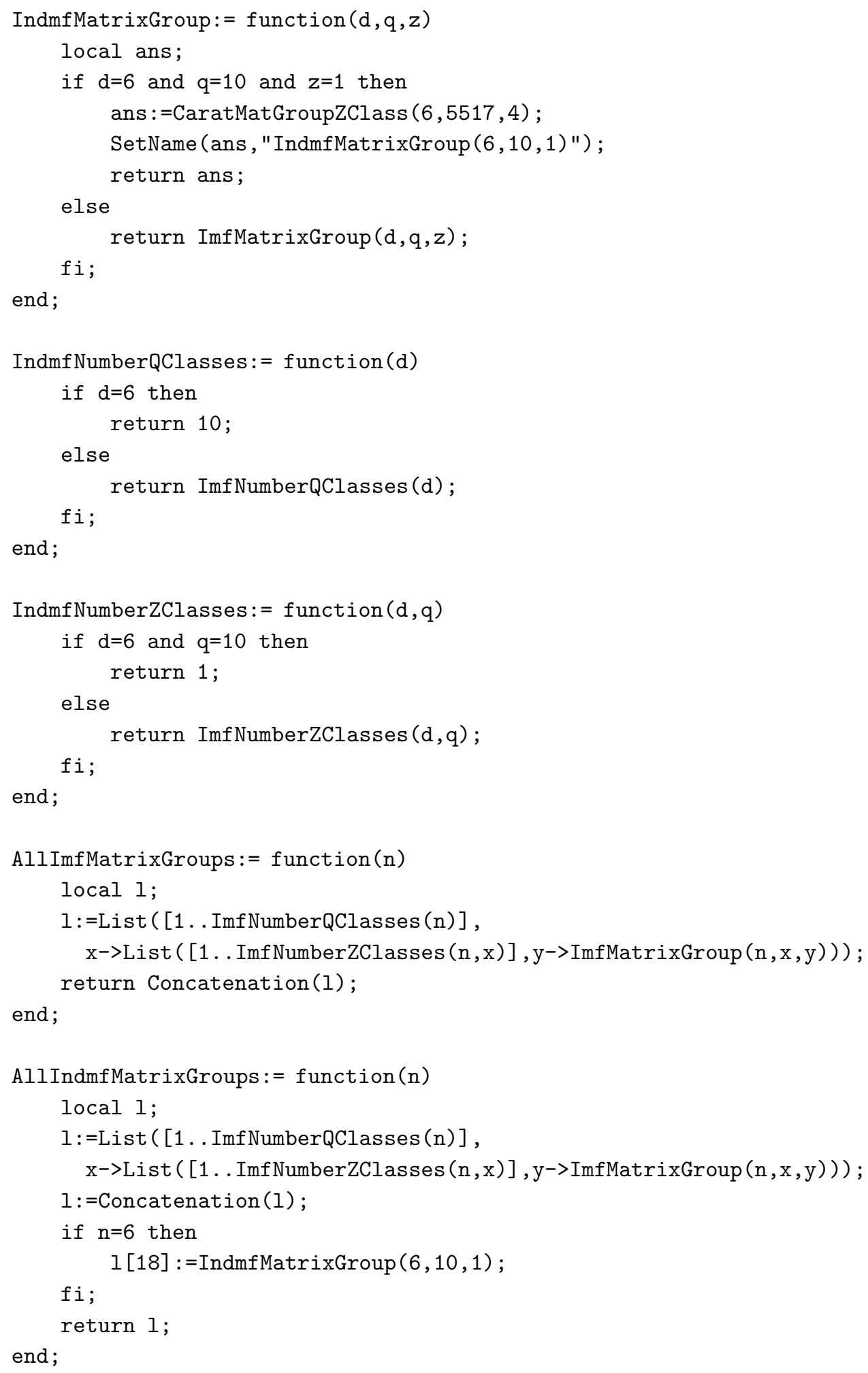

Example 4.7 (All the indecomposable maximal finite groups of dimension $n \leq 6$ ). 
gap> Imf 2:=AllImfMatrixGroups(2); \# Imf2=Indmf2: 2 groups

[ $\operatorname{ImfMatrixGroup}(2,1,1), \operatorname{ImfMatrix} \operatorname{Mroup}(2,2,1)]$

gap> Length $(\operatorname{Imf} 2)$;

2

gap> List (Imf2, CrystCatZClass);

$[[2,3,2,1],[2,4,4,1]]$

gap> List (Imf2,Size);

$[8,12]$

gap> List ([1...ImfNumberQClasses (2)]

$>\mathrm{x}->\operatorname{List}([1 \ldots \operatorname{ImfNumberZClasses}(2, \mathrm{x})], \mathrm{y}->\operatorname{Imf} \operatorname{Invariants}(2, \mathrm{x}, \mathrm{y})$.isomorphismType));

[ [ "C2 wr C2 = D8"], [ "C2 x S3 = C2 x W(A2) = D12" ] ]

gap> Imf 3:=AllImfMatrixGroups (3); \# Imf3=Indmf3: 3 groups

[ ImfMatrixGroup $(3,1,1), \operatorname{ImfMatrixGroup}(3,1,2), \operatorname{ImfMatrixGroup}(3,1,3)]$

gap> Length $(\operatorname{Imf} 3)$;

3

gap> List (Imf 3, CrystCatZClass);

$[[3,7,5,1],[3,7,5,2],[3,7,5,3]]$

gap> List (Imf3,Size);

[ $48,48,48]$

gap> List ([1...ImfNumberQClasses (3)],

$>\mathrm{x}->\operatorname{List}([1 \ldots \operatorname{ImfNumberZClasses}(3, \mathrm{x})], \mathrm{y}->\operatorname{Imf} \operatorname{Invariants}(3, \mathrm{x}, \mathrm{y})$.isomorphismType)) ;

[ [ "C2 $\mathrm{wr} \mathrm{S} 3=\mathrm{C} 2 \mathrm{x} \mathrm{S} 4=\mathrm{W}(\mathrm{B} 3) "$, "C2 $\mathrm{wr} \mathrm{S} 3=\mathrm{C} 2 \times \mathrm{S} 4=\mathrm{C} 2 \mathrm{x} \mathrm{W}(\mathrm{A} 3) "$,

"C2 wr S3 = C2 x S4 = C2 $2 \mathrm{~W}(\mathrm{~A} 3) "$ ] ]

gap> Imf 4:=AllImfMatrixGroups(4); \# Imf4=Indmf 4: 6 groups

[ ImfMatrixGroup (4,1,1), ImfMatrixGroup (4,2,1), $\operatorname{ImfMatrixGroup}(4,3,1)$,

$\operatorname{ImfMatrixGroup}(4,3,2), \operatorname{ImfMatrixGroup}(4,4,1), \operatorname{ImfMatrixGroup}(4,5,1)]$

gap> Length $(\operatorname{Imf} 4)$;

6

gap> List ( Imf 4, CrystCatZClass);

$[[4,33,16,1],[4,30,13,1],[4,31,7,1]$,

$[4,31,7,2],[4,32,21,1],[4,29,9,1]]$

gap> List(Imf4,Size); \# the first one is the Wyle group W(F4) of type F4

[ $1152,288,240,240,384,144$ ]

gap> List ([1...ImfNumberQClasses (4)],

$>\mathrm{x}->\operatorname{List}\left([1 \ldots \operatorname{ImfNumberZClasses}(4, \mathrm{x})], \mathrm{y}^{->} \operatorname{Imf} \operatorname{Invariants}(4, \mathrm{x}, \mathrm{y})\right.$. isomorphismType)) ;

[ [ "W(F4)"], [ "D12 wr C2 = (C2 x W(A2)) wr C2" ],

[ "C2 2 S5 = C2 $\mathrm{xW}(\mathrm{A} 4) ", " \mathrm{C} 2 \times \mathrm{S} 5=\mathrm{C} 2 \mathrm{xW}(\mathrm{A} 4) "],[" \mathrm{C} 2 \mathrm{wr} \mathrm{S} 4=\mathrm{W}(\mathrm{B} 4) "]$,

[ "(D12 Y D12):C2" ] ]

gap> Imf 5:=AllImfMatrixGroups (5); \# Imf5=Indmf5: 7 groups

[ ImfMatrixGroup $(5,1,1), \operatorname{ImfMatrixGroup}(5,1,2), \operatorname{ImfMatrixGroup}(5,1,3)$, $\operatorname{ImfMatrixGroup}(5,2,1), \operatorname{ImfMatrixGroup}(5,2,2), \operatorname{ImfMatrixGroup}(5,2,3)$,

$\operatorname{ImfMatrixGroup}(5,2,4)]$

gap> Length $(\operatorname{Imf} 5)$;

7

gap> List ( Imf 5, CaratZClass);

$[[5,942,1],[5,942,2],[5,942,3]$,

$[5,949,1],[5,949,4],[5,949,2]$,

$[5,949,3]]$

gap> List ( $\operatorname{Imf5,Size);~}$

[ $3840,3840,3840,1440,1440,1440,1440$ ]

gap> List ([1...ImfNumberQClasses (5)],

$>\mathrm{x}->\operatorname{List}\left([1 \ldots \operatorname{ImfNumberZClasses}(5, \mathrm{x})], \mathrm{y}^{->} \operatorname{Imf} \operatorname{Invariants}(5, \mathrm{x}, \mathrm{y})\right.$. isomorphismType $\left.)\right)$; 
[ [ "C2 $\mathrm{wr} \mathrm{S} 5=\mathrm{W}(\mathrm{B} 5) ", " \mathrm{C} 2 \mathrm{wr} \mathrm{S} 5=\mathrm{C} 2 \mathrm{x} \mathrm{W}(\mathrm{D} 5) ", " \mathrm{C} 2$ wr S5 = C2 $\mathrm{x}$ W(D5)" ], [ "C2 x S6", "C2 x S6", "C2 x S6", "C2 x S6" ] ]

gap> Imf6:=AllImfMatrixGroups (6); \# Imf6: 17 groups

[ $\operatorname{ImfMatrixGroup}(6,1,1), \operatorname{ImfMatrixGroup}(6,1,2), \operatorname{ImfMatrixGroup}(6,1,3)$, $\operatorname{ImfMatrixGroup}(6,2,1), \operatorname{ImfMatrixGroup}(6,3,1), \operatorname{ImfMatrixGroup}(6,3,2)$, $\operatorname{ImfMatrixGroup}(6,4,1), \operatorname{ImfMatrixGroup}(6,4,2), \operatorname{ImfMatrixGroup}(6,5,1)$, $\operatorname{ImfMatrixGroup}(6,6,1), \operatorname{ImfMatrixGroup}(6,6,2), \operatorname{ImfMatrixGroup}(6,6,3)$, $\operatorname{ImfMatrixGroup}(6,7,1), \operatorname{ImfMatrixGroup}(6,7,2), \operatorname{ImfMatrixGroup}(6,8,1)$, $\operatorname{ImfMatrixGroup}(6,9,1), \operatorname{ImfMatrixGroup}(6,9,2)]$

gap> Length $(\operatorname{Imf} 6)$;

17

gap> List (Imf6, CaratZClass);

$[[6,2773,1],[6,2773,3],[6,2773,2]$, $[6,2803,1],[6,2804,2],[6,2804,1]$, $[6,2932,1],[6,2932,2],[6,2945,1]$, $[6,2952,1],[6,2952,3],[6,2952,2]$, $[6,2772,2],[6,2772,5],[6,2750,4]$, $[6,2866,2],[6,2866,3]]$

gap> List $(\operatorname{Imf} 6$, Size) ;

[ $46080,46080,46080,10368,103680,103680,10080,10080,672,240$, $240,240,4608,4608,23040,288,288]$

gap> List ([1..ImfNumberQClasses (6)], $>\mathrm{x}->\operatorname{List}([1 \ldots \operatorname{ImfNumberZClasses}(6, \mathrm{x})], \mathrm{y}->\operatorname{Imf} \operatorname{Invariants}(6, \mathrm{x}, \mathrm{y})$.isomorphismType));

[ [ "C2 $\mathrm{wr} \mathrm{S} 6=\mathrm{W}(\mathrm{B} 6) ", " \mathrm{C} 2$ wr S6 = C2 x W(D6)", "C2 wr S6 = C2 x W(D6)"], [ "(C2 x S3) wr S3 = (C2 x W(A2)) wr S3 = D12 wr S3" ], [ "C2 x W(E6)", "C2 x W(E6)" ], [ "C2 x S7 = C2 $2 \mathrm{~W}(\mathrm{~A} 6) ", " \mathrm{C} 2 \times \mathrm{S} 7=\mathrm{C} 2 \times \mathrm{W}(\mathrm{A} 6) "],[$ "C2 $\mathrm{x}$ PGL $(2,7) "]$, [ "C2 x S5", "C2 x S5", "C2 x S5"], [ "(C2 x S4) wr C2 = (C2 x W(A3)) wr C2", "(C2 x S4) wr C2 = (C2 x W(A3)) wr C2" ], [ "subgroup of index 2 of $\mathrm{C} 2 \mathrm{wr} \mathrm{S} 6 "$ ],

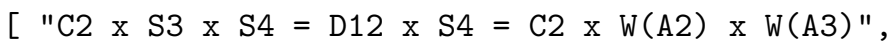
$" \mathrm{C} 2 \times \mathrm{S} 3 \times \mathrm{S} 4=\mathrm{D} 12 \times \mathrm{S} 4=\mathrm{C} 2 \times \mathrm{W}(\mathrm{A} 2) \times \mathrm{W}(\mathrm{A} 3) "]]$

gap> Indmf 2:=AllIndmfMatrixGroups (2); ;

gap> Indmf 3:=AllIndmfMatrixGroups (3); ;

gap> Indmf 4:=AllIndmfMatrixGroups (4); ;

gap> Indmf 5:=AllIndmfMatrixGroups (5); ;

gap> Indmf 6:=AllIndmfMatrixGroups (6); ;

gap $>$ List ([Indmf $2, \operatorname{Indmf} 3, \operatorname{Indmf} 4, \operatorname{Indmf} 5, \operatorname{Indmf} 6]$, Length) ;

$[2,3,6,7,18]$

gap $>[\operatorname{Imf} 2=\operatorname{Indmf} 2, \operatorname{Imf} 3=\operatorname{Indmf} 3, \operatorname{Imf} 4=\operatorname{Indmf} 4, \operatorname{Imf} 5=\operatorname{Indmf} 5, \operatorname{Imf} 6=\operatorname{Indmf} 6]$;

[ true, true, true, true, false ]

gap> Indmf6; \# Indmf6: $18(=17+1)$ groups

[ $\operatorname{ImfMatrixGroup}(6,1,1), \operatorname{ImfMatrixGroup}(6,1,2), \operatorname{ImfMatrixGroup}(6,1,3)$, $\operatorname{ImfMatrixGroup}(6,2,1), \operatorname{ImfMatrixGroup}(6,3,1), \operatorname{ImfMatrixGroup}(6,3,2)$, $\operatorname{ImfMatrixGroup}(6,4,1), \operatorname{ImfMatrixGroup}(6,4,2), \operatorname{ImfMatrixGroup}(6,5,1)$, $\operatorname{ImfMatrixGroup}(6,6,1), \operatorname{ImfMatrixGroup}(6,6,2), \operatorname{ImfMatrixGroup}(6,6,3)$, $\operatorname{ImfMatrixGroup}(6,7,1), \operatorname{ImfMatrixGroup}(6,7,2), \operatorname{ImfMatrixGroup}(6,8,1)$, $\operatorname{ImfMatrixGroup}(6,9,1), \operatorname{ImfMatrixGroup}(6,9,2), \operatorname{IndmfMatrixGroup}(6,10,1)]$ gap> CaratZClass (IndmfMatrixGroup $(6,10,1))$;

$[6,5517,4]$

gap> Size (IndmfMatrixGroup $(6,10,1))$;

2304

gap> StructureDescription (IndmfMatrixGroup $(6,10,1))$; 
4.1. Krull-Schmidt theorem (1). We will determine all the possible decompositions of $M_{G}$ into indecomposable ones for all finite subgroups $G$ of $\operatorname{GL}(n, \mathbb{Z})$ with $n \leq 6$ (see Examples 4.9 and Examples 4.13 below). Note that if a $G$-lattice $M$ splits into indecomposable $G$-lattices $M_{1}$ and $M_{2}$ of $\operatorname{rank} i$ and $j$, then $G$ is a subgroup of $G_{1} \times G_{2}$ where $G_{1}$ and $G_{2}$ are some indecomposable maximal finite groups of $\mathrm{GL}(i, \mathbb{Z})$ and $\operatorname{GL}(j, \mathbb{Z})$ respectively.

LatticeDecompositions(n) returns the list $\mathcal{L}=\left\{l_{1}, \ldots, l_{s}\right\}$ of the lists $l_{i}$ of the GAP IDs whose $i$-th list $l_{i}$ contains all the GAP IDs of the groups $G$ whose corresponding $G$-lattice $M_{G}$ is decomposable into the indecomposable $G$-lattices $M \simeq M_{1} \oplus \cdots \oplus M_{m}$ with rank $M_{j}=r_{j}$ where $\left(r_{1}, \ldots, r_{m}\right)$ corresponds to the $i$-th partitions Partitions(n) [i] of the integer $2 \leq n \leq 4$.

LatticeDecompositions ( $\mathrm{n}$ :Carat) returns the same as LatticeDecompositions ( $\mathrm{n}$ ) but using the CARAT ID instead of the GAP ID. This algorithm is valid for $1 \leq n \leq 6$.

LatticeDecompositions ( $\mathrm{n}$ :Carat, FromPerm) returns the same as LatticeDecompositions ( $\mathrm{n}$ :Carat) but using ConjugacyClassesSubgroupsFromPerm(G) instead of ConjugacyClassesSubgroups2(G) (see below).

In order to construct conjugacy classes of subgroups of a group $G$, we use the following GAP function ConjugacyClassesSubgroups2(G) because the ordering of the conjugacy classes of subgroups of $G$ by the built-in function ConjugacyClassesSubgroups $(G)$ is not fixed for some groups.

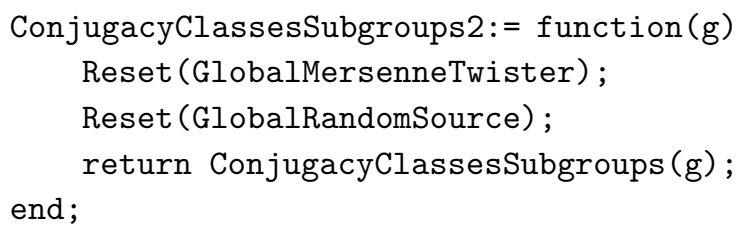

If a group $G$ is too big, ConjugacyClassesSubgroups2(G) may not work well. For example, we should use ConjugacyClassesSubgroupsFromPerm $(G)$ instead of ConjugacyClassesSubgroups2(G) for the 2nd $\mathbb{Z}$-class of the 1st $\mathbb{Q}$-class of the irreducible maximal finite group $\operatorname{Imf}(6,1,2) \simeq C_{2}^{6} \rtimes S_{6}$ of dimension 6 of order 46080 .

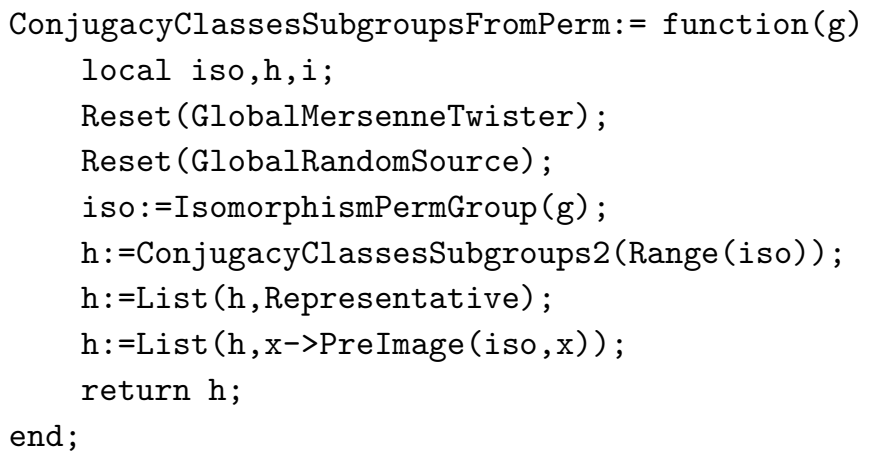

Algorithm KS1 (All the decomposable $G$-lattices $M_{G}$ of rank $n$ ). (The following algorithm needs the CARAT package of GAP and caratnumber.gap).

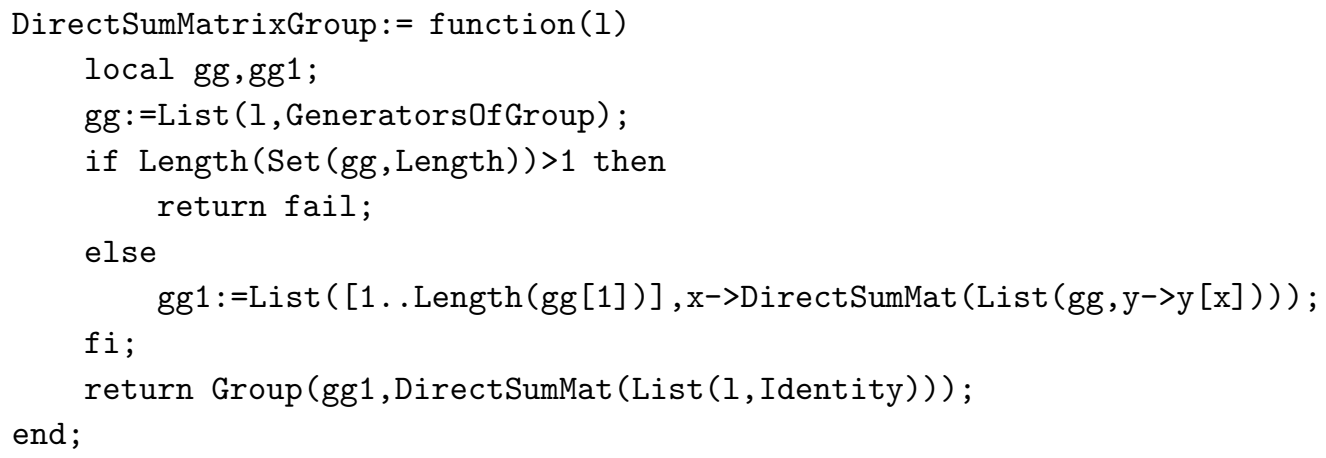




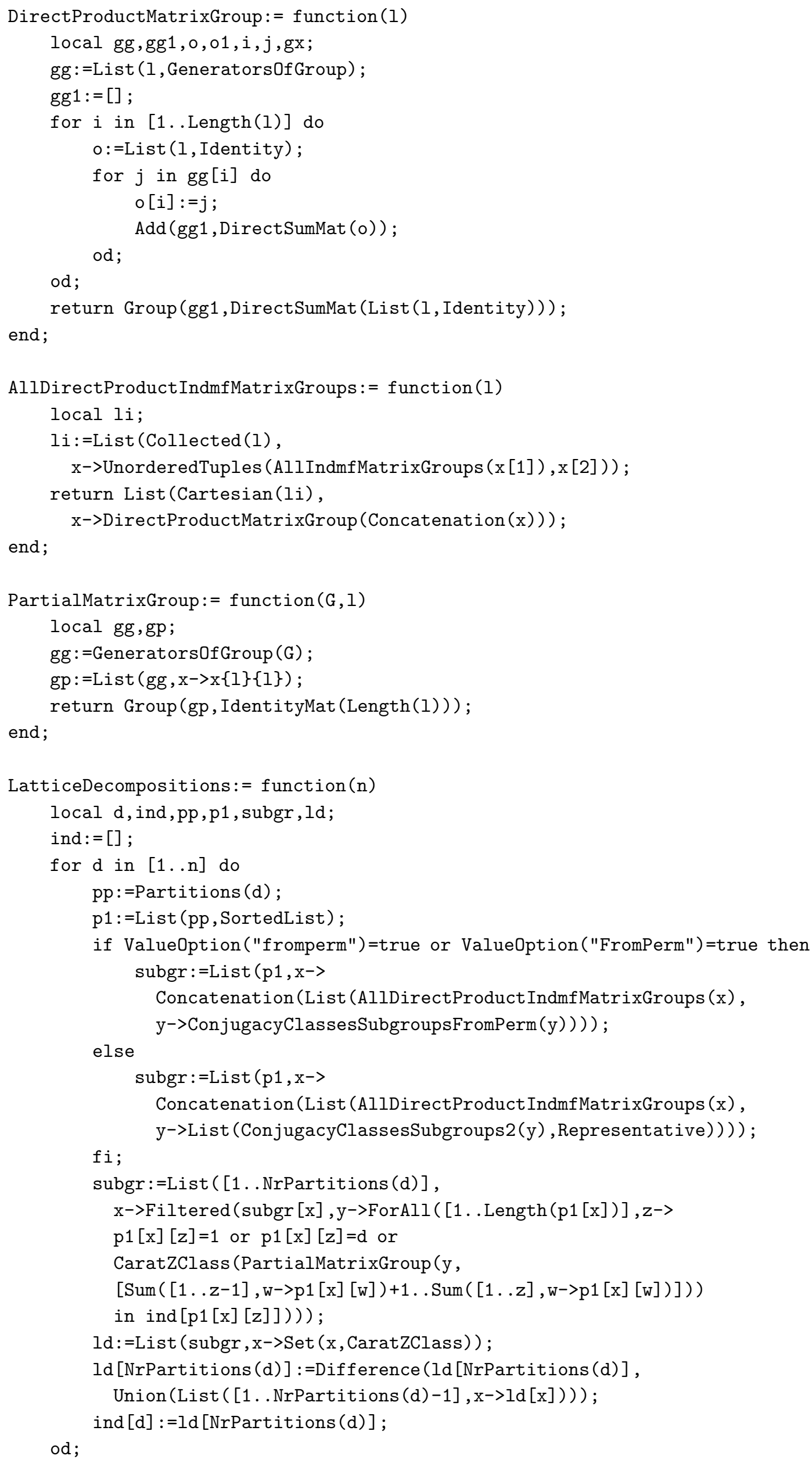




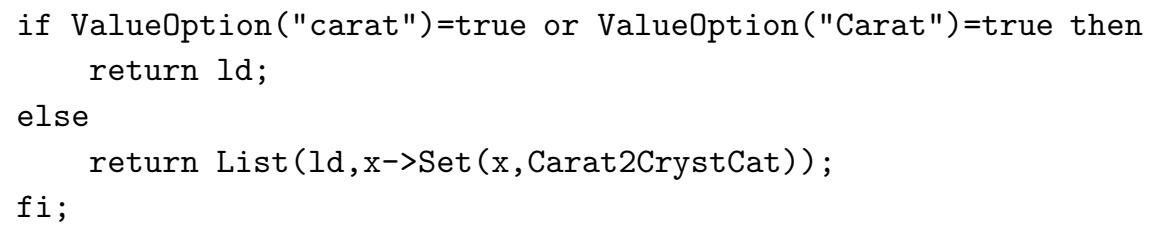

Example 4.8 (Functions DirectSumMatrixGroup(1) and DirectProductMatrixGroup (1)).

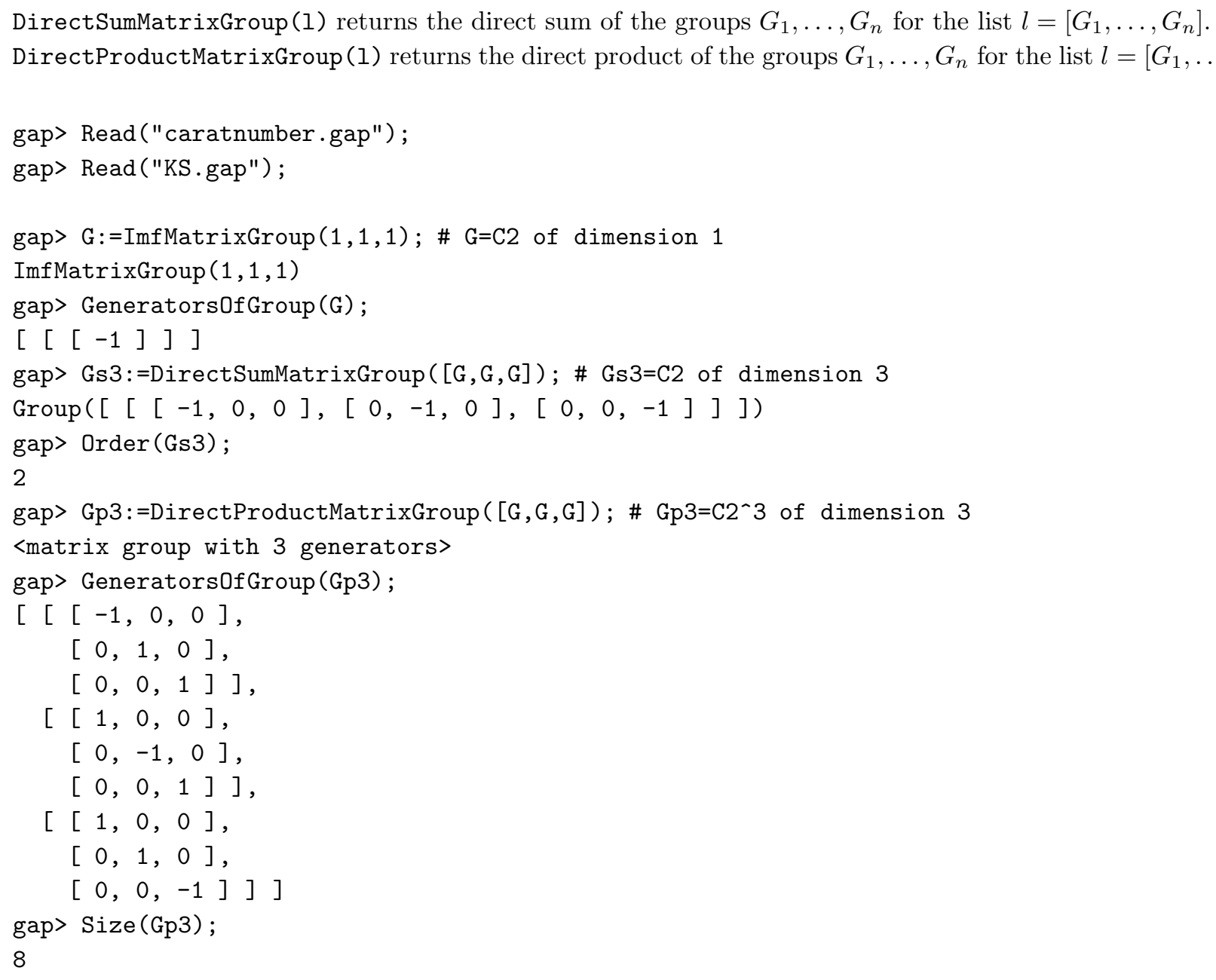

Example 4.9 (Algorithm KS1). By Algorithm KS1, we may check that the condition (KS1) holds when rank $M_{G}=n \leq 4$ and fails for the 11 cases when $\operatorname{rank} M_{G}=5$ and the 131 cases when rank $M_{G}=6$ as in Theorem 4.6. The decomposition types of the $11 G$-lattices $M_{G}$ of rank 5 are $(3,2)$ and $(4,1)$ and $G$ is a subgroup of the group $C_{2} \times D_{6}$ of the CARAT ID $(5,205,6)$. The decomposition types of the former 51 cases (resp. the latter 80 cases) out of the 131 cases are $(3,2,1)$ and $(4,1,1)$ (resp. $(3,3)$ and $(5,1))$ and $G$ is a subgroup of the group $C_{2}^{2} \times D_{6}$ of the CARAT ID $(6,2139,9)$ (resp. $D_{6} \times D_{4}$ of the CARAT ID $(6,145,4)$ ). For the groups $G_{1}$ and $G_{2}$ of the CARAT IDs $(6,2139,9)$ and $(5,205,6)$, we see that $M_{G_{1}} \simeq M_{G_{2}} \oplus N$ for some $G$-lattice $N$ of rank 1.

The computation in this example also confirms that the 2 (resp. $3,6,7$ ) irreducible maximal finite groups $\operatorname{Imf}(n, i, j)$ of dimension 2 (resp. $3,4,5$ ) become the indecomposable maximal finite groups, and the 18 groups given in Subsection 4.0 are the indecomposable maximal finite groups of dimension 6 (see also Example 9.2). Indeed, we get the 18996 indecomposable (conjugacy classes of) subgroups of $\operatorname{GL}(6, \mathbb{Z})$ as the subgroups of the 18 indecomposable maximal finite groups although we obtain only 14348 subgroups if we use the 17 irreducible ones. 


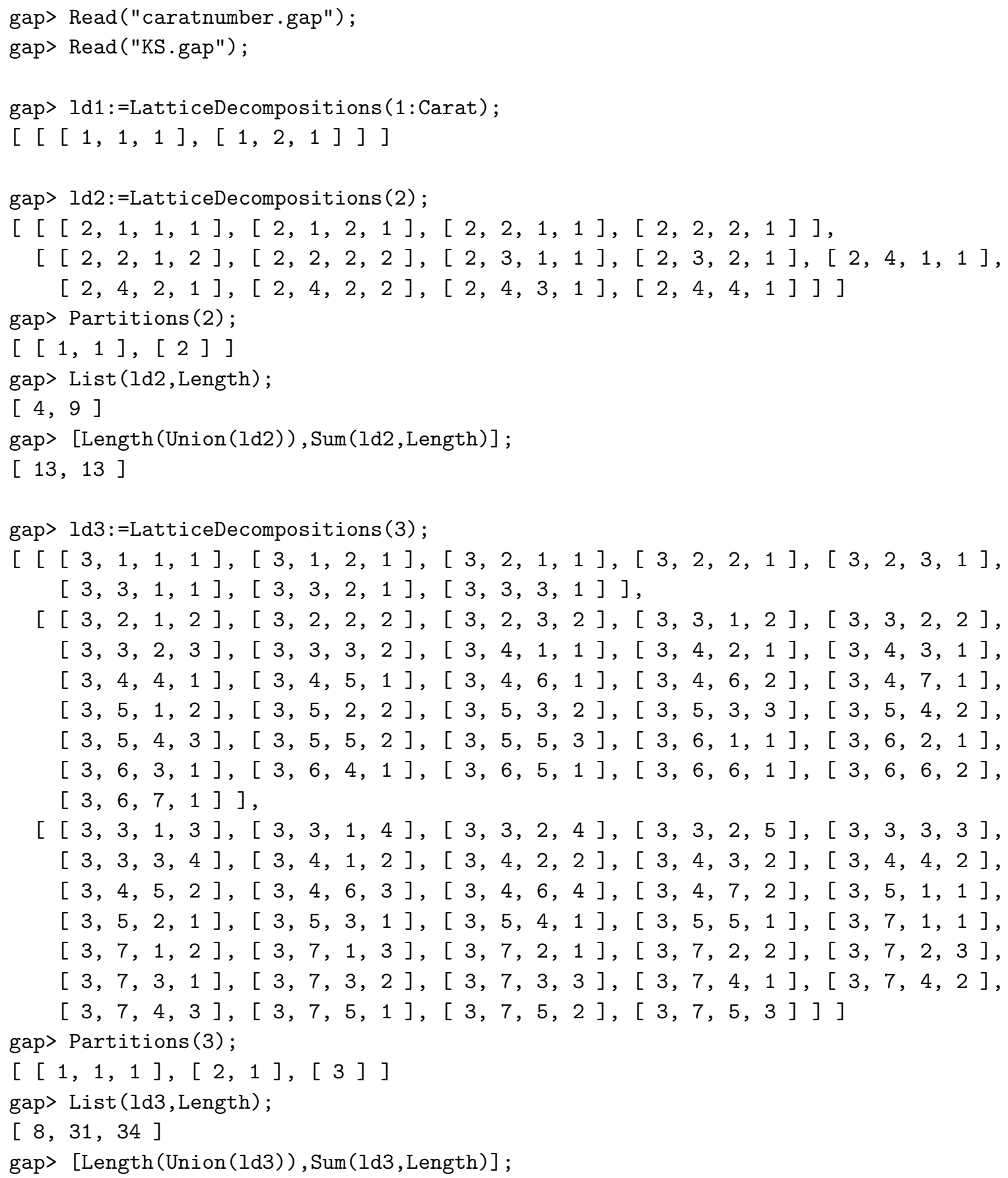

$[[3,3,1,3],[3,3,1,4],[3,3,2,4],[3,3,2,5],[3,3,3,3]$, $[3,3,3,4],[3,4,1,2],[3,4,2,2],[3,4,3,2],[3,4,4,2]$, $[3,4,5,2],[3,4,6,3],[3,4,6,4],[3,4,7,2],[3,5,1,1]$, $[3,5,2,1],[3,5,3,1],[3,5,4,1],[3,5,5,1],[3,7,1,1]$, $[3,7,1,2],[3,7,1,3],[3,7,2,1],[3,7,2,2],[3,7,2,3]$, $[3,7,3,1],[3,7,3,2],[3,7,3,3],[3,7,4,1],[3,7,4,2]$, $[3,7,4,3],[3,7,5,1],[3,7,5,2],[3,7,5,3]]]$

gap> Partitions (3);

[ [ $1,1,1],[2,1],[3]]$

gap> List (ld3, Length);

$[8,31,34]$

gap> [Length(Union(ld3)), Sum(ld3, Length)];

$[73,73]$

gap> 1d4:=LatticeDecompositions(4); ;

gap> Partitions (4);

$[[1,1,1,1],[2,1,1],[2,2],[3,1],[4]]$

gap> List (ld4, Length);

$[16,96,175,128,295]$

gap> [Length(Union(ld4)), Sum(ld4, Length)] ;

$[710,710]$

gap> 1d5:=LatticeDecompositions (5:Carat); ;

gap> Partitions (5);

$[[1,1,1,1,1],[2,1,1,1],[2,2,1],[3,1,1],[3,2],[4,1],[5]]$ gap> List (ld5, Length);

$[32,280,1004,442,1480,1400,1452]$

gap> [Length(Union(ld5)), Sum(ld5, Length)] ; 


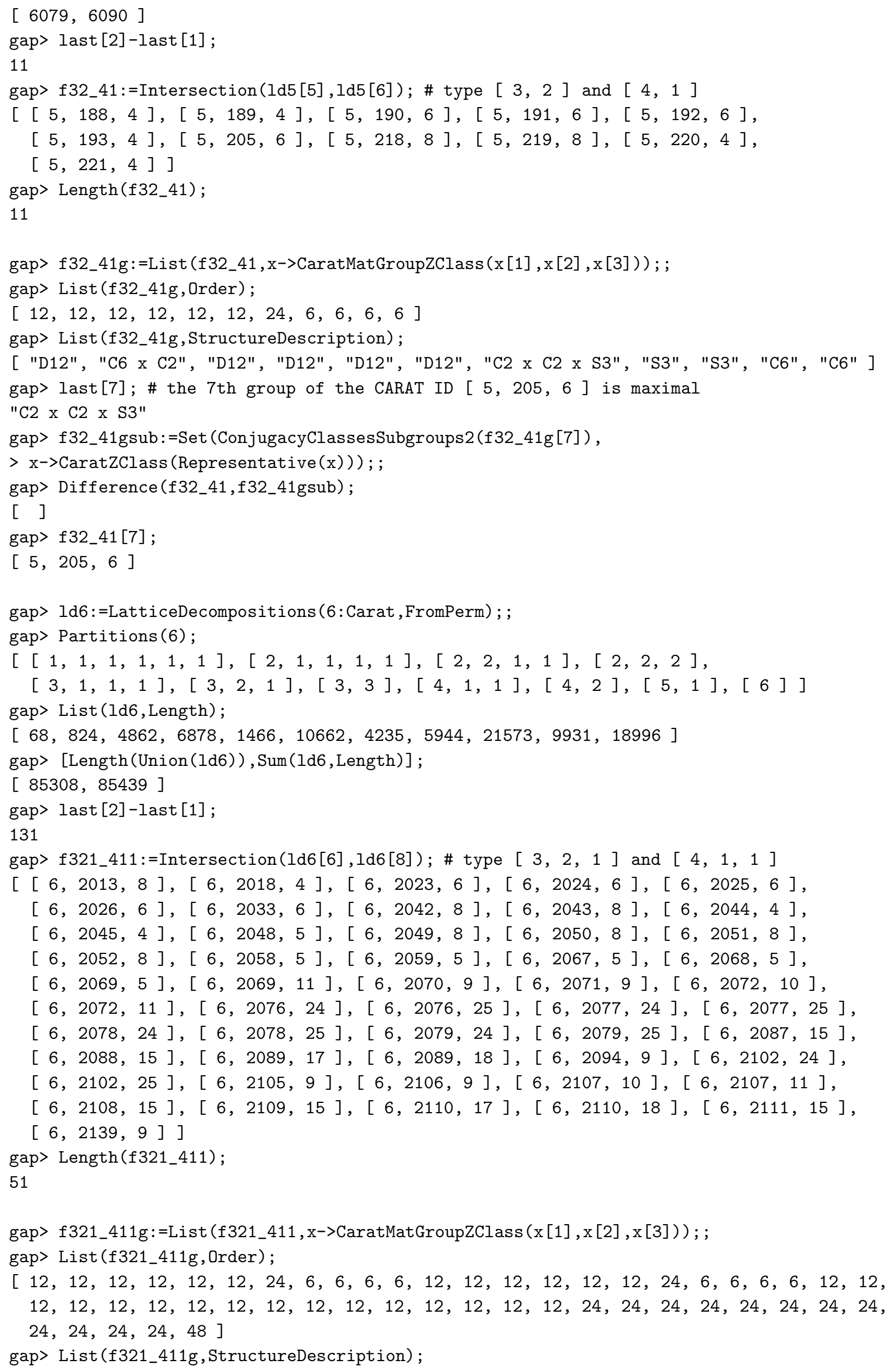


[ "D12", "C6 x C2", "D12", "D12", "D12", "D12", "C2 x C2 x S3", "S3", "S3", "C6", "C6", "C6 x C2", "D12", "D12", "D12", "D12", "D12", "C2 x C2 x S3", "C6", "C6", "S3", "S3", "C6 x C2", "C6 x C2", "C6 x C2", "C6 x C2", "D12", "D12", "D12", "D12", "D12", "D12", "D12", "D12", "D12", "D12", "D12", "D12", "C6 x C2 x C2", "C2 x C2 x S3", "C2 x C2 x S3", "C2 x C2 x S3", "C2 x C2 x S3", "C2 x C2 x S3", "C2 x C2 x S3", "C2 x C2 x S3",

"C2 x C2 x S3", "C2 x C2 x S3", "C2 x C2 x S3", "C2 x C2 x S3", "C2 x C2 x C2 x S3" ] gap> last [51]; \# the 51th group of the CARAT ID [6, 2139, 9 ] is maximal "C2 x C2 x C2 x S3" gap> f321_411gsub:=Set (ConjugacyClassesSubgroups2(f321_411g[51]), $>\mathrm{x}->$ CaratZClass (Representative $(\mathrm{x}))$ ) ; ; gap> Difference(f321_411,f321_411gsub);

[ ]

gap> f321_411[51];

$[6,2139,9]$

gap> gg:=GeneratorsOfGroup (CaratMatGroupZClass $(5,205,6))$; ;

gap> gg: $=$ List $(g g, x->\operatorname{DirectSumMat}([[1]], \mathrm{x}))$; ;

gap> Add (gg,-IdentityMat (6));

gap> CaratZClass (Group(gg));

$[6,2139,9]$

gap> f33_51:=Intersection(Id6[7],Id6[10]); \# type $[3,3]$ and $[5,1]$ $[[6,40,4],[6,41,4],[6,44,6],[6,45,6],[6,47,4]$, $[6,53,4],[6,54,4],[6,54,8],[6,55,4],[6,63,4]$, $[6,64,6],[6,65,4],[6,66,6],[6,67,6],[6,75,4]$, $[6,75,8],[6,76,8],[6,76,12],[6,77,8],[6,77,12]$, $[6,78,4],[6,78,8],[6,79,6],[6,80,4],[6,81,8]$, $[6,81,12],[6,90,4],[6,99,4],[6,108,4],[6,108,8]$, $[6,109,8],[6,109,12],[6,110,4],[6,111,6],[6,112,8]$, $[6,112,12],[6,113,4],[6,114,6],[6,115,6],[6,145,4]$, $[6,2070,10],[6,2070,11],[6,2071,10],[6,2071,11],[6,2072,12]$, $[6,2072,13],[6,2076,26],[6,2076,27],[6,2077,26],[6,2077,27]$, $[6,2078,26],[6,2078,27],[6,2079,26],[6,2079,27],[6,2087,16]$, $[6,2087,17],[6,2088,16],[6,2088,17],[6,2089,19],[6,2089,20]$, $[6,2094,10],[6,2094,11],[6,2102,26],[6,2102,27],[6,2105,10]$, $[6,2105,11],[6,2106,10],[6,2106,11],[6,2107,12],[6,2107,13]$, $[6,2108,16],[6,2108,17],[6,2109,16],[6,2109,17],[6,2110,19]$, $[6,2110,20],[6,2111,16],[6,2111,17],[6,2139,10],[6,2139,11]]$ gap> Length(f33_51);

80

gap> f33_51g:=List (f33_51, $x->$ CaratMatGroupZClass $(x[1], x[2], x[3])) ;$;

gap> List (f33_51g,Order);

$[12,12,12,12,24,24,24,24,24,24,24,24,24,24,24,24,24,24,24,24,24,24$, $24,24,24,24,48,48,48,48,48,48,48,48,48,48,48,48,48,96,12,12,12,12$, $12,12,12,12,12,12,12,12,12,12,12,12,12,12,12,12,24,24,24,24,24,24$, $24,24,24,24,24,24,24,24,24,24,24,24,48,48]$

gap> List(f33_51g, StructureDescription);

[ "C12", "C12", "C3 : C4", "C3 : C4", "C12 x C2", "C3 x D8", "C3 x D8", "C3 x D8", "C3 x D8", "C4 x S3", "C4 x S3", "C4 x S3", "C4 x S3", "C2 x (C3 : C4)", "(C6 x C2) : C2",

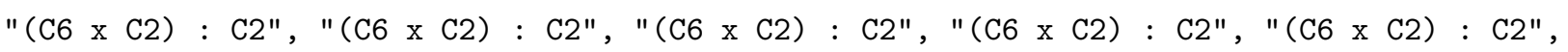
"(C6 x C2) : C2", "(C6 x C2) : C2", "D24", "D24", "D24", "D24", "C6 x D8", "C2 x C4 x S3", "D8 x S3", "D8 x S3", "C2 x ( (C6 x C2) : C2 $) "$, "C2 x ( (C6 x C2) : C2)", "D8 x S3", "D8 x S3", "D8 x S3", "D8 x S3", "D8 x S3", "D8 x S3", "C2 x D24", "C2 x S3 x D8", "C6 x C2", "C6 x C2", "C6 x C2", "C6 x C2", "C6 x C2", "C6 x C2", "D12", "D12", "D12", 


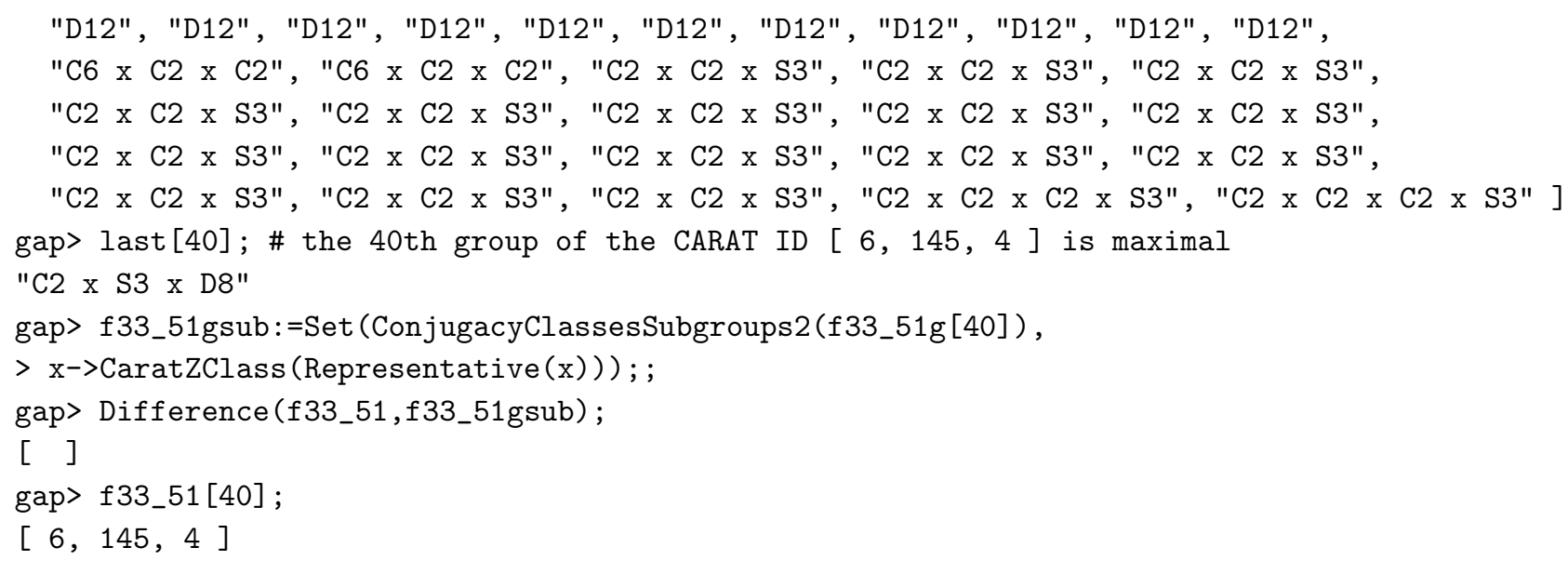

Example 4.10 (The generators of the exceptional 11 groups $G \leq \mathrm{GL}(5, \mathbb{Z})$ whose corresponding $G$-lattices $M_{G} \simeq M_{1} \oplus M_{2} \simeq N_{1} \oplus N_{2}$ with rank $M_{1}=4$, rank $M_{2}=1$, rank $N_{1}=3$ and $\left.\operatorname{rank} N_{2}=2\right)$. Let $I$ be the identity matrix of rank 5 . Let

$$
X=\left(\begin{array}{cccc|c}
0 & 1 & 0 & 1 & 0 \\
1 & 0 & 0 & 1 & 0 \\
0 & 0 & 0 & 1 & 0 \\
0 & 0 & -1 & -1 & 0 \\
\hline 0 & 0 & 0 & 0 & 1
\end{array}\right), \quad Y=\left(\begin{array}{cccc|c}
1 & 0 & 0 & 0 & 0 \\
0 & 1 & 0 & 0 & 0 \\
0 & 0 & 0 & -1 & 0 \\
0 & 0 & -1 & 0 & 0 \\
\hline 0 & 0 & 0 & 0 & 1
\end{array}\right) .
$$

Then the CARAT ID and the generators of the exceptional 11 groups $G \leq \mathrm{GL}(5, \mathbb{Z})$ are given as

\begin{tabular}{lll}
$G$ & CARAT ID & Generators \\
\hline$S_{3} \times C_{2} \simeq D_{6}$ & $(5,188,4)$ & $\left\langle X^{2}, X Y,-I\right\rangle$ \\
$C_{2} \times C_{6}$ & $(5,189,4)$ & $\langle X,-I\rangle$ \\
$D_{6}$ & $(5,190,6)$ & $\langle-X, Y\rangle$ \\
$D_{6}$ & $(5,191,6)$ & $\langle-X, X Y\rangle$ \\
$D_{6}$ & $(5,192,6)$ & $\langle X, Y\rangle$ \\
$D_{6}$ & $(5,193,4)$ & $\langle X,-Y\rangle$ \\
$D_{6} \times C_{2}$ & $(5,205,6)$ & $\langle X, Y,-I\rangle$ \\
$S_{3}$ & $(5,218,8)$ & $\left\langle X^{2}, X Y\right\rangle$ \\
$S_{3}$ & $(5,219,8)$ & $\left\langle X^{2},-X Y\right\rangle$ \\
$C_{6}$ & $(5,220,4)$ & $\langle X\rangle$ \\
$C_{6}$ & $(5,221,4)$ & $\langle-X\rangle$
\end{tabular}

The group $\langle X, Y\rangle \leq \mathrm{GL}(5, \mathbb{Z})$ may be regarded as the group embedded directly by the group $G \leq \mathrm{GL}(4, \mathbb{Z})$ of the GAP ID $(4,14,8,2)$. We also see that

$$
P^{-1} X P=\left(\begin{array}{ccc|cc}
0 & 1 & 0 & 0 & 0 \\
0 & 0 & 1 & 0 & 0 \\
1 & 0 & 0 & 0 & 0 \\
\hline 0 & 0 & 0 & 0 & 1 \\
0 & 0 & 0 & 1 & 0
\end{array}\right), \quad P^{-1} Y P=\left(\begin{array}{ccc|cc}
0 & 1 & 0 & 0 & 0 \\
1 & 0 & 0 & 0 & 0 \\
0 & 0 & 1 & 0 & 0 \\
\hline 0 & 0 & 0 & 1 & 0 \\
0 & 0 & 0 & 0 & 1
\end{array}\right) \text { where } P=\left(\begin{array}{ccccc}
0 & 0 & 1 & 0 & 1 \\
0 & 0 & 1 & 1 & 0 \\
0 & -1 & 1 & 0 & 0 \\
1 & 0 & -1 & 0 & 0 \\
1 & 1 & 1 & 1 & 1
\end{array}\right) \text {. }
$$

This shows that $M_{G} \simeq M_{1} \oplus M_{2} \simeq N_{1} \oplus N_{2}$ where $M_{1}, M_{2}, N_{1}$ and $N_{2}$ are indecomposable $G$-lattices with rank $M_{1}=4, \operatorname{rank} M_{2}=1, \operatorname{rank} N_{1}=3$ and $\operatorname{rank} N_{2}=2$.

4.2. Krull-Schmidt theorem (2). We will determine whether the condition (KS2) holds.

Algorithm KS2 (Determination whether the condition (KS2) holds).

(The following algorithm needs the CARAT package of GAP and caratnumber.gap).

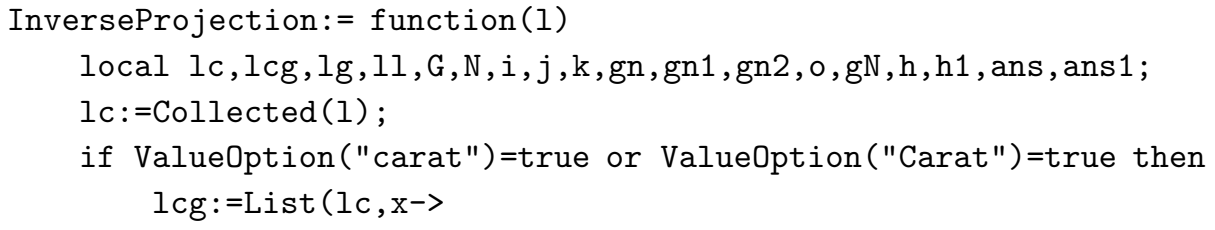




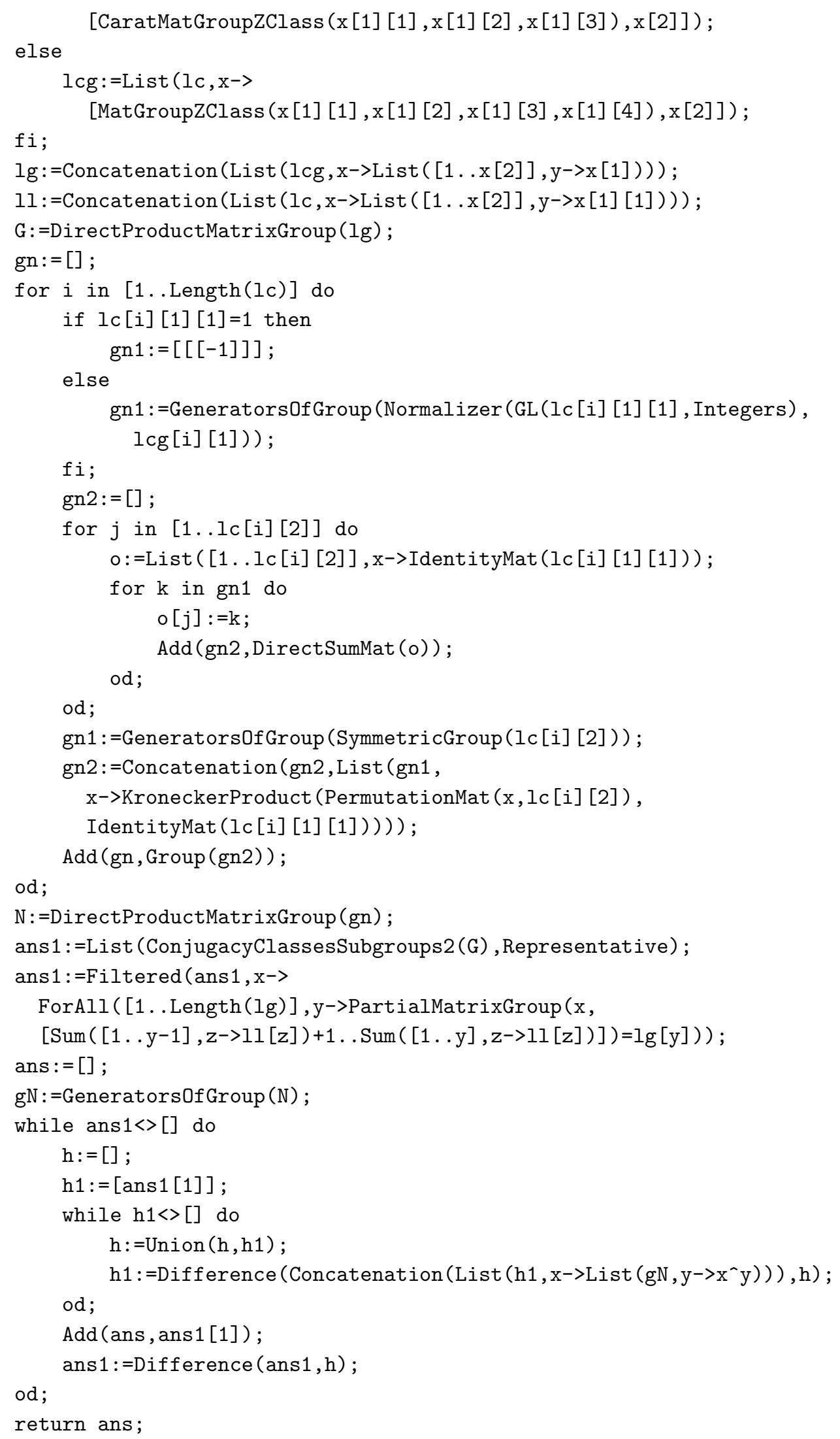

Example 4.11 (InverseProjection([11,12])). Let $G_{1} \simeq C_{4} \leq \mathrm{GL}(2, \mathbb{Z})\left(\right.$ resp. $G_{2} \simeq D_{6} \leq \mathrm{GL}(2, \mathbb{Z})$ ) be a cyclic group of order 4 (resp. dihedral group of order 12 ) of the GAP ID $l_{1}=(2,3,1,1)$ (resp. $\left.l_{2}=(2,4,4,1)\right)$. 
InverseProjection ( $[11,12]$ ) returns the list of all groups $G \leq \mathrm{GL}(4, \mathbb{Z})$ such that $M_{G} \simeq M_{G_{1}} \oplus M_{G_{2}}$ and the GAP ID of $G_{1}$ (resp. $G_{2}$ ) is $l_{1}$ (resp. $l_{2}$ ).

InverseProjection( $[11,12]$ : Carat) returns the same as InverseProjection( $[11,12])$ but with respect to the CARAT ID $l_{1}$ and $l_{2}$ instead of the GAP ID.

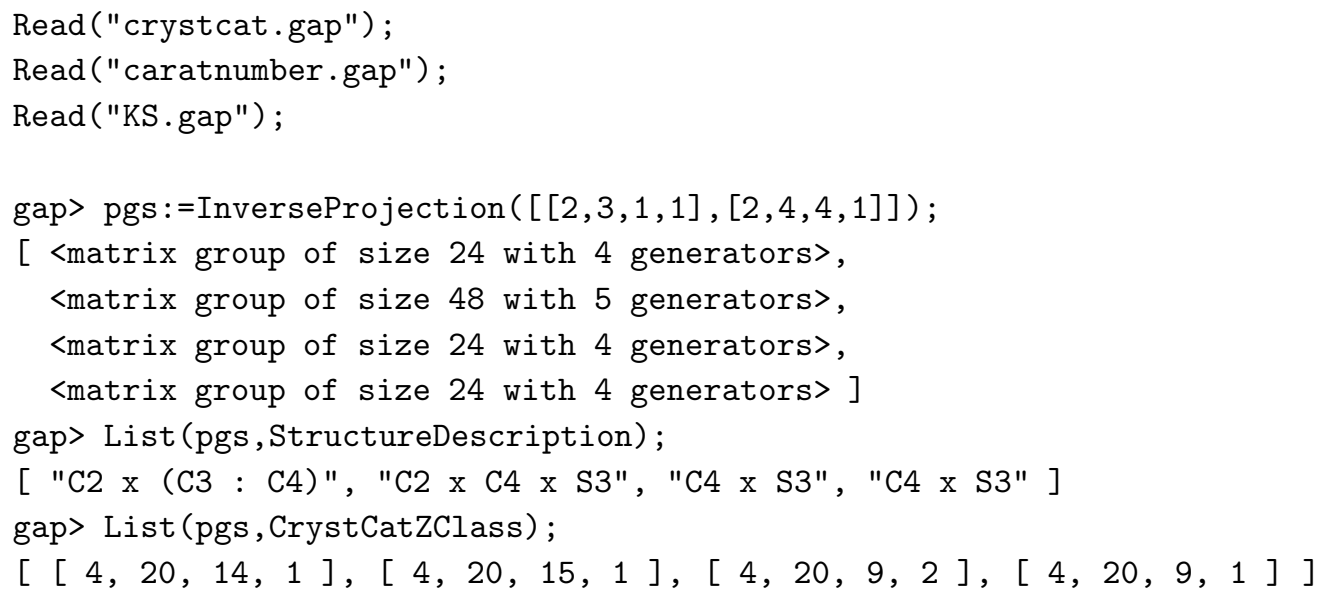

Example 4.12 (Verification of $\left[M_{G}\right]^{f l} \neq 0$ and that $\left[M_{G}\right]^{f l}$ is not invertible where $M_{G} \simeq M_{G_{1}} \oplus M_{G_{2}}$ is a decomposable $G$-lattice). By using InverseProjection in Algorithm KS2, we may obtain Table 3 of Theorems 1.9 and 1.27 and Tables 11 to 14 in Theorems 1.12 and 1.28 .

Let $G \leq \mathrm{GL}(4, \mathbb{Z})$ where $M_{G} \simeq M_{G_{1}} \oplus M_{G_{2}}$ is a decomposable $G$-lattice of rank 4 with $G_{1} \leq \mathrm{GL}(3, \mathbb{Z})$ and $G_{2} \leq \mathrm{GL}(1, \mathbb{Z})$. Let $\mathcal{N}_{3}$ be the set of the 15 GAP IDs as in Table 1. By Lemma 2.14 we have $\left[M_{G}\right]^{f l}=\left[M_{1}\right]^{f l}$. Hence, by Theorem 1.2 and Theorem 2.12, $L\left(M_{G}\right)^{G}$ is not retract $k$-rational if and only if $L\left(M_{G}\right)^{G}$ is not stably $k$-rational if and only if the GAP ID of $G_{1}$ is $l \in \mathcal{N}_{3}$.

All the GAP IDs $\mathcal{N}_{31}$, as in Table 3 of Theorem [1.9, of such groups $G$ which satisfy $M_{G} \simeq M_{G_{1}} \oplus M_{G_{2}}$, $G_{1} \leq \mathrm{GL}(3, \mathbb{Z})$ of the GAP ID $l \in \mathcal{N}_{3}$ and $G_{2} \leq \mathrm{GL}(1, \mathbb{Z})$ may be obtained as follows.

Similarly, by using the result $\mathcal{I}_{4}$ and $\mathcal{N}_{4}$ as in Tables 2 and 4 of Theorems 1.9 and 1.27, we may obtain the CARAT IDs $\mathcal{I}_{41}, \mathcal{N}_{311}, \mathcal{N}_{32}$ and $\mathcal{N}_{41}$ as in Tables 11 to 14 of Theorems 1.12 and 1.28

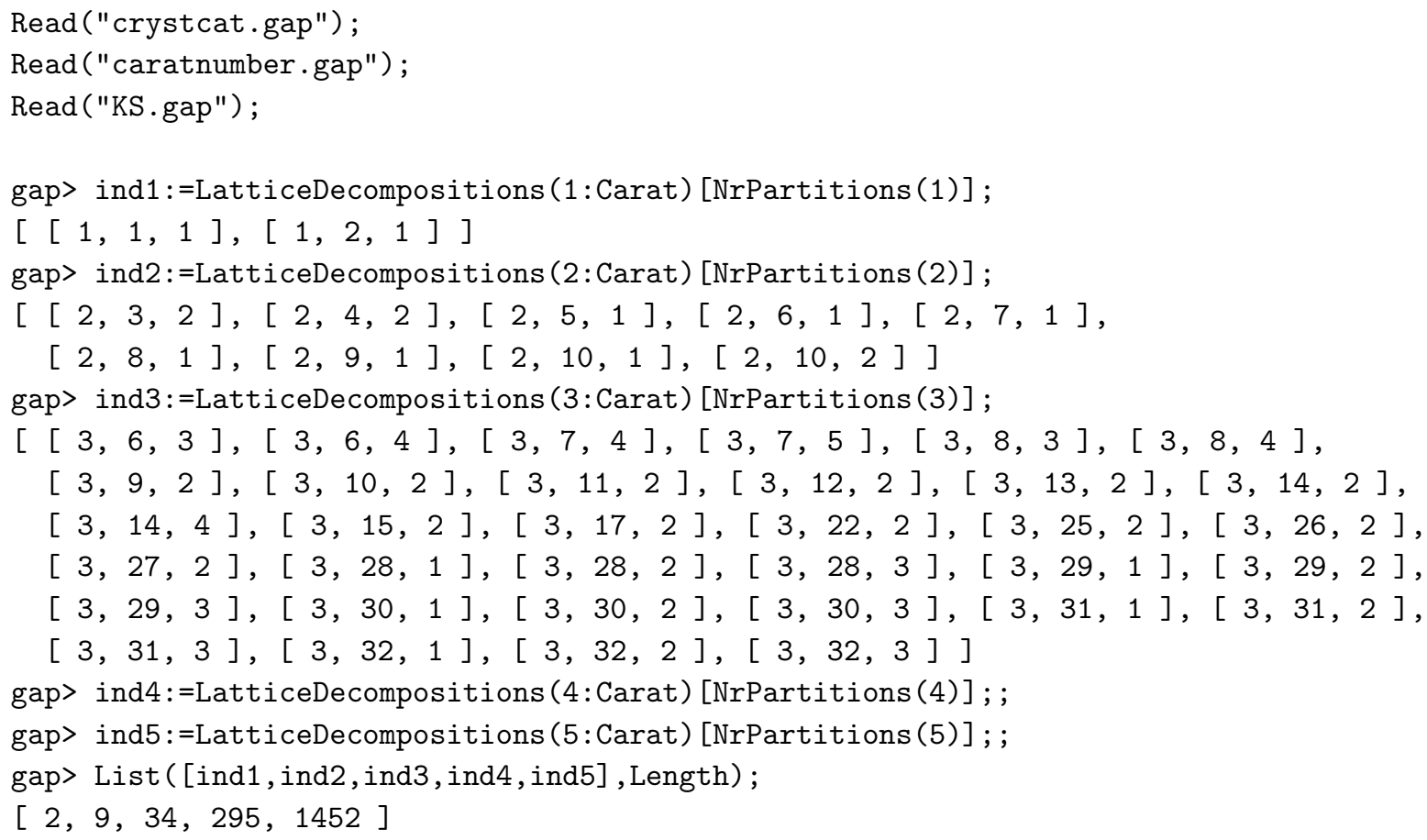


$>[3,7,3,2],[3,7,3,3],[3,7,4,2],[3,7,5,2],[3,7,5,3]] ;$; gap> Length(N3);

15

gap> N3c: $=$ List (N3, CrystCat2Carat);

$[[3,6,3],[3,8,3],[3,8,4],[3,15,2],[3,13,2]$,

$[3,14,4],[3,9,2],[3,28,1],[3,29,1],[3,29,3]$,

$[3,31,1],[3,31,3],[3,30,1],[3,32,1],[3,32,3]]$

gap> N31g:=List (Cartesian ([N3c,ind1]), $\mathrm{x}->$ InverseProjection ( $\mathrm{x}$ :Carat)); ;

gap> N31:=Set (Concatenation (N31g), CrystCatZClass);

$[[4,4,3,6],[4,4,4,4],[4,4,4,6],[4,5,1,9],[4,5,2,4]$,

$[4,5,2,7],[4,6,1,4],[4,6,1,8],[4,6,2,4],[4,6,2,8]$,

$[4,6,2,9],[4,6,3,3],[4,6,3,6],[4,7,3,2],[4,7,4,3]$,

$[4,7,5,2],[4,7,7,2],[4,12,2,4],[4,12,3,7],[4,12,4,6]$,

$[4,12,4,8],[4,12,4,9],[4,12,5,6],[4,12,5,7],[4,13,1,3]$,

$[4,13,2,4],[4,13,3,4],[4,13,4,3],[4,13,5,3],[4,13,6,3]$,

$[4,13,7,6],[4,13,7,7],[4,13,7,8],[4,13,8,3],[4,13,8,4]$,

$[4,13,9,3],[4,13,10,3],[4,24,1,5],[4,24,2,3],[4,24,2,5]$,

$[4,24,3,5],[4,24,4,3],[4,24,4,5],[4,24,5,3],[4,24,5,5]$,

$[4,25,1,2],[4,25,1,4],[4,25,2,4],[4,25,3,2],[4,25,3,4]$,

$[4,25,4,4],[4,25,5,2],[4,25,5,4],[4,25,6,2],[4,25,6,4]$,

$[4,25,7,2],[4,25,7,4],[4,25,8,2],[4,25,8,4],[4,25,9,4]$,

$[4,25,10,2],[4,25,10,4],[4,25,11,2],[4,25,11,4]]$

gap> Length(N31);

64

gap $>I 4:=[[4,31,1,3],[4,31,1,4],[4,31,2,2],[4,31,4,2]$,

$>[4,31,5,2],[4,31,7,2],[4,33,2,1]] ;$;

gap> Length(I4);

7

gap $>N 4:=[[4,5,1,12],[4,5,2,5],[4,5,2,8],[4,5,2,9],[4,6,1,6]$,

$[4,6,1,11],[4,6,2,6],[4,6,2,10],[4,6,2,12],[4,6,3,4]$,

$[4,6,3,7],[4,6,3,8],[4,12,2,5],[4,12,2,6],[4,12,3,11]$,

$[4,12,4,10],[4,12,4,11],[4,12,4,12],[4,12,5,8],[4,12,5,9]$,

$[4,12,5,10],[4,12,5,11],[4,13,1,5],[4,13,2,5],[4,13,3,5]$,

$[4,13,4,5],[4,13,5,4],[4,13,5,5],[4,13,6,5],[4,13,7,9]$,

$[4,13,7,10],[4,13,7,11],[4,13,8,5],[4,13,8,6],[4,13,9,4]$,

$[4,13,9,5],[4,13,10,4],[4,13,10,5],[4,18,1,3],[4,18,2,4]$,

$[4,18,2,5],[4,18,3,5],[4,18,3,6],[4,18,3,7],[4,18,4,4]$,

$[4,18,4,5],[4,18,5,5],[4,18,5,6],[4,18,5,7],[4,19,1,2]$,

$[4,19,2,2],[4,19,3,2],[4,19,4,3],[4,19,4,4],[4,19,5,2]$,

$[4,19,6,2],[4,22,1,1],[4,22,2,1],[4,22,3,1],[4,22,4,1]$,

$[4,22,5,1],[4,22,5,2],[4,22,6,1],[4,22,7,1],[4,22,8,1]$,

$[4,22,9,1],[4,22,10,1],[4,22,11,1],[4,24,2,4],[4,24,2,6]$,

$[4,24,4,4],[4,24,5,4],[4,24,5,6],[4,25,1,3],[4,25,2,3]$,

$[4,25,2,5],[4,25,3,3],[4,25,4,3],[4,25,5,3],[4,25,5,5]$,

$[4,25,6,3],[4,25,6,5],[4,25,7,3],[4,25,8,3],[4,25,9,3]$,

$[4,25,9,5],[4,25,10,3],[4,25,10,5],[4,25,11,3],[4,25,11,5]$,

$[4,29,1,1],[4,29,1,2],[4,29,2,1],[4,29,3,1],[4,29,3,2]$,

$[4,29,3,3],[4,29,4,1],[4,29,4,2],[4,29,5,1],[4,29,6,1]$,

$[4,29,7,1],[4,29,7,2],[4,29,8,1],[4,29,8,2],[4,29,9,1]$,

$[4,32,1,2],[4,32,2,2],[4,32,3,2],[4,32,4,2],[4,32,5,2]$,

$[4,32,5,3],[4,32,6,2],[4,32,7,2],[4,32,8,2],[4,32,9,4]$,

$[4,32,9,5],[4,32,10,2],[4,32,11,2],[4,32,11,3],[4,32,12,2]$,

$[4,32,13,3],[4,32,13,4],[4,32,14,3],[4,32,14,4],[4,32,15,2]$,

$>[4,32,16,2],[4,32,16,3],[4,32,17,2],[4,32,18,2],[4,32,18,3]$, 
$>[4,32,19,2],[4,32,19,3],[4,32,20,2],[4,32,20,3],[4,32,21,2]$,

$>[4,32,21,3],[4,33,1,1],[4,33,3,1],[4,33,4,1],[4,33,5,1]$,

$>[4,33,6,1],[4,33,7,1],[4,33,8,1],[4,33,9,1],[4,33,10,1]$,

$>[4,33,11,1],[4,33,12,1],[4,33,13,1],[4,33,14,1],[4,33,14,2]$,

$>[4,33,15,1],[4,33,16,1]] ;$;

gap> Length(N4);

152

gap> I4c:=List (I4, $\mathrm{x}->$ CaratZClass (MatGroupZClass (x [1] $\mathrm{x}[2], \mathrm{x}[3], \mathrm{x}[4]))$ ); ;

gap> I41g:=List (Cartesian ([I4c,ind1]), $\mathrm{x}->$ InverseProjection( $\mathrm{x}$ :Carat)); ;

gap> I41:=Set (Concatenation (I41g), CaratZClass); ;

$[[5,692,1],[5,693,1],[5,736,1],[5,911,1],[5,912,1]$,

$[5,914,1],[5,916,1],[5,917,1],[5,917,5],[5,918,1]$,

$[5,918,5],[5,919,1],[5,921,1],[5,922,1],[5,923,1]$,

$[5,924,1],[5,925,1],[5,926,1],[5,926,3],[5,927,1]$,

$[5,928,1],[5,928,3],[5,929,1],[5,930,1],[5,932,1]]$

gap> Length(I41);

25

gap> N311g:=List (Cartesian ([UnorderedTuples (N3c,1), UnorderedTuples (ind1,2)] ),

$>\mathrm{x}->$ InverseProjection (Concatenation (x):Carat)); ;

gap> N311:=Set (Concatenation(N311g), CaratZClass); ;

gap> Length (N311);

245

gap> N32g: =List (Cartesian ( [N3c, ind2]), $\mathrm{x}->$ InverseProjection( $\mathrm{x}$ :Carat)); ;

gap> N32: =Set (Concatenation (N32g), CaratZClass); ;

gap> Length(N32);

849

gap> N4C: $=\operatorname{List}(N 4, x->C \operatorname{aratZClass}(\operatorname{MatGroupZClass}(\mathrm{x}[1], \mathrm{x}[2], \mathrm{x}[3], \mathrm{x}[4])))$; ;

gap> N41g:=List (Cartesian ([N4C,ind1]), $\mathrm{x}->$ InverseProjection ( $\mathrm{x}$ :Carat)); ;

gap> $\mathrm{N} 41:=\operatorname{Set}$ (Concatenation $(\mathrm{N} 41 \mathrm{~g})$, CaratZClass); ;

Length (N41);

768

Example 4.13 (Verification of the condition (KS2)). By using Algorithm KS2, we may check that the condition (KS2) holds for $n \leq 5$. For $n=6$, there exist exactly 10 (resp. 8) $G$-lattices of decomposition type $(4,2)$ (resp. $(5,1))$ which are decomposable in two different ways $M_{G} \simeq M_{1} \oplus M_{2} \simeq N_{1} \oplus N_{2}$ as follows:

\begin{tabular}{ll|ll|ll|ll|ll}
\multicolumn{2}{l}{$M_{G} \simeq M_{1} \oplus M_{2} \simeq N_{1} \oplus N_{2}$} & $M_{1}$ & & $M_{2}$ & & $N_{1}$ & $N_{2}$ \\
\hline$(6,2072,14)$ & $C_{2} \times C_{6}$ & $(2,2,1,2)$ & $C_{2}$ & $(4,14,4,2)$ & $C_{2} \times C_{6}$ & $(2,2,2,2)$ & $C_{2}^{2}$ & $(4,14,1,2)$ & $C_{6}$ \\
$(6,2076,28)$ & $D_{6}$ & $(2,2,1,2)$ & $C_{2}$ & $(4,14,9,2)$ & $D_{6}$ & $(2,2,2,2)$ & $C_{2}^{2}$ & $(4,14,3,4)$ & $S_{3}$ \\
$(6,2077,28)$ & $D_{6}$ & $(2,2,2,2)$ & $C_{2}^{2}$ & $(4,14,5,2)$ & $D_{6}$ & $(2,2,2,2)$ & $C_{2}^{2}$ & $(4,14,7,2)$ & $D_{6}$ \\
$(6,2078,28)$ & $D_{6}$ & $(2,2,1,2)$ & $C_{2}$ & $(4,14,5,2)$ & $D_{6}$ & $(2,2,2,2)$ & $C_{2}^{2}$ & $(4,14,6,2)$ & $D_{6}$ \\
$(6,2079,28)$ & $D_{6}$ & $(2,2,1,2)$ & $C_{2}$ & $(4,14,8,2)$ & $D_{6}$ & $(2,2,1,2)$ & $C_{2}$ & $(4,14,3,3)$ & $S_{3}$ \\
$(6,2089,21)$ & $D_{6}$ & $(2,2,1,2)$ & $C_{2}$ & $(4,14,7,2)$ & $D_{6}$ & $(2,2,2,2)$ & $C_{2}^{2}$ & $(4,14,6,2)$ & $D_{6}$ \\
$(6,2102,28)$ & $C_{2} \times D_{6}$ & $(2,2,2,2)$ & $C_{2}^{2}$ & $(4,14,5,2)$ & $D_{6}$ & $(2,2,2,2)$ & $C_{2}^{2}$ & $(4,14,10,2)$ & $C_{2} \times D_{6}$ \\
$(6,2107,14)$ & $C_{2} \times D_{6}$ & $(2,2,2,2)$ & $C_{2}^{2}$ & $(4,14,6,2)$ & $D_{6}$ & $(2,2,1,2)$ & $C_{2}$ & $(4,14,10,2)$ & $C_{2} \times D_{6}$ \\
$(6,2110,21)$ & $C_{2} \times D_{6}$ & $(2,2,2,2)$ & $C_{2}^{2}$ & $(4,14,7,2)$ & $D_{6}$ & $(2,2,2,2)$ & $C_{2}^{2}$ & $(4,14,10,2)$ & $C_{2} \times D_{6}$ \\
$(6,2295,2)$ & $D_{6}$ & $(2,4,2,2)$ & $S_{3}$ & $(4,21,3,1)$ & $D_{6}$ & $(2,4,2,1)$ & $S_{3}$ & $(4,21,3,2)$ & $D_{6}$
\end{tabular}




\begin{tabular}{ll|ll|ll|ll|ll}
$M_{G} \simeq M_{1} \oplus M_{2} \simeq N_{1} \oplus N_{2}$ & $M_{1}$ & & $M_{2}$ & & $N_{1}$ & & $N_{2}$ \\
\hline$(6,3045,3)$ & $C_{2} \times A_{5}$ & $(1,2,1)$ & $C_{2}$ & $(5,910,3)$ & $C_{2} \times A_{5}$ & $(1,2,1)$ & $C_{2}$ & $(5,910,4)$ & $C_{2} \times A_{5}$ \\
$(6,3046,3)$ & $S_{5}$ & $(1,1,1)$ & $\{1\}$ & $(5,911,3)$ & $S_{5}$ & $(1,1,1)$ & $\{1\}$ & $(5,911,4)$ & $S_{5}$ \\
$(6,3047,3)$ & $S_{5}$ & $(1,2,1)$ & $C_{2}$ & $(5,912,3)$ & $S_{5}$ & $(1,2,1)$ & $C_{2}$ & $(5,912,4)$ & $S_{5}$ \\
$(6,3052,5)$ & $F_{20}$ & $(1,2,1)$ & $C_{2}$ & $(5,917,3)$ & $F_{20}$ & $(1,2,1)$ & $C_{2}$ & $(5,917,4)$ & $F_{20}$ \\
$(6,3053,5)$ & $F_{20}$ & $(1,1,1)$ & $\{1\}$ & $(5,918,3)$ & $F_{20}$ & $(1,1,1)$ & $\{1\}$ & $(5,918,4)$ & $F_{20}$ \\
$(6,3054,3)$ & $C_{2} \times S_{5}$ & $(1,2,1)$ & $C_{2}$ & $(5,919,3)$ & $C_{2} \times S_{5}$ & $(1,2,1)$ & $C_{2}$ & $(5,919,4)$ & $C_{2} \times S_{5}$ \\
$(6,3061,5)$ & $C_{2} \times F_{20}$ & $(1,2,1)$ & $C_{2}$ & $(5,926,5)$ & $C_{2} \times F_{20}$ & $(1,2,1)$ & $C_{2}$ & $(5,926,6)$ & $C_{2} \times F_{20}$ \\
$(6,3066,3)$ & $A_{5}$ & $(1,1,1)$ & $\{1\}$ & $(5,931,3)$ & $A_{5}$ & $(1,1,1)$ & $\{1\}$ & $(5,931,4)$ & $A_{5}$
\end{tabular}

In particular, we see that the $3 G$-lattices $M_{G}$ for the groups $S_{5}, F_{20}$ and $A_{5}$ of the CARAT IDs $(5,911,4)$, $(5,918,4)$ and $(5,931,4)$ respectively are not permutation but stably permutation because the $G$-lattices $M_{G}$ for the groups $S_{5}, F_{20}$ and $A_{5}$ of the CARAT IDs $(5,911,3),(5,918,3)$ and $(5,931,3)$ are permutation. We will study this 3 cases in Section 6 again (see Theorem 6.2 (iii), Table 8 and Example 6.5).

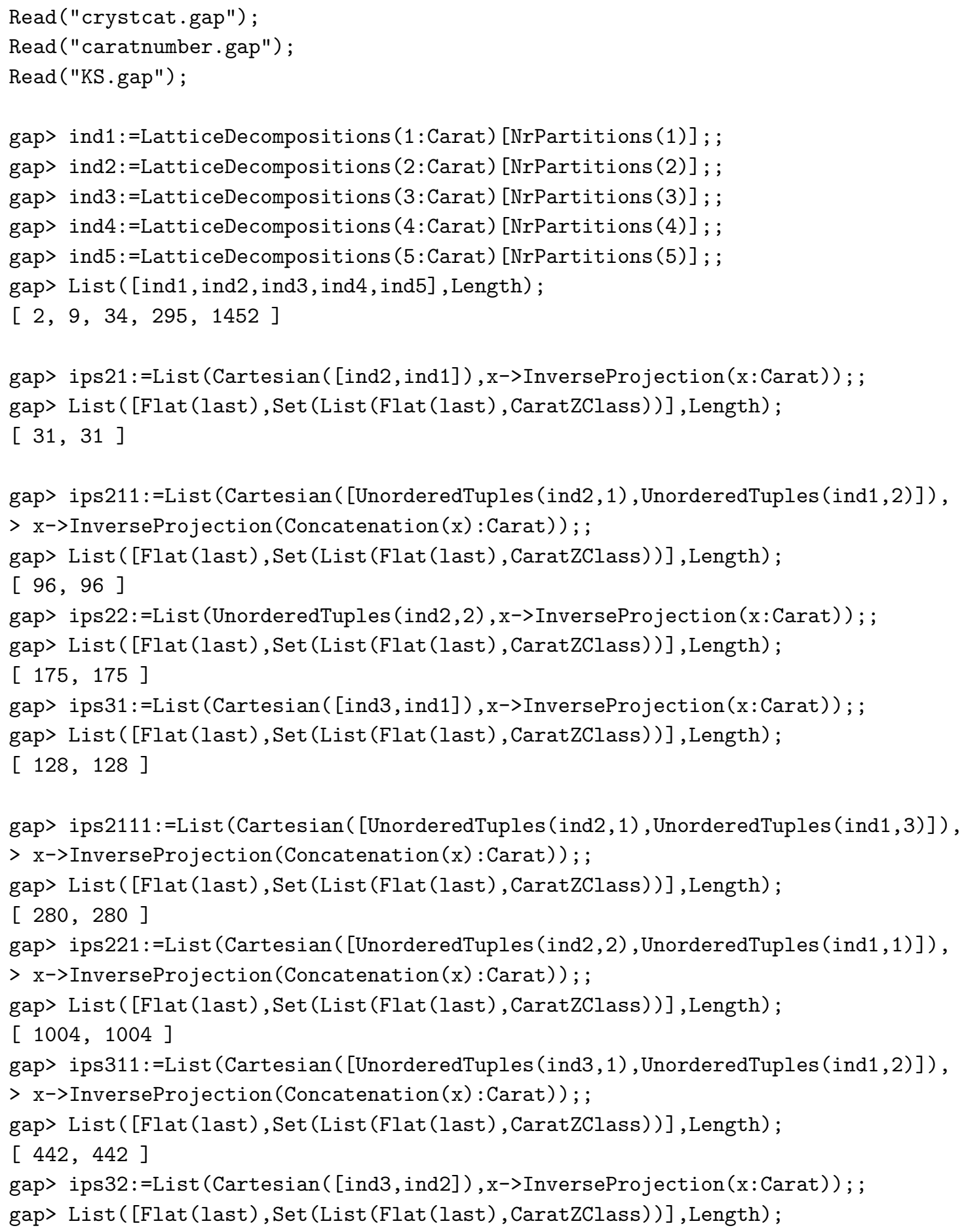


[ 1480,1480$]$

gap> ips41:=List(Cartesian([ind4,ind1]), $\mathrm{x}->$ InverseProjection( $\mathrm{x}$ :Carat)); ;

gap> List([Flat(last), Set (List(Flat(last), CaratZClass))], Length);

$[1400,1400]$

gap> ips21111:= List(Cartesian([UnorderedTuples (ind2,1), UnorderedTuples (ind 1,4)]),

$>\mathrm{x}->$ InverseProjection (Concatenation ( $\mathrm{x})$ : Carat)) ; ;

gap> List([Flat(last), Set (List(Flat(last), CaratZClass))], Length);

[ 824, 824]

gap> ips2211:= List (Cartesian([UnorderedTuples (ind2, 2), UnorderedTuples (ind1,2)]),

$>\mathrm{x}$->InverseProjection(Concatenation (x):Carat)); ;

gap> List ([Flat (last), Set (List (Flat(last), CaratZClass))], Length);

[ 4862,4862 ]

gap> ips222:= List(UnorderedTuples (ind2,3), $\mathrm{x}$->InverseProjection( $\mathrm{x}$ :Carat)) ;

gap> List([Flat(last), Set(List(Flat(last), CaratZClass))], Length);

[ 6878, 6878]

gap> ips3111:= List(Cartesian([UnorderedTuples(ind3,1), UnorderedTuples (ind1,3)]),

$>\mathrm{x}$->InverseProjection(Concatenation( $\mathrm{x})$ :Carat)); ;

gap> List ([Flat (last), Set (List (Flat (last), CaratZClass))], Length);

$[1466,1466]$

gap> ips321:= List(Cartesian([ind3, ind2, ind1]), $\mathrm{x}->$ InverseProjection( $\mathrm{x}$ :Carat)); ;

gap> List ([Flat (last), Set (List (Flat(last), CaratZClass))], Length);

[ 10662, 10662 ]

gap> ips33:= List(UnorderedTuples(ind3,2), $\mathrm{x}->$ InverseProjection( $\mathrm{x}$ :Carat)) ; ;

gap> List ([Flat (last), Set (List(Flat(last), CaratZClass))], Length);

[ 4235, 4235 ]

gap> ips411:= List (Cartesian([UnorderedTuples (ind4,1), UnorderedTuples (ind1,2)]),

$>\mathrm{x}$->InverseProjection (Concatenation( $\mathrm{x}$ ):Carat)); ;

gap> List([Flat(last), Set(List(Flat(last), CaratZClass))], Length);

[ 5944,5944$]$

gap> ips42:= List(Cartesian([ind4,ind2]), $\mathrm{x}->$ InverseProjection( $\mathrm{x}$ :Carat)); ;

gap> List([Flat (last), Set (List(Flat(last), CaratZClass))], Length);

[ 21583, 21573]

gap> last [1]-last [2];

10

gap> ips51:= List(Cartesian([ind5,ind1]), $\mathrm{x}->$ InverseProjection( $\mathrm{x}$ :Carat)); ;

gap> List([Flat (last), Set (List(Flat(last), CaratZClass))], Length);

[ 9939, 9931$]$

gap> last [1]-last [2];

8

gap> Filtered(Collected(List(Flat(ips42), CaratZClass)), $\left.x^{->x}[2]>1\right)$; \# type [4,2]

$[[[6,2072,14], 2],[[6,2076,28], 2],[[6,2077,28], 2]$,

$[[6,2078,28], 2],[[6,2079,28], 2],[[6,2089,21], 2]$,

$[[6,2102,28], 2],[[6,2107,14], 2],[[6,2110,21], 2]$,

$[[6,2295,2], 2]]$

gap> KSfail42:= List (last, $\mathrm{x}->\mathrm{x}[1])$;

$[[6,2072,14],[6,2076,28],[6,2077,28],[6,2078,28],[6,2079,28]$,

$[6,2089,21],[6,2102,28],[6,2107,14],[6,2110,21],[6,2295,2]]$

gap> Length(KSfail42);

10

gap> KSfail42g:=List(KSfail42, $\mathrm{x}->$ CaratMatGroupZClass (x[1] ,x[2] , $\mathrm{x}[3]$ )); ;

gap> List (KSfail42g, Order);

[ $12,12,12,12,12,12,24,24,24,12]$

gap> List(KSfail42g, StructureDescription); 
[ "C6 x C2", "D12", "D12", "D12", "D12", "D12", "C2 x C2 x S3", "C2 x C2 x S3", "C2 x C2 x S3", "D12" ]

gap> [last [7],last[8],last[9], last[10]]; \# 7th, 8th, 9th, 10th groups are maximal

[ "C2 x C2 x S3", "C2 x C2 x S3", "C2 x C2 x S3", "D12" ]

gap> KSfail42gsub1:=Set (ConjugacyClassesSubgroups2(KSfail42g[7]),

$>\mathrm{x}$->CaratZClass (Representative $(\mathrm{x}))$ ) ; ;

gap> KSfail42gsub2:=Set (ConjugacyClassesSubgroups2(KSfail42g[8]),

$>\mathrm{x}->$ CaratZClass (Representative (x))); ;

gap> KSfail42gsub3:=Set (ConjugacyClassesSubgroups2(KSfail42g[9]),

$>\mathrm{x}->$ CaratZClass (Representative $(\mathrm{x}))$ ) ; ;

gap> List(KSfail42, $\mathrm{x}^{->\mathrm{x}}$ in KSfail42gsub1);

[ false, true, true, true, true, false, true, false, false, false ]

gap> List(KSfail42, $\mathrm{x}^{->\mathrm{x}}$ in KSfail42gsub2);

[ true, true, false, true, false, true, false, true, false, false ]

gap> List(KSfail42, $\mathrm{x}->\mathrm{x}$ in KSfail42gsub3);

[ true, false, true, false, true, true, false, false, true, false ]

gap> ips42f:=Flat (ips42); ;

gap> ips42fc:=List (ips42f, CaratZClass); ;

gap> KSfail42i:=List (KSfail42,x->Filtered ([1..21583],y->ips42fc [y] =x));

[ [ 9822, 10444], [ 10162, 10496], [ 9901, 9960 ], [ 9890, 10028 ], [ 10095, 10518 ],

$[9956,10033],[9895,10257],[10030,10230],[9963,10248],[10647,10720]$ ]

gap> KSfail42gg:=List (KSfail42i,x->[ips42f[x[1]],ips42f [x[2]]]) ; ;

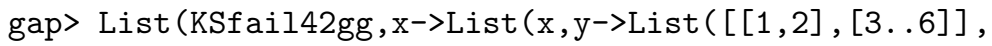

$>$ z->CrystCatZClass (PartialMatrixGroup (y,z)))));

$[[[[2,2,1,2],[4,14,4,2]],[[2,2,2,2],[4,14,1,2]]]$,

$[[[2,2,1,2],[4,14,9,2]],[[2,2,2,2],[4,14,3,4]]]$,

$[[[2,2,2,2],[4,14,5,2]],[[2,2,2,2],[4,14,7,2]]]$,

$[[[2,2,1,2],[4,14,5,2]],[[2,2,2,2],[4,14,6,2]]]$,

$[[[2,2,1,2],[4,14,8,2]],[[2,2,1,2],[4,14,3,3]]]$,

$[[[2,2,1,2],[4,14,7,2]],[[2,2,2,2],[4,14,6,2]]]$,

$[[[2,2,2,2],[4,14,5,2]],[[2,2,2,2],[4,14,10,2]]]$,

$[[[2,2,2,2],[4,14,6,2]],[[2,2,1,2],[4,14,10,2]]]$,

$[[[2,2,2,2],[4,14,7,2]],[[2,2,2,2],[4,14,10,2]]]$,

$[[[2,4,2,2],[4,21,3,1]],[[2,4,2,1],[4,21,3,2]]]]$ gap> List (last, $\mathrm{y}^{->\operatorname{List}}([\mathrm{y}[1][1], \mathrm{y}[1][2], \mathrm{y}[2][1], \mathrm{y}[2][2]]$,

$>\mathrm{x}->\operatorname{StructureDescription}(\operatorname{MatGroupZClass}(\mathrm{x}$ [1], $\mathrm{x}[2], \mathrm{x}[3], \mathrm{x}$ [4] )))) ;

[ [ "C2", "C6 x C2", "C2 x C2", "C6" ],

[ "C2", "D12", "C2 x C2", "S3" ],

[ "C2 x C2", "D12", "C2 x C2", "D12" ],

[ "C2", "D12", "C2 x C2", "D12"],

[ "C2", "D12", "C2", "S3" ],

[ "C2", "D12", "C2 x C2", "D12" ],

[ "C2 x C2", "D12", "C2 x C2", "C2 x C2 x S3" ],

[ "C2 x C2", "D12", "C2", "C2 x C2 x S3"],

[ "C2 x C2", "D12", "C2 x C2", "C2 x C2 x S3" ],

[ "S3", "D12", "S3", "D12" ] ]

gap> Filtered(Collected(List(Flat(ips51), CaratZClass)), x->x [2]>1); \# type [5,1]

$[[[6,3045,3], 2],[[6,3046,3], 2],[[6,3047,3], 2]$,

$[[6,3052,5], 2],[[6,3053,5], 2],[[6,3054,3], 2]$,

$[[6,3061,5], 2],[[6,3066,3], 2]]$

gap> KSfail51:= List (last, $\mathrm{x}->\mathrm{x}[1])$;

$[[6,3045,3],[6,3046,3],[6,3047,3],[6,3052,5]$,

$[6,3053,5],[6,3054,3],[6,3061,5],[6,3066,3]]$

gap> Length(KSfail51); 
8

gap> KSfail51g:=List (KSfail51,x->CaratMatGroupZClass (x [1],x[2],x[3])); ;

gap> List (KSfail51g,Order);

[ $120,120,120,20,20,240,40,60$ ]

gap> List (KSfail51g, StructureDescription);

[ "C2 x A5", "S5", "S5", "C5 : C4", "C5 : C4", "C2 x S5", "C2 x (C5 : C4)", "A5" ]

gap> last [6]; \# the 6th group of the CARAT ID [6,3054, 3 ] is maximal

"C2 x S5"

gap> KSfail51gsub:=Set (ConjugacyClassesSubgroups2 (KSfail51g[6]),

$>\mathrm{x}->$ CaratZClass (Representative $(\mathrm{x}))$ ); ;

gap> Difference(KSfail51,KSfail51gsub);

[ ]

gap> ips51f:=Flat (ips51); ;

gap> ips51fc:=List (ips51f,CaratZClass); ;

gap> KSfail51i:=List (KSfail51,x->Filtered ([1..9939],y->ips51fc[y]=x)) ;

[ [9630,9633], [ 9635,9638], [9642, 9645], [9648, 9651$]$,

[9653,9656 ], [9660,9665], [9670,9675 ], [9679,9681 ] ]

gap> KSfail51gg:=List (KSfail51i,x->[ips51f[x[1]],ips51f[x[2]]]) ; ;

gap> List (KSfail51gg, $\mathrm{x}->\operatorname{List}(\mathrm{x}, \mathrm{y}->\operatorname{List}([[1],[2.6]]$,

$>$ z->CaratZClass (PartialMatrixGroup $(y, z))))$ );

$[[[[1,2,1],[5,910,3]],[[1,2,1],[5,910,4]]]$,

$[[[1,1,1],[5,911,3]],[[1,1,1],[5,911,4]]]$,

$[[[1,2,1],[5,912,3]],[[1,2,1],[5,912,4]]]$,

$[[[1,2,1],[5,917,3]],[[1,2,1],[5,917,4]]]$,

$[[[1,1,1],[5,918,3]],[[1,1,1],[5,918,4]]]$,

$[[[1,2,1],[5,919,3]],[[1,2,1],[5,919,4]]]$,

$[[[1,2,1],[5,926,5]],[[1,2,1],[5,926,6]]]$,

$[[[1,1,1],[5,931,3]],[[1,1,1],[5,931,4]]]]$

gap> List (last, $\mathrm{y}^{->\operatorname{List}}([\mathrm{y}[1][1], \mathrm{y}[1][2], \mathrm{y}[2][1], \mathrm{y}[2][2]]$,

$>\mathrm{x}->\operatorname{StructureDescription}(\operatorname{CaratMatGroupZClass}(\mathrm{x}[1], \mathrm{x}[2], \mathrm{x}[3])))$ ));

[ [ "C2", "C2 x A5", "C2", "C2 x A5" ],

[ "1", "S5", "1", "S5"],

[ "C2", "S5", "C2", "S5" ],

[ "C2", "C5 : $\mathrm{C} 4 "$, "C2", "C5 : $\mathrm{C} 4 "]$,

[ "1", "C5 : C4", "1", "C5 : C4" ],

[ "C2", "C2 x S5", "C2", "C2 x S5"],

[ "C2", "C2 x (C5 : $\mathrm{C} 4) "$, "C2", "C2 x (C5 : C4)"],

[ "1", "A5", "1", "A5" ] ]

4.3. Maximal finite groups $G \leq \operatorname{GL}(n, \mathbb{Z})$ of dimension $n \leq 6$. In this subsection, we consider not only maximal irreducible (resp. indecomposable) groups $G \leq \mathrm{GL}(n, \mathbb{Z})$ but also reducible (resp. decomposable) ones, i.e. we consider all the maximal groups $G \leq \mathrm{GL}(n, \mathbb{Z})$.

Let $L$ be a set of $\mathbb{Z}$-classes of finite groups $G$ in $\operatorname{GL}(n, \mathbb{Z})$. The following algorithm enable us to determine which $\mathbb{Z}$-classes are maximal ones in $L$.

MaximalGroupsID (L) returns the list of the GAP IDs of the maximal $\mathbb{Z}$-classes in the groups of the GAP IDs $L$. MaximalGroupsID (L:Carat) returns the same as MaximalGroupsID(L) but using the CARAT ID instead of the GAP ID.

MaximalGroupsID (L:FromPerm) (resp. MaximalGroupsID (L:Carat,FromPerm)) returns the same as MaximalGroupsID (L) (resp. MaximalGroupsID (L:Carat)) but using ConjugacyClassesSubgroupsFromPerm(G) instead of ConjugacyClassesSubgroups (G).

MaximalGroupsID:= function $(\mathrm{L})$

local G, O,m,mo,r,ri,ro,i,j,id,sg;

if ValueOption ("carat")=true or ValueOption ("Carat")=true then 


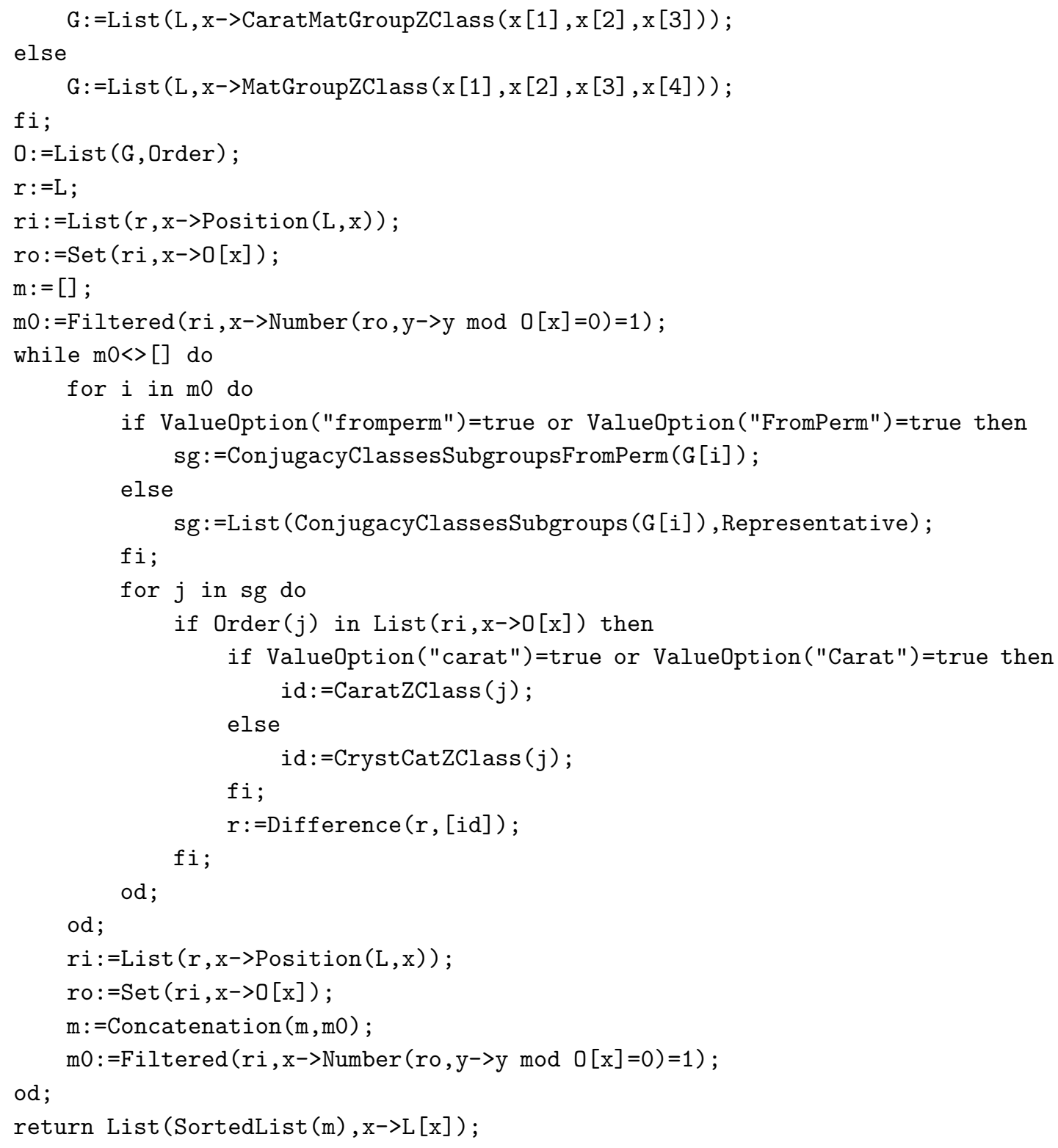

Example 4.14 (Maximal finite groups $G \leq \mathrm{GL}(n, \mathbb{Z})$ of dimension $n \leq 6$ ). Using MaximalGroupsID(L) for all $\mathbb{Z}$-classes $L$ of finite subgroups $G \leq \mathrm{GL}(n, \mathbb{Z})$, we can determine all the maximal finite groups $G \leq \mathrm{GL}(n, \mathbb{Z})$ of dimension $n \leq 6$. There exist 2 (resp. $4,9,17,39$ ) maximal groups $G \leq \operatorname{GL}(n, \mathbb{Z})$ (ZZ-classes) of dimension $n=2$ (resp. 3, 4, 5, 6) (see Dad65 for $n=4$, Rys72a, Rys72b, RL80] for $n=5$, Lor05, Section 1.10] for $n=6$ ).

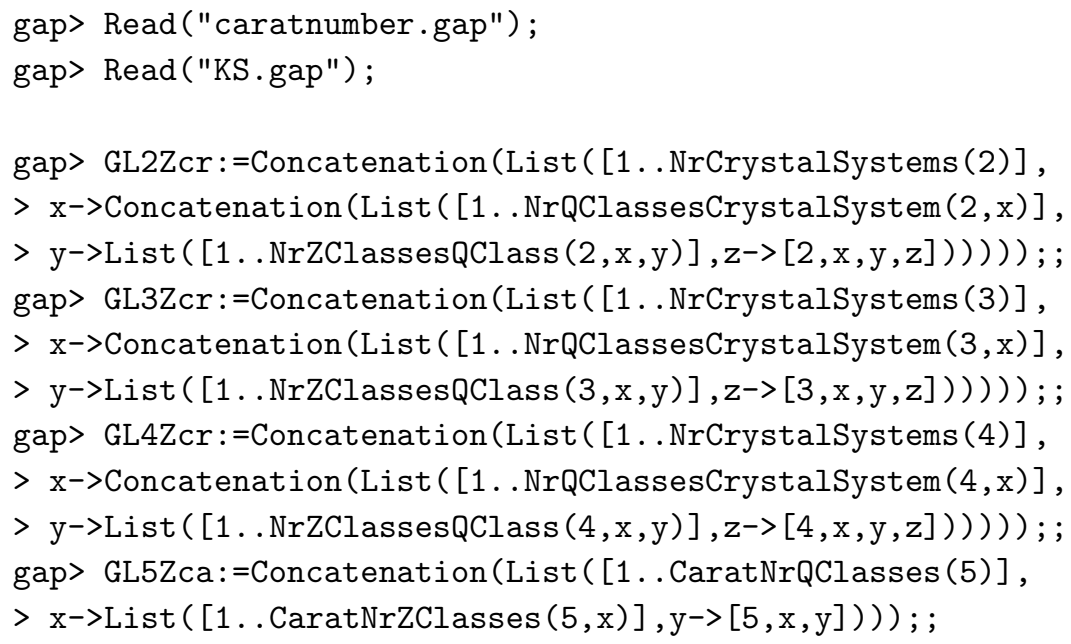




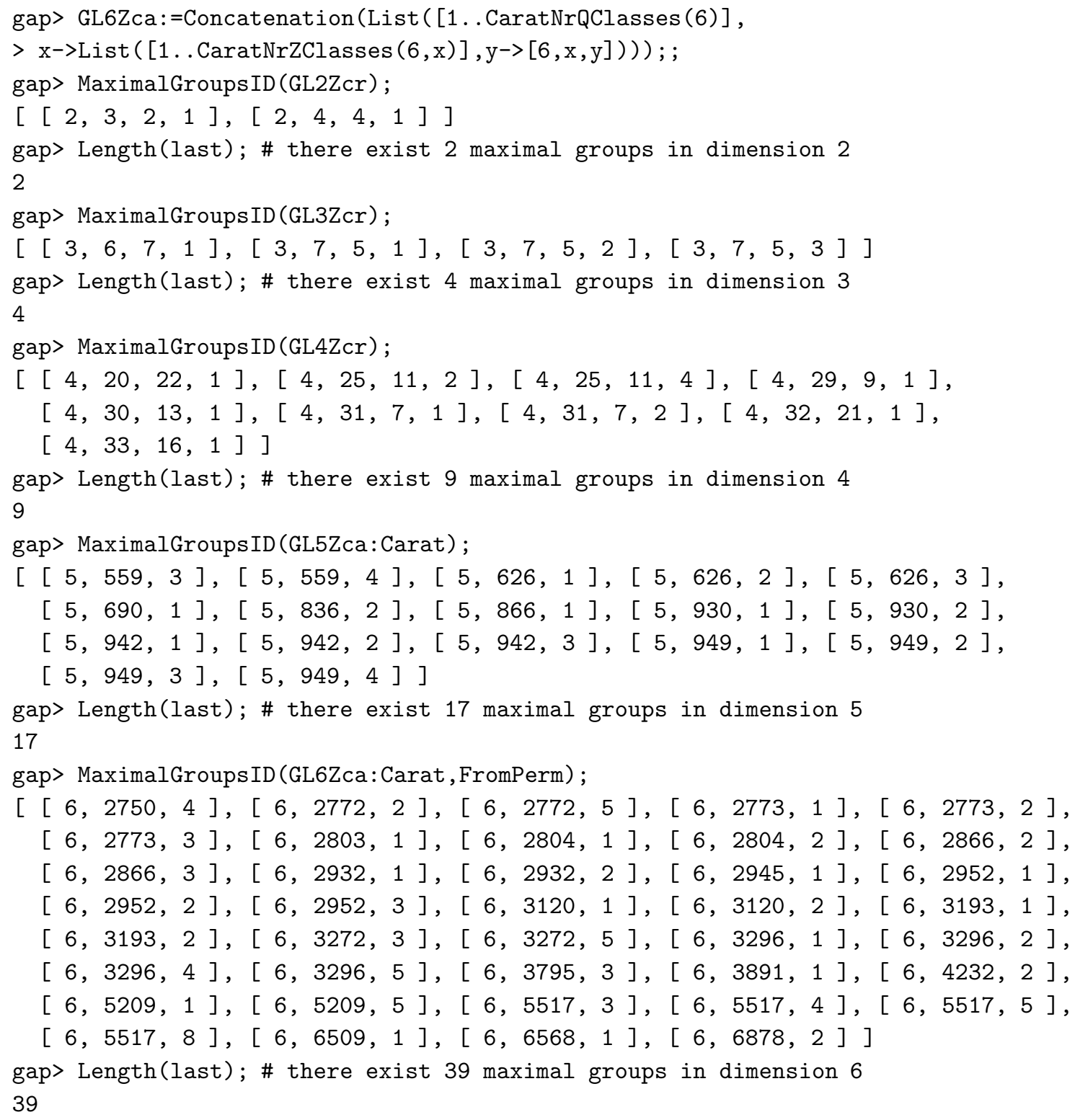

\subsection{Bravais groups of dimension $n \leq 6$ and corresponding quadratic forms.}

Definition 4.15 (Bravais group). Let $G \leq \mathrm{GL}(n, \mathbb{Z})$ be finite subgroup and $\mathbb{R}_{\mathrm{sym}}^{n \times n}$ be the set of $n \times n$ symmetric matrices (which correspond to quadratic forms) whose entries are in $\mathbb{R}$.

(i) $\mathcal{F}(G)=\left\{F \in \mathbb{R}_{\mathrm{sym}}^{n \times n} \mid g F^{t} g=F\right.$ (for any $g \in G$ ) $\}$ is called the space of invariant forms of $G$;

(ii) For $\mathcal{F} \leq \mathbb{R}_{\text {sym }}^{n \times n}, \mathcal{B}(\mathcal{F})=\left\{g \in \mathrm{GL}(n, \mathbb{Z}) \mid g F^{t} g=F\right.$ (for any $\left.\left.F \in \mathcal{F}\right)\right\}$ is called the Bravais group of $\mathcal{F}$;

(iii) $B(G):=\mathcal{B}(\mathcal{F}(G))$ is called the Bravais group of $G$;

(iv) $G \leq \mathrm{GL}(n, \mathbb{Z})$ is simply called a Bravais group of dimension $n$ if $B(G)=G$.

Note that if $\mathcal{F}$ contains a positive definite matrix, then $\mathcal{B}(\mathcal{F})$ becomes a finite set. By definitions, $\mathcal{F}(G)$ contains a positive definite matrix, and hence $G \leq B(G) \leq \mathrm{GL}(n, \mathbb{Z})$ is finite. In particular, all the maximal finite groups $G \leq \mathrm{GL}(n, \mathbb{Z})$ (as in the last subsection) are Bravais groups.

Example 4.16 (Bravais groups $G \leq \mathrm{GL}(n, \mathbb{Z}$ ) where $n \leq 6$ ). By using CARAT (Carat), e.g. the build-in function BravaisGroupsCrystalFamily, we may obtain the GAP ID and the Carat ID of all the $\mathbb{Z}$-classes of Bravais groups $G \leq \mathrm{GL}(n, \mathbb{Z})$ for $n \leq 6$ as follows. There exists 1 (resp. 5, 14, 64, 189, 841) 4 Bravais group of dimension $n=1$ (resp. 2, 3, 4, 5, 6) (see [BBNWZ78, Section 1.3], [PP77], Ple81, [PH84, [OPS98]).

\footnotetext{
${ }^{4}$ In PH84, the number, 826, of Bravais groups of dimension 6 was not correct. This is modified by OPS98 with the correct number 841.
} 
gap> Read ("caratnumber.gap");

gap> b1:=Flat (List (CaratCrystalFamilies [1], BravaisGroupsCrystalFamily)); ; gap> b2:=Flat (List (CaratCrystalFamilies [2], BravaisGroupsCrystalFamily)); ; gap> b3:=Flat (List (CaratCrystalFamilies [3], BravaisGroupsCrystalFamily)); ; gap> b4:=Flat (List (CaratCrystalFamilies [4], BravaisGroupsCrystalFamily)); ; gap> b5:=Flat (List (CaratCrystalFamilies [5], BravaisGroupsCrystalFamily)); ; gap> b6:=Flat (List (CaratCrystalFamilies [6], BravaisGroupsCrystalFamily)); ; gap> List ( [b1, b2, b3, b4 , b5, b6], Length);

$[1,5,14,64,189,841]$

gap> b1ca:=List (b1, CaratZClass);

$[1,2,1]]$

gap> Length(last); \# there exists 1 Bravais group of dimension 1

1

gap> b2cr:=List (b2, CrystCatZClass);

$[[2,1,2,1],[2,2,2,1],[2,2,2,2],[2,3,2,1],[2,4,4,1]]$ gap> Length(last); \# there exist 5 Bravais groups of dimension 2

5

gap> b3cr:=List (b3, CrystCatZClass);

$[[3,1,2,1],[3,2,3,1],[3,2,3,2],[3,3,3,1],[3,3,3,2]$,

$[3,3,3,3],[3,3,3,4],[3,4,7,1],[3,4,7,2],[3,6,7,1]$,

$[3,5,5,1],[3,7,5,3],[3,7,5,1],[3,7,5,2]]$

gap> Length(last); \# there exist 14 Bravais groups of dimension 3

14

gap> b4cr:=List (b4, CrystCatZClass) ;

$[[4,1,2,1],[4,2,3,1],[4,2,3,2],[4,3,2,1],[4,3,2,2]$,

$[4,3,2,3],[4,4,4,1],[4,4,4,3],[4,4,4,2],[4,4,4,6]$,

$[4,4,4,5],[4,4,4,4],[4,6,3,1],[4,6,3,2],[4,6,3,6]$,

$[4,6,3,8],[4,6,3,5],[4,6,3,3],[4,6,3,7],[4,6,3,4]$,

$[4,5,2,1],[4,10,1,1],[4,16,1,1],[4,16,1,2],[4,16,1,3]$,

$[4,7,7,1],[4,7,7,2],[4,13,10,1],[4,13,10,3],[4,13,10,2]$,

$[4,13,10,5],[4,13,10,4],[4,12,5,1],[4,19,6,1],[4,19,6,2]$,

$[4,18,5,1],[4,20,22,1],[4,11,2,1],[4,17,2,1],[4,17,2,2]$,

$[4,9,7,1],[4,8,5,1],[4,15,12,1],[4,15,12,2],[4,14,10,1]$,

$[4,14,10,2],[4,23,11,1],[4,22,11,1],[4,21,4,1],[4,25,11,2]$,

$[4,25,11,3],[4,24,5,1],[4,25,11,1],[4,25,11,5],[4,25,11,4]$,

$[4,32,21,1],[4,33,16,1],[4,26,2,1],[4,30,13,1],[4,29,9,1]$,

$[4,28,2,1],[4,31,7,1],[4,31,7,2],[4,27,4,1]]$

gap> Length(last); \# there exist 64 Bravais groups of dimension 4

64

gap> b5ca:=List (b5, CaratZClass);

$[[5,2,1],[5,5,1],[5,5,2],[5,8,1],[5,8,2]$,

$[5,8,3],[5,12,1],[5,12,2],[5,12,3],[5,12,4]$,

$[5,12,5],[5,12,6],[5,16,1],[5,16,2],[5,16,4]$,

$[5,16,3],[5,16,5],[5,16,6],[5,16,8],[5,16,7]$,

$[5,16,9],[5,17,1],[5,17,2],[5,17,3],[5,17,4]$,

$[5,17,5],[5,17,6],[5,17,7],[5,17,10],[5,17,8]$,

$[5,17,11],[5,17,9],[5,17,12],[5,17,13],[5,17,14]$,

$[5,17,17],[5,17,16],[5,17,15],[5,20,18],[5,29,1]$,

$[5,29,2],[5,29,3],[5,29,4],[5,29,5],[5,29,6]$,

$[5,29,8],[5,29,10],[5,29,7],[5,29,9],[5,29,11]$,

$[5,29,12],[5,29,14],[5,29,13],[5,29,15],[5,29,16]$,

$[5,25,31],[5,25,32],[5,25,33],[5,35,1],[5,35,2]$,

$[5,36,4],[5,36,5],[5,36,1],[5,36,2],[5,36,3]$, 
$[5,36,6],[5,36,7],[5,40,1],[5,40,2],[5,56,1]$, $[5,56,2],[5,56,4],[5,56,5],[5,56,3],[5,56,6]$,

$[5,56,7],[5,48,15],[5,97,1],[5,97,2],[5,97,3]$,

$[5,97,4],[5,97,5],[5,97,6],[5,97,7],[5,97,9]$,

$[5,97,8],[5,97,10],[5,97,11],[5,97,12],[5,88,39]$,

$[5,88,40],[5,88,41],[5,104,1],[5,104,2],[5,104,4]$,

$[5,104,3],[5,104,5],[5,151,17],[5,151,18],[5,151,19]$,

$[5,273,1],[5,273,2],[5,372,5],[5,372,6],[5,163,1]$,

$[5,167,2],[5,172,2],[5,171,2],[5,172,1],[5,171,4]$,

$[5,180,1],[5,176,3],[5,215,1],[5,215,2],[5,207,5]$,

$[5,207,6],[5,205,5],[5,205,6],[5,266,1],[5,266,2]$,

$[5,266,3],[5,257,10],[5,266,4],[5,257,12],[5,257,14]$,

$[5,257,13],[5,257,15],[5,421,1],[5,394,2],[5,465,6]$,

$[5,395,2],[5,465,7],[5,460,2],[5,416,4],[5,517,4]$,

$[5,517,5],[5,511,6],[5,517,2],[5,517,3],[5,517,1]$,

$[5,518,8],[5,518,9],[5,518,10],[5,518,11],[5,540,15]$,

$[5,540,16],[5,518,12],[5,540,17],[5,518,3],[5,518,4]$,

$[5,518,5],[5,518,6],[5,518,7],[5,518,1],[5,518,2]$,

$[5,559,4],[5,559,5],[5,559,1],[5,559,2],[5,559,3]$,

$[5,626,3],[5,626,2],[5,638,4],[5,626,1],[5,638,2]$,

$[5,643,1],[5,643,2],[5,772,4],[5,772,5],[5,690,1]$,

$[5,772,3],[5,793,1],[5,866,1],[5,801,3],[5,836,2]$,

$[5,801,6],[5,908,1],[5,904,3],[5,930,2],[5,919,3]$,

$[5,919,4],[5,930,1],[5,942,1],[5,942,3],[5,942,2]$,

$[5,949,4],[5,949,1],[5,949,3],[5,949,2]]$

gap> Length(last); \# there exist 189 Bravais groups of dimension 5

189

gap> b6ca: $=$ List (b6, CaratZClass);

$[[6,2710,1],[6,3,1],[6,3,2],[6,6,1],[6,6,2]$,

$[6,6,3],[6,10,1],[6,10,2],[6,10,3],[6,10,4]$,

$[6,10,6],[6,10,5],[6,12,1],[6,12,2],[6,12,3]$,

$[6,12,4],[6,17,1],[6,17,2],[6,17,3],[6,17,4]$,

$[6,17,5],[6,17,6],[6,17,7],[6,17,8],[6,17,9]$,

$[6,17,10],[6,17,11],[6,17,12],[6,4617,1],[6,4617,2]$,

$[6,4617,4],[6,4617,6],[6,4617,3],[6,4617,5],[6,4617,8]$,

$[6,4617,11],[6,4617,14],[6,4617,9],[6,4617,7],[6,4617,13]$,

$[6,4617,10],[6,4617,12],[6,4617,18],[6,4617,16],[6,4617,17]$,

$[6,4617,15],[6,4620,19],[6,4627,1],[6,4627,2],[6,4627,3]$,

$[6,4627,6],[6,4627,4],[6,4627,7],[6,4627,8],[6,4627,5]$,

$[6,4627,9],[6,4627,11],[6,4627,10],[6,4627,12],[6,4628,1]$,

$[6,4628,2],[6,4628,6],[6,4628,3],[6,4628,4],[6,4628,7]$,

$[6,4628,5],[6,4628,12],[6,4628,9],[6,4628,14],[6,4628,8]$,

$[6,4628,13],[6,4628,19],[6,4628,23],[6,4628,16],[6,4628,11]$,

$[6,4628,21],[6,4628,10],[6,4628,15],[6,4628,18],[6,4628,22]$,

$[6,4628,17],[6,4628,20],[6,4628,26],[6,4628,30],[6,4628,25]$,

$[6,4628,27],[6,4628,31],[6,4628,29],[6,4628,33],[6,4628,35]$,

$[6,4628,24],[6,4628,28],[6,4628,32],[6,4628,34],[6,4631,36]$,

$[6,4628,37],[6,4628,36],[6,4631,39],[6,4643,1],[6,4643,2]$,

$[6,4643,3],[6,4643,4],[6,4643,5],[6,4643,6],[6,4643,7]$,

$[6,4643,8],[6,4643,11],[6,4643,14],[6,4643,10],[6,4643,13]$,

$[6,4643,16],[6,4643,12],[6,4643,15],[6,4643,20],[6,4643,18]$,

$[6,4643,22],[6,4643,24],[6,4643,25],[6,4643,26],[6,4643,23]$,

$[6,4643,27],[6,4643,9],[6,4643,19],[6,4643,17],[6,4643,21]$,

$[6,4643,34],[6,4643,39],[6,4643,35],[6,4643,36],[6,4643,40]$,

$[6,4643,44],[6,4643,45],[6,4643,29],[6,4643,33],[6,4643,32]$, 
$[6,4643,37],[6,4643,38],[6,4643,30],[6,4643,43],[6,4643,31]$, $[6,4643,42],[6,4643,28],[6,4643,41],[6,4637,98],[6,4637,99]$, $[6,4636,50],[6,4636,51],[6,4643,48],[6,4643,47],[6,4643,49]$, $[6,4643,46],[6,4637,101],[6,4637,100],[6,4636,52],[6,4636,53]$, $[6,4663,1],[6,4663,2],[6,4663,3],[6,4663,4],[6,4663,5]$, $[6,4663,6],[6,4663,8],[6,4663,10],[6,4663,12],[6,4663,9]$, $[6,4663,11],[6,4663,13],[6,4663,15],[6,4663,14],[6,4663,16]$, $[6,4663,17],[6,4663,7],[6,4663,24],[6,4663,25],[6,4663,26]$, $[6,4663,28],[6,4663,27],[6,4663,29],[6,4663,19],[6,4663,23]$, $[6,4663,21],[6,4663,20],[6,4663,22],[6,4663,18],[6,4659,107]$, $[6,4659,109],[6,4659,112],[6,4663,33],[6,4663,34],[6,4663,31]$, $[6,4663,35],[6,4663,32],[6,4663,30],[6,4659,127],[6,4659,131]$, $[6,4659,128],[6,4659,132],[6,4657,107],[6,4659,129],[6,4659,133]$,

$[6,4663,36],[6,4658,37],[6,4659,134],[6,4657,109],[6,4666,1]$, $[6,4669,1],[6,4669,2],[6,4669,3],[6,4670,1],[6,4670,2]$, $[6,4670,3],[6,4670,4],[6,4670,6],[6,4670,5],[6,4672,7]$, $[6,4678,1],[6,4678,2],[6,4677,3],[6,25,1],[6,4680,2]$, $[6,4680,1],[6,4680,3],[6,4681,1],[6,4681,2],[6,4681,3]$, $[6,4681,4],[6,4681,5],[6,4681,6],[6,4681,7],[6,4681,8]$, $[6,4681,9],[6,4690,1],[6,4690,2],[6,4690,3],[6,4690,4]$, $[6,4690,6],[6,4690,5],[6,4689,34],[6,4689,35],[6,4690,7]$, $[6,4690,8],[6,4690,9],[6,4690,10],[6,4690,11],[6,4690,12]$, $[6,4690,14],[6,4690,13],[6,4690,15],[6,4690,16],[6,4689,21]$, $[6,4690,17],[6,4690,18],[6,4690,19],[6,4690,20],[6,4690,21]$, $[6,4689,27],[6,4688,22],[6,4704,1],[6,4704,2],[6,4700,3]$, $[6,4700,4],[6,4704,3],[6,4704,4],[6,4704,5],[6,4700,11]$, $[6,4704,6],[6,4704,7],[6,4700,18],[6,4701,13],[6,39,2]$, $[6,39,1],[6,39,3],[6,4705,1],[6,4705,2],[6,4721,1]$, $[6,4721,3],[6,4721,2],[6,4721,4],[6,4721,5],[6,4721,7]$, $[6,4721,6],[6,4719,8],[6,4742,1],[6,4742,2],[6,4742,3]$, $[6,4742,4],[6,4742,6],[6,4742,5],[6,4742,7],[6,4742,8]$, $[6,4737,9],[6,4742,9],[6,4737,11],[6,4800,1],[6,4800,5]$, $[6,4800,2],[6,4800,6],[6,4800,7],[6,4800,3],[6,4800,4]$, $[6,4800,8],[6,4800,9],[6,4800,19],[6,4800,14],[6,4800,20]$, $[6,4800,11],[6,4800,17],[6,4800,12],[6,4800,10],[6,4800,13]$, $[6,4800,21],[6,4800,22],[6,4800,15],[6,4800,18],[6,4800,23]$, $[6,4800,16],[6,4787,34],[6,4787,35],[6,4798,24],[6,4798,26]$, $[6,4800,25],[6,4800,26],[6,4800,27],[6,4800,24],[6,4787,41]$, $[6,4787,40],[6,4798,30],[6,4798,31],[6,4815,1],[6,4815,2]$, $[6,4815,3],[6,4815,4],[6,4815,5],[6,4815,6],[6,4815,7]$, $[6,4815,8],[6,4815,10],[6,4815,12],[6,4815,9],[6,4815,11]$, $[6,4815,13],[6,4815,16],[6,4815,17],[6,4815,14],[6,4815,18]$, $[6,4815,15],[6,4815,19],[6,4815,20],[6,4815,21],[6,4880,54]$, $[6,4880,56],[6,4880,59],[6,4815,23],[6,4815,25],[6,4815,22]$, $[6,4815,26],[6,4815,24],[6,4815,27],[6,4815,29],[6,4815,28]$, $[6,4880,76],[6,4880,80],[6,4880,77],[6,4880,81],[6,4880,82]$, $[6,4880,78],[6,4881,31],[6,4881,32],[6,4875,58],[6,4815,30]$, $[6,4880,83],[6,4881,33],[6,4882,31],[6,4875,61],[6,2319,1]$, $[6,2319,2],[6,2319,3],[6,2319,4],[6,2319,5],[6,2366,5]$, $[6,2366,7],[6,2319,6],[6,2366,9],[6,2366,10],[6,2425,1]$, $[6,2425,5],[6,2425,2],[6,2425,6],[6,2425,3],[6,2425,4]$, $[6,2425,7],[6,2425,12],[6,2425,9],[6,2425,14],[6,2379,15]$, $[6,2379,16],[6,2379,17],[6,2425,10],[6,2425,8],[6,2425,11]$, $[6,2425,13],[6,2382,19],[6,2382,21],[6,2538,25],[6,2379,21]$, $[6,2425,15],[6,2379,22],[6,2379,19],[6,2379,20],[6,2379,23]$, 
$[6,2379,24],[6,2382,22],[6,2384,50],[6,2379,25],[6,2538,30]$, $[6,2436,35],[6,2665,1],[6,2665,2],[6,2665,3],[6,2665,4]$, $[6,2636,5],[6,2636,7],[6,2604,15],[6,2637,5],[6,2636,8]$, $[6,2700,12],[6,2604,17],[6,748,1],[6,748,2],[6,684,7]$, $[6,1985,1],[6,2293,2],[6,2293,1],[6,46,1],[6,46,2]$, $[6,145,3],[6,145,4],[6,251,1],[6,251,3],[6,251,2]$, $[6,251,4],[6,164,12],[6,164,16],[6,164,15],[6,164,14]$, $[6,164,17],[6,251,5],[6,184,6],[6,164,18],[6,498,27]$, $[6,1343,1],[6,1616,4],[6,1487,2],[6,1966,1],[6,1967,1]$, $[6,1971,2],[6,1971,3],[6,1976,1],[6,1976,2],[6,1972,3]$, $[6,1972,4],[6,1999,1],[6,1992,2],[6,1986,2],[6,2255,2]$, $[6,2255,1],[6,2261,1],[6,2257,5],[6,2257,6],[6,2261,2]$, $[6,2257,2],[6,2277,3],[6,2277,4],[6,2271,9],[6,2271,10]$, $[6,2277,1],[6,2277,2],[6,2271,3],[6,2271,4],[6,2270,5]$, $[6,2270,6],[6,2296,2],[6,2313,8],[6,2300,4],[6,2296,1]$, $[6,2313,3],[6,2300,2],[6,2006,1],[6,2002,3],[6,2041,1]$, $[6,2041,2],[6,2033,5],[6,2031,3],[6,2033,6],[6,2031,4]$, $[6,2066,1],[6,2066,2],[6,2059,3],[6,2059,5],[6,2066,3]$, $[6,2059,6],[6,2153,1],[6,2153,3],[6,2153,2],[6,2153,4]$, $[6,2139,5],[6,2152,13],[6,2139,9],[6,2139,7],[6,2139,10]$, $[6,2152,18],[6,2152,17],[6,2152,15],[6,2152,19],[6,2153,6]$, $[6,2153,5],[6,2139,12],[6,2139,11],[6,2152,21],[6,2152,20]$, $[6,2158,1],[6,2158,2],[6,2158,3],[6,2158,4],[6,2243,19]$, $[6,2243,23],[6,2243,26],[6,2243,27],[6,2243,28],[6,2243,29]$, $[6,2158,6],[6,2158,5],[6,2158,7],[6,2243,32],[6,2243,31]$, $[6,2243,33],[6,2243,34],[6,2243,35],[6,2158,8],[6,2254,9]$, $[6,2243,36],[6,2235,26],[6,904,1],[6,878,4],[6,876,2]$, $[6,951,7],[6,943,2],[6,899,4],[6,951,8],[6,951,9]$, $[6,1198,1],[6,1198,2],[6,1112,3],[6,1113,7],[6,998,5]$, $[6,1113,8],[6,1112,4],[6,998,8],[6,1342,2],[6,1190,6]$, $[6,998,9],[6,1001,9],[6,998,10],[6,1001,10],[6,1098,10]$, $[6,1342,4],[6,1190,8],[6,1098,12],[6,1758,1],[6,1956,2]$, $[6,1872,3],[6,1846,2],[6,1915,3],[6,1748,4],[6,1872,4]$, $[6,1870,5],[6,1915,4],[6,4897,4],[6,4897,2],[6,4897,5]$, $[6,4897,1],[6,4897,3],[6,4897,6],[6,4894,3],[6,4894,7]$, $[6,4894,9],[6,4913,1],[6,4913,2],[6,4912,3],[6,4913,3]$, $[6,4913,4],[6,4913,5],[6,4914,10],[6,4914,12],[6,4914,11]$, $[6,4914,13],[6,4914,14],[6,4952,7],[6,4952,8],[6,4914,15]$, $[6,4914,16],[6,4952,10],[6,4952,11],[6,4964,6],[6,4964,8]$, $[6,4964,10],[6,4964,11],[6,4914,1],[6,4914,3],[6,4914,2]$, $[6,4914,4],[6,4914,5],[6,4914,7],[6,4914,6],[6,4914,8]$, $[6,4914,9],[6,4979,13],[6,4979,14],[6,4979,15],[6,4979,16]$, $[6,4979,17],[6,4979,18],[6,4966,10],[6,4966,12],[6,4966,15]$, $[6,4979,19],[6,4979,20],[6,4979,21],[6,4979,22],[6,4979,23]$, $[6,4966,22],[6,4966,26],[6,4966,23],[6,4966,27],[6,4966,24]$, $[6,4966,28],[6,4979,24],[6,4966,29],[6,4978,25],[6,4979,1]$, $[6,4979,2],[6,4979,3],[6,4979,4],[6,4979,5],[6,4979,6]$, $[6,4979,8],[6,4979,7],[6,4979,9],[6,4979,10],[6,4979,11]$, $[6,4979,12],[6,4979,25],[6,4979,26],[6,4979,27],[6,4979,28]$, $[6,5144,9],[6,5144,11],[6,5144,10],[6,5144,12],[6,5144,13]$, $[6,5116,6],[6,5116,8],[6,5144,14],[6,5118,21],[6,5116,9]$, $[6,5144,1],[6,5144,3],[6,5144,2],[6,5144,4],[6,5144,5]$, $[6,5144,6],[6,5144,7],[6,5144,8],[6,5209,1],[6,5209,2]$, $[6,5448,3],[6,5447,3],[6,5448,4],[6,5347,11],[6,5209,3]$, $[6,5310,2],[6,5209,4],[6,5448,7],[6,5310,4],[6,5491,14]$, 
$[6,5448,8],[6,5491,16],[6,5209,5],[6,5310,8],[6,5448,12]$, $[6,5491,24],[6,5517,6],[6,5517,7],[6,5503,8],[6,5557,11]$, $[6,5517,3],[6,5517,4],[6,5517,8],[6,5517,1],[6,5517,2]$, $[6,5529,2],[6,5529,4],[6,5533,5],[6,5517,5],[6,5529,8]$, $[6,5517,9],[6,5529,13],[6,5533,17],[6,5557,15],[6,5533,18]$, $[6,5566,1],[6,5566,2],[6,5582,1],[6,5582,2],[6,5582,3]$, $[6,5582,4],[6,5582,5],[6,5578,11],[6,5584,1],[6,5584,2]$, $[6,5599,5],[6,5601,1],[6,5749,3],[6,5749,4],[6,5667,1]$, $[6,5749,2],[6,5700,3],[6,5830,8],[6,5830,9],[6,5830,10]$, $[6,5830,11],[6,5830,12],[6,5889,11],[6,5993,1],[6,5830,4]$, $[6,5993,2],[6,5830,6],[6,6090,6],[6,5830,7],[6,6098,8]$, $[6,6322,1],[6,6322,2],[6,6404,5],[6,6509,1],[6,6322,5]$, $[6,6213,11],[6,6878,2],[6,6568,1],[6,6722,2],[6,3313,1]$, $[6,3328,1],[6,3328,2],[6,3330,1],[6,3332,2],[6,3358,1]$, $[6,3362,2],[6,3446,1],[6,3376,3],[6,3416,3],[6,3376,6]$, $[6,3478,1],[6,3478,2],[6,3635,5],[6,3635,6],[6,3513,7]$, $[6,3513,8],[6,3719,5],[6,3719,6],[6,3635,11],[6,3635,12]$, $[6,3891,1],[6,3988,2],[6,3988,4],[6,3795,3],[6,4349,1]$, $[6,4586,4],[6,4616,2],[6,4232,2],[6,4586,8],[6,4528,7]$, $[6,2965,1],[6,2961,3],[6,2988,1],[6,2988,2],[6,2982,5]$, $[6,2982,6],[6,2989,1],[6,3020,1],[6,3065,2],[6,3054,3]$, $[6,3065,1],[6,3113,3],[6,3113,4],[6,3097,5],[6,3097,6]$, $[6,3097,7],[6,3097,8],[6,3113,1],[6,3113,2],[6,3120,2]$, $[6,3120,1],[6,3127,2],[6,3193,2],[6,3193,1],[6,3272,1]$, $[6,3272,2],[6,3272,5],[6,3272,3],[6,3272,4],[6,3258,5]$, $[6,3296,5],[6,3296,6],[6,3282,8],[6,3282,9],[6,3296,1]$, $[6,3296,4],[6,3282,5],[6,3296,2],[6,3296,3],[6,2773,1]$, $[6,2773,2],[6,2773,3],[6,2772,5],[6,2772,2],[6,2750,4]$, $[6,2803,1],[6,2804,1],[6,2804,2],[6,2866,3],[6,2866,2]$, $[6,2930,1],[6,2932,2],[6,2932,1],[6,2945,1],[6,2949,1]$, $[6,2952,1],[6,2952,2],[6,2952,3],[6,2953,2],[6,2953,3]$, $[6,2953,1]]$

gap> Length(last); \# there exist 841 Bravais groups of dimension 6 841

Remark 4.17. We can get all the maximal group $G \leq \mathrm{GL}(n, \mathbb{Z})(n \leq 6)$ by the function MaximalGroupsID(L) as in Subsection 4.3 applying to only the Bravais groups $L$ (cf. Subsection 4.3 ). For example, we get the following for $n=6$ which agrees the result in Subsection 4.3 ;

gap> Read("caratnumber.gap");

gap> Read ("KS.gap");

gap> MaximalGroupsID (b6ca: Carat, FromPerm);

$[[6,5209,1],[6,5209,5],[6,5517,3],[6,5517,4],[6,5517,8]$, $[6,5517,5],[6,6509,1],[6,6878,2],[6,6568,1],[6,3891,1]$, $[6,3795,3],[6,4232,2],[6,3120,2],[6,3120,1],[6,3193,2]$, $[6,3193,1],[6,3272,5],[6,3272,3],[6,3296,5],[6,3296,1]$, $[6,3296,4],[6,3296,2],[6,2773,1],[6,2773,2],[6,2773,3]$, $[6,2772,5],[6,2772,2],[6,2750,4],[6,2803,1],[6,2804,1]$, $[6,2804,2],[6,2866,3],[6,2866,2],[6,2932,2],[6,2932,1]$,

$[6,2945,1],[6,2952,1],[6,2952,2],[6,2952,3]]$

gap> Length(last); \# there exist 39 maximal groups in dimension 6 
Voskresenskii (see Vos83, Vos98, Section 8]) investigated the rationality problem for algebraic tori $T$ which correspond to Bravais groups $G \leq \operatorname{GL}(n, \mathbb{Z})$. Using the classification of Bravais groups above, we will show that $H^{1}\left(G,\left[M_{G}\right]^{f l}\right)=0$ for any Bravais group $G$ of dimension $n \leq 6$ in Section 7 (see Theorem 7.1).

By using the algorithms below, one can obtain (positive definite) invariant quadratic forms $f$ (or corresponding invariant forms $F \in \mathbb{R}_{\mathrm{sym}}^{n \times n}$ ) under the action of Bravais group $G \leq \mathrm{GL}(n, \mathbb{Z})$. We will present the result only for the maximal groups $G \leq \mathrm{GL}(n, \mathbb{Z})$ and for $n \leq 6$ in the end of this section. For the maximal cases, see also Dad65 for $n=4$, Rys72a, Rys72b, RL80 for $n=5$, [PP77 for $n=6$ (irreducible cases). The following algorithms are available from http://math.h.kyoto-u.ac.jp/ yamasaki/Algorithm/as Bravais.gap.

InvariantFormsMatrix(G) returns a basis of the space of invariant forms $\mathcal{F}(G)=\left\{F \in \mathbb{R}_{\mathrm{sym}}^{n \times n} \mid g F^{t} g=\right.$ $F$ (for any $g \in G)\}$ for a finite group $G \leq \mathrm{GL}(n, \mathbb{Z})$.

InvariantQuadraticForms $(G)$ returns a basis of the set of all invariant quadratic forms under the action of $G \leq \operatorname{GL}(n, \mathbb{Z})$.

AutomorphismGroupDfMatrix(F) returns the automorphism group $G=\left\{g \in \mathrm{GL}(n, \mathbb{Z}) \mid g F^{t} g=F\right\}$ of a positive definite symmetric matrix $F \in \mathbb{R}_{\text {sym }}^{n \times n}$.

QuadraticFormToMatrix (f) returns a symmetric matrix $F$ which corresponds to quadratic form $f$. QuadraticFormToMatrix $(f, R)$, (resp. QuadraticFormToMatrix $\left(f,\left[x_{1}, \ldots, x_{n}\right]\right)$ ) returns the same as QuadraticFormToMatrix $(\mathrm{f})$ but with respect to the base ring $R$ (resp. variables $x_{1}, \ldots, x_{n}$ ).

MatrixToQuadraticForm(F) returns a quadratic form $f$ which corresponds to a symmetric matrix $F$. MatrixToQuadraticForm $(F, R)$, (resp. MatrixToQuadraticForm $\left(F,\left[x_{1}, \ldots, x_{n}\right]\right)$ ) returns the same as MatrixToQuadraticForm(F) but with respect to the base ring $R$ (resp. variables $x_{1}, \ldots, x_{n}$ ). IsPositiveDefinite(F) returns whether a symmetric matrix $F$ is positive definite or not.

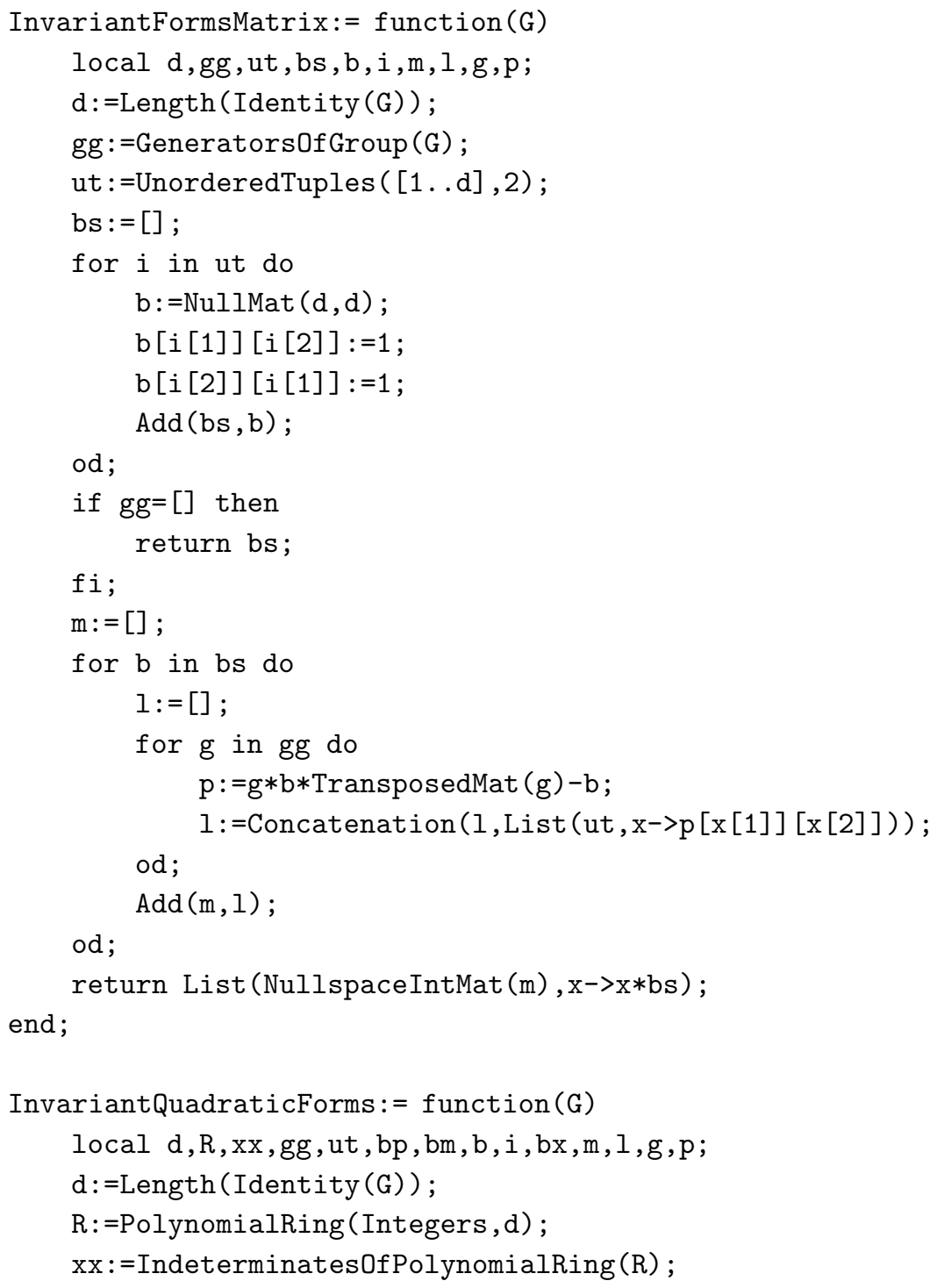




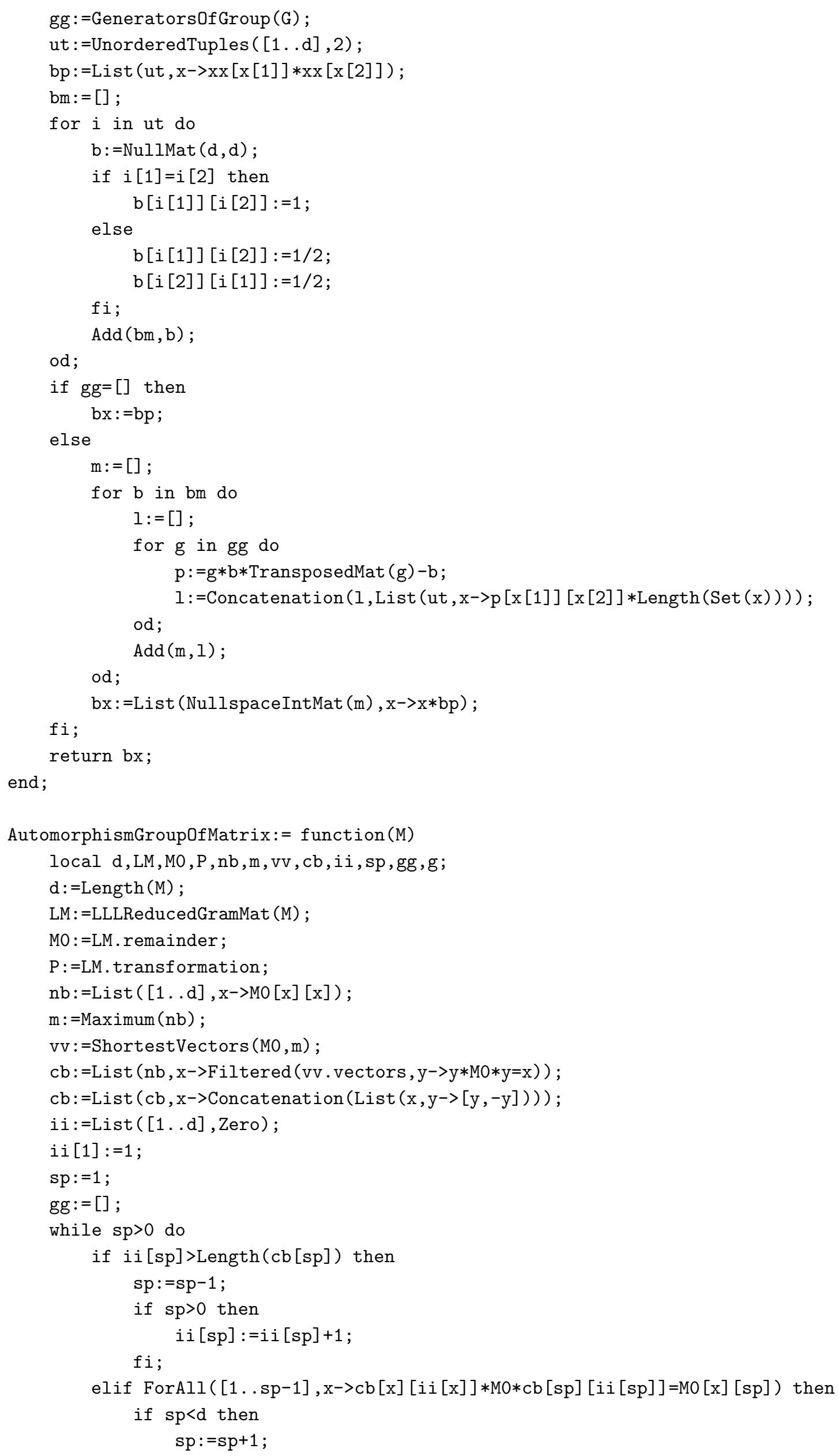




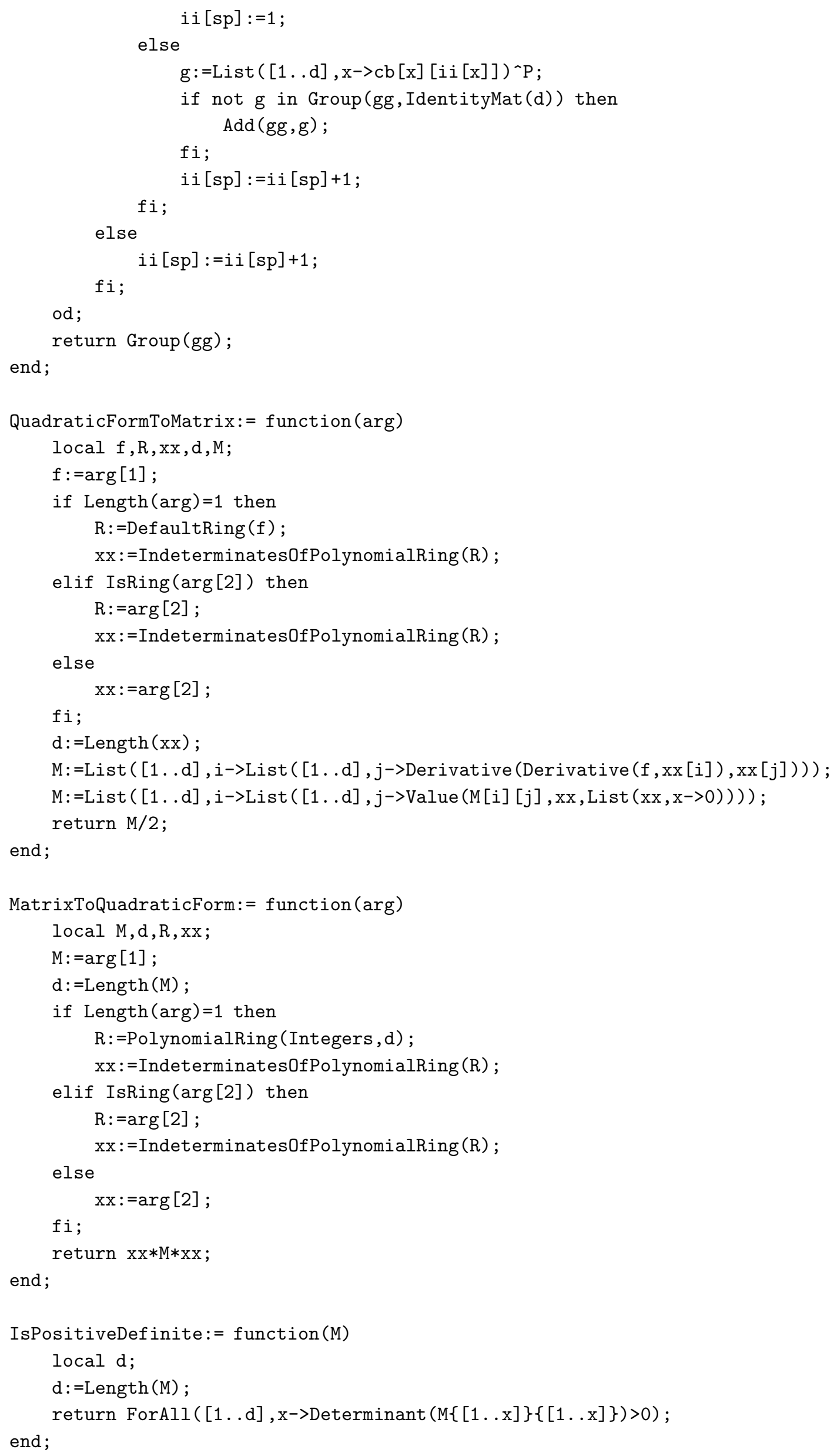


Example 4.18 (Functions related to Bravais groups $G \leq \mathrm{GL}(n, \mathbb{Z})$ in Bravais gap).

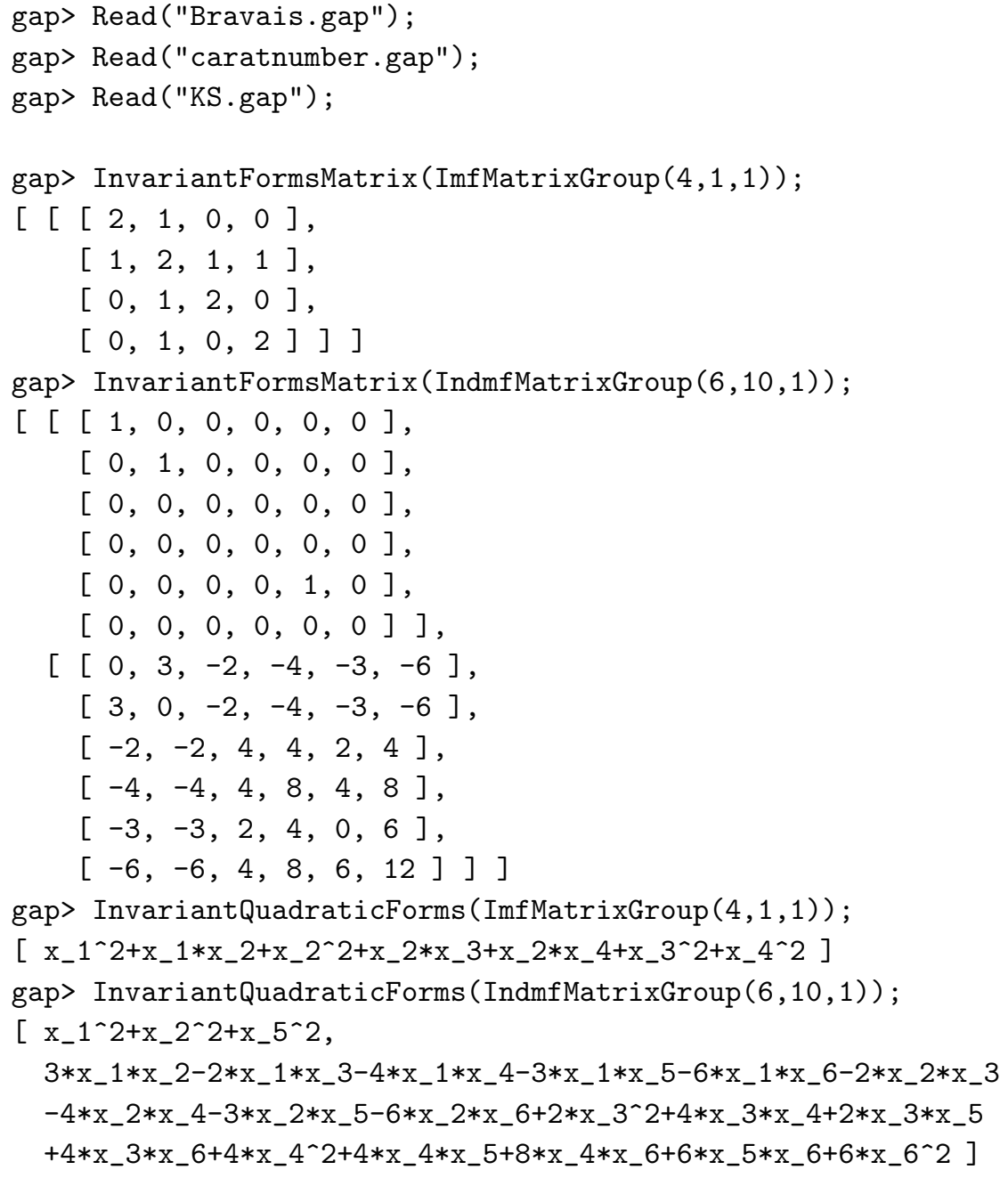


gap> MatrixToQuadraticForm(IdentityMat (4), R);

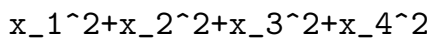

gap> MatrixToQuadraticForm(IdentityMat (4), [R.1,R.2,R.3,R.4]);

$\mathrm{x}_{-} 1^{\wedge} 2+\mathrm{x}_{-} 22^{\wedge} 2+\mathrm{x}_{-} 3^{\wedge} 2+\mathrm{x}_{-} 4^{\wedge} 2$

gap> IsPositiveDefinite([[2,1], $[1,2]])$;

true

gap> IsPositiveDefinite([[2,2], $[2,2]])$;

false 
GAP ID/

CARAT ID Imf/Indmf

Invariant (positive definite) quadratic form of maximal group $G \leq \mathrm{GL}(n, \mathbb{Z})$

\begin{tabular}{|c|c|}
\hline$(1,2,1)$ & $\operatorname{Imf}(1,1,1)$ \\
\hline$(2,3,2,1)$ & $\operatorname{Imf}(2,1,1)$ \\
\hline$(2,4,4,1)$ & $\operatorname{Imf}(2,2,1)$ \\
\hline$(3,6,7,1)$ & $\operatorname{Imf}(2,2,1) \times \operatorname{Imf}(1,1,1)$ \\
\hline$(3,7,5,1)$ & $\operatorname{Imf}(3,1,1)$ \\
\hline$(3,7,5,2)$ & $\operatorname{Imf}(3,1,2)$ \\
\hline$(3,7,5,3)$ & $\operatorname{Imf}(3,1,3)$ \\
\hline$(4,20,22,1)$ & $\operatorname{Imf}(2,1,1) \times \operatorname{Imf}(2,2,1)$ \\
\hline$(4,25,11,2)$ & $\operatorname{Imf}(3,1,3) \times \operatorname{Imf}(1,1,1)$ \\
\hline$(4,25,11,4)$ & $\operatorname{Imf}(3,1,2) \times \operatorname{Imf}(1,1,1)$ \\
\hline$(4,29,9,1)$ & $\operatorname{Imf}(4,5,1)$ \\
\hline$(4,30,13,1)$ & $\operatorname{Imf}(4,2,1)$ \\
\hline$(4,31,7,1)$ & $\operatorname{Imf}(4,3,1)$ \\
\hline$(4,31,7,2)$ & $\operatorname{Imf}(4,3,2)$ \\
\hline$(4,32,21,1)$ & $\operatorname{Imf}(4,4,1)$ \\
\hline$(4,33,16,1)$ & $\operatorname{Imf}(4,1,1)$ \\
\hline$(5,559,3)$ & $\overline{\operatorname{Imf}}(3,1,2) \times \operatorname{Imf}(2,1,1)$ \\
\hline$(5,559,4)$ & $\operatorname{Imf}(3,1,3) \times \operatorname{Imf}(2,1,1)$ \\
\hline$(5,626,1)$ & $\operatorname{Imf}(3,1,2) \times \operatorname{Imf}(2,2,1)$ \\
\hline$(5,626,2)$ & $\operatorname{Imf}(3,1,1) \times \operatorname{Imf}(2,2,1)$ \\
\hline$(5,626,3)$ & $\operatorname{Imf}(3,1,3) \times \operatorname{Imf}(2,2,1)$ \\
\hline$(5,690,1)$ & $\operatorname{Imf}(4,1,1) \times \operatorname{Imf}(1,1,1)$ \\
\hline$(5,836,2)$ & $\operatorname{Imf}(4,5,1) \times \operatorname{Imf}(1,1,1)$ \\
\hline
\end{tabular}

$(5,866,1)$

$\operatorname{Imf}(4,2,1) \times \operatorname{Imf}(1,1,1)$

$(5,930,1)$

$\operatorname{Imf}(4,3,2) \times \operatorname{Imf}(1,1,1)$

$(5,930,2)$

$\operatorname{Imf}(4,3,1) \times \operatorname{Imf}(1,1,1)$

$(5,942,1)$

$\operatorname{Imf}(5,1,1)$

$(5,942,2)$

$\operatorname{Imf}(5,1,2)$

$(5,942,3)$

$\operatorname{Imf}(5,1,3)$

$a_{1} x_{1}^{2}\left(a_{1}>0\right)$

$a_{1}\left(x_{1}^{2}+x_{2}^{2}\right)\left(a_{1}>0\right)$

$a_{1}\left(x_{1}^{2}-x_{1} x_{2}+x_{2}^{2}\right)\left(a_{1}>0\right)$

$(5,949,1) \quad \operatorname{Imf}(5,2,1)$

$a_{1}\left(x_{1}^{2}+x_{2}^{2}+x_{3}^{2}\right)\left(a_{1}>0\right)$

$a_{1}\left(3 x_{1}^{2}-2 x_{1} x_{2}-2 x_{1} x_{3}+3 x_{2}^{2}-2 x_{2} x_{3}+3 x_{3}^{2}\right)\left(a_{1}>0\right)$

$a_{1}\left(x_{1}^{2}+x_{1} x_{2}+x_{1} x_{3}+x_{2}^{2}+x_{2} x_{3}+x_{3}^{2}\right)\left(a_{1}>0\right)$

$a_{1}\left(x_{1}^{2}+x_{2}^{2}\right)+a_{2}\left(x_{3}^{2}-x_{3} x_{4}+x_{4}^{2}\right)\left(a_{1}, a_{2}>0\right)$

$a_{1}\left(x_{1}^{2}+x_{1} x_{2}+x_{1} x_{3}+x_{2}^{2}+x_{2} x_{3}+x_{3}^{2}\right)+a_{2} x_{4}^{2}\left(a_{1}, a_{2}>0\right)$

$a_{1}\left(3 x_{1}^{2}-2 x_{1} x_{2}-2 x_{1} x_{3}+3 x_{2}^{2}-2 x_{2} x_{3}+3 x_{3}^{2}\right)+a_{2} x_{4}^{2}\left(a_{1}, a_{2}>0\right)$

$a_{1}\left(2 x_{1}^{2}-2 x_{1} x_{2}-2 x_{1} x_{3}+x_{1} x_{4}+2 x_{2}^{2}+x_{2} x_{3}-2 x_{2} x_{4}+2 x_{3}^{2}-2 x_{3} x_{4}+2 x_{4}^{2}\right)\left(a_{1}>0\right)$

$a_{1}\left(x_{1}^{2}-x_{1} x_{2}+x_{2}^{2}+x_{3}^{2}-x_{3} x_{4}+x_{4}^{2}\right)\left(a_{1}>0\right)$

$a_{1}\left(x_{1}^{2}+x_{1} x_{2}+x_{1} x_{3}+x_{1} x_{4}+x_{2}^{2}+x_{2} x_{3}+x_{2} x_{4}+x_{3}^{2}+x_{3} x_{4}+x_{4}^{2}\right)\left(a_{1}>0\right)$

$a_{1}\left(2 x_{1}^{2}-x_{1} x_{2}-x_{1} x_{3}-x_{1} x_{4}+2 x_{2}^{2}-x_{2} x_{3}-x_{2} x_{4}+2 x_{3}^{2}-x_{3} x_{4}+2 x_{4}^{2}\right)\left(a_{1}>0\right)$

$a_{1}\left(x_{1}^{2}+x_{2}^{2}+x_{3}^{2}+x_{4}^{2}\right)\left(a_{1}>0\right)$

$a_{1}\left(x_{1}^{2}+x_{1} x_{2}+x_{2}^{2}+x_{2} x_{3}+x_{2} x_{4}+x_{3}^{2}+x_{4}^{2}\right)\left(a_{1}>0\right)$

$a_{1}\left(3 x_{1}^{2}-2 x_{1} x_{2}-2 x_{1} x_{3}+3 x_{2}^{2}-2 x_{2} x_{3}+3 x_{3}^{2}\right)+a_{2}\left(x_{4}^{2}+x_{5}^{2}\right)\left(a_{1}, a_{2}>0\right)$

$a_{1}\left(x_{1}^{2}+x_{1} x_{2}+x_{1} x_{3}+x_{2}^{2}+x_{2} x_{3}+x_{3}^{2}\right)+a_{2}\left(x_{4}^{2}+x_{5}^{2}\right)\left(a_{1}, a_{2}>0\right)$

$a_{1}\left(3 x_{1}^{2}-2 x_{1} x_{2}-2 x_{1} x_{3}+3 x_{2}^{2}-2 x_{2} x_{3}+3 x_{3}^{2}\right)+a_{2}\left(x_{4}^{2}-x_{4} x_{5}+x_{5}^{2}\right)\left(a_{1}, a_{2}>0\right)$

$a_{1}\left(x_{1}^{2}+x_{2}^{2}+x_{3}^{2}\right)+a_{2}\left(x_{4}^{2}-x_{4} x_{5}+x_{5}^{2}\right)\left(a_{1}, a_{2}>0\right)$

$a_{1}\left(x_{1}^{2}+x_{1} x_{2}+x_{1} x_{3}+x_{2}^{2}+x_{2} x_{3}+x_{3}^{2}\right)+a_{2}\left(x_{4}^{2}-x_{4} x_{5}+x_{5}^{2}\right)\left(a_{1}, a_{2}>0\right)$

$a_{1}\left(x_{1}^{2}+x_{1} x_{2}+x_{2}^{2}+x_{2} x_{3}+x_{2} x_{4}+x_{3}^{2}+x_{4}^{2}\right)+a_{2} x_{5}^{2}\left(a_{1}, a_{2}>0\right)$

$a_{1}\left(2 x_{1}^{2}-2 x_{1} x_{2}-2 x_{1} x_{3}+x_{1} x_{4}+2 x_{2}^{2}+x_{2} x_{3}-2 x_{2} x_{4}+2 x_{3}^{2}-2 x_{3} x_{4}+2 x_{4}^{2}\right)$

$+a_{2} x_{5}^{2}\left(a_{1}, a_{2}>0\right)$

$a_{1}\left(x_{1}^{2}-x_{1} x_{2}+x_{2}^{2}+x_{3}^{2}-x_{3} x_{4}+x_{4}^{2}\right)+a_{2} x_{5}^{2}\left(a_{1}, a_{2}>0\right)$

$a_{1}\left(2 x_{1}^{2}-x_{1} x_{2}-x_{1} x_{3}-x_{1} x_{4}+2 x_{2}^{2}-x_{2} x_{3}-x_{2} x_{4}+2 x_{3}^{2}-x_{3} x_{4}+2 x_{4}^{2}\right)$

$+a_{2} x_{5}^{2}\left(a_{1}, a_{2}>0\right)$

$a_{1}\left(x_{1}^{2}+x_{1} x_{2}+x_{1} x_{3}+x_{1} x_{4}+x_{2}^{2}+x_{2} x_{3}+x_{2} x_{4}+x_{3}^{2}+x_{3} x_{4}+x_{4}^{2}\right)+a_{2} x_{5}^{2}$

$\left(a_{1}, a_{2}>0\right)$

$a_{1}\left(x_{1}^{2}+x_{2}^{2}+x_{3}^{2}+x_{4}^{2}+x_{5}^{2}\right)\left(a_{1}>0\right)$

$a_{1}\left(x_{1}^{2}+x_{1} x_{2}+x_{2}^{2}+x_{2} x_{3}+x_{3}^{2}+x_{3} x_{4}+x_{3} x_{5}+x_{4}^{2}+x_{5}^{2}\right)\left(a_{1}>0\right)$

$a_{1}\left(4 x_{1}^{2}+4 x_{1} x_{5}+4 x_{2}^{2}+4 x_{2} x_{5}+4 x_{3}^{2}+4 x_{3} x_{5}+4 x_{4}^{2}+4 x_{4} x_{5}+5 x_{5}^{2}\right)\left(a_{1}>0\right)$

$a_{1}\left(5 x_{1}^{2}-2 x_{1} x_{2}-2 x_{1} x_{3}-2 x_{1} x_{4}-2 x_{1} x_{5}+5 x_{2}^{2}-2 x_{2} x_{3}-2 x_{2} x_{4}-2 x_{2} x_{5}\right.$

$\left.+5 x_{3}^{2}-2 x_{3} x_{4}-2 x_{3} x_{5}+5 x_{4}^{2}-2 x_{4} x_{5}+5 x_{5}^{2}\right)\left(a_{1}>0\right)$

$(5,949,2) \quad \operatorname{Imf}(5,2,3)$

$a_{1}\left(2 x_{1}^{2}+x_{1} x_{2}-2 x_{1} x_{3}-2 x_{1} x_{4}-2 x_{1} x_{5}+2 x_{2}^{2}+x_{2} x_{3}-2 x_{2} x_{4}+x_{2} x_{5}\right.$

$\left.+2 x_{3}^{2}+x_{3} x_{4}+x_{3} x_{5}+2 x_{4}^{2}+x_{4} x_{5}+2 x_{5}^{2}\right)\left(a_{1}>0\right)$

$a_{1}\left(3 x_{1}^{2}+2 x_{1} x_{2}-2 x_{1} x_{3}-2 x_{1} x_{4}-2 x_{1} x_{5}+3 x_{2}^{2}+2 x_{2} x_{3}-2 x_{2} x_{4}+2 x_{2} x_{5}\right.$

$\left.+3 x_{3}^{2}+2 x_{3} x_{4}+2 x_{3} x_{5}+3 x_{4}^{2}+2 x_{4} x_{5}+3 x_{5}^{2}\right)\left(a_{1}>0\right)$

$a_{1}\left(x_{1}^{2}+x_{1} x_{2}+x_{1} x_{3}+x_{1} x_{4}+x_{1} x_{5}+x_{2}^{2}+x_{2} x_{3}+x_{2} x_{4}+x_{2} x_{5}\right.$

$\left.+x_{3}^{2}+x_{3} x_{4}+x_{3} x_{5}+x_{4}^{2}+x_{4} x_{5}+x_{5}^{2}\right)\left(a_{1}>0\right)$

$\begin{array}{ll}(6,5209,1) & \operatorname{Imf}(3,1,3) \times \operatorname{Imf}(2,2,1) \\ & \times \operatorname{Imf}(1,1,1) \\ (6,5209,5) & \operatorname{Imf}(3,1,2) \times \operatorname{Imf}(2,2,1) \\ & \times \operatorname{Imf}(1,1,1) \\ (6,5517,3) & \operatorname{Imf}(3,1,1) \times \operatorname{Imf}(3,1,3) \\ (6,5517,4) & \operatorname{Indmf}(6,10,1)\end{array}$

$(6,5517,8) \quad \operatorname{Imf}(3,1,2) \times \operatorname{Imf}(3,1,3)$

$(6,5517,5)$

$(6,6509,1)$

$\operatorname{Imf}(3,1,1) \times \operatorname{Imf}(3,1,2)$

$\operatorname{Imf}(4,1,1) \times \operatorname{Imf}(2,1,1)$

$(6,6878,2)$

$\operatorname{Imf}(4,4,1) \times \operatorname{Imf}(2,2,1)$

$(6,6568,1)$

$\operatorname{Imf}(4,1,1) \times \operatorname{Imf}(2,2,1)$

$(6,3891,1)$

$\operatorname{Imf}(4,2,1) \times \operatorname{Imf}(2,1,1)$

$(6,3795,3)$

$\operatorname{Imf}(4,5,1) \times \operatorname{Imf}(2,1,1)$

$a_{1}\left(x_{1}^{2}+x_{1} x_{2}+x_{1} x_{3}+x_{2}^{2}+x_{2} x_{3}+x_{3}^{2}\right)+a_{2}\left(x_{4}^{2}-x_{4} x_{5}+x_{5}^{2}\right)+a_{3} x_{6}^{2}$

$\left(a_{1}, a_{2}, a_{3}>0\right)$

$a_{1}\left(3 x_{1}^{2}-2 x_{1} x_{2}-2 x_{1} x_{3}+3 x_{2}^{2}-2 x_{2} x_{3}+3 x_{3}^{2}\right)+a_{2}\left(x_{4}^{2}-x_{4} x_{5}+x_{5}^{2}\right)+a_{3} x_{6}^{2}$

$\left(a_{1}, a_{2}>0\right)$

$a_{1}\left(x_{1}^{2}+x_{2}^{2}+x_{3}^{2}\right)+a_{2}\left(x_{4}^{2}+x_{4} x_{5}+x_{4} x_{6}+x_{5}^{2}+x_{5} x_{6}+x_{6}^{2}\right)\left(a_{1}, a_{2}>0\right)$

$a_{1}\left(x_{1}^{2}+x_{2}^{2}+x_{5}^{2}\right)+a_{2}\left(3 x_{1} x_{2}-2 x_{1} x_{3}-4 x_{1} x_{4}-3 x_{1} x_{5}-6 x_{1} x_{6}\right.$

$-2 x_{2} x_{3}-4 x_{2} x_{4}-3 x_{2} x_{5}-6 x_{2} x_{6}+2 x_{3}^{2}+4 x_{3} x_{4}+2 x_{3} x_{5}+4 x_{3} x_{6}$

$\left.+4 x_{4}^{2}+4 x_{4} x_{5}+8 x_{4} x_{6}+6 x_{5} x_{6}+6 x_{6}^{2}\right)\left(\frac{2}{3} a_{1}>a_{2}>0\right)$

$a_{1}\left(3 x_{1}^{2}-2 x_{1} x_{2}-2 x_{1} x_{3}+3 x_{2}^{2}-2_{2} x_{3}+3 x_{3}^{2}\right)$

$+a_{2}\left(x_{4}^{2}+x_{4} x_{5}+x_{4} x_{6}+x_{5}^{2}+x_{5} x_{6}+x_{6}^{2}\right)\left(a_{1}, a_{2}>0\right)$

$a_{1}\left(x_{1}^{2}+x_{2}^{2}+x_{3}^{2}\right)+a_{2}\left(3 x_{4}^{2}-2 x_{4} x_{5}-2 x_{4} x_{6}+3 x_{5}^{2}-2 x_{5} x_{6}+3 x_{6}^{2}\right)\left(a_{1}, a_{2}>0\right)$

$a_{1}\left(x_{1}^{2}+x_{1} x_{2}+x_{2}^{2}+x_{2} x_{3}+x_{2} x_{4}+x_{3}^{2}+x_{4}^{2}\right)+a_{2}\left(x_{5}^{2}+x_{6}^{2}\right)\left(a_{1}, a_{2}>0\right)$

$a_{1}\left(x_{1}^{2}+x_{2}^{2}+x_{3}^{2}+x_{4}^{2}\right)+a_{2}\left(x_{5}^{2}-x_{5} x_{6}+x_{6}^{2}\right)\left(a_{1}, a_{2}>0\right)$

$a_{1}\left(x_{1}^{2}+x_{1} x_{2}+x_{2}^{2}+x_{2} x_{3}+x_{2} x_{4}+x_{3}^{2}+x_{4}^{2}\right)+a_{2}\left(x_{5}^{2}-x_{5} x_{6}+x_{6}^{2}\right)\left(a_{1}, a_{2}>0\right)$

$a_{1}\left(x_{1}^{2}-x_{1} x_{2}+x_{2}^{2}+x_{3}^{2}-x_{3} x_{4}+x_{4}^{2}\right)+a_{2}\left(x_{5}^{2}+x_{6}^{2}\right)\left(a_{1}, a_{2}>0\right)$

$a_{1}\left(2 x_{1}^{2}-2 x_{1} x_{2}-2 x_{1} x_{3}+x_{1} x_{4}+2 x_{2}^{2}+x_{2} x_{3}-2 x_{2} x_{4}\right.$

$\left.+2 x_{3}^{2}-2 x_{3} x_{4}+2 x_{4}^{2}\right)+a_{2}\left(x_{5}^{2}+x_{6}^{2}\right)\left(a_{1}, a_{2}>0\right)$ 
$(6,4232,2)$

$\operatorname{Imf}(4,5,1) \times \operatorname{Imf}(2,2,1)$

$(6,3120,2)$

$\operatorname{Imf}(4.3,1) \times \operatorname{Imf}(2,1,1)$

$(6,3120,1)$

$\operatorname{Imf}(4,3,2) \times \operatorname{Imf}(2,1,1)$

$(6,3193,2)$

$\operatorname{Imf}(4,3,1) \times \operatorname{Imf}(2,2,1)$

$(6,3193,1)$

$(6,3272,5)$

$(6,3272,3)$

$(6,3296,5)$

$(6,3296,1)$

$(6,3296,4)$

$(6,3296,2)$

$(6,2773,1)$

$(6,2773,2)$

$(6,2773,3)$

$(6,2772,5)$

$(6,2772,2)$

$\operatorname{Imf}(6,7,1)$

$(6,2750,4) \quad \operatorname{Imf}(6,8,1)$

$(6,2803,1) \quad \operatorname{Imf}(6,2,1)$

$(6,2804,1) \quad \operatorname{Imf}(6,3,2)$

$(6,2804,2) \quad \operatorname{Imf}(6,3,1)$

$(6,2866,3) \quad \operatorname{Imf}(6,9,2)$

$(6,2866,2) \quad \operatorname{Imf}(6,9,1)$

$(6,2932,2) \quad \operatorname{Imf}(6,4,2)$

$(6,2932,1) \quad \operatorname{Imf}(6,4,1)$

$(6,2945,1) \quad \operatorname{Imf}(6,5,1)$

$(6,2952,1) \quad \operatorname{Imf}(6,6,1)$

$(6,2952,2) \quad \operatorname{Imf}(6,6,3)$

$(6,2952,3) \quad \operatorname{Imf}(6,6,2)$ $a_{1}\left(2 x_{1}^{2}-2 x_{1} x_{2}-2 x_{1} x_{3}+x_{1} x_{4}+2 x_{2}^{2}+x_{2} x_{3}-2 x_{2} x_{4}+2 x_{3}^{2}-2 x_{3} x_{4}+2 x_{4}^{2}\right)$ $+a_{2}\left(x_{5}^{2}-x_{5} x_{6}+x_{6}^{2}\right)\left(a_{1}, a_{2}>0\right)$

$a_{1}\left(x_{1}^{2}+x_{1} x_{2}+x_{1} x_{3}+x_{1} x_{4}+x_{2}^{2}+x_{2} x_{3}+x_{2} x_{4}+x_{3}^{2}+x_{3} x_{4}+x_{4}^{2}\right)$ $+a_{2}\left(x_{5}^{2}+x_{6}^{2}\right)\left(a_{1}, a_{2}>0\right)$

$a_{1}\left(2 x_{1}^{2}-x_{1} x_{2}-x_{1} x_{3}-x_{1} x_{4}+2 x_{2}^{2}-x_{2} x_{3}-x_{2} x_{4}+2 x_{3}^{2}-x_{3} x_{4}+2 x_{4}^{2}\right)$

$+a_{2}\left(x_{5}^{2}+x_{6}^{2}\right)\left(a_{1}, a_{2}>0\right)$

$a_{1}\left(x_{1}^{2}+x_{1} x_{2}+x_{1} x_{3}+x_{1} x_{4}+x_{2}^{2}+x_{2} x_{3}+x_{2} x_{4}+x_{3}^{2}+x_{3} x_{4}+x_{4}^{2}\right)$

$+a_{2}\left(x_{5}^{2}-x_{5} x_{6}+x_{6}^{2}\right)\left(a_{1}, a_{2}>0\right)$

$a_{1}\left(2 x_{1}^{2}-x_{1} x_{2}-x_{1} x_{3}-x_{1} x_{4}+2 x_{2}^{2}-x_{2} x_{3}-x_{2} x_{4}+2 x_{3}^{2}-x_{3} x_{4}+2 x_{4}^{2}\right)$

$+a_{2}\left(x_{5}^{2}-x_{5} x_{6}+x_{6}^{2}\right)\left(a_{1}, a_{2}>0\right)$

$a_{1}\left(4 x_{1}^{2}+4 x_{1} x_{5}+4 x_{2}^{2}+4 x_{2} x_{5}+4 x_{3}^{2}+4 x_{3} x_{5}+4 x_{4}^{2}+4 x_{4} x_{5}+5 x_{5}^{2}\right)$ $+a_{2} x_{6}^{2}\left(a_{1}, a_{2}>0\right)$

$a_{1}\left(x_{1}^{2}+x_{1} x_{2}+x_{2}^{2}+x_{2} x_{3}+x_{3}^{2}+x_{3} x_{4}+x_{3} x_{5}+x_{4}^{2}+x_{5}^{2}\right)+a_{2} x_{6}^{2}\left(a_{1}, a_{2}>0\right)$

$a_{1}\left(x_{1}^{2}+x_{1} x_{2}+x_{1} x_{3}+x_{1} x_{4}+x_{1} x_{5}+x_{2}^{2}+x_{2} x_{3}+x_{2} x_{4}+x_{2} x_{5}\right.$

$\left.+x_{3}^{2}+x_{3} x_{4}+x_{3} x_{5}+x_{4}^{2}+x_{4} x_{5}+x_{5}^{2}\right)+a_{2} x_{6}^{2}\left(a_{1}, a_{2}>0\right)$

$a_{1}\left(5 x_{1}^{2}-2 x_{1} x_{2}-2 x_{1} x_{3}-2 x_{1} x_{4}-2 x_{1} x_{5}+5 x_{2}^{2}-2 x_{2} x_{3}-2 x_{2} x_{4}-2 x_{2} x_{5}\right.$

$\left.+5 x_{3}^{2}-2 x_{3} x_{4}-2 x_{3} x_{5}+5 x_{4}^{2}-2 x_{4} x_{5}+5 x_{5}^{2}\right)+a_{2} x_{6}^{2}\left(a_{1}, a_{2}>0\right)$

$a_{1}\left(3 x_{1}^{2}+2 x_{1} x_{2}-2 x_{1} x_{3}-2 x_{1} x_{4}-2 x_{1} x_{5}+3 x_{2}^{2}+2 x_{2} x_{3}-2 x_{2} x_{4}+2 x_{2} x_{5}\right.$

$\left.+3 x_{3}^{2}+2 x_{3} x_{4}+2 x_{3} x_{5}+3 x_{4}^{2}+2 x_{4} x_{5}+3 x_{5}^{2}\right)+a_{2} x_{6}^{2}\left(a_{1}, a_{2}>0\right)$

$a_{1}\left(2 x_{1}^{2}+x_{1} x_{2}-2 x_{1} x_{3}-2 x_{1} x_{4}-2 x_{1} x_{5}+2 x_{2}^{2}+x_{2} x_{3}-2 x_{2} x_{4}+x_{2} x_{5}\right.$

$\left.+2 x_{3}^{2}+x_{3} x_{4}+x_{3} x_{5}+2 x_{4}^{2}+x_{4} x_{5}+2 x_{5}^{2}\right)+a_{2} x_{6}^{2}\left(a_{1}, a_{2}>0\right)$

$a_{1}\left(x_{1}^{2}+x_{2}^{2}+x_{3}^{2}+x_{4}^{2}+x_{5}^{2}+x_{6}^{2}\right)\left(a_{1}>0\right)$

$a_{1}\left(2 x_{1}^{2}+2 x_{1} x_{6}+2 x_{2}^{2}+2 x_{2} x_{6}+2 x_{3}^{2}+2 x_{3} x_{6}+2 x_{4}^{2}+2 x_{4} x_{6}\right.$

$\left.+2 x_{5}^{2}+2 x_{5} x_{6}+3 x_{6}^{2}\right)\left(a_{1}>0\right)$

$a_{1}\left(x_{1}^{2}+x_{1} x_{2}+x_{2}^{2}+x_{2} x_{3}+x_{3}^{2}+x_{3} x_{4}+x_{4}^{2}+x_{4} x_{5}+x_{4} x_{6}+x_{5}^{2}+x_{6}^{2}\right)\left(a_{1}>0\right)$

$a_{1}\left(3 x_{1}^{2}-2 x_{1} x_{2}-2 x_{1} x_{3}+3 x_{2}^{2}-2 x_{2} x_{3}+3 x_{3}^{2}+3 x_{4}^{2}-2 x_{4} x_{5}-2 x_{4} x_{6}\right.$

$\left.+3 x_{5}^{2}-2 x_{5} x_{6}+3 x_{6}^{2}\right)\left(a_{1}>0\right)$

$a_{1}\left(x_{1}^{2}+x_{1} x_{2}+x_{1} x_{3}+x_{2}^{2}+x_{2} x_{3}+x_{3}^{2}+x_{4}^{2}+x_{4} x_{5}+x_{4} x_{6}\right.$

$\left.+x_{5}^{2}+x_{5} x_{6}+x_{6}^{2}\right)\left(a_{1}>0\right)$

$a_{1}\left(3 x_{1}^{2}+2 x_{1} x_{2}+2 x_{1} x_{3}+2 x_{1} x_{4}+2 x_{1} x_{5}+2 x_{1} x_{6}+3 x_{2}^{2}+2 x_{2} x_{3}\right.$

$+2 x_{2} x_{4}+2 x_{2} x_{5}+2 x_{2} x_{6}+3 x_{3}^{2}+2 x_{3} x_{4}+2 x_{3} x_{5}-2 x_{3} x_{6}+3 x_{4}^{2}$

$\left.+2 x_{4} x_{5}-2 x_{4} x_{6}+3 x_{5}^{2}+2 x_{5} x_{6}+3 x_{6}^{2}\right)\left(a_{1}>0\right)$

$a_{1}\left(x_{1}^{2}-x_{1} x_{2}+x_{2}^{2}+x_{3}^{2}-x_{3} x_{4}+x_{4}^{2}+x_{5}^{2}-x_{5} x_{6}+x_{6}^{2}\right)\left(a_{1}>0\right)$

$a_{1}\left(2 x_{1}^{2}+x_{1} x_{2}-2 x_{1} x_{3}-2 x_{1} x_{4}+x_{1} x_{5}+x_{1} x_{6}+2 x_{2}^{2}+x_{2} x_{3}-2 x_{2} x_{4}\right.$

$-2 x_{2} x_{5}+x_{2} x_{6}+2 x_{3}^{2}+x_{3} x_{4}-2 x_{3} x_{5}-2 x_{3} x_{6}$

$\left.+2 x_{4}^{2}+x_{4} x_{5}-2 x_{4} x_{6}+2 x_{5}^{2}+x_{5} x_{6}+2 x_{6}^{2}\right)\left(a_{1}>0\right)$

$a_{1}\left(x_{1}^{2}-x_{1} x_{3}+x_{2}^{2}-x_{2} x_{4}+x_{3}^{2}-x_{3} x_{4}+x_{4}^{2}-x_{4} x_{5}+x_{5}^{2}-x_{5} x_{6}+x_{6}^{2}\right)\left(a_{1}>0\right)$

$a_{1}\left(3 x_{1}^{2}-2 x_{1} x_{2}-2 x_{1} x_{3}-3 x_{1} x_{4}+x_{1} x_{5}+x_{1} x_{6}+3 x_{2}^{2}-2 x_{2} x_{3}+x_{2} x_{4}\right.$

$-3 x_{2} x_{5}+x_{2} x_{6}+3 x_{3}^{2}+x_{3} x_{4}+x_{3} x_{5}-3 x_{3} x_{6}$

$\left.+3 x_{4}^{2}-2 x_{4} x_{5}-2 x_{4} x_{6}+3 x_{5}^{2}-2 x_{5} x_{6}+3 x_{6}^{2}\right)\left(a_{1}>0\right)$

$a_{1}\left(2 x_{1}^{2}+2 x_{1} x_{2}+2 x_{1} x_{3}-2 x_{1} x_{4}-x_{1} x_{5}-x_{1} x_{6}+2 x_{2}^{2}+2 x_{2} x_{3}-x_{2} x_{4}\right.$

$-2 x_{2} x_{5}-x_{2} x_{6}+2 x_{3}^{2}-x_{3} x_{4}-x_{3} x_{5}-2 x_{3} x_{6}+2 x_{4}^{2}+2 x_{4} x_{5}+2 x_{4} x_{6}$

$\left.+2 x_{5}^{2}+2 x_{5} x_{6}+2 x_{6}^{2}\right)\left(a_{1}>0\right)$

$a_{1}\left(x_{1}^{2}+x_{1} x_{2}+x_{1} x_{3}+x_{1} x_{4}+x_{1} x_{5}+x_{1} x_{6}+x_{2}^{2}+x_{2} x_{3}+x_{2} x_{4}+x_{2} x_{5}+x_{2} x_{6}\right.$ $\left.+x_{3}^{2}+x_{3} x_{4}+x_{3} x_{5}+x_{3} x_{6}+x_{4}^{2}+x_{4} x_{5}+x_{4} x_{6}+x_{5}^{2}+x_{5} x_{6}+x_{6}^{2}\right)\left(a_{1}>0\right)$ $a_{1}\left(3 x_{1}^{2}-x_{1} x_{2}-x_{1} x_{3}-x_{1} x_{4}-x_{1} x_{5}-x_{1} x_{6}+3 x_{2}^{2}-x_{2} x_{3}-x_{2} x_{4}-x_{2} x_{5}\right.$ $-x_{2} x_{6}+3 x_{3}^{2}-x_{3} x_{4}-x_{3} x_{5}-x_{3} x_{6}+3 x_{4}^{2}-x_{4} x_{5}-x_{4} x_{6}+3 x_{5}^{2}-x_{5} x_{6}$ $\left.+3 x_{6}^{2}\right)\left(a_{1}>0\right)$

$a_{1}\left(2 x_{1}^{2}-x_{1} x_{2}-2 x_{1} x_{3}+x_{1} x_{4}+x_{1} x_{5}-2 x_{1} x_{6}+2 x_{2}^{2}-x_{2} x_{3}-2 x_{2} x_{4}+\right.$ $x_{2} x_{5}+x_{2} x_{6}+2 x_{3}^{2}-x_{3} x_{4}-2 x_{3} x_{5}+x_{3} x_{6}+2 x_{4}^{2}-x_{4} x_{5}-2 x_{4} x_{6}+2 x_{5}^{2}$ $\left.-x_{5} x_{6}+2 x_{6}^{2}\right)\left(a_{1}>0\right)$

$a_{1}\left(3 x_{1}^{2}-2 x_{1} x_{2}-2 x_{1} x_{3}+2 x_{1} x_{4}+2 x_{1} x_{5}+3 x_{2}^{2}-2 x_{2} x_{3}-2 x_{2} x_{4}+2 x_{2} x_{6}\right.$ $\left.+3 x_{3}^{2}-2 x_{3} x_{5}-2 x_{3} x_{6}+3 x_{4}^{2}-2 x_{4} x_{5}+2 x_{4} x_{6}+3 x_{5}^{2}-2 x_{5} x_{6}+3 x_{6}^{2}\right)\left(a_{1}>0\right)$

$a_{1}\left(5 x_{1}^{2}+2 x_{1} x_{2}-2 x_{1} x_{3}-2 x_{1} x_{4}+2 x_{1} x_{5}+4 x_{1} x_{6}+5 x_{2}^{2}+2 x_{2} x_{3}-2 x_{2} x_{4}\right.$ $-2 x_{2} x_{5}+4 x_{2} x_{6}+5 x_{3}^{2}+2 x_{3} x_{4}-2 x_{3} x_{5}+4 x_{3} x_{6}+5 x_{4}^{2}+2 x_{4} x_{5}+4 x_{4} x_{6}$ $\left.+5 x_{5}^{2}+4 x_{5} x_{6}+5 x_{6}^{2}\right)\left(a_{1}>0\right)$

$a_{1}\left(2 x_{1}^{2}+x_{1} x_{2}+2 x_{1} x_{3}+2 x_{1} x_{4}+x_{1} x_{5}+2 x_{2}^{2}+x_{2} x_{3}+2 x_{2} x_{4}+2 x_{2} x_{5}+x_{2} x_{6}\right.$ $\left.+2 x_{3}^{2}+x_{3} x_{4}+2 x_{3} x_{5}-x_{3} x_{6}+2 x_{4}^{2}+x_{4} x_{5}-x_{4} x_{6}+2 x_{5}^{2}+x_{5} x_{6}+2 x_{6}^{2}\right)\left(a_{1}>0\right)$ 


\section{GAP algorithms: The Flabby Class $\left[M_{G}\right]^{f l}$}

Let $G$ be a finite subgroup of $\operatorname{GL}(n, \mathbb{Z})$ and $M=M_{G}$ be the $G$-lattice as in Definition 1.26 . When $G \simeq \operatorname{Gal}(L / k)$ for a Galois extension $L / k$, the field $L\left(M_{G}\right)^{G}$ may be regarded as the function field of an algebraic $k$-torus $T$ which splits $L$ via (1) (see Section 1).

In this section, we provide some GAP algorithms for computing the flabby class $[M]^{f l}$ of $M$ and for verifying whether $[M]^{f l}$ is invertible and $[M]^{f l}=0$. The following flow chart shows the structure of the GAP algorithms:

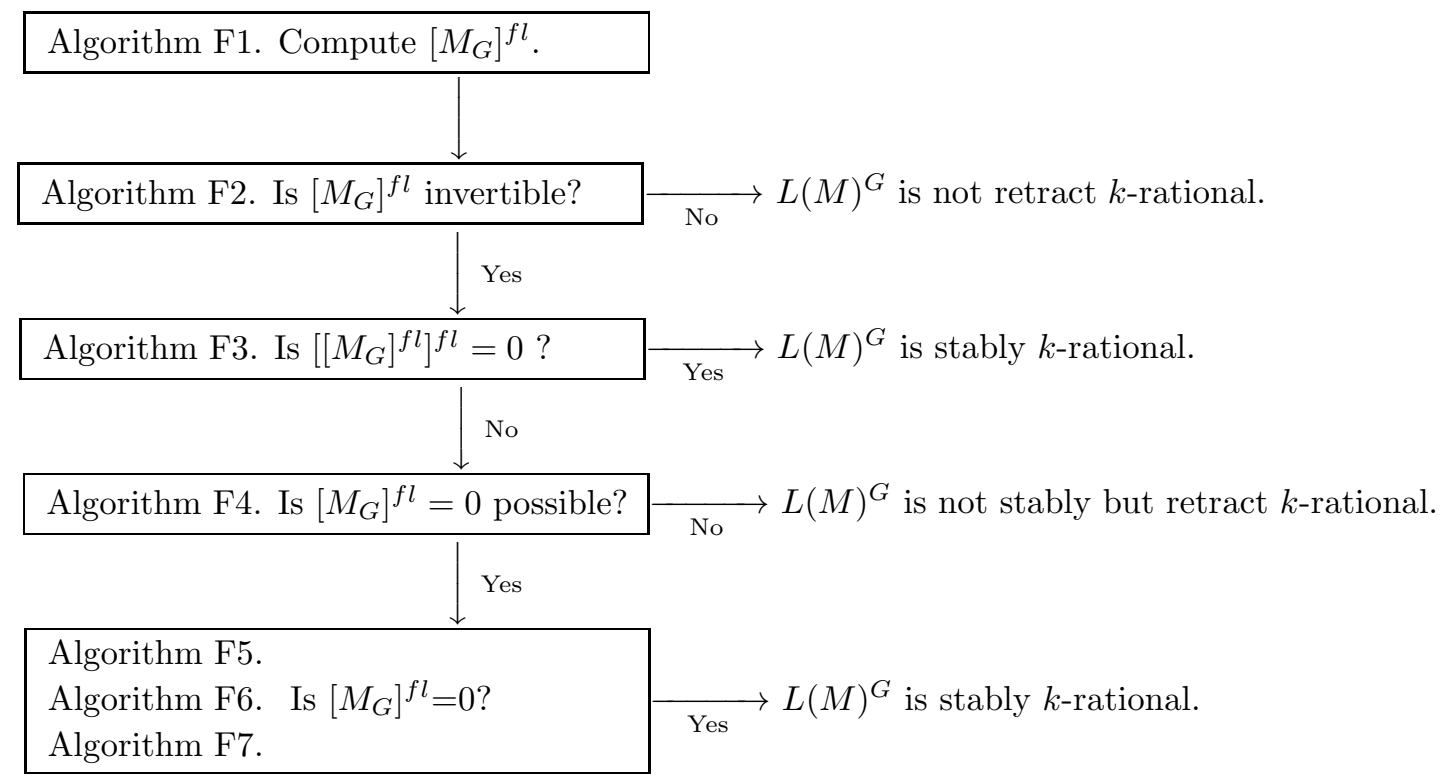

\subsection{Determination whether $M_{G}$ is flabby (coflabby).}

Let $G$ be a finite subgroup of $\mathrm{GL}(n, \mathbb{Z})$ and $M=M_{G}$ be the corresponding $G$-lattice of rank $n$ as in Definition 1.26. We provide GAP some algorithms for computing $\widehat{H}^{-1}\left(G, M_{G}\right)$ and $H^{1}\left(G, M_{G}\right)$ and verifying whether the $G$-lattice $M_{G}$ is flabby (resp. coflabby).

Hminus1(G) returns the Tate cohomology group $\widehat{H}^{-1}\left(G, M_{G}\right)$.

H1 (G) returns the cohomology group $H^{1}\left(G, M_{G}\right)$.

IsFlabby (G) returns whether $G$-lattice $M_{G}$ is flabby or not.

IsCoflabby (G) returns whether $G$-lattice $M_{G}$ is coflabby or not.

Note that in the algorithms below we use the formulas $\left(\widehat{B}^{-1}(G, M) \otimes_{\mathbb{Z}} \mathbb{Q}\right) \cap M=\widehat{Z}^{-1}(G, M),\left(\widehat{B}^{0}(G, M) \otimes_{\mathbb{Z}}\right.$ $\mathbb{Q}) \cap M=\widehat{Z}^{0}(G, M)$ and $\left(B^{1}(G, M) \otimes_{\mathbb{Z}} \mathbb{Q}\right) \cap C^{1}(G, M)=Z^{1}(G, M)$ to compute $\widehat{H}^{-1}(G, M), \widehat{H}^{0}(G, M)$ and $H^{1}(G, M)$ respectively.

Algorithm Fo (Determination whether $M_{G}$ is flabby (coflabby)).

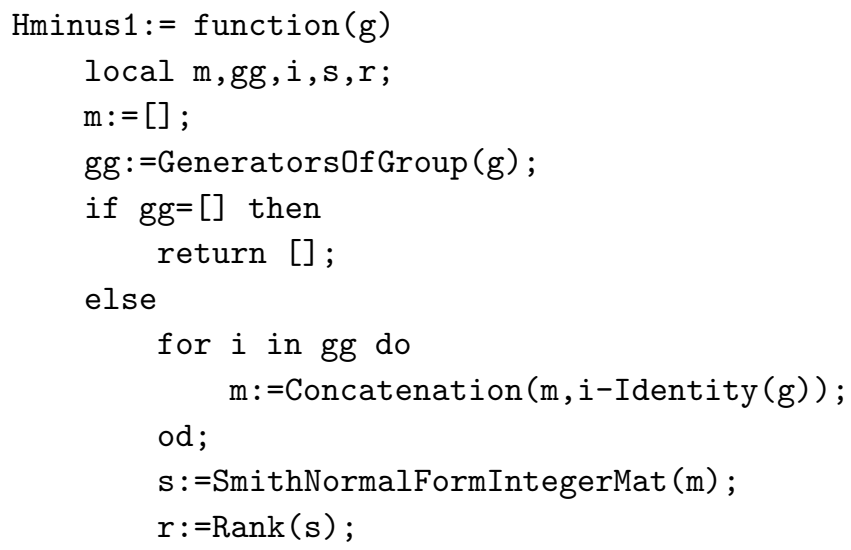


return $\operatorname{List}\left([1 \ldots r], x^{->s}[x][x]\right)$;

fi;

end;

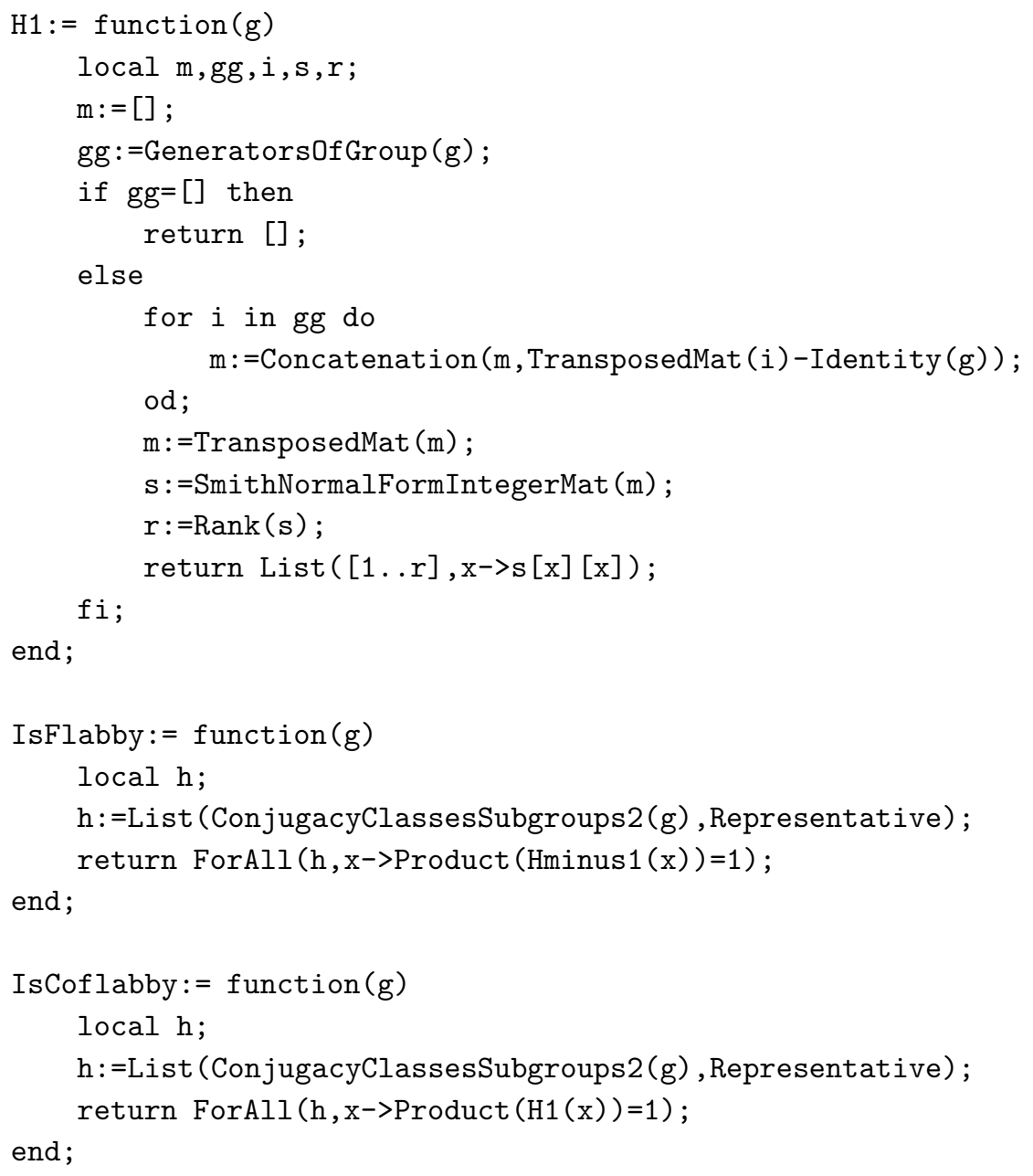

\subsection{Construction of the flabby class $\left[M_{G}\right]^{f l}$ of the $G$-lattice $M_{G}$.}

Let $G$ be a finite subgroup of $\mathrm{GL}(n, \mathbb{Z})$ and $M=M_{G}$ be the corresponding $G$-lattice of rank $n$ as in Definition 1.26. We want to construct a flabby resolution $0 \rightarrow M \rightarrow P \rightarrow F \rightarrow 0$ with rank $F$ not too large. If $0 \rightarrow Q \rightarrow$ $R \rightarrow M^{\circ} \rightarrow 0$ is a coflabby resolution of $M^{\circ}=\operatorname{Hom}_{\mathbb{Z}}(M, \mathbb{Z})$ where $R$ is permutation and $Q$ is coflasque, we can get a flasque resolution by taking a dual exact sequence $0 \rightarrow M \rightarrow \operatorname{Hom}_{\mathbb{Z}}(R, \mathbb{Z}) \rightarrow \operatorname{Hom}_{\mathbb{Z}}(Q, \mathbb{Z}) \rightarrow 0$.

We construct a coflabby resolution of $M^{\circ}$ first. Let $P^{\circ}$ be a permutation $G$-lattice and $P^{\circ} \stackrel{f}{\rightarrow} M^{\circ}$ be a $G$-homomorphism. For a subgroup $H$ of $G$, suppose that $f$ maps $\left(P^{\circ}\right)^{H}$ surjectively to $\left(M^{\circ}\right)^{H}$, i.e.

$$
\left(P^{\circ}\right)^{H} \stackrel{\left.f\right|_{\left(P^{\circ}\right)^{H}}}{\longrightarrow}\left(M^{\circ}\right)^{H} \rightarrow 0 \text { is exact. }
$$

Then $\widehat{H}^{0}\left(H, P^{\circ}\right) \rightarrow \widehat{H}^{0}\left(H, M^{\circ}\right)$ is surjective, so that $\widehat{H}^{1}(H, Q)=0$ where $Q=\operatorname{Ker} f$ by Lemma 2.5. In order to get a coflabby resolution of $M^{\circ}$, it is enough to construct a permutation $G$-lattice $P^{\circ}$ and a $G$-homomorphism $f$ such that (2) is satisfied for all subgroups $H$ of $G$.

Let $\mathcal{P}^{\circ}$ be a finite subset of $M^{\circ}$ which is closed under the action of $G$. Let $P^{\circ}$ be a free $\mathbb{Z}$-module over $\mathcal{P}^{\circ}$, i.e. $P^{\circ}=\mathbb{Z}\left[\mathcal{P}^{\circ}\right]$. The $G$-lattice $P^{\circ}$ is permutation naturally, and for $p \in \mathcal{P}^{\circ}, P^{\circ} \ni p \mapsto p \in \mathcal{P}^{\circ} \subset M^{\circ}$ defines a $G$-homomorphism $f: P^{\circ} \rightarrow M^{\circ}$. If $\mathcal{P}^{\circ}$ contains a $\mathbb{Z}$-basis of $\left(M^{\circ}\right)^{H}$ for all subgroups $H$ of $G$, then the condition (2) is satisfied for all subgroups $H \subset G$.

The condition (2) is consistent with the conjugation. Namely, (2) is satisfied for $H$ if and only if it is satisfied for $H^{g}=g^{-1} H g$. Thus it is enough to consider only the subgroups $H$ not mutually conjugate. 


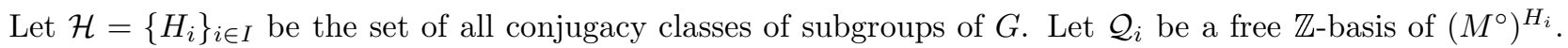
Then

$$
\mathcal{P}^{\circ}=\bigcup_{r \in \mathcal{R}} \operatorname{Orb}_{G}(r), \mathcal{R}=\bigcup_{i \in I} \mathcal{Q}_{i}
$$

provides $P^{\circ}=\mathbb{Z}\left[\mathcal{P}^{\circ}\right]$ which satisfies (2) for all $H_{i} \subset G$. Therefore we may obtain a coflabby resolution of $M$ :

$$
0 \rightarrow \operatorname{Ker} f \rightarrow P^{\circ} \stackrel{f}{\rightarrow} M^{\circ} \rightarrow 0 .
$$

The following algorithm (Algorithm F1) tries to find some "reduced" subset $\mathcal{R}^{\prime} \subset \mathcal{R}$ such that $P^{\circ}=\mathbb{Z}\left[\mathcal{P}^{\circ}\right]$ satisfies the condition (2) where

$$
\mathcal{P}^{\circ}=\bigcup_{r \in \mathcal{R}^{\prime}} \operatorname{Orb}_{G}(r) .
$$

This trial is performed in a computer calculation as follows:

The following algorithms construct a "reduced" flabby resolution of $M_{G}: 0 \rightarrow M_{G} \stackrel{\iota}{\rightarrow} P \stackrel{\phi}{\rightarrow} F \rightarrow 0$.

Z0lattice $(G)$ returns a $\mathbb{Z}$-basis of $\left(M_{G}\right)^{G}$.

FindCoflabbyResolutionBase (G,HH) returns a $\mathbb{Z}$-basis of a smaller permutation lattice $P^{\circ}$ satisfying (2) for any $H \in \mathcal{H}$.

FlabbyResolution(G) returns a flabby resolution of $M_{G}$ as follows:

FlabbyResolution $(G)$. actionP returns the matrix representation of the action of $G$ on $P$;

FlabbyResolution(G).actionF returns the matrix representation of the action of $G$ on $F$;

FlabbyResolution $(G)$. injection returns the matrix which corresponds to the injection $\iota: M_{G} \rightarrow P$;

FlabbyResolution $(G)$. surjection returns the matrix which corresponds to the surjection $\phi: P \rightarrow F$.

Algorithm F1 (Construction of the flabby class $\left[M_{G}\right]^{f l}$ of the $G$-lattice $M_{G}$ ).

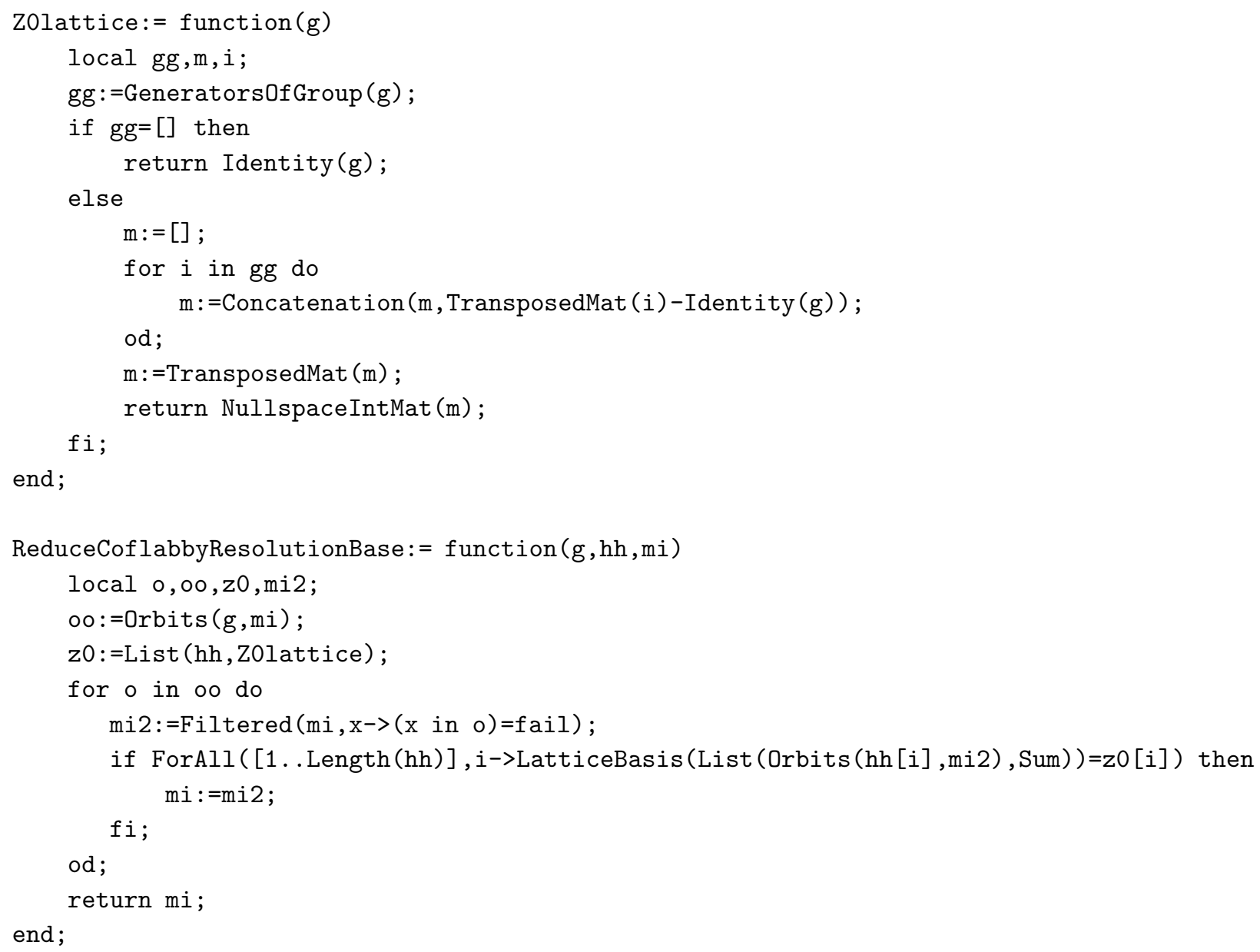




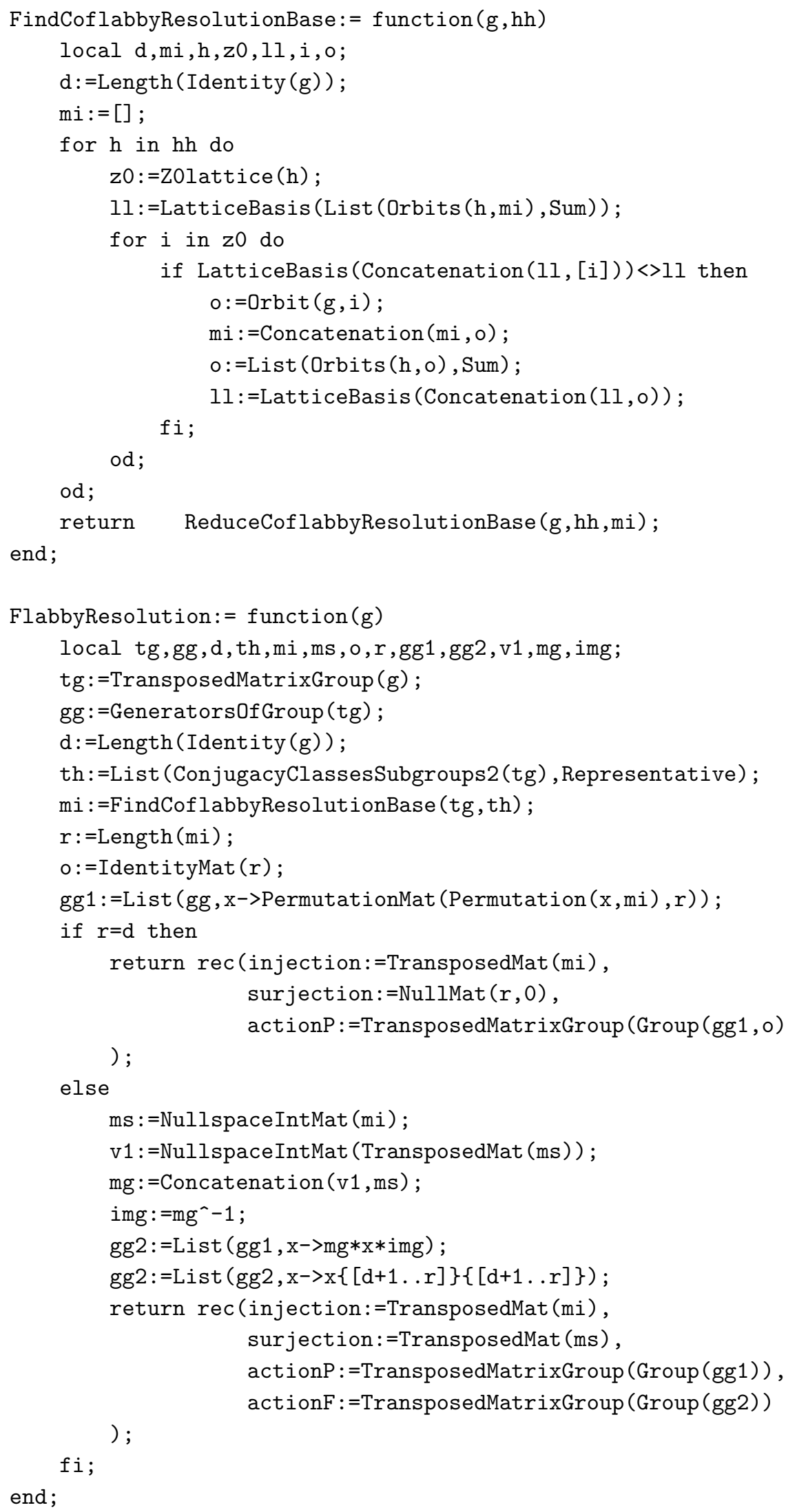

Example 5.1 (Algorithm[F1). The $G$-lattice $M_{G}$ has the trivial flabby class $\left[M_{G}\right]^{f l}=0$ for $G=\left\langle\left(\begin{array}{cc}0 & 1 \\ -1 & -1\end{array}\right)\right\rangle \simeq C_{3}$. Read("FlabbyResolution.gap");

gap $>\mathrm{G}:=\operatorname{Group}([[[0,1],[-1,-1]]])$; 


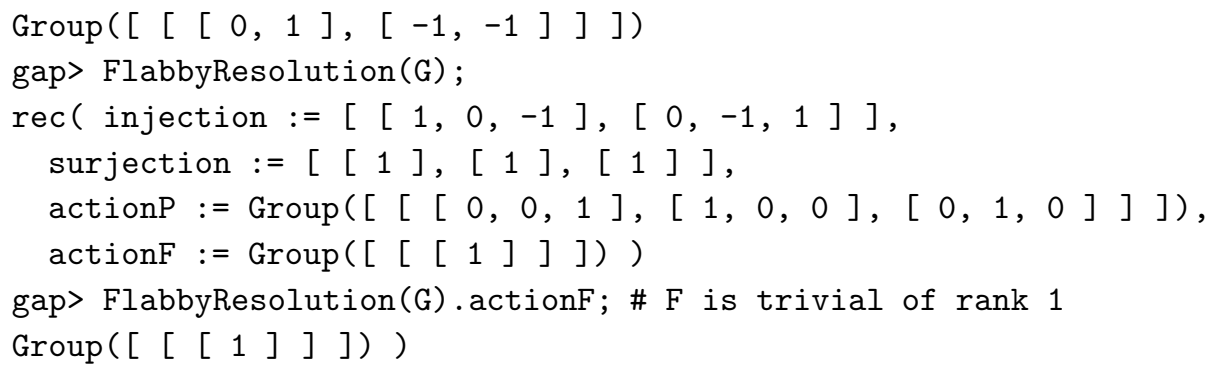

\subsection{Determination whether $\left[M_{G}\right]^{f l}$ is invertible.}

Let $G$ be a finite subgroup of $\operatorname{GL}(n, \mathbb{Z})$ and $M=M_{G}$ be the corresponding $G$-lattice of rank $n$ as in Definition 1.26. We provide the algorithm (Algorithm F2) for the determination whether $\left[M_{G}\right]^{f l}$ is invertible. First, take a flabby resolution of $M$ :

$$
0 \rightarrow M \rightarrow P \rightarrow F \rightarrow 0
$$

If $F$ is not coflabby, then $[M]^{f l}$ is not invertible. If $F$ is coflabby, then take a flabby resolution

$$
0 \rightarrow F \stackrel{\iota}{\rightarrow} Q \rightarrow E \rightarrow 0 .
$$

By Lemma 2.11, we find that $[M]^{f l}$ is invertible $\Longleftrightarrow F$ is invertible $\Longleftrightarrow E$ is invertible $\Longleftrightarrow$ (5) splits. Thus it remains to check whether (5) splits, i.e. whether there exists $\pi: Q \rightarrow F$ such that $\pi \circ \iota=\operatorname{id}_{F}$.

We divide the standard basis of $Q$ into $G$-orbits, and take a complete representative system $\left\{x_{\lambda}\right\}$ of the $G$ orbits. Let $H_{\lambda}$ be the stabilizer of $x_{\lambda}$ in $G$. Then $\pi\left(x_{\lambda}\right)$ in $F^{H_{\lambda}}$. Conversely if we fix an element of $F^{H}$ as $\pi\left(x_{\lambda}\right)$, then $\pi$ is determined by the representatives $\left\{x_{\lambda}\right\}$.

We may have the necessary and the sufficient condition for $\pi \circ \iota=\mathrm{id}_{M}$, and this becomes a system of linear equations. Hence this system of linear equations has an integer solution $\Longleftrightarrow$ the section $\pi: Q \rightarrow F$ exists $\Longleftrightarrow$ $[M]^{f l}$ is invertible. This computation is performed in GAP as follows:

IsInvertibleF (G) returns whether $\left[M_{G}\right]^{f l}$ is invertible.

Algorithm F2 (Determination whether $\left[M_{G}\right]^{f l}$ is invertible).

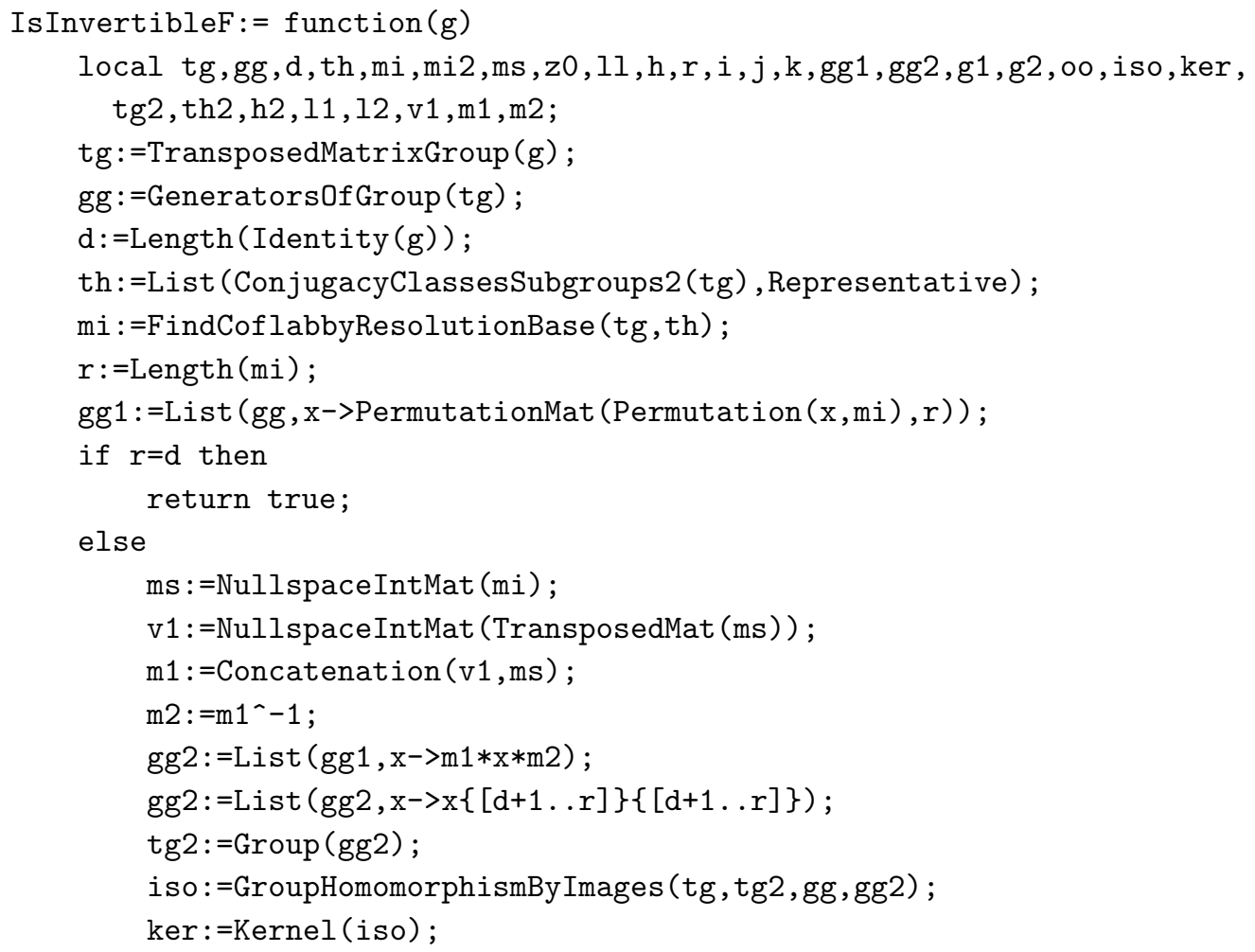




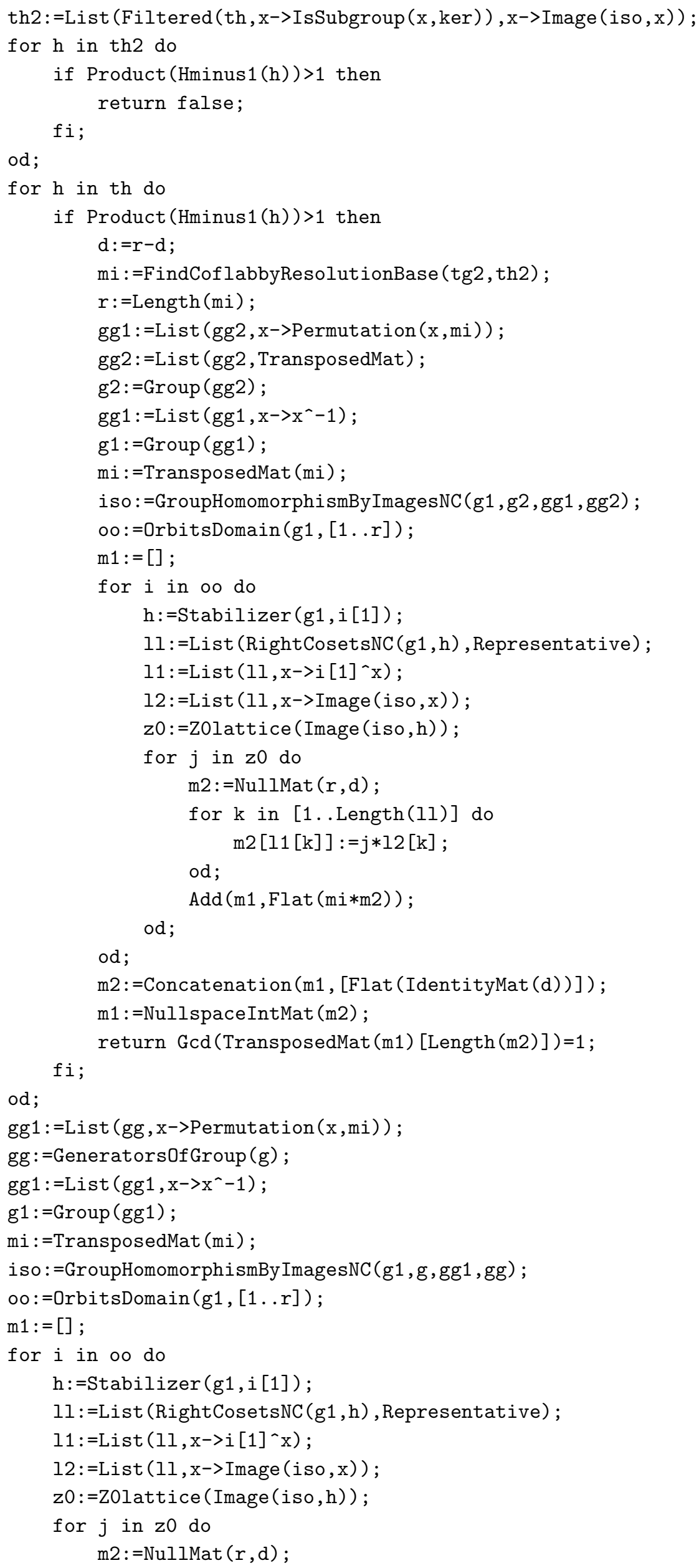




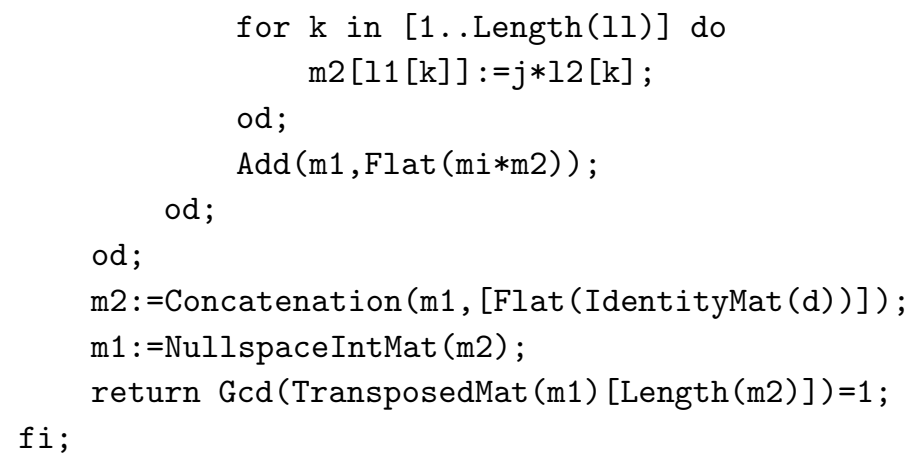

\subsection{Computation of $E$ with $\left[\left[M_{G}\right]^{f l}\right]^{f l}=[E]$.}

Let $G$ be a finite subgroup of $\operatorname{GL}(n, \mathbb{Z})$ and $M=M_{G}$ be the corresponding $G$-lattice of rank $n$ as in Definition 1.26. By a result of Section 5.2 (Algorithm $[$ 2 $)$, we assume that $[M]^{f l}$ is invertible. The next step is to determine whether $[M]^{f l}=0$. First, we give a sufficient condition for $[M]^{f l}=0$. Let $0 \rightarrow M \rightarrow P \rightarrow F \rightarrow 0$ be a flabby resolution of $M$. By Algorithm F2, we can compute $F$, and by the assumption $F$ is invertible. If $F$ is still complicated, we take a flabby resolution of $F: 0 \rightarrow F \rightarrow Q \rightarrow E \rightarrow 0$. Then $E=0 \Longrightarrow F=0 \Longrightarrow[M]^{f l}=0$. By the same way, we define $[M]^{f l^{n}}:=\left[[M]^{f l^{n-1}}\right]^{f l}$ inductively. Then we obtain a sufficient condition for $[M]^{f l}=0$, namely, $[M]^{f l^{n}}=0 \Longrightarrow[M]^{f l}=0$. The following algorithm may compute $E$ with $[E]=\left[[M]^{f l}\right]^{f l}$ effectively.

$\mathrm{flfl}(\mathrm{G})$ returns the $G$-lattice $E$ with $\left[\left[M_{G}\right]^{f l}\right]^{f l}=[E]$.

Algorithm F3 (Computation of $E$ with $\left[\left[M_{G}\right]^{f l}\right]^{f l}=[E]$ ).

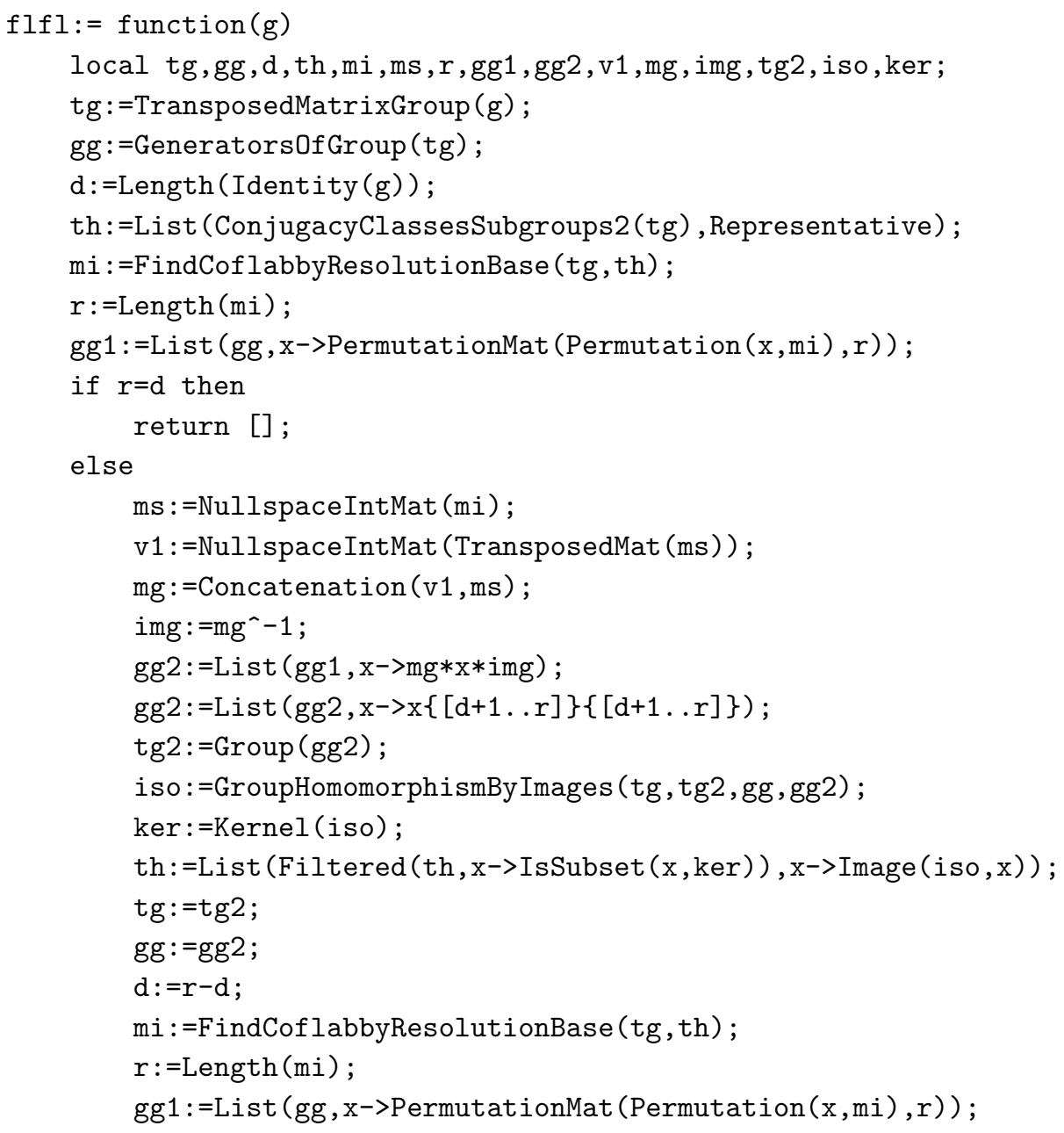




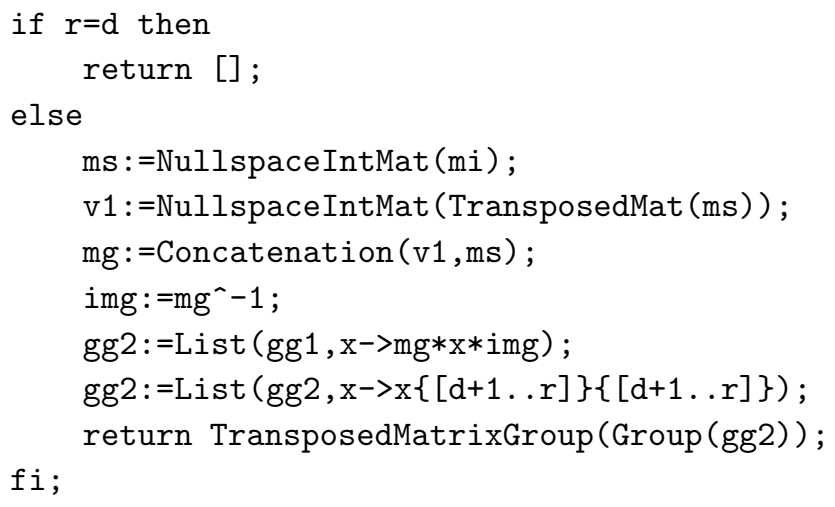

Example 5.2 (Algorithm F3). Let $\operatorname{Imf}(n, i, j) \leq \mathrm{GL}(n, \mathbb{Z})$ be the $j$-th $\mathbb{Z}$-class of the $i$-th $\mathbb{Q}$-class of the irreducible maximal finite group of dimension $n$ (which corresponds to the GAP command ImfMatrixGroup ( $n, i, j)$ ). Note that the maximal irreducible finite groups $\operatorname{Imf}(n, i, j)$ coincide with the maximal indecomposable finite groups $\operatorname{Indmf}(n, i, j)$ when $n \leq 5$ (see Subsection 4.0).

By using the algorithm $f l f l$, we may confirm that $\left[M_{G}\right]^{f l}=0$ for $G=\operatorname{Imf}(4,4,1)$ and $\operatorname{Imf}(5,1,1)$. By Lemma 2.17 we obtain $\left[M_{H}\right]^{f l}=0$ for any subgroups $H$ of $G$. There are 193 (resp. 953) conjugacy classes of subgroups of $\operatorname{Imf}(4,4,1)($ resp. $\operatorname{Imf}(5,1,1))$.

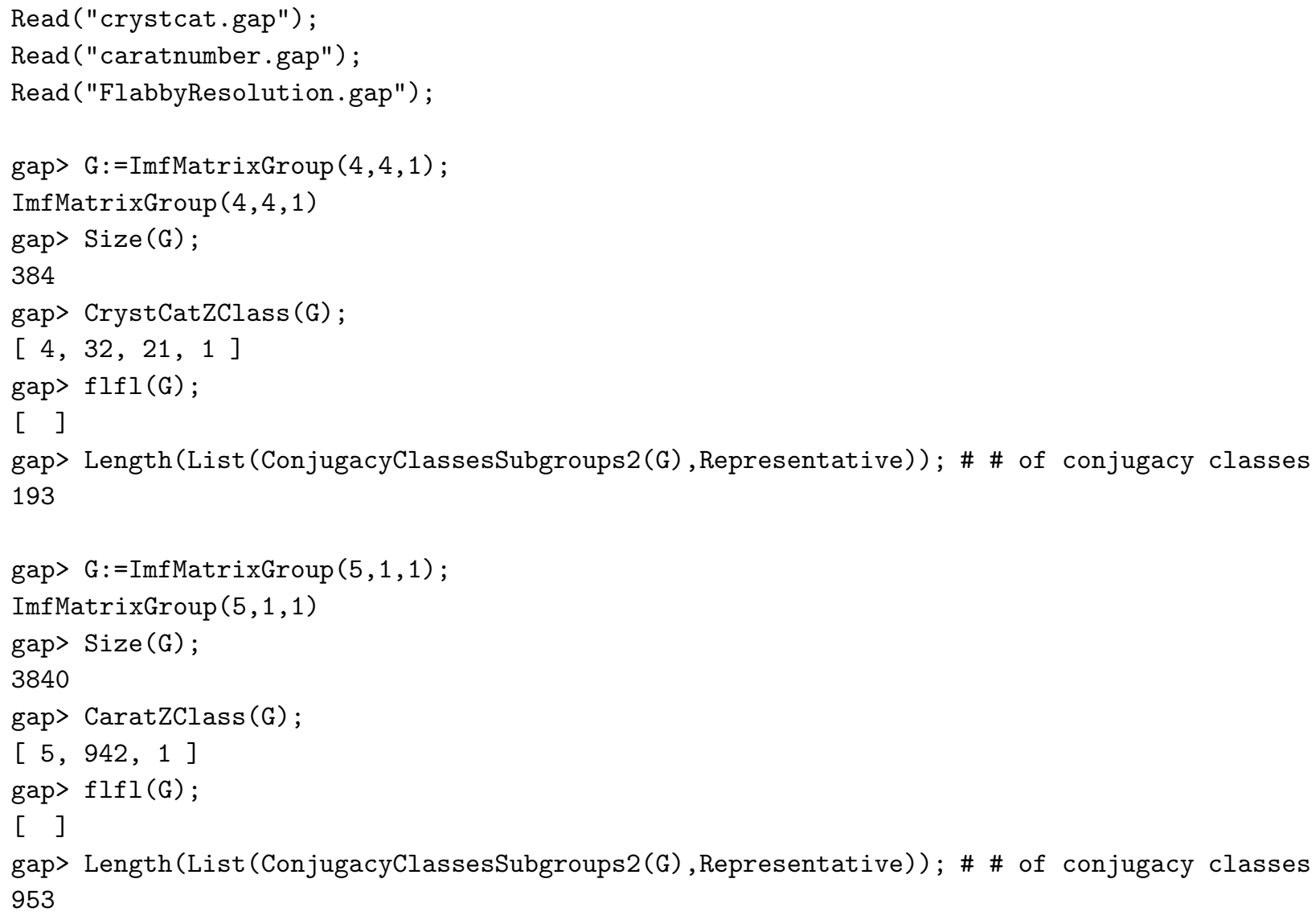

Example 5.3 (Kunyavskii's birational classification of algebraic $k$-tori of dimension 3 (Theorem 1.2)) . By Voskresenskii's theorem (Theorem[1.1), $\left[M_{G}\right]^{f l}=0$ for all $G$-lattices $M_{G}$ of rank $\leq 2$. Using the algorithm flfl, we may confirm Kunyavskii's theorem (Theorem 1.2). There exist 39 (resp. 34) decomposable (resp. indecomposable) $G$-lattices $M_{G}$ of rank 3 (see Example 4.9).

By Voskresenskii's theorem and Lemma 2.14, $\left[M_{G}\right]^{f l}=0$ for 39 decomposable $G$-lattices $M_{G}$. 
Using flfl and by Lemma 2.17, we may see that $\left[M_{G}\right]^{f l}=0$ for any subgroups $G$ of $\operatorname{Imf}(3,1,1) \simeq C_{2} \times S_{4}$, the group $G_{1} \simeq D_{4}$ of the GAP ID $(3,4,5,2)$ and the group $G_{2} \simeq S_{4}$ of the $\operatorname{GAP} \operatorname{ID}(3,7,4,3)$. Namely, $L\left(M_{G}\right)^{G}$ is stably $k$-rational. Note that Kunyavskii's theorem claims not only the stably $k$-rationality but also the $k$-rationality, and we could not confirm the $k$-rationality by this method.

There exist exactly 15 groups which are not subgroups of $\operatorname{Imf}(3,1,1), G_{1}$ and $G_{2}$ (see Table 2 in Theorem 1.1). Indeed, using the algorithm IsInvertibleF, we may confirm that $\left[M_{G}\right]^{f l}$ is not invertible for all the 15 groups $G$. Namely, $L\left(M_{G}\right)^{G}$ is not retract $k$-rational.

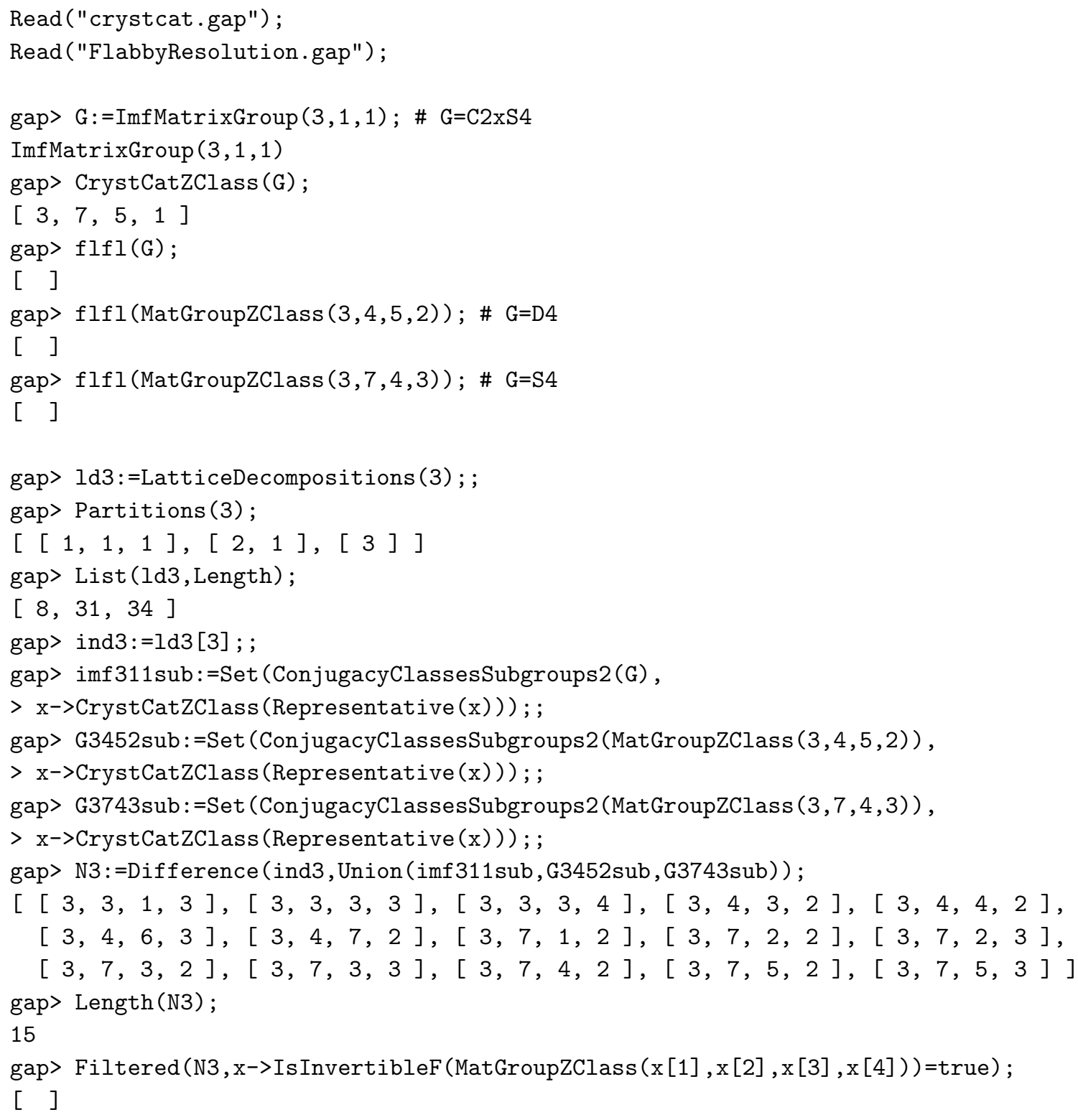

5.4. Possibility for $\left[M_{G}\right]^{f l}=0$.

We will devise an algorithm which, in a favorable situation, will enable us to show that some $G$-lattices are not stably permutation.

Let $G$ be a finite subgroup of $\operatorname{GL}(n, \mathbb{Z})$ and $M=M_{G}$ be the corresponding $G$-lattice of rank $n$ as in Definition 1.26 By a result of Section 5.2 (Algorithm $\left[\right.$ 22), we assume that $[M]^{f l}$ is invertible.

Each isomorphism class of irreducible permutation $G$-lattices corresponds to a conjugacy class of subgroup $H$ of $G$ by $H \leftrightarrow \mathbb{Z}[G / H]$. Let $H_{1}, \ldots, H_{r}$ be conjugacy classes of subgroups of $G$ whose ordering corresponds to the GAP function ConjugacyClassesSubgroups2(G) (see Section 4). Let $F$ be the flabby class of $M_{G}$. 
We assume that $F$ is stably permutation, i.e. for $x_{r+1}= \pm 1$,

$$
\left(\bigoplus_{i=1}^{r} \mathbb{Z}\left[G / H_{i}\right]^{\oplus x_{i}}\right) \oplus F^{\oplus x_{r+1}} \simeq \bigoplus_{i=1}^{r} \mathbb{Z}\left[G / H_{i}\right]^{\oplus y_{i}} .
$$

Define $a_{i}=x_{i}-y_{i}$ and $b_{1}=x_{r+1}$. Then we have for $b_{1}= \pm 1$,

$$
\bigoplus_{i=1}^{r} \mathbb{Z}\left[G / H_{i}\right]^{\oplus a_{i}} \simeq F^{\oplus\left(-b_{1}\right)}
$$

By computing some $\mathbb{Z}$-class invariants, we will give a necessary condition for $\left[M_{G}\right]^{f l}=0$.

Let $\left\{c_{1}, \ldots, c_{r}\right\}$ be a set of complete representatives of the conjugacy classes of $G$. Let $A_{i}\left(c_{j}\right)$ be the matrix representation of the factor coset action of $c_{j} \in G$ on $\mathbb{Z}\left[G / H_{i}\right]$ and $B\left(c_{j}\right)$ be the matrix representation of the action of $c_{j} \in G$ on $F$. By (6), for each $c_{j} \in G$, we have

$$
\sum_{i=1}^{r} a_{i} \operatorname{tr} A_{i}\left(c_{j}\right)+b_{1} \operatorname{tr} B\left(c_{j}\right)=0
$$

where $\operatorname{tr} A$ is the trace of the matrix $A$. Similarly, we consider the rank of $H^{0}=\widehat{Z}^{0}$. For each $H_{j}$, we get

$$
\sum_{i=1}^{r} a_{i} \operatorname{rank} \widehat{Z}^{0}\left(H_{j}, \mathbb{Z}\left[G / H_{i}\right]\right)+b_{1} \operatorname{rank} \widehat{Z}^{0}\left(H_{j}, F\right)=0 .
$$

Finally, we compute $\widehat{H}^{0}$. Let $S y_{p}(A)$ be a $p$-Sylow subgroup of an abelian group $A$. $S y_{p}(A)$ can be written as a direct product of cyclic groups uniquely. Let $n_{p, e}\left(S y_{p}(A)\right)$ be the number of direct summands of cyclic groups of order $p^{e}$. For each $H_{j}, p, e$, we get

$$
\sum_{i=1}^{r} a_{i} n_{p, e}\left(S y_{p}\left(\widehat{H}^{0}\left(H_{j}, \mathbb{Z}\left[G / H_{i}\right]\right)\right)\right)+b_{1} n_{p, e}\left(S y_{p}\left(\widehat{H}^{0}\left(H_{j}, F\right)\right)\right)=0 .
$$

By the equalities (7), (8) and (9), we may get a system of linear equations in $a_{1}, \ldots, a_{r}, b_{1}$ over $\mathbb{Z}$. Namely, we have that $\left[M_{G}\right]^{f l}=0 \Longrightarrow$ there exist $a_{1}, \ldots, a_{r} \in \mathbb{Z}$ and $b_{1}= \pm 1$ which satisfy (6) $\Longrightarrow$ this system of linear equations has a integer solution in $a_{1}, \ldots, a_{r}$ with $b_{1}= \pm 1$. In particular, if this system of linear equations has no integer solutions, then we conclude that $\left[M_{G}\right]^{f l} \neq 0$.

HO (G) returns the Tate cohomology group $\widehat{H}^{0}\left(G, M_{G}\right)$.

PossibilityOfStablyPermutationF(G) returns a basis $\mathcal{L}=\left\{l_{1}, \ldots, l_{s}\right\}$ of the solution space of the system of linear equations which is obtained by the equalities (7), (8) and (9).

PossibilityOfStablyPermutationM(G) returns the same as PossibilityOfStablyPermutationF(G) but with respect to $M_{G}$ instead of $F$. (We will use this in Section [6)

Algorithm F4 (Possibility for $\left.\left[M_{G}\right]^{f l}=0\right)$.

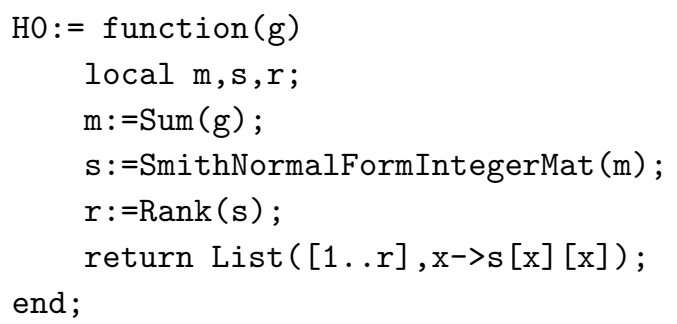




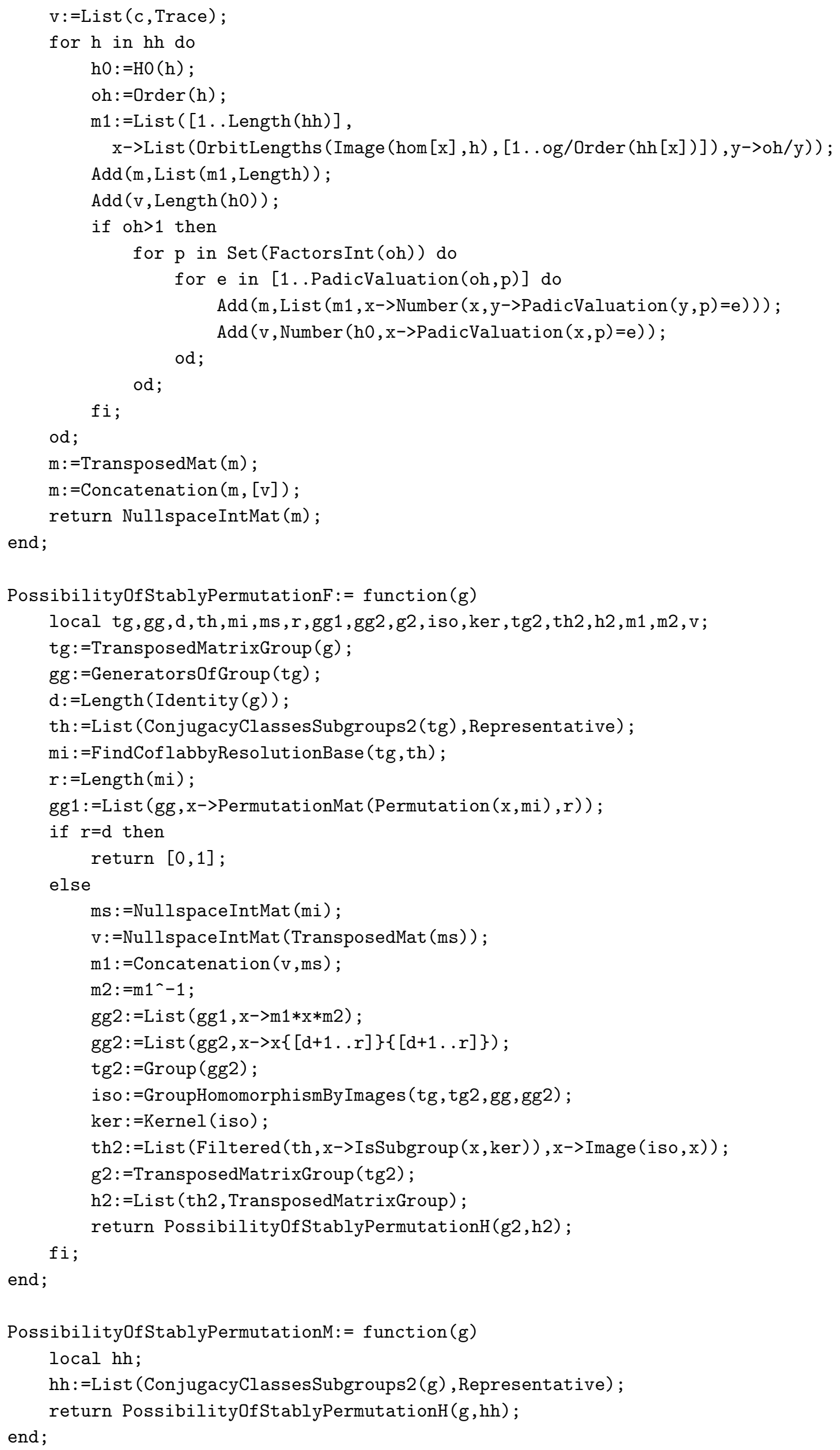


Example 5.4 (Algorithm F4 Possibility for $\left[M_{G}\right]^{f l}=0$ ). Let $G \simeq F_{20}$ be the Frobenius group of order 20 of the GAP ID $(4,31,1,3)$ or $(4,31,1,4)$ as in Table 2. By Algorithm F2, we may check that $\left[M_{G}\right]^{f l}$ is invertible. We will show that $\left[M_{G}\right]^{f l} \neq 0$. There exist 6 conjugacy classes of subgroups $\{1\}, H_{2} \simeq C_{2}, H_{3} \simeq C_{4}, H_{4} \simeq C_{5}$, $H_{5} \simeq D_{5}$ and $G$ of $G$ of order $1,2,4,5,10$ and 20 respectively. The corresponding permutation $G$-lattices are $\mathbb{Z}[G], \mathbb{Z}\left[G / H_{2}\right], \mathbb{Z}\left[G / H_{3}\right], \mathbb{Z}\left[G / H_{4}\right], \mathbb{Z}\left[G / H_{5}\right]$ and $\mathbb{Z}$ of rank $20,10,5,4,2$ and 1 (this ordering is determined by the GAP function ConjugacyClassesSubgroups2(G) (see Section 4). By Algorithm F1, we may obtain the flabby $G$-lattice $F$ of rank 16 via the function FlabbyResolution(G).actionF where $0 \rightarrow M_{G} \rightarrow P \rightarrow F$ is a flabby resolution of $M_{G}$.

Let $\mathcal{L}=\left\{l_{1}, \ldots, l_{s}\right\}$ be a list of lists obtained by the GAP function PossibilityOfStablyPermutationF(G). Put $\mathcal{U}=\mathbb{Z} l_{1}+\cdots+\mathbb{Z} l_{s}$ i.e. the set of all linear combinations of $\mathcal{L}$ over $\mathbb{Z}$. When $\left[a_{1}, \ldots, a_{r}, b_{1}\right] \in \mathcal{U}$, there is a possibility that

$$
\bigoplus_{i=1}^{r} \mathbb{Z}\left[G / H_{i}\right]^{\oplus a_{i}} \simeq F^{\oplus\left(-b_{1}\right)}
$$

By $\mathcal{U}=\langle[1,1,0,1,-1,0,-2]\rangle$, there is a possibility that

$$
\mathbb{Z}[G] \oplus \mathbb{Z}\left[G / H_{2}\right] \oplus \mathbb{Z}\left[G / H_{4}\right] \simeq \mathbb{Z}\left[G / H_{5}\right] \oplus F^{\oplus 2} .
$$

However, $b_{1}$ should be \pm 1 . This implies that $F$ is not stably permutation, hence $\left[M_{G}\right]^{f l} \neq 0$. In other words, $L\left(M_{G}\right)^{G}$ is not stably but retract $k$-rational.

By $H^{2}(G, \mathbb{Z}[G])=0, H^{2}\left(G, \mathbb{Z}\left[G / H_{2}\right]\right)=\mathbb{Z} / 2 \mathbb{Z}$ and $H^{2}\left(G, \mathbb{Z}\left[G / H_{4}\right]\right)=\mathbb{Z} / 5 \mathbb{Z}$ while $H^{2}\left(G, \mathbb{Z}\left[G / H_{5}\right]\right)=\mathbb{Z} / 2 \mathbb{Z}$ and $H^{2}(G, F)=0$, we also see that $F^{\oplus k}$ is not stably permutation for all $k \geq 1$.

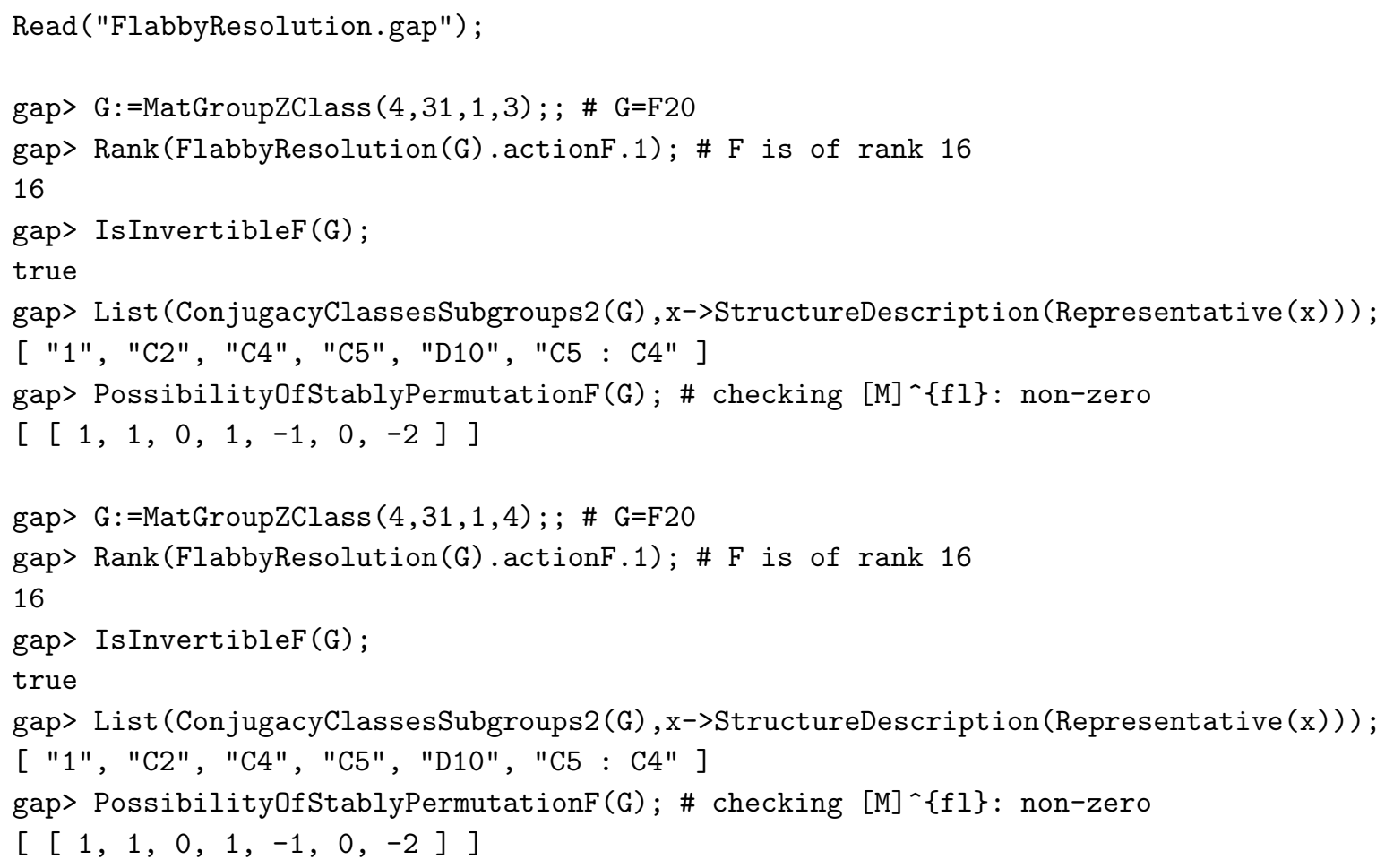

\subsection{Verification of $\left[M_{G}\right]^{f l}=0$ : Method I.}

Let $G$ be a finite subgroup of $\operatorname{GL}(n, \mathbb{Z})$ and $M=M_{G}$ be the corresponding $G$-lattice of rank $n$ as in Definition 1.26. Next we try to check whether the possibility of the isomorphism as in (6) actually holds. The following algorithm tries to find the isomorphism using the GAP function RepresentativeAction(GL (n, Integers), G1, G2).

Nlist(1) returns the negative part of the list $l$.

Plist (1) returns the positive part of the list $l$.

StablyPermutationFCheck (G, L1, L2) returns the matrix $P$ which satisfies $G_{1} P=P G_{2}$ where $G_{1}$ (resp. $G_{2}$ ) is 
the matrix representation group of the action of $G$ on $\left(\oplus_{i=1}^{r} \mathbb{Z}\left[G / H_{i}\right]^{\oplus a_{i}}\right) \oplus F^{\oplus b_{1}}\left(\right.$ resp. $\left.\left(\oplus_{i=1}^{r} \mathbb{Z}\left[G / H_{i}\right]^{\oplus a_{i}^{\prime}}\right) \oplus F^{\oplus b_{1}^{\prime}}\right)$ with the isomorphism

$$
\left(\bigoplus_{i=1}^{r} \mathbb{Z}\left[G / H_{i}\right]^{\oplus a_{i}}\right) \oplus F^{\oplus b_{1}} \simeq\left(\bigoplus_{i=1}^{r} \mathbb{Z}\left[G / H_{i}\right]^{\oplus a_{i}^{\prime}}\right) \oplus F^{\oplus b_{1}^{\prime}}
$$

for lists $L_{1}=\left[a_{1}, \ldots, a_{r}, b_{1}\right]$ and $L_{2}=\left[a_{1}^{\prime}, \ldots, a_{r}^{\prime}, b_{1}^{\prime}\right]$, if $P$ exists. If such $P$ does not exist, this returns false. StablyPermutationMCheck(G,L1,L2) returns the same as StablyPermutationFCheck(G,L1,L2) but with respect to $M_{G}$ instead of $F$. (We will use this in Section 6.)

If the rank of $F$ is small enough, StablyPermutationFCheck works well. However, if the rank of $F$ is not small, StablyPermutationFCheck does not return the answer in a suitable time, and then we need to make more efforts. We will explain this in the next section (Section [5.6).

Algorithm F5 (Verification of $\left[M_{G}\right]^{f l}=0$ : Method I). (The following algorithm needs the CARAT package, in particular for the command RepresentativeAction.)

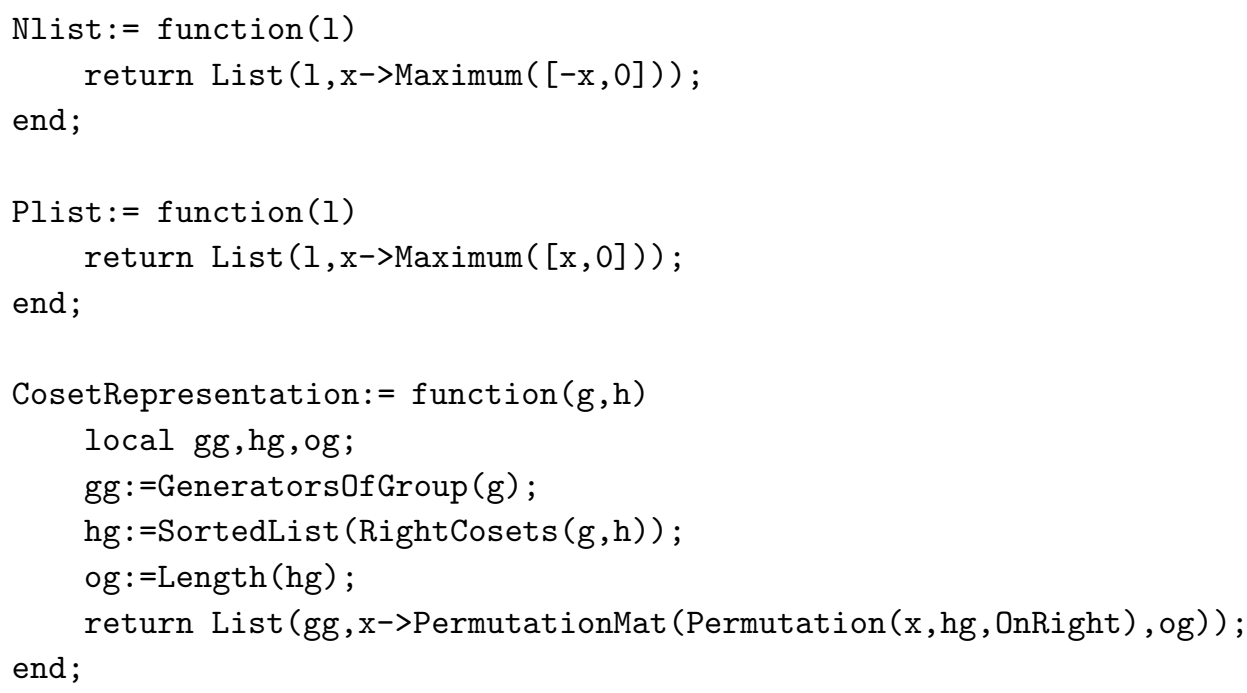


$f i$;

end;

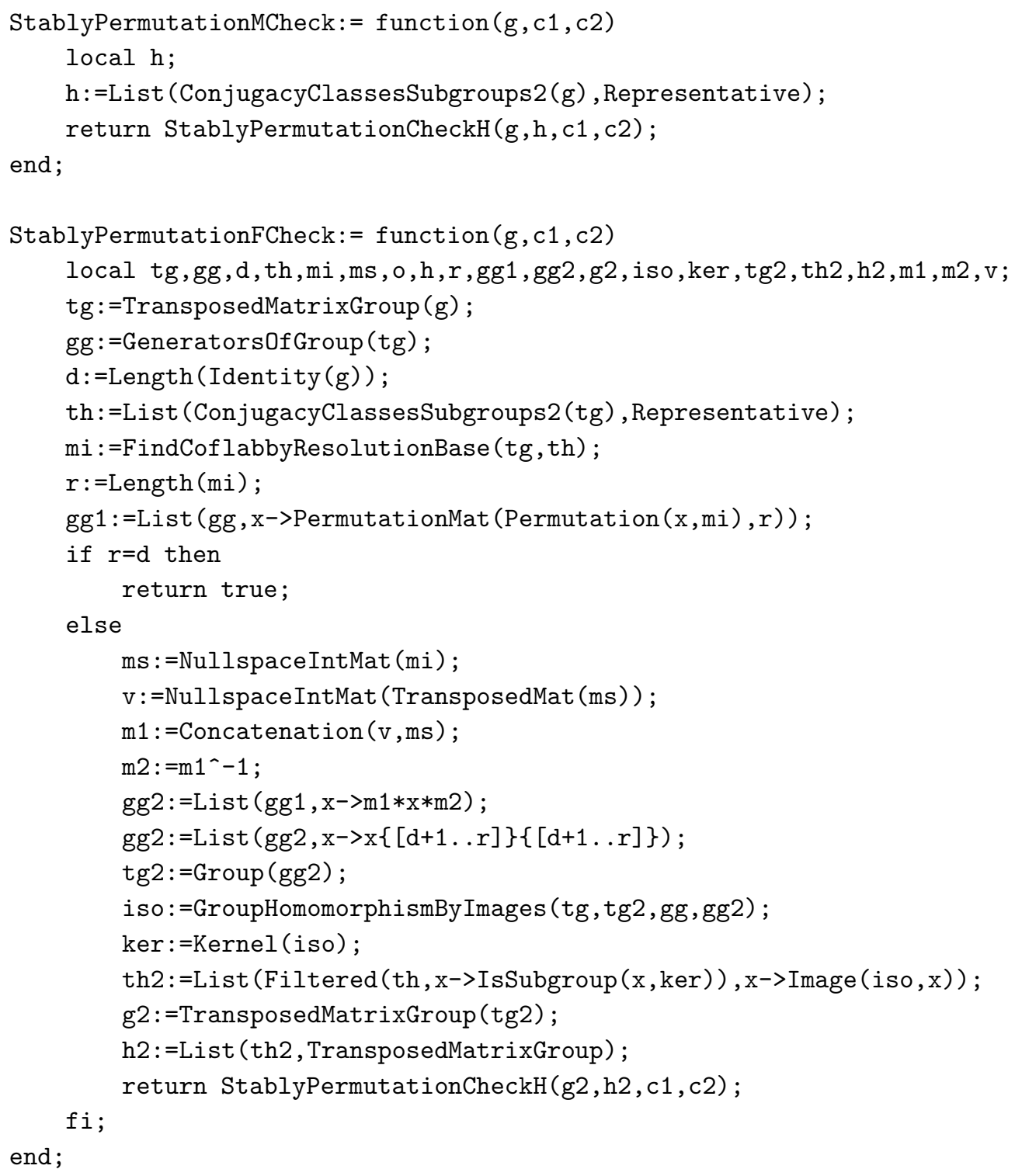

Example 5.5 (Algorithm F5] Method I (1)). Let $G=\operatorname{Imf}(4,3,1) \simeq S_{5} \times C_{2}$ be the group of order 240 of the GAP ID $(4,31,7,1)$. By Algorithm F1, the rank of the flabby class $F$ of $G$ is 6 . By Algorithm F5, the following possibility of the isomorphism exists: $\mathbb{Z}\left[G / H_{52}\right] \oplus \mathbb{Z}\left[G / H_{54}\right] \simeq \mathbb{Z} \oplus F$ on which the matrix representation groups of the action of $G$ both sides are $G_{1}$ and $G_{2}$ respectively where $H_{52} \simeq S_{4} \times C_{2}$ and $H_{54} \simeq S_{5}$. We may confirm the isomorphism via StablyPermutationFCheck(G,Nlist(1), Plist(1)) which returns the matrix $P$ with $G_{1} P=P G_{2}$. This implies $\left[M_{G}\right]^{f l}=0$, and hence $L\left(M_{G}\right)^{G}$ is stably $k$-rational.

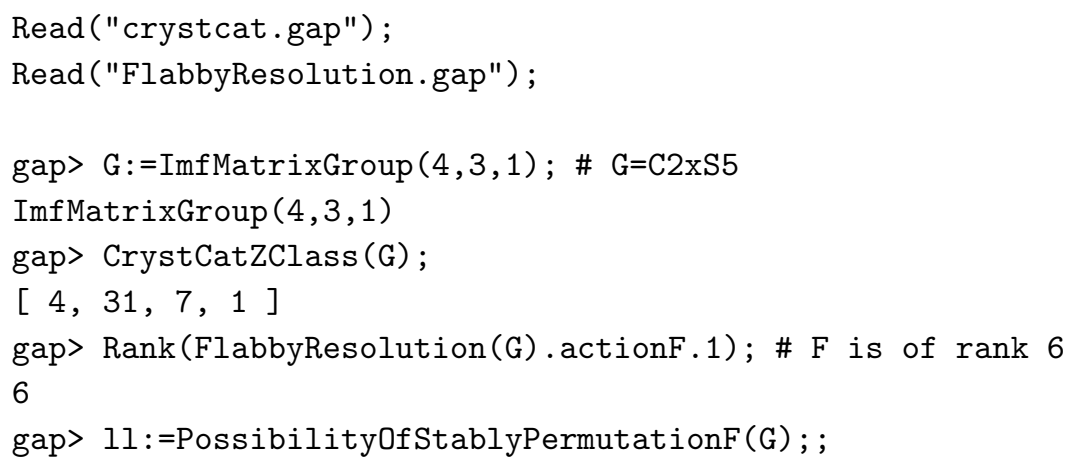




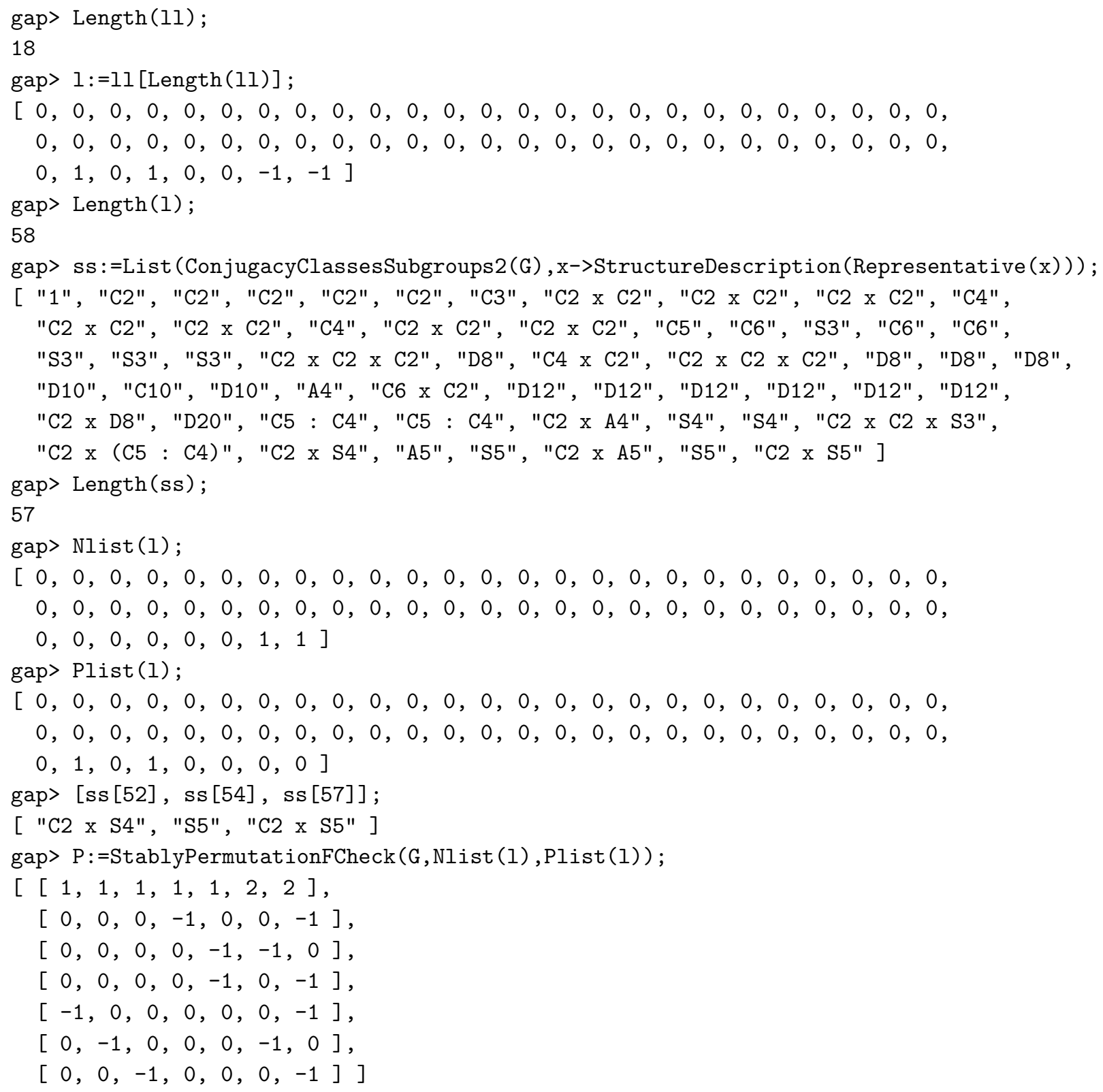

Example 5.6 (Algorithm F5, Method I (2)). It may be needed to add some more $G$-lattices to make the both hand side of $G$-lattices isomorphic before applying StablePermutationFCheck as in Method I. The following example shows that $F \oplus \mathbb{Z} \simeq \mathbb{Z}\left[S_{5} / S_{4}\right] \oplus \mathbb{Z}$ for the group $G \simeq S_{5}$ of the CARAT ID $(5,946,2)$ and $F$ is of rank 5 which satisfies $[F]=\left[M_{G}\right]^{f l}$ although $F \not \mathbb{Z}\left[S_{5} / S_{4}\right]$.

Read ("caratnumber.gap");

Read ("FlabbyResolution.gap");

gap> G:=CaratMatGroupZClass $(5,946,2) ;$; \# G=S5

gap> Rank(FlabbyResolution(G).actionF.1); \#F is of rank 5

5

gap> CaratZClass (FlabbyResolution(G) .actionF);

[ 5, 911, 4 ]

gap> 11:=PossibilityOfStablyPermutationF(G) ;

$[[1,0,0,-1,0,0,-4,0,-2,1,2,0,-1,-1,0,4,0,1,-4,4]$,

$[0,1,0,0,0,-1,-1,0,-1,0,0,0,0,0,1,1,0,0,-1,1]$,

$[0,0,1,0,0,0,-2,0,-1,0,1,0,-1,-1,0,2,0,1,-2,2]$,

$[0,0,0,0,1,2,-2,0,-2,1,2,-2,-1,-2,-2,2,0,1,-2,4]$, 


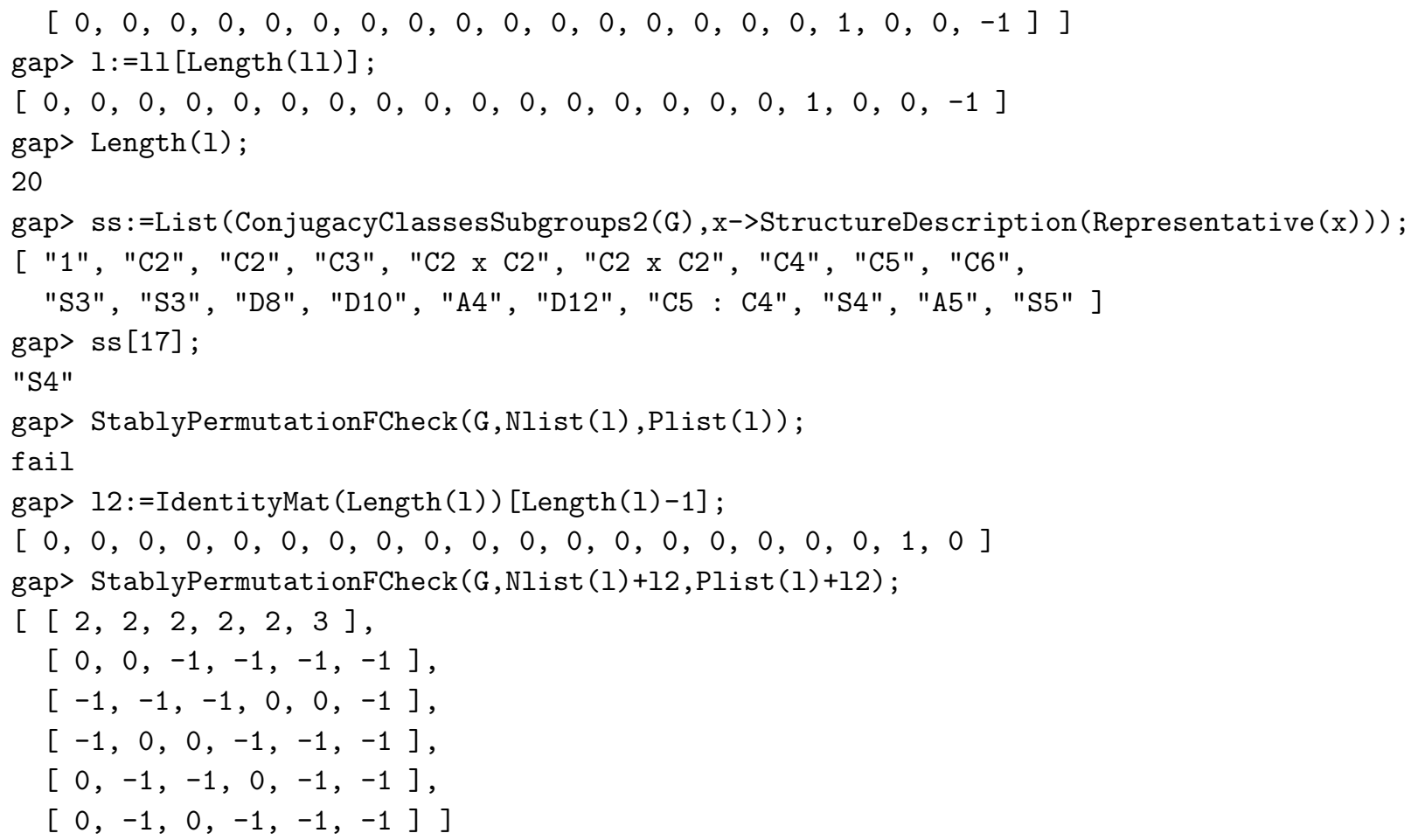

\subsection{Verification of $\left[M_{G}\right]^{f l}=0$ : Method II.}

Let $G$ be a finite subgroup of $\operatorname{GL}(n, \mathbb{Z})$ and $M=M_{G}$ be the corresponding $G$-lattice of rank $n$ as in Definition 1.26 The function RepresentativeAction(GL ( $n$, Integers) $\left., G_{1}, G_{2}\right)$ in Algorithm F5 may not work well when $n$ is not small. We will provide another method in order to confirm the isomorphism (10), i.e.

$$
\left(\bigoplus_{i=1}^{r} \mathbb{Z}\left[G / H_{i}\right]^{\oplus a_{i}}\right) \oplus F^{\oplus b_{1}} \simeq\left(\bigoplus_{i=1}^{r} \mathbb{Z}\left[G / H_{i}\right]^{\oplus a_{i}^{\prime}}\right) \oplus F^{\oplus b_{1}^{\prime}}
$$

although it is needed by trial and error.

Our aim is to find the matrix $P$ which satisfies $G_{1} P=P G_{2}$ rapidly. If we can choose a matrix with determinant det $P= \pm 1, G_{1}$ and $G_{2}$ are $\mathrm{GL}(n, \mathbb{Z})$-conjugate, and hence the isomorphism (10) established. This implies that the flabby class $\left[M_{G}\right]^{f l}=0$.

StablyPermutationFCheckP (G, L1, L2) returns a basis $\mathcal{P}=\left\{P_{1}, \ldots, P_{m}\right\}$ of the solution space of $G_{1} P=P G_{2}$ where $G_{1}$ (resp. $G_{2}$ ) is the matrix representation group of the action of $G$ on $\left(\oplus_{i=1}^{r} \mathbb{Z}\left[G / H_{i}\right]^{\oplus a_{i}}\right) \oplus F^{\oplus b_{1}}$ (resp. $\left.\left(\oplus_{i=1}^{r} \mathbb{Z}\left[G / H_{i}\right]^{\oplus a_{i}^{\prime}}\right) \oplus F^{\oplus b_{1}^{\prime}}\right)$ with the isomorphism (10) for lists $L_{1}=\left[a_{1}, \ldots, a_{r}, b_{1}\right]$ and $L_{2}=\left[a_{1}^{\prime}, \ldots, a_{r}^{\prime}, b_{1}^{\prime}\right]$, if $P$ exists. If such $P$ does not exist, this returns [ ].

StablyPermutationMCheckP (G, L1, L2) returns the same as StablyPermutationFCheckP (G, L1, L2) but with respect to $M_{G}$ instead of $F$. (We will use these in Section [6)

StablyPermutationFCheckMat (G, L1, L2, P) returns true if $G_{1} P=P G_{2}$ and $\operatorname{det} P= \pm 1$ where $G_{1}$ (resp. $G_{2}$ ) is the matrix representation group of the action of $G$ on $\left(\oplus_{i=1}^{r} \mathbb{Z}\left[G / H_{i}\right]^{\oplus a_{i}}\right) \oplus F^{\oplus b_{1}}\left(\right.$ resp. $\left.\left(\oplus_{i=1}^{r} \mathbb{Z}\left[G / H_{i}\right]^{\oplus a_{i}^{\prime}}\right) \oplus F^{\oplus b_{1}^{\prime}}\right)$ with the isomorphism (10) for lists $L_{1}=\left[a_{1}, \ldots, a_{r}, b_{1}\right]$ and $L_{2}=\left[a_{1}^{\prime}, \ldots, a_{r}^{\prime}, b_{1}^{\prime}\right]$. If not, this returns false.

StablyPermutationMCheckMat (G,L1,L2,P) returns the same as StablyPermutationFCheckMat (G, L1, L2, P) but with respect to $M_{G}$ instead of $F$. (We will use these in Section 6 )

StablyPermutationFCheckGen(G,L1,L2) returns the list $\left[\mathcal{M}_{1}, \mathcal{M}_{2}\right]$ where $\mathcal{M}_{1}=\left[g_{1}, \ldots, g_{t}\right]$ (resp. $\mathcal{M}_{2}=$ $\left.\left[g_{1}^{\prime}, \ldots, g_{t}^{\prime}\right]\right)$ is a list of the generators of $G_{1}$ (resp. $G_{2}$ ) which is the matrix representation group of the action of $G$ on $\left(\oplus_{i=1}^{r} \mathbb{Z}\left[G / H_{i}\right]^{\oplus a_{i}}\right) \oplus F^{\oplus b_{1}}$ (resp. $\left(\oplus_{i=1}^{r} \mathbb{Z}\left[G / H_{i}\right]^{\oplus a_{i}^{\prime}}\right) \oplus F^{\oplus b_{1}^{\prime}}$ ) with the isomorphism (10) for lists $L_{1}=\left[a_{1}, \ldots, a_{r}, b_{1}\right]$ and $L_{2}=\left[a_{1}^{\prime}, \ldots, a_{r}^{\prime}, b_{1}^{\prime}\right]$.

StablyPermutationMCheckGen (G,L1,L2) returns the same as StablyPermutationFCheckGen(G,L1,L2) but with respect to $M_{G}$ instead of $F$. (We will use these in Section $[6$ ) 


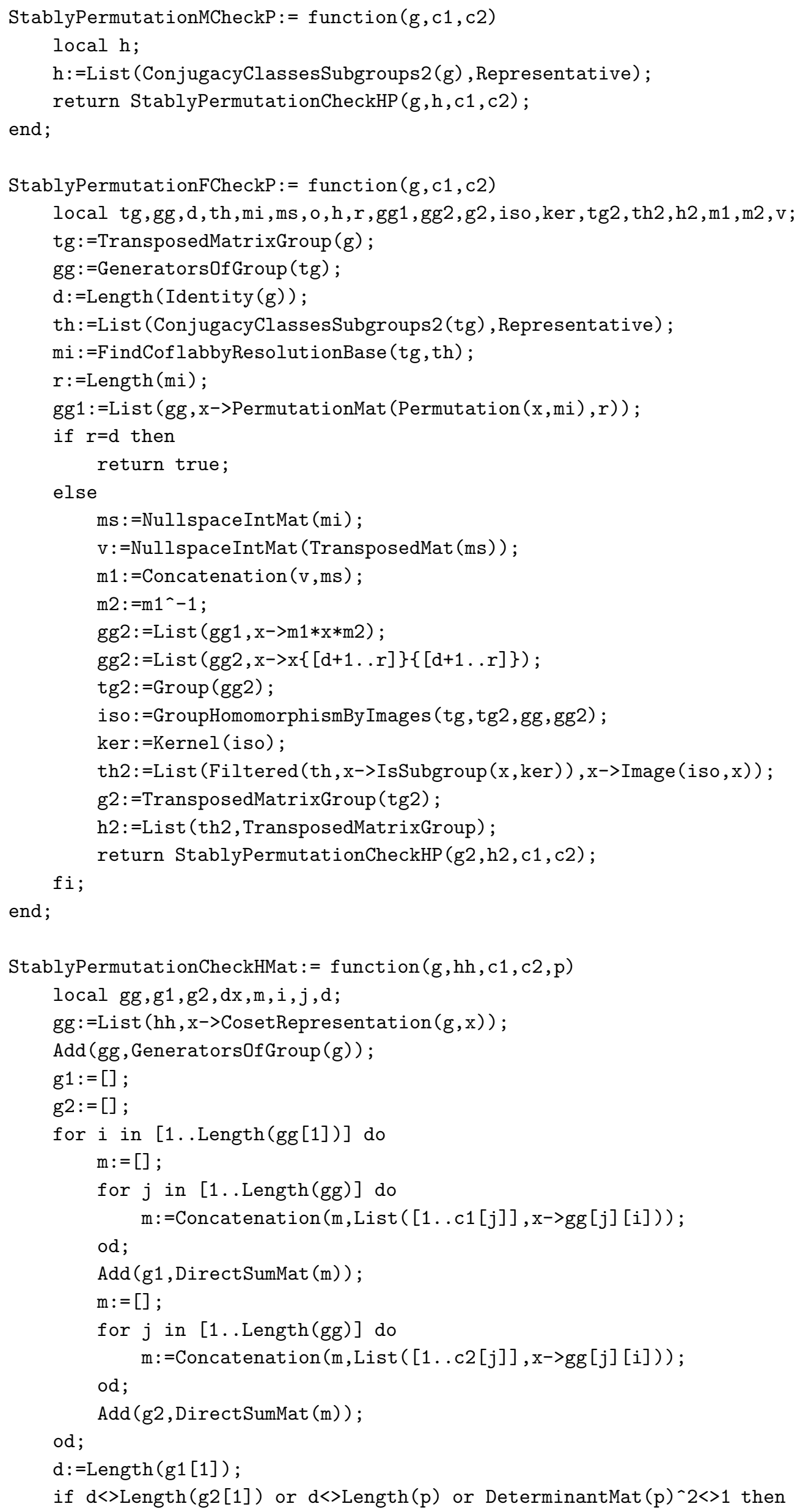




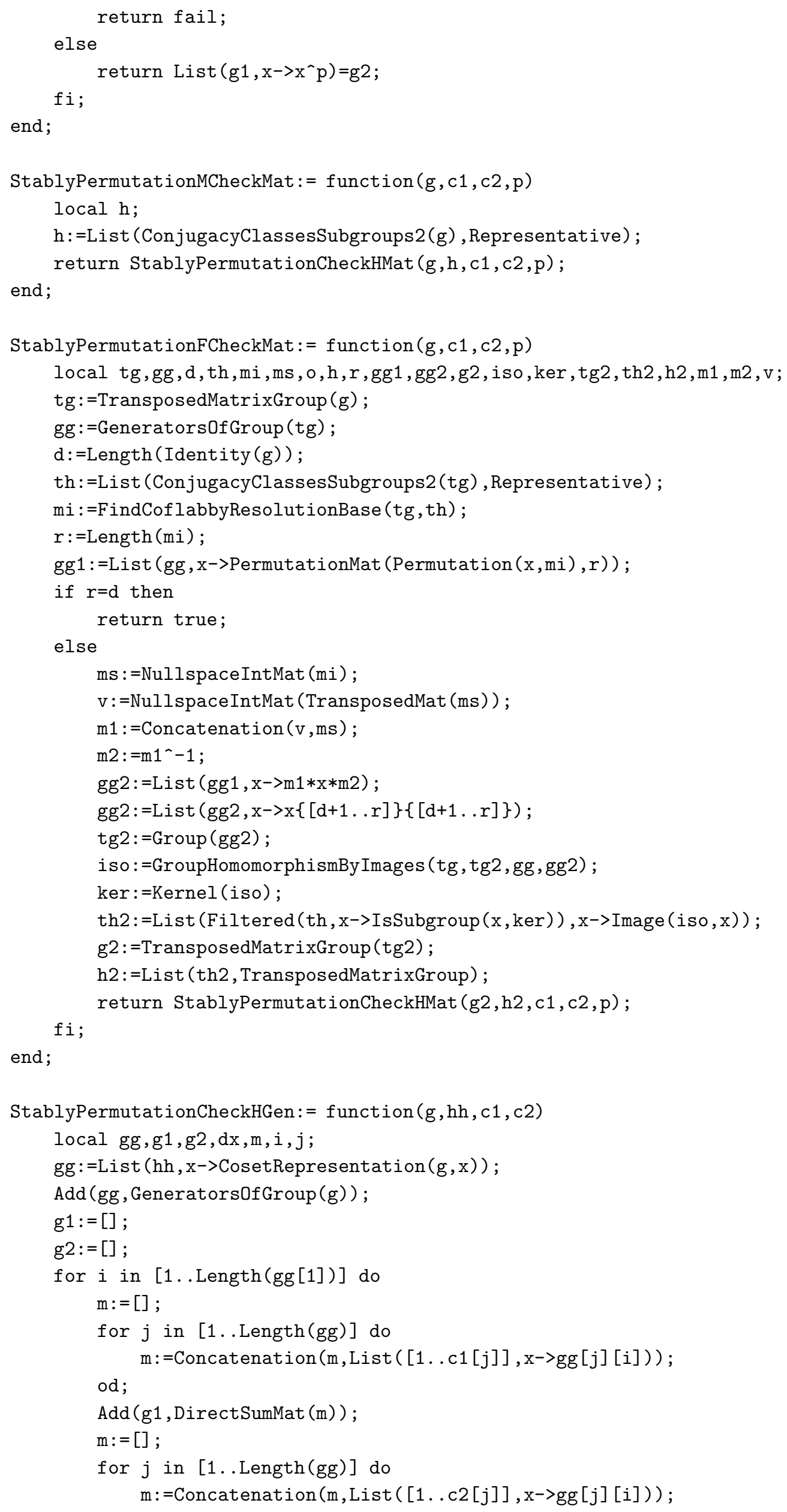




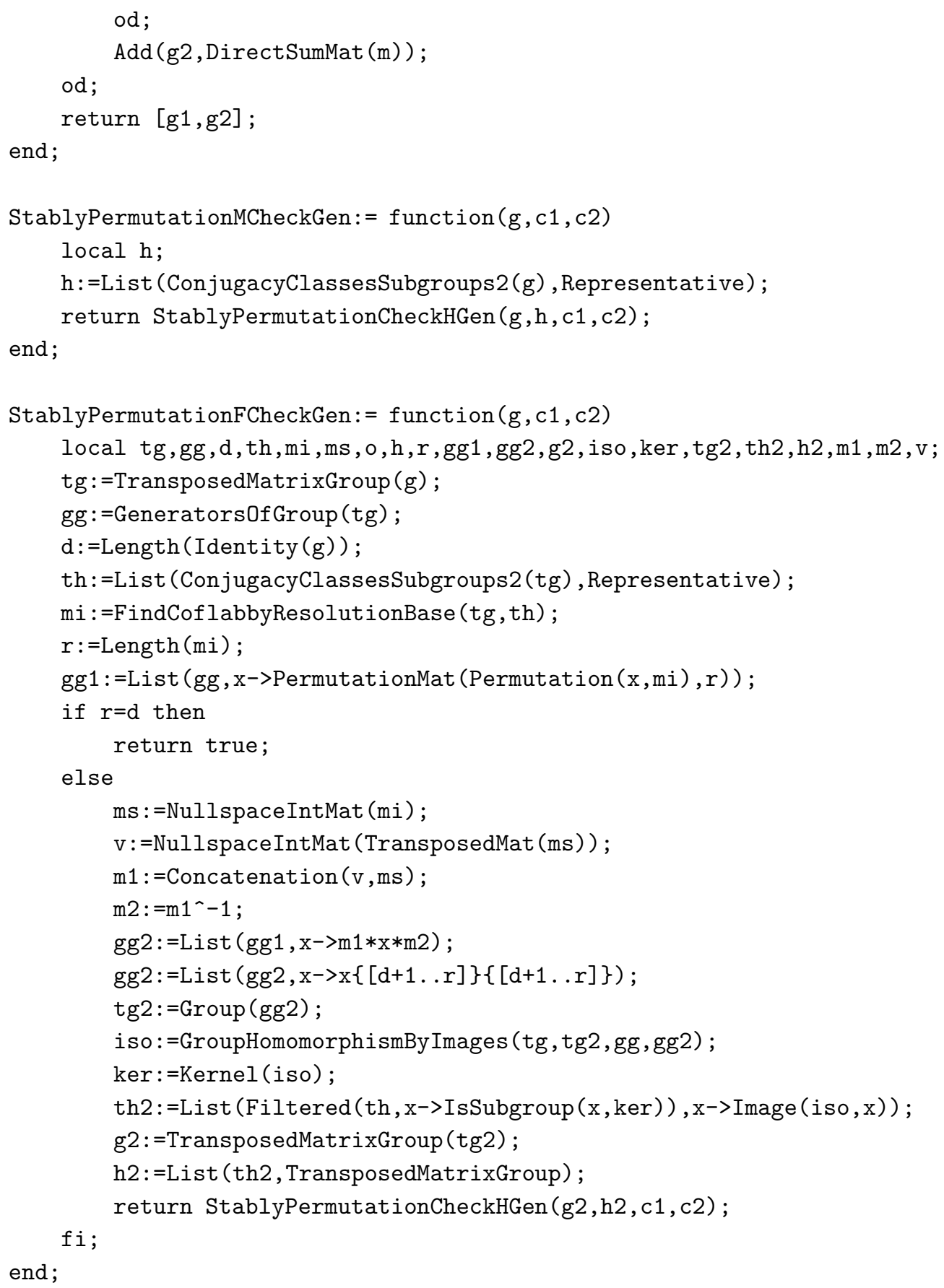

Example 5.7 (Algorithm [F6] Method II). Let $M_{G}$ be the $G$-lattice where $G=\operatorname{Imf}(4,2,1) \simeq D_{6}^{2} \rtimes C_{2}$ is the group of order 288 of the GAP ID $(4,30,13,1)$. We will show that $[M]^{f l}=[F]=0$. Indeed, we can verify that $F$ is of rank 8 and

$$
\mathbb{Z}\left[G / H_{196}\right] \oplus \mathbb{Z}\left[G / H_{212}\right] \simeq F \oplus \mathbb{Z}\left[G / H_{217}\right]
$$

where $H_{196} \simeq C_{2}^{2} \times D_{6}, H_{212} \simeq C_{2} \times S_{3}^{2}$ and $H_{217} \simeq D_{6}^{2}$ (the rank of the both sides is $6+4=8+2=10$ ). By comparing with Algorithm F5. Method I, we may obtain the matrix representations of $G_{1}$ and $G_{2}$ which corresponds to the action of $G$ on $\mathbb{Z}\left[G / H_{196}\right] \oplus \mathbb{Z}\left[G / H_{212}\right]$ and $F \oplus \mathbb{Z}\left[G / H_{217}\right]$ respectively and the matrix $P$ which satisfies $\sigma_{1} P=P \sigma_{2}$ for any $\sigma_{1} \in G_{1}$ and $\sigma_{2} \in G_{2}$.

gap> Read("crystcat.gap");

gap> Read("FlabbyResolution.gap");

gap> G:=ImfMatrixGroup $(4,2,1)$;

$\operatorname{ImfMatrixGroup}(4,2,1)$ 


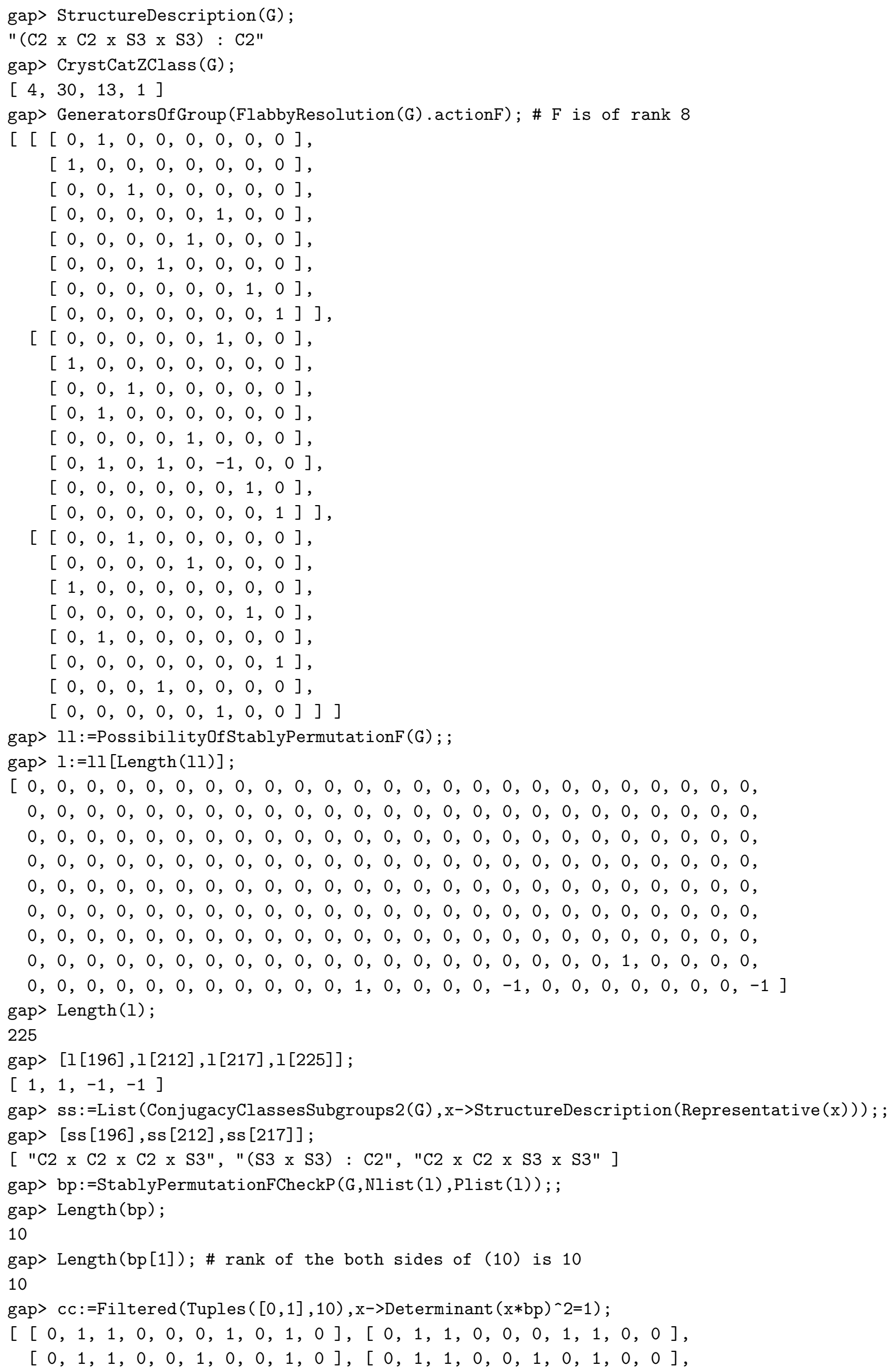


$[0,1,1,0,1,0,1,0,1,1],[0,1,1,0,1,0,1,1,0,1]$, $[0,1,1,0,1,1,0,0,1,1],[0,1,1,0,1,1,0,1,0,1]$, $[1,0,0,1,0,0,1,0,1,0],[1,0,0,1,0,0,1,1,0,0]$, $[1,0,0,1,0,1,0,0,1,0],[1,0,0,1,0,1,0,1,0,0]$, $[1,0,0,1,1,0,1,0,1,1],[1,0,0,1,1,0,1,1,0,1]$, $[1,0,0,1,1,1,0,0,1,1],[1,0,0,1,1,1,0,1,0,1]]$ gap> $\mathrm{p}:=\mathrm{cc}[1] * \mathrm{bp}$;

$[[0,1,0,0,1,1,1,1,0,0]$, $[1,0,1,1,0,0,0,0,1,1]$, $[0,0,0,0,0,1,0,1,0,0]$, $[0,0,0,0,1,0,1,0,0,0]$, $[0,0,0,1,0,0,0,0,0,1]$, $[0,1,0,0,0,0,0,1,0,0]$, $[0,0,1,0,0,0,0,0,1,0]$, $[0,1,0,0,0,0,1,0,0,0]$, $[1,0,0,0,0,0,0,0,0,1]$, $[1,0,0,0,0,0,0,0,1,0]]$ gap> Determinant $(p)$;

1 gap> gg:=StablyPermutationFCheckGen (G,Nlist (1), Plist(1));

$[[[[1,0,0,0,0,0,0,0,0,0],[0,1,0,0,0,0,0,0,0,0]$, $[0,0,0,1,0,0,0,0,0,0],[0,0,1,0,0,0,0,0,0,0]$, $[0,0,0,0,1,0,0,0,0,0],[0,0,0,0,0,0,0,1,0,0]$, $[0,0,0,0,0,0,1,0,0,0],[0,0,0,0,0,1,0,0,0,0]$, $[0,0,0,0,0,0,0,0,1,0],[0,0,0,0,0,0,0,0,0,1]]$,

$[[1,0,0,0,0,0,0,0,0,0],[0,1,0,0,0,0,0,0,0,0]$, $[0,0,0,0,0,0,0,1,0,0],[0,0,1,0,0,0,0,0,0,0]$, $[0,0,0,0,1,0,0,0,0,0],[0,0,0,1,0,0,0,0,0,0]$, $[0,0,0,0,0,0,1,0,0,0],[0,0,0,1,0,1,0,-1,0,0]$, $[0,0,0,0,0,0,0,0,1,0],[0,0,0,0,0,0,0,0,0,1]]$,

$[[0,1,0,0,0,0,0,0,0,0],[1,0,0,0,0,0,0,0,0,0]$, $[0,0,0,0,1,0,0,0,0,0],[0,0,0,0,0,0,1,0,0,0]$, $[0,0,1,0,0,0,0,0,0,0],[0,0,0,0,0,0,0,0,1,0]$, $[0,0,0,1,0,0,0,0,0,0],[0,0,0,0,0,0,0,0,0,1]$, $[0,0,0,0,0,1,0,0,0,0],[0,0,0,0,0,0,0,1,0,0]]]$,

$[[[1,0,0,0,0,0,0,0,0,0],[0,1,0,0,0,0,0,0,0,0]$, $[0,0,1,0,0,0,0,0,0,0],[0,0,0,1,0,0,0,0,0,0]$, $[0,0,0,0,0,1,0,0,0,0],[0,0,0,0,1,0,0,0,0,0]$, $[0,0,0,0,0,0,0,1,0,0],[0,0,0,0,0,0,1,0,0,0]$, $[0,0,0,0,0,0,0,0,1,0],[0,0,0,0,0,0,0,0,0,1]]$,

$[[1,0,0,0,0,0,0,0,0,0],[0,0,0,0,1,0,0,0,0,0]$, $[0,0,1,0,0,0,0,0,0,0],[0,0,0,1,0,0,0,0,0,0]$, $[0,0,0,0,0,1,0,0,0,0],[0,1,0,0,0,0,0,0,0,0]$, $[0,0,0,0,0,0,0,1,0,0],[0,0,0,0,0,0,1,0,0,0]$, $[0,0,0,0,0,0,0,0,1,0],[0,0,0,0,0,0,0,0,0,1]]$,

$[[0,1,0,0,0,0,0,0,0,0],[1,0,0,0,0,0,0,0,0,0]$, $[0,0,0,0,1,0,0,0,0,0],[0,0,0,0,0,1,0,0,0,0]$, $[0,0,1,0,0,0,0,0,0,0],[0,0,0,1,0,0,0,0,0,0]$, $[0,0,0,0,0,0,0,0,1,0],[0,0,0,0,0,0,0,0,0,1]$, $[0,0,0,0,0,0,1,0,0,0],[0,0,0,0,0,0,0,1,0,0]]]]$ gap> List $\left(\mathrm{gg}[1], \mathrm{x}^{-}>\mathrm{p}^{\wedge}-1 * \mathrm{x} * \mathrm{p}\right)=\mathrm{gg}[2]$;

true

gap> StablyPermutationFCheckMat (G,Nlist (l), Plist (l), p);

true 


\subsection{Verification of $\left[M_{G}\right]^{f l}=0$ : Method III.}

Let $G$ be a finite subgroup of $\operatorname{GL}(n, \mathbb{Z})$ and $M=M_{G}$ be the corresponding $G$-lattice of rank $n$ as in Definition 1.26 In order to confirm that $[M]^{f l}=0$, Method I and Method II in the previous two section gave how to find the explicit isomorphism

$$
\left(\bigoplus_{i=1}^{r} \mathbb{Z}\left[G / H_{i}\right]^{\oplus a_{i}}\right) \oplus F^{\oplus b_{1}} \simeq\left(\bigoplus_{i=1}^{r} \mathbb{Z}\left[G / H_{i}\right]^{\oplus a_{i}^{\prime}}\right) \oplus F^{\oplus b_{1}^{\prime}} .
$$

However, if the rank of the $G$-lattices in the both sides of (10) is large, the algorithms in Method I and Method II do not work. In this case, we should try to reduce the rank of the $G$-lattices in (10). The following algorithm (Algorithm F7) can search suitable base change of the permutation $G$-lattice $P^{\circ}$ in a coflabby resolution $0 \rightarrow$ Ker $f \rightarrow P^{\circ} \stackrel{f}{\rightarrow} M^{\circ} \rightarrow 0$ of $M^{\circ}$ as in (3) in order to reduce the rank of Ker $f$. Then we may get a "reduced" flabby resolution of $M: 0 \rightarrow M \rightarrow P \rightarrow(\operatorname{Ker} f)^{\circ} \rightarrow 0$.

SearchCoflabbyResolutionBase (G, b) returns a list $\mathcal{L}=\left\{m_{1}, \ldots, m_{s}\right\}$ where the $m_{i}$ 's are all of the lists which satisfy that $m_{i}=\mathcal{P}^{\circ}, P^{\circ}=\mathbb{Z}\left[\mathcal{P}^{\circ}\right]$ and $P^{\circ}$ satisfies (4) with $\# \mathcal{R}^{\prime} \leq b$.

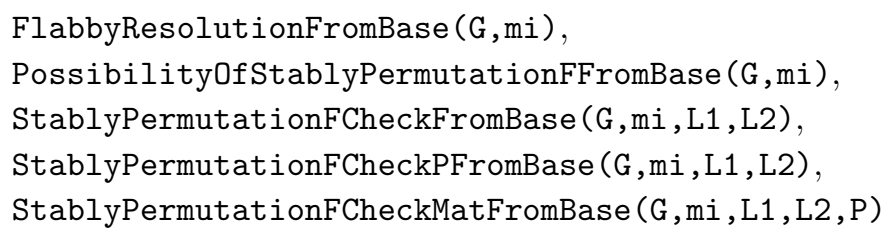

return the same as

FlabbyResolution(G) (in Algorithm F1),

PossibilityOfStablyPermutationF(G) (in Algorithm F4),

StablyPermutationFCheck (G,L1,L2) (in Algorithm F5 Method I),

StablyPermutationFCheckP(G,L1,L2) (in Algorithm[F6, Method II),

StablyPermutationFCheckMat (G,L1,L2,P) (in Algorithm F6. Method II)

respectively but with respect to $m_{i}=\mathcal{P}^{\circ}$ instead of the original $\mathcal{P}^{\circ}$ as in (4).

Algorithm F7 (Base change of a flabby resolution of $M_{G}$ : Method III).

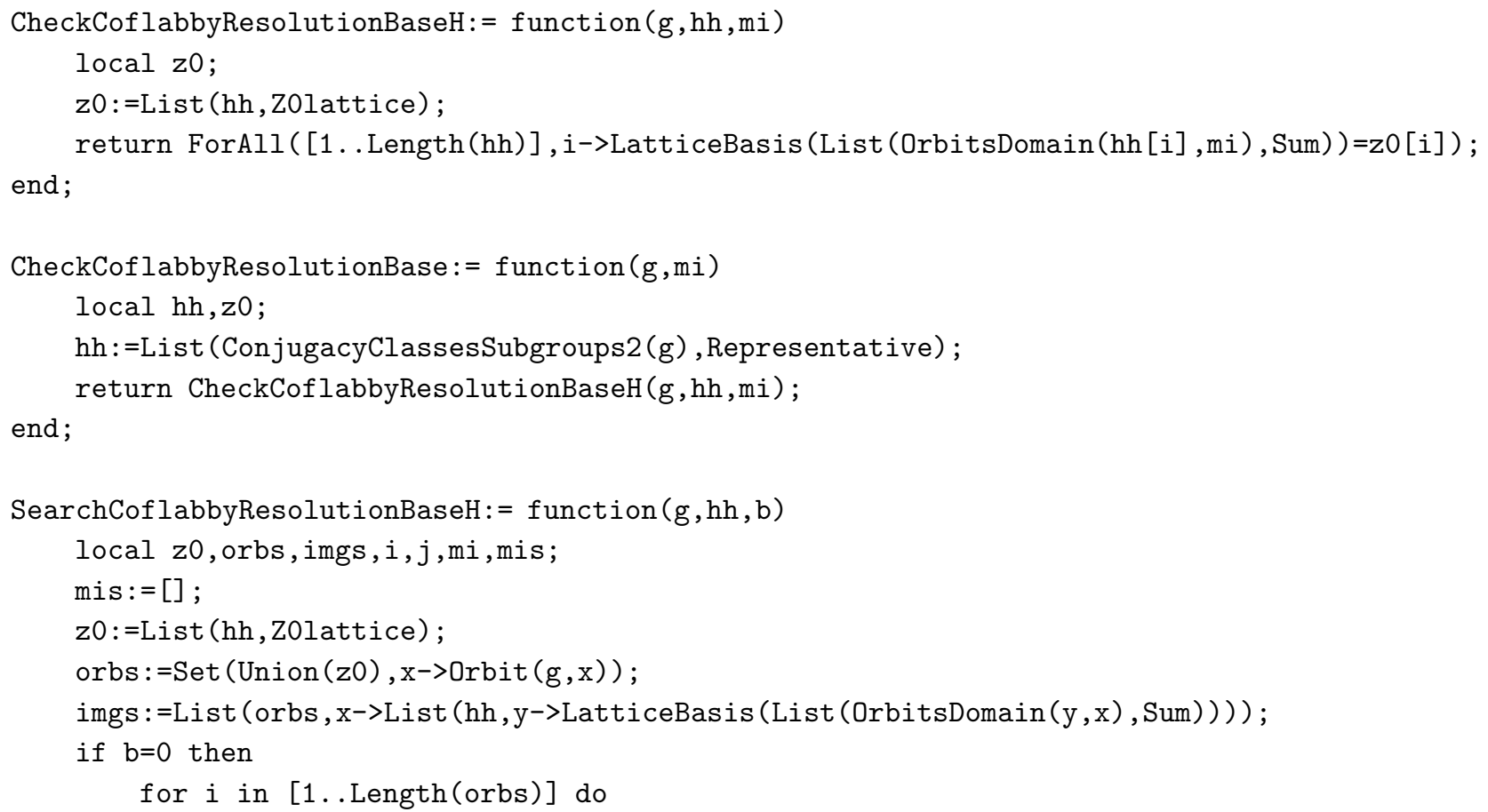




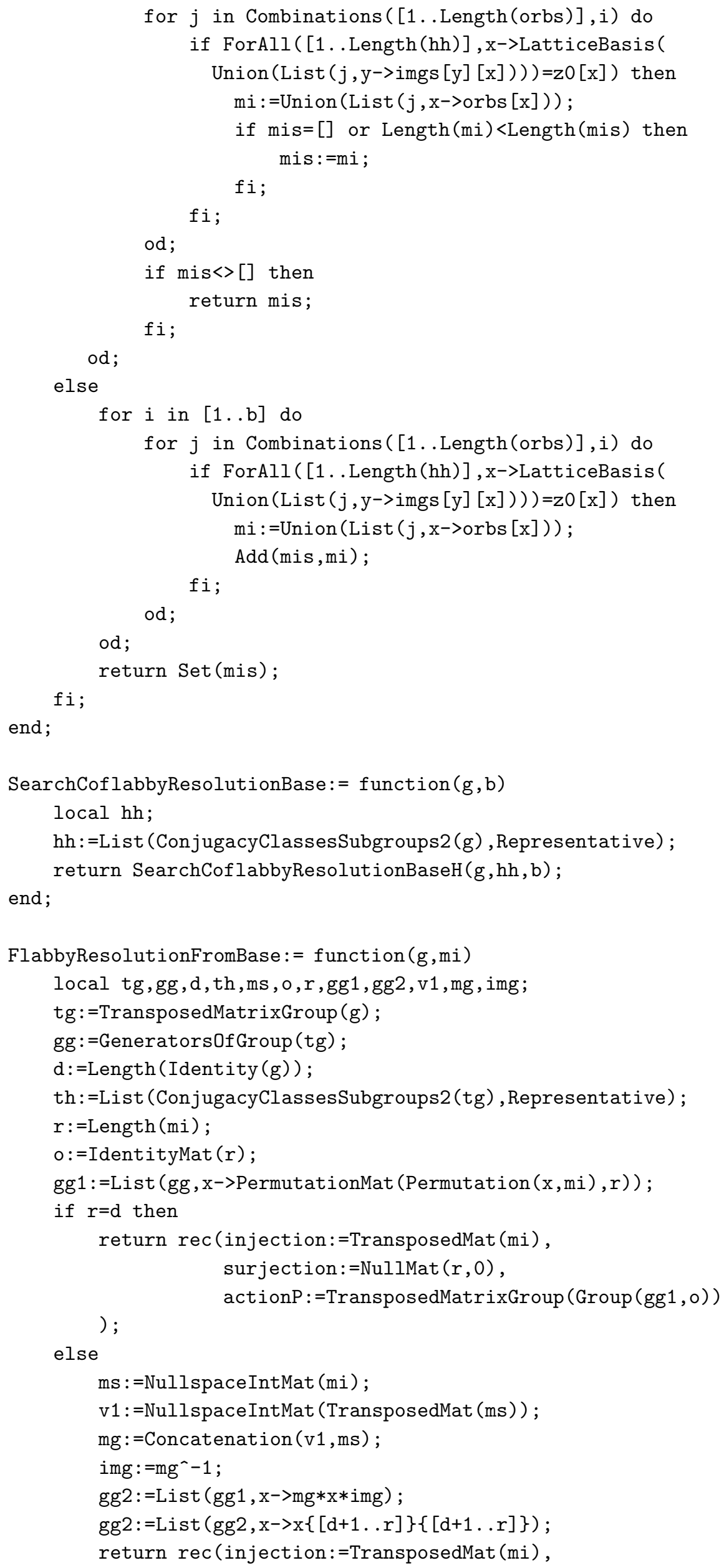




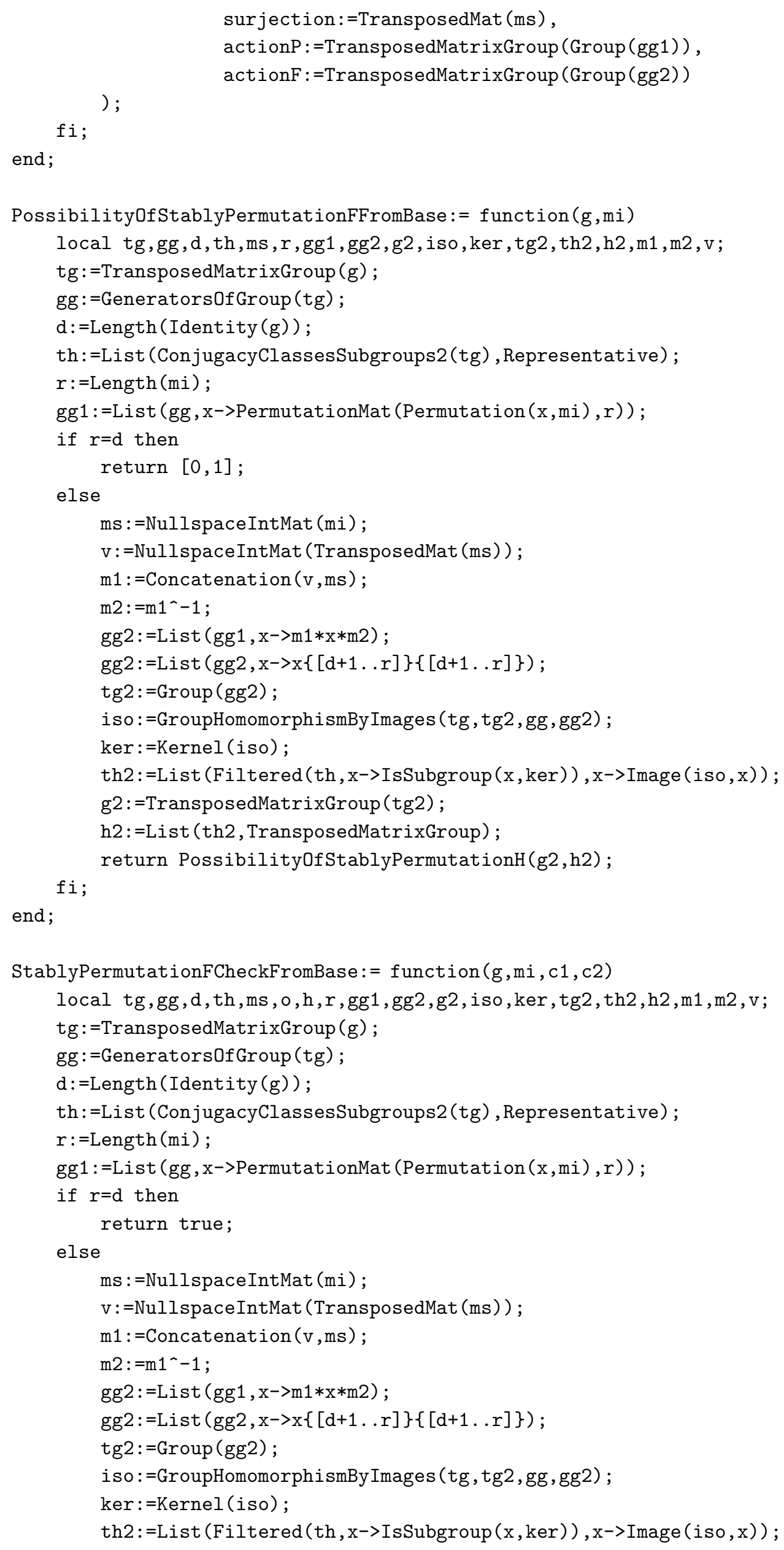


g2:=TransposedMatrixGroup(tg2);

h2:=List (th2, TransposedMatrixGroup);

return StablyPermutationCheckH (g2,h2,c1,c2);

$\mathrm{fi}$;

end;

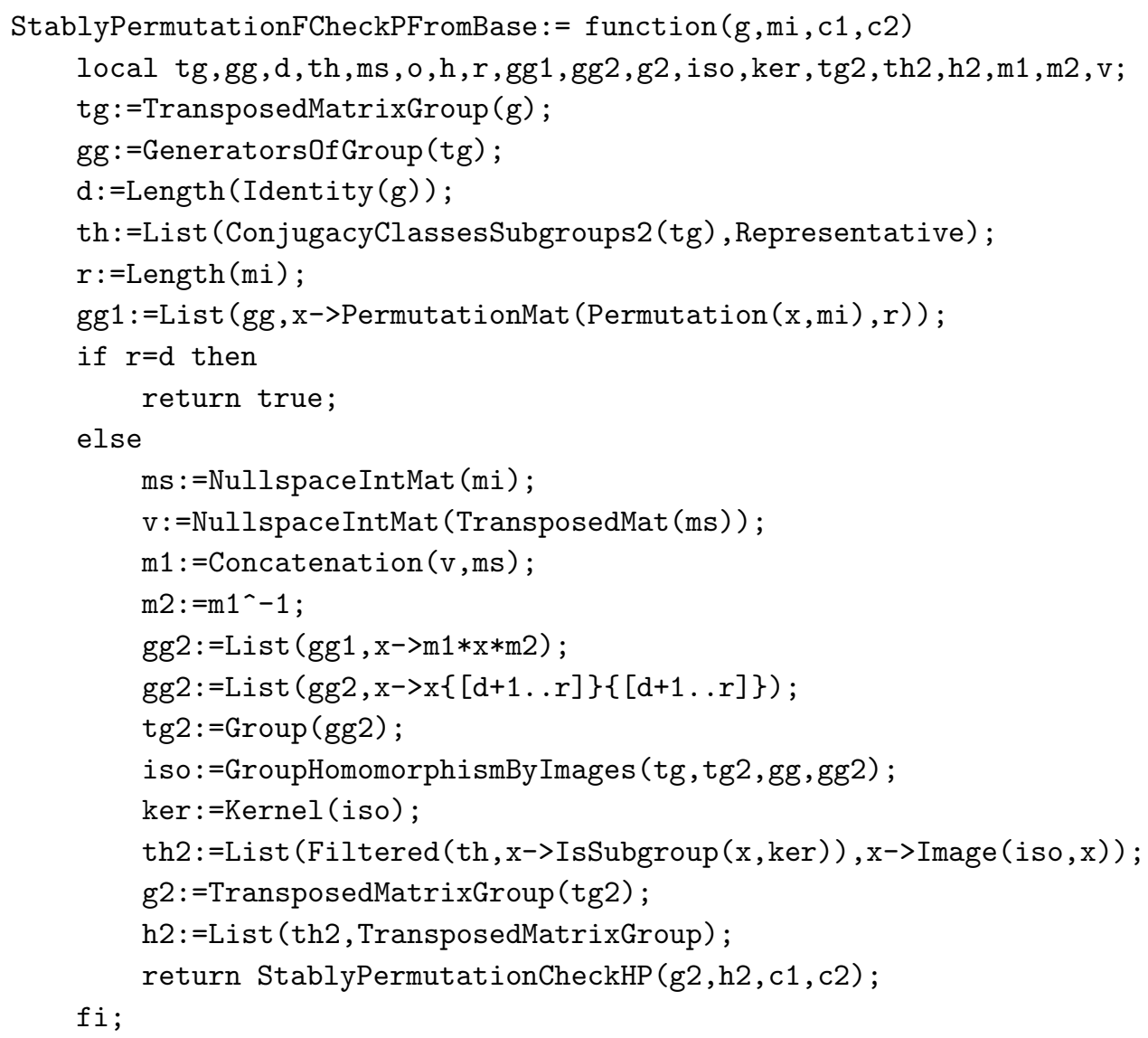




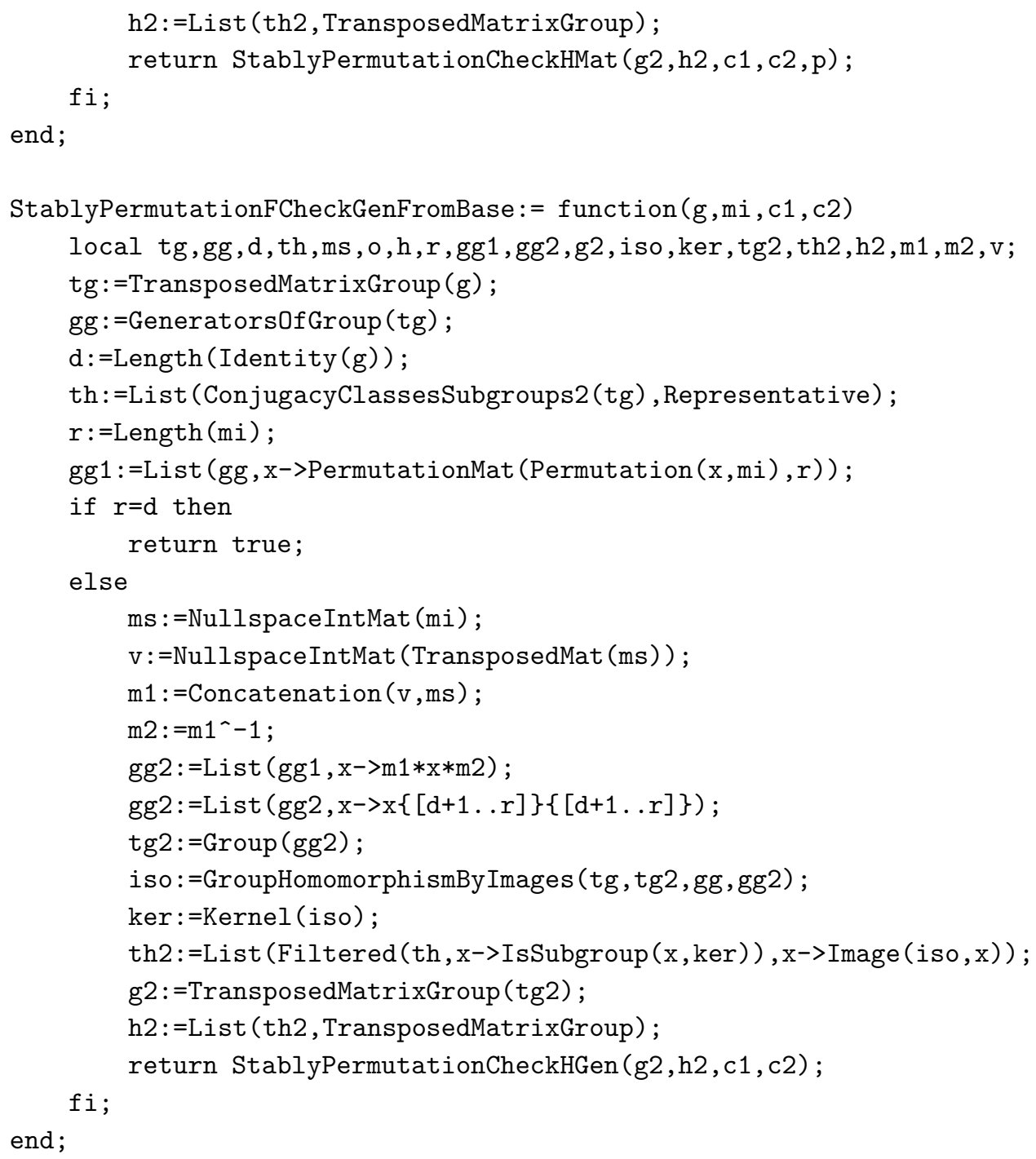

Example 5.8 (Algorithm F7, Method III). Let $M_{G}$ be the $G$-lattice where $G \simeq C_{2} \times S_{4}$ is the group of order 48 of the CARAT ID $(5,533,8)$. By using FlabbyResolution(G) actionF as in Algorithm F1, we obtain a flabby resolution $0 \rightarrow M_{G} \rightarrow P \rightarrow F \rightarrow 0$ of $M_{G}$. However, the flabby $G$-lattice $F$ is of rank 44 .

By FlabbyResolutionFromBase(G,mi).actionF, we get a suitable flabby resolution of $M_{G}: 0 \rightarrow M_{G} \rightarrow$ $P^{\prime} \rightarrow F^{\prime} \rightarrow 0$ where the flabby $G$-lattice $F^{\prime}$ is of rank 10 .

By using PossibilityOfStablyPermutationf(G), it may be possible that the isomorphism $\mathbb{Z}\left[G / H_{20}\right] \oplus$ $\mathbb{Z}\left[G / H_{22}\right] \oplus \mathbb{Z} \simeq \mathbb{Z}\left[G / H_{29}\right] \oplus F^{\prime}$ occurs where $H_{20} \simeq C_{2}^{3}, H_{22} \simeq D_{4}$ and $H_{29} \simeq C_{2} \times D_{4}$.

In order to confirm the isomorphism, we will use Mersenne Twister (cf. [MN98]) to search an appropriate coefficients $c_{i}$ to get a transformation matrix $P=\sum_{i} c_{i} P_{i}$ which satisfies $G_{1} P=P G_{2}$ as in (10) (one can use the classical global random generator via IsGlobalRandomSource which is given in [Knu98, Algorithm A in 3.2.2 with lag 30]). We should choose suitable integers $n_{1}, n_{2}, n_{3}$ in

$$
\text { rr: }=\operatorname{List}([1 \ldots \mathrm{n} 1], \mathrm{x}->\operatorname{List}([1 \ldots 20], \mathrm{y}->\operatorname{Random}(\mathrm{rs},[\mathrm{n} 2 \ldots \mathrm{n} 3])))
$$

to get the desired coefficients.

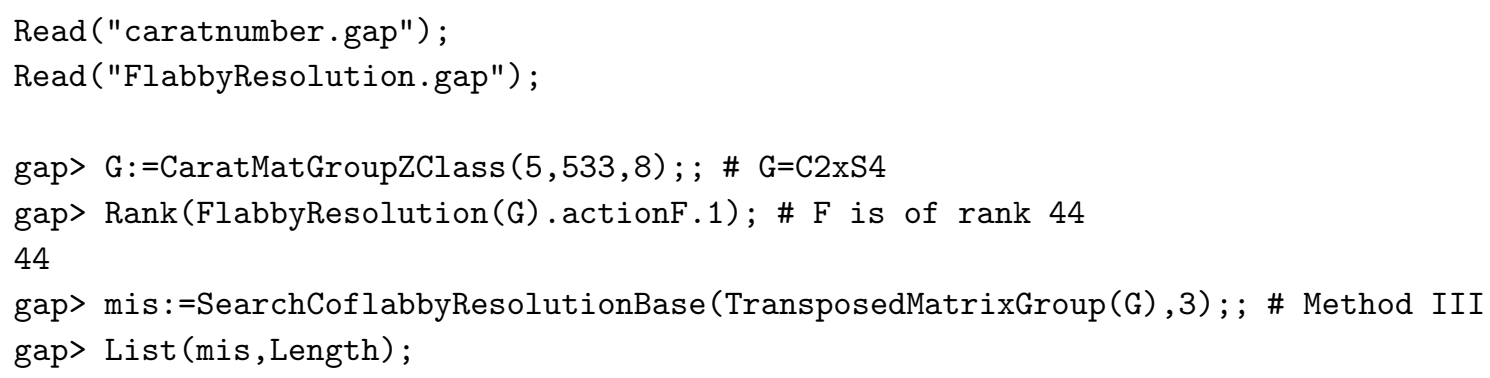


$[29,29,15,15,15,15]$

gap> mi : =mis [3] ;

$[[-1,-1,0,-1,0],[-1,-1,0,0,-1],[0,-1,0,-1,0]$,

$[0,-1,0,0,-1],[0,-1,1,-1,0],[0,-1,1,0,-1]$,

$[0,0,0,-1,0],[0,0,0,-1,1],[0,0,0,0,-1]$,

$[0,0,0,1,-1],[0,0,1,-1,0],[0,0,1,0,-1]$,

$[0,1,-1,1,1],[1,0,1,-1,0],[1,0,1,0,-1]]$

gap> FF:=FlabbyResolutionFromBase $(\mathrm{G}, \mathrm{mi})$. actionF ;

<matrix group with 2 generators>

gap> Rank(FF.1); \# FF is of rank $10(=15-5)$

10

gap> $11:=$ PossibilityOfStablyPermutationFFromBase (G,mi);

$[[1,0,0,0,0,-2,-1,0,0,0,0,0,0,0,0,0,0,0,2,0,0,1,0,0$,

$1,-1,0,0,1,1,-1,1,-1,-1]$,

$[0,1,0,0,0,0,0,0,0,0,0,0,0,0,0,-2,0,-1,0,0,0,1,0,0$,

$0,0,0,2,1,1,0,0,-1,-1]$,

$[0,0,0,0,1,-1,0,0,0,0,0,0,0,0,0,0,-1,0,1,0,0,0,0,0$, $1,-1,0,0,0,0,-1,1,0,0]$,

$[0,0,0,0,0,0,0,1,0,0,0,0,0,0,0,0,0,0,0,0,0,1,0,0$, $-1,-1,-1,0,1,1,1,1,-1,-1]$,

$[0,0,0,0,0,0,0,0,0,0,0,0,0,0,0,0,0,0,0,1,0,1,0,0$, $0,0,0,0,-1,0,0,0,1,-1]]$

gap> $1:=11[$ Length $(11)]$;

$[0,0,0,0,0,0,0,0,0,0,0,0,0,0,0,0,0,0,0,1$,

$0,1,0,0,0,0,0,0,-1,0,0,0,1,-1]$

gap> Length $(1)$;

34

gap> $[1[20], 1[22], 1[33], 1[29], 1[34]]$;

$[1,1,1,-1,-1]$

gap> ss:=List (ConjugacyClassesSubgroups2(G), $\mathrm{x}$->StructureDescription(Representative(x)));

[ "1", "C2", "C2", "C2", "C2", "C2", "C3", "C2 x C2", "C2 x C2", "C2 x C2", "C2 x C2",

"C2 x C2", "C4", "C4", "C2 x C2", "C2 x C2", "C6", "S3", "S3", "C2 x C2 x C2", "D8",

"D8", "C2 x C2 x C2", "C4 x C2", "D8", "D8", "A4", "D12", "C2 x D8", "C2 x A4",

"S4", "S4", "C2 x S4" ]

gap> [ss [20], ss [22], ss [33], ss [29]];

[ "C2 x C2 x C2", "D8", "C2 x S4", "C2 x D8" ]

gap> bp:=StablyPermutationFCheckPFromBase (G,mi,Nlist(1),Plist(1)); ;

gap> Length (bp);

20

gap> Length(bp[1]); \# rank of the both sides of (10) is 13

13

gap> rs:=RandomSource (IsMersenneTwister);

$<$ RandomSource in IsMersenneTwister>

gap> rr:=List ([1 . 1000], $\mathrm{x}->\operatorname{List}([1 \ldots 20], \mathrm{y}->\operatorname{Random}(\mathrm{rs},[0 \ldots 1])))$; ;

gap> Filtered $(r r, x->$ Determinant $(x * b p) \wedge 2=1)$; \# MT found 3 solutions

[ $[1,1,0,1,1,1,1,1,0,1,1,1,1,1,0,1,1,0,1,1]$,

$[0,1,1,0,0,1,1,0,1,0,0,1,1,1,0,1,1,0,1,1]$,

$[0,1,1,1,1,1,1,1,1,1,1,0,1,0,1,1,1,1,1,1]]$

gap> p: =last $[1] * \mathrm{bp}$;

$[[1,1,1,1,0,0,1,1,1,1,1,1,1]$,

$[0,1,1,0,1,1,1,1,1,1,1,1,1]$,

$[1,0,0,1,1,1,1,1,1,1,1,1,1]$,

$[1,1,0,1,1,1,1,1,1,0,1,1,1]$,

$[0,1,1,1,1,1,1,1,0,1,1,1,1]$,

$[1,1,1,1,0,1,1,1,1,1,1,0,1]$, 


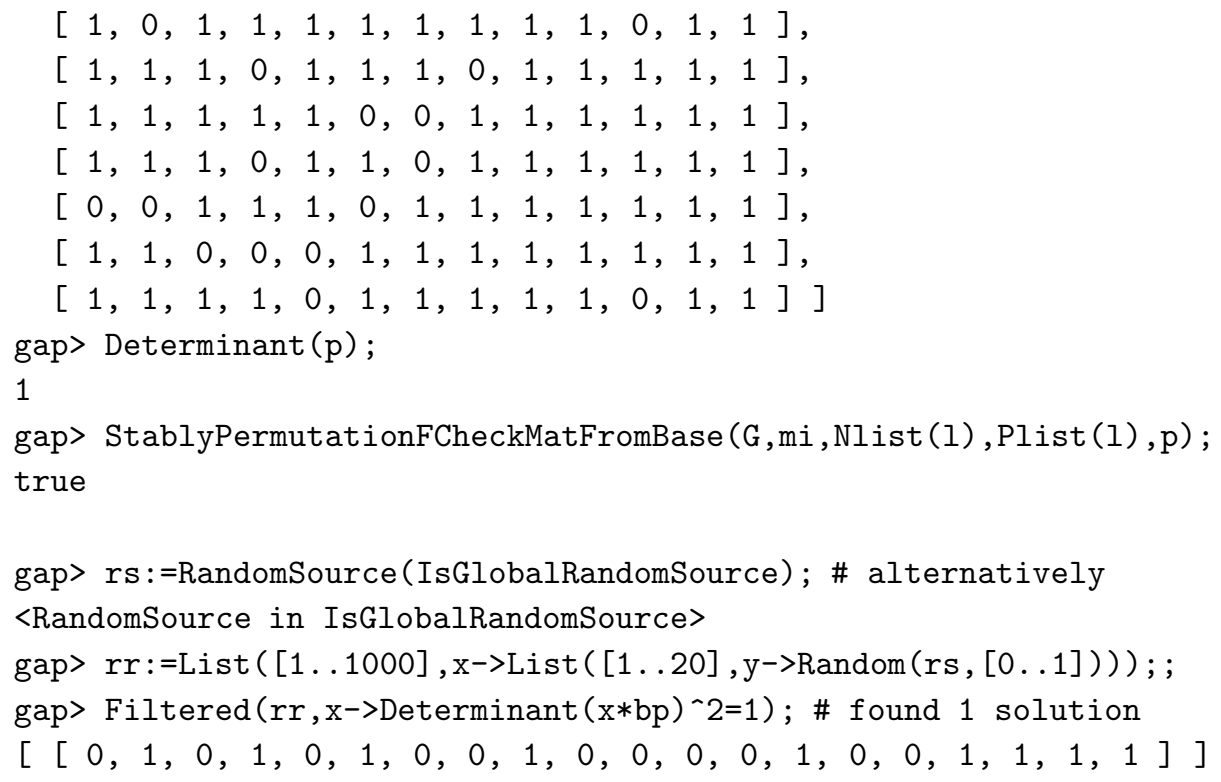

\section{Flabby and Coflabby $G$-Lattices}

In this section, we will determine all the flabby and coflabby $G$-lattices $M$ of rank $M \leq 6$ by using the algorithms IsFlabby and IsCoflabby which are given in Section 5 .

First we make a list of all $\mathbb{Z}$-class of $\mathrm{GL}(n, \mathbb{Z})$ and we filter off the groups $G$ such that $H^{-1}(G) \neq 0$ or $H^{1}(G) \neq 0$ form the list. Next we use the derived subgroup $D(G)=[G, G]$, the center $Z(G)$ and a 2-Sylow subgroup $S y_{2}(G)$ of $G$ to filter the groups off. Finally we filter off the groups which are not flabby or not coflabby. Because IsFlabby(G) and IsCoflabby(G) are much slower than Hminus1(G) and H1(G), we use Hminus (G) and H1(G) to the specified subgroups of $G$ as above. The following algorithms are available from http://math.h.kyoto-u.ac.jp/〜yamasaki/Algorithm/ as KS.gap.

AllFlabbyCoflabbyZClasses (n) returns the list of all the GAP IDs of $G$ such that $M_{G}$ is a flabby and coflabby $G$-lattice of rank $n$ when $2 \leq n \leq 4$.

AllFlabbyCoflabbyZClasses(n:Carat) returns the same as AllFlabbyCoflabbyZClasses(n) but using the CARAT ID instead of the GAP ID when $1 \leq n \leq 6$.

AllPermutationZClasses (n) returns the list of all the GAP IDs of $G$ such that $M_{G}$ is a permutation $G$-lattice when $2 \leq n \leq 4$.

AllPermutationZClasses ( $\mathrm{n}$ : Carat) returns the same as AllPermutation(n) but using the CARAT ID instead of the GAP ID when $1 \leq n \leq 6$.

Algorithm FC (Flabby and coflabby $G$-lattices).

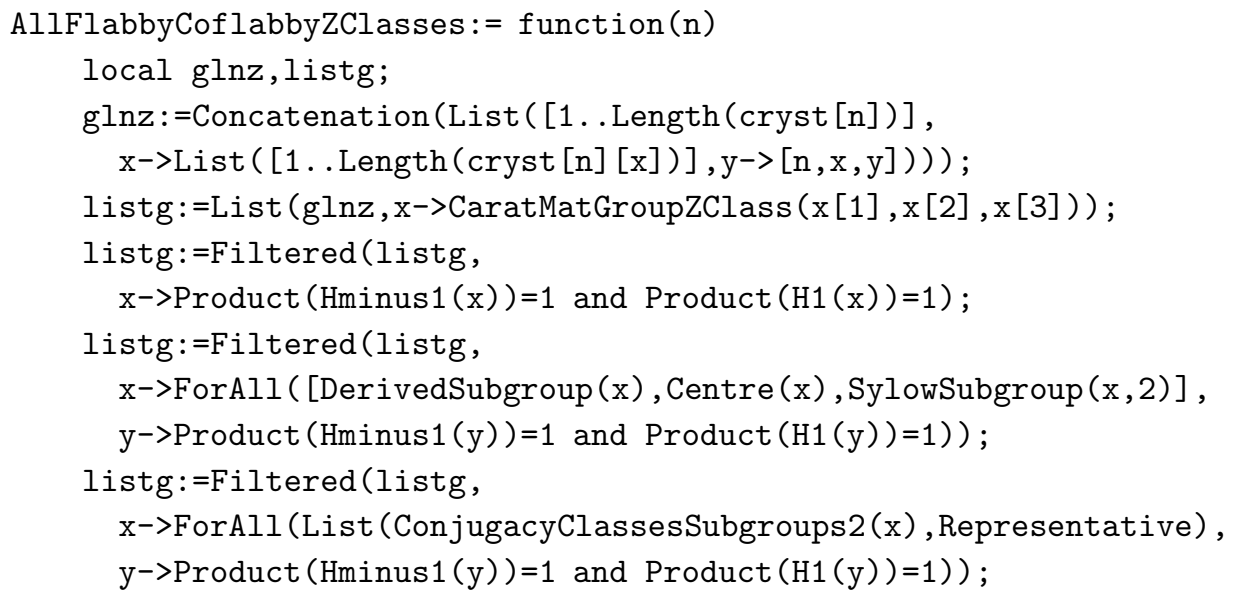




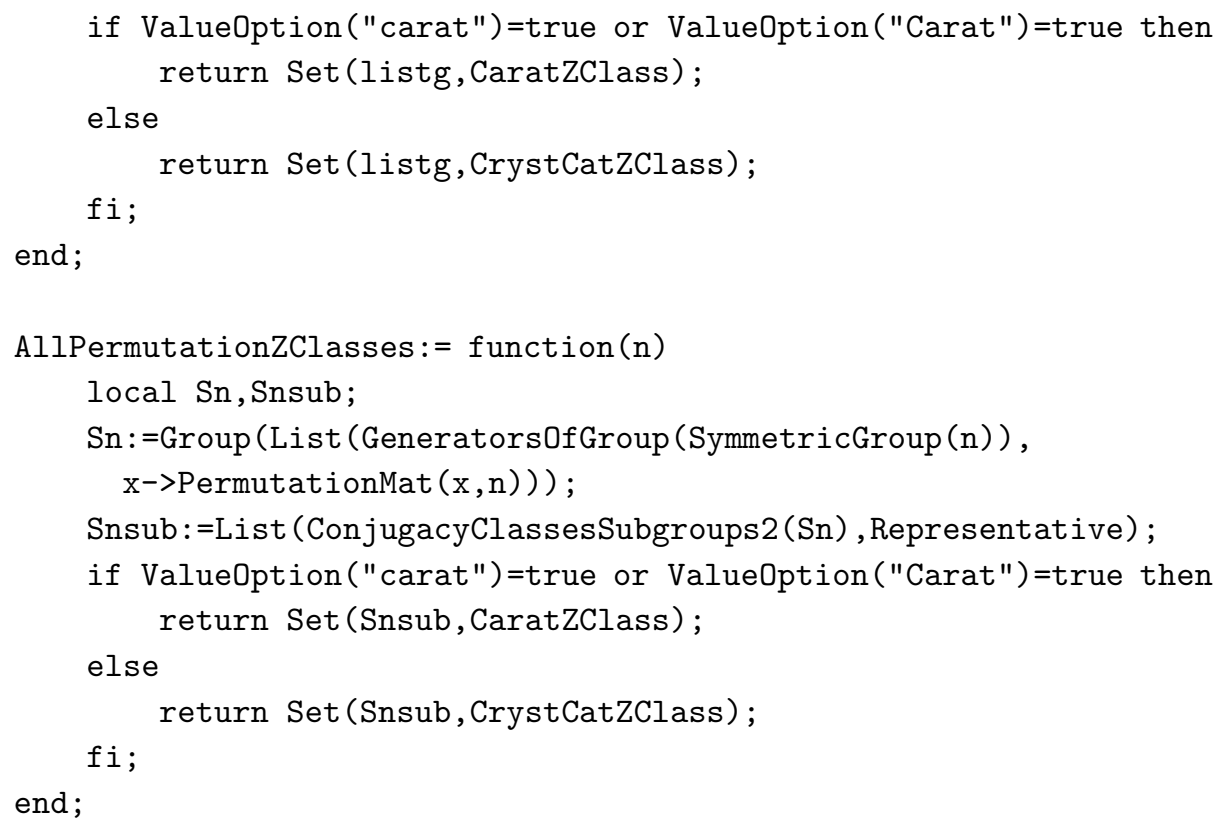

Example 6.1 (All flabby and coflabby $G$-lattices of rank $n \leq 6$ which are not permutation). We may compute all the permutation $G$-lattices of rank $n \leq 6$ and all the flabby and coflabby $G$-lattices by using Algorithm 6 ,

There exist 2 (resp. 4, 11, 19, 56) permutation $G$-lattices of rank 2 (resp. 3, 4, 5, 6). All the permutation $G$-lattices of rank $2 \leq n \leq 5$ are given as follows:

\begin{tabular}{l|lllllll}
$G$ : permutation & $\{1\}$ & $C_{2}$ & & & \\
\\
\cline { 1 - 3 } GAP ID & $(2,1,1,1)$ & $(2,2,1,2)$ & & & \\
$G$ : permutation & $\{1\}$ & $C_{2}$ & $C_{3}$ & $S_{3}$ & & \\
\cline { 1 - 3 } GAP ID & $(3,1,1,1)$ & $(3,2,2,2)$ & $(3,5,1,1)$ & $(3,5,4,1)$ & & \\
$G$ : permutation & $\{1\}$ & $C_{2}$ & $C_{2}$ & $C_{2}^{2}$ & $C_{2}^{2}$ & $C_{3}$ \\
\hline GAP ID & $(4,1,1,1)$ & $(4,2,1,2)$ & $(4,3,1,3)$ & $(4,4,1,5)$ & $(4,5,1,1)$ & $(4,8,1,1)$ \\
$G:$ permutation & $S_{3}$ & $C_{4}$ & $D_{4}$ & $A_{4}$ & $S_{4}$ & \\
\hline GAP ID & $(4,8,3,1)$ & $(4,12,1,1)$ & $(4,12,3,1)$ & $(4,24,1,1)$ & $(4,24,3,1)$ \\
$G:$ permutation & $\{1\}$ & $C_{2}$ & $C_{2}$ & $C_{2}^{2}$ & $C_{2}^{2}$ & $C_{4}$ & $D_{4}$ \\
\hline CARAT ID & $(5,1,1)$ & $(5,4,2)$ & $(5,7,3)$ & $(5,9,6)$ & $(5,19,18)$ & $(5,58,8)$ & $(5,62,8)$ \\
$G:$ permutation & $C_{3}$ & $S_{3}$ & $D_{6}$ & $S_{3}$ & $C_{6}$ & $A_{4}$ \\
\hline CARAT ID & $(5,181,2)$ & $(5,186,2)$ & $(5,192,6)$ & $(5,218,8)$ & $(5,220,4)$ & $(5,502,3)$ \\
$G:$ permutation & $C_{4}$ & $D_{5}$ & $C_{5}$ & $S_{5}$ & $F_{20}$ & $A_{5}$ \\
\hline CARAT ID & $(5,506,6)$ & $(5,901,3)$ & $(5,909,2)$ & $(5,911,3)$ & $(5,918,3)$ & $(5,931,3)$
\end{tabular}

Read ("caratnumber gap");

Read ("KS.gap") ;

gap> 12f:=AllFlabbyCoflabbyZClasses (2);

$[[2,1,1,1],[2,2,1,2]]$

gap> 12p:=AllPermutationZClasses (2);

$[[2,1,1,1],[2,2,1,2]]$

gap> 13f: =AllFlabbyCoflabbyZClasses (3);

$[[3,1,1,1],[3,2,2,2],[3,5,1,1],[3,5,4,1]]$

gap> 13p:=AllPermutationZClasses (3);

$[[3,1,1,1],[3,2,2,2],[3,5,1,1],[3,5,4,1]]$ 
$[[4,1,1,1],[4,2,1,2],[4,3,1,3],[4,4,1,5],[4,5,1,1]$,

$[4,8,1,1],[4,8,3,1],[4,12,1,1],[4,12,3,1],[4,14,2,2]$,

$[4,14,3,3],[4,14,3,4],[4,14,8,2],[4,24,1,1],[4,24,3,1]]$ gap> Length(14f);

15

gap> 14p:=AllPermutationZClasses (4); ;

gap> Length(14p);

11

gap> Difference $(14 f, 14 p)$;

$[[4,14,2,2],[4,14,3,3],[4,14,3,4],[4,14,8,2]]$

gap> Length(last);

4

gap> 15f: $=A l l F l$ abbyCoflabbyZClasses ( 5 :Carat);

$[[5,1,1],[5,4,2],[5,7,3],[5,9,6],[5,19,18]$,

$[5,58,8],[5,62,8],[5,181,2],[5,186,2],[5,192,6]$,

$[5,218,4],[5,218,8],[5,220,4],[5,502,3],[5,506,6]$,

$[5,901,3],[5,909,2],[5,911,3],[5,911,4],[5,918,3]$,

$[5,918,4],[5,931,3],[5,931,4]]$

gap> Length(15f);

23

gap> 15p:=AllPermutationZClasses (5:Carat); ;

gap> Length (15p);

19

gap> Difference $(15 f, 15 p)$;

$[[5,218,4],[5,911,4],[5,918,4],[5,931,4]]$

gap> Length(last);

4

gap> 16f: $=$ AllFlabbyCoflabbyZClasses $(6:$ Carat $)$;

$[[6,2,2],[6,4,3],[6,8,6],[6,11,4],[6,15,12]$,

$[6,159,14],[6,161,14],[6,161,28],[6,197,14],[6,226,14]$,

$[6,226,40],[6,231,39],[6,238,27],[6,246,21],[6,366,27]$,

$[6,891,7],[6,894,6],[6,927,9],[6,984,6],[6,993,16]$,

$[6,1087,20],[6,1090,18],[6,1142,8],[6,1199,16],[6,1968,3]$,

$[6,2007,2],[6,2010,3],[6,2026,6],[6,2043,4],[6,2043,8]$,

$[6,2044,4],[6,2051,9],[6,2068,6],[6,2069,6],[6,2069,12]$,

$[6,2070,12],[6,2079,14],[6,2079,28],[6,2088,18],[6,2105,12]$,

$[6,2154,26],[6,2156,40],[6,2156,80],[6,2188,39],[6,2263,6]$,

$[6,2278,8],[6,2709,1],[6,2958,3],[6,2966,2],[6,2968,4]$,

$[6,2969,4],[6,2969,8],[6,2977,6],[6,3046,3],[6,3053,5]$,

$[6,3066,3],[6,3068,7],[6,3068,8],[6,3071,7],[6,3071,8]$,

$[6,3073,7],[6,3073,8],[6,3073,15],[6,3073,16],[6,3076,7]$,

$[6,3076,8],[6,3091,11],[6,3091,12],[6,3276,9],[6,3297,9]$,

$[6,3299,9],[6,3302,9],[6,3575,8],[6,3662,8],[6,3663,12]$,

$[6,3749,10],[6,4618,18],[6,4618,19],[6,4621,18],[6,4630,52]$,

$[6,4647,101],[6,4722,8],[6,4733,8],[6,4743,13],[6,4750,13]$,

$[6,4762,41],[6,4807,41],[6,4811,41],[6,4814,82],[6,4898,3]$,

$[6,4904,3],[6,4915,20],[6,4919,20],[6,4929,11],[6,5210,8]$,

$[6,5210,14],[6,5262,11],[6,5311,16],[6,5318,8],[6,5321,6]$,

$[6,5321,14],[6,5421,6],[6,5424,16],[6,5475,6],[6,5477,11]$,

$[6,5487,11]]$

gap> Length(16f);

106

gap> 16p:=AllPermutationZClasses (6:Carat); 


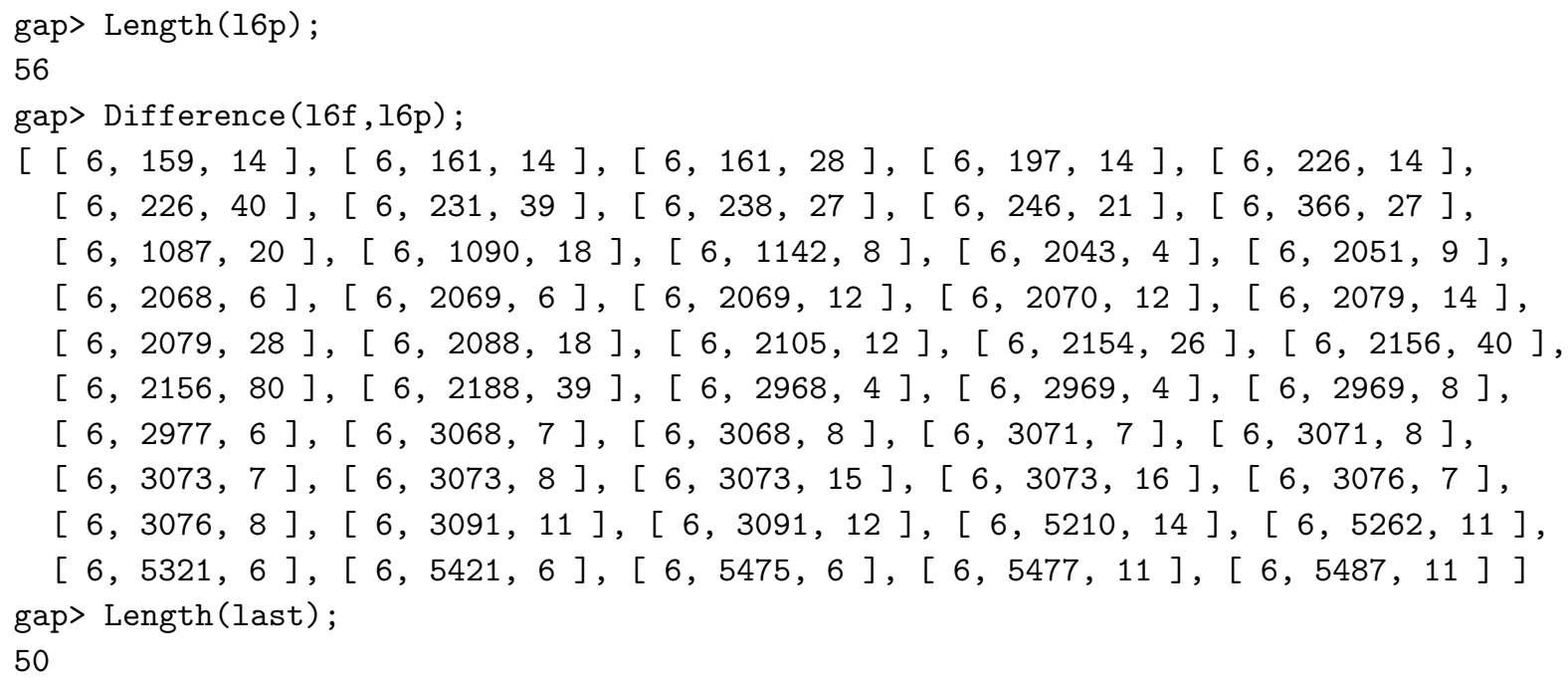

Theorem 6.2. Let $G$ be a finite subgroup of $\mathrm{GL}(n, \mathbb{Z})$ and $M_{G}$ be the $G$-lattice as in Definition 1.26 .

(i) When $n \leq 3, M_{G}$ is flabby and coflabby if and only if $M_{G}$ is permutation.

(ii) When $n=4, M_{G}$ is flabby and coflabby if and only if $M_{G}$ is permutation or the GAP ID of $G$ is one of $(4,14,2,2),(4,14,3,3),(4,14,3,4),(4,14,8,2)$.

(There are 11 conjugacy classes of subgroups of $S_{4}$ and hence 15 flabby and coflabby $G$-lattices of rank 4 in total.)

(iii) When $n=5, M_{G}$ is flabby and coflabby if and only if $M_{G}$ is permutation or the CARAT ID of $G$ is one of $(5,218,4),(5,911,4),(5,918,4),(5,931,4)$.

(There are 19 conjugacy classes of subgroups of $S_{5}$ and hence 23 flabby and coflabby $G$-lattices of rank 5 in total.) (iv) When $n=6, M_{G}$ is flabby and coflabby if and only if $M_{G}$ is permutation or the CARAT ID of $G$ is one of the 50 triples

$\begin{array}{lllll}(6,159,14), & (6,161,14), & (6,161,28), & (6,197,14), & (6,226,14), \\ (6,226,40), & (6,231,39), & (6,238,27), & (6,246,21), & (6,366,27), \\ (6,1087,20), & (6,1090,18), & (6,1142,8), & (6,2043,4), & (6,2051,9), \\ (6,2068,6), & (6,2069,6), & (6,2069,12), & (6,2070,12), & (6,2079,14), \\ (6,2079,28), & (6,2088,18), & (6,2105,12), & (6,2154,26), & (6,2156,40), \\ (6,2156,80), & (6,2188,39), & (6,2968,4), & (6,2969,4), & (6,2969,8), \\ (6,2977,6), & (6,3068,7), & (6,3068,8), & (6,3071,7), & (6,3071,8), \\ (6,3073,7), & (6,3073,8), & (6,3073,15), & (6,3073,16), & (6,3076,7), \\ (6,3076,8), & (6,3091,11), & (6,3091,12), & (6,5210,14), & (6,5262,11), \\ (6,5321,6), & (6,5421,6), & (6,5475,6), & (6,5477,11), & (6,5487,11) .\end{array}$

(There are 56 conjugacy classes of subgroups of $S_{6}$ and hence 106 flabby and coflabby G-lattices of rank 6 in total.)

By Theorem 1.20, when any $p$-Sylow subgroup of $G$ is cyclic for odd $p$ and cyclic or dihedral (including Klein's four group) for $p=2, G$-lattice $M$ is flabby and coflabby if and only if $M$ is invertible. Moreover, for rank $M \leq 6$, $M$ is flabby and coflabby if and only if $M$ is stably permutation.

Theorem 6.3. Let $G$ be a finite subgroup of $\operatorname{GL}(n, \mathbb{Z})$ and $M_{G}$ be the G-lattice as in Definition 1.26 . When $n \leq 6, M_{G}$ is flabby and coflabby if and only if $M_{G}$ is stably permutation. Indeed, flabby and coflabby G-lattices $M_{G}$ which are not permutation as in Theorem 6.2 are stably permutation as in Table 8. 
Table 8: flabby and coflabby $G$-lattices $M=M_{G}$ of rank $\leq 6$ which are not permutation

\begin{tabular}{|c|c|c|c|}
\hline GAP/CARAT ID & $G$ & $M$ is stably permutation, $H_{i}$ & is the $i$ th conjugacy class of subgroups of $G$ \\
\hline$(4,14,2,2)$ & $C_{6}$ & $M \oplus \mathbb{Z}$ & $\simeq \mathbb{Z}\left[G / H_{2}\right] \oplus \mathbb{Z}\left[G / H_{3}\right]$ \\
\hline$(4,14,3,3)$ & $S_{3}$ & $M \oplus \mathbb{Z}$ & $\mathbb{Z}\left[G / H_{2}\right] \oplus \mathbb{Z}\left[G / H_{3}\right]$ \\
\hline$(4,14,3,4)$ & $S_{3}$ & $M \oplus \mathbb{Z}\left[G / H_{2}\right]$ & $\mathbb{Z}[G] \oplus \mathbb{Z}$ \\
\hline$(4,14,8,2)$ & $D_{6}$ & $M \oplus \mathbb{Z}$ & $\mathbb{Z}\left[G / H_{6}\right] \oplus \mathbb{Z}\left[G / H_{9}\right]$ \\
\hline$(5,218,4)$ & $S_{3}$ & $M \oplus \mathbb{Z}\left[G / H_{2}\right]$ & $\mathbb{Z}[G] \oplus \mathbb{Z}^{\oplus 2}$ \\
\hline$(5,911,4)$ & $S_{5}$ & $M \oplus \mathbb{Z}$ & $\mathbb{Z}\left[G / H_{17}\right] \oplus \mathbb{Z}$ \\
\hline$(5,918,4)$ & $F_{20}$ & $M \oplus \mathbb{Z}$ & $\mathbb{Z}\left[G / H_{3}\right] \oplus \mathbb{Z}$ \\
\hline$(5,931,4)$ & $A_{5}$ & $M \oplus \mathbb{Z}$ & $\mathbb{Z}\left[G / H_{8}\right] \oplus \mathbb{Z}$ \\
\hline$(6,159,14)$ & $C_{12}$ & $M \oplus \mathbb{Z}$ & $\mathbb{Z}\left[G / H_{3}\right] \oplus \mathbb{Z}\left[G / H_{4}\right]$ \\
\hline$(6,161,14)$ & $Q_{12}$ & $M \oplus \mathbb{Z}\left[G / H_{4}\right] \oplus \mathbb{Z}\left[G / H_{5}\right]$ & $\simeq \mathbb{Z}\left[G / H_{2}\right] \oplus \mathbb{Z}\left[G / H_{3}\right] \oplus \mathbb{Z}$ \\
\hline$(6,161,28)$ & $Q_{12}$ & $M \oplus \mathbb{Z}$ & $\mathbb{Z}\left[G / H_{3}\right] \oplus \mathbb{Z}\left[G / H_{4}\right]$ \\
\hline$(6,197,14)$ & $D_{4} \times C_{3}$ & $M \oplus \mathbb{Z}$ & $\mathbb{Z}\left[G / H_{10}\right] \oplus \mathbb{Z}\left[G / H_{12}\right]$ \\
\hline$(6,226,14)$ & $C_{3} \rtimes D_{4}$ & $M \oplus \mathbb{Z}\left[G / H_{12}\right] \oplus \mathbb{Z}\left[G / H_{13}\right]$ & $\mathbb{Z}\left[G / H_{6}\right] \oplus \mathbb{Z}\left[G / H_{10}\right] \oplus \mathbb{Z}$ \\
\hline$(6,226,40)$ & $C_{3} \rtimes D_{4}$ & $M \oplus \mathbb{Z}$ & $\mathbb{Z}\left[G / H_{10}\right] \oplus \mathbb{Z}\left[G / H_{12}\right]$ \\
\hline$(6,231,39)$ & $D_{12}$ & $M \oplus \mathbb{Z}$ & $\mathbb{Z}\left[G / H_{10}\right] \oplus \mathbb{Z}\left[G / H_{12}\right]$ \\
\hline$(6,238,27)$ & $C_{3} \rtimes D_{4}$ & $M \oplus \mathbb{Z}$ & $\mathbb{Z}\left[G / H_{11}\right] \oplus \mathbb{Z}\left[G / H_{12}\right]$ \\
\hline$(6,246,21)$ & $S_{3} \times C_{4}$ & $M \oplus \mathbb{Z}$ & $\mathbb{Z}\left[G / H_{11}\right] \oplus \mathbb{Z}\left[G / H_{12}\right]$ \\
\hline$(6,366,27)$ & $D_{4} \times S_{3}$ & $M \oplus \mathbb{Z}$ & $\mathbb{Z}\left[G / H_{43}\right] \oplus \mathbb{Z}\left[G / H_{46}\right]$ \\
\hline$(6,1087,20)$ & $S_{3} \times C_{3}$ & $M \oplus \mathbb{Z}\left[G / H_{7}\right]$ & $\mathbb{Z}\left[G / H_{4}\right] \oplus \mathbb{Z}\left[G / H_{6}\right]$ \\
\hline$(6,1090,18)$ & $C_{3}^{2} \rtimes C_{2}$ & $M \oplus \mathbb{Z}\left[G / H_{9}\right]$ & $\mathbb{Z}\left[G / H_{5}\right] \oplus \mathbb{Z}\left[G / H_{10}\right]$ \\
\hline$(6,1142,8)$ & $S_{3} \times S_{3}$ & $M \oplus \mathbb{Z}\left[G / H_{17}\right]$ & $\mathbb{Z}\left[G / H_{10}\right] \oplus \mathbb{Z}\left[G / H_{18}\right]$ \\
\hline$(6,2043,4)$ & $S_{3}$ & $M \oplus \mathbb{Z}\left[G / H_{2}\right]$ & $\mathbb{Z}[G] \oplus \mathbb{Z}^{\oplus 3}$ \\
\hline$(6,2051,9)$ & $D_{6}$ & $M \oplus \mathbb{Z}$ & $\mathbb{Z}\left[G / H_{6}\right] \oplus \mathbb{Z}\left[G / H_{9}\right]^{\oplus 2}$ \\
\hline$(6,2068,6)$ & $C_{6}$ & $M \oplus \mathbb{Z}$ & $\mathbb{Z}\left[G / H_{2}\right] \oplus \mathbb{Z}\left[G / H_{3}\right]^{\oplus 2}$ \\
\hline$(6,2069,6)$ & $S_{3}$ & $M \oplus \mathbb{Z}\left[G / H_{2}\right]$ & $\mathbb{Z}[G] \oplus \mathbb{Z}\left[G / H_{3}\right] \oplus \mathbb{Z}$ \\
\hline$(6,2069,12)$ & $S_{3}$ & $M \oplus \mathbb{Z}$ & $\mathbb{Z}\left[G / H_{2}\right] \oplus \mathbb{Z}\left[G / H_{3}\right]^{\oplus 2}$ \\
\hline$(6,2070,12)$ & $C_{6} \times C_{2}$ & $M \oplus \mathbb{Z}$ & $\mathbb{Z}\left[G / H_{6}\right] \oplus \mathbb{Z}\left[G / H_{8}\right] \oplus \mathbb{Z}\left[G / H_{9}\right]$ \\
\hline$(6,2079,14)$ & $D_{6}$ & $M \oplus \mathbb{Z}\left[G / H_{6}\right]$ & $\mathbb{Z}\left[G / H_{2}\right] \oplus \mathbb{Z}\left[G / H_{9}\right] \oplus \mathbb{Z}$ \\
\hline$(6,2079,28)$ & $D_{6}$ & $M \oplus \mathbb{Z}$ & $\mathbb{Z}\left[G / H_{6}\right] \oplus \mathbb{Z}\left[G / H_{7}\right] \oplus \mathbb{Z}\left[G / H_{9}\right]$ \\
\hline$(6,2088,18)$ & $D_{6}$ & $M \oplus \mathbb{Z}$ & $\simeq \mathbb{Z}\left[G / H_{6}\right] \oplus \mathbb{Z}\left[G / H_{8}\right] \oplus \mathbb{Z}\left[G / H_{9}\right]$ \\
\hline$(6,2105,12)$ & $D_{6} \times C_{2}$ & $M \oplus \mathbb{Z}$ & $\simeq \mathbb{Z}\left[G / H_{24}\right] \oplus \mathbb{Z}\left[G / H_{29}\right] \oplus \mathbb{Z}\left[G / H_{31}\right]$ \\
\hline$(6,2154,26)$ & $C_{6} \times C_{2}$ & $M \oplus \mathbb{Z}$ & $\mathbb{Z}\left[G / H_{5}\right] \oplus \mathbb{Z}\left[G / H_{6}\right]$ \\
\hline$(6,2156,40)$ & $D_{6}$ & $M \oplus \mathbb{Z}\left[G / H_{6}\right] \oplus \mathbb{Z}\left[G / H_{9}\right]$ & $\simeq \mathbb{Z}\left[G / H_{2}\right] \oplus \mathbb{Z}\left[G / H_{5}\right] \oplus \mathbb{Z}$ \\
\hline$(6,2156,80)$ & $D_{6}$ & $M \oplus \mathbb{Z}$ & $\mathbb{Z}\left[G / H_{5}\right] \oplus \mathbb{Z}\left[G / H_{6}\right]$ \\
\hline$(6,2188,39)$ & $D_{6} \times C_{2}$ & $M \oplus \mathbb{Z}$ & $\mathbb{Z}\left[G / H_{19}\right] \oplus \mathbb{Z}\left[G / H_{24}\right]$ \\
\hline$(6,2968,4)$ & $C_{10}$ & $M \oplus \mathbb{Z}$ & $\simeq \mathbb{Z}\left[G / H_{2}\right] \oplus \mathbb{Z}\left[G / H_{3}\right]$ \\
\hline$(6,2969,4)$ & $D_{5}$ & $M \oplus \mathbb{Z}$ & $\mathbb{Z}\left[G / H_{2}\right] \oplus \mathbb{Z}\left[G / H_{3}\right]$ \\
\hline$(6,2969,8)$ & $D_{5}$ & $M \oplus \mathbb{Z}\left[G / H_{2}\right]$ & $\mathbb{Z}[G] \oplus \mathbb{Z}$ \\
\hline$(6,2977,6)$ & $D_{10}$ & $M \oplus \mathbb{Z}$ & $\mathbb{Z}\left[G / H_{5}\right] \oplus \mathbb{Z}\left[G / H_{8}\right]$ \\
\hline$(6,3068,7)$ & $A_{5} \times C_{2}$ & $M \oplus \mathbb{Z}$ & $\simeq \mathbb{Z}\left[G / H_{20}\right] \oplus \mathbb{Z}\left[G / H_{21}\right]$ \\
\hline$(6,3068,8)$ & $A_{5} \times C_{2}$ & $M \oplus \mathbb{Z}$ & $\simeq \mathbb{Z}\left[G / H_{20}\right] \oplus \mathbb{Z}\left[G / H_{21}\right]$ \\
\hline$(6,3071,7)$ & $S_{5}$ & $M \oplus \mathbb{Z}$ & $\simeq \mathbb{Z}\left[G / H_{17}\right] \oplus \mathbb{Z}\left[G / H_{18}\right]$ \\
\hline$(6,3071,8)$ & $S_{5}$ & $M \oplus \mathbb{Z}$ & $\simeq \mathbb{Z}\left[G / H_{17}\right] \oplus \mathbb{Z}\left[G / H_{18}\right]$ \\
\hline$(6,3073,7)$ & $F_{20}$ & $M \oplus \mathbb{Z}$ & $\simeq \mathbb{Z}\left[G / H_{3}\right] \oplus \mathbb{Z}\left[G / H_{5}\right]$ \\
\hline$(6,3073,8)$ & $F_{20}$ & $M \oplus \mathbb{Z}$ & $\mathbb{Z}\left[G / H_{3}\right] \oplus \mathbb{Z}\left[G / H_{5}\right]$ \\
\hline$(6,3073,15)$ & $F_{20}$ & $M \oplus \mathbb{Z}\left[G / H_{3}\right]$ & $\simeq \mathbb{Z}\left[G / H_{2}\right] \oplus \mathbb{Z}$ \\
\hline$(6,3073,16)$ & $F_{20}$ & $M \oplus \mathbb{Z}\left[G / H_{3}\right]$ & $\simeq \mathbb{Z}\left[G / H_{2}\right] \oplus \mathbb{Z}$ \\
\hline$(6,3076,7)$ & $S_{5} \times C_{2}$ & $M \oplus \mathbb{Z}$ & $\simeq \mathbb{Z}\left[G / H_{52}\right] \oplus \mathbb{Z}\left[G / H_{56}\right]$ \\
\hline$(6,3076,8)$ & $S_{5} \times C_{2}$ & $M \oplus \mathbb{Z}$ & $\simeq \mathbb{Z}\left[G / H_{52}\right] \oplus \mathbb{Z}\left[G / H_{56}\right]$ \\
\hline$(6,3091,11)$ & $F_{20} \times C_{2}$ & $M \oplus \mathbb{Z}$ & $\simeq \mathbb{Z}\left[G / H_{9}\right] \oplus \mathbb{Z}\left[G / H_{15}\right]$ \\
\hline$(6,3091,12)$ & $F_{20} \times C_{2}$ & $M \oplus \mathbb{Z}$ & $\simeq \mathbb{Z}\left[G / H_{9}\right] \oplus \mathbb{Z}\left[G / H_{15}\right]$ \\
\hline$(6,5210,14)$ & $A_{4}$ & $M \oplus \mathbb{Z}$ & $\simeq \mathbb{Z}\left[G / H_{3}\right] \oplus \mathbb{Z}\left[G / H_{4}\right]$ \\
\hline$(6,5262,11)$ & $S_{4} \times S_{3}$ & $M \oplus \mathbb{Z}$ & $\simeq \mathbb{Z}\left[G / H_{64}\right] \oplus \mathbb{Z}\left[G / H_{65}\right]$ \\
\hline$(6,5321,6)$ & $S_{4}$ & $M \oplus \mathbb{Z}$ & $\simeq \mathbb{Z}\left[G / H_{8}\right] \oplus \mathbb{Z}\left[G / H_{9}\right]$ \\
\hline$(6,5421,6)$ & $A_{4} \times C_{3}$ & $M \oplus \mathbb{Z}$ & $\simeq \mathbb{Z}\left[G / H_{9}\right] \oplus \mathbb{Z}\left[G / H_{13}\right]$ \\
\hline$(6,5475,6)$ & $S_{4} \times C_{3}$ & $M \oplus \mathbb{Z}$ & $\simeq \mathbb{Z}\left[G / H_{20}\right] \oplus \mathbb{Z}\left[G / H_{21}\right]$ \\
\hline$(6,5477,11)$ & $A_{4} \times S_{3}$ & $M \oplus \mathbb{Z}$ & $\simeq \mathbb{Z}\left[G / H_{22}\right] \oplus \mathbb{Z}\left[G / H_{24}\right]$ \\
\hline$(6,5487,11)$ & $\left(A_{4} \times A_{3}\right) \rtimes C_{2}$ & $M \oplus \mathbb{Z}$ & $\mathbb{Z}\left[G / H_{24}\right] \oplus \mathbb{Z}\left[G / H_{28}\right]$ \\
\hline
\end{tabular}


Note that in Table 8, $H_{i}$ is the $i$-th conjugacy class of subgroups of $G$ which is determined by the function ConjugacyClassesSubgroups2 in GAP (see Section 4).

All cases can be done by using Method I in Section 5.5 and Method II in Section 5.6. We give the following 3 typical examples instead of the full proof of Theorem 6.3

Example 6.4 (Method I (1)). We use PossibilityOfStablyPermutationM as in Algorithm F4 to get a possibility of the isomorphism. Then we use StablyPermutationMCheck as in Algorithm F5. Method I to get the actual isomorphism.

Let $G \simeq C_{6}$ be the group of the GAP ID $(4,14,2,2)$ which is generated by the matrix $\sigma=\left(\begin{array}{cccc}0 & 1 & 0 & 1 \\ 1 & 0 & 0 & 1 \\ 0 & 0 & 0 & 1 \\ 0 & 0 & -1 & -1\end{array}\right)$. There exist 4 conjugacy classes of subgroups $\{1\}, H_{2}, H_{3}$ and $G$ of $G$ which are isomorphic to cyclic groups of order $1,2,3$ and 6 . Corresponding $G$-lattices $M_{G}$ are isomorphic to $\mathbb{Z}[G], \mathbb{Z}\left[G /\left\langle\sigma^{3}\right\rangle\right], \mathbb{Z}\left[G /\left\langle\sigma^{2}\right\rangle\right]$ and $\mathbb{Z}$ of rank $6,3,2$ and 1 respectively.

Let $G_{1}$ (resp. $G_{2}$ ) be the matrix representation group of the action of $G$ on $\mathbb{Z} \oplus M_{G}$ (resp. $\mathbb{Z}\left[G /\left\langle\sigma^{3}\right\rangle\right] \oplus$ $\mathbb{Z}\left[G /\left\langle\sigma^{2}\right\rangle\right]$. StablyPermutaionMCheck(G,Nlist(1), Plist(1)) shows that $G_{1} P=P G_{2}$ with the isomorphism

$$
\mathbb{Z} \oplus M_{G} \simeq \mathbb{Z}\left[G /\left\langle\sigma^{3}\right\rangle\right] \oplus \mathbb{Z}\left[G /\left\langle\sigma^{2}\right\rangle\right]
$$

where

$$
P=\left(\begin{array}{ccccc}
1 & 1 & 1 & 1 & 1 \\
0 & 0 & -1 & 0 & -1 \\
0 & 0 & -1 & -1 & 0 \\
1 & 0 & -1 & 0 & 0 \\
0 & -1 & 1 & 0 & 0
\end{array}\right)
$$

Similar examples for the groups $S_{3}, S_{3}$ and $D_{6}$ of the CARAT IDs $(4,14,3,3),(4,14,3,4)$ and $(4,14,8,2)$ are given below.

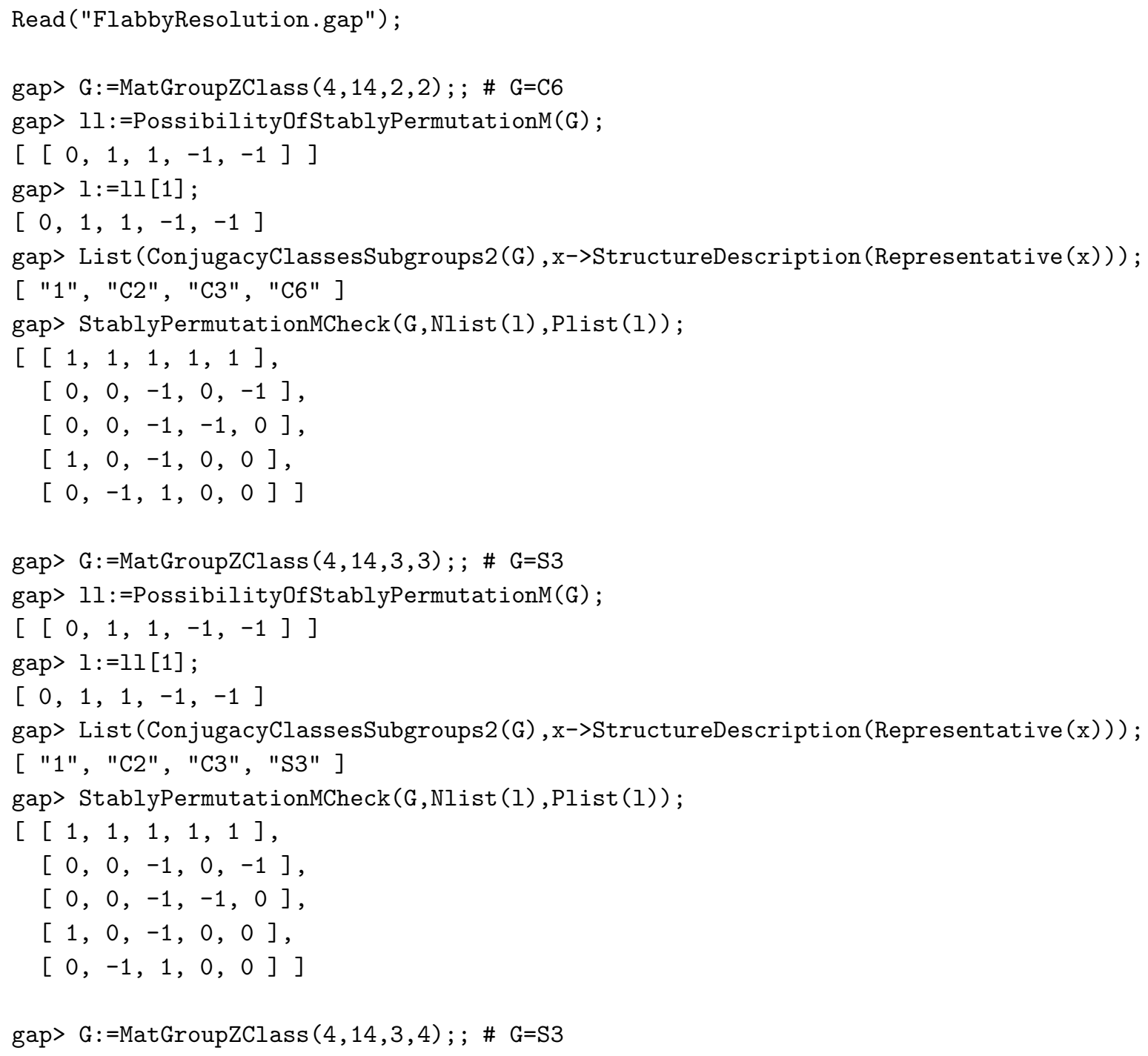




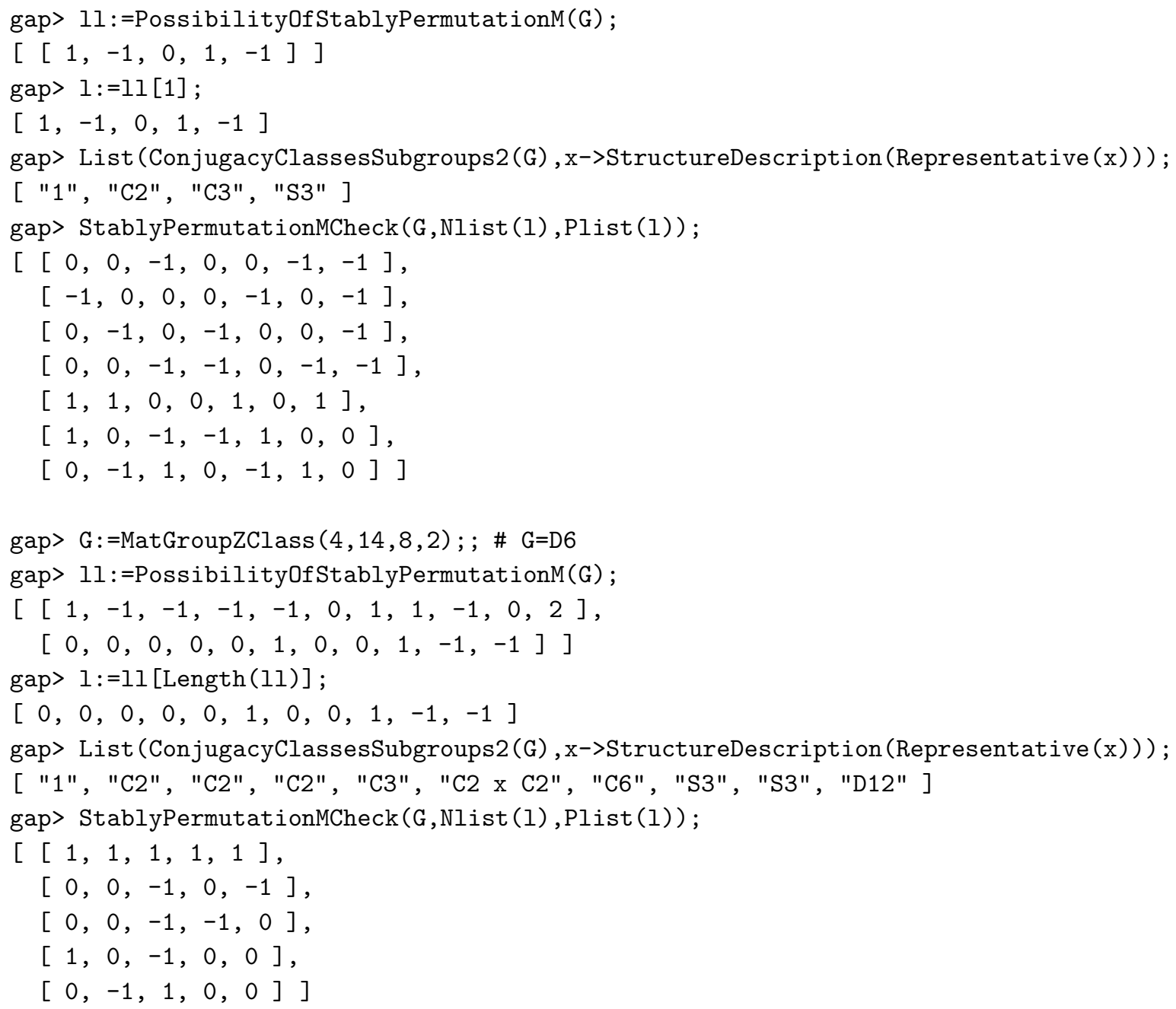

Example 6.5 (Method I (2)). Before applying StablePermutationMCheck in Method I, in some cases, we have to add some more $G$-lattices to make both hand side of $G$-lattices isomorphic. We will show that for the group $G \simeq S_{5}$ of the CARAT ID $(5,911,4), M_{G} \not{Z} \mathbb{Z}\left[S_{5} / S_{4}\right]$ but $M_{G} \oplus \mathbb{Z} \simeq \mathbb{Z}\left[S_{5} / S_{4}\right] \oplus \mathbb{Z}$.

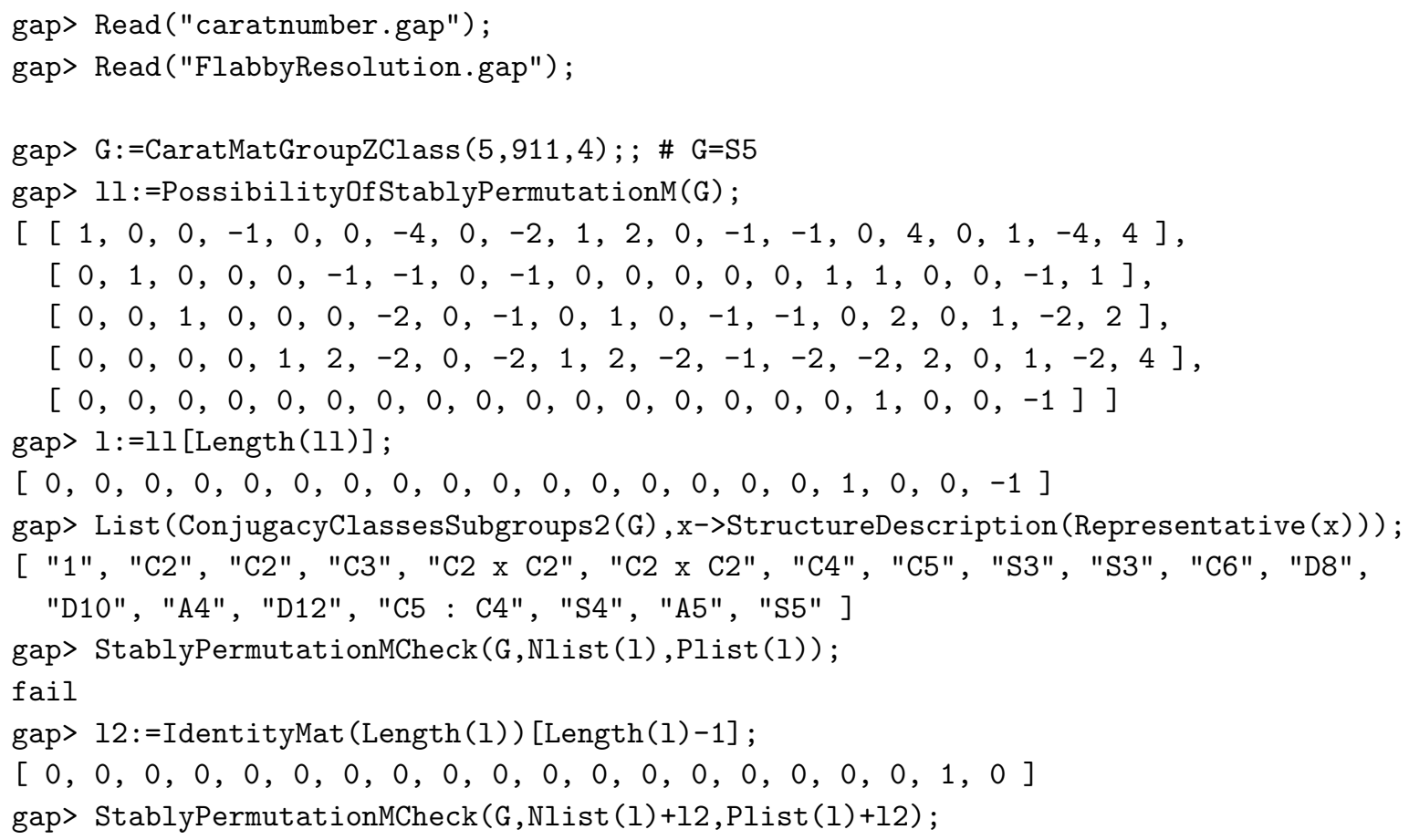


$[[2,2,2,2,2,3]$,

$[0,-1,0,-1,-1,-1]$,

$[0,1,-1,0,0,0]$,

$[1,1,0,0,1,1]$,

$[1,0,1,1,0,1]$,

$[-1,-2,-1,-1,-1,-2]]$

Example 6.6 (Method II). When StablyPermutationMCheck does not return any result in an appropriate time, we may use the command StablyPermutatinoCheakP.

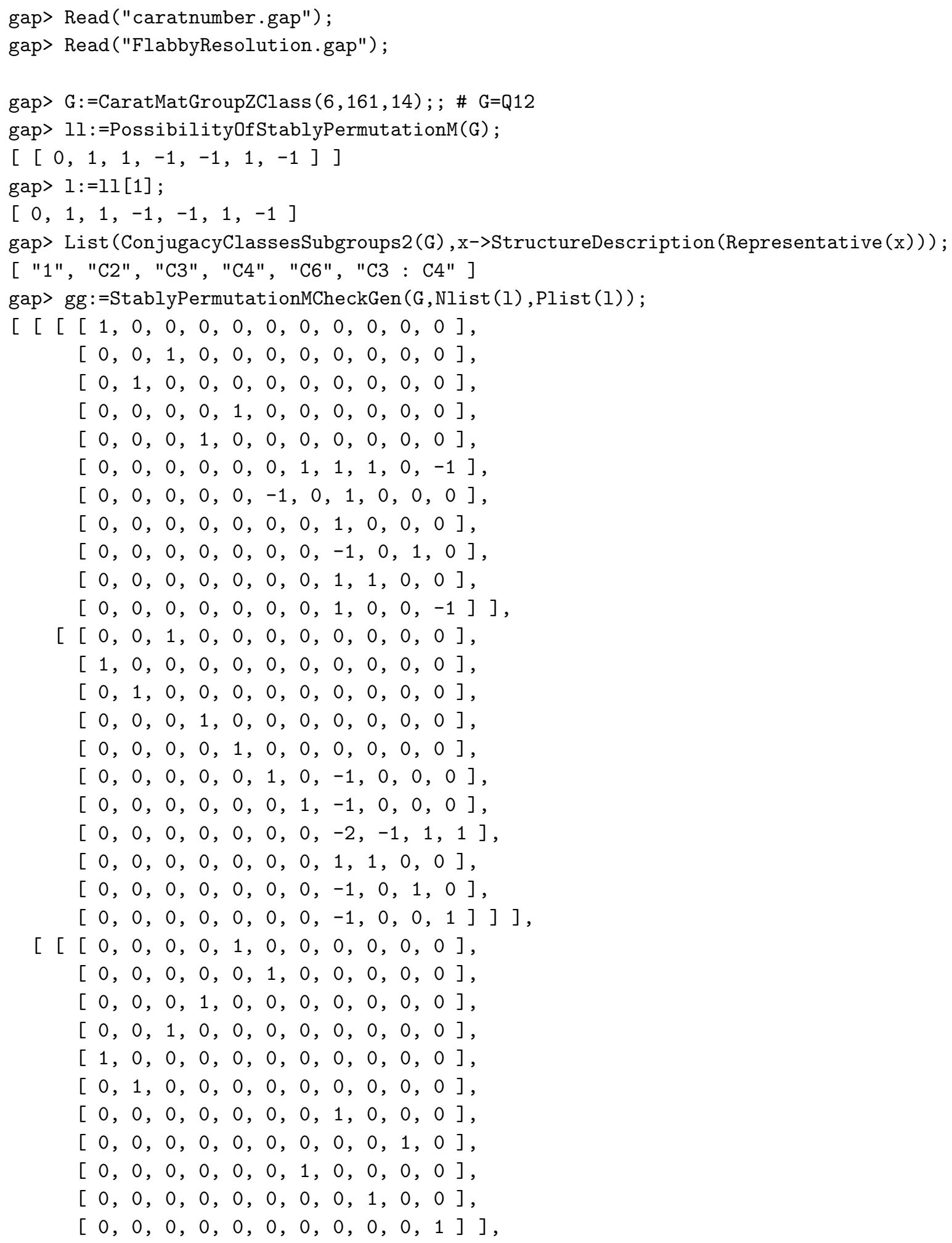




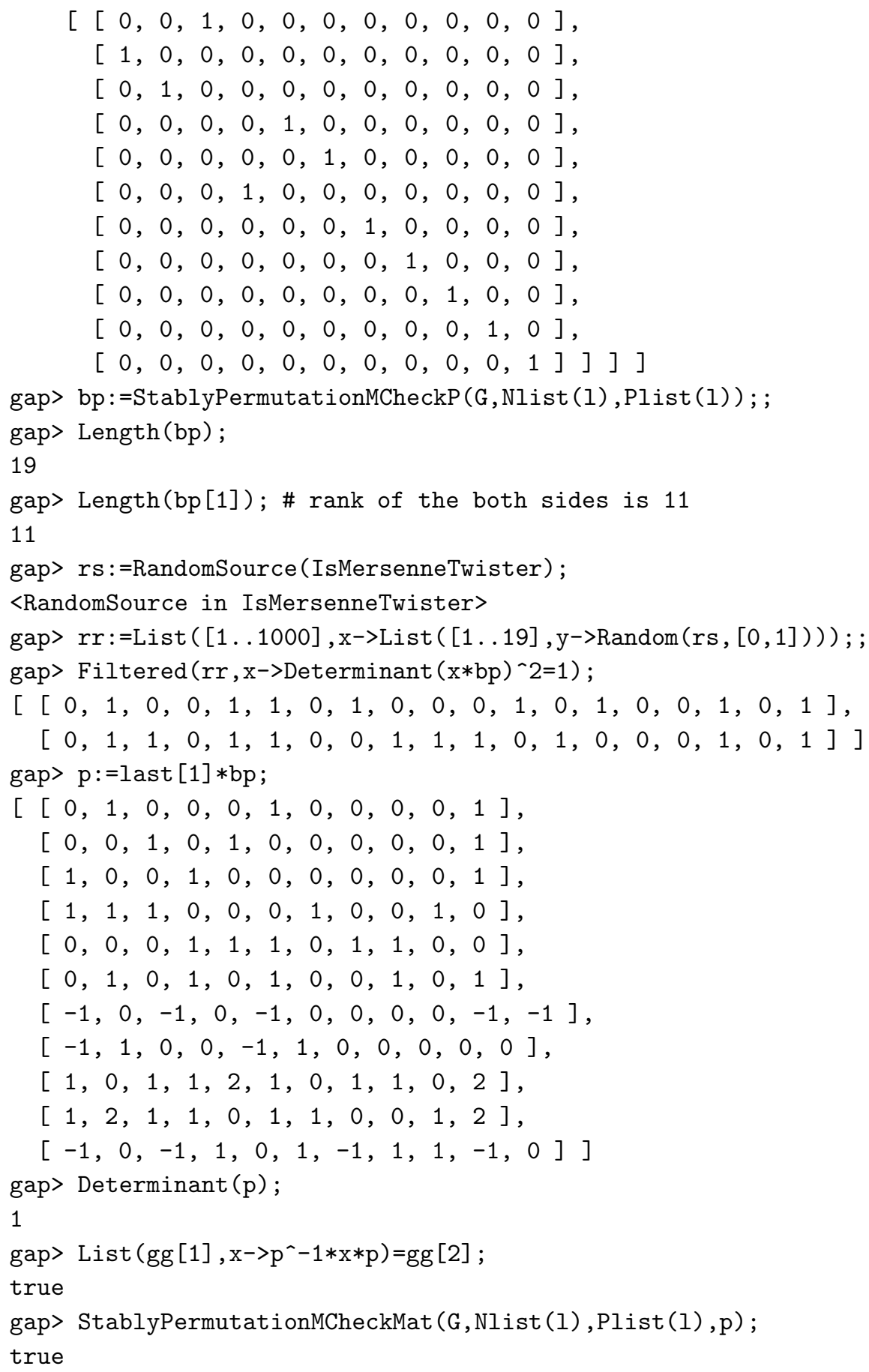

As an application of Example 6.4, we see that Krull-Schmidt theorem fails for permutation $D_{6}$-lattices by constructing explicit isomorphism. Let $M_{G}$ be the $G$-lattice where $G \simeq D_{6} \leq \mathrm{GL}(4, \mathbb{Z})$ is the group of the GAP ID $(4,14,8,2)$. Example 6.4 confirms that the isomorphism

$$
M_{G} \oplus \mathbb{Z} \simeq \mathbb{Z}\left[G / H_{6}\right] \oplus \mathbb{Z}\left[G / H_{9}\right]
$$

holds. Note that $\mathbb{Z}[G / H]$ is indecomposable for any $H \leq G$ ([CR87, Theorem 32.4]). We also see by Example 6.4 that it is possible that

$$
M_{G}^{\oplus 2} \oplus \mathbb{Z}[G] \oplus \mathbb{Z}\left[G / H_{7}\right] \oplus \mathbb{Z}\left[G / H_{8}\right] \simeq \mathbb{Z}\left[G / H_{2}\right] \oplus \mathbb{Z}\left[G / H_{3}\right] \oplus \mathbb{Z}\left[G / H_{4}\right] \oplus \mathbb{Z}\left[G / H_{5}\right] \oplus \mathbb{Z}\left[G / H_{9}\right] .
$$

If the isomorphism (12) holds, then it follows from (11) and (12) that the Krull-Schmidt theorem fails for the permutation $D_{6}$-lattices (the rank of the both sides is $12+2 \times 3+2+2+2=6+6+6+4+2 \times 1=24$ ):

$$
\mathbb{Z}[G] \oplus \mathbb{Z}\left[G / H_{6}\right]^{\oplus 2} \oplus \mathbb{Z}\left[G / H_{7}\right] \oplus \mathbb{Z}\left[G / H_{8}\right] \oplus \mathbb{Z}\left[G / H_{9}\right] \simeq \mathbb{Z}\left[G / H_{2}\right] \oplus \mathbb{Z}\left[G / H_{3}\right] \oplus \mathbb{Z}\left[G / H_{4}\right] \oplus \mathbb{Z}\left[G / H_{5}\right] \oplus \mathbb{Z} \oplus 2 .
$$

We may check that this isomorphism actually holds (see Example 6.8 below), namely we have: 
Proposition 6.7 (The Krull-Schmidt theorem fails for permutation $D_{6}$-lattices). Let $D_{6}$ be the dihedral group of order 12 and $\{1\}, C_{2}^{(1)}, C_{2}^{(2)}, C_{2}^{(3)}, C_{3}, C_{2}^{2}, C_{6}, S_{3}^{(1)}, S_{3}^{(2)}$ and $D_{6}$ be the conjugacy classes of subgroups of $D_{6}$. Then the following isomorphism holds:

$$
\begin{aligned}
& \mathbb{Z}\left[D_{6}\right] \oplus \mathbb{Z}\left[D_{6} / C_{2}^{2}\right]^{\oplus 2} \oplus \mathbb{Z}\left[D_{6} / C_{6}\right] \oplus \mathbb{Z}\left[D_{6} / S_{3}^{(1)}\right] \oplus \mathbb{Z}\left[D_{6} / S_{3}^{(2)}\right] \\
\simeq & \mathbb{Z}\left[D_{6} / C_{2}^{(1)}\right] \oplus \mathbb{Z}\left[D_{6} / C_{2}^{(2)}\right] \oplus \mathbb{Z}\left[D_{6} / C_{2}^{(3)}\right] \oplus \mathbb{Z}\left[D_{6} / C_{3}\right] \oplus \mathbb{Z}^{\oplus 2} .
\end{aligned}
$$

Example 6.8 (Verification of Proposition 6.7 the Krull-Schmidt theorem fails for permutation $D_{6}$-lattices).

\section{Read("FlabbyResolution.gap");}

gap> G:=MatGroupZClass $(4,14,8,2) ;$; \# G=D6

gap> $11:=$ PossibilityOfStablyPermutationM(G);

$[[1,-1,-1,-1,-1,0,1,1,-1,0,2]$,

$[0,0,0,0,0,1,0,0,1,-1,-1]]$

gap> $1:=11[1]+2 * 11[2]$;

$[1,-1,-1,-1,-1,2,1,1,1,-2,0]$

gap> List (ConjugacyClassesSubgroups2(G), $\mathrm{x}$->StructureDescription(Representative(x))) ;

[ "1", "C2", "C2", "C2", "C3", "C2 x C2", "C6", "S3", "S3", "D12" ]

gap> bp:=StablyPermutationMCheckP(G,Nlist(l),Plist(1)); ;

gap> Length(bp);

68

gap> Length(bp[1]); \# rank of the both sides is 24

24

\section{\# after some efforts we may get}

gap> $\mathrm{n}:=[$

$>1,-1,-1,-1,-1,-1,-1,-1,0,-1,0,1,0,1,1,0,0,0,-1,1,1,-1,1,0,-1$,

$>1,-1,0,-1,0,1,1,0,1,0,0,-1,1,1,-1,-1,0,-1,0,-1,1,0,-1,-1,1$,

$>1,0,0,1,0,1,1,0,1,0,1,1,1,1,0,-1,-1,0] ;$;

gap> $\mathrm{p}:=\mathrm{n} * \mathrm{bp}$;

$[[1,-1,-1,-1,-1,-1,1,-1,-1,-1,-1,-1,-1,-1,0,-1,0,1,0,1,1,1,0,0]$,

$[-1,1,-1,-1,-1,-1,-1,1,-1,-1,-1,-1,-1,-1,0,0,-1,1,1,0,1,1,0,0]$,

$[-1,-1,1,-1,-1,-1,-1,-1,1,-1,-1,-1,0,-1,-1,1,0,-1,1,0,1,1,0,0]$,

$[-1,-1,-1,1,-1,-1,-1,-1,-1,1,-1,-1,0,-1,-1,1,-1,0,0,1,1,1,0,0]$,

$[-1,-1,-1,-1,1,-1,-1,-1,-1,-1,1,-1,-1,0,-1,0,1,-1,0,1,1,1,0,0]$,

$[-1,-1,-1,-1,-1,1,-1,-1,-1,-1,-1,1,-1,0,-1,-1,1,0,1,0,1,1,0,0]$,

$[0,0,-1,1,1,-1,-1,1,1,-1,0,0,1,0,0,-1,1,1,-1,-1,0,-1,0,0]$,

$[0,0,1,-1,-1,1,1,-1,0,0,1,-1,0,1,0,1,-1,1,-1,-1,-1,0,0,0]$,

$[-1,1,0,0,-1,1,1,-1,-1,1,0,0,0,0,1,1,1,-1,-1,-1,-1,0,0,0]$,

$[1,-1,0,0,1,-1,0,0,1,-1,-1,1,0,1,0,1,-1,1,-1,-1,0,-1,0,0]$,

$[1,-1,-1,1,0,0,-1,1,0,0,-1,1,0,0,1,1,1,-1,-1,-1,0,-1,0,0]$,

$[-1,1,1,-1,0,0,0,0,-1,1,1,-1,1,0,0,-1,1,1,-1,-1,-1,0,0,0]$,

$[1,1,0,0,1,1,1,0,0,0,0,1,-1,1,1,1,-1,-1,-1,-1,0,0,-1,0]$,

$[1,1,1,1,0,0,0,1,0,1,0,0,1,-1,1,-1,1,-1,-1,-1,0,0,-1,0]$,

$[0,0,1,1,1,1,0,0,1,0,1,0,1,1,-1,-1,-1,1,-1,-1,0,0,-1,0]$,

$[1,0,0,0,0,1,1,1,0,0,1,1,-1,1,1,1,-1,-1,-1,-1,0,0,0,-1]$,

$[0,1,0,1,0,0,1,1,1,1,0,0,1,-1,1,-1,1,-1,-1,-1,0,0,0,-1]$,

$[0,0,1,0,1,0,0,0,1,1,1,1,1,1,-1,-1,-1,1,-1,-1,0,0,0,-1]$,

$[-1,1,1,-1,-1,1,0,-1,-1,0,0,-1,-1,-1,-1,1,1,1,1,0,0,1,0,1]$,

$[1,-1,-1,1,1,-1,-1,0,0,-1,-1,0,-1,-1,-1,1,1,1,0,1,1,0,0,1]$,

$[0,-1,-1,0,0,-1,-1,1,1,-1,-1,1,-1,-1,-1,1,1,1,1,0,1,0,1,0]$,

$[-1,0,0,-1,-1,0,1,-1,-1,1,1,-1,-1,-1,-1,1,1,1,0,1,0,1,1,0]$,

$[1,1,1,1,1,1,1,1,1,1,1,1,0,0,0,1,1,1,0,0,1,1,1,1]$, 
$[1,1,1,1,1,1,1,1,1,1,1,1,1,1,1,0,0,0,-1,-1,-1,-1,0,0]]$ gap> Determinant $(p)$;

1

gap> StablyPermutationMCheckMat(G,Nlist(1),Plist(1),p); true

\section{7. $H^{1}\left(G,\left[M_{G}\right]^{f l}\right)=0$ FOR ANY BRAVAIS GROUP $G$ OF DIMENSION $n \leq 6$}

Let $G$ be a finite subgroup of $\operatorname{GL}(n, \mathbb{Z})$ and $M_{G}$ be the $G$-lattice as in Definition 1.26 , The rationality problem for algebraic tori $T$ which correspond to Bravais groups $G \leq \operatorname{GL}(n, \mathbb{Z})$ was investigated by Voskresenskii ([Vos83], [Vos98, Section 8]). The referee of the paper pointed out that Voskresenskii asked a question whether $H^{1}\left(G,\left[M_{G}\right]^{f l}\right)=0$ for any Bravais group $G$ (or, at least maximal finite subgroup $G \leq \mathrm{GL}(n, \mathbb{Z})$ ).

By the classification of Bravais group $G$ of dimension $n \leq 6$ (see Subsection 4.4) and the computation of the flabby class $\left[M_{G}\right]^{f l}$ (see Subsection [5.1), we obtain the following:

Theorem 7.1. If $G$ is a Bravais group of dimension $n \leq 6$, then $H^{1}\left(G,\left[M_{G}\right]^{f l}\right)=0$. In particular, if $G$ is a maximal finite subgroup $G \leq \mathrm{GL}(n, \mathbb{Z})$ where $n \leq 6$, then $H^{1}\left(G,\left[M_{G}\right]^{f l}\right)=0$.

In dimension $n=6$, we should use the following FlabbyResolutionFromPerm(G) instead of FlabbyResolution(G) for the 821st and the 822nd Bravais group of dimension 6 (see Example 7.2 below):

FlabbyResolutionFromPerm(G) returns the same as FlabbyResolution(G) but using ConjugacyClassesSubgroupsFromPerm (G) instead of ConjugacyClassesSubgroups2(G).

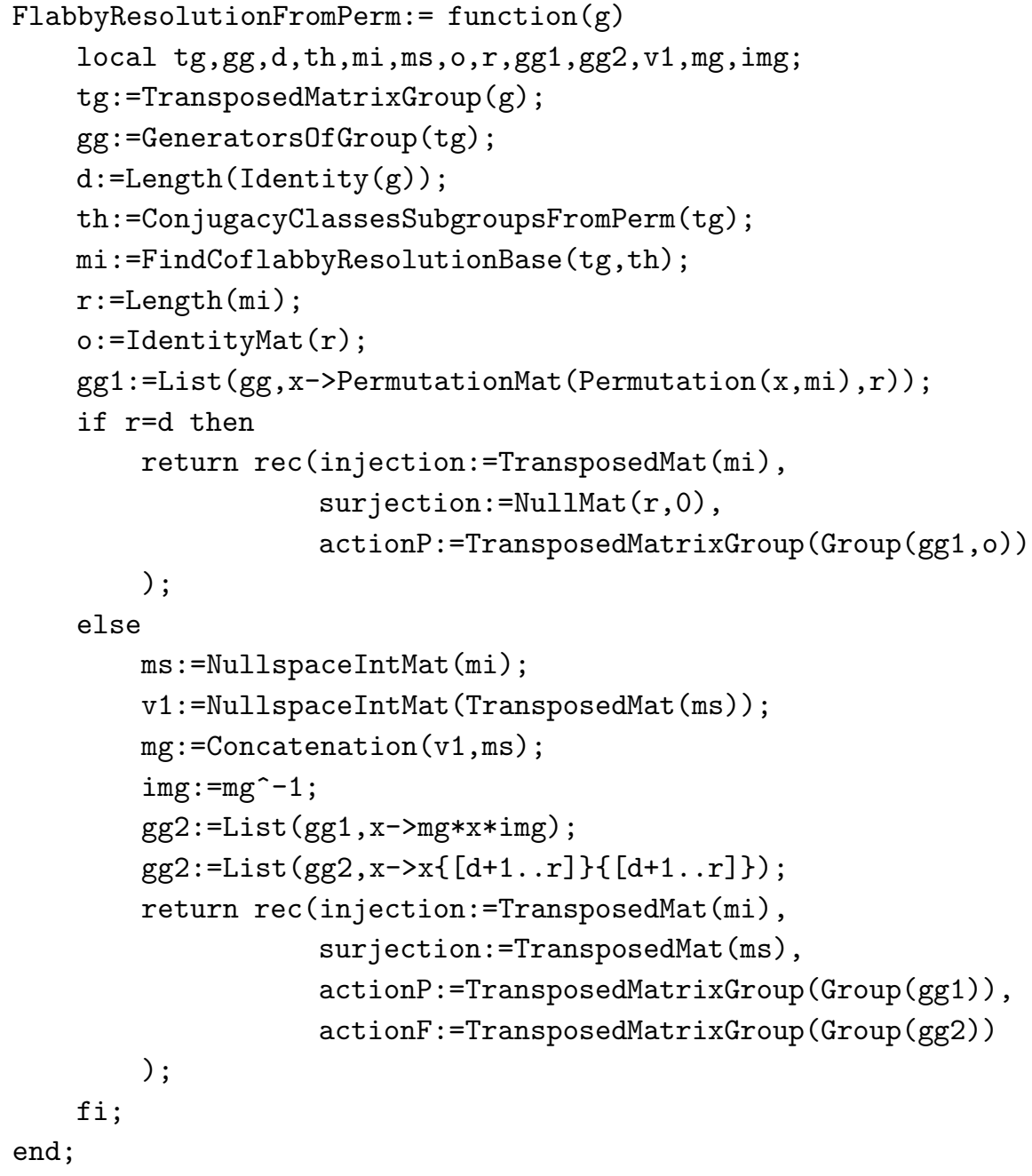


Example 7.2 (Verification of $H^{1}\left(G,\left[M_{G}\right]^{f l}\right)=0$ for any Bravais group of dimension $\left.n \leq 6\right)$.

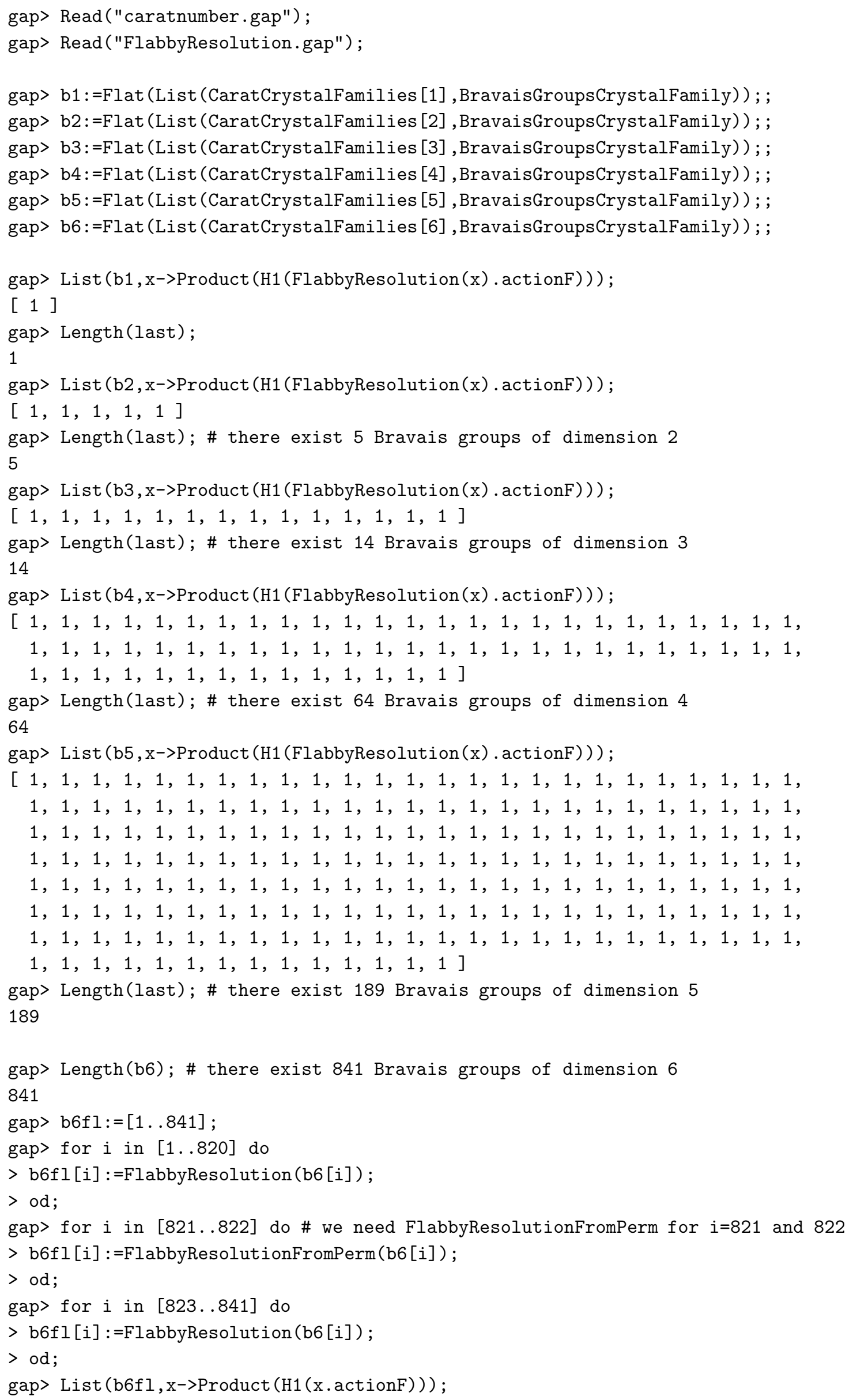


$[1,1,1,1,1,1,1,1,1,1,1,1,1,1,1,1,1,1,1,1,1,1,1,1,1$, $1,1,1,1,1,1,1,1,1,1,1,1,1,1,1,1,1,1,1,1,1,1,1,1,1$, $1,1,1,1,1,1,1,1,1,1,1,1,1,1,1,1,1,1,1,1,1,1,1,1,1$, $1,1,1,1,1,1,1,1,1,1,1,1,1,1,1,1,1,1,1,1,1,1,1,1,1$, $1,1,1,1,1,1,1,1,1,1,1,1,1,1,1,1,1,1,1,1,1,1,1,1,1$, $1,1,1,1,1,1,1,1,1,1,1,1,1,1,1,1,1,1,1,1,1,1,1,1,1$, $1,1,1,1,1,1,1,1,1,1,1,1,1,1,1,1,1,1,1,1,1,1,1,1,1$, $1,1,1,1,1,1,1,1,1,1,1,1,1,1,1,1,1,1,1,1,1,1,1,1,1$, $1,1,1,1,1,1,1,1,1,1,1,1,1,1,1,1,1,1,1,1,1,1,1,1,1$, $1,1,1,1,1,1,1,1,1,1,1,1,1,1,1,1,1,1,1,1,1,1,1,1,1$, $1,1,1,1,1,1,1,1,1,1,1,1,1,1,1,1,1,1,1,1,1,1,1,1,1$, $1,1,1,1,1,1,1,1,1,1,1,1,1,1,1,1,1,1,1,1,1,1,1,1,1$, $1,1,1,1,1,1,1,1,1,1,1,1,1,1,1,1,1,1,1,1,1,1,1,1,1$, $1,1,1,1,1,1,1,1,1,1,1,1,1,1,1,1,1,1,1,1,1,1,1,1,1$, $1,1,1,1,1,1,1,1,1,1,1,1,1,1,1,1,1,1,1,1,1,1,1,1,1$, $1,1,1,1,1,1,1,1,1,1,1,1,1,1,1,1,1,1,1,1,1,1,1,1,1$, $1,1,1,1,1,1,1,1,1,1,1,1,1,1,1,1,1,1,1,1,1,1,1,1,1$, $1,1,1,1,1,1,1,1,1,1,1,1,1,1,1,1,1,1,1,1,1,1,1,1,1$, $1,1,1,1,1,1,1,1,1,1,1,1,1,1,1,1,1,1,1,1,1,1,1,1,1$, $1,1,1,1,1,1,1,1,1,1,1,1,1,1,1,1,1,1,1,1,1,1,1,1,1$, $1,1,1,1,1,1,1,1,1,1,1,1,1,1,1,1,1,1,1,1,1,1,1,1,1$, $1,1,1,1,1,1,1,1,1,1,1,1,1,1,1,1,1,1,1,1,1,1,1,1,1$, $1,1,1,1,1,1,1,1,1,1,1,1,1,1,1,1,1,1,1,1,1,1,1,1,1$, $1,1,1,1,1,1,1,1,1,1,1,1,1,1,1,1,1,1,1,1,1,1,1,1,1$, $1,1,1,1,1,1,1,1,1,1,1,1,1,1,1,1,1,1,1,1,1,1,1,1,1$, $1,1,1,1,1,1,1,1,1,1,1,1,1,1,1,1,1,1,1,1,1,1,1,1,1$, $1,1,1,1,1,1,1,1,1,1,1,1,1,1,1,1,1,1,1,1,1,1,1,1,1$, $1,1,1,1,1,1,1,1,1,1,1,1,1,1,1,1,1,1,1,1,1,1,1,1,1$, $1,1,1,1,1,1,1,1,1,1,1,1,1,1,1,1,1,1,1,1,1,1,1,1,1$, $1,1,1,1,1,1,1,1,1,1,1,1,1,1,1,1,1,1,1,1,1,1,1,1,1$, $1,1,1,1,1,1,1,1,1,1,1,1,1,1,1,1,1,1,1,1,1,1,1,1,1$, $1,1,1,1,1,1,1,1,1,1,1,1,1,1,1,1,1,1,1,1,1,1,1,1,1$, $1,1,1,1,1,1,1,1,1,1,1,1,1,1,1,1,1,1,1,1,1,1,1,1,1$, $1,1,1,1,1,1,1,1,1,1,1,1,1,1,1,1]$

gap> Length(last);

841

\section{NORM ONE TORI}

Let $K / k$ be a separable field extension of degree $n$ and $L / k$ be the Galois closure of $K / k$. Let $G=$ $\operatorname{Gal}(L / k)$ and $H=\operatorname{Gal}(L / K)$. The Galois group $G$ may be regarded as a transitive subgroup of the symmetric group $S_{n}$ of degree $n$. Let $R_{K / k}^{(1)}\left(\mathbb{G}_{m}\right)$ be the norm one torus of $K / k$, i.e. the kernel of the norm map $R_{K / k}\left(\mathbb{G}_{m}\right) \rightarrow \mathbb{G}_{m}$. The norm one torus $R_{K / k}^{(1)}\left(\mathbb{G}_{m}\right)$ has the Chevalley module $J_{G / H}$ as its character module and the field $L\left(J_{G / H}\right)^{G}$ as its function field (see Section 1). The following algorithm is available from http://math.h.kyoto-u.ac.jp/〜yamasaki/Algorithm/as FlabbyResolution.gap.

Norm1TorusJ (d,m) returns the Chevalley module $J_{G / H}$ for the $m$-th transitive subgroup $G=d T m \leq S_{d}$ of degree $d$ where $H$ is the stabilizer of one of the letters in $G$.

Algorithm N1T (Construction of Chevalley module $J_{G / H}$ for the transitive subgroups $G=d T m \leq S_{d}$ ).

Norm1TorusJ:= function $(\mathrm{d}, \mathrm{m})$ 


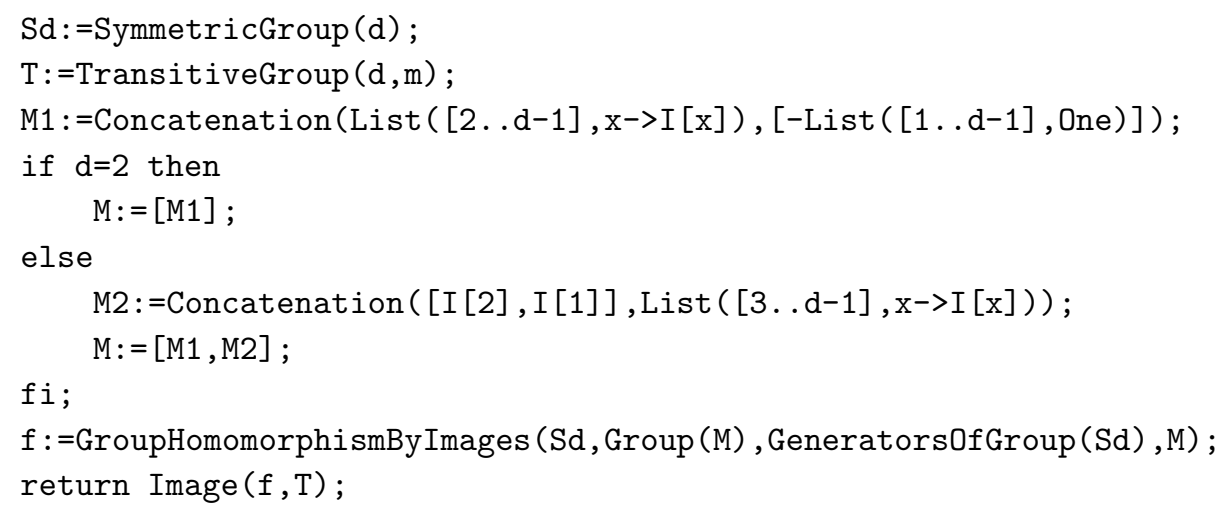

Example 8.1 $\left(\left[J_{G / H}\right]^{f l}=0\right.$ for $\left.G=5 T 4 \simeq A_{5}\right)$. By using Method II as in Algorithm F6, we may verify that $\left[J_{G / H}\right]^{f l}=0$ for $G=5 T 4 \simeq A_{5}$ and $H=A_{4}$.

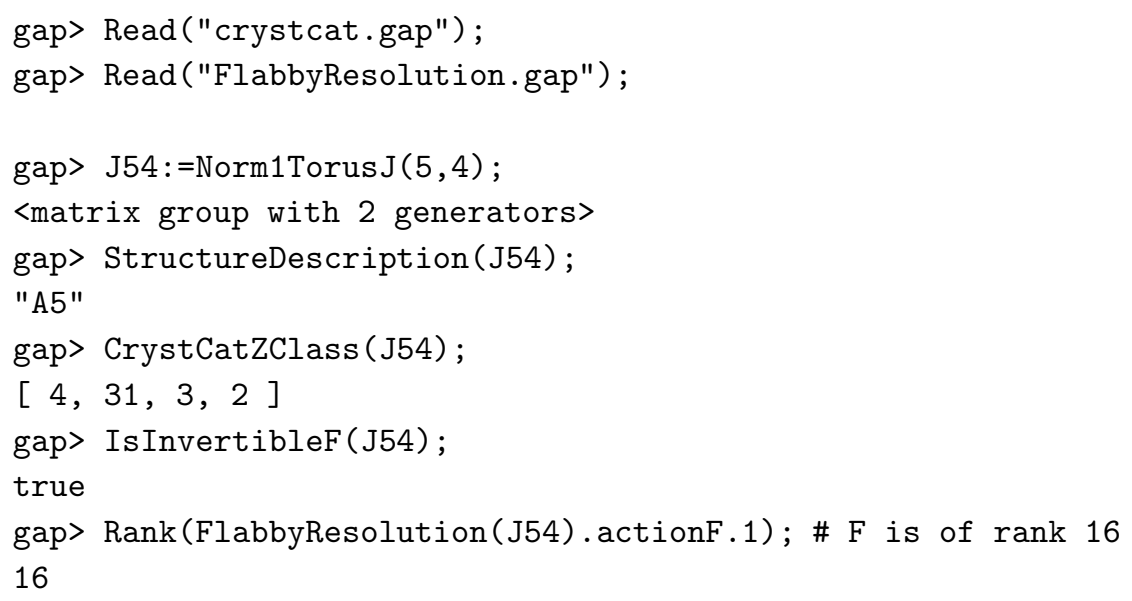


$[0,0,0,0,0,0,2,0,0,2,0,0,-1,-1,0,-1,0,0,-1,0,0,-1]$, $[-1,0,-1,1,1,0,-1,1,-1,-1,0,-1,0,1,0,1,0,0,0,0,1,1]$, $[1,-1,1,1,-1,-1,-1,-1,0,-1,0,0,1,0,0,0,0,1,1,0,0,1]$, $[-1,-1,0,-1,0,1,1,-1,-1,0,-1,1,0,0,1,0,1,0,0,1,0,1]$, $[1,1,-1,-1,0,1,-1,0,0,-1,-1,-1,0,1,0,1,0,0,1,1,0,0]$, $[-1,0,0,-1,-1,-1,1,1,-1,0,-1,1,0,0,1,0,1,0,1,0,1,0]$, $[0,-1,-1,0,-1,-1,0,-1,1,1,1,-1,1,0,0,0,0,1,0,1,1,0]$, $[-1,-1,0,0,0,1,-1,-1,-1,-1,1,1,1,0,0,1,1,0,0,0,0,1]$, $[-1,1,1,-1,-1,-1,0,0,-1,1,-1,0,0,0,1,0,1,0,1,0,1,0]$, $[-1,0,-1,1,-1,-1,1,1,-1,0,0,-1,0,0,1,1,0,1,0,0,1,0]$, $[0,-1,0,0,-1,-1,-1,-1,1,-1,1,1,1,0,0,0,0,1,1,0,0,1]$, $[0,-1,-1,-1,1,0,0,-1,1,1,-1,-1,0,1,0,0,1,1,0,1,0,0]$, $[0,0,0,-1,-1,-1,-1,1,1,-1,-1,1,1,1,1,0,0,0,1,0,0,0]$, $[-1,1,-1,0,-1,-1,0,0,-1,1,1,-1,0,0,1,1,0,1,0,0,1,0]$, $[1,-1,-1,1,-1,-1,1,-1,0,0,0,-1,1,0,0,0,0,1,0,1,1,0]$, $[0,0,-1,-1,1,0,-1,1,1,-1,-1,-1,0,1,0,1,0,0,1,1,0,0]$, $[1,-1,-1,-1,0,1,1,-1,0,0,-1,-1,0,1,0,0,1,1,0,1,0,0]]$ gap> Determinant $(p)$;

$-1$

gap> StablyPermutationFCheckMat (J54,Nlist (l), Plist (1),p);

true

Example 8.2 $\left(\left[J_{G / H}\right]^{f l}=0\right.$ for $\left.G=6 T 3 \simeq D_{6}\right)$. By using Method II as in Algorithm F6, we may verify that $\left[J_{G / H}\right]^{f l}=0$ for $G=6 T 3 \simeq D_{6}$ and $H=C_{2}$.

gap> Read ("caratnumber.gap");

gap> Read("FlabbyResolution.gap");

gap> J63:=Norm1TorusJ $(6,3)$;

<matrix group with 2 generators>

gap> StructureDescription(J63);

"D12"

gap> CaratZClass (J63);

$[5,391,4]$

gap> IsInvertibleF (J63);

true

gap> Rank(FlabbyResolution(J63).actionF.1); \# F is of rank 13

13

gap> mis:=SearchCoflabbyResolutionBase(TransposedMatrixGroup(J63),3);; \# Method III gap> List (mis, Length);

$[24,20,24,18,24,20,24,20,18,14,21,17,21,24,20,24,24,20$,

$24,18,30,26,24,20,18,20,14,24,20,18]$

gap> mi:=mis [Length(mis)-3]; \# (new) F is of rank 9 (=14-5)

$[[-1,0,0,0,0],[-1,1,-1,1,-1],[-1,1,0,0,0],[0,-1,1,0,0]$,

$[0,0,-1,1,0],[0,0,0,-1,1],[0,0,0,0,-1],[0,0,0,0,1]$,

$[0,0,0,1,-1],[0,0,1,-1,0],[0,1,-1,0,0],[1,-1,0,0,0]$,

$[1,-1,1,-1,1],[1,0,0,0,0]]$

gap> $11:=$ PossibilityOfStablyPermutationFFromBase $(\mathrm{J} 63, \mathrm{mi})$;

$[[1,-1,0,-1,0,2,0,1,1,-1,-1],[0,0,1,0,1,0,-1,0,0,1,-1]]$

gap> $1:=11[$ Length $(11)]$;

$[0,0,1,0,1,0,-1,0,0,1,-1]$

gap> bp:=StablyPermutationFCheckPFromBase (J63,mi,Nlist(1),Plist(1)); ;

gap> Length (bp);

17 


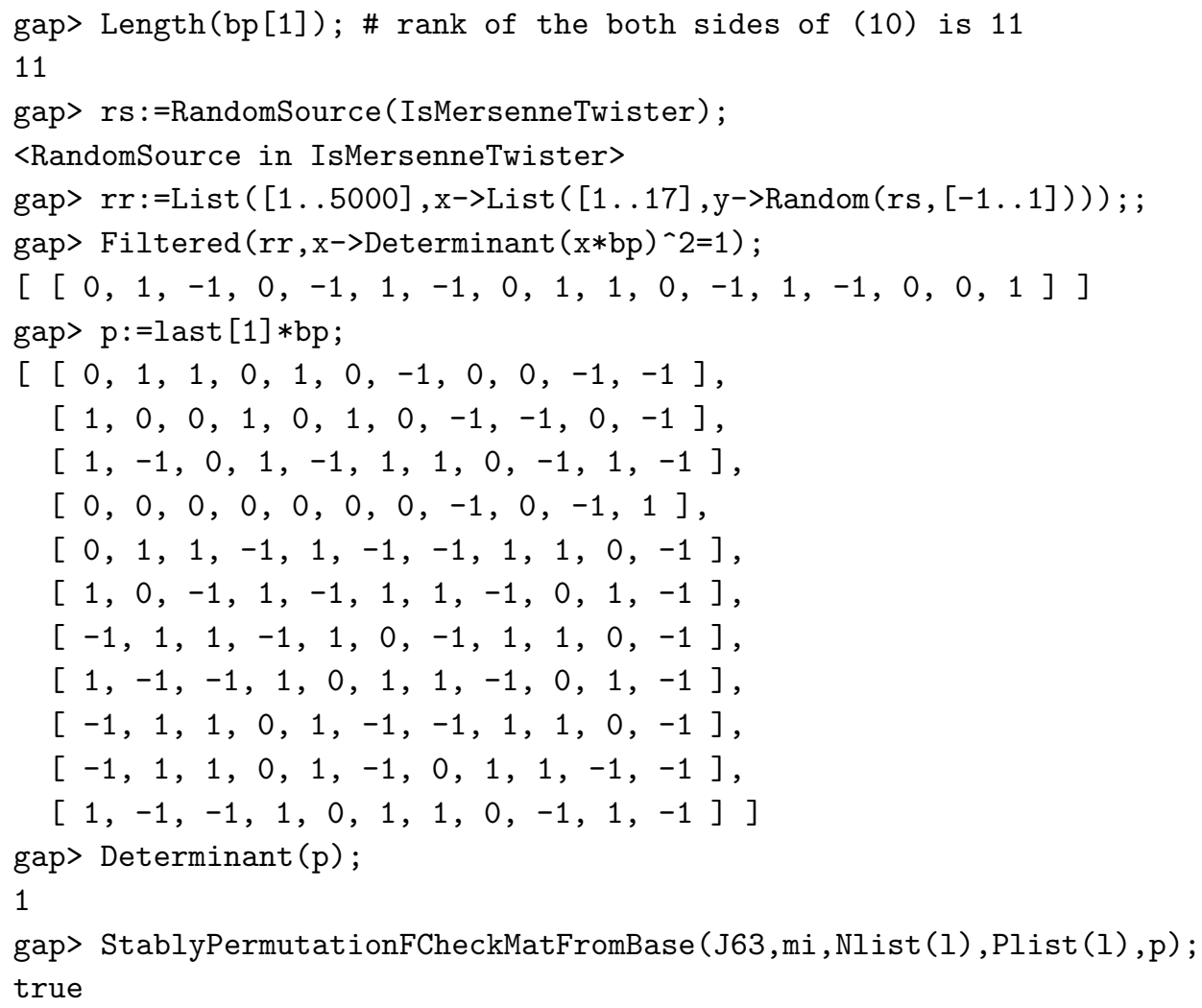

Example 8.3 (The flabby class $\left[J_{Q_{8}}\right]^{f l}$ is not invertible but flabby and coflabby). Let $J_{Q_{8}}$ be the Chevalley module of the quaternion group $Q_{8}$ of order 8 . The rank of $J_{Q_{8}}$ is 7 . Let $8 T 5$ be the 5 th transitive subgroup of $S_{8}$. Then $8 T 5 \simeq Q_{8}$. By Theorems [1.3, 1.17 (ii) and 1.18, the flabby class [ $\left.J_{Q_{8}}\right]^{f l}$ of $J_{Q_{8}}$ is not invertible but flabby and coflabby. Using FlabbyResolution as in Algorithm[F1, we may obtain the flabby class $\left[J_{Q_{8}}\right]^{f l}=[F]$ of $J_{Q_{8}}$ where $F$ is of rank 9 which satisfies $0 \rightarrow M_{Q_{8}} \rightarrow P \rightarrow F \rightarrow 0$.

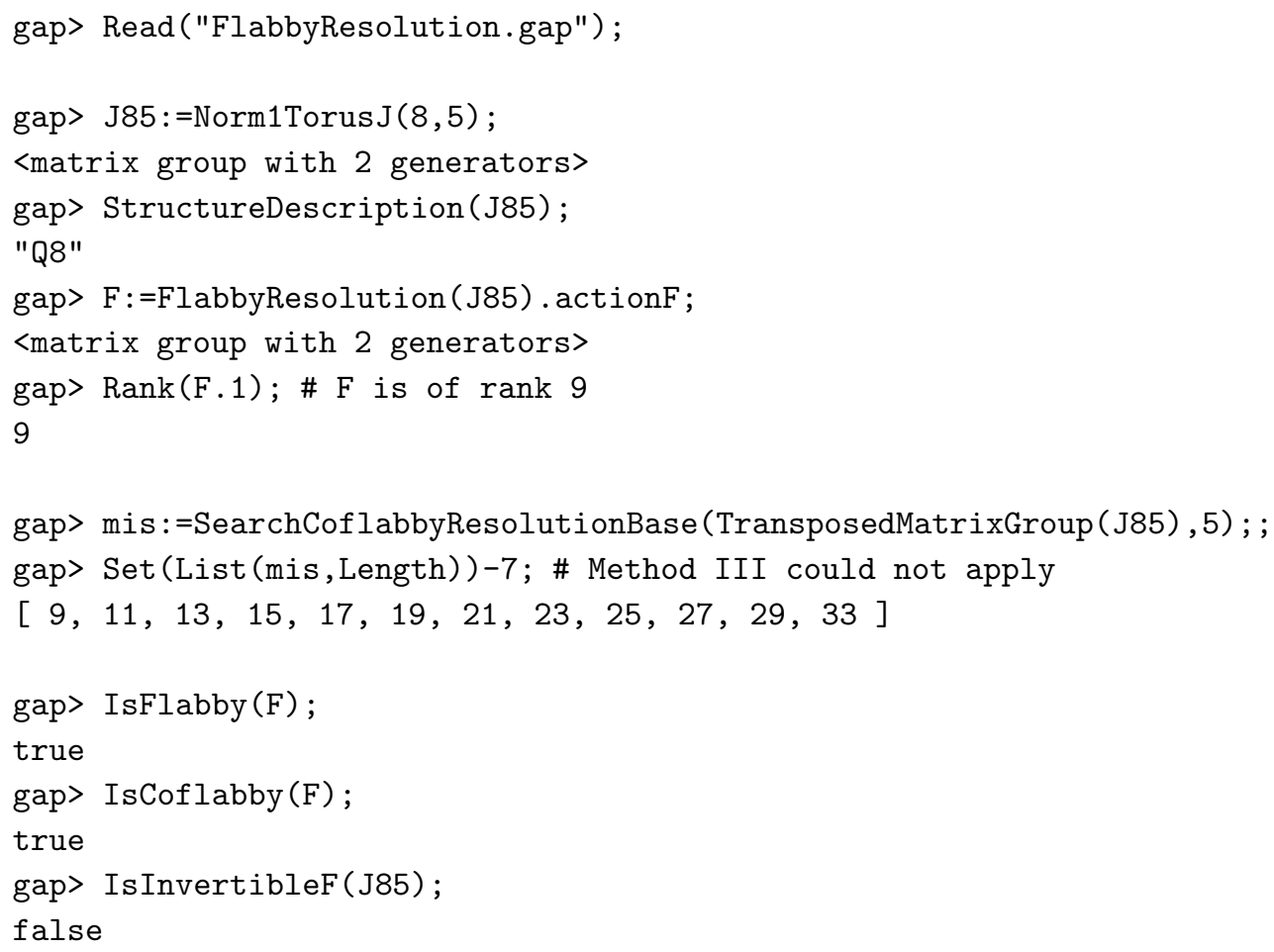

Example 8.4 $\left(\left[J_{G / H}\right]^{f l}\right.$ for $G=7 T m$ and $\left.G=11 T m\right)$. By Theorems 1.3, 1.4, 1.6, 1.7 and 1.8, and Lemma 2.17. we may obtain the stably rational classification of norm one tori $R_{K / k}^{(1)}\left(\mathbb{G}_{m}\right)$ of dimensions 6 and 10 via 
Algorithm N1T for $\left[J_{G / H}\right]^{f l}$ with $G=7 T m \leq S_{7}(1 \leq m \leq 7)$ and $G=11 T m \leq S_{11}(1 \leq m \leq 8)$ respectively. The result is as follows:

Theorem 8.5 (Stably rational classification of norm one tori of dimensions 6 and 10).

(i) $R_{K / k}^{(1)}\left(\mathbb{G}_{m}\right)$ is stably $k$-rational for $7 T 1 \simeq C_{7}$ and $7 T 2 \simeq D_{7}$;

(ii) $R_{K / k}^{(1)}\left(\mathbb{G}_{m}\right)$ is not stably but retract $k$-rational for $7 T 3 \simeq F_{21}, 7 T 4 \simeq F_{42}, 7 T 5 \simeq \operatorname{PSL}(2,7)$, $7 T 6 \simeq A_{7}$ and $7 T 7 \simeq S_{7} ;$

(iii) $R_{K / k}^{(1)}\left(\mathbb{G}_{m}\right)$ is stably $k$-rational for $11 T 1 \simeq C_{11}$ and $11 T 2 \simeq D_{11}$;

(iv) $R_{K / k}^{(1)}\left(\mathbb{G}_{m}\right)$ is not stably but retract $k$-rational for $11 T 3 \simeq F_{55}, 11 T 4 \simeq F_{110}, 11 T 5 \simeq \mathrm{PSL}(2,11), 11 T 6 \simeq M_{11}$, $11 T 7 \simeq A_{11}$ and $11 T 8 \simeq S_{11}$ where $M_{11}$ is the Mathieu group of degree 11 .

\section{TATE COHOMOlOGY: GAP COMPUtations}

In this section, we provide some algorithms of GAP for computing the Tate cohomology $\widehat{H}^{n}\left(G, M_{G}\right)$ by using the GAP package HAP ([HAP]). We will use this for showing the main theorem (Theorem 1.27) in Section 10, The following algorithms are available from http://math.h.kyoto-u.ac.jp/〜yamasaki/Algorithm/as Hn.gap.

TateCohomology $(\mathrm{G}, \mathrm{n})$ returns the Tate cohomology group $\widehat{H}^{n}\left(G, M_{G}\right)$ for $n \in \mathbb{Z}$.

Algorithm TC (Tate cohomology).

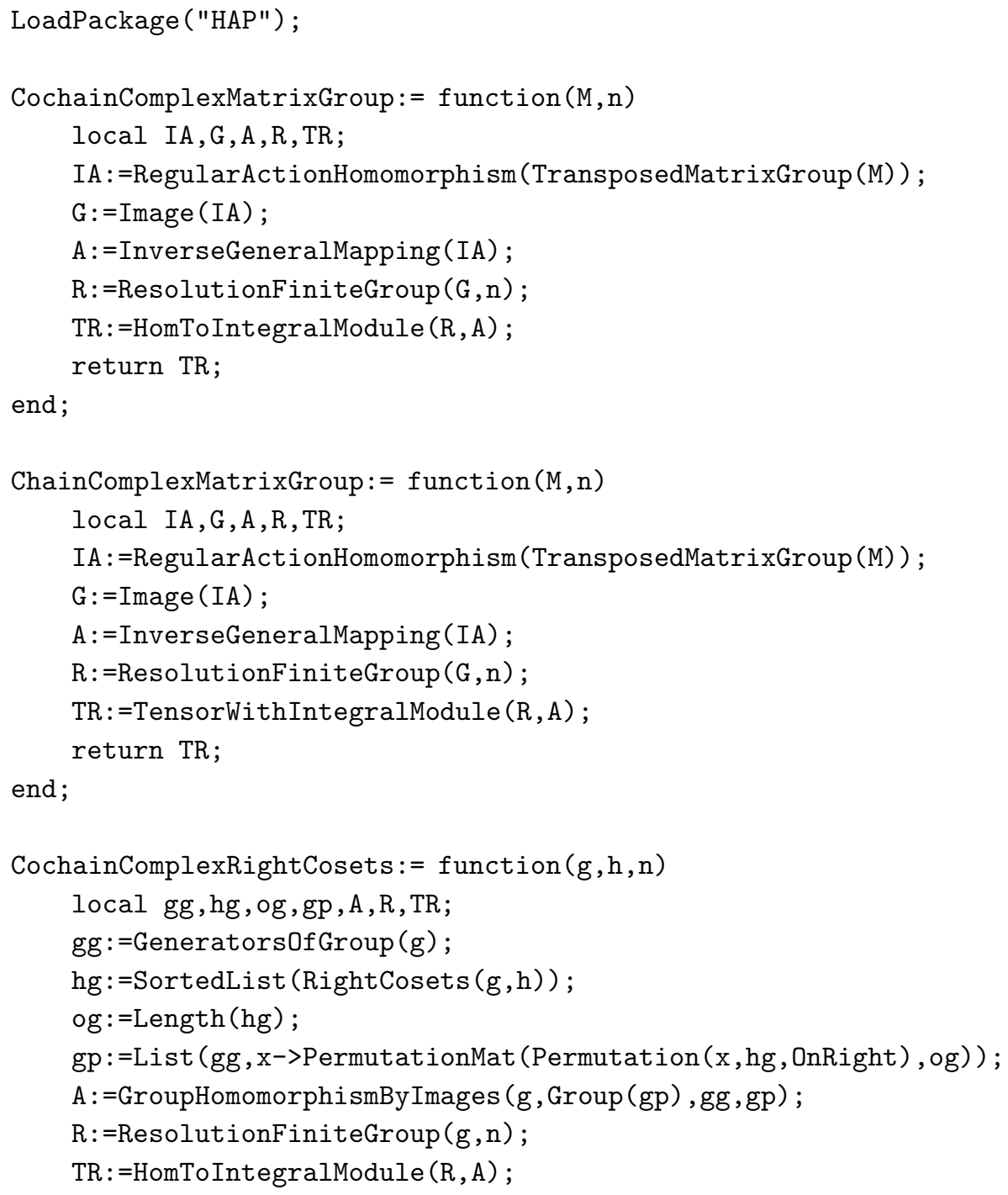


return TR;

end;

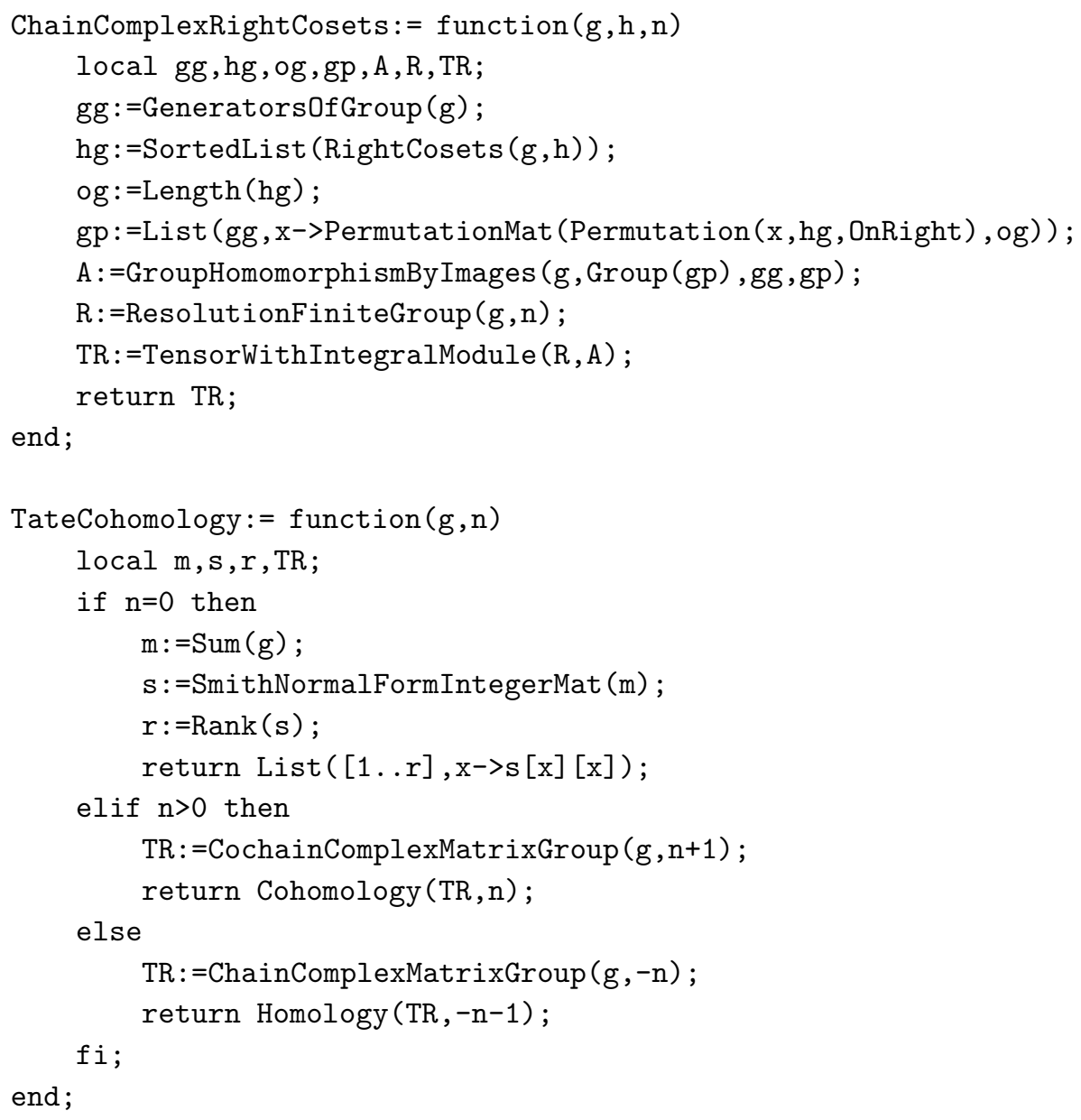

Example 9.1 (Tate cohomology $\widehat{H}^{n}\left(G, M_{G}\right)$ for the group $C_{2}^{3}$ of the GAP ID $(3,3,3,3)$ ). Let $G \simeq C_{2}^{3}$ be the group of the GAP ID $(3,3,3,3)$. We may obtain the Tate cohomologies $\widehat{H}^{n}\left(G, M_{G}\right)$ for $-7 \leq n \leq 7$ as follows:

\begin{tabular}{l|cccccccc}
$n$ & -7 & -6 & -5 & -4 & -3 & -2 & -1 & 0 \\
\hline$\widehat{H}^{n}\left(C_{2}^{3}, M_{C_{2}^{3}}\right)$ & $(\mathbb{Z} / 2 \mathbb{Z})^{\oplus 13}$ & $(\mathbb{Z} / 2 \mathbb{Z})^{\oplus 9}$ & $(\mathbb{Z} / 2 \mathbb{Z})^{\oplus 7}$ & $(\mathbb{Z} / 2 \mathbb{Z})^{\oplus 4}$ & $(\mathbb{Z} / 2 \mathbb{Z})^{\oplus 3}$ & $\mathbb{Z} / 2 \mathbb{Z}$ & $\mathbb{Z} / 2 \mathbb{Z}$ & 0 \\
$n$ & 1 & 2 & 3 & 4 & 5 & 6 & 7 \\
\hline$\widehat{H}^{n}\left(C_{2}^{3}, M_{C_{2}^{3}}\right)$ & $(\mathbb{Z} / 2 \mathbb{Z})^{\oplus 2}$ & $(\mathbb{Z} / 2 \mathbb{Z})^{\oplus 3}$ & $(\mathbb{Z} / 2 \mathbb{Z})^{\oplus 6}$ & $(\mathbb{Z} / 2 \mathbb{Z})^{\oplus 8}$ & $(\mathbb{Z} / 2 \mathbb{Z})^{\oplus 12}$ & $(\mathbb{Z} / 2 \mathbb{Z})^{\oplus 15}$ & $(\mathbb{Z} / 2 \mathbb{Z})^{\oplus 20}$
\end{tabular}

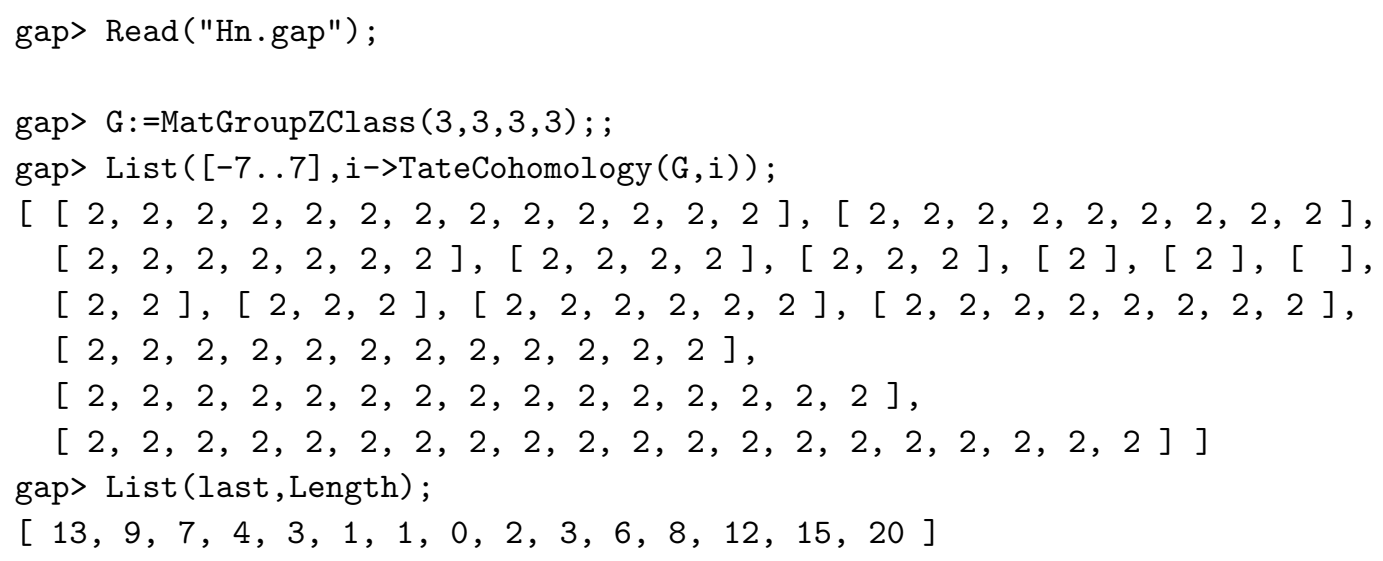


Example 9.2 (The group $\operatorname{Indmf}(6,10,1)$ is not a subgroup of the 17 irreducible maximal finite groups but is indecomposable). Let $G=\operatorname{Indmf}(6,10,1)$ be the indecomposable maximal finite group of the CARAT ID $(6,5517,4)$. We will confirm that $G$ is not a subgroup of all the 17 irreducible maximal finite groups $\operatorname{Imf}(6, i, j)$ of dimension 6 but is indecomposable by using the Tate cohomology $\widehat{H}^{-1}(G), \widehat{H}^{1}(G)$ and $\widehat{H}^{2}(G)$ where $\widehat{H}^{i}(G)=$ $\widehat{H}^{i}\left(G, M_{G}\right)$ and $M_{G}$ is the corresponding $G$-lattice as in Definition 1.26 .

Let $\mathrm{Sy}_{2}(G)$ be a 2-Sylow subgroup of $G$. We have the normalizer $N_{\mathrm{GL}(6, \mathbb{Z})}\left(\mathrm{Sy}_{2}(G)\right)$ of $\operatorname{Sy}_{2}(G)$ in $\mathrm{GL}(6, \mathbb{Z})$ is $\mathrm{Sy}_{2}(G)$. Suppose that there exists a group $G^{\prime}$ such that $G<G^{\prime}$ with even index. Then there exists a 2-Sylow subgroup $\operatorname{Sy}_{2}\left(G^{\prime}\right)$ of $G^{\prime}$ such that $\operatorname{Sy}_{2}(G)<\operatorname{Sy}_{2}\left(G^{\prime}\right)$. But this is impossible because $N_{\mathrm{GL}(6, \mathbb{Z})}\left(\operatorname{Sy}_{2}(G)\right)=\operatorname{Sy}_{2}(G)$. Hence such a group $G<G^{\prime}$ with even index does not exist.

Only two groups $G^{\prime}=\operatorname{Imf}(6,3,1)$ and $G^{\prime \prime}=\operatorname{Imf}(6,3,2)$ out of the 17 groups $\operatorname{Imf}(6, i, j)$ may have a subgroup $G$ with odd index. However, these two does not occur because $\widehat{H}^{1}\left(\operatorname{Sy}_{2}(G)\right)=\mathbb{Z} / 2 \mathbb{Z} \oplus \mathbb{Z} / 2 \mathbb{Z}, \widehat{H}^{1}\left(\operatorname{Sy}_{2}\left(G^{\prime}\right)\right)=\mathbb{Z} / 2 \mathbb{Z}$ and $\widehat{H}^{1}\left(\mathrm{Sy}_{2}\left(G^{\prime \prime}\right)\right)=\mathbb{Z} / 2 \mathbb{Z}$. This implies that $G$ is not a subgroup of all the 17 irreducible maximal finite groups $\operatorname{Imf}(6, i, j)$ of dimension 6 .

Next, we will show that $G$ is indecomposable. Over the field $\mathbb{C}$ of complex numbers, $G$ splits into 2 irreducible direct summands of degree 3. Indeed, we also see that the 6 groups of the CARAT IDs $(6,5517,1),(6,5517,3)$, $(6,5517,5),(6,5517,6),(6,5517,8)$ and $(6,5517,9)$ are in the same $\mathbb{Q}$-class of $G$ but splits into 2 irreducible direct summands of degree 3 in $\mathrm{GL}(3, \mathbb{Z})$. This implies that $G$ splits into them in $\mathrm{GL}(3, \mathbb{Q})$.

We will confirm that $G$ is indecomposable in $\operatorname{GL}(6, \mathbb{Z})$. Because of the order of $G$ is $2304=48^{2}$, we see that $G$ should be a direct product of 2 groups among 3 groups $G_{1}=\operatorname{Imf}(3,1,1), G_{2}=\operatorname{Imf}(3,1,2)$ and $G_{3}=\operatorname{Imf}(3,1,3)$ which are isomorphic to the group $C_{2} \times S_{4}$ of order 48 . By computing the Tate cohomologies $\widehat{H}^{i}$, we have

$$
\begin{array}{llll}
\widehat{H}^{-1}(G)=\mathbb{Z} / 2 \mathbb{Z}, & \widehat{H}^{-1}\left(G_{1} \times G_{1}\right)=(\mathbb{Z} / 2 \mathbb{Z})^{\oplus 2}, & \widehat{H}^{-1}\left(G_{2} \times G_{2}\right)=(\mathbb{Z} / 2 \mathbb{Z})^{\oplus 2}, & \widehat{H}^{-1}\left(G_{3} \times G_{3}\right)=0, \\
& \widehat{H}^{-1}\left(G_{1} \times G_{2}\right)=(\mathbb{Z} / 2 \mathbb{Z})^{\oplus 2}, & \widehat{H}^{-1}\left(G_{1} \times G_{3}\right)=\mathbb{Z} / 2 \mathbb{Z}, & \widehat{H}^{-1}\left(G_{2} \times G_{3}\right)=\mathbb{Z} / 2 \mathbb{Z}, \\
\widehat{H}^{1}(G)=\mathbb{Z} / 2 \mathbb{Z}, & \widehat{H}^{1}\left(G_{1} \times G_{1}\right)=(\mathbb{Z} / 2 \mathbb{Z})^{\oplus 2}, & \widehat{H}^{1}\left(G_{2} \times G_{2}\right)=0, & \widehat{H}^{1}\left(G_{3} \times G_{3}\right)=(\mathbb{Z} / 2 \mathbb{Z})^{\oplus 2}, \\
& \widehat{H}^{1}\left(G_{1} \times G_{2}\right)=\mathbb{Z} / 2 \mathbb{Z}, & \widehat{H}^{1}\left(G_{1} \times G_{3}\right)=(\mathbb{Z} / 2 \mathbb{Z})^{\oplus 2}, & \widehat{H}^{1}\left(G_{2} \times G_{3}\right)=\mathbb{Z} / 2 \mathbb{Z}, \\
\widehat{H}^{2}(G)=(\mathbb{Z} / 2 \mathbb{Z})^{\oplus 4}, & \widehat{H}^{2}\left(G_{1} \times G_{1}\right)=(\mathbb{Z} / 2 \mathbb{Z})^{\oplus 8}, & \widehat{H}^{2}\left(G_{2} \times G_{2}\right)=(\mathbb{Z} / 2 \mathbb{Z})^{\oplus 2}, & \widehat{H}^{2}\left(G_{3} \times G_{3}\right)=(\mathbb{Z} / 2 \mathbb{Z})^{\oplus 8}, \\
& \widehat{H}^{2}\left(G_{1} \times G_{2}\right)=(\mathbb{Z} / 2 \mathbb{Z})^{\oplus 5}, & \widehat{H}^{2}\left(G_{1} \times G_{3}\right)=(\mathbb{Z} / 2 \mathbb{Z})^{\oplus 8}, & \widehat{H}^{2}\left(G_{2} \times G_{3}\right)=(\mathbb{Z} / 2 \mathbb{Z})^{\oplus 5} .
\end{array}
$$

Hence $G$ is indecomposable in $\operatorname{GL}(6, \mathbb{Z})$.

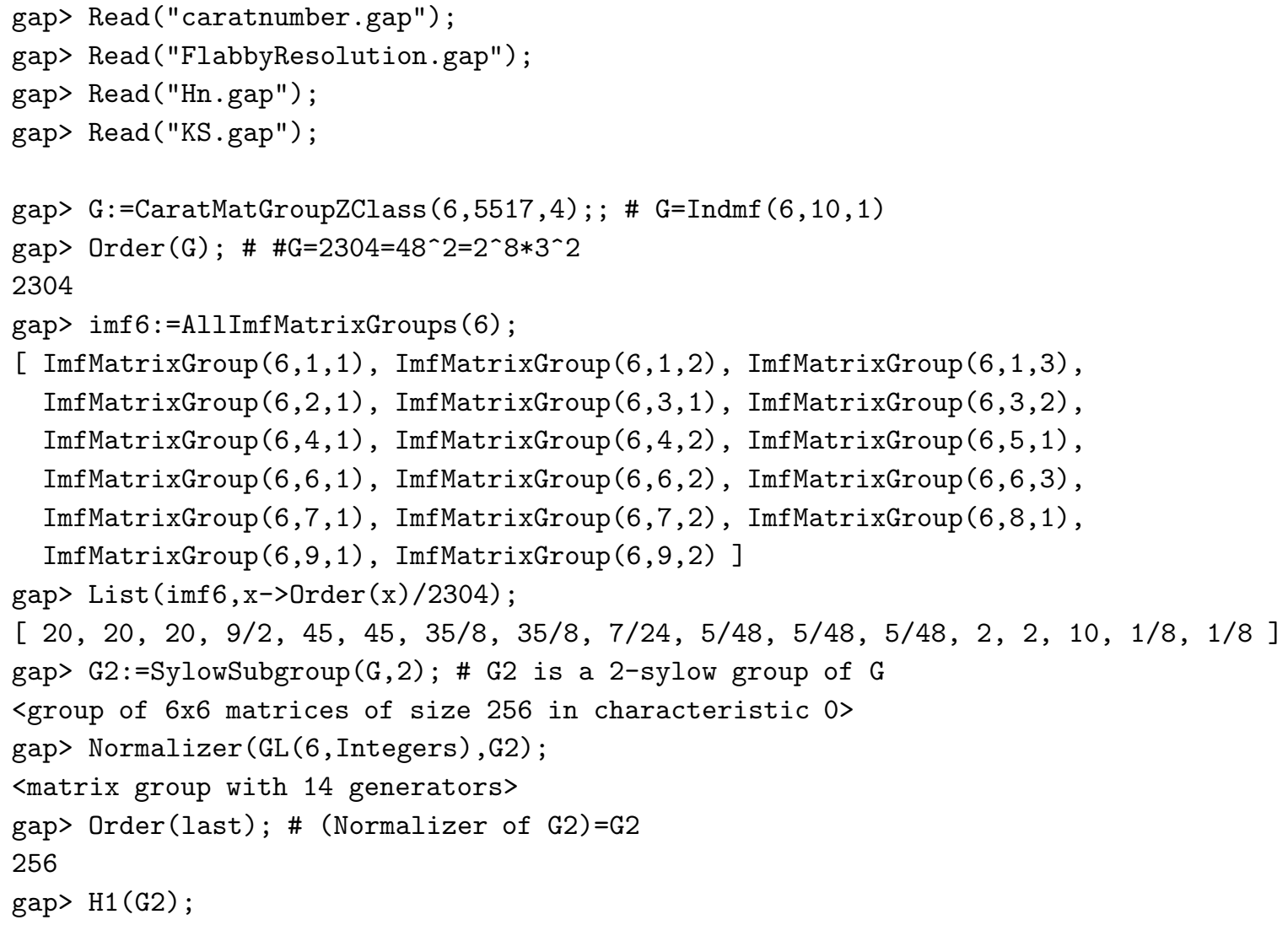


$[1,1,1,1,2,2]$

gap> H1 (SylowSubgroup (ImfMatrixGroup $(6,3,1), 2)$ ) ;

$[1,1,1,1,1,2]$

gap> H1 (SylowSubgroup (ImfMatrixGroup $(6,3,2), 2)$ ) ;

$[1,1,1,1,1,2]$

gap> Set (AllDirectProductIndmfMatrixGroups $([3,3])$, CaratZClass);

$[[6,5517,1],[6,5517,3],[6,5517,5]$,

$[6,5517,6],[6,5517,8],[6,5517,9]]$

gap> ig:=IrreducibleRepresentations $(G) ;$;

gap> List (ig, $\mathrm{x}->\operatorname{Sum}(\mathrm{G}, \mathrm{y}->\operatorname{Trace}(\mathrm{y}) * \operatorname{Trace}(\operatorname{Image}(\mathrm{x}, \mathrm{y}))))$;

$[0,0,0,0,0,0,0,0,0,0,0,0,0,0,0,0,0,0,0,0$,

$0,0,0,0,0,0,0,0,0,0,0,0,0,0,0,0,0,0,0,0$,

$0,0,0,2304,0,0,0,0,0,0,0,0,0,0,0,0,0,0,0,0$,

$0,0,0,0,2304,0,0,0,0,0,0,0,0,0,0,0,0,0,0,0$,

$0,0,0,0,0,0,0,0,0,0,0,0,0,0,0,0,0,0,0,0]$

gap> Filtered([1..Length(ig) $], x->$ last $[x]>0)$;

$[44,65]$

gap> ig [44];

CompositionMapping $([(2,6)(5,8)(7,11),(5,8),(1,12)(2,6)(4,5,9,8)(7,11),(6,7)$,

$(1,2,6)(3,4,8)(5,10,9)(7,12,11),(1,2,6)(7,12,11),(4,9)(5,8),(1,12)(6,7),(2,11)(6,7)$, $(3,10)(4,9)] \rightarrow$

$[[[-1,0,0],[0,1,0],[0,0,1]],[[-1,0,0],[0,1,0],[0,0,1]]$, $[[0,1,0],[-1,0,0],[0,0,1]],[[1,0,0],[0,1,0],[0,0,1]]$,

$[[0,0,1],[1,0,0],[0,1,0]],[[1,0,0],[0,1,0],[0,0,1]]$,

$[[-1,0,0],[0,-1,0],[0,0,1]],[[1,0,0],[0,1,0],[0,0,1]]$,

$[[1,0,0],[0,1,0],[0,0,1]],[[1,0,0],[0,-1,0],[0,0,-1]]]$, <action isomorphism>)

gap> ig[65] ;

CompositionMapping $([(2,6)(5,8)(7,11),(5,8),(1,12)(2,6)(4,5,9,8)(7,11),(6,7)$,

$(1,2,6)(3,4,8)(5,10,9)(7,12,11),(1,2,6)(7,12,11),(4,9)(5,8),(1,12)(6,7),(2,11)(6,7)$, $(3,10)(4,9)] \rightarrow$

$[[[1,0,0],[0,0,1],[0,1,0]],[[1,0,0],[0,1,0],[0,0,1]]$, $[[-1,0,0],[0,0,1],[0,1,0]],[[1,0,0],[0,-1,0],[0,0,1]]$,

$[[0,0,1],[1,0,0],[0,1,0]],[[0,0,1],[1,0,0],[0,1,0]]$,

$[[1,0,0],[0,1,0],[0,0,1]],[[-1,0,0],[0,-1,0],[0,0,1]]$,

$[[1,0,0],[0,-1,0],[0,0,-1]],[[1,0,0],[0,1,0],[0,0,1]]]$, <action isomorphism>)

gap> Imf 3:=AllImfMatrixGroups (3);

[ ImfMatrixGroup (3,1,1), ImfMatrixGroup $(3,1,2), \operatorname{ImfMatrixGroup}(3,1,3)]$

gap> List (Imf3,Size);

[ $48,48,48]$

gap> G11:=DirectProductMatrixGroup $([\operatorname{Imf} 3[1], \operatorname{Imf} 3[1]]) ;$;

gap> G12:=DirectProductMatrixGroup ([Imf3[1], $\operatorname{Imf} 3[2]]) ;$;

gap> G13:=DirectProductMatrixGroup ([Imf3[1], $\operatorname{Imf} 3[3]]) ;$;

gap> G22:=DirectProductMatrixGroup ([Imf3[2], $\operatorname{Imf} 3[2]]) ;$;

gap> G23:=DirectProductMatrixGroup $([\operatorname{Imf} 3[2], \operatorname{Imf} 3[3]]) ;$;

gap> G33:=DirectProductMatrixGroup ([Imf3[3], $\operatorname{Imf} 3[3]]) ;$;

gap> List ( [G11, G22, G33, G12, G13, G23], CaratZClass);

$[[6,5517,1],[6,5517,9],[6,5517,6]$,

$[6,5517,5],[6,5517,3],[6,5517,8]]$

gap> List ( $[\mathrm{G}, \mathrm{G} 11, \mathrm{G} 22, \mathrm{G} 33, \mathrm{G12}, \mathrm{G13}, \mathrm{G} 23], \mathrm{x}->$ TateCohomology $(\mathrm{x},-1)$ ) ;

[ [ 2$],[2,2],[2,2],[],[2,2],[2],[2]]$

gap> List ( $[\mathrm{G}, \mathrm{G} 11, \mathrm{G} 22, \mathrm{G} 33, \mathrm{G} 12, \mathrm{G} 13, \mathrm{G} 23], \mathrm{x}->$ TateCohomology $(\mathrm{x}, 1)$ ); 
$[[2],[2,2],[],[2,2],[2],[2,2],[2]]$

gap> List ( $[\mathrm{G}, \mathrm{G} 11, \mathrm{G} 22, \mathrm{G} 33, \mathrm{G12}, \mathrm{G13}, \mathrm{G} 23], \mathrm{x}->$ TateCohomology $(\mathrm{x}, 2))$;

$[[2,2,2,2],[2,2,2,2,2,2,2,2],[2,2],[2,2,2,2,2,2,2,2]$,

$[2,2,2,2,2],[2,2,2,2,2,2,2,2],[2,2,2,2,2]]$

gap> List (last, Length);

$[4,8,2,8,5,8,5]$

\section{Proof of Theorem 1.27}

Let $G$ be a finite subgroup of $\mathrm{GL}(4, \mathbb{Z})$ and $M=M_{G}$ be the corresponding $G$-lattice of rank 4 as in Definition 1.26

Step 1. The case where $M_{G}$ is decomposable.

We assume that $M_{G} \simeq M_{1} \oplus M_{2}$ is decomposable with rank $M_{1} \geq \operatorname{rank} M_{2} \geq 1$. Thus rank $M_{1} \leq 3$ and rank $M_{2} \leq 2$. By Theorem [1.1 we have $\left[M_{2}\right]^{f l}=0$. It follows from Lemma 2.14 that $\left[M_{G}\right]^{f l}=\left[M_{1}\right]^{f l}$. By Theorem 1.2 and Lemma 2.14. $\left[M_{1}\right]^{f l} \neq 0 \Longleftrightarrow\left[M_{1}\right]^{f l}$ is not invertible $\Longleftrightarrow G / N_{1}$ is conjugate to one of the 15 groups as in Table 1 where $N_{1}=\left\{\sigma \in G \mid \sigma(v)=v\right.$ for any $\left.v \in M_{1}\right\}$. There exist exactly 64 such $G$-lattices $M_{G} \simeq M_{1} \oplus M_{2}$ with $\left[M_{G}\right]^{f l} \neq 0$ (equivalently $\left[M_{G}\right]^{f l}$ is not invertible in this case) whose GAP IDs $\mathcal{N}_{31}$ are computed in Example 4.12 (see also Table 3).

Step 2. $\left[M_{G}\right]^{f l}=0$ for $G=\operatorname{Imf}(4,2,1), \operatorname{Imf}(4,3,1)$ and $\operatorname{Imf}(4,4,1)$.

We assume that $M_{G}$ is indecomposable. There exist 295 indecomposable $G$-lattices $M_{G}$ of rank 4 (see Example 4.9). Let $\operatorname{Imf}(4, i, j) \leq \mathrm{GL}(4, \mathbb{Z})$ be the $j$-th $\mathbb{Z}$-class of the $i$-th $\mathbb{Q}$-class of the irreducible maximal finite group of dimension 4 which corresponds to the GAP command $\operatorname{ImfMatrixGroup}(4, i, j)$. There exist exactly 6 such maximal groups $\operatorname{Imf}(4,1,1), \operatorname{Imf}(4,2,1), \operatorname{Imf}(4,3,1), \operatorname{Imf}(4,3,2), \operatorname{Imf}(4,4,1)$ and $\operatorname{Imf}(4,5,1)$ of order 1152, 288, 240, 240, 384 and 144 respectively. They also form the maximal indecomposable finite groups of dimension 4 (see Subsection 4.0.

By using flfl in Algorithm [F3, we get $\left[M_{G}\right]^{f l}=0$ for $G=\operatorname{Imf}(4,4,1)$ (see Example [5.2).

We may also verify that $\left[M_{G}\right]^{f l}=0$ for $G=\operatorname{Imf}(4,2,1)$ and $\operatorname{Imf}(4,3,1)$ by using StablyPermutationFCheckP in Algorithm[F6: Method II and StablyPermutationFCheck in Algorithm[F5. Method I (1) respectively (see Example 5.7 and Example [5.5). Hence, by Lemma 2.17 $\left[M_{H}\right]^{f l}=0$ for any subgroup $H$ of $\operatorname{Imf}(4,2,1), \operatorname{Imf}(4,3,1)$ and $\operatorname{Imf}(4,4,1)$.

Step 3. Determination of all $G$-lattices $M_{G}$ whose flabby class $\left[M_{G}\right]^{f l}$ is not invertible.

Assume that $M_{G}$ is indecomposable. By Step 2, $\left[M_{H}\right]^{f l}$ is invertible for any subgroups $H$ of $\operatorname{Imf}(4,2,1)$, $\operatorname{Imf}(4,3,1)$ and $\operatorname{Imf}(4,4,1)$. We will check whether $\left[M_{H}\right]^{f l}$ is invertible for subgroups $H$ of $\operatorname{Imf}(4,1,1), \operatorname{Imf}(4,3,2)$ and $\operatorname{Imf}(4,5,1)$.

There exist 182, 36 and 50 indecomposable $G$-lattices $M_{G}$ where $G$ is (a conjugacy class of) a subgroup of $\operatorname{Imf}(4,1,1) \operatorname{Imf}(4,3,2)$ and $\operatorname{Imf}(4,5,1)$ respectively. Some of them are also subgroups of $\operatorname{Imf}(4,2,1), \operatorname{Imf}(4,3,1)$ or $\operatorname{Imf}(4,4,1)$ as in Step 2. Then we should treat 166, 8 and 27 indecomposable $G$-lattices $M_{G}$ where $G$ is a subgroup of $\operatorname{Imf}(4,1,1) \operatorname{Imf}(4,3,2)$ and $\operatorname{Imf}(4,5,1)$ respectively but not a subgroup of $\operatorname{Imf}(4,2,1), \operatorname{Imf}(4,3,1)$ and $\operatorname{Imf}(4,4,1)$. We denote the set of the corresponding GAP IDs of $M_{G}$ in each case by $\widetilde{\mathcal{U}}_{411}, \widetilde{\mathcal{U}}_{432}$ and $\widetilde{\mathcal{U}}_{451}$, i.e. $\# \widetilde{\mathcal{U}}_{411}=166, \# \widetilde{\mathcal{U}}_{432}=8, \# \widetilde{\mathcal{U}}_{451}=27$, (see Example 10.1 below).

By using IsInvertibleF in Algorithm F2, we may determine all of the $G$-lattices $M_{G}$ whose flabby class $\left[M_{G}\right]^{f l}$ is not invertible. There exist 137, 0, $27 G$-lattices $M_{G}$ with $\left[M_{G}\right]^{f l}$ not invertible where the GAP ID of $G$ is $l \in \widetilde{\mathcal{U}}_{411}, \widetilde{\mathcal{U}}_{432}, \widetilde{\mathcal{U}}_{451}$ respectively. Note that $\left[M_{G}\right]^{f l}$ is invertible for $G=\operatorname{Imf}(4,3,2)$. Because the $12 G$-lattices of them are overlapped, there exist $152 G$-lattices $M_{G}$ with $\left[M_{G}\right]^{f l}$ not invertible (see Example 10.1 below and Table 4).

Step 4. Determination whether $\left[M_{G}\right]^{f l}=0$. 
We should determine whether $\left[M_{G}\right]^{f l}=0$ for the remaining cases $\mathcal{U}_{411}$ and $\mathcal{U}_{432}$ of $\widetilde{\mathcal{U}}_{411}$ and $\widetilde{\mathcal{U}}_{432}$ as in Step 3. We see that $\# \mathcal{U}_{411}=29, \# \mathcal{U}_{432}=8$ and $\mathcal{U}_{411}$ and $\mathcal{U}_{432}$ are given by

$$
\begin{aligned}
& \mathcal{U}_{411}=\{(4,5,1,7),(4,5,1,11),(4,5,1,13),(4,6,1,10),(4,6,1,12),(4,6,2,11),(4,12,1,6),(4,12,1,7), \\
&(4,12,3,10),(4,12,3,12),(4,12,3,13),(4,12,4,13),(4,13,1,6),(4,13,2,6),(4,13,3,6),(4,13,4,6), \\
&(4,13,6,6),(4,13,7,12),(4,24,1,4),(4,24,1,6),(4,24,3,4),(4,24,3,6),(4,24,4,6),(4,25,1,5), \\
&(4,25,3,5),(4,25,4,5),(4,25,7,5),(4,25,8,5),(4,33,2,1)\}, \\
& \mathcal{U}_{432}=\{(4,31,1,3),(4,31,1,4),(4,31,2,2),(4,31,3,2),(4,31,4,2),(4,31,5,2),(4,31,6,2),(4,31,7,2)\} .
\end{aligned}
$$

We will show that there exist exactly 7 groups $G$ of the GAP IDs $(4,33,2,1) \in \mathcal{U}_{411}$ and $(4,31,1,3),(4,31,1,4)$, $(4,31,2,2),(4,31,4,2),(4,31,5,2)$ and $(4,31,7,2) \in \mathcal{U}_{432}$ such that $\left[M_{G}\right]^{f l} \neq 0$.

First we treat the case of $\mathcal{U}_{411}$. Each group $G$ of the GAP ID $l \in \mathcal{U}_{411}$ is contained in at least one of the groups of the 21st, 27 th, 28 th and 29 th GAP IDs, i.e. $(4,24,3,4),(4,25,7,5),(4,25,8,5)$ and $(4,33,2,1)$, in $\mathcal{U}_{411}$ (see Example 10.2 below). By using flfl in Algorithm[F3, (resp. StablyPermutationFCheckP in Algorithm F6, Method II), we may confirm that $\left[M_{G}\right]^{f l}=0$ for the 21st and 27th (resp. 28th) groups $G$ of the GAP ID $(4,24,3,4)$ and $(4,25,7,5)$ (resp. $(4,25,8,5))$ (see Example 10.2 below). None of the groups $G$ of the GAP ID $l \in \mathcal{U}_{411}$ is contained in the 29th group $G$ other than itself. By Lemma 2.17 $\left[M_{G}\right]^{f l}=0$ for all groups $G$ of the GAP ID $l \in \mathcal{U}_{411}$ except for the 29th group $G$ of the GAP ID $(4,33,2,1)$. By using PossibilityOfStablyPermutationF in Algorithm F5, we may see that $\left[M_{G}\right]^{f l} \neq 0$ for the 29th group $G$ (see Example 10.2 below).

Next we will consider the case of $\mathcal{U}_{432}$. By using PossibilityOfStablyPermutationF in Algorithm F5, we see that $\left[M_{G}\right]^{f l} \neq 0$ for the 1 st and the 2 nd groups $G$ of the GAP IDs $(4,31,1,3)$ and $(4,31,1,4)$ (see Example 5.4 and Example 10.3 below). Because the 3rd, 5th, 6 th and 8 th groups $G$ of the GAP IDs $(4,31,2,2),(4,31,4,2),(4,31,5,2)$ and $(4,31,7,2)$ contain the 1 st or 2 nd ones, it follows from Lemma 2.17 that $\left[M_{G}\right]^{f l} \neq 0$. We may confirm that $\left[M_{G}\right]^{f l}=0$ for the 4 th and 7 th groups $G$ of the GAP IDs $(4,31,3,2)$ and $(4,31,6,2)$ by using StablyPermutationFCheckP in Algorithm F6, Method II (see Example 10.3 below).

Step 5. $\left[M_{G}\right]^{f l}=0$ if and only if $\left[M_{G}\right]^{f l}$ is of finite order in $\mathcal{C}(G) / \mathcal{S}(G)$.

We should show that if $\left[M_{G}\right]^{f l} \neq 0$, then $\left[M_{G}\right]^{f l}$ is of infinite order in $\mathcal{C}(G) / \mathcal{S}(G)$. Note that $\left[M_{G}\right]^{f l}$ is of finite order if and only if there exist permutation $G$-lattices $P, P^{\prime}$ such that $\left[\left(M_{G}\right)^{\oplus r}\right]^{f l} \oplus P \simeq P^{\prime}$ for some $r \geq 1$. Thus if $\left[M_{G}\right]^{f l}$ is not invertible, then $\left[M_{G}\right]^{f l}$ is of infinite order. Hence it is enough to verify that $\left[\left(M_{G}\right)^{\oplus r}\right]^{f l} \neq 0$ for the 7 cases as in Table 2, i.e. the 7 cases where $\left[M_{G}\right]^{f l}$ is not zero but invertible. By Step 4, we should show that $\left[\left(M_{G}\right)^{\oplus r}\right]^{f l} \neq 0$ for only the 3 groups $G$ of the GAP IDs $(4,31,1,3),(4,31,1,4)$ and $(4,33,2,1)$ because the remaining 4 groups contain the 1st or the 2 nd one.

By using PossibilityOfStablyPermutationF as in Algorithm F4, for 2 groups $G \simeq F_{20}$ of the GAP IDs $(4,31,1,3)$ and $(4,31,1,4)$, we get the flabby class $[F]=\left[M_{G}\right]^{f l}$ with rank 16 and see that the only possibility of the isomorphism (10) is

$$
\mathbb{Z}[G] \oplus \mathbb{Z}\left[G / C_{2}\right] \oplus \mathbb{Z}\left[G / C_{5}\right] \simeq \mathbb{Z}\left[G / D_{5}\right] \oplus F^{\oplus 2}
$$

(the rank of the both sides is $20+10+4=2+2 \times 16=34$ ). By using the algorithm TateCohomology as in Section 9, however, for the group $G$ of the GAP ID $(4,31,1,3)$, we see that the 2nd (Tate) cohomologies of the both sides are $\widehat{H}^{2}\left(G_{1}, M_{G_{1}}\right)=\mathbb{Z} / 2 \mathbb{Z}$ and $\widehat{H}^{2}\left(G_{2}, M_{G_{2}}\right)=\mathbb{Z} / 10 \mathbb{Z}$ where $G_{1}$ (resp. $\left.G_{2}\right)$ is the matrix representation group of the action of $G$ on the left (resp. right) hand side of (13). Thus the isomorphism (13) is impossible. Hence $\left[F^{\oplus r}\right] \neq 0$ for any $r \geq 1$. Similarly, for the group $G$ of the GAP ID $(4,31,1,4)$, we see that $\widehat{H}^{2}\left(G_{1}, M_{G_{1}}\right)=\mathbb{Z} / 5 \mathbb{Z} \oplus \mathbb{Z} / 10 \mathbb{Z}$ and $\widehat{H}^{2}\left(G_{2}, M_{G_{2}}\right)=\mathbb{Z} / 10 \mathbb{Z}$ (see Example 10.4 below).

For the group $G \simeq C_{3} \rtimes C_{8}$ of the GAP ID $(4,33,2,1)$, by using PossibilityOfStablyPermutationF as in Algorithm F4, the only possibility of (10) is

$$
\mathbb{Z}[G] \oplus \mathbb{Z}\left[G / C_{2}\right] \oplus \mathbb{Z}\left[G / C_{3}\right] \simeq \mathbb{Z}\left[G / C_{6}\right] \oplus F^{\oplus 2}
$$

where $F$ is of rank 20 with $[F]=\left[M_{G}\right]^{f l}$ (the rank of the both sides is $24+12+8=4+2 \times 20=44$ ). However, the Tate cohomologies of the both sides $\widehat{H}^{n}\left(G_{1}, M_{G_{1}}\right) \simeq \widehat{H}^{n}\left(G_{2}, M_{G_{2}}\right)$ coincide for $-7 \leq n \leq 7$ (see Example 10.4 below). Thus we need another observation.

Assume that the isomorphism (14) holds. Let $G_{1}$ (resp. $G_{2}$ ) be the matrix representation group of the action of $G$ on the left (resp. right) hand side of (14). By using StablyPermutationFCheckGen as in Algorithm F6. Method II, we may obtain $G_{1}$ and $G_{2}$ of rank 44 (see Example 10.4 below). We choose the generators 
$a, b$ of $G$ such that $G=\left\langle a, b \mid a^{3}=b^{8}=1, b^{-1} a b=a^{-1}\right\rangle \simeq\langle a\rangle \rtimes\langle b\rangle$. We take the reduction $\overline{G_{1}}=\left\langle\overline{a_{1}}, \overline{b_{1}}\right\rangle$, $\overline{G_{2}}=\left\langle\overline{a_{2}}, \overline{b_{2}}\right\rangle \leq \mathrm{GL}\left(44, \mathbb{F}_{3}\right)$ over the field $\mathbb{F}_{3}$ of 3 elements. Because the subgroup $\langle a\rangle \simeq C_{3}$ is normal in $G$, $v_{i}=\left\{\left(\overline{a_{i}-I_{44}}\right)^{2} \mid a_{i} \in G_{i}\right\}$ is $\overline{G_{i}}$-invariant $(i=1,2)$. Consider the action of $b$ on the $\overline{G_{i}}$-invariant space $v_{i}$ over the field $\mathbb{F}_{9}$ of 9 elements and compare the dimensions of each eigen spaces of the action matrix of $b$ on $v_{i}$ $(i=1,2)$ (we see $\operatorname{dim}\left(v_{1}\right)=\operatorname{dim}\left(v_{2}\right)=12$ ). Then we get the (multi-)set of such dimensions $\{2,2,2,2,2,0,2,0\}$ and $\{2,2,1,2,1,1,2,1\}$ for $v_{1}$ and $v_{2}$ respectively (see Example 10.4 below). This yields a contradiction. Hence the isomorphism (14) is impossible.

Step 6. $\left[M_{G}\right]^{f l}=-\left[J_{S_{5} / S_{4}}\right]^{f l}$ for the group $G \simeq S_{5}$ of the GAP ID $(4,32,5,2)$.

Let $G_{1}, G_{2} \simeq S_{5} \leq \mathrm{GL}(4, \mathbb{Z})$ be the groups of the GAP IDs $(4,31,4,2)$ and $(4,31,5,2)$. Note that $M_{G_{1}} \simeq$ $J_{S_{5} / S_{4}}$. Let $M_{G} \simeq M_{G_{1}} \oplus M_{G_{2}}$ be the $S_{5}$-lattice of rank 8. By Step 4, $\left[M_{G_{1}}\right]^{f l}$ and $\left[M_{G_{2}}\right]^{f l}$ is not zero but invertible, and we will show that $\left[M_{G}\right]^{f l}=[F]=0$.

By using StablyPermutationFCheckGen as in Algorithm F6: Method II, it is possible that

$$
\mathbb{Z}\left[G / H_{4}\right] \oplus \mathbb{Z}\left[G / H_{13}\right] \oplus F \simeq \mathbb{Z}\left[G / H_{8}\right] \oplus \mathbb{Z}\left[G / H_{9}\right] \oplus \mathbb{Z}\left[G / H_{10}\right] \oplus \mathbb{Z}\left[G / H_{11}\right]
$$

where $H_{4} \simeq C_{3}, H_{13} \simeq D_{5}, H_{8} \simeq C_{5}, H_{9} \simeq C_{6}, H_{10} \simeq H_{11} \simeq S_{3}$ (the rank of the both sides is $40+12+32=$ $24+20+20+20=84)$. However, we could not establish the isomorphism (15).

As in Example 5.6 (Algorithm F5, Method I (2)), after adding $\mathbb{Z}$ to the both sides of (15), we may confirm that the isomorphism

$$
\mathbb{Z}\left[G / C_{3}\right] \oplus \mathbb{Z}\left[G / D_{5}\right] \oplus F \oplus \mathbb{Z} \simeq \mathbb{Z}\left[G / C_{5}\right] \oplus \mathbb{Z}\left[G / C_{6}\right] \oplus \mathbb{Z}\left[G / S_{3}^{(1)}\right] \oplus \mathbb{Z}\left[G / S_{3}^{(2)}\right] \oplus \mathbb{Z}
$$

holds (see Example 10.5 below)

Step 7. $\left[M_{G}\right]^{f l}=-\left[J_{F_{20} / C_{4}}\right]^{f l}$ for the group $G \simeq F_{20}$ of the GAP ID $(4,31,1,4)$.

Let $G_{1}^{\prime}, G_{2}^{\prime} \simeq F_{20} \leq \mathrm{GL}(4, \mathbb{Z})$ be the groups of the GAP IDs $(4,31,1,3)$ and $(4,31,1,4)$. Note that $M_{G_{1}^{\prime}} \simeq$ $J_{F_{20} / C_{4}}$. Let $M_{G} \simeq M_{G_{1}^{\prime}} \oplus M_{G_{2}^{\prime}}$ be the $F_{20}$-lattice of rank 8. By Step 4, $\left[M_{G_{1}^{\prime}}\right]^{f l}$ and $\left[M_{G_{2}^{\prime}}\right]^{f l}$ is not zero but invertible. We should show that $\left[M_{G}\right]^{f l}=[F]=0$. Because $G_{1}^{\prime}$ (resp. $G_{2}^{\prime}$ ) is a subgroup of $G_{1}$ (resp. $G_{2}$ ) where $G_{1}$ (resp. $G_{2}$ ) is the group $S_{5}$ as in Step 6, the assertion follows from Step 6 and Lemma 2.17

We will give another (direct) proof below. By using StablyPermutationFCheckGen as in Algorithm F6. Method II, it is possible that

$$
\mathbb{Z}[G] \oplus \mathbb{Z}\left[G / C_{2}\right] \oplus \mathbb{Z}\left[G / C_{5}\right] \simeq \mathbb{Z}\left[G / D_{5}\right] \oplus F
$$

(the rank of the both sides is $20+10+4=2+32=34$ ). And, after some efforts, we may confirm that the isomorphism (16) actually holds (see Example 10.6 below).

Example 10.1 (Determination whether $\left[M_{G}\right]^{f l}$ is invertible).

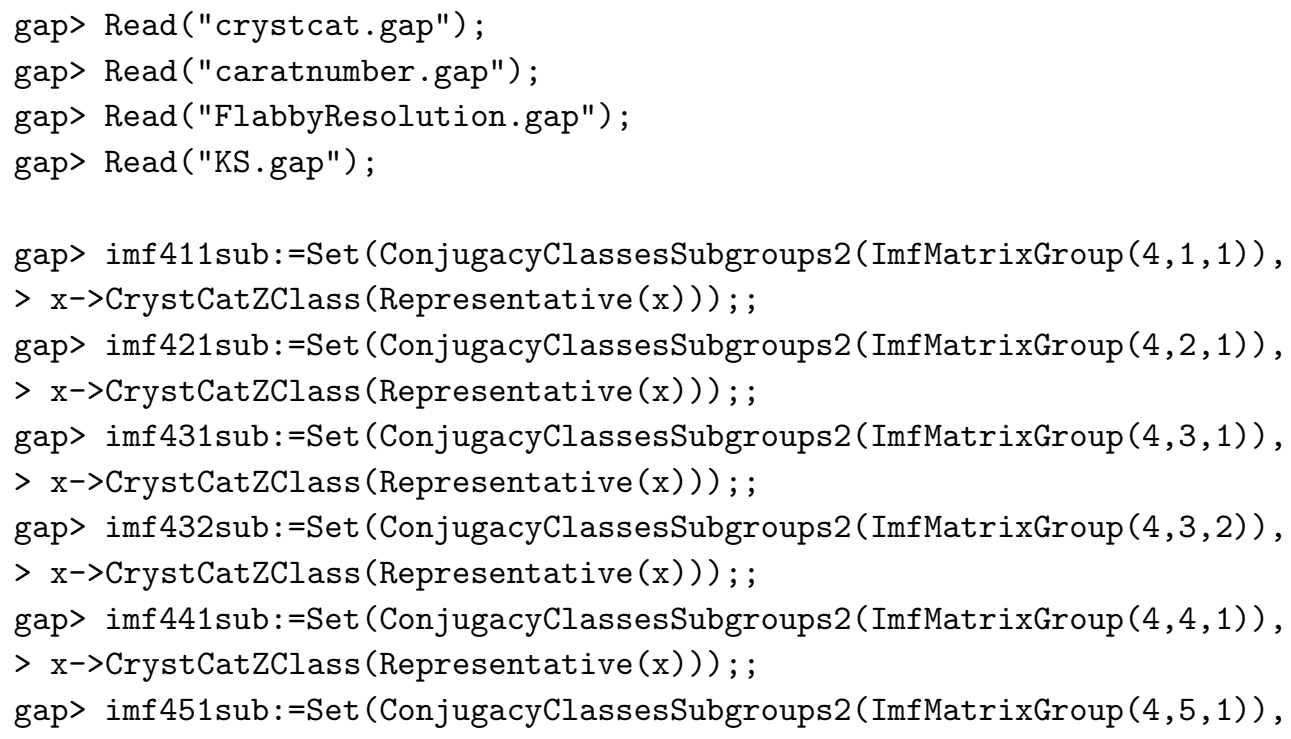


> x->CrystCatZClass (Representative (x))); ;

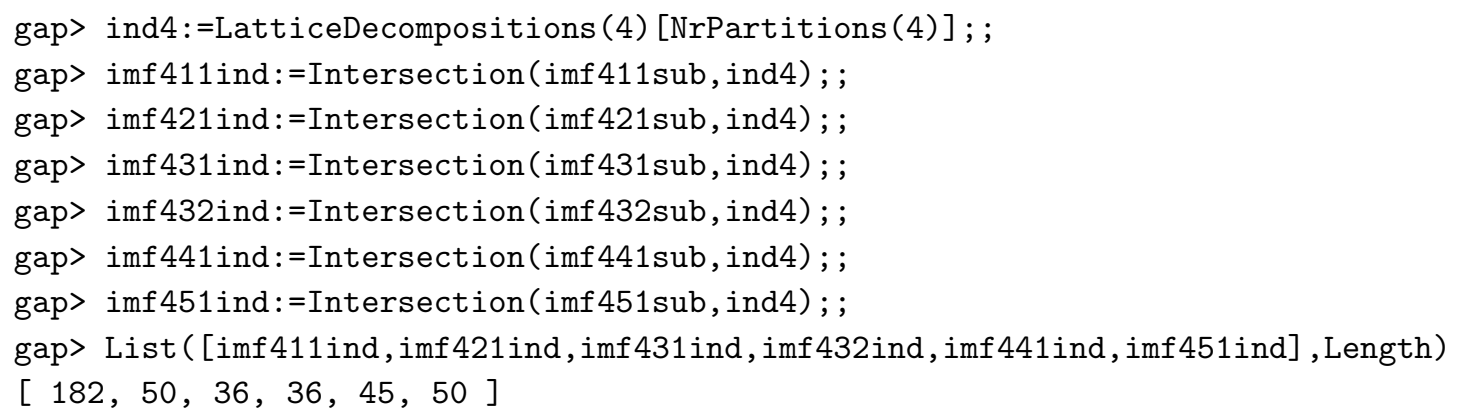

Example 10.2 (Determination whether $\left[M_{G}\right]^{f l}=0$ for the GAP ID $l \in \mathcal{U}_{411}$ ). 
gap> U411inc:=List (U411sub, $\mathrm{x}^{->\operatorname{Set}}$ (Intersection ( $\left.\mathrm{x}, \mathrm{U} 411\right), \mathrm{y}->$ Position (U411,y))) ;

$[[1],[2],[3],[2,4],[3,5],[2,6],[7],[8],[1,7,9]$,

$[2,8,10],[3,8,11],[3,8,12],[8,13],[8,14],[2,3,15]$,

$[2,3,16],[2,3,4,5,8,10,11,13,16,17]$,

$[2,3,5,6,8,10,12,14,15,18],[1,19],[3,20]$,

$[1,7,9,19,21],[3,8,11,20,22],[3,8,12,20,23]$,

$[3,5,20,24],[2,3,16,20,25],[2,3,15,20,26]$,

$[2,3,4,5,8,10,11,13,16,17,20,22,24,25,27]$,

$[2,3,5,6,8,10,12,14,15,18,20,23,24,26,28],[29]]$

gap> U411[21]; \# checking $[\mathrm{M}]-\{\mathrm{fl}\}=0$

$[4,24,3,4]$

gap> G:=MatGroupZClass $(4,24,3,4) ; ; \# \mathrm{G}=\mathrm{S} 4$

gap> $f l f l(G)$;

[ ]

gap> U411[27]; \# checking $[\mathrm{M}] \sim\{f 1\}=0$

$[4,25,7,5]$

gap> G:=MatGroupZClass $(4,25,7,5) ;$; \# G=C2xS4

gap $>f l f(G)$;

[ ]

gap> U411[28]; \# checking $[\mathrm{M}] \wedge\{\mathrm{fl}\}=0$

$[4,25,8,5]$

gap> G:=MatGroupZClass $(4,25,8,5) ;$; \# G=C2xS4

gap> Rank(FlabbyResolution(G).actionF.1); \#F is of rank 10

10

gap> mis:=SearchCoflabbyResolutionBase (TransposedMatrixGroup (G) ,5) ; ;

gap> Set(List(mis, Length))-4; \# Method III could not apply

$[10,16,18,22,24,26,28,30,32,34,36,38,40,42,44,46,50]$

gap> 11:=Possibility0fStablyPermutationF $(G)$;

$[[1,0,0,0,0,-2,-1,0,0,0,0,0,0,0,0,0,0,2,0,0,1,1,0,-1,0$, $0,0,0,1,1,-1,1,-1,-1]$

$[0,1,0,0,0,0,0,0,0,0,0,0,0,0,0,-2,-1,0,0,0,1,0,0,0,0$, $0,0,2,1,1,0,0,-1,-1]$,

$[0,0,0,0,1,-1,0,0,0,0,0,0,0,0,0,0,0,1,-1,0,0,1,0,-1,0$, $0,0,0,0,0,-1,1,0,0]$,

$[0,0,0,0,0,0,0,1,0,0,0,0,0,0,0,0,0,0,0,0,1,-1,0,-1,0$, $0,-1,0,1,1,1,1,-1,-1]$,

$[0,0,0,0,0,0,0,0,0,0,0,0,0,0,0,0,0,0,0,1,1,0,0,0,0$, $0,0,0,-1,0,0,0,1,-1]]$

gap> $1:=11[$ Length $(11)]$;

$[0,0,0,0,0,0,0,0,0,0,0,0,0,0,0,0,0,0,0,1$,

$1,0,0,0,0,0,0,0,-1,0,0,0,1,-1]$

gap> bp:=StablyPermutationFCheckP(G,Nlist (1),Plist (1)); ;

gap> Length (bp);

20

gap> Length(bp[1]); \# rank of the both sides of (10) is 13

13

gap> rs:=RandomSource (IsMersenneTwister);

<RandomSource in IsMersenneTwister>

gap > rr:=List $\left([1 \ldots 1000], x^{->} \operatorname{List}\left([1 \ldots 20], y^{->} \operatorname{Random}(r s,[0,1])\right)\right)$; ;

gap> Filtered ( $r r, x->$ Determinant $(x * b p) ` 2=1)$; 
$[[0,1,1,1,1,1,0,1,1,1,0,0,1,0,0,1,0,0,0,0]$,

$[0,1,0,1,1,1,0,1,0,1,0,1,0,0,1,1,0,0,0,1]]$

gap> $\mathrm{p}:=$ last $[1] * \mathrm{bp}$;

$[[0,1,1,1,0,1,1,1,1,1,1,1,1]$,

$[1,0,1,0,1,1,1,1,1,1,1,1,1]$,

$[1,1,0,1,1,0,1,1,1,1,1,1,1]$,

$[0,0,0,1,1,1,1,1,1,1,1,1,1]$,

$[1,1,1,0,0,0,1,1,1,1,1,1,1]$,

$[0,0,1,0,0,2,1,0,0,0,0,0,0]$,

$[0,2,0,1,0,0,1,0,0,0,0,0,0]$,

$[1,0,0,0,2,0,0,1,0,0,0,0,0]$,

$[0,0,1,0,0,2,0,0,0,0,0,1,0]$,

$[0,0,2,0,0,1,0,1,0,0,0,0,0]$,

$[2,0,0,0,1,0,0,0,1,0,0,0,0]$,

$[1,0,0,0,2,0,0,0,0,0,1,0,0]$,

$[2,0,0,0,1,0,0,0,0,1,0,0,0]]$

gap> Determinant $(p)$;

1

gap> StablyPermutationFCheckMat (G,Nlist (1), Plist(1),p);

true

gap> U411[29]; \# checking [M]^\{fl\}: non-zero

[ 4, 33, 2, 1 ]

gap> G:=MatGroupZClass $(4,33,2,1) ; ;$ \# G=C3:C8

gap> PossibilityOfStablyPermutationF $(G)$;

$[[1,1,1,0,-1,0,0,0,-2]]$

gap> i411: $=[\mathrm{U} 411[29]]$;

$[[4,33,2,1]]$

Example 10.3 (Determination whether $\left[M_{G}\right]^{f l}=0$ for the GAP ID $l \in \mathcal{U}_{432}$ ).

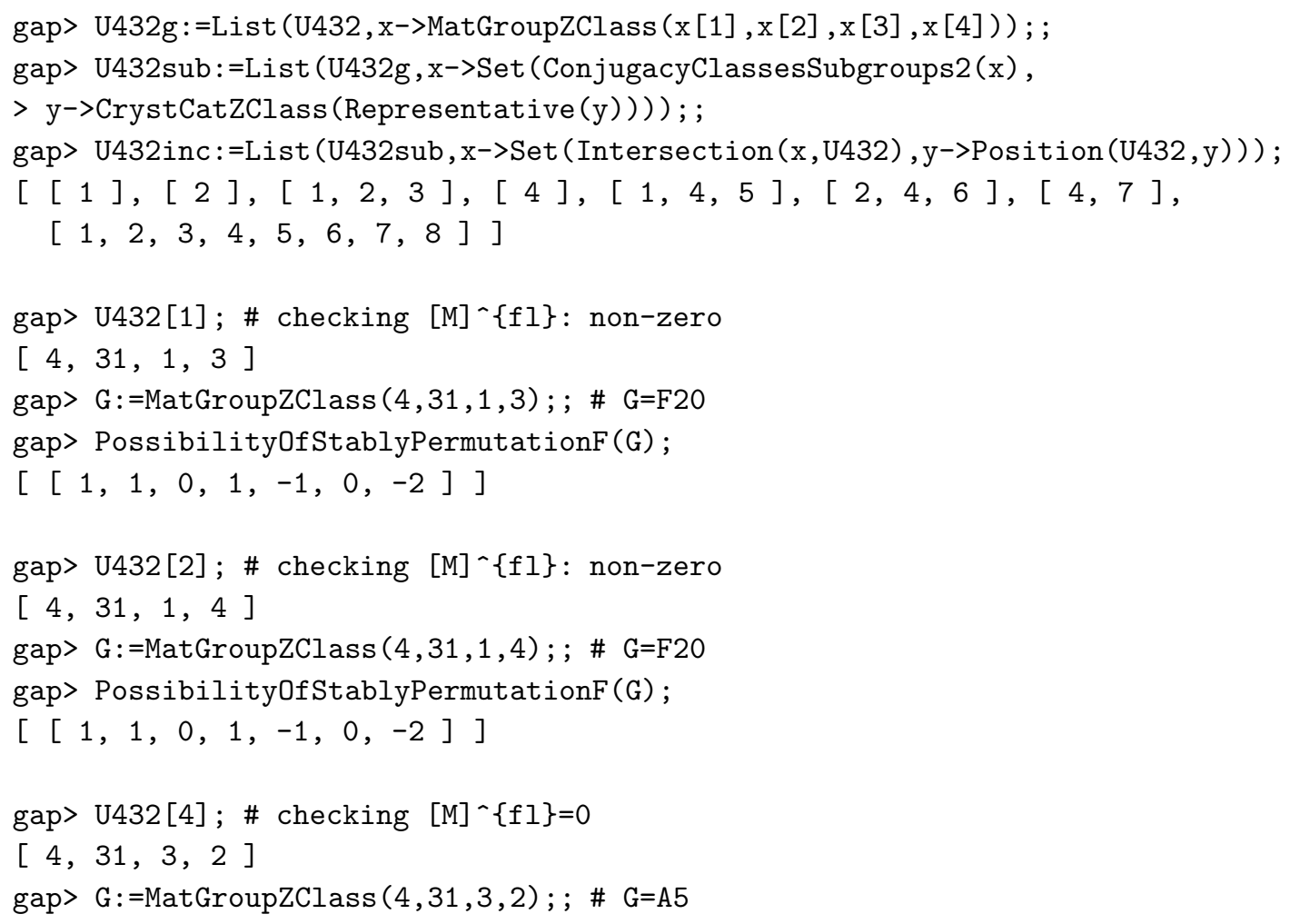


gap> Rank(FlabbyResolution(G).actionF.1); \#F is of rank 16 16

gap> mis:=SearchCoflabbyResolutionBase (TransposedMatrixGroup (G) ,5); ;

gap> Set(List(mis, Length))-4; \# Method III could not apply

$[16,21,26,31,36,41,46,51,56,61,66,71,76,81$ ]

gap> 1l:=Possibility0fStablyPermutationF (G);

$[[1,-2,-1,0,0,1,1,1,-1,0],[0,0,0,0,1,1,-1,0,0,-1]]$

gap> 1:=11[Length $(11)]$;

$[0,0,0,0,1,1,-1,0,0,-1]$

gap> bp:=StablyPermutationFCheckP(G,Nlist (1),Plist(1)); ;

gap> Length(bp);

11

gap> Length(bp[1]); \# rank of the both sides of (10) is 22

22

gap> rs:=RandomSource (IsMersenneTwister);

<RandomSource in IsMersenneTwister>

gap> $r r:=\operatorname{List}([1 \ldots 100000], \mathrm{x}->\operatorname{List}([1 \ldots 11], \mathrm{y}->\operatorname{Random}(\mathrm{rs},[-1 \ldots 2])))$; ;

gap> Filtered $\left(r r, x->\operatorname{Determinant}(\mathrm{x} * \mathrm{bp}){ }^{\wedge} 2=1\right)$;

$[[2,0,2,-1,0,0,1,0,0,1,1],[2,0,0,1,-1,1,0,1,0,1,1]]$ gap> p:=last $[1] * \mathrm{bp}$;

$[[2,0,0,2,0,0,0,0,0,0,0,0,2,-1,2,2,2,-1,2,-1,-1,-1]$, $[0,2,0,0,0,2,0,0,0,0,0,0,2,2,-1,2,-1,2,-1,-1,-1,2]$, $[0,0,2,0,0,0,0,0,0,2,0,0,-1,2,2,2,-1,-1,-1,2,2,-1]$, $[0,0,0,0,2,0,0,0,0,0,2,0,2,-1,-1,-1,2,2,-1,2,2,-1]$, $[0,0,0,0,0,0,2,0,0,0,0,2,-1,2,-1,-1,2,-1,2,2,-1,2]$, $[0,0,0,0,0,0,0,2,2,0,0,0,-1,-1,2,-1,-1,2,2,-1,2,2]$, $[0,0,0,1,0,0,0,1,0,1,0,0,0,0,1,1,0,0,1,0,1,0]$, $[0,0,0,1,0,0,1,1,0,0,0,0,0,0,1,0,1,0,1,0,0,1]$, $[0,1,1,0,0,0,1,0,0,0,0,0,0,1,0,1,0,0,0,1,0,1]$, $[1,0,0,0,0,0,0,0,1,0,0,1,0,0,1,0,1,0,1,0,0,1]$, $[0,0,0,1,1,0,1,0,0,0,0,0,1,0,0,0,1,0,1,1,0,0]$, $[0,0,1,0,1,0,1,0,0,0,0,0,0,1,0,0,1,0,0,1,1,0]$, $[1,0,1,0,0,0,0,0,1,0,0,0,0,0,1,1,0,0,1,0,1,0]$, $[0,0,1,0,1,0,0,0,1,0,0,0,0,0,1,0,0,1,0,1,1,0]$, $[0,0,0,0,1,1,0,0,1,0,0,0,1,0,0,0,0,1,0,0,1,1]$, $[0,0,0,0,0,1,0,0,0,1,0,1,0,1,0,1,0,0,0,1,0,1]$, $[1,0,0,0,0,0,0,0,0,0,1,1,1,0,0,0,1,0,1,1,0,0]$, $[0,0,0,0,0,0,0,0,0,1,1,1,0,1,0,0,1,0,0,1,1,0]$, $[0,0,0,1,1,1,0,0,0,0,0,0,1,0,0,1,1,1,0,0,0,0]$, $[1,1,1,0,0,0,0,0,0,0,0,0,1,1,1,1,0,0,0,0,0,0]$, $[0,0,0,0,0,0,0,1,0,1,1,0,0,0,1,0,0,1,0,1,1,0]$, $[0,0,0,0,0,1,0,0,1,0,0,1,0,1,0,0,0,1,1,0,0,1]]$ gap> Determinant $(p)$;

1

gap> StablyPermutationFCheckMat (G,Nlist(1),Plist (1),p);

true

gap> U432[7]; \# checking $[M] \wedge\{f 1\}=0$

$[4,31,6,2]$

gap> G:=MatGroupZClass $(4,31,6,2) ; ; \# \mathrm{G}=\mathrm{C} 2 \times \mathrm{xA} 5$

gap> Rank(FlabbyResolution(G).actionF.1); \#F is of rank 16 16 
gap> mis: =SearchCoflabbyResolutionBase (TransposedMatrixGroup(G),5); ;

gap> Set(List(mis,Length)) 4 ; \# Method III could not apply

$[16,26,36,46,56,66,76,86,96,106,116,126,136,146,156,166$ ]

gap> $11:=$ PossibilityOfStablyPermutationF $(G)$;

[ [ $1,0,0,-2,0,0,0,0,-1,0,-1,1,0,0,1,0,-1,-1,1,2,1,-2,1]$,

$[0,1,0,0,0,0,0,-2,0,0,-1,0,0,0,0,0,0,1,1,1,0,-1,0]$,

$[0,0,1,-1,0,0,0,0,0,0,0,1,0,0,0,-1,-1,-1,1,1,1,-1,0]$,

$[0,0,0,0,1,0,0,0,-1,-1,-1,-1,0,0,1,1,0,1,-1,0,0,0,1]$,

$[0,0,0,0,0,0,0,0,0,0,0,0,0,1,0,0,0,1,-1,0,0,0,-1]]$

gap> $1:=11$ [Length $(11)]$;

$[0,0,0,0,0,0,0,0,0,0,0,0,0,1,0,0,0,1,-1,0,0,0,-1]$

gap> bp:=StablyPermutationFCheckP(G,Nlist(1), Plist(1)); ;

gap> Length(bp);

11

gap> Length(bp[1]); \# rank of the both sides of (10) is 22

22

gap> rs:=RandomSource (IsMersenneTwister);

<RandomSource in IsMersenneTwister>

gap $>\mathrm{rr}:=\operatorname{List}([1 \ldots 100000], \mathrm{x}->\operatorname{List}([1 \ldots 11], \mathrm{y}->\operatorname{Random}(\mathrm{rs},[-1 \ldots 2])))$; ;

gap $>$ Filtered $\left(r r, x->\right.$ Determinant $\left.(x * b p)^{\wedge} 2=1\right)$;

$[[2,1,0,1,0,0,-1,0,0,0,-1],[2,0,1,0,1,-1,0,1,0,1,1]$,

$[2,0,1,0,1,-1,0,1,0,1,1],[2,0,0,1,1,-1,-1,0,0,-1,-1]$,

$[2,1,2,-1,0,0,-1,0,-1,1,2],[2,1,-1,0,0,0,0,-1,0,0,1]]$ gap> p:=last $[1] * \mathrm{bp}$;

[ $[2,1,1,1,1,1,2,1,1,1,1,1,0,1,0,1,0,0,0,1,1,1]$,

$[1,2,1,1,1,1,1,1,1,1,1,2,1,0,0,1,1,0,1,0,1,0]$,

$[1,1,2,1,2,1,1,1,1,1,1,1,0,0,1,0,1,0,1,1,0,1]$,

$[1,1,1,2,1,1,1,1,1,2,1,1,1,1,0,0,0,1,1,1,0,0]$,

$[1,1,1,1,1,2,1,1,2,1,1,1,1,0,1,1,0,1,0,0,0,1]$,

$[1,1,1,1,1,1,1,2,1,1,2,1,0,1,1,0,1,1,0,0,1,0]$,

$[0,0,-1,0,0,0,0,-1,0,0,0,-1,0,0,0,0,-1,0,0,0,0,0]$,

$[-1,0,0,0,-1,0,0,0,-1,0,0,0,0,0,0,0,0,0,0,0,0,-1]$,

$[-1,0,0,0,-1,0,0,0,0,-1,0,0,0,0,0,0,0,0,0,-1,0,0]$,

$[0,0,0,-1,0,0,0,0,-1,0,-1,0,0,0,0,0,0,-1,0,0,0,0]$,

$[0,0,-1,-1,0,0,0,0,0,0,0,-1,0,0,0,0,0,0,-1,0,0,0]$,

$[0,0,0,-1,0,0,0,0,-1,0,0,-1,-1,0,0,0,0,0,0,0,0,0]$,

$[0,0,-1,0,0,-1,0,-1,0,0,0,0,0,0,-1,0,0,0,0,0,0,0]$,

$[0,0,-1,0,0,-1,-1,0,0,0,0,0,0,0,0,0,0,0,0,0,0,-1]$,

$[0,-1,0,0,0,-1,-1,0,0,0,0,0,0,0,0,-1,0,0,0,0,0,0]$,

$[0,0,0,-1,0,0,-1,0,0,0,-1,0,0,-1,0,0,0,0,0,0,0,0]$,

$[0,0,0,0,-1,0,0,0,-1,0,-1,0,0,0,-1,0,0,0,0,0,0,0]$,

$[-1,0,0,0,0,0,0,-1,0,-1,0,0,0,-1,0,0,0,0,0,0,0,0]$,

$[0,-1,0,0,-1,0,0,0,0,-1,0,0,0,0,0,0,0,0,-1,0,0,0]$,

$[-1,0,0,0,0,0,0,-1,0,0,0,-1,0,0,0,0,0,0,0,0,-1,0]$,

$[0,-1,0,0,0,-1,0,0,0,-1,0,0,-1,0,0,0,0,0,0,0,0,0]$,

$[0,-1,0,0,0,0,-1,0,0,0,-1,0,0,0,0,0,0,0,0,0,-1,0]]$

gap> Determinant $(p)$;

$-1$

gap> StablyPermutationFCheckMat (G,Nlist(l),Plist(l),p);

true

gap> i432:=Difference(U432, [U432 [4], U432[7]] );

$[[4,31,1,3],[4,31,1,4],[4,31,2,2],[4,31,4,2]$,

$[4,31,5,2],[4,31,7,2]]$ 


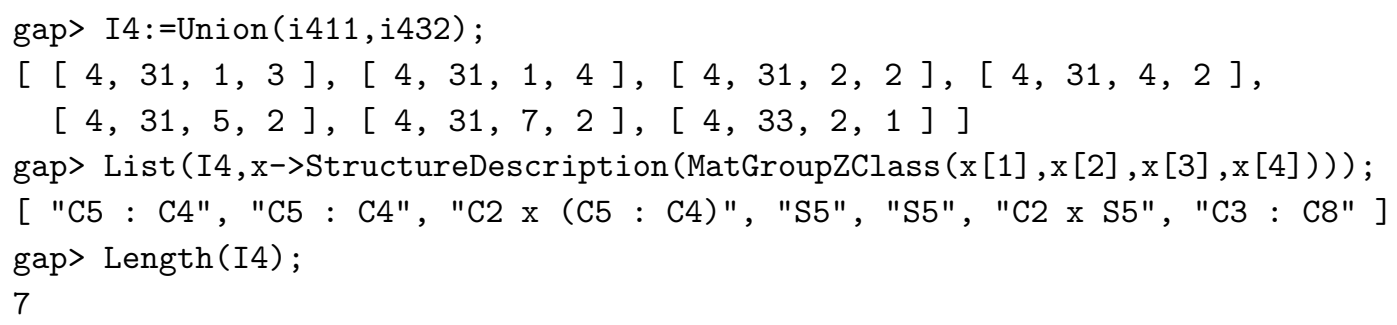

Example 10.4 $\left(\left[M_{G}\right]^{f l}=0\right.$ if and only if $\left[M_{G}\right]^{f l}$ is of finite order in $\mathcal{C}(G) / \mathcal{S}(G)$ for the groups $G$ of the GAP IDs $(4,31,1,3),(4,31,1,4)$ and $(4,33,2,1))$.

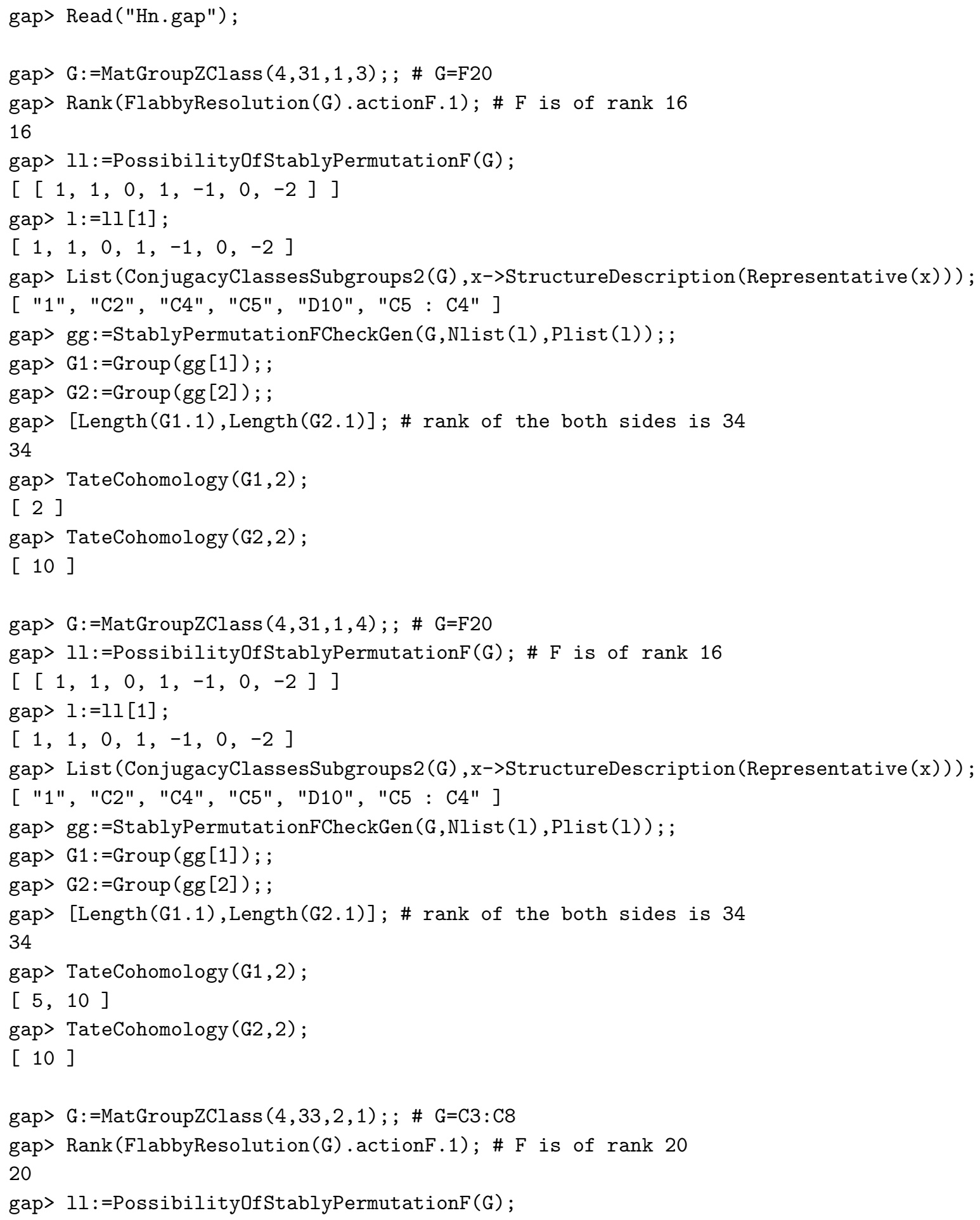


$[[1,1,1,0,-1,0,0,0,-2]]$

gap> $1:=11[1]$;

$[1,1,1,0,-1,0,0,0,-2]$

gap> List (ConjugacyClassesSubgroups2(G), $\mathrm{x}->$ StructureDescription(Representative(x))) ;

[ "1", "C2", "C3", "C4", "C6", "C8", "C12", "C3 : C8" ]

gap> gg:=StablyPermutationFCheckGen(G,Nlist(1), Plist(1)); ;

gap> G1:=Group $(\operatorname{gg}[1])$;

<matrix group with 2 generators>

gap> G2:=Group (gg[2]);

<matrix group with 2 generators>

gap> [Length(G1.1), Length(G2.1)]; \# rank of the both sides is 44

$[44,44]$

gap> List $([-7 . .7], i->$ TateCohomology $($ G1,i)); \# Tate cohomologies are coincide

$[[],[6],[],[6],[],[6],[0,0,0],[1,1,6]$,

[]$,[6],[],[6],[],[6],[]]$

gap> List $([-7 \ldots 7], i->$ TateCohomology $(G 2, i))$;

$[[],[6],[],[6],[],[6],[0,0,0],[1,1,6]$,

[]$,[6],[],[6],[],[6],[]]$

gap> Length(GeneratorsOfGroup $(G))$; \# number of generators of $G$ is 2

2

gap> List([G.1,G.2],Order); \# order of two generators are 8, 12

$[8,12]$

gap> a : =G.2^ 4 ;

$[[-1,-1,-1,1],[1,0,0,-1],[0,0,-1,1],[0,0,-1,0]]$

gap> b:=G.1;

$[[0,0,-1,0],[-1,0,0,0],[1,1,1,-2],[0,1,0,-1]]$

gap> List $([\mathrm{a}, \mathrm{b}]$, Order $)$;

$[3,8]$

gap> $a^{\wedge} b=a^{\wedge} 2$;

true

gap> $G=\operatorname{Group}(a, b)$;

true

gap> G1_3:=Group $(\mathrm{gg}[1] * \mathrm{Z}(3)) ;$;

gap> G2_3:=Group (gg [2]*Z(3)); ;

gap> v1:=VectorSpace (GF(3^2), (G1_3.2^4-IdentityMat (44)*Z(3)^0)^2);

<vector space over GF(3^2), with 44 generators>

gap> v2:=VectorSpace (GF (3^2), (G2_3.2^4-IdentityMat (44)*Z(3)^0)^2);

<vector space over $\mathrm{GF}\left(3^{\wedge} 2\right)$, with 44 generators>

gap> List([v1,v2], Dimension);

$[12,12]$

gap> Eigenvalues $\left(\mathrm{GF}\left(3^{\wedge} 2\right), \mathrm{G} 1 \_3.1\right)$;

$\left[\mathrm{Z}(3), \mathrm{Z}(3)^{\wedge} 0, \mathrm{Z}\left(3^{\wedge} 2\right)^{\wedge} 5, \mathrm{Z}\left(3^{\wedge} 2\right)^{\wedge} 6, \mathrm{Z}\left(3^{\wedge} 2\right)^{\wedge} 7, \mathrm{Z}\left(3^{\wedge} 2\right), \mathrm{Z}\left(3^{\wedge} 2\right)^{\wedge} 2, \mathrm{Z}^{\wedge}\left(3^{\wedge} 2\right)^{\wedge} 3\right]$

gap> Eigenvalues $\left(\mathrm{GF}\left(3^{\wedge} 2\right), \mathrm{G} 2 \_3.1\right)$;

$\left[\mathrm{Z}(3), \mathrm{Z}(3)^{\wedge} 0, \mathrm{Z}\left(3^{\wedge} 2\right)^{\wedge} 5, \mathrm{Z}\left(3^{\wedge} 2\right)^{\wedge} 6, \mathrm{Z}\left(3^{\wedge} 2\right)^{\wedge} 7, \mathrm{Z}\left(3^{\wedge} 2\right), \mathrm{Z}\left(3^{\wedge} 2\right)^{\wedge} 2, \mathrm{Z}\left(3^{\wedge} 2\right)^{\wedge} 3\right]$

gap> e1:=Eigenspaces $\left(\mathrm{GF}\left(3^{\wedge} 2\right), \mathrm{G} 1 \_3.1\right)$;

[ <vector space over GF( $\left(3^{\wedge} 2\right)$, with 7 generators>,

<vector space over GF( $\left.3^{\wedge} 2\right)$, with 7 generators>,

<vector space over GF(3^2), with 4 generators>,

<vector space over GF( $\left.3^{\wedge} 2\right)$, with 7 generators>,

<vector space over GF( $\left.3^{\wedge} 2\right)$, with 4 generators>,

<vector space over GF(3^2), with 4 generators>, 


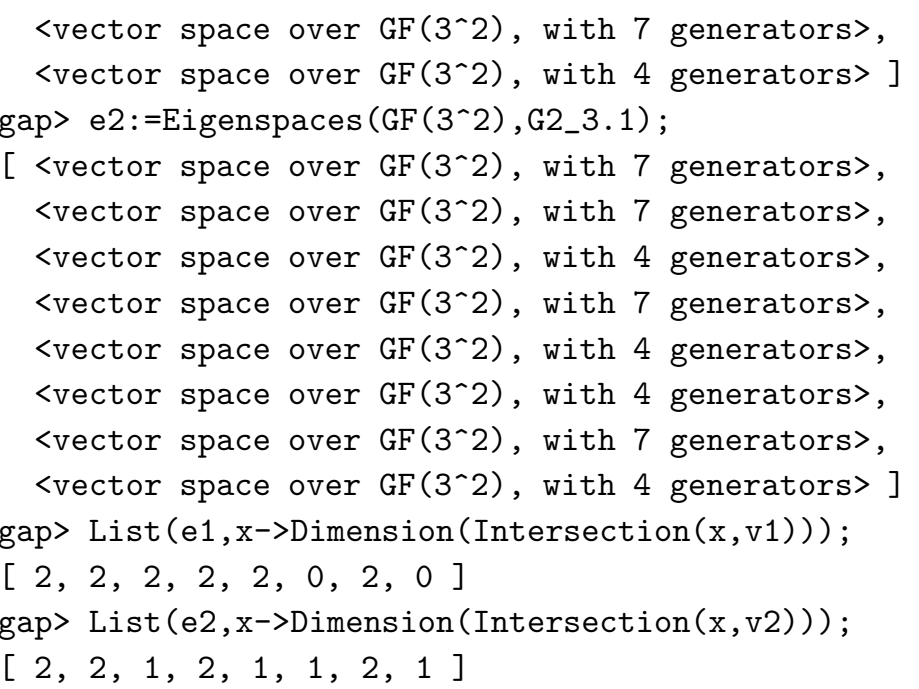

Example 10.5 $\left(\left[M_{G}\right]^{f l}+\left[J_{S_{5} / S_{4}}\right]^{f l}=0\right.$ for the group $G \simeq S_{5}$ of the GAP ID $\left.(4,31,5,2)\right)$.

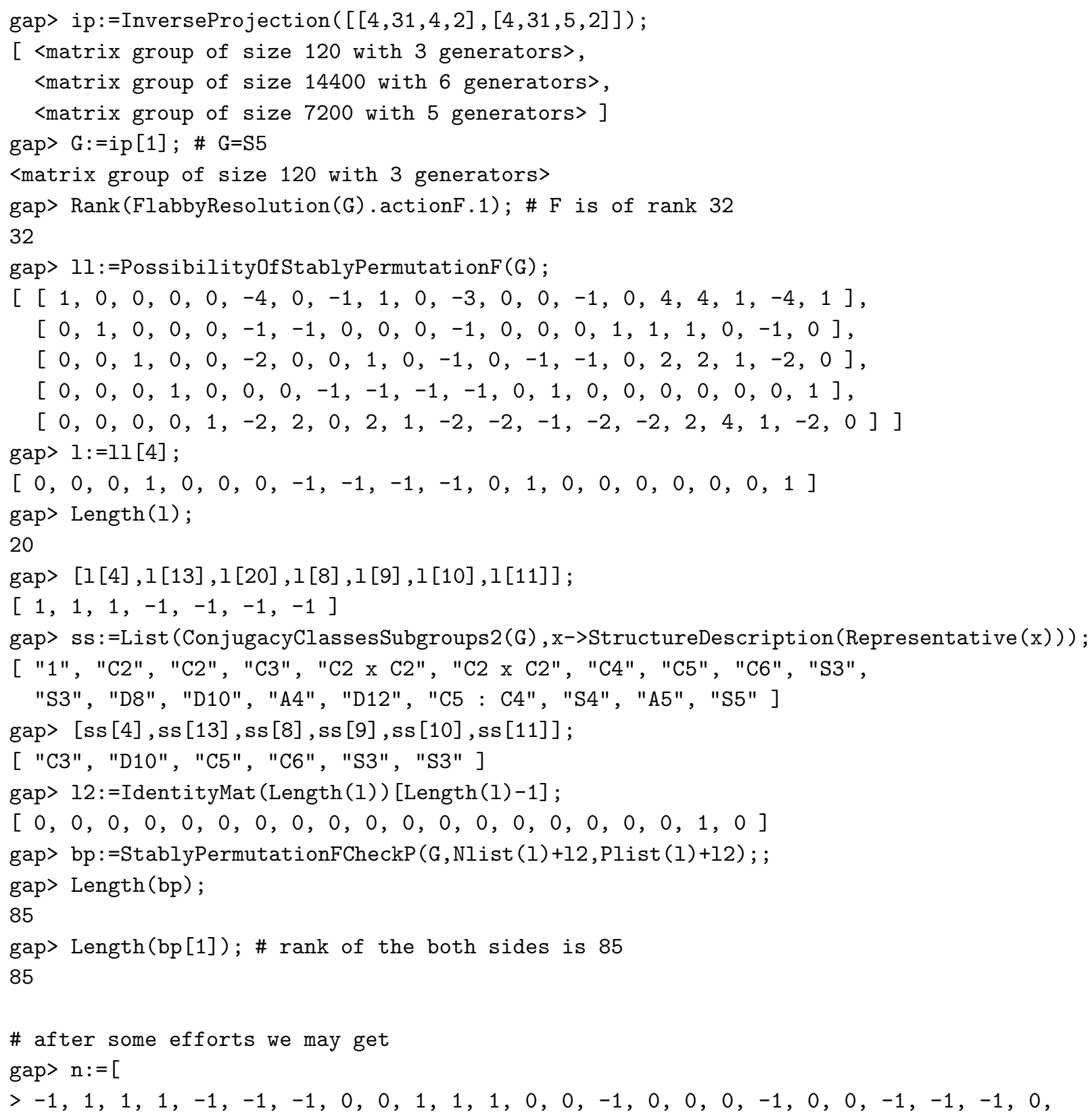




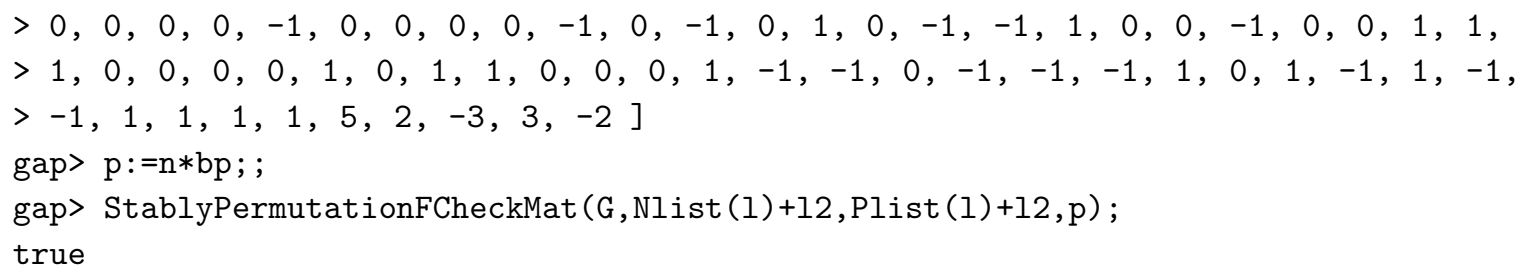

Example 10.6 $\left(\left[M_{G}\right]^{f l}+\left[J_{F_{20} / C_{4}}\right]^{f l}=0\right.$ for the group $G \simeq F_{20}$ of the GAP ID $\left.(4,31,1,4)\right)$.

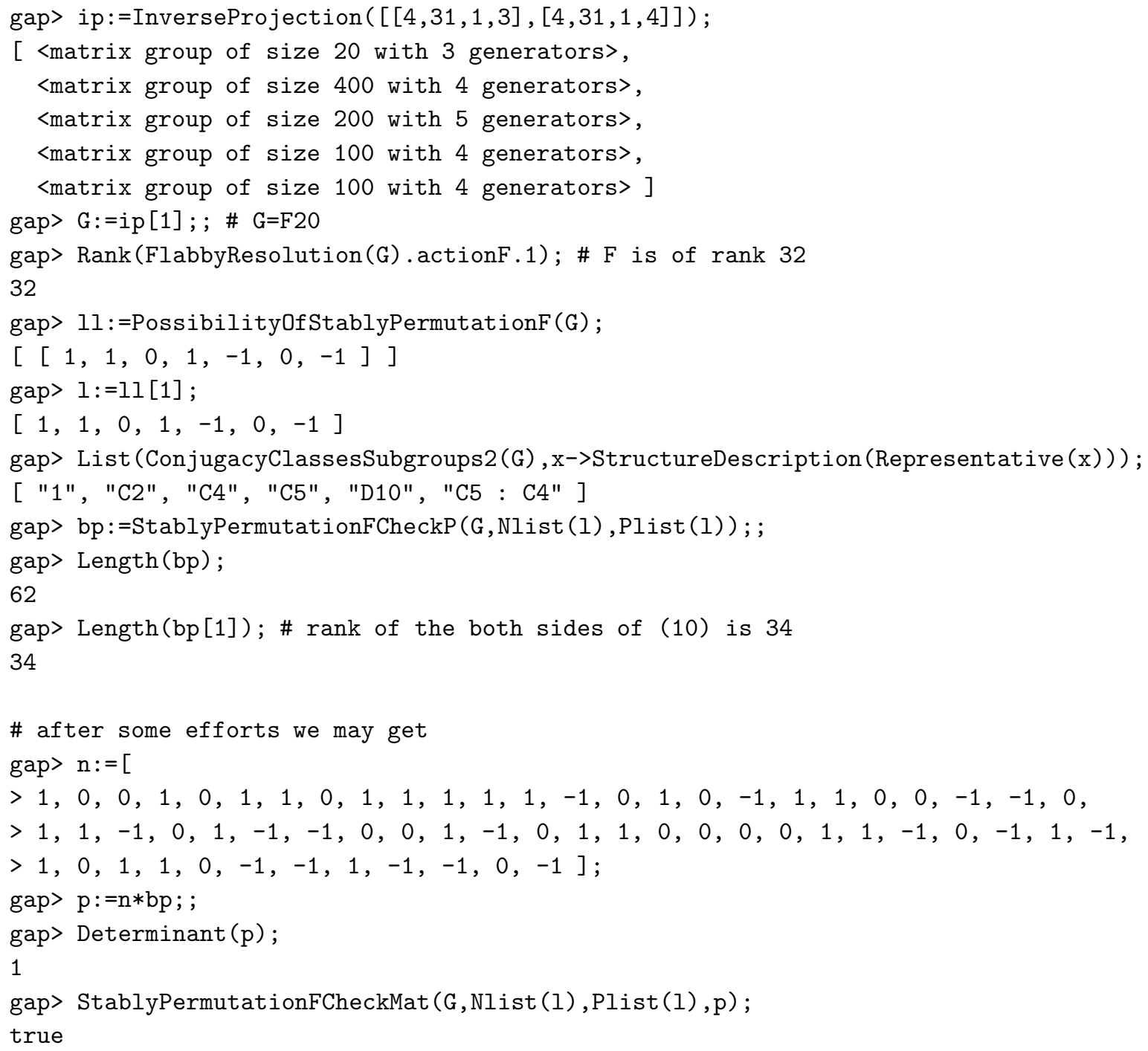

\section{Proof of Theorem 1.28}

Let $G$ be a finite subgroup of $\mathrm{GL}(5, \mathbb{Z})$ and $M=M_{G}$ be the corresponding $G$-lattice of rank 5 as in Definition 1.26

Step 1. The case where $M_{G}$ is decomposable.

We assume that $M_{G} \simeq M_{1} \oplus M_{2}$ is decomposable with $\operatorname{rank} M_{1} \geq \operatorname{rank} M_{2} \geq 1$. Hence rank $M_{1} \leq 4$ and rank $M_{2} \leq 2$. It follows from Theorem 1.1 and Lemma 2.14 that $\left[M_{2}\right]^{f l}=0$ and $\left[M_{G}\right]^{f l}=\left[M_{1}\right]^{f l}$.

There exist exactly $25 G$-lattices $M_{G} \simeq M_{1} \oplus M_{2}$ with $\left[M_{G}\right]^{f l} \neq 0$ but invertible whose GAP IDs $\mathcal{I}_{41}$ are computed in Example 4.12 (see also Table 11).

There exist exactly 245 (resp. 849, 768) $G$-lattices $M_{G} \simeq M_{1} \oplus M_{2} \oplus M_{3}$ with rank $M_{1}=3$, rank $M_{2}=1$, rank $M_{3}=1$ (resp. $M_{G} \simeq M_{1} \oplus M_{2}$ with rank $M_{1}=3$, rank $M_{2}=2, M_{G} \simeq M_{1} \oplus M_{2}$ with rank $M_{1}=4$, rank 
$M_{2}=1$ ) and $\left[M_{G}\right]^{f l} \neq 0$ whose GAP IDs $\mathcal{N}_{311}$ (resp. $\mathcal{N}_{32}, \mathcal{N}_{41}$ ) are computed in Example 4.12 (see also Tables 12,13 and 14).

Step 2. Determination of all $G$-lattices $M_{G}$ whose flabby class $\left[M_{G}\right]^{f l}$ is not invertible.

We assume that $M_{G}$ is indecomposable. There exist 1452 indecomposable $G$-lattices $M_{G}$ of rank 5 (see Example 4.9). By using IsInvertibleF as in Algorithm F2, we see that there exist exactly $1141 G$-lattices $M_{G}$ with $\left[M_{G}\right]^{f l}$ not invertible (see Example 11.1 below). In the next step, we will show that all the remaining 311 cases satisfy $\left[M_{G}\right]^{f l}=0$.

Step 3. Verification of $\left[M_{G}\right]^{f l}=0$ for all the remaining 311 cases.

First, we see that for all the remaining $311 G$-lattices $M_{G} G$ is a subgroup of at least one of the 18 groups of the CARAT IDs as in Table 9 (see Example 11.2 below). Hence by Lemma 2.17 it is enough to show that $\left[M_{G}\right]^{f l}=0$ for the 18 groups $G$ in Table 9 .

Table 9: the maximal 18 groups in the remaining 311 cases

\begin{tabular}{|c|c|c|c|c|c|c|c|}
\hline CARAT ID & $G$ & $\# G$ & Method & CARAT ID & $G$ & $\# G$ & Method \\
\hline$(5,942,1)$ & $\operatorname{Imf}(5,1,1)$ & 3840 & flfl & $(5,947,2)$ & $S_{5}$ & 120 & Method III $+\alpha$ \\
\hline$(5,953,4)$ & $S_{6}$ & 720 & $f l f l$ & $(5,337,12)$ & $D_{4} \times S_{3}$ & 48 & Method III \\
\hline$(5,726,4)$ & & 384 & $f l f l$ & $(5,341,6)$ & $D_{4} \times S_{3}$ & 48 & Method III \\
\hline$(5,919,4)$ & $C_{2} \times S_{5}$ & 240 & Method III & $(5,531,13)$ & $C_{2} \times S_{4}$ & 48 & Method III \\
\hline$(5,801,3)$ & $C_{2} \times\left(S_{3}^{2} \rtimes C_{2}\right)$ & 144 & Method III & $(5,533,8)$ & $C_{2} \times S_{4}$ & 48 & Method III \\
\hline$(5,655,4)$ & $D_{4}^{2} \rtimes C_{2}$ & 128 & $f l f l$ & $(5,623,4)$ & $C_{2} \times S_{4}$ & 48 & Method III \\
\hline$(5,911,4)$ & $S_{5}$ & 120 & Method I (1) & $(5,245,12)$ & $C_{2}^{2} \times S_{3}$ & 24 & Method III \\
\hline$(5,946,2)$ & $S_{5}$ & 120 & Method I (2) & $(5,81,42)$ & $C_{2} \times D_{4}$ & 16 & $f l f l$ \\
\hline$(5,946,4)$ & $S_{5}$ & 120 & Method II $+\alpha$ & $(5,81,48)$ & $C_{2} \times D_{4}$ & 16 & $f l f l$ \\
\hline
\end{tabular}

Using $f l f l$ as in Algorithm F3 once or twice, we see that $\left[M_{G}\right]^{f l}=0$ for the 6 groups $G$ of the CARAT IDs $(5,942,1),(5,953,4),(5,726,4),(5,655,4),(5,81,42)$ and $(5,81,48)$ (see Example 11.3 below).

Using functions in Algorithm [F7 Method III, we may confirm that $\left[M_{G}\right]^{f l}=0$ for the 8 groups $G$ of the CARAT IDs $(5,919,4),(5,801,3),(5,337,12),(5,341,6),(5,531,13),(5,533,8),(5,623,4)$ and $(5,245,12)$ (see Example 11.4 below). Thus there are 4 remaining cases which are groups $G \simeq S_{5}$ of order 120 of the CARAT IDs $(5,911,4),(5,946,2),(5,946,4),(5,947,2)$.

For two group $G$ of the CARAT IDs $(5,911,4)$ and $(5,946,2)$, by Method I (1) and Method I (2) respectively, we have that $\left[M_{G}\right]^{f l}=0$ (see Example 11.5 below and Example [5.6). Indeed, for the group $G$ of the CARAT ID $(5,911,4)$, we see that $M_{G}$ is flabby and coflabby and hence stably permutation by Theorem 6.2. This implies $\left[M_{G}\right]^{f l}=0$. We also see that for the group $G$ of the CARAT ID $(5,946,2)$, the CARAT ID of $F$ with $[F]=\left[M_{G}\right]^{f l}$ is $(5,911,4)$. Hence we confirm that $\left[M_{G}\right]^{f l}=0$.

For the group $G \simeq S_{5}$ of the CARAT ID $(5,947,2)$, using Algorithm F7 Method III, we may find the flabby class $\left[M_{G}\right]^{f l}=[F]$ where $F$ is of rank 21 . However, the rank of the both sides of (10) becomes 81 . Thus we take another $F$ of rank 25 (see Example 11.6 below). Then the rank of the both sides of (10) becomes 55. By using PossibilityOfStablyPermutationFFromBase, it is possible that

$$
\mathbb{Z}\left[G / H_{6}\right] \oplus \mathbb{Z}\left[G / H_{11}\right] \oplus \mathbb{Z}\left[G / H_{17}\right] \simeq \mathbb{Z}\left[G / H_{10}\right] \oplus \mathbb{Z}\left[G / H_{15}\right] \oplus F
$$

where $H_{6} \simeq C_{2}^{2}, H_{11} \simeq S_{3}, H_{17} \simeq S_{4}, H_{10} \simeq S_{3}$ and $H_{15} \simeq D_{6}$ (the rank of the both sides is $30+20+5=$ $20+10+25=55$ ). We could not establish the isomorphism (17). However, as in Example 5.6 (Algorithm F5. Method I (2)), after adding $\mathbb{Z}$ to the both sides of (17), we may confirm that the isomorphism

$$
\mathbb{Z}\left[G / H_{6}\right] \oplus \mathbb{Z}\left[G / H_{11}\right] \oplus \mathbb{Z}\left[G / H_{17}\right] \oplus \mathbb{Z} \simeq \mathbb{Z}\left[G / H_{10}\right] \oplus \mathbb{Z}\left[G / H_{15}\right] \oplus F \oplus \mathbb{Z}
$$

holds (see Example 11.6).

For the group $G \simeq S_{5}$ of the CARAT ID $(5,946,4)$, Method III does not work well. We may obtain $\left[M_{G}\right]^{f l}=[F]$ with $F$ rank 17 by FlabbyResolution(G) . actionF. Using PossibilityOfStablyPermutationF, it turns out that 
the isomorphism

$$
\begin{aligned}
& \mathbb{Z}\left[G / H_{6}\right] \oplus \mathbb{Z}\left[H_{9}\right] \oplus \mathbb{Z}\left[G / H_{11}\right] \oplus \mathbb{Z}\left[G / H_{16}\right] \oplus \mathbb{Z}\left[G / H_{17}\right]^{\oplus 2} \oplus \mathbb{Z}\left[G / H_{18}\right] \\
& \simeq \mathbb{Z}\left[G / H_{7}\right] \oplus \mathbb{Z}\left[G / H_{10}\right] \oplus \mathbb{Z}\left[G / H_{14}\right] \oplus \mathbb{Z} \oplus F
\end{aligned}
$$

may occur where $H_{6} \simeq C_{2}^{2}, H_{9} \simeq H_{11} \simeq S_{3}, H_{16} \simeq F_{20}, H_{17} \simeq S_{4}, H_{18} \simeq A_{5}, H_{7} \simeq C_{4}, H_{10} \simeq C_{6}, H_{14} \simeq A_{4}$ and $H_{15} \simeq D_{6}$ (the rank of the both sides is $30+20+20+6+2 \times 5+2=30+20+10+10+1+17=88$ ). After some efforts, we see that the isomorphism (18) actually holds (see Example 11.7 below).

Step 4. $\left[M_{G}\right]^{f l}=0$ if and only if $\left[M_{G}\right]^{f l}$ is of finite order in $\mathcal{C}(G) / \mathcal{S}(G)$.

We should show that if $G$ is one of the 25 groups as in Table 11, i.e. the 25 cases where $\left[M_{G}\right]^{f l}$ is not zero but invertible, then $\left[\left(M_{G}\right)^{\oplus r}\right]^{f l} \neq 0$ for any $r \geq 1$ (see also Step 5 of Section 10). By Remark 1.13, $\left[M_{G}\right]^{f l}=\left[M_{1}\right]^{f l}$ where $M_{G} \simeq M_{1} \oplus M_{2}, M_{1}$ is a $G / N_{1}$-lattice of rank 4 which is one of the 7 cases as in Table 2 . Note that $\left[M_{1}\right]^{f l}=\rho_{G}\left(M_{1}\right)=0$ if and only if $\rho_{G / N_{1}}\left(M_{1}\right)=0$ by Lemma 2.14. Hence the assertion follows from Step 5 of Section 10.

Example 11.1 (Determination of all the cases where $\left[M_{G}\right]^{f l}$ is not invertible).

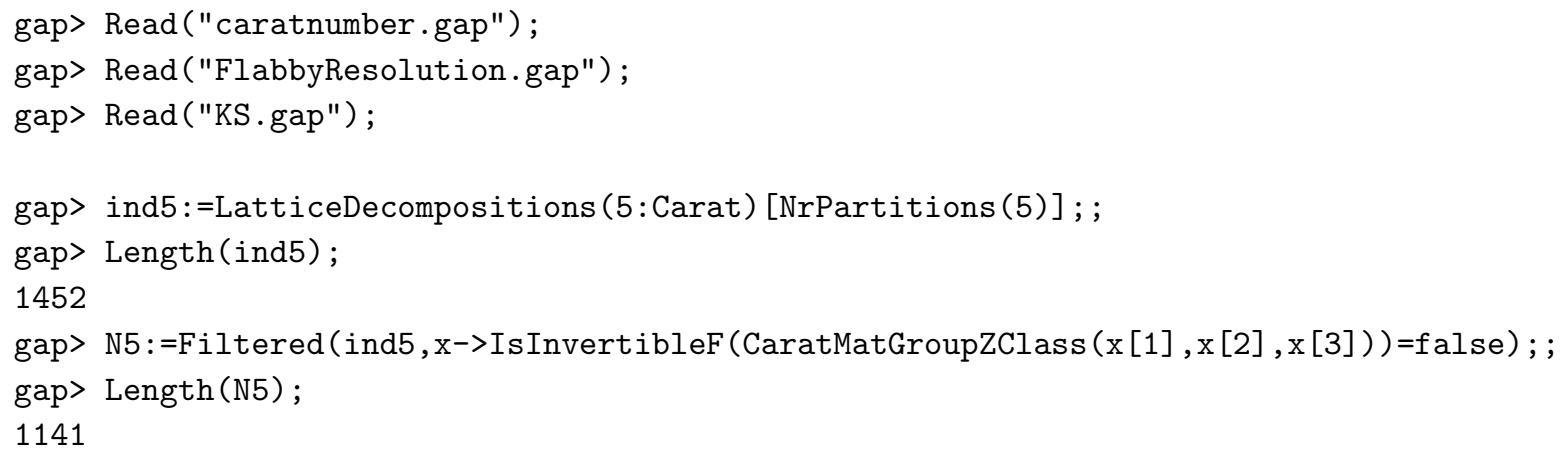

Example 11.2 (The maximal 18 groups in the remaining 311 cases ).

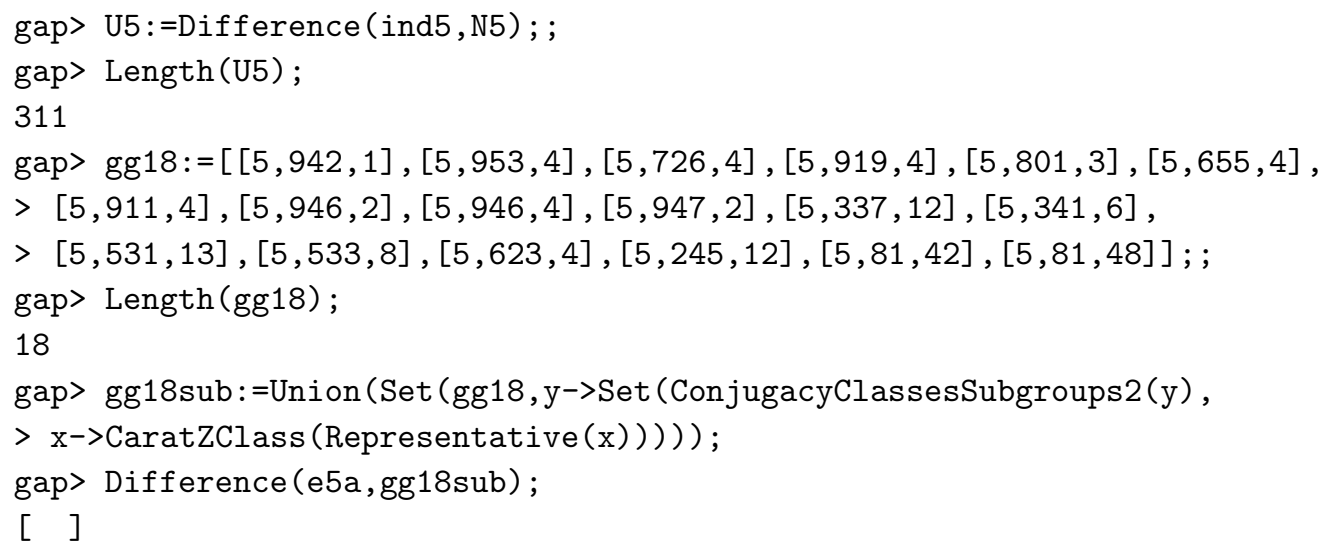

Example 11.3 (Verification of $\left[M_{G}\right]^{f l}=0$ for the 6 groups $G$ of the CARAT ID $(5,942,1),(5,953,4),(5,726,4)$, $(5,655,4),(5,81,42)$ and $(5,81,48)$ : Method $f l f l)$.

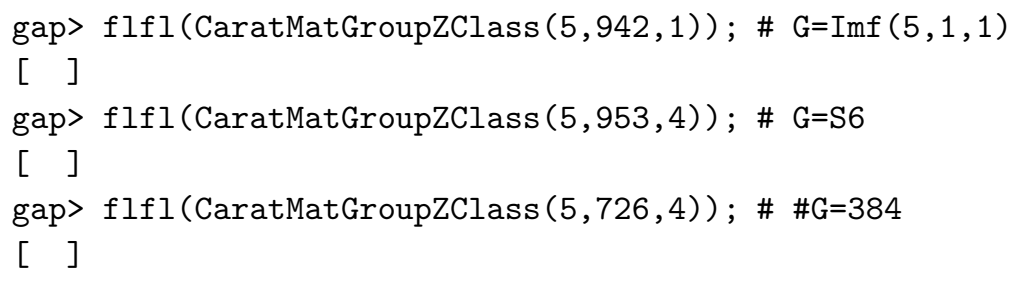




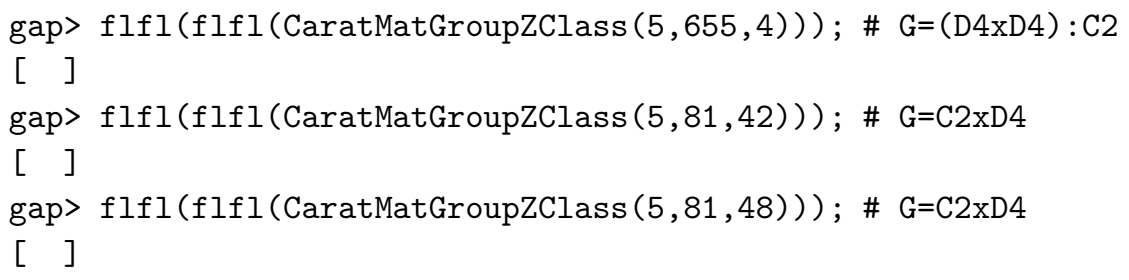

Example 11.4 (Verification of $\left[M_{G}\right]^{f l}=0$ for the 8 groups $G$ of the CARAT IDs $(5,919,4),(5,801,3),(5,337,12)$, $(5,341,6),(5,531,13),(5,533,8),(5,623,4)$ and $(5,245,12)$ : Method III).

gap> G:=CaratMatGroupZClass $(5,919,4) ;$; \# G=C2xS5

gap> Rank(FlabbyResolution(G).actionF.1); \#F is of rank 27

27

gap> mis:=SearchCoflabbyResolutionBase(TransposedMatrixGroup(G),3);; \# Method III gap> List (mis, Length);

$[42,32,42,82,72,42,32,32,42,32,12]$

gap> mi:=mis $[$ Length (mis)]; \# (new) $F$ is of rank $7(=12-5)$

$[[-1,0,0,0,-1],[-1,0,1,1,-2],[-1,1,1,0,-1],[0,-1,0,1,0]$,

$[0,0,-1,0,1],[0,0,0,-1,1],[0,0,0,1,-1],[0,0,1,0,-1]$,

$[0,1,0,-1,0],[1,-1,-1,0,1],[1,0,-1,-1,2],[1,0,0,0,1]]$ gap> 1l:=PossibilityOfStablyPermutationFFromBase (G,mi); ;

gap> Length (11);

18

gap> 1:=11[Length $(11)]$;

$[0,0,0,0,0,0,0,0,0,0,0,0,0,0,0,0,0,0,0,0,0,0,0,0,0$,

$0,0,0,0,0,0,0,0,0,0,0,0,0,0,0,0,0,0,0,0,0,0,0,0,0$,

$0,1,0,0,0,1,0,-1]$

gap> StablyPermutationFCheckFromBase (G,mi,Nlist(1),Plist(1));

$[[0,0,0,-1,0,0,-1]$,

$[1,1,1,1,1,1,3]$,

$[-1,0,0,0,0,0,-1]$,

$[0,-1,0,0,0,0,-1]$,

$[0,0,-1,0,0,-1,0]$,

$[0,0,0,0,-1,-1,0]$,

$[0,0,0,0,-1,0,-1]]$

gap> G:=CaratMatGroupZClass $(5,801,3) ;$; \# \#G=144

gap> Rank(FlabbyResolution(G).actionF.1); \#F is of rank 25

25

gap> mis:=SearchCoflabbyResolutionBase(TransposedMatrixGroup(G),3); \# Method III gap> List (mis, Length);

$[48,48,32,32,66,66,50,50,48,48,32,32,32,48,42,42,30,32,48,42$, $30,32,26,26,14,32,26,14]$

gap> mi:=mis $[$ Length $($ mis) $]$; \# (new) $F$ is of rank $9(=14-5)$

$[[-1,-1,0,1,0],[-1,-1,1,1,-1],[-1,0,0,0,0],[0,-1,0,0,0]$,

$[0,0,-1,0,0],[0,0,0,-1,0],[0,0,0,0,-1],[0,0,0,0,1]$,

$[0,0,0,1,0],[0,0,1,0,0],[0,1,0,0,0],[1,0,0,0,0]$,

$[1,1,-1,-1,1],[1,1,0,-1,0]]$

gap> 1l:=PossibilityOfStablyPermutationFFromBase (G,mi); ;

gap> Length(11);

32

gap> 1:=11 [Length (11)];

$[0,0,0,0,0,0,0,0,0,0,0,0,0,0,0,0,0,0,0,0,0,0,0,0,0$, $0,0,0,0,0,0,0,0,0,0,0,0,0,0,0,0,0,0,0,0,0,0,0,0,0$, 


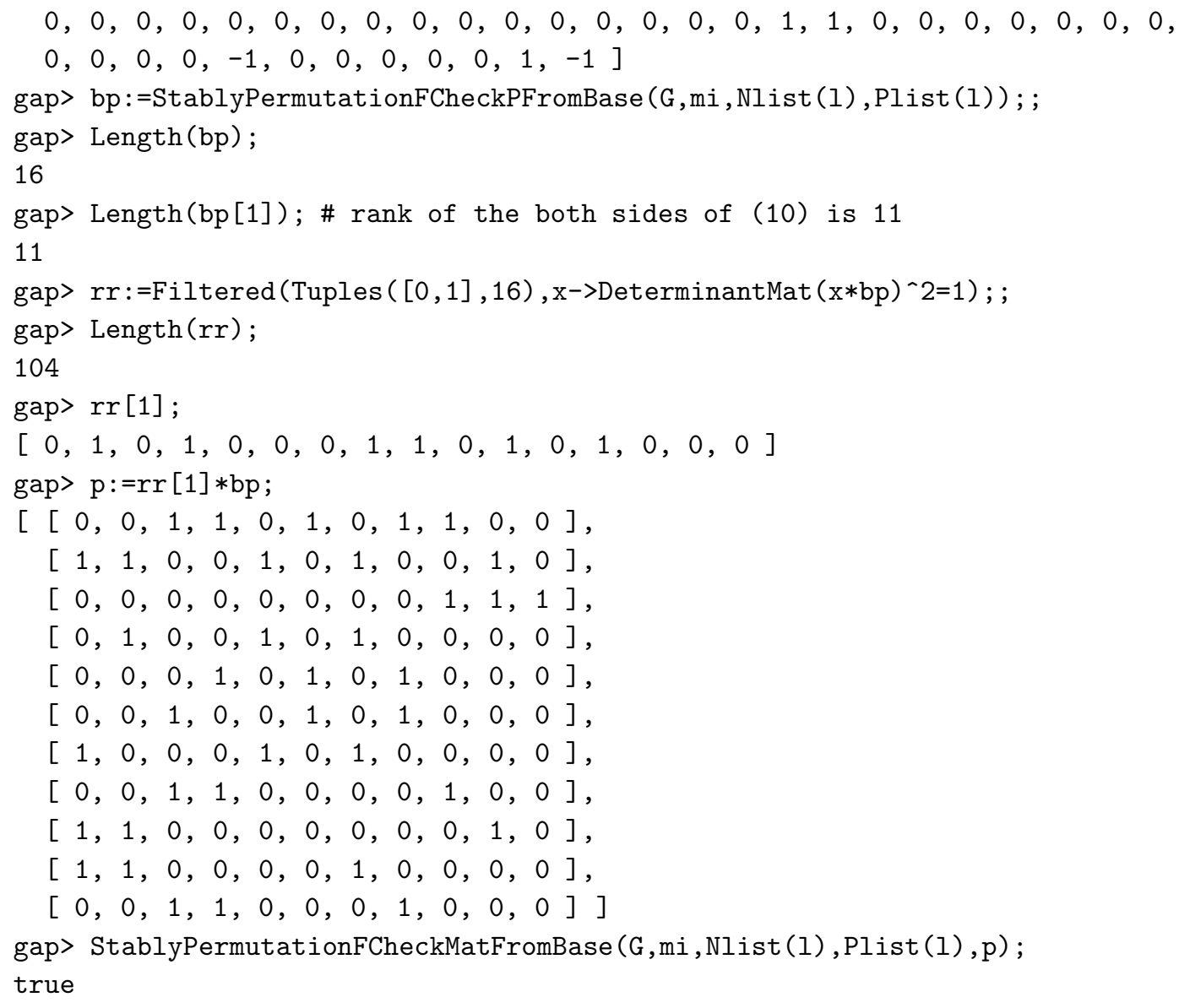


gap> mi:=mis [Length(mis)-2]; \# (new) F is of rank 2 (=7-5)

$[[-1,0,1,1,-1],[-1,1,1,1,-1],[-1,1,1,1,0],[0,0,0,1,-1]$,

$[0,0,1,0,-1],[0,0,1,1,-1],[0,1,1,1,-1]]$

gap> FlabbyResolutionFromBase(G,mi).actionF; \# (new) F is permutation

$\operatorname{Group}([[[1,0],[0,1]],[[1,0],[0,1]],[[0,1],[1,0]]])$

gap> G:=CaratMatGroupZClass $(5,531,13) ; ; \# \mathrm{G}=\mathrm{C} 2 \mathrm{xS} 4$

gap> Rank(FlabbyResolution(G).actionF.1); \#F is of rank 15

15

gap> mis:=SearchCoflabbyResolutionBase (TransposedMatrixGroup(G),3); \# Method III gap> List (mis, Length);

$[18,18,14,14,14,14,12,12,10,12,6,7,12,6,7]$

gap> mi:=mis [Length(mis)-1]; \# (new) F is trivial of rank 1 (=6-5)

$[[0,0,0,1,0],[0,0,1,1,1],[0,1,0,1,1]$,

$[1,-1,-1,0,-1],[1,0,0,0,-1],[1,0,0,0,0]]$

gap> FlabbyResolutionFromBase(G,mi).actionf; \# (new) F is trivial of rank 1

$\operatorname{Group}\left(\left[\left[\left[\begin{array}{ll}1 & ]\end{array}\right],\left[\left[\begin{array}{ll}1 & ]\end{array}\right]\right)\right.\right.\right.$

gap> G:=CaratMatGroupZClass $(5,533,8) ;$; \# G=C2xS4

gap> Rank(FlabbyResolution(G).actionF.1); \#F is of rank 44

44

gap> mis:=SearchCoflabbyResolutionBase (TransposedMatrixGroup(G),3); ; Method III gap> List (mis, Length);

$[29,29,15,15,15,15]$

gap> mi:=mis [Length(mis)]; \# (new) $F$ is of rank 10 (=15-5)

$[[-1,0,-1,0,1],[-1,0,-1,1,0],[0,0,-1,0,1],[0,0,-1,1,0]$,

$[0,0,0,0,1],[0,0,0,1,0],[0,1,-1,0,1],[0,1,-1,0,2]$,

$[0,1,-1,1,0],[0,1,-1,1,1],[0,1,-1,2,0],[0,1,0,0,1]$,

$[0,1,0,1,0],[1,1,0,0,1],[1,1,0,1,0]]$

gap> 11:=PossibilityOfStablyPermutationFFromBase (G,mi); ;

gap> Length $(11)$;

5

gap> $1:=11[$ Length $(11)]$;

$[0,0,0,0,0,0,0,0,0,0,0,0,0,0,0,0,0,0,0,1,0,1,0,0,0,0,0$,

$0,-1,0,0,0,1,-1]$

gap> bp:=StablyPermutationFCheckPFromBase (G,mi,Nlist (1),Plist(1)); ;

gap> Length (bp);

20

gap> Length(bp[1]); \# rank of the both sides of (10) is 13

13

gap> rr:=Filtered (Tuples $([0,1], 20), x->$ DeterminantMat $(x * b p) ` 2=1)$; ;

gap> Length(rr);

2448

gap> $\operatorname{rr}[1]$;

$[0,0,1,0,0,1,0,0,0,0,1,0,0,0,0,1,0,1,0,1]$

gap> p: $=\operatorname{rr}[1] * \mathrm{bp}$;

$[[0,0,1,1,0,0,0,0,0,0,0,0,1]$,

$[1,0,0,0,0,1,0,0,0,0,0,0,1]$,

$[0,1,0,0,1,0,0,0,0,0,0,0,1]$,

$[0,0,0,0,1,0,0,0,0,0,0,1,0]$,

$[0,0,1,0,0,0,0,0,0,0,1,0,0]$,

$[0,0,0,1,0,0,0,1,0,0,0,0,0]$,

$[1,0,0,0,0,0,1,0,0,0,0,0,0]$,

$[0,0,0,0,0,1,0,0,0,1,0,0,0]$,

$[0,1,0,0,0,0,0,0,1,0,0,0,0]$, 
$[0,0,0,0,0,1,0,0,1,0,0,0,0]$,

$[1,1,1,0,0,0,0,0,0,0,0,0,1]$,

$[-2,-2,-2,-2,-2,-2,-1,-1,-1,-1,-1,-1,-2]$,

$[0,0,0,1,0,0,1,0,0,0,0,0,0]]$

gap> StablyPermutationFCheckMatFromBase (G,mi,Nlist(1),Plist (1),p);

true

gap> G:=CaratMatGroupZClass $(5,623,4) ; ; \# \mathrm{G}=\mathrm{C} 2 \mathrm{xS} 4$

gap> Rank(FlabbyResolution(G).actionF.1); \#F is of rank 13

13

gap> mis:=SearchCoflabbyResolutionBase(TransposedMatrixGroup(G),3);; \# Method III

gap> List (mis, Length);

$[42,30,24,33,21,15,36,38,42,33,42,36,30,24,18,26,20,30,24,21$,

$15,24,18,12]$

gap> mi:=mis [Length(mis)]; \# (new) F is of rank $7(=12-5)$

$[[-1,-1,1,0,0],[-1,0,1,0,0],[-1,1,-1,1,-1],[-1,1,0,1,-1]$,

$[0,-1,1,-1,1],[0,0,1,-1,1],[0,1,-1,1,-1],[0,1,0,1,-1]$,

$[1,-1,0,-1,1],[1,-1,0,0,1],[1,0,-1,-1,0],[1,0,-1,0,0]]$

gap> $11:=$ PossibilityOfStablyPermutationFFromBase $(\mathrm{G}, \mathrm{mi})$;

$[0,2,1,-1,-1]]$

gap $>1:=11[1]$;

$[0,2,1,-1,-1]$

gap> bp:=StablyPermutationFCheckPFromBase (G,mi, Nlist (1),Plist(1)); ;

gap> Length(bp);

14

gap> Length(bp[1]); \# rank of the both sides of (10) is 8

8

gap> rr:=Filtered $(\operatorname{Tuples}([0,1], 14), \mathrm{x}->$ DeterminantMat $(\mathrm{x} * \mathrm{bp}) \wedge 2=1) ;$;

gap> Length $(r r)$;

224

gap> $r r[1]$;

$[0,1,1,1,0,0,0,0,0,0,0,1,0,1]$

gap> $\mathrm{p}:=\mathrm{rr}[1] * \mathrm{bp}$;

$[[0,0,0,1,1,1,1,1]$,

$[1,0,0,0,0,0,0,0]$,

$[0,0,1,0,1,0,0,1]$,

$[0,1,0,0,0,0,0,0]$,

$[0,0,1,1,0,0,1,0]$,

$[0,1,0,0,0,1,1,0]$,

$[1,0,0,0,0,1,0,1]$,

$[0,1,0,1,0,0,0,1]]$

gap> StablyPermutationFCheckMatFromBase (G,mi,Nlist(1),Plist(1),p);

true

gap> G:=CaratMatGroupZClass $(5,245,12) ; ; \# \mathrm{G}=\mathrm{C} 22^{\wedge} 2 \mathrm{xS} 3$

gap> Rank(FlabbyResolution(G).actionF.1); \# F is of rank 16

16

gap> mis:=SearchCoflabbyResolutionBase(TransposedMatrixGroup(G),3);; \# Method III

gap> List (mis, Length);

[ 11 ]

gap> mi:=mis[1]; \# (new) F is of rank 6 (=11-5)

$[[-1,-1,1,-2,0],[-1,0,1,-2,-1],[0,0,-1,1,1],[0,0,0,-1,-1]$,

$[0,0,0,1,1],[0,0,1,-2,-1],[0,0,1,-1,-1],[0,1,-1,2,1]$,

$[0,1,0,0,0],[1,1,-1,2,0],[1,1,-1,2,1]]$

gap> ll:=PossibilityOfStablyPermutationFFromBase(G,mi); ; 


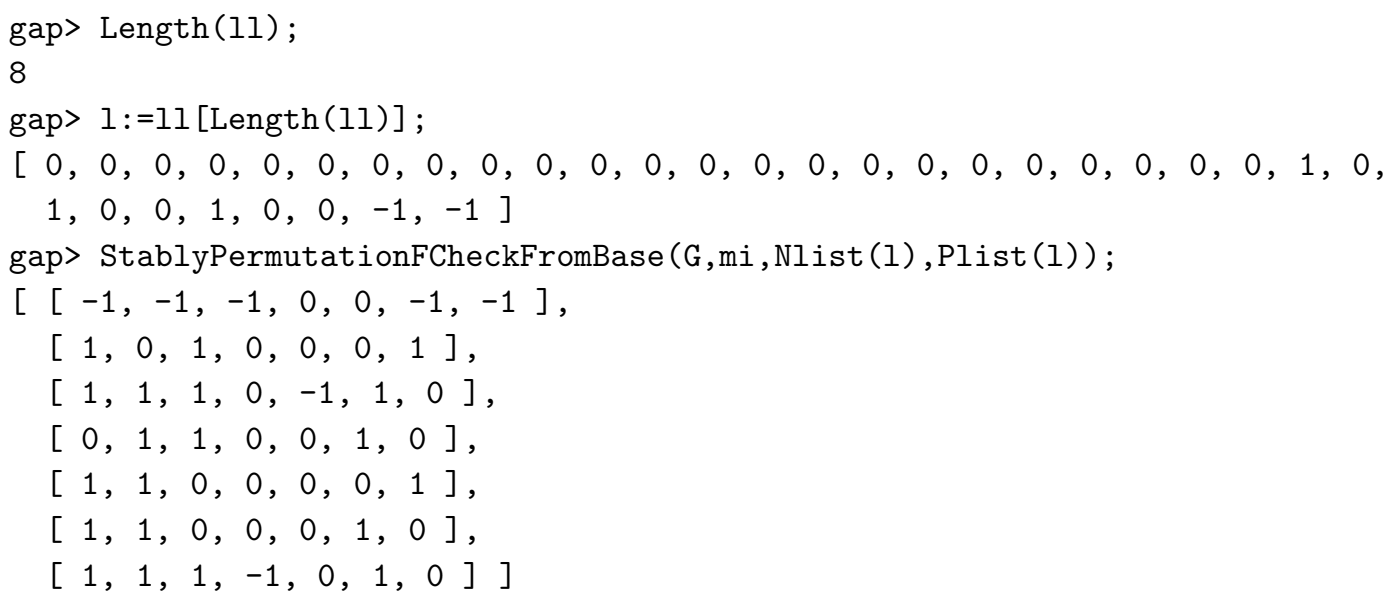

Example 11.5 (Verification of $\left[M_{G}\right]^{f l}=0$ for two groups $G \simeq S_{5}$ of the CARAT IDs $(5,911,4)$ and $(5,946,2)$ ).

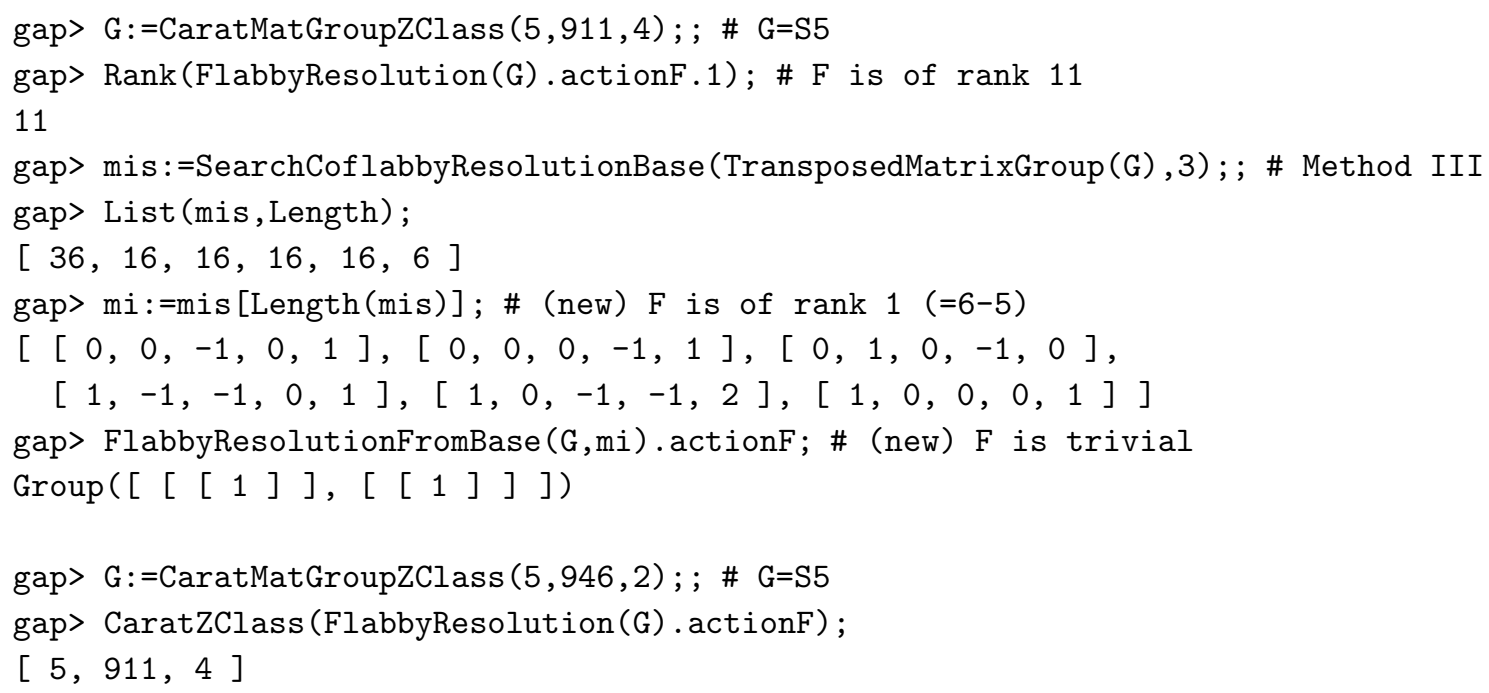

Example 11.6 (Verification of $\left[M_{G}\right]^{f l}=0$ for the group $G \simeq S_{5}$ of the CARAT ID $(5,947,2)$ ).

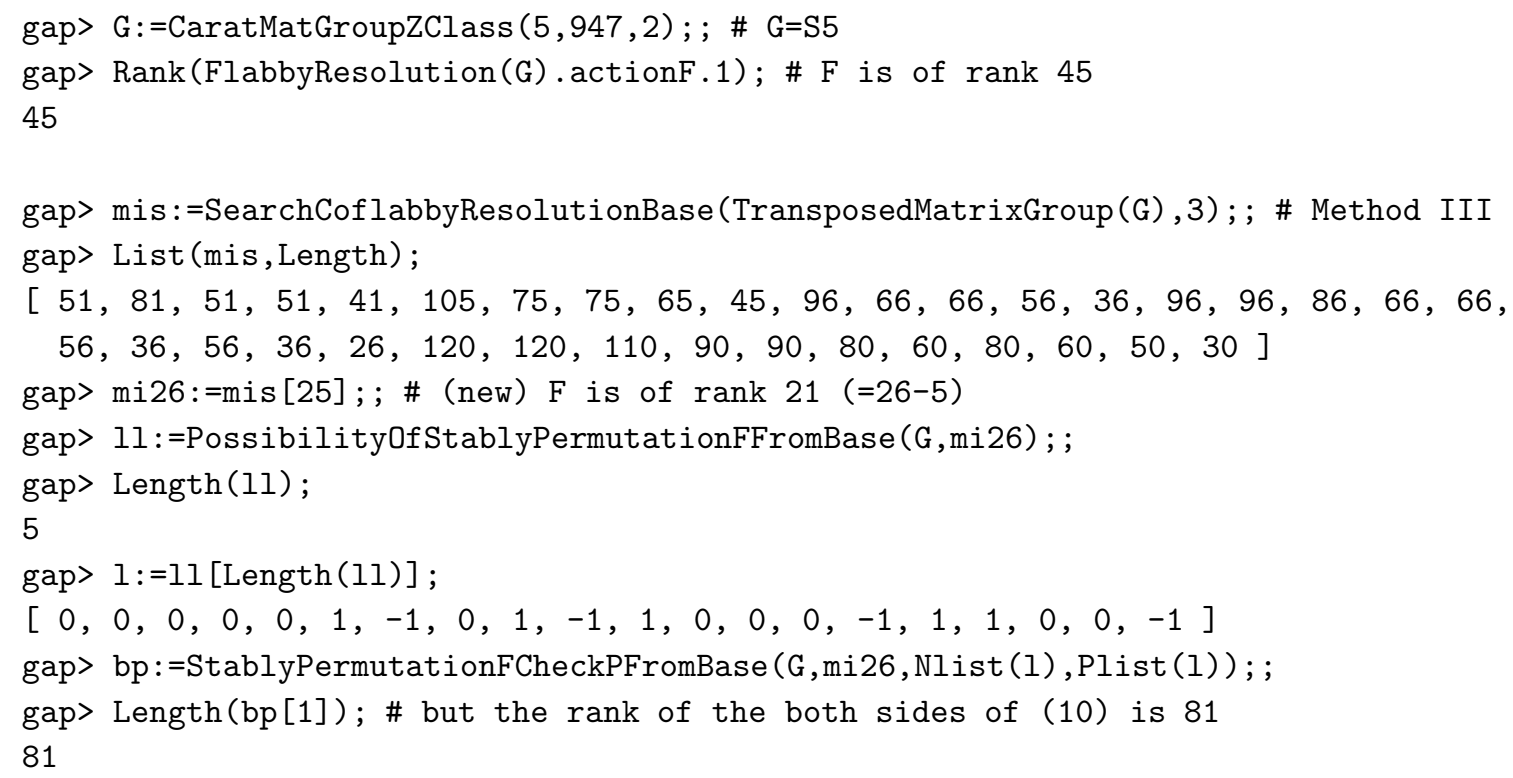


gap> mi:=mis [Length(mis)]; \# (new) F is of rank 25 (=30-5)

$[[-2,-1,-1,0,1],[-2,0,-1,1,1],[-1,-2,0,-1,1],[-1,-1,-1,-1,1]$, $[-1,-1,-1,0,0],[-1,-1,0,-1,0],[-1,0,0,1,1],[-1,1,-1,1,1]$, $[-1,1,-1,2,0],[-1,1,0,1,0],[0,-2,1,-1,1],[0,-1,1,0,1]$, $[0,0,-1,-1,1],[0,0,-1,1,-1],[0,0,1,-1,-1],[0,1,-1,0,1]$, $[0,1,-1,2,-1],[0,1,1,0,-1],[1,-1,1,-1,1],[1,-1,1,0,0]$, $[1,-1,2,-1,0],[1,0,0,-1,1],[1,0,0,1,-1],[1,0,2,-1,-1]$, $[1,1,-1,0,0],[1,1,-1,1,-1],[1,1,0,-1,0],[1,1,0,1,-2]$,

$[1,1,1,-1,-1],[1,1,1,0,-2]]$

gap> ll:=PossibilityOfStablyPermutationFFromBase (G,mi);

$[[1,0,0,-1,0,0,-4,0,1,-2,2,0,-1,-1,0,4,4,1,-4,0]$,

$[0,1,0,0,0,0,-1,0,0,-2,1,0,0,0,0,1,2,0,-1,-1]$,

$[0,0,1,0,0,0,-2,0,0,-1,1,0,-1,-1,0,2,2,1,-2,0]$,

$[0,0,0,0,1,0,-2,0,1,0,0,-2,-1,-2,0,2,2,1,-2,2]$,

$[0,0,0,0,0,1,0,0,0,-1,1,0,0,0,-1,0,1,0,0,-1]]$

gap> $1:=11[$ Length $(11)]$;

$[0,0,0,0,0,1,0,0,0,-1,1,0,0,0,-1,0,1,0,0,-1]$

gap> Length (1);

20

gap> $[1[6], 1[11], 1[17], 1[10], 1[15], 1[20]]$;

$[1,1,1,-1,-1,-1]$

gap> ss:=List (ConjugacyClassesSubgroups $2(G), x->$ StructureDescription(Representative(x))) ;

[ "1", "C2", "C2", "C3", "C2 x C2", "C2 x C2", "C4", "C5", "C6", "S3", "S3",

"D8", "D10", "A4", "D12", "C5 : C4", "S4", "A5", "S5" ]

gap> Length(ss);

19

gap> [ss [6] ,ss [11], ss [17], ss [10] ,ss [15]];

[ "C2 x C2", "S3", "S4", "S3", "D12" ]

gap> bp:=StablyPermutationFCheckPFromBase (G, mi,Nlist(1),Plist(1)); ;

gap> Length(bp);

40

gap> Length(bp[1]); \# rank of the both sides of (10) is 55

55

gap> $12:=$ IdentityMat (Length (1)) [Length $(1)-1]$;

$[0,0,0,0,0,0,0,0,0,0,0,0,0,0,0,0,0,0,1,0]$

gap> bp:=StablyPermutationFCheckPFromBase (G, mi, Nlist(1)+12, Plist(1)+12); ;

gap> Length (bp);

47

gap> Length(bp[1]); \# rank of the both sides of (10) is 56

56

\# after some efforts we may get

gap> $\mathrm{n}:=[$

$>1,0,-1,0,-1,-1,-1,1,0,0,-1,1,-1,0,-1,1,1,1,1,0,0,1,0,1,0$,

$>-1,-1,-1,0,-1,1,2,0,-1,0,0,1,1,1,-1,-1,0,-1,0,1,0,1]$;

gap> $\mathrm{p}:=\mathrm{n} * \mathrm{bp} ;$;

gap> Determinant $(p)$;

$-1$

gap> StablyPermutationFCheckMatFromBase (G, mi,Nlist (1)+12, Plist (1) +12,p));

true

Example 11.7 (Verification of $\left[M_{G}\right]^{f l}=0$ for the group $G \simeq S_{5}$ of the CARAT ID $(5,946,4)$ ). 
gap> G:=CaratMatGroupZClass $(5,946,4) ; ;$ \# G=S5

gap> Rank(FlabbyResolution(G).actionF.1); \#F is of rank 17

17

gap> mis:=SearchCoflabbyResolutionBase (TransposedMatrixGroup (G) ,5) ; ;

gap> Set(List(mis, Length))-5; \# Method III could not apply

$[17,32,35,47,50,62,65,77,80,92,95]$

gap> 11:=PossibilityOfStablyPermutationF (G) ;

$[[1,0,0,-1,0,0,-4,0,1,-2,2,0,-1,-1,0,4,4,1,-4,0]$,

$[0,1,0,0,0,0,-2,0,1,-2,1,0,0,-1,0,2,3,1,-2,-1]$,

$[0,0,1,0,0,0,-2,0,0,-1,1,0,-1,-1,0,2,2,1,-2,0]$,

$[0,0,0,0,1,0,0,0,-1,0,0,-2,-1,0,0,0,0,-1,0,2]$,

$[0,0,0,0,0,1,-1,0,1,-1,1,0,0,-1,-1,1,2,1,-1,-1]]$

gap> $1:=11[$ Length $(11)]$;

$[0,0,0,0,0,1,-1,0,1,-1,1,0,0,-1,-1,1,2,1,-1,-1]$

gap> Length (1);

20

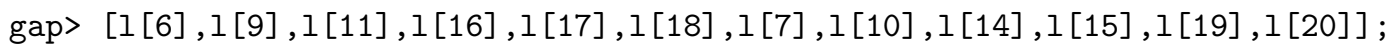

$[1,1,1,1,2,1,-1,-1,-1,-1,-1,-1]$

gap> ss:=List (ConjugacyClassesSubgroups2(G), $\mathrm{x}->$ StructureDescription (Representative (x))) ;

[ "1", "C2", "C2", "C3", "C2 x C2", "C2 x C2", "C4", "C5", "S3", "C6", "S3",

"D8", "D10", "A4", "D12", "C5 : C4", "S4", "A5", "S5" ]

gap> [ss [6] , ss [9] , ss [11] , ss [16] , ss [17] , ss [18] , ss [7] , ss [10] , ss [14] , ss [15] , ss [19]] ;

[ "C2 x C2", "S3", "S3", "C5 : C4", "S4", "A5", "C4", "C6", "A4", "D12", "S5" ]

gap> bp:=StablyPermutationFCheckP(G,Nlist (1),Plist(1)); ;

gap> Length (bp);

122

gap> Length(bp[1]); \# rank of the both sides of (10) is 88

88

\# after some efforts we may get

gap> $\mathrm{n}:=[$

$>-1,1,1,0,0,1,0,1,0,0,1,1,1,-1,1,-1,0,-1,0,0,-1,1,0,-1,1$,

$>1,1,0,0,0,-1,-1,0,-1,1,0,1,1,-1,0,1,1,0,-1,1,0,1,1,-1,0$,

$>1,1,-1,0,-1,1,-1,-1,0,1,1,0,1,-1,1,-1,-1,0,1,-1,0,0,0,-1,1$,

$>0,-1,-1,-1,-1,0,-1,-1,1,1,1,0,2,-2,4,0,1,3,-1,-1,-1,-1,-1,0,-1$,

$>-1,-1,-1,1,0,1,-1,0,-1,1,0,-1,-1,1,-1,0,0,-1,1,1,-1,1] ;$;

gap $>\mathrm{p}:=\mathrm{n} * \mathrm{bp} ;$;

gap> Determinant $(p)$;

$-1$

gap> StablyPermutationFCheckMat (G,Nlist(1),Plist(1),p);

true

12. Proof of Theorem 12.3

Theorem 12.1 (Yamasaki Yam12, Lemma 4.3]). Let $k$ be a field of char $k \neq 2$ and $k(x, y, z)$ be the rational function field over $k$ with variables $x, y, z$. Let $\sigma$ be a $k$-involution on $k(x, y, z)$ defined by

$$
\sigma: x \mapsto-x, \quad y \mapsto \frac{a}{y}, \quad z \mapsto \frac{-b x^{2}+c}{z} \quad\left(a, b, c \in k^{\times}\right)
$$

(i) $k(x, y, z)^{\langle\sigma\rangle}=k\left(z_{0}, z_{1}, z_{2}, z_{3}\right)$ where

$$
z_{0}^{2}=\left(z_{1}^{2}-a\right)\left(z_{2}^{2}-b\right)\left(z_{3}^{2}-c\right)
$$


(ii) The fixed field $k(x, y, z)^{\langle\sigma\rangle}$ is $k$-rational if and only if $[k(\sqrt{a}, \sqrt{b}, \sqrt{c}): k] \leq 2$ or $[k(\sqrt{a}, \sqrt{b}, \sqrt{c}): k]=4$ with $a b c \notin k^{\times 2}$. In particular, if $k(x, y, z)^{\langle\sigma\rangle}$ is not $k$-rational, then $k(x, y, z)^{\langle\sigma\rangle}$ is not retract $k$-rational.

Example 12.2 (Another proof of Theorem 12.1 not retract $k$-rational cases). Assume that $[k(\sqrt{a}, \sqrt{b}, \sqrt{c}): k]=$ 4 and $a b c \in k^{\times 2}$. We will show that $k(x, y, z)^{\langle\sigma\rangle}$ is not retract $k$-rational by using Is InvertibleF in Algorithm F2. This also implies that $k(x, y, z)^{\langle\sigma\rangle}$ is not retract $k$-rational when $[k(\sqrt{a}, \sqrt{b}, \sqrt{c}): k]=8$.

We may assume that $c=a b$. Put $\alpha=\sqrt{a}, \beta=\sqrt{b}$ and $L=k(\alpha, \beta)$. Then $L=k(\sqrt{a}, \sqrt{b}, \sqrt{c})$ and $[L: k]=4$. Put $y^{\prime}:=\frac{y-\alpha}{y+\alpha}, z^{\prime}:=\frac{z-\beta x-\alpha \beta}{z+\beta x+\alpha \beta}$. Then $L(x, y, z)=L\left(x, y^{\prime}, z^{\prime}\right)$ and $\sigma$ acts on $L\left(x, y^{\prime}, z^{\prime}\right)$ by $\sigma: x \mapsto-x, y^{\prime} \mapsto-y^{\prime}$, $z^{\prime} \mapsto-z^{\prime}$. Put $y_{1}:=x^{2}, y_{2}:=x y^{\prime}, y_{3}:=x z^{\prime}$. Then $k(x, y, z)^{\langle\sigma\rangle}=\left(L(x, y, z)^{\langle\sigma\rangle}\right)^{\left\langle\rho_{a}, \rho_{b}\right\rangle}=L\left(y_{1}, y_{2}, y_{3}\right)^{\left\langle\rho_{a}, \rho_{b}\right\rangle}$ is $L$-rational where

$$
\begin{aligned}
& \rho_{a}: \alpha \mapsto-\alpha, y_{1} \mapsto y_{1}, y_{2} \mapsto \frac{y_{1}}{y_{2}}, y_{3} \mapsto \frac{y_{1}\left(y_{3}+\alpha\right)}{y_{1}+\alpha y_{3}}, \\
& \rho_{b}: \beta \mapsto-\beta, y_{1} \mapsto y_{1}, y_{2} \mapsto y_{2}, y_{3} \mapsto \frac{y_{1}}{y_{3}} .
\end{aligned}
$$

Let $G=\operatorname{Gal}(L / k)=\left\langle\rho_{a}, \rho_{b}\right\rangle \simeq C_{2} \times C_{2}$. We consider the $G$-lattice $M=\left\langle y_{1}, y_{2}, y_{3}, t_{1}, t_{2}, t_{3}\right\rangle$ of rank 6 where $\left(t_{1}, t_{2}, t_{3}\right)=\left(y_{1}-a, y_{1}+\alpha y_{3}, y_{3}+\alpha\right)$. The action of $G$ on $L(M)$ is given by

$$
\begin{aligned}
& \rho_{a}: \alpha \mapsto-\alpha, y_{1} \mapsto y_{1}, y_{2} \mapsto \frac{y_{1}}{y_{2}}, y_{3} \mapsto \frac{y_{1} t_{3}}{t_{2}}, t_{1} \mapsto t_{1}, t_{2} \mapsto \frac{y_{1} t_{1}}{t_{2}}, t_{3} \mapsto \frac{y_{3} t_{1}}{t_{2}}, \\
& \rho_{b}: \beta \mapsto-\beta, y_{1} \mapsto y_{1}, y_{2} \mapsto y_{2}, y_{3} \mapsto \frac{y_{1}}{y_{3}}, t_{1} \mapsto t_{1}, t_{2} \mapsto \frac{y_{1} t_{3}}{y_{3}}, t_{3} \mapsto \frac{t_{2}}{y_{3}} .
\end{aligned}
$$

The actions of $\rho_{a}$ and $\rho_{b}$ on $M$ are represented as matrices

$$
\left(\begin{array}{cccccc}
1 & 0 & 0 & 0 & 0 & 0 \\
1 & -1 & 0 & 0 & 0 & 0 \\
1 & 0 & 0 & 0 & -1 & 1 \\
0 & 0 & 0 & 1 & 0 & 0 \\
1 & 0 & 0 & 1 & -1 & 0 \\
0 & 0 & 1 & 1 & -1 & 0
\end{array}\right),\left(\begin{array}{cccccc}
1 & 0 & 0 & 0 & 0 & 0 \\
0 & 1 & 0 & 0 & 0 & 0 \\
1 & 0 & -1 & 0 & 0 & 0 \\
0 & 0 & 0 & 1 & 0 & 0 \\
1 & 0 & -1 & 0 & 0 & 1 \\
0 & 0 & -1 & 0 & 1 & 0
\end{array}\right) .
$$

By Algorithm [F2, we see that $[M]^{f l}$ is not invertible. Hence $L(M)^{G}$ is not retract $k$-rational. It follows from Yam12, Theorem 2.10] that $k(x, y, z)^{\langle\sigma\rangle}$ is not retract $k$-rational (cf. [Yam12, Case 3 in the proof of Lemma 4.3], in particular, we do not need to enlarge $M$ in order to vanish $\left.H^{1}\right)$.

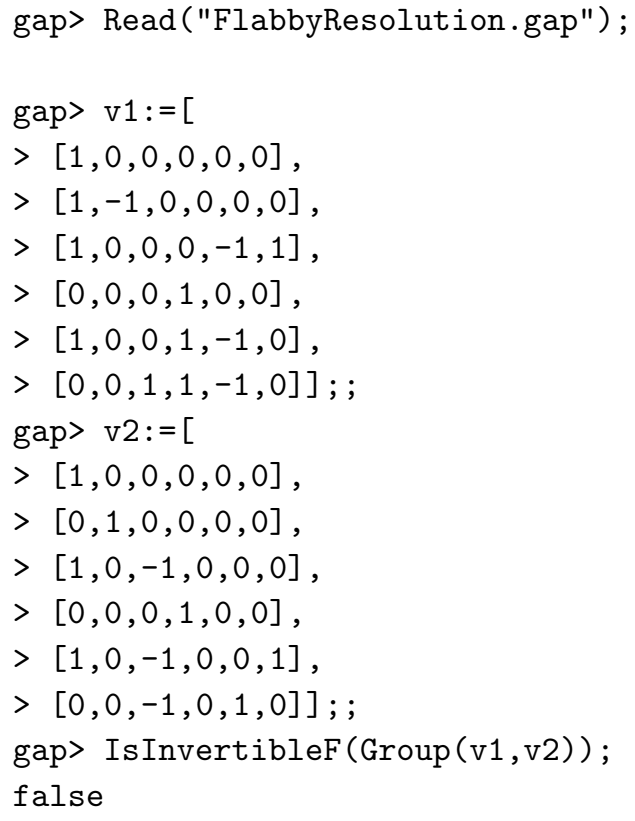

We generalize Theorem 12.1 as follows:

Theorem 12.3. Let $k$ be a field of char $k \neq 2$ and $k(x, y, z)$ be the rational function field over $k$ with variables $x, y, z$. Let $\sigma_{a, b, c, d}$ be a $k$-involution on $k(x, y, z)$ defined by

$$
\sigma_{a, b, c, d}: x \mapsto-x, \quad y \mapsto \frac{-a x^{2}+b}{y}, \quad z \mapsto \frac{-c x^{2}+d}{z} \quad\left(a, b, c, d \in k^{\times}\right)
$$


and $m=[k(\sqrt{a}, \sqrt{b}, \sqrt{c}, \sqrt{d}): k]$.

(i) $k(x, y, z)^{\left\langle\sigma_{a, b, c, d}\right\rangle}=k\left(t_{1}, t_{2}, t_{3}, t_{4}\right)$ where $t_{1}, t_{2}, t_{3}, t_{4}$ satisfy the relation

$$
\left(t_{1}^{2}-a\right)\left(t_{4}^{2}-d\right)=\left(t_{2}^{2}-b\right)\left(t_{3}^{2}-c\right) .
$$

(ii) $k(x, y, z)^{\left\langle\sigma_{a, b, c, d}\right\rangle}$ is $k$-isomorphic to $k(x, y, z)^{\left\langle\sigma_{\tau(a), \tau(b), \tau(c), \tau(d)}\right\rangle}$ for $\tau \in D_{4}$ where $D_{4}=\langle(a b d c),(a b)(c d)\rangle$ is the permutation group on the set $\{a, b, c, d\}$ which is isomorphic to the dihedral group of order 8 .

(iii) If one of the following conditions holds, then $k(x, y, z)^{\left\langle\sigma_{a, b, c, d}\right\rangle}$ is not retract $k$-rational:

(C15) $m=4$, (1) $a b, a c d \in k^{\times 2}$; (2) $b d, a b c \in k^{\times 2}$; (3) $c d, a b d \in k^{\times 2}$; (4) $a c, b c d \in k^{\times 2}$;

(C16) $m=4$, (1) $a d, a b c \in k^{\times 2} ;(2) b c, a b d \in k^{\times 2}$;

(C18) $m=8$, (1) $a b \in k^{\times 2}$; (2) $a c \in k^{\times 2}$; (3) $b d \in k^{\times 2}$; (4) $c d \in k^{\times 2}$;

(C19) $m=8$, (1) $a d \in k^{\times 2} ;(2) b c \in k^{\times 2}$;

(C20) $m=8$, (1) $a b c \in k^{\times 2} ;(2) b c d \in k^{\times 2} ;$ (3) $a b d \in k^{\times 2} ;$ (4) $a c d \in k^{\times 2}$;

(C21) $m=8, a b c d \in k^{\times 2}$;

(C22) $m=16$.

Proof of Theorem 12.3. We prove the assertion (i). Put

$$
\begin{aligned}
& t_{1}:=\frac{1}{2 x}\left(y-\frac{-a x^{2}+b}{y}\right), t_{2}:=\frac{1}{2}\left(y+\frac{-a x^{2}+b}{y}\right), \\
& t_{3}:=\frac{1}{2 x}\left(z-\frac{-c x^{2}+d}{z}\right), t_{4}:=\frac{1}{2}\left(z+\frac{-c x^{2}+d}{z}\right) .
\end{aligned}
$$

Then we see that $k\left(t_{1}, t_{2}, t_{3}, t_{4}\right) \subset k(x, y, z)^{\left\langle\sigma_{a, b, c, d}\right\rangle}$. It follows from the equalities

$$
y=t_{2}+t_{1} x, z=t_{4}+t_{3} x, x^{2}\left(t_{3}^{2}-c\right)-\left(t_{4}^{2}-d\right)=0
$$

that $\left[k(x, y, z): k\left(t_{1}, t_{2}, t_{3}, t_{4}\right)\right] \leq 2$. Hence we get $k(x, y, z)^{\left\langle\sigma_{a, b, c, d}\right\rangle}=k\left(t_{1}, t_{2}, t_{3}, t_{4}\right)$. The relation $\left(t_{1}^{2}-a\right)\left(t_{4}^{2}-d\right)=$ $\left(t_{2}^{2}-b\right)\left(t_{3}^{2}-c\right)$ may be obtained by the direct calculation. The assertion (ii) follows from (i). We will prove the assertion (iii).

The case (C15): $m=4$. By (ii), we should show only the case (1) $a b, a c d \in k^{\times 2}$. Define $Y:=\frac{a y}{a x+\sqrt{a b}}$. Then $k(x, y, z)=k(x, Y, z)$ and $\sigma_{a, b, c, d}$ acts on $k(x, Y, z)$ by

$$
\sigma_{a, b, c, d}: x \mapsto-x, \quad Y \mapsto \frac{a}{Y}, \quad z \mapsto \frac{-c x^{2}+d}{z} .
$$

By Theorem 12.1 (ii), $k(x, y, z)^{\left\langle\sigma_{a, b, c, d}\right\rangle}$ is not retract $k$-rational.

The case (C16): $m=4$. By (ii), we should treat only the case (1) ad, abc $\in k^{\times 2}$. Let $L=k(\alpha, \beta, \gamma, \delta)$ where $\alpha^{2}=a, \beta^{2}=b, \gamma^{2}=c, \delta^{2}=d$. Then $L=k(\alpha, \beta)$ and $[L: k]=4$. Put $y^{\prime}:=(\alpha x+\beta) / y, z^{\prime}:=(\gamma x+\delta) / z$. Then $L(x, y, z)=L\left(x, y^{\prime}, z^{\prime}\right)$ and $\sigma_{a, b, c, d}$ acts on $L\left(x, y^{\prime}, z^{\prime}\right)$ by

$$
\sigma_{a, b, c, d}: x \mapsto x, y^{\prime} \mapsto \frac{1}{y^{\prime}}, z^{\prime} \mapsto \frac{1}{z^{\prime}} .
$$

We put

$$
y_{1}:=x^{2}, y_{2}:=x \frac{1-y^{\prime}}{1+y^{\prime}}=x \frac{y-\alpha x-\beta}{y+\alpha x+\beta}, y_{3}:=x \frac{1-z^{\prime}}{1+z^{\prime}}=x \frac{z-\gamma x-\delta}{y+\gamma x+\delta} .
$$

By the assumptions $a d, a b c \in k^{\times 2}$, there exist $e, f \in k^{\times}$such that $\gamma=\alpha \beta e$ and $\delta=\alpha f$. Then $k(x, y, z)^{\left\langle\sigma_{a, b, c, d}\right\rangle}=$ $\left(L(x, y, z)^{\left\langle\sigma_{a, b, c, d}\right\rangle}\right)^{\left\langle\rho_{a}, \rho_{b}\right\rangle}=L\left(y_{1}, y_{2}, y_{3}\right)^{\left\langle\rho_{a}, \rho_{b}\right\rangle}$, where

$$
\begin{aligned}
& \rho_{a}: \alpha \mapsto-\alpha, y_{1} \mapsto y_{1}, y_{2} \mapsto \frac{\alpha y_{1}+\beta y_{2}}{\alpha y_{2}+\beta}, y_{3} \mapsto \frac{y_{1}}{y_{3}}, \\
& \rho_{b}: \beta \mapsto-\beta, y_{1} \mapsto y_{1}, y_{2} \mapsto \frac{y_{1}\left(\alpha y_{2}+\beta\right)}{\alpha y_{1}+\beta y_{2}}, y_{3} \mapsto \frac{\beta e y_{1}+f y_{3}}{\beta e y_{3}+f} .
\end{aligned}
$$

Let $G=\operatorname{Gal}(L / k)=\left\langle\rho_{a}, \rho_{b}\right\rangle \simeq C_{2} \times C_{2}$. We consider the $G$-lattice $M=\left\langle y_{1}, y_{2}, y_{3}, t_{1}, t_{2}, t_{3}, t_{4}, u_{1}, u_{2}\right\rangle$ of rank 9 where

$$
\left(t_{1}, t_{2}, t_{3}, t_{4}, u_{1}, u_{2}\right)=\left(\alpha y_{1}+\beta y_{2}, \alpha y_{2}+\beta, \beta e y_{1}+f y_{3}, \beta e y_{3}+f, a y_{1}-b,-b e^{2} y_{1}+f^{2}\right)
$$


The action of $G$ on $L(M)$ is given by

$$
\begin{aligned}
\rho_{a}: \alpha \mapsto-\alpha, y_{2} \mapsto \frac{t_{1}}{t_{2}}, y_{3} \mapsto \frac{y_{1}}{y_{3}}, t_{1} \mapsto-\frac{u_{1} y_{2}}{t_{2}}, t_{2} \mapsto-\frac{u_{1}}{t_{2}}, t_{3} \mapsto \frac{y_{1} t_{4}}{y_{3}}, t_{4} \mapsto \frac{t_{3}}{y_{3}}, \\
\rho_{b}: \beta \mapsto-\beta, y_{2} \mapsto \frac{y_{1} t_{2}}{t_{1}}, y_{3} \mapsto \frac{t_{3}}{t_{4}}, t_{1} \mapsto \frac{y_{1} u_{1}}{t_{1}}, t_{2} \mapsto \frac{u_{1} y_{2}}{t_{1}}, t_{3} \mapsto \frac{u_{2} y_{3}}{t_{4}}, t_{4} \mapsto \frac{u_{2}}{t_{4}}
\end{aligned}
$$

where $y_{1}, u_{1}, u_{2}$ are invariants under the action of $G$. The actions of $\rho_{a}$ and $\rho_{b}$ on $M$ are represented as matrices

$\left(\begin{array}{ccccccccc}1 & 0 & 0 & 0 & 0 & 0 & 0 & 0 & 0 \\ 0 & 0 & 0 & 1 & -1 & 0 & 0 & 0 & 0 \\ 1 & 0 & -1 & 0 & 0 & 0 & 0 & 0 & 0 \\ 0 & 1 & 0 & 0 & -1 & 0 & 0 & 1 & 0 \\ 0 & 0 & 0 & 0 & -1 & 0 & 0 & 1 & 0 \\ 1 & 0 & -1 & 0 & 0 & 0 & 1 & 0 & 0 \\ 0 & 0 & -1 & 0 & 0 & 1 & 0 & 0 & 0 \\ 0 & 0 & 0 & 0 & 0 & 0 & 0 & 1 & 0 \\ 0 & 0 & 0 & 0 & 0 & 0 & 0 & 0 & 1\end{array}\right),\left(\begin{array}{ccccccccc}1 & 0 & 0 & 0 & 0 & 0 & 0 & 0 & 0 \\ 1 & 0 & 0 & -1 & 1 & 0 & 0 & 0 & 0 \\ 0 & 0 & 0 & 0 & 0 & 1 & -1 & 0 & 0 \\ 1 & 0 & 0 & -1 & 0 & 0 & 0 & 1 & 0 \\ 0 & 1 & 0 & -1 & 0 & 0 & 0 & 1 & 0 \\ 0 & 0 & 1 & 0 & 0 & 0 & -1 & 0 & 1 \\ 0 & 0 & 0 & 0 & 0 & 0 & -1 & 0 & 1 \\ 0 & 0 & 0 & 0 & 0 & 0 & 0 & 1 & 0 \\ 0 & 0 & 0 & 0 & 0 & 0 & 0 & 0 & 1\end{array}\right)$.

By Algorithm $\mathrm{F} 2$, we see that $[M]^{f l}$ is not invertible (see Example 12.4 below). Hence $L(M)^{G}$ is not retract $k$-rational. It follows from [Yam12, Theorem 2.10] that $k(x, y, z)^{\left\langle\sigma_{a, b, c, d}\right\rangle}$ is not retract $k$-rational.

The cases $(\mathrm{C} 18),(\mathrm{C} 19)$ and $(\mathrm{C} 20)$. By the result of $(\mathrm{C} 15)$ and $(\mathrm{C} 16), k(x, y, z)^{\left\langle\sigma_{a, b, c, d}\right\rangle}$ is not retract rational over some quadratic extension of $k$, hence not retract $k$-rational.

The case (C21): $m=8, a b c d \in k^{\times 2}$. Let $L=k(\sqrt{a}, \sqrt{b}, \sqrt{c}, \sqrt{d})$. We assume $[L: k]=8$ and $a b c d \in k^{\times 2}$, and hence $d=a b c e^{2}$ for some $e \in k^{\times}$. We put $\alpha:=\sqrt{a}, \beta:=\sqrt{b}, \gamma:=\sqrt{c}$. Then $L=k(\alpha, \beta, \gamma)$. We first see that $L(x, y, z)^{\left\langle\sigma_{a, b, c, d}\right\rangle}$ is $L$-rational as follows. Put

$$
y_{1}:=x^{2}, y_{2}:=x \frac{y-\alpha x-\beta}{y+\alpha x+\beta}, y_{3}:=x \frac{z-\gamma x-\alpha \beta \gamma e}{z+\gamma x+\alpha \beta \gamma e} .
$$

Then $L(x, y, z)=L\left(x, y_{2}, y_{3}\right)$ and the action of $\sigma_{a, b, c, d}$ on $L\left(x, y_{2}, y_{3}\right)$ is given by $\sigma_{a, b, c, d}: x \mapsto-x, y_{2} \mapsto y_{2}, y_{3} \mapsto$ $y_{3}$. Hence the field $L(x, y, z)^{\left\langle\sigma_{a, b, c, d}\right\rangle}=L\left(y_{1}, y_{2}, y_{3}\right)$ is $L$-rational. Let $G=\left\langle\rho_{a}, \rho_{b}, \rho_{c}\right\rangle \simeq C_{2} \times C_{2} \times C_{2}$ be the Galois group $\operatorname{Gal}(L / k)$ of $L / k$ where

$$
\begin{aligned}
& \rho_{a}: \alpha \mapsto-\alpha, \beta \mapsto \beta, \gamma \mapsto \gamma, \\
& \rho_{b}: \alpha \mapsto \alpha, \beta \mapsto-\beta, \gamma \mapsto \gamma, \\
& \rho_{c}: \alpha \mapsto \alpha, \beta \mapsto \beta, \gamma \mapsto-\gamma
\end{aligned}
$$

Then we get $k(x, y, z)^{\left\langle\sigma_{a, b, c, d}\right\rangle}=\left(L(x, y, z)^{\left\langle\sigma_{a, b, c, d}\right\rangle}\right)^{G}=L\left(y_{1}, y_{2}, y_{3}\right)^{G}$. The action of $G$ on $L\left(y_{1}, y_{2}, y_{3}\right)$ is given by

$$
\begin{aligned}
& \rho_{a}: \alpha \mapsto-\alpha, y_{1} \mapsto y_{1}, y_{2} \mapsto \frac{\alpha y_{1}+\beta y_{2}}{\alpha y_{2}+\beta}, y_{3} \mapsto \frac{y_{1}\left(y_{3}+\alpha \beta e\right)}{y_{1}+\alpha \beta e y_{3}}, \\
& \rho_{b}: \beta \mapsto-\beta, y_{1} \mapsto y_{1}, y_{2} \mapsto \frac{y_{1}\left(\alpha y_{2}+\beta\right)}{\alpha y_{1}+\beta y_{2}}, y_{3} \mapsto \frac{y_{1}\left(y_{3}+\alpha \beta e\right)}{y_{1}+\alpha \beta e y_{3}}, \\
& \rho_{c}: \gamma \mapsto-\gamma, y_{1} \mapsto y_{1}, y_{2} \mapsto y_{2}, y_{3} \mapsto \frac{y_{1}}{y_{3}} .
\end{aligned}
$$

We consider the $G$-lattice $M=\left\langle y_{1}, y_{2}, y_{3}, t_{1}, t_{2}, t_{3}, t_{4}, u_{1}, u_{2}\right\rangle$ of rank 9 where

$$
\left(t_{1}, t_{2}, t_{3}, t_{4}, u_{1}, u_{2}\right)=\left(\alpha y_{1}+\beta y_{2}, \alpha y_{2}+\beta, y_{3}+\alpha \beta e, y_{1}+\alpha \beta e y_{3}, b-a y_{1}, y_{1}-a b e^{2}\right) .
$$

The action of $G$ on $L(M)$ is given by

$$
\begin{aligned}
& \rho_{a}: \alpha \mapsto-\alpha, y_{2} \mapsto \frac{t_{1}}{t_{2}}, y_{3} \mapsto \frac{y_{1} t_{3}}{t_{4}}, t_{1} \mapsto \frac{u_{1} y_{2}}{t_{2}}, t_{2} \mapsto \frac{u_{1}}{t_{2}}, t_{3} \mapsto \frac{u_{2} y_{3}}{t_{4}}, t_{4} \mapsto \frac{y_{1} u_{2}}{t_{4}}, \\
& \rho_{b}: \beta \mapsto-\beta, y_{2} \mapsto \frac{y_{1} t_{2}}{t_{1}}, y_{3} \mapsto \frac{y_{1} t_{3}}{t_{4}}, t_{1} \mapsto-\frac{y_{1} u_{2}}{t_{1}}, t_{2} \mapsto-\frac{u_{1} y_{2}}{t_{1}}, t_{3} \mapsto \frac{u_{2} y_{3}}{t_{4}}, t_{4} \mapsto \frac{y_{1} u_{2}}{t_{4}}, \\
& \rho_{c}: \gamma \mapsto-\gamma, y_{2} \mapsto y_{2}, y_{3} \mapsto \frac{y_{1}}{y_{3}}, t_{1} \mapsto t_{1}, t_{2} \mapsto t_{2}, t_{3} \mapsto \frac{t_{4}}{y_{3}}, t_{4} \mapsto \frac{y_{1} t_{3}}{y_{3}}
\end{aligned}
$$

where $y_{1}, u_{1}, u_{2}$ are invariants under the action of $G$. The actions of $\rho_{a}, \rho_{b}$ and $\rho_{c}$ on $M$ are represented as matrices 


$$
\left(\begin{array}{ccccccccc}
1 & 0 & 0 & 0 & 0 & 0 & 0 & 0 & 0 \\
0 & 0 & 0 & 1 & -1 & 0 & 0 & 0 & 0 \\
1 & 0 & 0 & 0 & 0 & 1 & -1 & 0 & 0 \\
0 & 1 & 0 & 0 & -1 & 0 & 0 & 1 & 0 \\
0 & 0 & 0 & 0 & -1 & 0 & 0 & 1 & 0 \\
0 & 0 & 1 & 0 & 0 & 0 & -1 & 0 & 1 \\
1 & 0 & 0 & 0 & 0 & 0 & -1 & 0 & 1 \\
0 & 0 & 0 & 0 & 0 & 0 & 0 & 1 & 0 \\
0 & 0 & 0 & 0 & 0 & 0 & 0 & 0 & 1
\end{array}\right),\left(\begin{array}{ccccccccc}
1 & 0 & 0 & 0 & 0 & 0 & 0 & 0 & 0 \\
1 & 0 & 0 & -1 & 1 & 0 & 0 & 0 & 0 \\
1 & 0 & 0 & 0 & 0 & 1 & -1 & 0 & 0 \\
1 & 0 & 0 & -1 & 0 & 0 & 0 & 1 & 0 \\
0 & 1 & 0 & -1 & 0 & 0 & 0 & 1 & 0 \\
0 & 0 & 1 & 0 & 0 & 0 & -1 & 0 & 1 \\
1 & 0 & 0 & 0 & 0 & 0 & -1 & 0 & 1 \\
0 & 0 & 0 & 0 & 0 & 0 & 0 & 1 & 0 \\
0 & 0 & 0 & 0 & 0 & 0 & 0 & 0 & 1
\end{array}\right),\left(\begin{array}{ccccccccc}
1 & 0 & 0 & 0 & 0 & 0 & 0 & 0 & 0 \\
0 & 1 & 0 & 0 & 0 & 0 & 0 & 0 & 0 \\
1 & 0 & -1 & 0 & 0 & 0 & 0 & 0 & 0 \\
0 & 0 & 0 & 1 & 0 & 0 & 0 & 0 & 0 \\
0 & 0 & 0 & 0 & 1 & 0 & 0 & 0 & 0 \\
0 & 0 & -1 & 0 & 0 & 0 & 1 & 0 & 0 \\
1 & 0 & -1 & 0 & 0 & 1 & 0 & 0 & 0 \\
0 & 0 & 0 & 0 & 0 & 0 & 0 & 1 & 0 \\
0 & 0 & 0 & 0 & 0 & 0 & 0 & 0 & 1
\end{array}\right) .
$$

By Algorithm [F2, we obtain that $[M]^{f l}$ is not invertible (see Example 12.4 below). Hence $L(M)^{G}$ is not retract $k$-rational. By Yam12, Theorem 2.10], $k(x, y, z)^{\left\langle\sigma_{a, b, c, d}\right\rangle}$ is not retract $k$-rational.

The case $(\mathrm{C} 22): m=16$. By the result of $(\mathrm{C} 21), k(x, y, z)^{\left\langle\sigma_{a, b, c, d}\right\rangle}$ is not retract rational over a quadratic extension of $k$, hence not retract $k$-rational.

Example 12.4 ([M] $]^{f l}$ is not invertible for the cases (C16) and (C21)). The following GAP computation confirms that $[M]^{f l}$ is not invertible hence $L(M)^{G}$ is not retract $k$-rational as in the cases $(\mathrm{C} 16)$ and $(\mathrm{C} 21)$ of the proof of Theorem 12.3 above.

gap> Read("FlabbyResolution.gap");

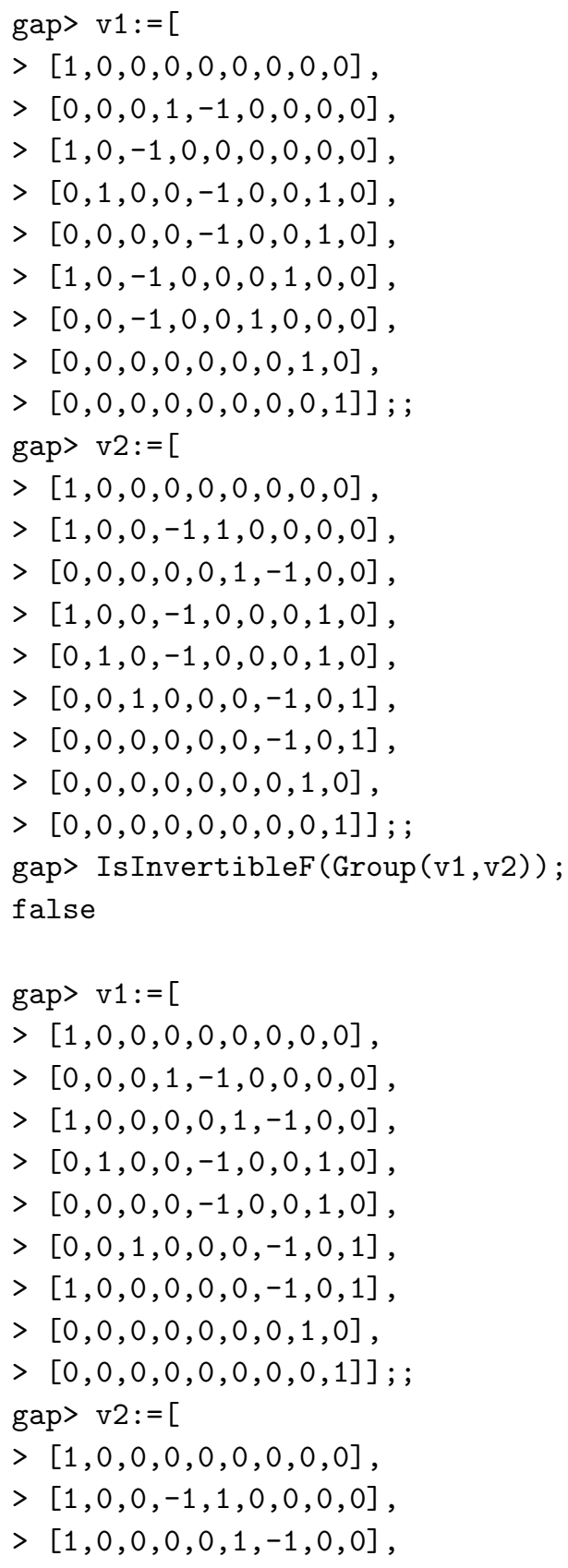




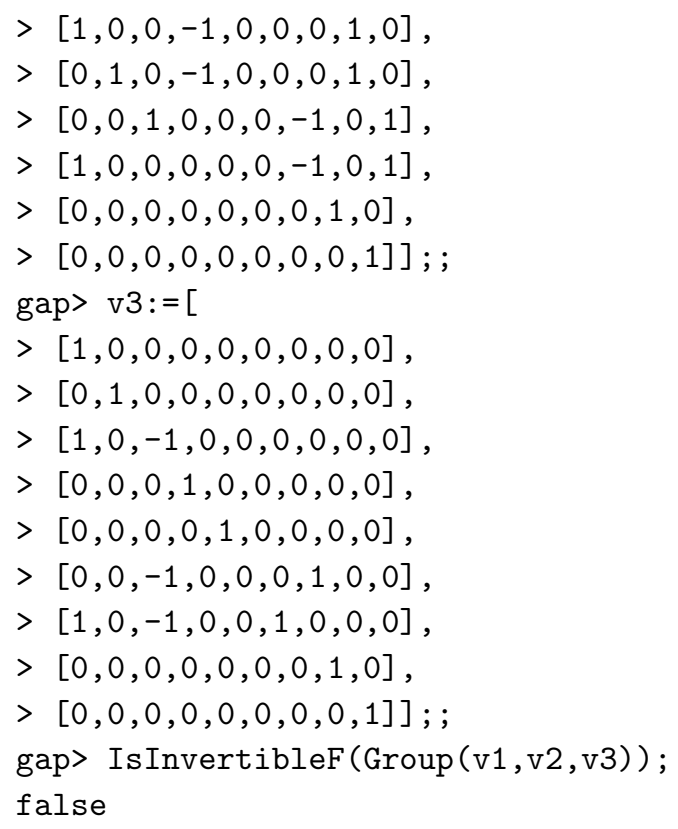

Lemma 12.5. Let $k$ be a field of char $k \neq 2$ and $k(x, y, z)$ be the rational function field over $k$ with variables $x, y, z$. Let $\sigma_{a, b, c, d}$ be a k-involution on $k(x, y, z)$ defined by

$$
\sigma_{a, b, c, d}: x \mapsto-x, \quad y \mapsto \frac{-a x^{2}+b}{y}, \quad z \mapsto \frac{-c x^{2}+d}{z} \quad\left(a, b, c, d \in k^{\times}\right)
$$

and $m=[k(\sqrt{a}, \sqrt{b}, \sqrt{c}, \sqrt{d}): k]$. If one of the following conditions holds, then $k(x, y, z)^{\left\langle\sigma_{a, b, c, d}\right\rangle}$ is $k$-rational:

(C1) $m=1$;

(C2) $m=2$, (1) $a, b, c \in k^{\times 2}$; (2) $a, b, d \in k^{\times 2}$; (3) $a, c, d \in k^{\times 2}$; (4) b, c, $d \in k^{\times 2}$;

(C3) $m=2$, (1) $a, b, c d \in k^{\times 2}$; (2) b, d, ac $\in k^{\times 2}$; (3) $d, c, a b \in k^{\times 2}$; (4) $c, a, b d \in k^{\times 2}$;

(C5) $m=2$, (1) $a, b d, c d \in k^{\times 2}$; (2) b, cd, ac $\in k^{\times 2}$; (3) $d, a c, a b \in k^{\times 2}$; (4) $c, a b, b d \in k^{\times 2}$;

(C6) $m=2, a b, a c, a d \in k^{\times 2}$;

(C7) $m=4$, (1) $a, b \in k^{\times 2}$; (2) b, $d \in k^{\times 2}$; (3) $d, c \in k^{\times 2}$; (4) $c, a \in k^{\times 2}$;

(C10) $m=4$, (1) $a, b d \in k^{\times 2}$; (2) b, dc $\in k^{\times 2}$; (3) $d, a c \in k^{\times 2}$; (4) $c, a b \in k^{\times 2}$;

(5) $a, c d \in k^{\times 2}$; (6) $b, a c \in k^{\times 2} ;$ (7) $d, a b \in k^{\times 2}$; (8) $c, b d \in k^{\times 2}$;

(C12) $m=4$, (1) $a b, c d \in k^{\times 2} ;(2) b d, a c \in k^{\times 2}$;

(C13) $m=4$, (1) $a b, a c \in k^{\times 2} ;(2) b d, a b \in k^{\times 2} ;(3) c d, b d \in k^{\times 2} ;$ (4) $a c, c d \in k^{\times 2}$.

Proof. The case (C7): $m=4$. By Theorem 12.3 (ii), we should show only the case (1) $a, b \in k^{\times 2}$. Take $\alpha, \beta \in k$ with $\alpha^{2}=a$ and $\beta^{2}=b$. The equation (19) becomes

$$
\frac{\left(t_{1}+\alpha\right)\left(t_{1}-\alpha\right)}{\left(t_{2}+\beta\right)\left(t_{2}-\beta\right)}=\frac{t_{3}^{2}-c}{t_{4}^{2}-d}
$$

Define $T_{1}:=\left(t_{1}+\alpha\right) /\left(t_{2}+\beta\right), T_{2}:=\left(t_{1}-\alpha\right) /\left(t_{2}-\beta\right)$. Then $k(x, y, z)^{\left\langle\sigma_{a, b, c, d}\right\rangle}=k\left(t_{1}, t_{2}, t_{3}, t_{4}\right)=k\left(T_{1}, T_{2}, t_{3}, t_{4}\right)=$ $k\left(T_{1}, t_{3}, t_{4}\right)$ is $k$-rational.

The cases (C10), (C12) and (C13): $m=4$. By Theorem 12.3 (ii), we may assume that $a b \in k^{\times 2}$. Define $Y:=\frac{a y}{a x+\sqrt{a b}}$. Then $k(x, y, z)=k(x, Y, z)$ and $\sigma_{a, b, c, d}$ acts on $k(x, Y, z)$ by

$$
\sigma_{a, b, c, d}: x \mapsto-x, \quad Y \mapsto \frac{a}{Y}, \quad z \mapsto \frac{-c x^{2}+d}{z} .
$$

By Theorem 12.1 (ii), $k(x, y, z)^{\left\langle\sigma_{a, b, c, d}\right\rangle}$ is $k$-rational.

The cases (C1), (C2), (C3), (C5) and (C6). By the results of (C10), (C12) and (C13), k(x,y,z) $\left.{ }^{\left\langle\sigma_{a, b, c}, d\right.}\right\rangle$ is $k$-rational.

Remark 12.6. We do not know whether $k(x, y, z)^{\left\langle\sigma_{a, b, c, d}\right\rangle}$ is $k$-rational (resp. stably $k$-rational, retract $k$-rational) for the following cases:

(C4) $m=2$, (1) $a, d, b c \in k^{\times 2} ;(2) b, c, a d \in k^{\times 2}$;

(C8) $m=4$, (1) $a, d \in k^{\times 2}$; (2) b,c $\in k^{\times 2}$; 
(C9) $m=4$, (1) $a, b c \in k^{\times 2}$; (2) $b, a d \in k^{\times 2}$; (3) $d, b c \in k^{\times 2}$; (4) $c, a d \in k^{\times 2}$;

(C11) $m=4$, (1) $a, b c d \in k^{\times 2}$; (2) $b, a c d \in k^{\times 2}$; (3) $d, a b c \in k^{\times 2}$; (4) $c, a b d \in k^{\times 2}$;

(C14) $m=4, a d, b c \in k^{\times 2}$;

(C17) $m=8$, (1) $a \in k^{\times 2}$; (2) $b \in k^{\times 2}$; (3) $d \in k^{\times 2}$; (4) $c \in k^{\times 2}$.

For example, for the case of (C4) $m=2$, (1) $a, d, b c \in k^{\times 2}, k(x, y, z)^{\left\langle\sigma_{a, b, c, d}\right\rangle}$ may be obtained as follows. Let $L=k(\alpha, \beta, \gamma, \delta)$ where $\alpha^{2}=a, \beta^{2}=b, \gamma^{2}=c, \delta^{2}=d$. Then $L=k(\beta)$ and $L(x, y, z)=L\left(x, y^{\prime}, z^{\prime}\right)$ where $y^{\prime}=(\alpha x+\beta) / y$ and $z^{\prime}=(\gamma x+\delta) / z$. We see that $\sigma_{a, b, c, d}$ acts on $L\left(x, y^{\prime}, z^{\prime}\right)$ by

$$
\sigma_{a, b, c, d}: x \mapsto x, y^{\prime} \mapsto \frac{1}{y^{\prime}}, z^{\prime} \mapsto \frac{1}{z^{\prime}}
$$

Hence $L\left(x, y^{\prime}, z^{\prime}\right)^{\left\langle\sigma_{a, b, c, d}\right\rangle}=L\left(y_{1}, y_{2}, y_{3}\right)$ where

$$
y_{1}=x^{2}, y_{2}=x \frac{1-y^{\prime}}{1+y^{\prime}}=x \frac{y-\alpha x-\beta}{y+\alpha x+\beta}, y_{3}=x \frac{1-z^{\prime}}{1+z^{\prime}}=x \frac{z-\gamma x-\delta}{y+\gamma x+\delta} .
$$

By the assumption $b c \in k^{\times 2}$, there exists $e \in k^{\times}$such that $\gamma=\beta e$. Then $k(x, y, z)^{\left\langle\sigma_{a, b, c, d}\right\rangle}=\left(L(x, y, z)^{\left\langle\sigma_{a, b, c, d}\right\rangle}\right)^{\left\langle\rho_{b}\right\rangle}$ $=L\left(y_{1}, y_{2}, y_{3}\right)^{\left\langle\rho_{b}\right\rangle}$ and

$$
\rho_{b}: \beta \mapsto-\beta, y_{1} \mapsto y_{1}, y_{2} \mapsto \frac{y_{1}\left(\alpha y_{2}+\beta\right)}{\alpha y_{1}+\beta y_{2}}, y_{3} \mapsto \frac{\beta e y_{1}+\delta y_{3}}{\beta e y_{3}+\delta}
$$

Define

$$
z_{1}:=\beta e\left(\frac{\beta e y_{1}+\delta y_{3}}{\beta e y_{3}+\delta}-y_{3}\right)-2 \delta, z_{2}:=e\left(\frac{\beta e y_{1}+\delta y_{3}}{\beta e y_{3}+\delta}+y_{3}\right), z_{3}:=4 b e^{2}\left(\alpha y_{1}+\beta y_{2}\right) .
$$

Then $L\left(y_{1}, y_{2}, y_{3}\right)=L\left(z_{1}, z_{2}, z_{3}\right)$ and

$$
\rho_{b}: \beta \mapsto-\beta, z_{1} \mapsto z_{1}, z_{2} \mapsto z_{2}, z_{3} \mapsto \frac{f\left(z_{1}, z_{2}\right)}{z_{3}}
$$

where $f\left(z_{1}, z_{2}\right)=\left(z_{1}^{2}-b z_{2}^{2}-4 d\right)\left(a\left(z_{1}^{2}-b z_{2}^{2}-4 d\right)+4 b^{2} e^{2}\right)$. Define

$$
t_{1}:=\frac{1}{2}\left(z_{3}+\frac{f\left(z_{1}, z_{2}\right)}{z_{3}}\right), t_{2}:=\frac{1}{2 \beta}\left(z_{3}-\frac{f\left(z_{1}, z_{2}\right)}{z_{3}}\right) .
$$

Then $k(x, y, z)^{\left\langle\sigma_{a, b, c, d}\right\rangle}=k\left(t_{1}, t_{2}, z_{1}, z_{2}\right)$ where

$$
t_{1}^{2}-b t_{2}^{2}=f\left(z_{1}, z_{2}\right)=\left(z_{1}^{2}-b z_{2}^{2}-4 d\right)\left(a\left(z_{1}^{2}-b z_{2}^{2}-4 d\right)+4 b^{2} e^{2}\right) .
$$

However, we do not know whether $k(x, y, z)^{\left\langle\sigma_{a, b, c, d}\right\rangle}$ is $k$-rational (resp. stably $k$-rational, retract $k$-rational).

\section{Applichtion of Theorem 12.3}

Let $G$ be a finite group acting on the rational function field $k\left(x_{g} \mid g \in G\right)$ by $k$-automorphisms $h\left(x_{g}\right)=x_{h g}$ for any $g, h \in G$. We denote the fixed field $k\left(x_{g} \mid g \in G\right)^{G}$ by $k(G)$. Noether's problem asks whether $k(G)$ is $k$-rational (see a survey paper of Swan [Swa83] for Noether's problem for abelian groups). The unramified Brauer group $B r_{v, k}(L)$ of $L$ over $k$ is defined to be $B r_{v, k}(L)=\cap_{R} B r(R)$ where $B r(R)$ is the image of the injective map $\operatorname{Br}(R) \rightarrow \operatorname{Br}(L)$ of Brauer groups and $R$ runs over all discrete valuation domain with $k \subset R$ and the quotient field of $R$ is $L$ (cf. [Sal84b]).

Let $F$ be an algebraically closed field with $\operatorname{gcd}\{\operatorname{char} k,|G|\}=1$. By [Sal90, Theorem 12], Bog90, Theorem $1.3^{\prime}$ ], $B r_{v, F}(F(G))=\cap_{A} \operatorname{Ker}\left(\right.$ res : $\left.H^{2}(G, \mu) \rightarrow H^{2}(A, \mu)\right)$ where $\mu \subset F^{\times}$denotes the subgroup of roots of unity, res is the restriction map of cohomology groups and $A$ runs over abelian subgroups of $G$ of rank 1 or 2 . If $G$ is a 2 -group, this is valid not only over an algebraically closed field but also over a quadratically closed field $F$ (i.e. a field of $\operatorname{char} F \neq 2$ satisfying $\sqrt{a} \in F$ for any $a \in F$ ). We denote $B r_{v, F}(F(G))$ by $B_{0}(G)$ (cf. [BMP04, Kun10, CHKK10]). By [Sal84b, Proposition 3.2] and Sal87, Proposition 2.2], if $L_{1} / L_{2}(\supset k)$ is retract rational, then $B r_{v, k}\left(L_{1}\right) \simeq B r_{v, k}\left(L_{2}\right)$ and hence if $F(G)$ is retract $F$-rational, then $B_{0}(G)=0$.

Saltman [Sal84b] showed that for any prime $p$ there exists a meta-abelian $p$-group $G$ of order $p^{9}$ such that $B_{0}(G) \neq 0$. In particular, $F(G)$ is not retract $F$-rational. Moreover, Bogomolov Bog88 obtained that when char $F=0$ there exists a $p$-group $G$ of order $p^{6}$ such that $B_{0}(G) \neq 0$ for any prime $p$. Recently, Moravec [Mor12] proved that there exist exactly 3 groups of order $3^{5}$ such that $B_{0}(G) \neq 0$ by GAP computations. Hoshi, Kang and Kunyavskii HKK13. showed that there exist exactly $1+\operatorname{gcd}\{4, p-1\}+\operatorname{gcd}\{3, p-1\}$ groups of order $p^{5}$ such that $B_{0}(G) \neq 0$ for any prime $p \geq 5$. 
In the case where $p=2$, by $\mathrm{Chu}$ and Kang [CK01] and $\mathrm{Chu}$, Hu, Kang and Prokhorov [CHKP08, if $G$ is a group of order $\leq 32$, then $F(G)$ is $F$-rational. Moreover $\mathrm{Chu}$, Hu, Kang and Kunyavskii [CHKK10 investigated the case where $G$ is a group of order 64 as follows. There exist exactly 267 non-isomorphic groups of order 64 .

Let $C_{n}$ be the cyclic group of order $n, Z(G)$ be the center of the group $G$ and $[G, G]$ be the commutator subgroup of the group $G$. Let $G(n, i)$ be the $i$-th group of order $n$ in GAP [GAP].

Theorem 13.1 ([CHKK10, Theorem 1.8]). Let $G$ be a group of order 64 and $F$ be a quadratically closed field. Then the following conditions are equivalent:

(i) $B_{0}(G) \neq 0$;

(ii) $Z(G) \simeq C_{2}^{2},[G, G] \simeq C_{4} \times C_{2}, G /[G, G] \simeq C_{2}^{3}$, G has no abelian subgroup of index 2 , and $G$ has no faithful 4-dimensional representation over $\mathbb{C}$;

(iii) $G$ is isomorphic to one of the nine groups $G(64, i)$ where $i=149,150,151,170,171,172,177,178,182$.

Theorem 13.2 ([CHKK10, Theorem 1.9]). Let $G$ be a group of order 64 and $F$ be a quadratically closed field. If $B_{0}(G)=0$, then $F(G)$ is $F$-rational except possibly for groups $G$ which is isomorphic to one of the five groups $G(64, i)$ where $241 \leq i \leq 245$.

Theorem 13.3 (CHKK10, Proposition 6.3]). Let $G=G(64, i)$ where $241 \leq i \leq 245, F$ be a quadratically closed field and $H=\left\langle f_{1}, f_{2}\right\rangle \simeq C_{2} \times C_{2}$ act on the rational function field $F\left(x_{1}, x_{2}, x_{3}, y_{1}, y_{2}, y_{3}\right)$ by $F$-automorphisms defined by

$$
\begin{aligned}
& f_{1}: x_{1} \mapsto \frac{1}{x_{1}}, x_{2} \mapsto \frac{1}{x_{1} x_{3}}, x_{3} \mapsto \frac{x_{1}}{x_{2}}, y_{1} \mapsto y_{1}, y_{2} \mapsto \frac{y_{1}}{y_{2}}, y_{3} \mapsto \frac{1}{y_{1} y_{3}}, \\
& f_{2}: x_{1} \mapsto \frac{1}{x_{1}}, x_{2} \mapsto x_{3}, x_{3} \mapsto x_{2}, y_{1} \mapsto \frac{1}{y_{1}}, y_{2} \mapsto y_{3}, y_{3} \mapsto y_{2} .
\end{aligned}
$$

Let $L=F\left(x_{1}, x_{2}, x_{3}, y_{1}, y_{2}, y_{3}\right)^{H}$. Then there is an F-injective homomorphism $\varphi: L \rightarrow F(G)$ so that $F(G)$ is a rational extension over $\rho(L)$.

Let $k\left(x_{1}, \ldots, x_{n}\right)$ be the rational function field over $k$ with $n$ variables $x_{1}, \ldots, x_{n}$. Let $H$ be a finite subgroup of $\mathrm{GL}(n, \mathbb{Z})$ acting on $k\left(x_{1}, \ldots, x_{n}\right)$ by $k$-automorphisms

$$
\sigma\left(x_{j}\right)=c_{j}(\sigma) \prod_{i=1}^{n} x_{i}^{a_{i, j}}, \sigma=\left[a_{i, j}\right]_{1 \leq i, j \leq n} \in H, c_{j}(\sigma) \in k^{\times}, \text {for } 1 \leq j \leq n,
$$

where $k^{\times}=k \backslash\{0\}$. This action of $H$ on $k\left(x_{1}, \ldots, x_{n}\right)$ is said to be monomial. If $c_{j}(\sigma)=1$ for any $\sigma \in G$ and any $1 \leq j \leq n$, the action is said to be purely monomial. The rationality problem with respect to monomial action, i.e. whether $k\left(x_{1}, \ldots, x_{n}\right)^{G}$ is $k$-rational, is determined up to conjugacy in $\operatorname{GL}(n, \mathbb{Z})$ and solved affirmatively by Hajja Haj83, Haj87 when $n=2$. For $n=3$, the problem under purely monomial actions was solved affirmatively by Hajja and Kang [HK92, [HK94 and Hoshi and Rikuna HR08, (see also [KP10, HK10, Yam12] and HKY11] for non-purely monomial case).

It is remarked in [CHKK10, page 2537] that although $L_{0}:=F\left(x_{1}, x_{2}, x_{3}\right)^{H}=F\left(t_{1}, t_{2}, t_{3}\right)$ is $F$-rational, the field $L$ as in Theorem 13.3 may be regarded as $L=L_{0}(\alpha, \beta)\left(y_{1}, y_{2}, y_{3}\right)^{H}$ the function field of a 3-dimensional algebraic torus defined over $L_{0}$ and split over biquadratic Galois extension $F\left(x_{1}, x_{2}, x_{3}\right)=L_{0}(\alpha, \beta)$ of $L_{0}$ for some $\alpha, \beta$ with $\alpha^{2}, \beta^{2} \in L_{0}$. By Theorem 1.2 the field $L$ is not stably rational over $L_{0}=F\left(t_{1}, t_{2}, t_{3}\right)$. We restate Theorem 1.2 of Kunyavskii after adopting the definition of the action of $G$ via (20) (we should take $G$ instead of ${ }^{t} G$ and hence the corresponding GAP IDs may change, cf. Theorem 1.2).

Theorem 13.4 (Kunyavskii [Kun90]). Let $L / k$ be a Galois extension and $G \simeq \operatorname{Gal}(L / k)$ be a finite subgroup of $\mathrm{GL}(3, \mathbb{Z})$ which acts on $L\left(x_{1}, x_{2}, x_{3}\right)$ via (20). Then $L\left(x_{1}, x_{2}, x_{3}\right)^{G}$ is not $k$-rational if and only if $G$ is conjugate to one of the 15 groups which are given as in Table 10 . Moreover, if $L\left(x_{1}, x_{2}, x_{3}\right)^{G}$ is not $k$-rational, then it is

\begin{tabular}{|c|c|c|c|c|c|c|c|c|}
\hline$G$ in Kun90 & GAP ID & $G$ & $G$ in Kun90 & GAP ID & $G$ & $G$ in Kun90 & GAP ID & $G$ \\
\hline$U_{1}$ & $(3,3,1,4)$ & $C_{2}^{2}$ & $U_{6}$ & $(3,4,7,2)$ & $D_{4} \times C_{2}$ & $U_{11}$ & $(3,7,5,2)$ & $S_{4} \times C_{2}$ \\
\hline$U_{2}$ & $(3,3,3,4)$ & $C_{2}^{3}$ & $U_{7}$ & $(3,7,2,3)$ & $A_{4} \times C_{2}$ & $U_{12}$ & $(3,7,5,3)$ & $S_{4} \times C_{2}$ \\
\hline$U_{3}$ & $(3,4,4,2)$ & $D_{4}$ & $U_{8}$ & $(3,7,3,2)$ & $S_{4}$ & $W_{1}$ & $(3,4,3,2)$ & $C_{4} \times C_{2}$ \\
\hline$U_{4}$ & $(3,4,6,4)$ & $D_{4}$ & $U_{9}$ & $(3,7,3,3)$ & $S_{4}$ & $W_{2}$ & $(3,3,3,3)$ & $C_{2}^{3}$ \\
\hline$U_{5}$ & $(3,7,1,3)$ & $A_{4}$ & $U_{10}$ & $(3,7,4,3)$ & $S_{4}$ & $W_{3}$ & $(3,7,2,2)$ & $A_{4} \times C_{2}$ \\
\hline
\end{tabular}
not retract $k$-rational.

Table 10: $L(M)^{G}$ not retract $k$-rational, rank $M=3, M$ : indecomposable (15 cases) 
Let $H=\left\langle f_{1}, f_{2}\right\rangle \simeq C_{2} \times C_{2}$ act on $k\left(x_{1}, x_{2}, x_{3}, y_{1}, y_{2}, y_{3}\right)$ by $k$-automorphisms as the same in Theorem 13.3 . The purely monomial actions of $H$ on $k\left(x_{1}, x_{2}, x_{3}\right)$ and on $k\left(y_{1}, y_{2}, y_{3}\right)$ correspond to the same conjugacy class of the GAP ID $(3,3,1,4)$. Indeed, if we define $\left(X_{1}, X_{2}, X_{3}, Y_{1}, Y_{2}, Y_{3}\right):=\left(x_{1} / x_{2}, 1 /\left(x_{1} x_{3}\right), x_{3}, 1 /\left(y_{1} y_{3}\right), y_{1} / y_{2}, y_{3}\right)$, then $k\left(x_{1}, x_{2}, x_{3}, y_{1}, y_{2}, y_{3}\right)=k\left(X_{1}, X_{2}, X_{3}, Y_{1}, Y_{2}, Y_{3}\right)$ and the action of $H$ on $k\left(X_{1}, X_{2}, X_{3}, Y_{1}, Y_{2}, Y_{3}\right)$ is given by

$$
\begin{aligned}
\sigma_{1}: X_{1} \mapsto X_{3}, X_{2} \mapsto \frac{1}{X_{1} X_{2} X_{3}}, X_{3} \mapsto X_{1}, Y_{1} \mapsto Y_{3}, Y_{2} \mapsto \frac{1}{Y_{1} Y_{2} Y_{3}}, Y_{3} \mapsto Y_{1}, \\
\sigma_{2}: X_{1} \mapsto X_{2}, X_{2} \mapsto X_{1}, X_{3} \mapsto \frac{1}{X_{1} X_{2} X_{3}}, Y_{1} \mapsto Y_{2}, Y_{2} \mapsto Y_{1}, Y_{3} \mapsto \frac{1}{Y_{1} Y_{2} Y_{3}}
\end{aligned}
$$

We define

$$
s_{1}:=\frac{1+X_{1} X_{3}}{1-X_{1} X_{3}}, s_{2}:=\frac{1+X_{2} X_{3}}{1-X_{2} X_{3}}, s_{3}:=X_{3}, s_{4}:=\frac{1+Y_{1} Y_{3}}{1-Y_{1} Y_{3}}, s_{5}:=\frac{1+Y_{2} Y_{3}}{1-Y_{2} Y_{3}}, s_{6}:=Y_{3} .
$$

Then $k\left(X_{1}, X_{2}, X_{3}, Y_{1}, Y_{2}, Y_{3}\right)=k\left(s_{1}, \ldots, s_{6}\right)$ and the actions of $\sigma_{1}$ and $\sigma_{2}$ on $k\left(s_{1}, \ldots, s_{6}\right)$ are given by

$$
\begin{aligned}
& \sigma_{1}: s_{1} \mapsto s_{1}, s_{2} \mapsto-s_{2}, s_{3} \mapsto \frac{s_{1}-1}{s_{3}\left(s_{1}+1\right)}, s_{4} \mapsto s_{4}, s_{5} \mapsto-s_{5}, s_{6} \mapsto \frac{s_{4}-1}{s_{6}\left(s_{4}+1\right)}, \\
& \sigma_{2}: s_{1} \mapsto-s_{1}, s_{2} \mapsto-s_{2}, s_{3} \mapsto \frac{s_{3}\left(s_{1}+1\right)\left(s_{2}+1\right)}{\left(s_{1}-1\right)\left(s_{2}-1\right)}, s_{4} \mapsto-s_{4}, s_{5} \mapsto-s_{5}, s_{6} \mapsto \frac{s_{6}\left(s_{4}+1\right)\left(s_{5}+1\right)}{\left(s_{4}-1\right)\left(s_{5}-1\right)} .
\end{aligned}
$$

We also define

$$
t_{1}:=s_{1} s_{2}, t_{2}:=\frac{\left(s_{1} s_{2}+1\right) s_{3}}{\left(s_{1}-1\right)\left(s_{2}-1\right)}, t_{3}:=\frac{\left(s_{4} s_{5}+1\right) s_{6}}{\left(s_{4}-1\right)\left(s_{5}-1\right)}, t_{4}:=s_{2}^{2}, t_{5}:=\frac{s_{1}}{s_{4}}, t_{6}:=\frac{s_{2}}{s_{5}} .
$$

Then we have $k\left(s_{1}, \ldots, s_{6}\right)=k\left(t_{1}, t_{2}, t_{3}, s_{2}, t_{5}, t_{6}\right)$ and $\sigma_{2}: s_{2} \mapsto-s_{2}, t_{i} \mapsto t_{i}, 1 \leq i \leq 6$. It follows that $k\left(x_{1}, x_{2}, x_{3}, y_{1}, y_{2}, y_{3}\right)^{\left\langle\sigma_{2}\right\rangle}=k\left(t_{1}, \ldots, t_{6}\right)$ and the action of $\sigma_{1}$ on $k\left(t_{1}, \ldots, t_{6}\right)$ is given by

$$
\sigma_{1}: t_{1} \mapsto-t_{1}, t_{2} \mapsto \frac{\left(t_{1}^{2}-1\right) t_{4}}{t_{2}\left(t_{1}^{2}-t_{4}\right)\left(t_{4}-1\right)}, t_{3} \mapsto \frac{t_{4}\left(t_{5}^{2} t_{6}^{2}-t_{1}^{2}\right)}{t_{3}\left(t_{6}^{2}-t_{4}\right)\left(t_{1}^{2}-t_{4} t_{5}^{2}\right)}, t_{4} \mapsto t_{4}, t_{5} \mapsto t_{5}, t_{6} \mapsto t_{6} .
$$

Define

$$
u_{1}:=\frac{t_{1}}{t_{4}}, u_{2}:=\frac{t_{1}+1}{t_{2}}, u_{3}:=\frac{t_{5} t_{6}+t_{1}}{t_{3}}, u_{4}:=t_{4}, u_{5}:=t_{5}, u_{6}:=t_{6} .
$$

Then we get $k\left(t_{1}, \ldots, t_{6}\right)=k\left(u_{1}, \ldots, u_{6}\right)$ and the action of $\sigma_{1}$ on $k\left(u_{1}, \ldots, u_{6}\right)$ is given by

$$
\begin{aligned}
\sigma_{1}: u_{1} & \mapsto-u_{1}, u_{2} \mapsto-\frac{\left(u_{4}-1\right)\left(u_{1}^{2} u_{4}-1\right)}{u_{2}}, u_{3} \mapsto-\frac{\left(u_{4}-u_{6}^{2}\right)\left(u_{1}^{2} u_{4}-u_{5}^{2}\right)}{u_{3}}, \\
u_{4} & \mapsto u_{4}, u_{5} \mapsto u_{5}, u_{6} \mapsto u_{6} .
\end{aligned}
$$

As a consequence of Theorem 12.3, we have:

Proposition 13.5. Let $k$ be a field of char $k \neq 2$ and $\left\langle\sigma_{1}, \sigma_{2}\right\rangle \simeq C_{2} \times C_{2}$ act on $k\left(X_{1}, X_{2}, X_{3}, Y_{1}, Y_{2}, Y_{3}\right)$ by k-automorphisms

$$
\begin{aligned}
\sigma_{1}: X_{1} \mapsto X_{3}, X_{2} \mapsto \frac{1}{X_{1} X_{2} X_{3}}, X_{3} \mapsto X_{1}, Y_{1} \mapsto Y_{3}, Y_{2} \mapsto \frac{1}{Y_{1} Y_{2} Y_{3}}, Y_{3} \mapsto Y_{1}, \\
\sigma_{2}: X_{1} \mapsto X_{2}, X_{2} \mapsto X_{1}, X_{3} \mapsto \frac{1}{X_{1} X_{2} X_{3}}, Y_{1} \mapsto Y_{2}, Y_{2} \mapsto Y_{1}, Y_{3} \mapsto \frac{1}{Y_{1} Y_{2} Y_{3}} .
\end{aligned}
$$

There exist algebraically independent elements $u_{4}, u_{5}, u_{6} \in k\left(X_{1}, X_{2}, X_{3}, Y_{1}, Y_{2}, Y_{3}\right)^{\left\langle\sigma_{1}, \sigma_{2}\right\rangle}$ over $k$ and algebraically dependent elements $z_{1}, z_{2}, z_{3}, z_{4} \in k\left(X_{1}, X_{2}, X_{3}, Y_{1}, Y_{2}, Y_{3}\right)^{\left\langle\sigma_{1}, \sigma_{2}\right\rangle}$ over $k\left(u_{4}, u_{5}, u_{6}\right)$ which satisfy the following conditions:

(i) $k\left(X_{1}, X_{2}, X_{3}, Y_{1}, Y_{2}, Y_{3}\right)^{\left\langle\sigma_{1}, \sigma_{2}\right\rangle}=k\left(z_{1}, z_{2}, z_{3}, z_{4}, u_{4}, u_{5}, u_{6}\right)$ and

$$
\left(z_{1}^{2}-a\right)\left(z_{4}^{2}-d\right)=\left(z_{2}^{2}-b\right)\left(z_{3}^{2}-c\right)
$$

where $a=u_{4}\left(u_{4}-1\right), b=u_{4}-1, c=u_{4}\left(u_{4}-u_{6}^{2}\right), d=u_{5}^{2}\left(u_{4}-u_{6}^{2}\right)$;

(ii) $k\left(z_{1}, z_{2}, z_{3}, z_{4}, u_{4}, u_{5}, u_{6}\right)$ is not retract rational over $k\left(u_{4}, u_{5}, u_{6}\right)$.

Proof. If we take a field $k\left(u_{4}, u_{5}, u_{6}\right)$ as the base field, the assertion (i) follows from (21) and Theorem 12.3 (i). For (ii), we see that $a b c d \in k\left(u_{4}, u_{5}, u_{6}\right)^{\times 2}$ and $m=\left[k\left(u_{4}, u_{5}, u_{6}\right)(\sqrt{a}, \sqrt{b}, \sqrt{c}, \sqrt{d}): k\left(u_{4}, u_{5}, u_{6}\right)\right]=8$. Thus (ii) follows from Theorem 12.3 (iii). 
Furthermore, we define

$$
v_{1}:=u_{1}, v_{2}:=\frac{u_{2}}{1-u_{1}^{2} u_{4}}, v_{3}:=\frac{\left(u_{1}^{2}-1\right) u_{3}}{1-u_{1}^{2} u_{4}}, v_{4}:=\frac{u_{4}-1}{1-u_{1}^{2} u_{4}}, v_{5}:=u_{5}, v_{6}:=u_{6} .
$$

Then $k\left(X_{1}, X_{2}, X_{3}, Y_{1}, Y_{2}, Y_{3}\right)^{\left\langle\sigma_{2}\right\rangle}=k\left(u_{1}, \ldots, u_{6}\right)=k\left(v_{1}, \ldots, v_{6}\right)$ and

$$
\sigma_{1}: v_{1} \mapsto-v_{1}, v_{2} \mapsto \frac{v_{4}}{v_{2}}, v_{3} \mapsto-\frac{\left(v_{1}^{2} v_{4} v_{5}^{2}-v_{1}^{2} v_{4}-v_{1}^{2}+v_{5}^{2}\right)\left(v_{1}^{2} v_{4} v_{6}^{2}+v_{6}^{2}-v_{4}-1\right)}{v_{3}}, v_{4} \mapsto v_{4}, v_{5} \mapsto v_{5}, v_{6} \mapsto v_{6} .
$$

We also put

$$
w_{1}:=v_{1}, w_{2}:=v_{3}, w_{3}:=\frac{1}{2}\left(v_{2}+\frac{v_{4}}{v_{2}}\right), w_{4}:=\frac{1}{2 v_{1}}\left(v_{2}-\frac{v_{4}}{v_{2}}\right), w_{5}:=v_{5}, w_{6}:=v_{6} .
$$

Then $k\left(v_{1}, \ldots, v_{6}\right)=k\left(w_{1}, \ldots, w_{6}\right)$ and

$\sigma_{1}: w_{1} \mapsto-w_{1}, w_{2} \mapsto-\frac{\left(w_{4}^{2}\left(w_{5}^{2}-1\right) w_{1}^{4}+\left(w_{3}^{2}-w_{3}^{2} w_{5}^{2}+1\right) w_{1}^{2}-w_{5}^{2}\right)\left(w_{4}^{2} w_{6}^{2} w_{1}^{4}-\left(w_{4}^{2}+w_{3}^{2} w_{6}^{2}\right) w_{1}^{2}+w_{3}^{2}-w_{6}^{2}+1\right)}{w_{2}}$, $w_{3} \mapsto w_{3}, w_{4} \mapsto w_{4}, w_{5} \mapsto w_{5}, w_{6} \mapsto w_{6}$.

Define $t=w_{1}^{2}$. Then $k\left(X_{1}, X_{2}, X_{3}, Y_{1}, Y_{2}, Y_{3}\right)^{\left\langle\sigma_{1}, \sigma_{2}\right\rangle}=k\left(u, v, t, w_{3}, w_{4}, w_{5}, w_{6}\right)$ with the relation

$$
u^{2}-t v^{2}=-\left(w_{4}^{2}\left(w_{5}^{2}-1\right) t^{2}+\left(w_{3}^{2}-w_{3}^{2} w_{5}^{2}+1\right) t-w_{5}^{2}\right)\left(w_{4}^{2} w_{6}^{2} t^{2}-\left(w_{4}^{2}+w_{3}^{2} w_{6}^{2}\right) t+w_{3}^{2}-w_{6}^{2}+1\right)
$$

(cf. HKK14, Section 6]).

We do not know whether $k\left(X_{1}, X_{2}, X_{3}, Y_{1}, Y_{2}, Y_{3}\right)^{\left\langle\sigma_{1}, \sigma_{2}\right\rangle}$ is $k$-rational (resp. stably $k$-rational, retract $k$ rational). We may also obtain the following description of $k\left(X_{1}, X_{2}, X_{3}, Y_{1}, Y_{2}, Y_{3}\right)^{\left\langle\sigma_{1}, \sigma_{2}\right\rangle}$ although the proof is omitted.

Theorem 13.6. The field $k\left(X_{1}, X_{2}, X_{3}, Y_{1}, Y_{2}, Y_{3}\right)^{\left\langle\sigma_{1}, \sigma_{2}\right\rangle}=k\left(m_{0}, \ldots, m_{6}\right)$ where

$$
m_{0}^{2}=\left(4 m_{3}+m_{3} m_{4}^{2}+m_{4}^{2}\right)\left(m_{3}-m_{5}^{2}+1\right)\left(m_{1}^{2} m_{3}+m_{6}^{2}-1\right)\left(4 m_{3}+m_{1}^{2} m_{2}^{2} m_{3}+m_{2}^{2} m_{6}^{2}\right) .
$$




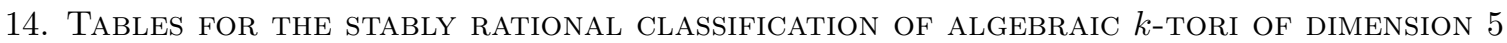

Table 11: $L(M)^{G}$ not stably but retract $k$-rational, $M=M_{1} \oplus M_{2}$, rank $M_{1}=4$, rank $M_{2}=1, M_{i}$ : indecomposable (25 cases)

\begin{tabular}{|c|c|c|c|c|c|c|c|c|c|}
\hline CARAT & $G$ & CARAT & $G$ & CARAT & $G$ & CARAT & G & CARAT & $G$ \\
\hline$(5,692,1)$ & $C_{3} \rtimes C_{8}$ & $(5,914,1)$ & $\overline{S_{5}}$ & $(5,918,5)$ & $\overline{F_{20}}$ & $(5,924,1)$ & $C_{2} \times S_{5}$ & $(5,928,1)$ & $C_{2} \times F_{20}$ \\
\hline$(5,693,1)$ & $C_{3} \rtimes C_{8}$ & $(5,916,1)$ & $S_{5}$ & $(5,919,1)$ & $C_{2} \times S_{5}$ & $(5,925,1)$ & $C_{2} \times S_{5}$ & $(5,928,3)$ & $C_{2} \times F_{20}$ \\
\hline$(5,736,1)$ & $C_{2} \times\left(C_{3} \rtimes C_{8}\right)$ & $(5,917,1)$ & $F_{20}$ & $(5,921,1)$ & $C_{2} \times S_{5}$ & $(5,926,1)$ & $C_{2} \times F_{20}$ & $(5,929,1)$ & $C_{2} \times F_{20}$ \\
\hline$(5,911,1)$ & $S_{5}$ & $(5,917,5)$ & $F_{20}$ & $(5,922,1)$ & $C_{2} \times S_{5}$ & $(5,926,3)$ & $C_{2} \times F_{20}$ & $(5,930,1)$ & $C_{2}^{2} \times S_{5}$ \\
\hline$(5,912,1)$ & $S_{5}$ & $(5,918,1)$ & $F_{20}$ & $(5,923,1)$ & $C_{2} \times S_{5}$ & $(5,927,1)$ & $C_{2} \times F_{20}$ & $(5,932,1)$ & $C_{2}^{2} \times F_{20}$ \\
\hline
\end{tabular}

Table 12: $L(M)^{G}$ not retract $k$-rational, $M=M_{1} \oplus M_{2} \oplus M_{3}$, $\operatorname{rank} M_{1}=3, \operatorname{rank} M_{2}=\operatorname{rank} M_{3}=1, M_{i}$ : indecomposable (245 cases)

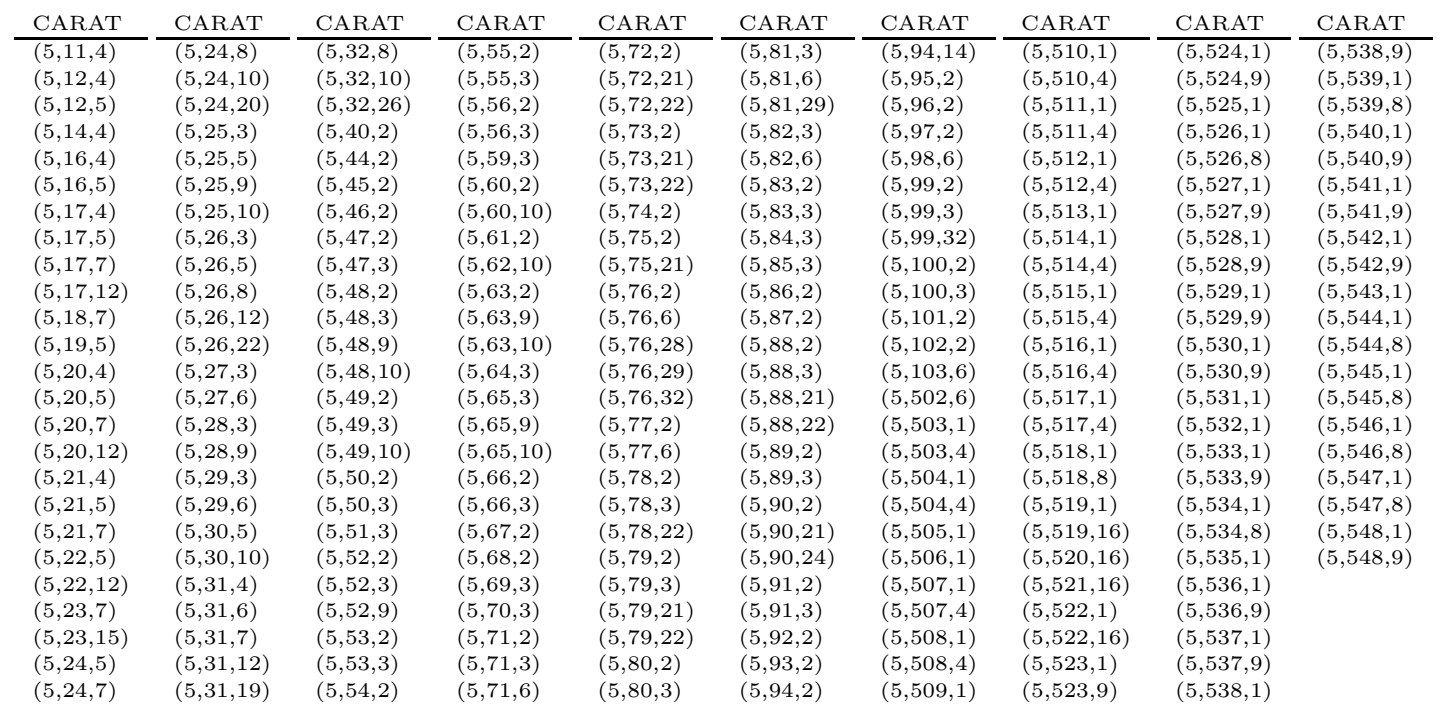


Table 13: $L(M)^{G}$ not retract $k$-rational, $M=M_{1} \oplus M_{2}$, rank $M_{1}=3$, rank $M_{2}=2, M_{i}$ : indecomposable (849 cases)

\begin{tabular}{|c|c|c|c|c|c|c|c|c|c|}
\hline CARAT & CARAT & CARAT & CARAT & CARAT & CARAT & CARAT & CARAT & CARAT & CARAT \\
\hline$(5,14,8)$ & $(5,73,35)$ & $(5,95,10)$ & $(5,140,16)$ & $(5,240,6)$ & $(5,299,4)$ & $(5,349,2)$ & $(5,528,12)$ & $(5,569,8)$ & $(5,603,3)$ \\
\hline$(5,16,8)$ & $(5,74,12)$ & $(5,96,5)$ & $(5,140,20)$ & $(5,240,14)$ & $(5,302,2)$ & $(5,349,4)$ & $(5,529,2)$ & $(5,569,9)$ & $(5,603,4)$ \\
\hline$(5,16,9)$ & $(5,75,12)$ & $(5,96,8)$ & $(5,140,22)$ & $(5,240,15)$ & $(5,302,10)$ & $(5,350,2)$ & $(5,529,12)$ & $(5,570,5)$ & $(5,603,6)$ \\
\hline$(5,17,11)$ & $(5,75,31)$ & $(5,96,10)$ & $(5,141,4)$ & $(5,241,4)$ & $(5,303,2)$ & $(5,350,4)$ & $(5,530,2)$ & $(5,570,6)$ & $(5,604,1)$ \\
\hline$(5,17,16)$ & $(5,76,11)$ & $(5,97,5)$ & $(5,141,10)$ & $(5,241,5)$ & $(5,303,6)$ & $(5,350,6)$ & $(5,530,12)$ & $(5,570,8)$ & $(5,604,5)$ \\
\hline$(5,18,19)$ & $(5,76,15)$ & $(5,97,8)$ & $(5,141,14)$ & $(5,241,9)$ & $(5,304,2)$ & $(5,350,8)$ & $(5,531,2)$ & $(5,570,9)$ & $(5,605,1)$ \\
\hline$(5,20,11)$ & $(5,76,20)$ & $(5,97,10)$ & $(5,141,16)$ & $(5,241,11)$ & $(5,304,10)$ & $(5,351,2)$ & $(5,532,2)$ & $(5,571,5)$ & $(5,606,1)$ \\
\hline$(5,20,16)$ & $(5,76,21)$ & $(5,98,20)$ & $(5,141,20)$ & $(5,242,4)$ & $(5,305,2)$ & $(5,351,4)$ & $(5,533,2)$ & $(5,571,8)$ & $(5,606,5)$ \\
\hline$(5,21,11)$ & $(5,76,37)$ & $(5,99,15)$ & $(5,141,22)$ & $(5,242,14)$ & $(5,305,6)$ & $(5,352,2)$ & $(5,533,12)$ & $(5,572,3)$ & $(5,607,1)$ \\
\hline$(5,21,16)$ & $(5,76,41)$ & $(5,99,18)$ & $(5,142,2)$ & $(5,244,4)$ & $(5,305,10)$ & $(5,353,2)$ & $(5,534,2)$ & $(5,573,3)$ & $(5,607,5)$ \\
\hline$(5,23,14)$ & $(5,76,44)$ & $(5,99,47)$ & $(5,142,4)$ & $(5,244,6)$ & $(5,305,12)$ & $(5,353,4)$ & $(5,534,10)$ & $(5,573,4)$ & $(5,608,1)$ \\
\hline$(5,23,26)$ & $(5,76,46)$ & $(5,100,11)$ & $(5,142,11)$ & $(5,244,14)$ & $(5,306,4)$ & $(5,354,2)$ & $(5,535,2)$ & $(5,574,5)$ & $(5,608,5)$ \\
\hline$(5,24,16)$ & $(5,76,47)$ & $(5,100,15)$ & $(5,142,13)$ & $(5,244,15)$ & $(5,306,10)$ & $(5,355,2)$ & $(5,536,2)$ & $(5,574,6)$ & $(5,608,7)$ \\
\hline$(5,24,19)$ & $(5,77,11)$ & $(5,100,18)$ & $(5,142,17)$ & $(5,246,5)$ & $(5,309,2)$ & $(5,357,4)$ & $(5,536,12)$ & $(5,574,8)$ & $(5,608,10)$ \\
\hline$(5,24,25)$ & $(5,77,15)$ & $(5,101,12)$ & $(5,142,19)$ & $(5,246,7)$ & $(5,309,4)$ & $(5,359,2)$ & $(5,537,2)$ & $(5,574,9)$ & $(5,609,1)$ \\
\hline$(5,25,15)$ & $(5,77,20)$ & $(5,102,8)$ & $(5,142,23)$ & $(5,246,17)$ & $(5,309,6)$ & $(5,359,6)$ & $(5,537,12)$ & $(5,575,3)$ & $(5,609,3)$ \\
\hline$(5,25,18)$ & $(5,77,21)$ & $(5,102,12)$ & $(5,142,25)$ & $(5,246,18)$ & $(5,309,8)$ & $(5,360,2)$ & $(5,538,2)$ & $(5,575,4)$ & $(5,610,1)$ \\
\hline$(5,25,24)$ & $(5,78,8)$ & $(5,103,20)$ & $(5,143,2)$ & $(5,247,3)$ & $(5,310,2)$ & $(5,360,4)$ & $(5,538,12)$ & $(5,576,3)$ & $(5,610,3)$ \\
\hline$(5,25,26)$ & $(5,78,11)$ & $(5,104,2)$ & $(5,143,4)$ & $(5,247,7)$ & $(5,310,6)$ & $(5,360,6)$ & $(5,539,2)$ & $(5,577,3)$ & $(5,611,1)$ \\
\hline$(5,26,17)$ & $(5,78,14)$ & $(5,105,2)$ & $(5,143,8)$ & $(5,248,3)$ & $(5,311,2)$ & $(5,360,8)$ & $(5,539,10)$ & $(5,577,4)$ & $(5,611,3)$ \\
\hline$(5,26,20)$ & $(5,78,27)$ & $(5,105,4)$ & $(5,143,10)$ & $(5,248,4)$ & $(5,311,4)$ & $(5,361,2)$ & $(5,540,2)$ & $(5,578,3)$ & $(5,612,1)$ \\
\hline$(5,26,25)$ & $(5,78,33)$ & $(5,105,8)$ & $(5,143,14)$ & $(5,248,7)$ & $(5,312,2)$ & $(5,361,4)$ & $(5,540,12)$ & $(5,578,4)$ & $(5,612,3)$ \\
\hline$(5,26,35)$ & $(5,79,8)$ & $(5,107,4)$ & $(5,143,16)$ & $(5,248,8)$ & $(5,312,6)$ & $(5,361,6)$ & $(5,541,2)$ & $(5,579,3)$ & $(5,612,4)$ \\
\hline$(5,26,37)$ & $(5,79,11)$ & $(5,109,2)$ & $(5,143,20)$ & $(5,249,4)$ & $(5,315,4)$ & $(5,361,8)$ & $(5,541,12)$ & $(5,579,4)$ & $(5,612,6)$ \\
\hline$(5,27,9)$ & $(5,79,14)$ & $(5,109,4)$ & $(5,143,22)$ & $(5,249,5)$ & $(5,315,8)$ & $(5,362,2)$ & $(5,542,2)$ & $(5,580,1)$ & $(5,613,1)$ \\
\hline$(5,27,13)$ & $(5,79,16)$ & $(5,109,8)$ & $(5,144,4)$ & $(5,249,9)$ & $(5,316,2)$ & $(5,362,4)$ & $(5,542,12)$ & $(5,581,1)$ & $(5,613,3)$ \\
\hline$(5,28,18)$ & $(5,79,27)$ & $(5,109,10)$ & $(5,144,8)$ & $(5,249,10)$ & $(5,317,2)$ & $(5,363,2)$ & $(5,543,2)$ & $(5,581,3)$ & $(5,613,4)$ \\
\hline$(5,28,24)$ & $(5,79,30)$ & $(5,110,4)$ & $(5,144,10)$ & $(5,251,4)$ & $(5,317,4)$ & $(5,363,4)$ & $(5,544,2)$ & $(5,582,1)$ & $(5,613,6)$ \\
\hline$(5,29,9)$ & $(5,79,33)$ & $(5,111,2)$ & $(5,145,2)$ & $(5,251,5)$ & $(5,318,2)$ & $(5,364,2)$ & $(5,544,10)$ & $(5,582,3)$ & $(5,614,1)$ \\
\hline$(5,29,13)$ & $(5,79,35)$ & $(5,111,8)$ & $(5,145,4)$ & $(5,251,9)$ & $(5,319,2)$ & $(5,364,4)$ & $(5,545,2)$ & $(5,583,1)$ & $(5,614,3)$ \\
\hline$(5,30,18)$ & $(5,80,8)$ & $(5,111,10)$ & $(5,145,8)$ & $(5,251,10)$ & $(5,320,2)$ & $(5,364,6)$ & $(5,545,10)$ & $(5,583,3)$ & $(5,615,1)$ \\
\hline$(5,30,26)$ & $(5,80,11)$ & $(5,112,2)$ & $(5,145,10)$ & $(5,253,4)$ & $(5,322,2)$ & $(5,364,8)$ & $(5,546,2)$ & $(5,584,1)$ & $(5,616,1)$ \\
\hline$(5,31,17)$ & $(5,80,14)$ & $(5,113,2)$ & $(5,146,4)$ & $(5,254,5)$ & $(5,323,2)$ & $(5,365,2)$ & $(5,546,10)$ & $(5,584,3)$ & $(5,616,3)$ \\
\hline$(5,31,23)$ & $(5,81,18)$ & $(5,115,2)$ & $(5,146,8)$ & $(5,254,6)$ & $(5,323,4)$ & $(5,365,6)$ & $(5,547,2)$ & $(5,585,1)$ & $(5,617,1)$ \\
\hline$(5,31,30)$ & $(5,81,20)$ & $(5,116,2)$ & $(5,146,10)$ & $(5,256,3)$ & $(5,324,2)$ & $(5,368,2)$ & $(5,547,10)$ & $(5,585,3)$ & $(5,617,3)$ \\
\hline$(5,31,38)$ & $(5,81,44)$ & $(5,116,4)$ & $(5,147,2)$ & $(5,256,4)$ & $(5,325,2)$ & $(5,368,4)$ & $(5,548,2)$ & $(5,586,1)$ & $(5,617,4)$ \\
\hline$(5,31,42)$ & $(5,82,18)$ & $(5,116,11)$ & $(5,148,2)$ & $(5,257,4)$ & $(5,326,2)$ & $(5,369,2)$ & $(5,548,12)$ & $(5,587,1)$ & $(5,617,6)$ \\
\hline$(5,32,17)$ & $(5,82,20)$ & $(5,116,17)$ & $(5,148,7)$ & $(5,257,5)$ & $(5,326,4)$ & $(5,369,4)$ & $(5,549,3)$ & $(5,587,3)$ & $(5,618,1)$ \\
\hline$(5,32,24)$ & $(5,83,8)$ & $(5,116,19)$ & $(5,149,2)$ & $(5,257,9)$ & $(5,327,2)$ & $(5,369,6)$ & $(5,549,4)$ & $(5,588,1)$ & $(5,618,3)$ \\
\hline$(5,32,50)$ & $(5,83,12)$ & $(5,116,23)$ & $(5,149,10)$ & $(5,257,11)$ & $(5,328,2)$ & $(5,369,8)$ & $(5,550,5)$ & $(5,588,3)$ & $(5,618,4)$ \\
\hline$(5,35,2)$ & $(5,83,14)$ & $(5,118,12)$ & $(5,149,12)$ & $(5,258,3)$ & $(5,328,4)$ & $(5,370,2)$ & $(5,550,6)$ & $(5,589,1)$ & $(5,618,6)$ \\
\hline$(5,36,2)$ & $(5,83,16)$ & $(5,118,16)$ & $(5,149,16)$ & $(5,258,4)$ & $(5,331,2)$ & $(5,371,2)$ & $(5,550,8)$ & $(5,589,3)$ & $(5,619,1)$ \\
\hline$(5,36,5)$ & $(5,84,14)$ & $(5,119,2)$ & $(5,150,2)$ & $(5,258,7)$ & $(5,332,2)$ & $(5,372,2)$ & $(5,550,9)$ & $(5,589,4)$ & $(5,619,3)$ \\
\hline$(5,38,5)$ & $(5,85,8)$ & $(5,119,7)$ & $(5,150,4)$ & $(5,258,8)$ & $(5,333,2)$ & $(5,372,4)$ & $(5,551,5)$ & $(5,589,6)$ & $(5,620,1)$ \\
\hline$(5,38,8)$ & $(5,85,14)$ & $(5,120,2)$ & $(5,151,2)$ & $(5,259,3)$ & $(5,333,4)$ & $(5,373,2)$ & $(5,551,6)$ & $(5,590,1)$ & $(5,621,1)$ \\
\hline$(5,39,2)$ & $(5,85,16)$ & $(5,120,10)$ & $(5,151,7)$ & $(5,260,3)$ & $(5,334,2)$ & $(5,373,4)$ & $(5,551,8)$ & $(5,591,1)$ & $(5,621,5)$ \\
\hline$(5,39,4)$ & $(5,86,5)$ & $(5,122,2)$ & $(5,151,9)$ & $(5,260,4)$ & $(5,334,4)$ & $(5,373,6)$ & $(5,551,9)$ & $(5,591,3)$ & $(5,622,1)$ \\
\hline$(5,47,7)$ & $(5,86,8)$ & $(5,122,13)$ & $(5,151,13)$ & $(5,261,3)$ & $(5,335,2)$ & $(5,373,8)$ & $(5,552,5)$ & $(5,591,4)$ & $(5,622,5)$ \\
\hline$(5,48,7)$ & $(5,87,5)$ & $(5,123,2)$ & $(5,152,2)$ & $(5,261,4)$ & $(5,335,4)$ & $(5,374,2)$ & $(5,552,6)$ & $(5,591,6)$ & $(5,623,1)$ \\
\hline$(5,48,14)$ & $(5,87,8)$ & $(5,123,4)$ & $(5,153,2)$ & $(5,261,7)$ & $(5,336,2)$ & $(5,374,4)$ & $(5,552,8)$ & $(5,592,1)$ & $(5,623,5)$ \\
\hline$(5,49,7)$ & $(5,87,10)$ & $(5,123,11)$ & $(5,153,4)$ & $(5,261,8)$ & $(5,336,4)$ & $(5,376,4)$ & $(5,552,9)$ & $(5,592,3)$ & $(5,623,7)$ \\
\hline$(5,49,14)$ & $(5,88,8)$ & $(5,123,13)$ & $(5,153,8)$ & $(5,262,4)$ & $(5,336,6)$ & $(5,377,2)$ & $(5,553,3)$ & $(5,592,4)$ & $(5,623,10)$ \\
\hline$(5,50,7)$ & $(5,88,12)$ & $(5,124,2)$ & $(5,153,10)$ & $(5,262,5)$ & $(5,336,10)$ & $(5,377,4)$ & $(5,553,4)$ & $(5,592,6)$ & $(5,624,1)$ \\
\hline$(5,51,7)$ & $(5,88,14)$ & $(5,124,4)$ & $(5,154,2)$ & $(5,264,4)$ & $(5,337,2)$ & $(5,378,2)$ & $(5,554,3)$ & $(5,593,1)$ & $(5,624,5)$ \\
\hline$(5,52,7)$ & $(5,88,16)$ & $(5,125,2)$ & $(5,154,7)$ & $(5,264,5)$ & $(5,337,6)$ & $(5,378,4)$ & $(5,554,4)$ & $(5,593,3)$ & $(5,624,7)$ \\
\hline$(5,53,7)$ & $(5,88,27)$ & $(5,125,4)$ & $(5,154,9)$ & $(5,265,4)$ & $(5,338,2)$ & $(5,379,2)$ & $(5,555,3)$ & $(5,593,4)$ & $(5,624,10)$ \\
\hline$(5,55,7)$ & $(5,88,31)$ & $(5,127,2)$ & $(5,154,13)$ & $(5,265,5)$ & $(5,338,4)$ & $(5,380,2)$ & $(5,556,3)$ & $(5,593,6)$ & $(5,625,1)$ \\
\hline$(5,56,7)$ & $(5,88,33)$ & $(5,127,4)$ & $(5,155,2)$ & $(5,265,9)$ & $(5,339,2)$ & $(5,380,4)$ & $(5,556,4)$ & $(5,594,1)$ & $(5,626,1)$ \\
\hline$(5,59,7)$ & $(5,88,35)$ & $(5,127,8)$ & $(5,157,2)$ & $(5,265,10)$ & $(5,339,4)$ & $(5,380,6)$ & $(5,557,3)$ & $(5,594,3)$ & $(5,626,3)$ \\
\hline$(5,60,14)$ & $(5,89,8)$ & $(5,127,10)$ & $(5,157,4)$ & $(5,266,3)$ & $(5,340,2)$ & $(5,380,8)$ & $(5,557,4)$ & $(5,594,4)$ & $(5,627,1)$ \\
\hline$(5,63,14)$ & $(5,89,12)$ & $(5,128,2)$ & $(5,157,8)$ & $(5,266,4)$ & $(5,340,4)$ & $(5,381,2)$ & $(5,558,3)$ & $(5,594,6)$ & $(5,627,3)$ \\
\hline$(5,64,7)$ & $(5,89,14)$ & $(5,128,8)$ & $(5,158,2)$ & $(5,273,2)$ & $(5,340,6)$ & $(5,381,4)$ & $(5,558,4)$ & $(5,595,1)$ & $(5,628,1)$ \\
\hline$(5,65,7)$ & $(5,89,16)$ & $(5,128,10)$ & $(5,158,4)$ & $(5,274,2)$ & $(5,340,10)$ & $(5,382,2)$ & $(5,559,3)$ & $(5,595,3)$ & $(5,628,3)$ \\
\hline$(5,65,14)$ & $(5,90,8)$ & $(5,129,4)$ & $(5,158,8)$ & $(5,275,2)$ & $(5,342,2)$ & $(5,383,2)$ & $(5,559,4)$ & $(5,595,4)$ & $(5,629,1)$ \\
\hline$(5,66,7)$ & $(5,90,12)$ & $(5,130,2)$ & $(5,158,10)$ & $(5,276,2)$ & $(5,342,6)$ & $(5,384,2)$ & $(5,560,3)$ & $(5,595,6)$ & $(5,630,1)$ \\
\hline$(5,69,7)$ & $(5,90,15)$ & $(5,130,4)$ & $(5,159,2)$ & $(5,278,2)$ & $(5,343,2)$ & $(5,384,4)$ & $(5,561,3)$ & $(5,596,1)$ & $(5,630,3)$ \\
\hline$(5,70,7)$ & $(5,90,27)$ & $(5,131,2)$ & $(5,159,4)$ & $(5,279,2)$ & $(5,343,4)$ & $(5,385,2)$ & $(5,561,4)$ & $(5,596,3)$ & $(5,631,1)$ \\
\hline$(5,71,11)$ & $(5,90,31)$ & $(5,131,7)$ & $(5,160,2)$ & $(5,280,2)$ & $(5,343,6)$ & $(5,518,2)$ & $(5,562,3)$ & $(5,597,1)$ & $(5,631,3)$ \\
\hline$(5,71,15)$ & $(5,90,33)$ & $(5,132,2)$ & $(5,224,4)$ & $(5,280,4)$ & $(5,343,8)$ & $(5,518,10)$ & $(5,562,4)$ & $(5,597,3)$ & $(5,632,1)$ \\
\hline$(5,71,18)$ & $(5,90,34)$ & $(5,132,7)$ & $(5,227,5)$ & $(5,281,2)$ & $(5,344,2)$ & $(5,519,4)$ & $(5,563,3)$ & $(5,597,4)$ & $(5,633,1)$ \\
\hline$(5,71,20)$ & $(5,91,8)$ & $(5,132,9)$ & $(5,228,3)$ & $(5,282,2)$ & $(5,344,6)$ & $(5,519,17)$ & $(5,564,3)$ & $(5,597,6)$ & $(5,634,1)$ \\
\hline$(5,71,21)$ & $(5,91,11)$ & $(5,132,13)$ & $(5,232,4)$ & $(5,285,2)$ & $(5,345,2)$ & $(5,520,17)$ & $(5,564,4)$ & $(5,598,1)$ & $(5,634,3)$ \\
\hline$(5,72,8)$ & $(5,91,14)$ & $(5,134,2)$ & $(5,232,9)$ & $(5,288,2)$ & $(5,345,4)$ & $(5,521,17)$ & $(5,565,5)$ & $(5,598,3)$ & $(5,635,1)$ \\
\hline$(5,72,12)$ & $(5,91,16)$ & $(5,134,7)$ & $(5,233,4)$ & $(5,289,2)$ & $(5,346,2)$ & $(5,522,4)$ & $(5,565,6)$ & $(5,599,1)$ & $(5,635,3)$ \\
\hline$(5,72,16)$ & $(5,92,12)$ & $(5,135,2)$ & $(5,233,6)$ & $(5,290,2)$ & $(5,346,4)$ & $(5,522,17)$ & $(5,565,8)$ & $(5,599,3)$ & $(5,635,4)$ \\
\hline$(5,72,27)$ & $(5,93,5)$ & $(5,136,2)$ & $(5,234,5)$ & $(5,290,4)$ & $(5,346,6)$ & $(5,523,2)$ & $(5,565,9)$ & $(5,599,4)$ & $(5,635,6)$ \\
\hline$(5,72,31)$ & $(5,93,8)$ & $(5,136,10)$ & $(5,234,7)$ & $(5,291,2)$ & $(5,346,8)$ & $(5,523,12)$ & $(5,566,3)$ & $(5,599,6)$ & $(5,636,1)$ \\
\hline$(5,72,33)$ & $(5,94,5)$ & $(5,136,12)$ & $(5,235,3)$ & $(5,291,4)$ & $(5,347,2)$ & $(5,524,2)$ & $(5,567,3)$ & $(5,600,1)$ & $(5,636,3)$ \\
\hline$(5,72,35)$ & $(5,94,8)$ & $(5,136,16)$ & $(5,235,4)$ & $(5,294,2)$ & $(5,347,4)$ & $(5,524,12)$ & $(5,567,4)$ & $(5,600,3)$ & $(5,636,4)$ \\
\hline$(5,73,8)$ & $(5,94,10)$ & $(5,139,2)$ & $(5,236,3)$ & $(5,294,4)$ & $(5,347,6)$ & $(5,525,2)$ & $(5,568,5)$ & $(5,600,4)$ & $(5,636,6)$ \\
\hline$(5,73,12)$ & $(5,94,17)$ & $(5,139,8)$ & $(5,236,4)$ & $(5,295,2)$ & $(5,347,8)$ & $(5,526,2)$ & $(5,568,6)$ & $(5,600,6)$ & $(5,637,1)$ \\
\hline$(5,73,16)$ & $(5,94,20)$ & $(5,139,10)$ & $(5,237,3)$ & $(5,295,4)$ & $(5,348,2)$ & $(5,526,10)$ & $(5,568,8)$ & $(5,601,1)$ & $(5,637,3)$ \\
\hline$(5,73,27)$ & $(5,94,22)$ & $(5,140,4)$ & $(5,239,4)$ & $(5,296,2)$ & $(5,348,4)$ & $(5,527,2)$ & $(5,568,9)$ & $(5,602,1)$ & $(5,638,1)$ \\
\hline$(5,73,31)$ & $(5,95,5)$ & $(5,140,10)$ & $(5,239,5)$ & $(5,296,4)$ & $(5,348,6)$ & $(5,527,12)$ & $(5,569,5)$ & $(5,602,3)$ & $(5,638,5)$ \\
\hline$(5,73,33)$ & $(5,95,8)$ & $(5,140,14)$ & $(5,240,4)$ & $(5,299,2)$ & $(5,348,8)$ & $(5,528,2)$ & $(5,569,6)$ & $(5,603,1)$ & \\
\hline
\end{tabular}


Table 14: $L(M)^{G}$ not retract $k$-rational,

$M=M_{1} \oplus M_{2}$, rank $M_{1}=4$, rank $M_{2}=1, M_{i}$ : indecomposable (768 cases)

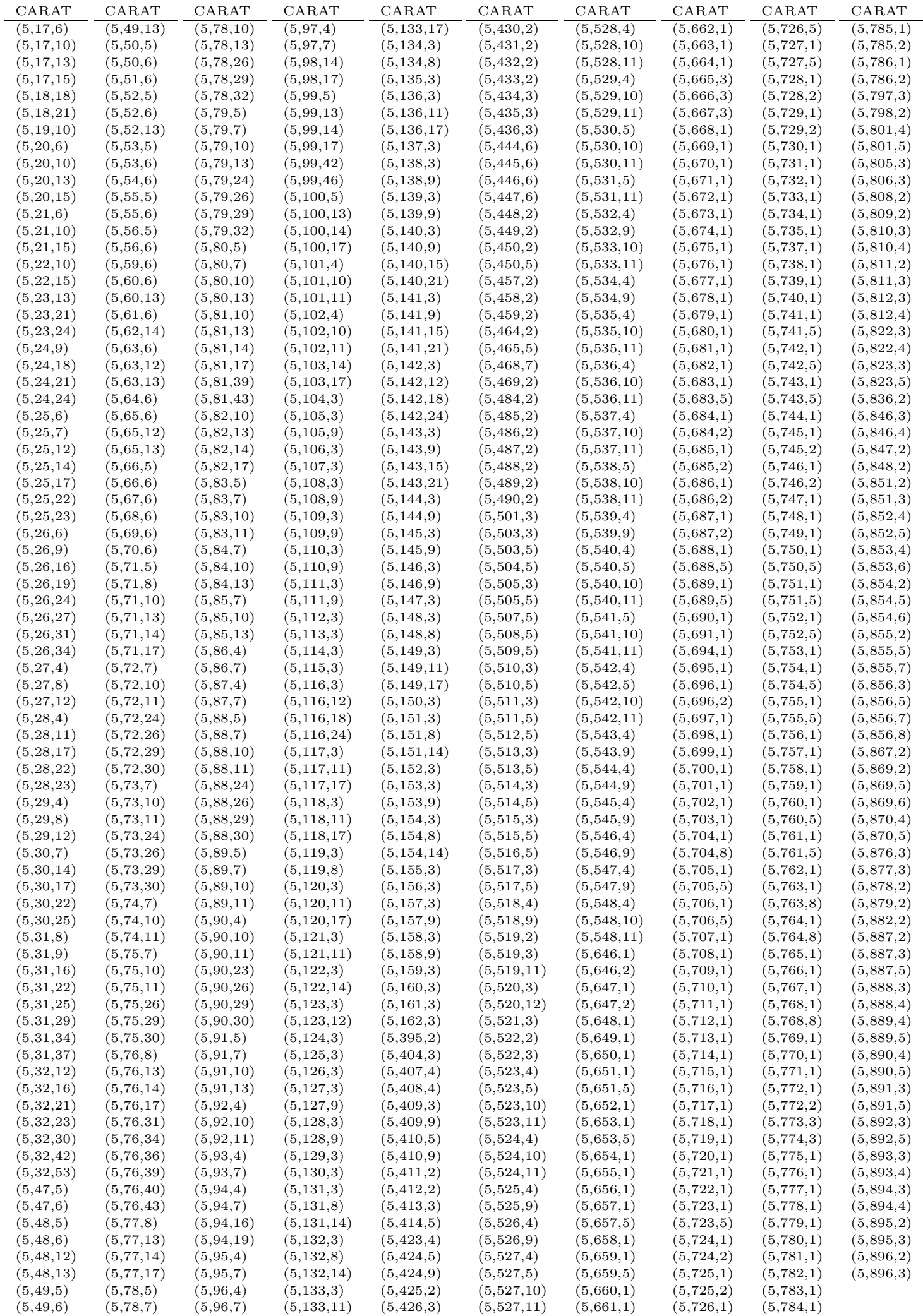


Table 15: $L(M)^{G}$ not retract $k$-rational, rank $M=5, M$ : indecomposable (1141 cases)

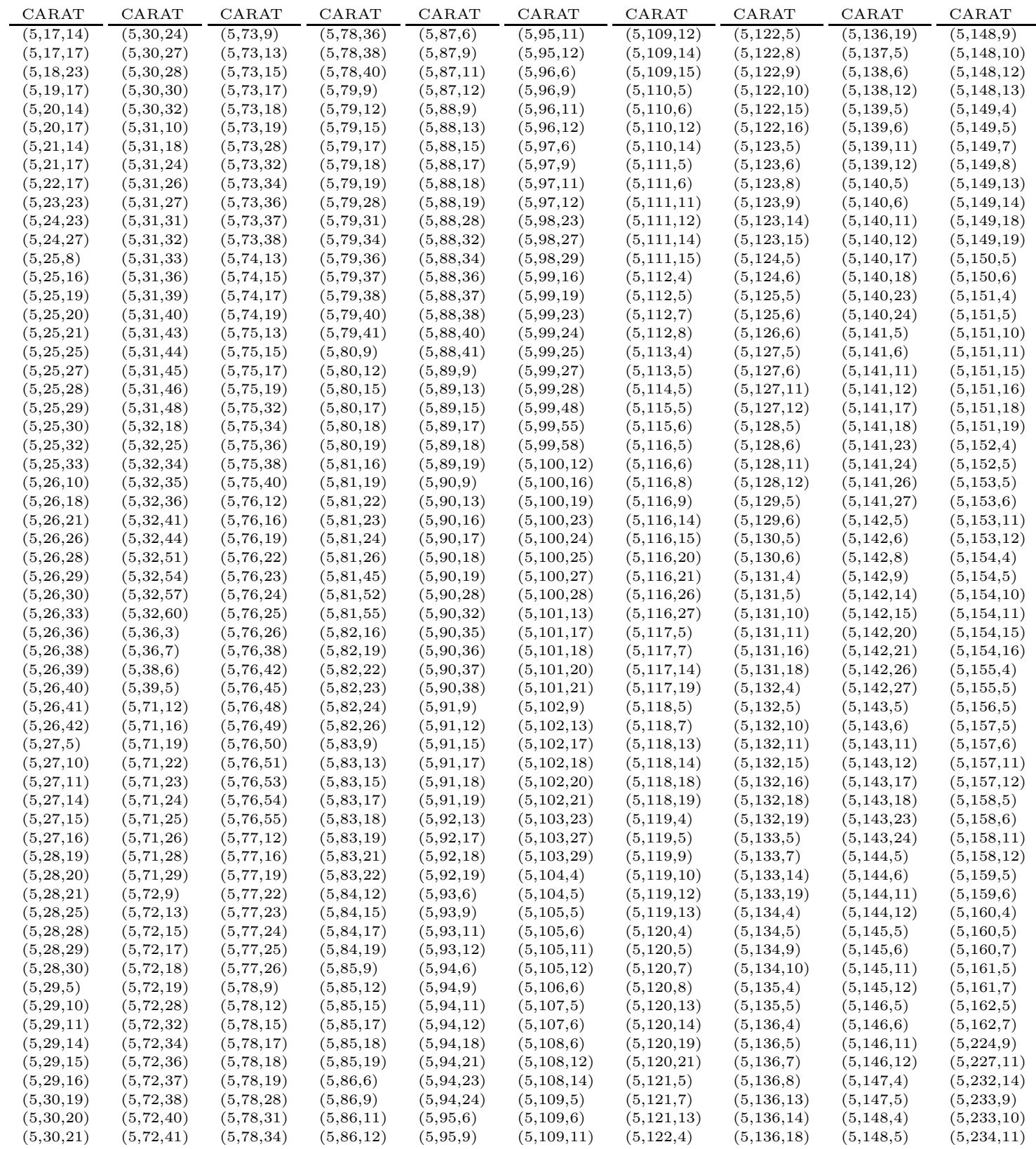


Table 15 (continued): $L(M)^{G}$ not retract $k$-rational, rank $M=5, M$ : indecomposable (1141 cases)

\begin{tabular}{|c|c|c|c|c|c|c|c|c|c|}
\hline CARAT & CARAT & CARAT & CARAT & CARAT & CARAT & CARAT & CARAT & CARAT & CARAT \\
\hline$(5,234,12)$ & $(5,519,15)$ & $(5,533,13)$ & $(5,547,11)$ & $(5,568,10)$ & $(5,639,2)$ & $(5,685,5)$ & $(5,723,2)$ & $(5,764,3)$ & $(5,939,2)$ \\
\hline$(5,240,9)$ & $(5,520,7)$ & $(5,533,14)$ & $(5,547,12)$ & $(5,569,2)$ & $(5,641,4)$ & $(5,686,3)$ & $(5,723,4)$ & $(5,764,5)$ & $(5,939,3)$ \\
\hline$(5,240,10)$ & $(5,520,9)$ & $(5,534,6)$ & $(5,548,7)$ & $(5,569,4)$ & $(5,642,2)$ & $(5,686,5)$ & $(5,724,3)$ & $(5,764,7)$ & $(5,940,2)$ \\
\hline$(5,241,14)$ & $(5,520,15)$ & $(5,534,7)$ & $(5,548,8)$ & $(5,569,7)$ & $(5,643,2)$ & $(5,687,3)$ & $(5,724,5)$ & $(5,764,9)$ & $(5,940,3)$ \\
\hline$(5,241,15)$ & $(5,521,7)$ & $(5,534,11)$ & $(5,548,13)$ & $(5,569,10)$ & $(5,651,2)$ & $(5,688,2)$ & $(5,725,3)$ & $(5,764,11)$ & $(5,941,2)$ \\
\hline$(5,242,9)$ & $(5,521,9)$ & $(5,534,12)$ & $(5,548,14)$ & $(5,570,2)$ & $(5,651,4)$ & $(5,688,4)$ & $(5,725,5)$ & $(5,765,2)$ & $(5,941,3)$ \\
\hline$(5,244,9)$ & $(5,522,5)$ & $(5,535,7)$ & $(5,549,2)$ & $(5,570,4)$ & $(5,651,6)$ & $(5,689,2)$ & $(5,726,2)$ & $(5,765,4)$ & $(5,942,2)$ \\
\hline$(5,244,10)$ & $(5,522,7)$ & $(5,535,8)$ & $(5,549,5)$ & $(5,570,7)$ & $(5,651,8)$ & $(5,696,3)$ & $(5,727,2)$ & $(5,766,2)$ & $(5,942,3)$ \\
\hline$(5,246,11)$ & $(5,522,8)$ & $(5,535,13)$ & $(5,550,2)$ & $(5,570,10)$ & $(5,652,2)$ & $(5,697,2)$ & $(5,727,4)$ & $(5,766,4)$ & $(5,943,2)$ \\
\hline$(5,246,12)$ & $(5,523,7)$ & $(5,535,14)$ & $(5,550,4)$ & $(5,571,2)$ & $(5,652,4)$ & $(5,697,4)$ & $(5,728,3)$ & $(5,767,2)$ & $(5,943,3)$ \\
\hline$(5,257,14)$ & $(5,523,8)$ & $(5,536,7)$ & $(5,550,7)$ & $(5,571,4)$ & $(5,653,2)$ & $(5,700,2)$ & $(5,728,5)$ & $(5,767,4)$ & $(5,944,2)$ \\
\hline$(5,257,15)$ & $(5,523,13)$ & $(5,536,8)$ & $(5,550,10)$ & $(5,571,7)$ & $(5,653,4)$ & $(5,701,2)$ & $(5,729,3)$ & $(5,768,2)$ & $(5,944,3)$ \\
\hline$(5,274,4)$ & $(5,523,14)$ & $(5,536,13)$ & $(5,551,2)$ & $(5,571,10)$ & $(5,653,6)$ & $(5,701,4)$ & $(5,729,5)$ & $(5,768,3)$ & $(5,945,2)$ \\
\hline$(5,275,4)$ & $(5,523,16)$ & $(5,536,14)$ & $(5,551,4)$ & $(5,572,2)$ & $(5,653,8)$ & $(5,702,2)$ & $(5,741,2)$ & $(5,768,5)$ & $(5,945,3)$ \\
\hline$(5,276,4)$ & $(5,523,17)$ & $(5,537,8)$ & $(5,551,7)$ & $(5,572,5)$ & $(5,654,2)$ & $(5,702,4)$ & $(5,741,4)$ & $(5,768,7)$ & $(5,946,1)$ \\
\hline$(5,290,6)$ & $(5,524,8)$ & $(5,537,13)$ & $(5,551,10)$ & $(5,573,2)$ & $(5,654,4)$ & $(5,703,2)$ & $(5,742,2)$ & $(5,768,9)$ & $(5,946,3)$ \\
\hline$(5,291,6)$ & $(5,524,13)$ & $(5,537,14)$ & $(5,552,2)$ & $(5,573,5)$ & $(5,655,2)$ & $(5,704,2)$ & $(5,743,2)$ & $(5,768,11)$ & $(5,947,1)$ \\
\hline$(5,294,6)$ & $(5,524,14)$ & $(5,538,8)$ & $(5,552,4)$ & $(5,574,2)$ & $(5,656,2)$ & $(5,704,3)$ & $(5,743,4)$ & $(5,769,2)$ & $(5,947,3)$ \\
\hline$(5,302,4)$ & $(5,525,6)$ & $(5,538,13)$ & $(5,552,7)$ & $(5,574,4)$ & $(5,657,2)$ & $(5,704,5)$ & $(5,749,2)$ & $(5,769,4)$ & $(5,948,1)$ \\
\hline$(5,303,4)$ & $(5,525,7)$ & $(5,538,14)$ & $(5,552,10)$ & $(5,574,7)$ & $(5,657,4)$ & $(5,704,9)$ & $(5,749,4)$ & $(5,770,2)$ & $(5,948,2)$ \\
\hline$(5,304,4)$ & $(5,525,11)$ & $(5,539,6)$ & $(5,553,2)$ & $(5,574,10)$ & $(5,657,6)$ & $(5,705,2)$ & $(5,750,2)$ & $(5,770,4)$ & $(5,948,3)$ \\
\hline$(5,305,4)$ & $(5,525,12)$ & $(5,539,7)$ & $(5,553,5)$ & $(5,575,2)$ & $(5,657,8)$ & $(5,705,6)$ & $(5,750,6)$ & $(5,772,3)$ & $(5,948,4)$ \\
\hline$(5,305,8)$ & $(5,526,6)$ & $(5,539,11)$ & $(5,554,2)$ & $(5,575,5)$ & $(5,658,2)$ & $(5,705,7)$ & $(5,751,2)$ & $(5,772,5)$ & $(5,949,1)$ \\
\hline$(5,306,12)$ & $(5,526,7)$ & $(5,539,12)$ & $(5,554,5)$ & $(5,576,2)$ & $(5,658,4)$ & $(5,706,2)$ & $(5,751,4)$ & $(5,773,4)$ & $(5,949,2)$ \\
\hline$(5,316,4)$ & $(5,526,11)$ & $(5,540,7)$ & $(5,555,2)$ & $(5,576,5)$ & $(5,659,2)$ & $(5,706,6)$ & $(5,751,6)$ & $(5,774,4)$ & $(5,949,3)$ \\
\hline$(5,326,6)$ & $(5,526,12)$ & $(5,540,8)$ & $(5,555,5)$ & $(5,577,2)$ & $(5,659,4)$ & $(5,706,7)$ & $(5,751,8)$ & $(5,785,3)$ & $(5,949,4)$ \\
\hline$(5,335,6)$ & $(5,527,7)$ & $(5,540,13)$ & $(5,556,2)$ & $(5,577,5)$ & $(5,659,6)$ & $(5,706,8)$ & $(5,752,2)$ & $(5,786,3)$ & $(5,950,1)$ \\
\hline$(5,336,8)$ & $(5,527,8)$ & $(5,540,14)$ & $(5,556,5)$ & $(5,578,2)$ & $(5,659,8)$ & $(5,706,10)$ & $(5,752,4)$ & $(5,786,5)$ & $(5,950,2)$ \\
\hline$(5,336,12)$ & $(5,527,13)$ & $(5,540,16)$ & $(5,557,2)$ & $(5,578,5)$ & $(5,660,2)$ & $(5,706,12)$ & $(5,752,6)$ & $(5,801,6)$ & $(5,950,3)$ \\
\hline$(5,337,8)$ & $(5,527,14)$ & $(5,540,17)$ & $(5,557,5)$ & $(5,579,2)$ & $(5,660,4)$ & $(5,707,2)$ & $(5,752,8)$ & $(5,822,5)$ & $(5,950,4)$ \\
\hline$(5,338,6)$ & $(5,527,16)$ & $(5,541,8)$ & $(5,558,2)$ & $(5,579,5)$ & $(5,664,2)$ & $(5,708,2)$ & $(5,753,2)$ & $(5,823,4)$ & $(5,951,1)$ \\
\hline$(5,339,6)$ & $(5,527,17)$ & $(5,541,13)$ & $(5,558,5)$ & $(5,580,2)$ & $(5,664,4)$ & $(5,708,4)$ & $(5,754,2)$ & $(5,846,5)$ & $(5,951,2)$ \\
\hline$(5,340,8)$ & $(5,528,7)$ & $(5,541,14)$ & $(5,559,2)$ & $(5,604,2)$ & $(5,665,4)$ & $(5,709,2)$ & $(5,754,6)$ & $(5,852,6)$ & $(5,951,3)$ \\
\hline$(5,340,12)$ & $(5,528,8)$ & $(5,542,7)$ & $(5,559,5)$ & $(5,604,4)$ & $(5,666,4)$ & $(5,709,3)$ & $(5,755,2)$ & $(5,853,5)$ & $(5,952,1)$ \\
\hline$(5,342,4)$ & $(5,528,13)$ & $(5,542,8)$ & $(5,560,2)$ & $(5,605,2)$ & $(5,667,2)$ & $(5,709,5)$ & $(5,755,4)$ & $(5,854,7)$ & $(5,952,3)$ \\
\hline$(5,372,6)$ & $(5,528,15)$ & $(5,542,13)$ & $(5,560,5)$ & $(5,605,4)$ & $(5,667,4)$ & $(5,710,2)$ & $(5,755,6)$ & $(5,855,6)$ & $(5,953,1)$ \\
\hline$(5,404,5)$ & $(5,528,16)$ & $(5,542,14)$ & $(5,561,2)$ & $(5,606,2)$ & $(5,668,2)$ & $(5,711,2)$ & $(5,755,8)$ & $(5,856,4)$ & $(5,953,2)$ \\
\hline$(5,409,5)$ & $(5,528,17)$ & $(5,543,6)$ & $(5,561,5)$ & $(5,607,2)$ & $(5,669,2)$ & $(5,712,2)$ & $(5,756,2)$ & $(5,869,7)$ & $(5,953,3)$ \\
\hline$(5,410,7)$ & $(5,529,8)$ & $(5,543,7)$ & $(5,562,2)$ & $(5,607,6)$ & $(5,669,4)$ & $(5,712,4)$ & $(5,757,2)$ & $(5,870,6)$ & $(5,954,1)$ \\
\hline$(5,413,6)$ & $(5,529,13)$ & $(5,543,11)$ & $(5,562,5)$ & $(5,608,2)$ & $(5,670,2)$ & $(5,713,2)$ & $(5,757,4)$ & $(5,889,6)$ & $(5,954,2)$ \\
\hline$(5,414,8)$ & $(5,529,15)$ & $(5,543,12)$ & $(5,563,2)$ & $(5,608,6)$ & $(5,670,3)$ & $(5,714,2)$ & $(5,758,2)$ & $(5,890,6)$ & $(5,954,3)$ \\
\hline$(5,424,7)$ & $(5,529,16)$ & $(5,544,6)$ & $(5,563,5)$ & $(5,621,2)$ & $(5,670,7)$ & $(5,714,4)$ & $(5,759,2)$ & $(5,891,4)$ & $(5,954,4)$ \\
\hline$(5,426,6)$ & $(5,530,7)$ & $(5,544,7)$ & $(5,564,2)$ & $(5,621,4)$ & $(5,671,2)$ & $(5,715,2)$ & $(5,759,4)$ & $(5,892,4)$ & $(5,955,1)$ \\
\hline$(5,434,5)$ & $(5,530,8)$ & $(5,544,11)$ & $(5,564,5)$ & $(5,622,2)$ & $(5,671,3)$ & $(5,716,2)$ & $(5,760,2)$ & $(5,933,2)$ & $(5,955,2)$ \\
\hline$(5,435,6)$ & $(5,530,13)$ & $(5,544,12)$ & $(5,565,2)$ & $(5,622,4)$ & $(5,671,7)$ & $(5,716,4)$ & $(5,760,6)$ & $(5,933,3)$ & $(5,955,3)$ \\
\hline$(5,436,5)$ & $(5,530,15)$ & $(5,545,6)$ & $(5,565,4)$ & $(5,623,2)$ & $(5,672,2)$ & $(5,717,2)$ & $(5,761,2)$ & $(5,934,2)$ & $(5,955,4)$ \\
\hline$(5,465,7)$ & $(5,530,16)$ & $(5,545,7)$ & $(5,565,7)$ & $(5,623,6)$ & $(5,673,2)$ & $(5,717,4)$ & $(5,761,4)$ & $(5,934,3)$ & \\
\hline$(5,501,5)$ & $(5,530,17)$ & $(5,545,11)$ & $(5,565,10)$ & $(5,624,2)$ & $(5,673,4)$ & $(5,718,2)$ & $(5,761,6)$ & $(5,935,2)$ & \\
\hline$(5,518,6)$ & $(5,531,8)$ & $(5,545,12)$ & $(5,566,2)$ & $(5,624,4)$ & $(5,674,2)$ & $(5,718,4)$ & $(5,761,8)$ & $(5,935,3)$ & \\
\hline$(5,518,7)$ & $(5,531,15)$ & $(5,546,6)$ & $(5,566,5)$ & $(5,624,6)$ & $(5,675,2)$ & $(5,719,2)$ & $(5,762,2)$ & $(5,936,2)$ & \\
\hline$(5,518,11)$ & $(5,531,17)$ & $(5,546,7)$ & $(5,567,2)$ & $(5,624,9)$ & $(5,675,4)$ & $(5,719,4)$ & $(5,763,2)$ & $(5,936,3)$ & \\
\hline$(5,518,12)$ & $(5,532,6)$ & $(5,546,11)$ & $(5,567,5)$ & $(5,625,2)$ & $(5,683,2)$ & $(5,720,2)$ & $(5,763,3)$ & $(5,937,2)$ & \\
\hline$(5,519,5)$ & $(5,532,7)$ & $(5,546,12)$ & $(5,568,2)$ & $(5,625,4)$ & $(5,683,4)$ & $(5,720,4)$ & $(5,763,5)$ & $(5,937,3)$ & \\
\hline$(5,519,7)$ & $(5,532,11)$ & $(5,547,6)$ & $(5,568,4)$ & $(5,638,2)$ & $(5,684,3)$ & $(5,721,2)$ & $(5,763,9)$ & $(5,938,2)$ & \\
\hline$(5,519,8)$ & $(5,532,12)$ & $(5,547,7)$ & $(5,568,7)$ & $(5,638,4)$ & $(5,685,3)$ & $(5,721,4)$ & $(5,764,2)$ & $(5,938,3)$ & \\
\hline
\end{tabular}


Table 16: stably rational classification of algebraic $k$-tori of dimension 5

\begin{tabular}{|c|c|c|c|c|c|c|c|c|c|}
\hline CARAT & $\#$ & {$[s, r, u]$} & $G(n, i)$ & & CARAT & $\#$ & {$[s, r, u]$} & $G(n, i)$ & \\
\hline$(5,1)$ & 1 & {$[1,0,0]$} & $G(1,1)$ & $\{1\}$ & $(5,81)$ & 55 & {$[34,0,21]$} & $G(16,11)$ & $C_{2} \times D_{4}$ \\
\hline$(5,2)$ & 1 & {$[1,0,0]$} & $G(2,1)$ & $C_{2}$ & $(5,82)$ & 26 & {$[12,0,14]$} & $G(16,11)$ & $C_{2} \times D_{4}$ \\
\hline$(5,3)$ & 2 & {$[2,0,0]$} & $G(2,1)$ & $C_{2}$ & $(5,83)$ & 22 & {$[4,0,18]$} & $G(16,10)$ & $C_{4} \times C_{2}^{2}$ \\
\hline$(5,4)$ & 2 & {$[2,0,0]$} & $G(2,1)$ & $C_{2}$ & $(5,84)$ & 19 & {$[10,0,9]$} & $G(16,10)$ & $C_{4} \times C_{2}^{2}$ \\
\hline$(5,5)$ & 2 & {$[2,0,0]$} & $G(4,2)$ & $C_{2}^{2}$ & $(5,85)$ & 19 & {$[6,0,13]$} & $G(16,10)$ & $C_{4} \times C_{2}^{\overline{2}}$ \\
\hline$(5,6)$ & 3 & {$[3,0,0]$} & $G(2,1)$ & $C_{2}$ & $(5,86)$ & 12 & {$[3,0,9]$} & $G(16,10)$ & $C_{4} \times C_{2}^{2}$ \\
\hline$(5,7)$ & 3 & {$[3,0,0]$} & $G(2,1)$ & $C_{2}$ & $(5,87)$ & 12 & {$[2,0,10]$} & $G(16,10)$ & $C_{4} \times C_{2}^{2}$ \\
\hline$(5,8)$ & 3 & {$[3,0,0]$} & $G(4,2)$ & $C_{2}^{2}$ & $(5,88)$ & 41 & {$[7,0,34]$} & $G(32,46)$ & $C_{2}^{2} \times D_{4}$ \\
\hline$(5,9)$ & 6 & {$[6,0,0]$} & $G(4,2)$ & $C_{2}^{2}$ & $(5,89)$ & 19 & {$[3,0,16]$} & $G(32,46)$ & $C_{2}^{2} \times D_{4}$ \\
\hline$(5,10)$ & 7 & {$[7,0,0]$} & $G(4,2)$ & $C_{2}^{2}$ & $(5,90)$ & 38 & {$[9,0,29]$} & $G(32,46)$ & $C_{2}^{2} \times D_{4}$ \\
\hline$(5,11)$ & 6 & {$[5,0,1]$} & $G(4,2)$ & $C_{2}^{2}$ & $(5,91)$ & 19 & {$[3,0,16]$} & $G(32,46)$ & $C_{2}^{2} \times D_{4}$ \\
\hline$(5,12)$ & 6 & {$[4,0,2]$} & $G(8,5)$ & $C_{2}^{3}$ & $(5,92)$ & 19 & {$[10,0,9]$} & $G(32,46)$ & $C_{2}^{2} \times D_{4}$ \\
\hline$(5,13)$ & 9 & {$[9,0,0]$} & $G(4,2)$ & $C_{2}^{2}$ & $(5,93)$ & 12 & {$[3,0,9]$} & $G(32,46)$ & $C_{2}^{2} \times D_{4}$ \\
\hline$(5,14)$ & 9 & {$[7,0,2]$} & $G(4,2)$ & $C_{2}^{2}$ & $(5,94)$ & 24 & {$[4,0,20]$} & $G(32,46)$ & $C_{2}^{2} \times D_{4}$ \\
\hline$(5,15)$ & 11 & {$[11,0,0]$} & $G(4,2)$ & $C_{2}^{2}$ & $(5,95)$ & 12 & {$[2,0,10]$} & $G(32,46)$ & $C_{2}^{2} \times D_{4}$ \\
\hline$(5,16)$ & 9 & {$[5,0,4]$} & $G(8,5)$ & $C_{2}^{3}$ & $(5,96)$ & 12 & {$[2,0,10]$} & $G(32,45)$ & $C_{4} \times C_{2}^{3}$ \\
\hline$(5,17)$ & 17 & {$[5,0,12]$} & $G(16,14)$ & $C_{2}^{4}$ & $(5,97)$ & 12 & {$[2,0,10]$} & $G(64,261)$ & $C_{2}^{3} \times D_{4}$ \\
\hline$(5,18)$ & 28 & {$[23,0,5]$} & $G(4,2)$ & $C_{2}^{2}$ & $(5,98)$ & 29 & {$[22,0,7]$} & $G(8,3)$ & $D_{4}$ \\
\hline$(5,19)$ & 18 & {$[15,0,3]$} & $G(4,2)$ & $C_{2}^{2}$ & $(5,99)$ & 58 & {$[36,0,22]$} & $G(8,3)$ & $\mathrm{D}_{4}$ \\
\hline$(5,20)$ & 18 & {$[6,0,12]$} & $G(8,5)$ & $C_{2}^{3}$ & $(5,100)$ & 29 & {$[12,0,17]$} & $G(8,3)$ & $D_{4}$ \\
\hline$(5,21)$ & 17 & {$[7,0,10]$} & $G(8,5)$ & $C_{2}^{3}$ & $(5,101)$ & 22 & {$[12,0,10]$} & $G(8,2)$ & $C_{4} \times C_{2}$ \\
\hline$(5,22)$ & 17 & {$[12,0,5]$} & $G(8,5)$ & $C_{2}^{3}$ & $(5,102)$ & 22 & {$[10,0,12]$} & $G(8,2)$ & $C_{4} \times C_{2}$ \\
\hline$(5,23)$ & 27 & {$[19,0,8]$} & $G(8,5)$ & $C_{2}^{3}$ & $(5,103)$ & 29 & {$[22,0,7]$} & $G(8,2)$ & $C_{4} \times C_{2}$ \\
\hline$(5,24)$ & 27 & {$[13,0,14]$} & $G(8,5)$ & $C_{2}^{3}$ & $(5,104)$ & 5 & {$[1,0,4]$} & $G(128,2194)$ & $C_{2} \times D_{4} \times D_{4}$ \\
\hline$(5,25)$ & 33 & {$[6,0,27]$} & $G(16,14)$ & $C_{2}^{4}$ & $(5,105)$ & 12 & {$[3,0,9]$} & $G(16,4)$ & $C_{4} \rtimes C_{4}$ \\
\hline$(5,26)$ & 42 & {$[10,0,32]$} & $G(16,14)$ & $C_{2}^{4}$ & $(5,106)$ & 6 & {$[4,0,2]$} & $G(16,4)$ & $C_{4} \rtimes C_{4}$ \\
\hline$(5,27)$ & 16 & {$[3,0,13]$} & $G(16,14)$ & $C_{2}^{\overline{4}}$ & $(5,107)$ & 6 & {$[2,0,4]$} & $G(16,4)$ & $C_{4} \rtimes C_{4}$ \\
\hline$(5,28)$ & 30 & {$[14,0,16]$} & $G(16,14)$ & $C_{2}^{4}$ & $(5,108)$ & 15 & {$[10,0,5]$} & $G(16,3)$ & $\left(C_{4} \times C_{2}\right) \rtimes C_{2}$ \\
\hline$(5,29)$ & 16 & {$[3,0,13]$} & $G(32,51)$ & $C_{2}^{5}$ & $(5,109)$ & 15 & {$[3,0,12]$} & $G(16,3)$ & $\left(C_{4} \times C_{2}\right) \rtimes C_{2}$ \\
\hline$(5,30)$ & 33 & {$[16,0,17]$} & $G(8,5)$ & $C_{2}^{3}$ & $(5,110)$ & 15 & {$[8,0,7]$} & $G(16,3)$ & $\left(C_{4} \times C_{2}\right) \rtimes C_{2}$ \\
\hline$(5,31)$ & 49 & {$[15,0,34]$} & $G(8,5)$ & $C_{2}^{3}$ & $(5,111)$ & 15 & {$[4,0,11]$} & $G(16,3)$ & $\left(C_{4} \times C_{2}\right) \rtimes C_{2}$ \\
\hline$(5,32)$ & 60 & {$[36,0,24]$} & $G(8,5)$ & $C_{2}^{\overline{3}}$ & $(5,112)$ & 8 & {$[2,0,6]$} & $G(16,10)$ & $C_{4} \times C_{2}^{2}$ \\
\hline$(5,33)$ & 2 & {$[2,0,0]$} & $G(4,1)$ & $C_{4}$ & $(5,113)$ & 5 & {$[1,0,4]$} & $G(16,2)$ & $C_{4} \times C_{4}$ \\
\hline$(5,34)$ & 2 & {$[2,0,0]$} & $G(4,1)$ & $C_{4}$ & $(5,114)$ & 5 & {$[3,0,2]$} & $G(16,2)$ & $C_{4} \times C_{4}$ \\
\hline$(5,35)$ & 2 & {$[1,0,1]$} & $G(8,2)$ & $C_{4} \times C_{2}$ & $(5,115)$ & 6 & {$[2,0,4]$} & $G(16,2)$ & $C_{4} \times C_{4}$ \\
\hline$(5,36)$ & 7 & {$[3,0,4]$} & $G(16,11)$ & $C_{2} \times D_{4}$ & $(5,116)$ & 27 & {$[7,0,20]$} & $G(16,3)$ & $\left(C_{4} \times C_{2}\right) \rtimes C_{2}$ \\
\hline$(5,37)$ & 7 & {$[7,0,0]$} & $G(8,3)$ & $D_{4}$ & $(5,117)$ & 19 & {$[12,0,7]$} & $G(16,3)$ & $\left(C_{4} \times C_{2}\right) \rtimes C_{2}$ \\
\hline$(5,38)$ & 10 & {$[7,0,3]$} & $G(8,3)$ & $D_{4}$ & $(5,118)$ & 19 & {$[8,0,11]$} & $G(16,3)$ & $\left(C_{4} \times C_{2}\right) \rtimes C_{2}$ \\
\hline$(5,39)$ & 7 & {$[4,0,3]$} & $G(8,3)$ & $\mathrm{D}_{4}$ & $(5,119)$ & 13 & {$[3,0,10]$} & $G(16,11)$ & $C_{2} \times D_{4}$ \\
\hline$(5,40)$ & 2 & {$[1,0,1]$} & $G(16,11)$ & $C_{2} \times D_{4}$ & $(5,120)$ & 22 & {$[9,0,13]$} & $G(16,11)$ & $C_{2} \times D_{4}$ \\
\hline$(5,41)$ & 2 & {$[2,0,0]$} & $G(4,1)$ & $C_{4}$ & $(5,121)$ & 13 & {$[8,0,5]$} & $G(16,11)$ & $C_{2} \times D_{4}$ \\
\hline$(5,42)$ & 2 & {$[2,0,0]$} & $G(4,1)$ & $C_{4}$ & $(5,122)$ & 16 & {$[5,0,11]$} & $G(16,11)$ & $C_{2} \times D_{4}$ \\
\hline$(5,43)$ & 2 & {$[2,0,0]$} & $G(8,3)$ & $D_{4}$ & $(5,123)$ & 15 & {$[3,0,12]$} & $G(32,22)$ & $C_{2} \times\left(\left(C_{4} \times C_{2}\right) \rtimes C_{2}\right)$ \\
\hline$(5,44)$ & 2 & {$[1,0,1]$} & $G(8,3)$ & $D_{4}$ & $(5,124)$ & 6 & {$[1,0,5]$} & $G(32,23)$ & $C_{2} \times\left(C_{4} \rtimes C_{4}\right)$ \\
\hline$(5,45)$ & 4 & {$[3,0,1]$} & $G(8,3)$ & $D_{4}$ & $(5,125)$ & 6 & {$[1,0,5]$} & $G(32,25)$ & $C_{4} \times D_{4}$ \\
\hline$(5,46)$ & 2 & {$[1,0,1]$} & $G(8,2)$ & $C_{4} \times C_{2}$ & $(5,126)$ & 6 & {$[4,0,2]$} & $G(32,25)$ & $C_{4} \times D_{4}$ \\
\hline$(5,47)$ & 7 & {$[2,0,5]$} & $G(16,11)$ & $C_{2} \times D_{4}$ & $(5,127)$ & 12 & {$[2,0,10]$} & $G(32,25)$ & $C_{4} \times D_{4}$ \\
\hline$(5,48)$ & 15 & {$[5,0,10]$} & $G(16,11)$ & $C_{2} \times D_{4}$ & $(5,128)$ & 12 & {$[3,0,9]$} & $G(32,25)$ & $C_{4} \times D_{4}$ \\
\hline$(5,49)$ & 14 & {$[6,0,8]$} & $G(16,11)$ & $C_{2} \times D_{4}$ & $(5,129)$ & 6 & {$[2,0,4]$} & $G(32,25)$ & $C_{4} \times D_{4}$ \\
\hline$(5,50)$ & 7 & {$[2,0,5]$} & $G(16,11)$ & $C_{2} \times D_{4}$ & $(5,130)$ & 6 & {$[1,0,5]$} & $G(32,25)$ & $C_{4} \times D_{4}$ \\
\hline$(5,51)$ & 7 & {$[4,0,3]$} & $G(16,11)$ & $C_{2} \times D_{4}$ & $(5,131)$ & 19 & {$[8,0,11]$} & $G(32,27)$ & $C_{2}^{4} \rtimes C_{2}$ \\
\hline$(5,52)$ & 14 & {$[7,0,7]$} & $G(16,11)$ & $C_{2} \times D_{4}$ & $(5,132)$ & 19 & {$[4,0,15]$} & $G(32,27)$ & $C_{2}^{4} \rtimes C_{2}$ \\
\hline$(5,53)$ & 7 & {$[2,0,5]$} & $G(16,11)$ & $C_{2} \times D_{4}$ & $(5,133)$ & 19 & {$[12,0,7]$} & $G(32,27)$ & $C_{2}^{4} \rtimes C_{2}$ \\
\hline$(5,54)$ & 7 & {$[5,0,2]$} & $G(16,11)$ & $C_{2} \times D_{4}$ & $(5,134)$ & 10 & {$[2,0,8]$} & $G(32,34)$ & $C_{4}^{2} \rtimes C_{2}$ \\
\hline$(5,55)$ & 7 & {$[2,0,5]$} & $G(16,10)$ & $C_{4} \times C_{2}^{2}$ & $(5,135)$ & 5 & {$[1,0,4]$} & $G(32,34)$ & $C_{4}^{2} \rtimes C_{2}$ \\
\hline$(5,56)$ & 7 & {$[2,0,5]$} & $G(32,46)$ & $C_{2}^{2} \times D_{4}$ & $(5,136)$ & 19 & {$[4,0,15]$} & $G(32,27)$ & $C_{2}^{4} \rtimes C_{2}$ \\
\hline$(5,57)$ & 8 & {$[8,0,0]$} & $G(4,1)$ & $C_{4}$ & $(5,137)$ & 5 & {$[3,0,2]$} & $G(32,34)$ & $C_{4}^{2} \rtimes C_{2}$ \\
\hline$(5,58)$ & 8 & {$[8,0,0]$} & $G(4,1)$ & $C_{4}$ & $(5,138)$ & 12 & {$[8,0,4]$} & $G(32,28)$ & $\left(C_{4} \times C_{2}^{2}\right) \rtimes C_{2}$ \\
\hline$(5,59)$ & 7 & {$[4,0,3]$} & $G(8,3)$ & $D_{4}$ & $(5,139)$ & 12 & {$[3,0,9]$} & $G(32,28)$ & $\left(C_{4} \times C_{2}^{2}\right) \rtimes C_{2}$ \\
\hline$(5,60)$ & 14 & {$[9,0,5]$} & $G(8,3)$ & $D_{4}$ & $(5,140)$ & 24 & {$[6,0,18]$} & $G(32,28)$ & $\left(C_{4} \times C_{2}^{2}\right) \rtimes C_{2}$ \\
\hline$(5,61)$ & 7 & {$[5,0,2]$} & $G(8,3)$ & $D_{4}$ & $(5,141)$ & 27 & {$[7,0,20]$} & $G(32,27)$ & $C_{2}^{4} \rtimes C_{2}$ \\
\hline$(5,62)$ & 15 & {$[13,0,2]$} & $G(8,3)$ & $D_{4}$ & $(5,142)$ & 27 & {$[5,0,22]$} & $G(32,27)$ & $C_{2}^{4} \rtimes C_{2}$ \\
\hline$(5,63)$ & 15 & {$[8,0,7]$} & $G(8,3)$ & $D_{4}$ & $(5,143)$ & 24 & {$[4,0,20]$} & $G(32,28)$ & $\left(C_{4} \times C_{2}^{2}\right) \rtimes C_{2}$ \\
\hline$(5,64)$ & 15 & {$[12,0,3]$} & $G(8,3)$ & $D_{4}$ & $(5,144)$ & 12 & {$[3,0,9]$} & $G(32,28)$ & $\left(C_{4} \times C_{2}^{2}\right) \rtimes C_{2}$ \\
\hline$(5,65)$ & 15 & {$[7,0,8]$} & $G(8,3)$ & $D_{4}$ & $(5,145)$ & 12 & {$[2,0,10]$} & $G(32,28)$ & $\left(C_{4} \times C_{2}^{2}\right) \rtimes C_{2}$ \\
\hline$(5,66)$ & 8 & {$[3,0,5]$} & $G(8,2)$ & $C_{4} \times C_{2}$ & $(5,146)$ & 12 & {$[3,0,9]$} & $G(32,34)$ & $C_{4}^{2} \rtimes C_{2}$ \\
\hline$(5,67)$ & 7 & {$[5,0,2]$} & $G(8,2)$ & $C_{4} \times C_{2}$ & $(5,147)$ & 5 & {$[1,0,4]$} & $G(32,21)$ & $C_{4} \times C_{4} \times C_{2}$ \\
\hline$(5,68)$ & 7 & {$[5,0,2]$} & $G(8,2)$ & $C_{4} \times C_{2}$ & $(5,148)$ & 13 & {$[3,0,10]$} & $G(32,46)$ & $C_{2}^{2} \times D_{4}$ \\
\hline$(5,69)$ & 7 & {$[4,0,3]$} & $G(8,2)$ & $C_{4} \times C_{2}$ & $(5,149)$ & 19 & {$[4,0,15]$} & $G(32,22)$ & $C_{2} \times\left(\left(C_{4} \times C_{2}\right) \rtimes C_{2}\right)$ \\
\hline$(5,70)$ & 7 & {$[4,0,3]$} & $G(8,2)$ & $C_{4} \times C_{2}$ & $(5,150)$ & 6 & {$[1,0,5]$} & $G(64,196)$ & $C_{2} \times C_{4} \times D_{4}$ \\
\hline$(5,71)$ & 29 & {$[5,0,24]$} & $G(16,11)$ & $C_{2} \times D_{4}$ & $(5,151)$ & 19 & {$[4,0,15]$} & $G(64,202)$ & $C_{2} \times\left(C_{2}^{4} \rtimes C_{2}\right)$ \\
\hline$(5,72)$ & 41 & {$[10,0,31]$} & $G(16,11)$ & $C_{2} \times D_{4}$ & $(5,152)$ & 5 & {$[1,0,4]$} & $G(64,211)$ & $C_{2} \times\left(C_{4}^{2} \rtimes C_{2}\right)$ \\
\hline$(5,73)$ & 38 & {$[9,0,29]$} & $G(16,11)$ & $C_{2} \times D_{4}$ & $(5,153)$ & 12 & {$[2,0,10]$} & $G(64,203)$ & $C_{2} \times\left(\left(C_{4} \times C_{2}^{2}\right) \rtimes C_{2}\right)$ \\
\hline$(5,74)$ & 19 & {$[10,0,9]$} & $G(16,11)$ & $C_{2} \times D_{4}$ & $(5,154)$ & 16 & {$[3,0,13]$} & $G(64,226)$ & $D_{4} \times D_{4}$ \\
\hline$(5,75)$ & 41 & {$[22,0,19]$} & $G(16,11)$ & $C_{2} \times D_{4}$ & $(5,155)$ & 5 & {$[1,0,4]$} & $G(64,226)$ & $D_{4} \times D_{4}$ \\
\hline$(5,76)$ & 55 & {$[13,0,42]$} & $G(16,11)$ & $C_{2} \times D_{4}$ & $(5,156)$ & 5 & {$[3,0,2]$} & $G(64,226)$ & $D_{4} \times D_{4}$ \\
\hline$(5,77)$ & 26 & {$[8,0,18]$} & $G(16,11)$ & $C_{2} \times D_{4}$ & $(5,157)$ & 12 & {$[3,0,9]$} & $G(64,226)$ & $D_{4} \times D_{4}$ \\
\hline$(5,78)$ & 41 & {$[14,0,27]$} & $G(16,11)$ & $C_{2} \times D_{4}$ & $(5,158)$ & 12 & {$[2,0,10]$} & $G(64,226)$ & $D_{4} \times D_{4}$ \\
\hline$(5,79)$ & 41 & {$[7,0,34]$} & $G(16,11)$ & $C_{2} \times D_{4}$ & $(5,159)$ & 6 & {$[1,0,5]$} & $G(64,226)$ & $D_{4} \times D_{4}$ \\
\hline$(5,80)$ & 19 & {$[4,0,15]$} & $G(16,11)$ & $C_{2} \times D_{4}$ & $(5,160)$ & 8 & {$[3,0,5]$} & $G(8,2)$ & $C_{4} \times C_{2}$ \\
\hline
\end{tabular}


Table 16 (continued): stably rational classification of algebraic $k$-tori of dimension 5

\begin{tabular}{|c|c|c|c|c|c|c|c|c|c|}
\hline CARAT & \# & {$[s, r, u]$} & $G(n, i)$ & & CARAT & \# & {$[s, r, u]$} & $G(n, i)$ & \\
\hline$(5,161)$ & 8 & {$[5,0,3]$} & $G(8,2)$ & $C_{4} \times C_{2}$ & $(5,241)$ & 15 & {$[9,0,6]$} & $G(24,14)$ & $C_{2}^{2} \times S_{3}$ \\
\hline$(5,162)$ & 8 & {$[5,0,3]$} & $G(8,2)$ & $C_{4} \times C_{2}$ & $(5,242)$ & 15 & {$[12,0,3]$} & $G(24,14)$ & $C_{2}^{2} \times S_{3}$ \\
\hline$(5,163)$ & 1 & {$[1,0,0]$} & $G(12,5)$ & $C_{6} \times C_{2}$ & $(5,243)$ & 15 & {$[15,0,0]$} & $G(24,14)$ & $C_{2}^{2} \times S_{3}$ \\
\hline$(5,164)$ & 2 & {$[2,0,0]$} & $G(3,1)$ & $C_{3}$ & $(5,244)$ & 15 & {$[9,0,6]$} & $G(24,14)$ & $C_{2}^{2} \times S_{3}$ \\
\hline$(5,165)$ & 1 & {$[1,0,0]$} & $G(6,2)$ & $C_{6}$ & $(5,245)$ & 18 & {$[18,0,0]$} & $G(24,14)$ & $C_{2}^{2} \times S_{3}$ \\
\hline$(5,166)$ & 1 & {$[1,0,0]$} & $G(6,2)$ & $C_{6}$ & $(5,246)$ & 18 & {$[12,0,6]$} & $G(24,14)$ & $C_{2}^{2} \times S_{3}$ \\
\hline$(5,167)$ & 2 & {$[2,0,0]$} & $G(6,2)$ & $C_{6}$ & $(5,247)$ & 8 & {$[6,0,2]$} & $G(24,14)$ & $C_{2}^{2} \times S_{3}$ \\
\hline$(5,168)$ & 2 & {$[2,0,0]$} & $G(12,4)$ & $D_{12}$ & $(5,248)$ & 8 & {$[4,0,4]$} & $G(24,14)$ & $C_{2}^{2} \times S_{3}$ \\
\hline$(5,169)$ & 2 & {$[2,0,0]$} & $G(12,4)$ & $D_{12}$ & $(5,249)$ & 10 & {$[6,0,4]$} & $G(24,14)$ & $C_{2}^{2} \times S_{3}$ \\
\hline$(5,170)$ & 3 & {$[3,0,0]$} & $G(12,4)$ & $D_{12}$ & $(5,250)$ & 10 & {$[10,0,0]$} & $G(24,14)$ & $C_{2}^{2} \times S_{3}$ \\
\hline$(5,171)$ & 5 & {$[5,0,0]$} & $G(12,4)$ & $D_{12}$ & $(5,251)$ & 10 & {$[6,0,4]$} & $G(24,14)$ & $C_{2}^{2} \times S_{3}$ \\
\hline$(5,172)$ & 2 & {$[2,0,0]$} & $G(24,14)$ & $C_{2}^{2} \times S_{3}$ & $(5,252)$ & 5 & {$[5,0,0]$} & $G(24,14)$ & $C_{2}^{2} \times S_{3}$ \\
\hline$(5,173)$ & 5 & {$[5,0,0]$} & $G(6,1)$ & $S_{3}$ & $(5,253)$ & 5 & {$[4,0,1]$} & $G(24,14)$ & $C_{2}^{2} \times S_{3}$ \\
\hline$(5,174)$ & 5 & {$[5,0,0]$} & $G(6,1)$ & $S_{3}$ & $(5,254)$ & 6 & {$[4,0,2]$} & $G(24,14)$ & $C_{2}^{2} \times S_{3}$ \\
\hline$(5,175)$ & 1 & {$[1,0,0]$} & $G(12,5)$ & $C_{6} \times C_{2}$ & $(5,255)$ & 6 & {$[6,0,0]$} & $G(24,14)$ & $C_{2}^{2} \times S_{3}$ \\
\hline$(5,176)$ & 3 & {$[3,0,0]$} & $G(12,4)$ & $D_{12}$ & $(5,256)$ & 4 & {$[2,0,2]$} & $G(48,52)$ & $C_{6} \times C_{2}^{3}$ \\
\hline$(5,177)$ & 2 & {$[2,0,0]$} & $G(12,4)$ & $D_{12}$ & $(5,257)$ & 15 & {$[9,0,6]$} & $G(48,51)$ & $C_{2}^{3} \times S_{3}$ \\
\hline$(5,178)$ & 1 & {$[1,0,0]$} & $G(12,4)$ & $D_{12}$ & $(5,258)$ & 8 & {$[4,0,4]$} & $G(48,51)$ & $C_{2}^{3} \times S_{3}$ \\
\hline$(5,179)$ & 1 & {$[1,0,0]$} & $G(12,4)$ & $D_{12}$ & $(5,259)$ & 4 & {$[3,0,1]$} & $G(48,51)$ & $C_{2}^{3} \times S_{3}$ \\
\hline$(5,180)$ & 1 & {$[1,0,0]$} & $G(24,14)$ & $C_{2}^{2} \times S_{3}$ & $(5,260)$ & 4 & {$[2,0,2]$} & $G(48,51)$ & $C_{2}^{3} \times S_{3}$ \\
\hline$(5,181)$ & 2 & {$[2,0,0]$} & $G(3,1)$ & $C_{3}$ & $(5,261)$ & 8 & {$[4,0,4]$} & $G(48,51)$ & $C_{2}^{3} \times S_{3}$ \\
\hline$(5,182)$ & 2 & {$[2,0,0]$} & $G(6,2)$ & $C_{6}$ & $(5,262)$ & 5 & {$[3,0,2]$} & $G(48,51)$ & $C_{2}^{3} \times S_{3}$ \\
\hline$(5,183)$ & 1 & {$[1,0,0]$} & $G(6,2)$ & $C_{6}$ & $(5,263)$ & 5 & {$[5,0,0]$} & $G(48,51)$ & $C_{2}^{3} \times S_{3}$ \\
\hline$(5,184)$ & 1 & {$[1,0,0]$} & $G(6,2)$ & $C_{6}$ & $(5,264)$ & 5 & {$[3,0,2]$} & $G(48,51)$ & $C_{2}^{3} \times S_{3}$ \\
\hline$(5,185)$ & 3 & {$[3,0,0]$} & $G(6,1)$ & $S_{3}$ & $(5,265)$ & 10 & {$[6,0,4]$} & $G(48,51)$ & $C_{2}^{3} \times S_{3}$ \\
\hline$(5,186)$ & 3 & {$[3,0,0]$} & $G(6,1)$ & $S_{3}$ & $(5,266)$ & 4 & {$[2,0,2]$} & $G(96,230)$ & $C_{2}^{4} \times S_{3}$ \\
\hline$(5,187)$ & 4 & {$[4,0,0]$} & $G(12,5)$ & $C_{6} \times C_{2}$ & $(5,267)$ & 4 & {$[4,0,0]$} & $G(12,2)$ & $C_{12}$ \\
\hline$(5,188)$ & 8 & {$[8,0,0]$} & $G(12,4)$ & $D_{12}$ & $(5,268)$ & 4 & {$[4,0,0]$} & $G(12,2)$ & $C_{12}$ \\
\hline$(5,189)$ & 4 & {$[4,0,0]$} & $G(12,5)$ & $C_{6} \times C_{2}$ & $(5,269)$ & 2 & {$[2,0,0]$} & $G(12,2)$ & $C_{12}$ \\
\hline$(5,190)$ & 6 & {$[6,0,0]$} & $G(12,4)$ & $D_{12}$ & $(5,270)$ & 2 & {$[2,0,0]$} & $G(12,2)$ & $C_{12}$ \\
\hline$(5,191)$ & 6 & {$[6,0,0]$} & $G(12,4)$ & $D_{12}$ & $(5,271)$ & 6 & {$[6,0,0]$} & $G(12,1)$ & $C_{3} \rtimes C_{4}$ \\
\hline$(5,192)$ & 6 & {$[6,0,0]$} & $G(12,4)$ & $D_{12}$ & $(5,272)$ & 6 & {$[6,0,0]$} & $G(12,1)$ & $C_{3} \rtimes C_{4}$ \\
\hline$(5,193)$ & 6 & {$[6,0,0]$} & $G(12,4)$ & $D_{12}$ & $(5,273)$ & 2 & {$[1,0,1]$} & $G(192,1514)$ & $C_{2}^{2} \times S_{3} \times D_{4}$ \\
\hline$(5,194)$ & 2 & {$[2,0,0]$} & $G(12,5)$ & $C_{6} \times C_{2}$ & $(5,274)$ & 4 & {$[2,0,2]$} & $G(24,9)$ & $C_{12} \times C_{2}$ \\
\hline$(5,195)$ & 2 & {$[2,0,0]$} & $G(12,5)$ & $C_{6} \times C_{2}$ & $(5,275)$ & 8 & {$[6,0,2]$} & $G(24,10)$ & $C_{3} \times D_{4}$ \\
\hline$(5,196)$ & 2 & {$[2,0,0]$} & $G(12,5)$ & $C_{6} \times C_{2}$ & $(5,276)$ & 4 & {$[2,0,2]$} & $G(24,10)$ & $C_{3} \times D_{4}$ \\
\hline$(5,197)$ & 2 & {$[2,0,0]$} & $G(12,5)$ & $C_{6} \times C_{2}$ & $(5,277)$ & 4 & {$[4,0,0]$} & $G(24,10)$ & $C_{3} \times D_{4}$ \\
\hline$(5,198)$ & 6 & {$[6,0,0]$} & $G(12,4)$ & $D_{12}$ & $(5,278)$ & 2 & {$[1,0,1]$} & $G(24,10)$ & $C_{3} \times D_{4}$ \\
\hline$(5,199)$ & 6 & {$[6,0,0]$} & $G(12,4)$ & $D_{12}$ & $(5,279)$ & 4 & {$[3,0,1]$} & $G(24,10)$ & $C_{3} \times D_{4}$ \\
\hline$(5,200)$ & 6 & {$[6,0,0]$} & $G(12,4)$ & $D_{12}$ & $(5,280)$ & 4 & {$[2,0,2]$} & $G(24,10)$ & $C_{3} \times D_{4}$ \\
\hline$(5,201)$ & 6 & {$[6,0,0]$} & $G(12,4)$ & $D_{12}$ & $(5,281)$ & 4 & {$[3,0,1]$} & $G(24,10)$ & $C_{3} \times D_{4}$ \\
\hline$(5,202)$ & 2 & {$[2,0,0]$} & $G(12,4)$ & $D_{12}$ & $(5,282)$ & 4 & {$[3,0,1]$} & $G(24,10)$ & $C_{3} \times D_{4}$ \\
\hline$(5,203)$ & 2 & {$[2,0,0]$} & $G(12,4)$ & $D_{12}$ & $(5,283)$ & 4 & {$[4,0,0]$} & $G(24,10)$ & $C_{3} \times D_{4}$ \\
\hline$(5,204)$ & 4 & {$[4,0,0]$} & $G(12,4)$ & $D_{12}$ & $(5,284)$ & 2 & {$[2,0,0]$} & $G(24,10)$ & $C_{3} \times D_{4}$ \\
\hline$(5,205)$ & 6 & {$[6,0,0]$} & $G(24,14)$ & $C_{2}^{2} \times S_{3}$ & $(5,285)$ & 2 & {$[1,0,1]$} & $G(24,9)$ & $C_{12} \times C_{2}$ \\
\hline$(5,206)$ & 2 & {$[2,0,0]$} & $G(24,15)$ & $C_{6} \times C_{2}^{2}$ & $(5,286)$ & 2 & {$[2,0,0]$} & $G(24,9)$ & $C_{12} \times C_{2}$ \\
\hline$(5,207)$ & 6 & {$[6,0,0]$} & $G(24,14)$ & $C_{2}^{2} \times S_{3}$ & $(5,287)$ & 2 & {$[2,0,0]$} & $G(24,9)$ & $C_{12} \times C_{2}$ \\
\hline$(5,208)$ & 2 & {$[2,0,0]$} & $G(24,14)$ & $C_{2}^{2} \times S_{3}$ & $(5,288)$ & 2 & {$[1,0,1]$} & $G(24,9)$ & $C_{12} \times C_{2}$ \\
\hline$(5,209)$ & 4 & {$[4,0,0]$} & $G(24,14)$ & $C_{2}^{2} \times S_{3}$ & $(5,289)$ & 2 & {$[1,0,1]$} & $G(24,9)$ & $C_{12} \times C_{2}$ \\
\hline$(5,210)$ & 4 & {$[4,0,0]$} & $G(24,14)$ & $C_{2}^{2} \times S_{3}$ & $(5,290)$ & 6 & {$[3,0,3]$} & $G(24,5)$ & $C_{4} \times S_{3}$ \\
\hline$(5,211)$ & 2 & {$[2,0,0]$} & $G(24,14)$ & $C_{2}^{2} \times S_{3}$ & $(5,291)$ & 6 & {$[3,0,3]$} & $G(24,5)$ & $C_{4} \times S_{3}$ \\
\hline$(5,212)$ & 2 & {$[2,0,0]$} & $G(24,14)$ & $C_{2}^{2} \times S_{3}$ & $(5,292)$ & 6 & {$[6,0,0]$} & $G(24,5)$ & $C_{4} \times S_{3}$ \\
\hline$(5,213)$ & 2 & {$[2,0,0]$} & $G(24,14)$ & $C_{2}^{2} \times S_{3}$ & $(5,293)$ & 6 & {$[6,0,0]$} & $G(24,5)$ & $C_{4} \times S_{3}$ \\
\hline$(5,214)$ & 2 & {$[2,0,0]$} & $G(24,14)$ & $C_{2}^{2} \times S_{3}$ & $(5,294)$ & 6 & {$[3,0,3]$} & $G(24,7)$ & $C_{2} \times\left(C_{3} \rtimes C_{4}\right)$ \\
\hline$(5,215)$ & 2 & {$[2,0,0]$} & $G(48,51)$ & $C_{2}^{3} \times S_{3}$ & $(5,295)$ & 4 & {$[2,0,2]$} & $G(24,5)$ & $C_{4} \times S_{3}$ \\
\hline$(5,216)$ & 4 & {$[4,0,0]$} & $G(6,2)$ & $C_{6}$ & $(5,296)$ & 4 & {$[2,0,2]$} & $G(24,5)$ & $C_{4} \times S_{3}$ \\
\hline$(5,217)$ & 4 & {$[4,0,0]$} & $G(6,2)$ & $C_{6}$ & $(5,297)$ & 4 & {$[4,0,0]$} & $G(24,5)$ & $C_{4} \times S_{3}$ \\
\hline$(5,218)$ & 8 & {$[8,0,0]$} & $G(6,1)$ & $S_{3}$ & $(5,298)$ & 4 & {$[4,0,0]$} & $G(24,5)$ & $C_{4} \times S_{3}$ \\
\hline$(5,219)$ & 8 & {$[8,0,0]$} & $G(6,1)$ & $S_{3}$ & $(5,299)$ & 4 & {$[2,0,2]$} & $G(24,7)$ & $C_{2} \times\left(C_{3} \rtimes C_{4}\right)$ \\
\hline$(5,220)$ & 4 & {$[4,0,0]$} & $G(6,2)$ & $C_{6}$ & $(5,300)$ & 2 & {$[2,0,0]$} & $G(24,7)$ & $C_{2} \times\left(C_{3} \rtimes C_{4}\right)$ \\
\hline$(5,221)$ & 4 & {$[4,0,0]$} & $G(6,2)$ & $C_{6}$ & $(5,301)$ & 2 & {$[2,0,0]$} & $G(24,7)$ & $C_{2} \times\left(C_{3} \rtimes C_{4}\right)$ \\
\hline$(5,222)$ & 10 & {$[10,0,0]$} & $G(12,5)$ & $C_{6} \times C_{2}$ & $(5,302)$ & 12 & {$[9,0,3]$} & $G(24,8)$ & $\left(C_{6} \times C_{2}\right) \rtimes C_{2}$ \\
\hline$(5,223)$ & 12 & {$[12,0,0]$} & $G(12,5)$ & $C_{6} \times C_{2}$ & $(5,303)$ & 6 & {$[3,0,3]$} & $G(24,6)$ & $D_{12}$ \\
\hline$(5,224)$ & 10 & {$[8,0,2]$} & $G(12,5)$ & $C_{6} \times C_{2}$ & $(5,304)$ & 12 & {$[9,0,3]$} & $G(24,6)$ & $D_{12}$ \\
\hline$(5,225)$ & 12 & {$[12,0,0]$} & $G(12,4)$ & $D_{12}$ & $(5,305)$ & 12 & {$[6,0,6]$} & $G(24,8)$ & $\left(C_{6} \times C_{2}\right) \rtimes C_{2}$ \\
\hline$(5,226)$ & 24 & {$[24,0,0]$} & $G(12,4)$ & $D_{12}$ & $(5,306)$ & 12 & {$[9,0,3]$} & $G(24,8)$ & $\left(C_{6} \times C_{2}\right) \rtimes C_{2}$ \\
\hline$(5,227)$ & 12 & {$[10,0,2]$} & $G(12,4)$ & $D_{12}$ & $(5,307)$ & 6 & {$[6,0,0]$} & $G(24,6)$ & $D_{12}$ \\
\hline$(5,228)$ & 4 & {$[3,0,1]$} & $G(12,5)$ & $C_{6} \times C_{2}$ & $(5,308)$ & 12 & {$[12,0,0]$} & $G(24,8)$ & $\left(C_{6} \times C_{2}\right) \rtimes C_{2}$ \\
\hline$(5,229)$ & 5 & {$[5,0,0]$} & $G(12,5)$ & $C_{6} \times C_{2}$ & $(5,309)$ & 8 & {$[4,0,4]$} & $G(24,8)$ & $\left(C_{6} \times C_{2}\right) \rtimes C_{2}$ \\
\hline$(5,230)$ & 15 & {$[15,0,0]$} & $G(12,4)$ & $D_{12}$ & $(5,310)$ & 8 & {$[6,0,2]$} & $G(24,6)$ & $D_{12}$ \\
\hline$(5,231)$ & 18 & {$[18,0,0]$} & $G(12,4)$ & $D_{12}$ & $(5,311)$ & 4 & {$[2,0,2]$} & $G(24,6)$ & $D_{12}$ \\
\hline$(5,232)$ & 15 & {$[12,0,3]$} & $G(12,4)$ & $D_{12}$ & $(5,312)$ & 8 & {$[6,0,2]$} & $G(24,8)$ & $\left(C_{6} \times C_{2}\right) \rtimes C_{2}$ \\
\hline$(5,233)$ & 10 & {$[6,0,4]$} & $G(24,15)$ & $C_{6} \times C_{2}^{2}$ & $(5,313)$ & 8 & {$[8,0,0]$} & $G(24,8)$ & $\left(C_{6} \times C_{2}\right) \rtimes C_{2}$ \\
\hline$(5,234)$ & 12 & {$[8,0,4]$} & $G(24,14)$ & $C_{2}^{2} \times S_{3}$ & $(5,314)$ & 4 & {$[4,0,0]$} & $G(24,6)$ & $D_{12}$ \\
\hline$(5,235)$ & 4 & {$[2,0,2]$} & $G(24,15)$ & $C_{6} \times C_{2}^{2}$ & $(5,315)$ & 8 & {$[6,0,2]$} & $G(24,8)$ & $\left(C_{6} \times C_{2}\right) \rtimes C_{2}$ \\
\hline$(5,236)$ & 4 & {$[2,0,2]$} & $G(24,15)$ & $C_{6} \times C_{2}^{2}$ & $(5,316)$ & 4 & {$[2,0,2]$} & $G(48,45)$ & $C_{6} \times D_{4}$ \\
\hline$(5,237)$ & 4 & {$[3,0,1]$} & $G(24,15)$ & $C_{6} \times C_{2}^{2}$ & $(5,317)$ & 4 & {$[2,0,2]$} & $G(48,45)$ & $C_{6} \times D_{4}$ \\
\hline$(5,238)$ & 5 & {$[5,0,0]$} & $G(24,15)$ & $C_{6} \times C_{2}^{2}$ & $(5,318)$ & 2 & {$[1,0,1]$} & $G(48,45)$ & $C_{6} \times D_{4}$ \\
\hline$(5,239)$ & 5 & {$[3,0,2]$} & $G(24,15)$ & $C_{6} \times C_{2}^{2}$ & $(5,319)$ & 4 & {$[3,0,1]$} & $G(48,45)$ & $C_{6} \times D_{4}$ \\
\hline$(5,240)$ & 15 & {$[9,0,6]$} & $G(24,14)$ & $C_{2}^{2} \times S_{3}$ & $(5,320)$ & 2 & {$[1,0,1]$} & $G(48,45)$ & $C_{6} \times D_{4}$ \\
\hline
\end{tabular}


Table 16 (continued): stably rational classification of algebraic $k$-tori of dimension 5

\begin{tabular}{|c|c|c|c|c|c|c|c|c|c|}
\hline CARAT & \# & {$[s, r, u]$} & $G(n, i)$ & & CARAT & \# & {$[s, r, u]$} & $G(n, i)$ & \\
\hline$(5,321)$ & 2 & {$[2,0,0]$} & $G(48,45)$ & $C_{6} \times D_{4}$ & $(5,401)$ & 2 & {$[2,0,0]$} & $G(144,192)$ & $C_{2}^{2} \times S_{3}^{2}$ \\
\hline$(5,322)$ & 2 & {$[1,0,1]$} & $G(48,45)$ & $C_{6} \times D_{4}$ & $(5,402)$ & 2 & {$[2,0,0]$} & $G(144,192)$ & $C_{2}^{2} \times S_{3}^{2}$ \\
\hline$(5,323)$ & 4 & {$[2,0,2]$} & $G(48,45)$ & $C_{6} \times D_{4}$ & $(5,403)$ & 1 & {$[1,0,0]$} & $G(144,192)$ & $C_{2}^{2} \times S_{3}^{2}$ \\
\hline$(5,324)$ & 2 & {$[1,0,1]$} & $G(48,45)$ & $C_{6} \times D_{4}$ & $(5,404)$ & 5 & {$[3,0,2]$} & $G(18,5)$ & $C_{6} \times C_{3}$ \\
\hline$(5,325)$ & 2 & {$[1,0,1]$} & $G(48,44)$ & $C_{12} \times C_{2}^{2}$ & $(5,405)$ & 2 & {$[2,0,0]$} & $G(18,5)$ & $C_{6} \times C_{3}$ \\
\hline$(5,326)$ & 6 & {$[3,0,3]$} & $G(48,35)$ & $C_{2} \times C_{4} \times S_{3}$ & $(5,406)$ & 2 & {$[2,0,0]$} & $G(18,5)$ & $C_{6} \times C_{3}$ \\
\hline$(5,327)$ & 2 & {$[1,0,1]$} & $G(48,42)$ & $C_{2}^{2} \times\left(C_{3} \rtimes C_{4}\right)$ & $(5,407)$ & 4 & {$[3,0,1]$} & $G(18,3)$ & $C_{3} \times S_{3}$ \\
\hline$(5,328)$ & 4 & {$[2,0,2]$} & $G(48,35)$ & $C_{2} \times C_{4} \times S_{3}$ & $(5,408)$ & 4 & {$[3,0,1]$} & $G(18,3)$ & $C_{3} \times S_{3}$ \\
\hline$(5,329)$ & 2 & {$[2,0,0]$} & $G(48,35)$ & $C_{2} \times C_{4} \times S_{3}$ & $(5,409)$ & 9 & {$[6,0,3]$} & $G(18,4)$ & $C_{3}^{2} \rtimes C_{2}$ \\
\hline$(5,330)$ & 2 & {$[2,0,0]$} & $G(48,35)$ & $C_{2} \times C_{4} \times S_{3}$ & $(5,410)$ & 9 & {$[6,0,3]$} & $G(18,4)$ & $C_{3}^{2} \rtimes C_{2}$ \\
\hline$(5,331)$ & 2 & {$[1,0,1]$} & $G(48,35)$ & $C_{2} \times C_{4} \times S_{3}$ & $(5,411)$ & 2 & {$[1,0,1]$} & $G(18,5)$ & $C_{6} \times C_{3}$ \\
\hline$(5,332)$ & 2 & {$[1,0,1]$} & $G(48,35)$ & $C_{2} \times C_{4} \times S_{3}$ & $(5,412)$ & 2 & {$[1,0,1]$} & $G(18,5)$ & $C_{6} \times C_{3}$ \\
\hline$(5,333)$ & 4 & {$[2,0,2]$} & $G(48,35)$ & $C_{2} \times C_{4} \times S_{3}$ & $(5,413)$ & 8 & {$[6,0,2]$} & $G(18,3)$ & $C_{3} \times S_{3}$ \\
\hline$(5,334)$ & 4 & {$[2,0,2]$} & $G(48,35)$ & $C_{2} \times C_{4} \times S_{3}$ & $(5,414)$ & 8 & {$[6,0,2]$} & $G(18,3)$ & $C_{3} \times S_{3}$ \\
\hline$(5,335)$ & 6 & {$[3,0,3]$} & $G(48,36)$ & $C_{2} \times D_{12}$ & $(5,415)$ & 2 & {$[2,0,0]$} & $G(24,15)$ & $C_{6} \times C_{2}^{2}$ \\
\hline$(5,336)$ & 12 & {$[6,0,6]$} & $G(48,43)$ & $C_{2} \times\left(\left(C_{6} \times C_{2}\right) \rtimes C_{2}\right)$ & $(5,416)$ & 6 & {$[6,0,0]$} & $G(24,14)$ & $C_{2}^{2} \times S_{3}$ \\
\hline$(5,337)$ & 12 & {$[9,0,3]$} & $G(48,38)$ & $D_{4} \times S_{3}$ & $(5,417)$ & 2 & {$[2,0,0]$} & $G(24,14)$ & $C_{2}^{2} \times S_{3}$ \\
\hline$(5,338)$ & 6 & {$[3,0,3]$} & $G(48,38)$ & $D_{4} \times S_{3}$ & $(5,418)$ & 2 & {$[2,0,0]$} & $G(24,14)$ & $C_{2}^{2} \times S_{3}$ \\
\hline$(5,339)$ & 6 & {$[3,0,3]$} & $G(48,38)$ & $D_{4} \times S_{3}$ & $(5,419)$ & 4 & {$[4,0,0]$} & $G(24,14)$ & $C_{2}^{2} \times S_{3}$ \\
\hline$(5,340)$ & 12 & {$[6,0,6]$} & $G(48,38)$ & $D_{4} \times S_{3}$ & $(5,420)$ & 4 & {$[4,0,0]$} & $G(24,14)$ & $C_{2}^{2} \times S_{3}$ \\
\hline$(5,341)$ & 6 & {$[6,0,0]$} & $G(48,38)$ & $D_{4} \times S_{3}$ & $(5,421)$ & 1 & {$[1,0,0]$} & $G(288,1040)$ & $C_{2}^{3} \times S_{3}^{2}$ \\
\hline$(5,342)$ & 6 & {$[3,0,3]$} & $G(48,38)$ & $D_{4} \times S_{3}$ & $(5,422)$ & 2 & {$[2,0,0]$} & $G(36,14)$ & $C_{6} \times C_{6}$ \\
\hline$(5,343)$ & 8 & {$[4,0,4]$} & $G(48,38)$ & $D_{4} \times S_{3}$ & $(5,423)$ & 4 & {$[3,0,1]$} & $G(36,12)$ & $C_{6} \times S_{3}$ \\
\hline$(5,344)$ & 8 & {$[6,0,2]$} & $G(48,38)$ & $D_{4} \times S_{3}$ & $(5,424)$ & 9 & {$[6,0,3]$} & $G(36,13)$ & $C_{2} \times\left(C_{3}^{2} \rtimes C_{2}\right)$ \\
\hline$(5,345)$ & 4 & {$[2,0,2]$} & $G(48,38)$ & $D_{4} \times S_{3}$ & $(5,425)$ & 2 & {$[1,0,1]$} & $G(36,14)$ & $C_{6} \times C_{6}$ \\
\hline$(5,346)$ & 8 & {$[4,0,4]$} & $G(48,38)$ & $D_{4} \times S_{3}$ & $(5,426)$ & 8 & {$[6,0,2]$} & $G(36,12)$ & $C_{6} \times S_{3}$ \\
\hline$(5,347)$ & 8 & {$[4,0,4]$} & $G(48,43)$ & $C_{2} \times\left(\left(C_{6} \times C_{2}\right) \rtimes C_{2}\right)$ & $(5,427)$ & 4 & {$[4,0,0]$} & $G(36,12)$ & $C_{6} \times S_{3}$ \\
\hline$(5,348)$ & 8 & {$[4,0,4]$} & $G(48,38)$ & $D_{4} \times S_{3}$ & $(5,428)$ & 2 & {$[2,0,0]$} & $G(36,12)$ & $C_{6} \times S_{3}$ \\
\hline$(5,349)$ & 4 & {$[2,0,2]$} & $G(48,38)$ & $D_{4} \times S_{3}$ & $(5,429)$ & 2 & {$[2,0,0]$} & $G(36,12)$ & $C_{6} \times S_{3}$ \\
\hline$(5,350)$ & 8 & {$[4,0,4]$} & $G(48,43)$ & $C_{2} \times\left(\left(C_{6} \times C_{2}\right) \rtimes C_{2}\right)$ & $(5,430)$ & 3 & {$[2,0,1]$} & $G(36,12)$ & $C_{6} \times S_{3}$ \\
\hline$(5,351)$ & 4 & {$[2,0,2]$} & $G(48,36)$ & $C_{2} \times D_{12}$ & $(5,431)$ & 3 & {$[2,0,1]$} & $G(36,12)$ & $C_{6} \times S_{3}$ \\
\hline$(5,352)$ & 4 & {$[3,0,1]$} & $G(48,43)$ & $C_{2} \times\left(\left(C_{6} \times C_{2}\right) \rtimes C_{2}\right)$ & $(5,432)$ & 3 & {$[2,0,1]$} & $G(36,12)$ & $C_{6} \times S_{3}$ \\
\hline$(5,353)$ & 4 & {$[2,0,2]$} & $G(48,43)$ & $C_{2} \times\left(\left(C_{6} \times C_{2}\right) \rtimes C_{2}\right)$ & $(5,433)$ & 3 & {$[2,0,1]$} & $G(36,12)$ & $C_{6} \times S_{3}$ \\
\hline$(5,354)$ & 4 & {$[3,0,1]$} & $G(48,36)$ & $C_{2} \times D_{12}$ & $(5,434)$ & 8 & {$[6,0,2]$} & $G(36,10)$ & $S_{3}^{2}$ \\
\hline$(5,355)$ & 2 & {$[1,0,1]$} & $G(48,36)$ & $C_{2} \times D_{12}$ & $(5,435)$ & 11 & {$[9,0,2]$} & $G(36,10)$ & $S_{3}^{2}$ \\
\hline$(5,356)$ & 2 & {$[2,0,0]$} & $G(48,36)$ & $C_{2} \times D_{12}$ & $(5,436)$ & 8 & {$[6,0,2]$} & $G(36,10)$ & $S_{3}^{2}$ \\
\hline$(5,357)$ & 4 & {$[3,0,1]$} & $G(48,43)$ & $C_{2} \times\left(\left(C_{6} \times C_{2}\right) \rtimes C_{2}\right)$ & $(5,437)$ & 1 & {$[1,0,0]$} & $G(36,14)$ & $C_{6} \times C_{6}$ \\
\hline$(5,358)$ & 4 & {$[4,0,0]$} & $G(48,43)$ & $C_{2} \times\left(\left(C_{6} \times C_{2}\right) \rtimes C_{2}\right)$ & $(5,438)$ & 1 & {$[1,0,0]$} & $G(36,14)$ & $C_{6} \times C_{6}$ \\
\hline$(5,359)$ & 8 & {$[6,0,2]$} & $G(48,38)$ & $D_{4} \times S_{3}$ & $(5,439)$ & 1 & {$[1,0,0]$} & $G(36,14)$ & $C_{6} \times C_{6}$ \\
\hline$(5,360)$ & 8 & {$[4,0,4]$} & $G(48,38)$ & $D_{4} \times S_{3}$ & $(5,440)$ & 3 & {$[3,0,0]$} & $G(36,12)$ & $C_{6} \times S_{3}$ \\
\hline$(5,361)$ & 8 & {$[4,0,4]$} & $G(48,38)$ & $D_{4} \times S_{3}$ & $(5,441)$ & 3 & {$[3,0,0]$} & $G(36,12)$ & $C_{6} \times S_{3}$ \\
\hline$(5,362)$ & 4 & {$[2,0,2]$} & $G(48,36)$ & $C_{2} \times D_{12}$ & $(5,442)$ & 3 & {$[3,0,0]$} & $G(36,12)$ & $C_{6} \times S_{3}$ \\
\hline$(5,363)$ & 4 & {$[2,0,2]$} & $G(48,36)$ & $C_{2} \times D_{12}$ & $(5,443)$ & 3 & {$[3,0,0]$} & $G(36,12)$ & $C_{6} \times S_{3}$ \\
\hline$(5,364)$ & 8 & {$[4,0,4]$} & $G(48,43)$ & $C_{2} \times\left(\left(C_{6} \times C_{2}\right) \rtimes C_{2}\right)$ & $(5,444)$ & 7 & {$[6,0,1]$} & $G(36,10)$ & $S_{3}^{2}$ \\
\hline$(5,365)$ & 8 & {$[6,0,2]$} & $G(48,38)$ & $D_{4} \times S_{3}$ & $(5,445)$ & 7 & {$[6,0,1]$} & $G(36,10)$ & $S_{3}^{2}$ \\
\hline$(5,366)$ & 8 & {$[8,0,0]$} & $G(48,38)$ & $D_{4} \times S_{3}$ & $(5,446)$ & 7 & {$[6,0,1]$} & $G(36,10)$ & $S_{3}^{2}$ \\
\hline$(5,367)$ & 4 & {$[4,0,0]$} & $G(48,38)$ & $D_{4} \times S_{3}$ & $(5,447)$ & 7 & {$[6,0,1]$} & $G(36,10)$ & $S_{3}^{2}$ \\
\hline$(5,368)$ & 4 & {$[2,0,2]$} & $G(48,38)$ & $D_{4} \times S_{3}$ & $(5,448)$ & 3 & {$[2,0,1]$} & $G(36,13)$ & $C_{2} \times\left(C_{3}^{2} \rtimes C_{2}\right)$ \\
\hline$(5,369)$ & 8 & {$[4,0,4]$} & $G(48,38)$ & $D_{4} \times S_{3}$ & $(5,449)$ & 3 & {$[2,0,1]$} & $G(36,13)$ & $C_{2} \times\left(C_{3}^{2} \rtimes C_{2}\right)$ \\
\hline$(5,370)$ & 2 & {$[1,0,1]$} & $G(96,221)$ & $C_{2} \times C_{6} \times D_{4}$ & $(5,450)$ & 5 & {$[3,0,2]$} & $G(36,13)$ & $C_{2} \times\left(C_{3}^{2} \rtimes C_{2}\right)$ \\
\hline$(5,371)$ & 2 & {$[1,0,1]$} & $G(96,206)$ & $C_{2}^{2} \times C_{4} \times S_{3}$ & $(5,451)$ & 2 & {$[2,0,0]$} & $G(36,12)$ & $C_{6} \times S_{3}$ \\
\hline$(5,372)$ & 6 & {$[3,0,3]$} & $G(96,209)$ & $C_{2} \times S_{3} \times D_{4}$ & $(5,452)$ & 1 & {$[1,0,0]$} & $G(36,12)$ & $C_{6} \times S_{3}$ \\
\hline$(5,373)$ & 8 & {$[4,0,4]$} & $G(96,209)$ & $C_{2} \times D_{4} \times S_{3}$ & $(5,453)$ & 1 & {$[1,0,0]$} & $G(36,12)$ & $C_{6} \times S_{3}$ \\
\hline$(5,374)$ & 4 & {$[2,0,2]$} & $G(96,209)$ & $C_{2} \times S_{3} \times D_{4}$ & $(5,454)$ & 3 & {$[3,0,0]$} & $G(36,13)$ & $C_{2} \times\left(C_{3}^{2} \rtimes C_{2}\right)$ \\
\hline$(5,375)$ & 2 & {$[2,0,0]$} & $G(96,209)$ & $C_{2} \times S_{3} \times D_{4}$ & $(5,455)$ & 3 & {$[3,0,0]$} & $G(36,13)$ & $C_{2} \times\left(C_{3}^{2} \rtimes C_{2}\right)$ \\
\hline$(5,376)$ & 4 & {$[3,0,1]$} & $G(96,209)$ & $C_{2} \times S_{3} \times D_{4}$ & $(5,456)$ & 6 & {$[6,0,0]$} & $G(36,13)$ & $C_{2} \times\left(C_{3}^{2} \rtimes C_{2}\right)$ \\
\hline$(5,377)$ & 4 & {$[2,0,2]$} & $G(96,209)$ & $C_{2} \times S_{3} \times D_{4}$ & $(5,457)$ & 5 & {$[4,0,1]$} & $G(36,10)$ & $S_{3}^{2}$ \\
\hline$(5,378)$ & 4 & {$[2,0,2]$} & $G(96,219)$ & $C_{2}^{2} \times\left(\left(C_{6} \times C_{2}\right) \rtimes C_{2}\right)$ & $(5,458)$ & 4 & {$[3,0,1]$} & $G(36,10)$ & $S_{3}^{2}$ \\
\hline$(5,379)$ & 2 & {$[1,0,1]$} & $G(96,209)$ & $C_{2} \times S_{3} \times D_{4}$ & $(5,459)$ & 4 & {$[3,0,1]$} & $G(36,10)$ & $S_{3}^{2}$ \\
\hline$(5,380)$ & 8 & {$[4,0,4]$} & $G(96,209)$ & $C_{2} \times S_{3} \times D_{4}$ & $(5,460)$ & 2 & {$[2,0,0]$} & $G(48,51)$ & $C_{2}^{3} \times S_{3}$ \\
\hline$(5,381)$ & 4 & {$[2,0,2]$} & $G(96,209)$ & $C_{2} \times S_{3} \times D_{4}$ & $(5,461)$ & 4 & {$[4,0,0]$} & $G(6,2)$ & $C_{6}$ \\
\hline$(5,382)$ & 2 & {$[1,0,1]$} & $G(96,209)$ & $C_{2} \times S_{3} \times D_{4}$ & $(5,462)$ & 4 & {$[4,0,0]$} & $G(6,2)$ & $C_{6}$ \\
\hline$(5,383)$ & 2 & {$[1,0,1]$} & $G(96,207)$ & $C_{2}^{2} \times D_{12}$ & $(5,463)$ & 2 & {$[2,0,0]$} & $G(72,48)$ & $C_{2} \times C_{6} \times S_{3}$ \\
\hline$(5,384)$ & 4 & {$[2,0,2]$} & $G(96,209)$ & $C_{2} \times D_{4} \times S_{3}$ & $(5,464)$ & 3 & {$[2,0,1]$} & $G(72,48)$ & $C_{2} \times C_{6} \times S_{3}$ \\
\hline$(5,385)$ & 2 & {$[1,0,1]$} & $G(96,209)$ & $C_{2} \times S_{3} \times D_{4}$ & $(5,465)$ & 8 & {$[6,0,2]$} & $G(72,46)$ & $C_{2} \times S_{3}^{2}$ \\
\hline$(5,386)$ & 2 & {$[2,0,0]$} & $G(12,5)$ & $C_{6} \times C_{2}$ & $(5,466)$ & 1 & {$[1,0,0]$} & $G(72,50)$ & $C_{6} \times C_{6} \times C_{2}$ \\
\hline$(5,387)$ & 2 & {$[2,0,0]$} & $G(12,5)$ & $C_{6} \times C_{2}$ & $(5,467)$ & 3 & {$[3,0,0]$} & $G(72,48)$ & $C_{2} \times C_{6} \times S_{3}$ \\
\hline$(5,388)$ & 2 & {$[2,0,0]$} & $G(12,5)$ & $C_{6} \times C_{2}$ & $(5,468)$ & 7 & {$[6,0,1]$} & $G(72,46)$ & $C_{2} \times S_{3}^{2}$ \\
\hline$(5,389)$ & 6 & {$[6,0,0]$} & $G(12,4)$ & $D_{12}$ & $(5,469)$ & 3 & {$[2,0,1]$} & $G(72,49)$ & $C_{2}^{2} \times\left(C_{3}^{2} \rtimes C_{2}\right)$ \\
\hline$(5,390)$ & 6 & {$[6,0,0]$} & $G(12,4)$ & $D_{12}$ & $(5,470)$ & 1 & {$[1,0,0]$} & $G(72,48)$ & $C_{2} \times C_{6} \times S_{3}$ \\
\hline$(5,391)$ & 12 & {$[12,0,0]$} & $G(12,4)$ & $D_{12}$ & $(5,471)$ & 1 & {$[1,0,0]$} & $G(72,48)$ & $C_{2} \times C_{6} \times S_{3}$ \\
\hline$(5,392)$ & 4 & {$[4,0,0]$} & $G(12,5)$ & $C_{6} \times C_{2}$ & $(5,472)$ & 2 & {$[2,0,0]$} & $G(72,48)$ & $C_{2} \times C_{6} \times S_{3}$ \\
\hline$(5,393)$ & 1 & {$[1,0,0]$} & $G(144,195)$ & $C_{2}^{2} \times C_{6} \times S_{3}$ & $(5,473)$ & 1 & {$[1,0,0]$} & $G(72,48)$ & $C_{2} \times C_{6} \times S_{3}$ \\
\hline$(5,394)$ & 3 & {$[3,0,0]$} & $G(144,192)$ & $C_{2}^{2} \times S_{3}^{2}$ & $(5,474)$ & 1 & {$[1,0,0]$} & $G(72,48)$ & $C_{2} \times C_{6} \times S_{3}$ \\
\hline$(5,395)$ & 4 & {$[3,0,1]$} & $G(144,192)$ & $C_{2}^{2} \times S_{3}^{2}$ & $(5,475)$ & 1 & {$[1,0,0]$} & $G(72,48)$ & $C_{2} \times C_{6} \times S_{3}$ \\
\hline$(5,396)$ & 1 & {$[1,0,0]$} & $G(144,196)$ & $C_{2}^{3} \times\left(C_{3}^{2} \rtimes C_{2}\right)$ & $(5,476)$ & 2 & {$[2,0,0]$} & $G(72,48)$ & $C_{2} \times C_{6} \times S_{3}$ \\
\hline$(5,397)$ & 1 & {$[1,0,0]$} & $G(144,192)$ & $C_{2}^{2} \times S_{3}^{2}$ & $(5,477)$ & 3 & {$[3,0,0]$} & $G(72,49)$ & $C_{2}^{2} \times\left(C_{3}^{2} \rtimes C_{2}\right)$ \\
\hline$(5,398)$ & 1 & {$[1,0,0]$} & $G(144,192)$ & $C_{2}^{2} \times S_{3}^{2}$ & $(5,478)$ & 3 & {$[3,0,0]$} & $G(72,46)$ & $C_{2} \times S_{3}^{2}$ \\
\hline$(5,399)$ & 3 & {$[3,0,0]$} & $G(144,192)$ & $C_{2}^{2} \times S_{3}^{2}$ & $(5,479)$ & 6 & {$[6,0,0]$} & $G(72,46)$ & $C_{2} \times S_{3}^{2}$ \\
\hline$(5,400)$ & 2 & {$[2,0,0]$} & $G(144,192)$ & $C_{2}^{2} \times S_{3}^{2}$ & $(5,480)$ & 6 & {$[6,0,0]$} & $G(72,46)$ & $C_{2} \times S_{3}^{2}$ \\
\hline
\end{tabular}


Table 16 (continued): stably rational classification of algebraic $k$-tori of dimension 5

\begin{tabular}{|c|c|c|c|c|c|c|c|c|c|}
\hline CARAT & $\#$ & {$[s, r, u]$} & $G(n, i)$ & & CARAT & $\#$ & {$[s, r, u]$} & $G(n, i)$ & \\
\hline$(5,481)$ & 3 & {$[3,0,0]$} & $G(72,46)$ & $C_{2} \times S_{3}^{2}$ & $(5,561)$ & 5 & {$[1,0,4]$} & $G(48,31)$ & $C_{4} \times A_{4}$ \\
\hline$(5,482)$ & 3 & {$[3,0,0]$} & $G(72,46)$ & $C_{2} \times S_{3}^{2}$ & $(5,562)$ & 5 & {$[1,0,4]$} & $G(48,30)$ & $A_{4} \rtimes C_{4}$ \\
\hline$(5,483)$ & 3 & {$[3,0,0]$} & $G(72,46)$ & $C_{2} \times S_{3}^{2}$ & $(5,563)$ & 5 & {$[2,0,3]$} & $G(48,30)$ & $A_{4} \rtimes C_{4}$ \\
\hline$(5,484)$ & 5 & {$[4,0,1]$} & $G(72,46)$ & $C_{2} \times S_{3}^{2}$ & $(5,564)$ & 5 & {$[1,0,4]$} & $G(96,197)$ & $D_{4} \times A_{4}$ \\
\hline$(5,485)$ & 4 & {$[3,0,1]$} & $G(72,46)$ & $C_{2} \times S_{3}^{2}$ & $(5,565)$ & 10 & {$[2,0,8]$} & $G(96,197)$ & $D_{4} \times A_{4}$ \\
\hline$(5,486)$ & 4 & {$[3,0,1]$} & $G(72,46)$ & $C_{2} \times S_{3}^{2}$ & $(5,566)$ & 5 & {$[2,0,3]$} & $G(96,197)$ & $A_{4} \times D_{4}$ \\
\hline$(5,487)$ & 4 & {$[3,0,1]$} & $G(72,46)$ & $C_{2} \times S_{3}^{2}$ & $(5,567)$ & 5 & {$[1,0,4]$} & $G(96,196)$ & $C_{2} \times C_{4} \times A_{4}$ \\
\hline$(5,488)$ & 5 & {$[4,0,1]$} & $G(72,46)$ & $C_{2} \times S_{3}^{2}$ & $(5,568)$ & 10 & {$[2,0,8]$} & $G(96,195)$ & $\left(C_{2}^{2} \times A_{4}\right) \rtimes C_{2}$ \\
\hline$(5,489)$ & 4 & {$[3,0,1]$} & $G(72,46)$ & $C_{2} \times S_{3}^{2}$ & $(5,569)$ & 10 & {$[2,0,8]$} & $G(96,195)$ & $\left(C_{2}^{2} \times A_{4}\right) \rtimes C_{2}$ \\
\hline$(5,490)$ & 4 & {$[3,0,1]$} & $G(72,46)$ & $C_{2} \times S_{3}^{2}$ & $(5,570)$ & 10 & {$[2,0,8]$} & $G(96,195)$ & $\left(C_{2}^{2} \times A_{4}\right) \rtimes C_{2}$ \\
\hline$(5,491)$ & 2 & {$[2,0,0]$} & $G(72,49)$ & $C_{2}^{2} \times\left(C_{3}^{2} \rtimes C_{2}\right)$ & $(5,571)$ & 10 & {$[4,0,6]$} & $G(96,195)$ & $\left(C_{2}^{\overline{2}} \times A_{4}\right) \rtimes C_{2}$ \\
\hline$(5,492)$ & 1 & {$[1,0,0]$} & $G(72,49)$ & $C_{2}^{2} \times\left(C_{3}^{2} \rtimes C_{2}\right)$ & $(5,572)$ & 5 & {$[2,0,3]$} & $G(96,187)$ & $\left(C_{2} \times S_{4}\right) \rtimes C_{2}$ \\
\hline$(5,493)$ & 1 & {$[1,0,0]$} & $G(72,49)$ & $C_{2}^{2} \times\left(C_{3}^{2} \rtimes C_{2}\right)$ & $(5,573)$ & 5 & {$[1,0,4]$} & $G(96,187)$ & $\left(C_{2} \times S_{4}\right) \rtimes C_{2}$ \\
\hline$(5,494)$ & 2 & {$[2,0,0]$} & $G(72,46)$ & $C_{2} \times S_{3}^{2}$ & $(5,574)$ & 10 & {$[2,0,8]$} & $G(96,187)$ & $\left(C_{2} \times S_{4}\right) \rtimes C_{2}$ \\
\hline$(5,495)$ & 2 & {$[2,0,0]$} & $G(72,46)$ & $C_{2} \times S_{3}^{2}$ & $(5,575)$ & 5 & {$[1,0,4]$} & $G(96,186)$ & $C_{4} \times S_{4}$ \\
\hline$(5,496)$ & 2 & {$[2,0,0]$} & $G(72,46)$ & $C_{2} \times S_{3}^{2}$ & $(5,576)$ & 5 & {$[2,0,3]$} & $G(96,186)$ & $C_{4} \times S_{4}$ \\
\hline$(5,497)$ & 2 & {$[2,0,0]$} & $G(72,46)$ & $C_{2} \times S_{3}^{2}$ & $(5,577)$ & 5 & {$[1,0,4]$} & $G(96,186)$ & $C_{4} \times S_{4}$ \\
\hline$(5,498)$ & 4 & {$[4,0,0]$} & $G(72,46)$ & $C_{2} \times S_{3}^{2}$ & $(5,578)$ & 5 & {$[1,0,4]$} & $G(96,186)$ & $C_{4} \times S_{4}$ \\
\hline$(5,499)$ & 4 & {$[4,0,0]$} & $G(72,46)$ & $C_{2} \times S_{3}^{2}$ & $(5,579)$ & 5 & {$[1,0,4]$} & $G(96,194)$ & $C_{2} \times\left(A_{4} \rtimes C_{4}\right)$ \\
\hline$(5,500)$ & 2 & {$[2,0,0]$} & $G(72,49)$ & $C_{2}^{2} \times\left(C_{3}^{2} \rtimes C_{2}\right)$ & $(5,580)$ & 5 & {$[3,0,2]$} & $G(12,3)$ & $A_{4}$ \\
\hline$(5,501)$ & 5 & {$[3,0,2]$} & $G(9,2)$ & $C_{3} \times C_{3}$ & $(5,581)$ & 3 & {$[1,0,2]$} & $G(144,193)$ & $C_{2} \times C_{6} \times A_{4}$ \\
\hline$(5,502)$ & 6 & {$[5,0,1]$} & $G(12,3)$ & $A_{4}$ & $(5,582)$ & 3 & {$[1,0,2]$} & $G(144,188)$ & $C_{6} \times S_{4}$ \\
\hline$(5,503)$ & 6 & {$[2,0,4]$} & $G(24,13)$ & $C_{2} \times A_{4}$ & $(5,583)$ & 3 & {$[1,0,2]$} & $G(144,188)$ & $C_{6} \times S_{4}$ \\
\hline$(5,504)$ & 5 & {$[2,0,3]$} & $G(24,13)$ & $C_{2} \times A_{4}$ & $(5,584)$ & 3 & {$[1,0,2]$} & $G(144,188)$ & $C_{6} \times S_{4}$ \\
\hline$(5,505)$ & 5 & {$[2,0,3]$} & $G(24,13)$ & $C_{2} \times A_{4}$ & $(5,585)$ & 3 & {$[1,0,2]$} & $G(144,188)$ & $C_{6} \times S_{4}$ \\
\hline$(5,506)$ & 6 & {$[5,0,1]$} & $G(24,12)$ & $S_{4}$ & $(5,586)$ & 3 & {$[2,0,1]$} & $G(144,188)$ & $C_{6} \times S_{4}$ \\
\hline$(5,507)$ & 6 & {$[3,0,3]$} & $G(24,12)$ & $S_{4}$ & $(5,587)$ & 3 & {$[1,0,2]$} & $G(144,188)$ & $C_{6} \times S_{4}$ \\
\hline$(5,508)$ & 5 & {$[2,0,3]$} & $G(24,12)$ & $S_{4}$ & $(5,588)$ & 3 & {$[1,0,2]$} & $G(144,190)$ & $C_{2} \times A_{4} \times S_{3}$ \\
\hline$(5,509)$ & 5 & {$[3,0,2]$} & $G(24,12)$ & $S_{4}$ & $(5,589)$ & 6 & {$[2,0,4]$} & $G(144,190)$ & $C_{2} \times A_{4} \times S_{3}$ \\
\hline$(5,510)$ & 5 & {$[1,0,4]$} & $G(48,49)$ & $C_{2}^{2} \times A_{4}$ & $(5,590)$ & 3 & {$[2,0,1]$} & $G(144,190)$ & $C_{2} \times A_{4} \times S_{3}$ \\
\hline$(5,511)$ & 6 & {$[2,0,4]$} & $G(48,48)$ & $C_{2} \times S_{4}$ & $(5,591)$ & 6 & {$[2,0,4]$} & $G(144,190)$ & $C_{2} \times A_{4} \times S_{3}$ \\
\hline$(5,512)$ & 5 & {$[2,0,3]$} & $G(48,48)$ & $C_{2} \times S_{4}$ & $(5,592)$ & 6 & {$[2,0,4]$} & $G(144,183)$ & $S_{4} \times S_{3}$ \\
\hline$(5,513)$ & 5 & {$[2,0,3]$} & $G(48,48)$ & $C_{2} \times S_{4}$ & $(5,593)$ & 6 & {$[2,0,4]$} & $G(144,183)$ & $S_{4} \times S_{3}$ \\
\hline$(5,514)$ & 5 & {$[1,0,4]$} & $G(48,48)$ & $C_{2} \times S_{4}$ & $(5,594)$ & 6 & {$[2,0,4]$} & $G(144,189)$ & $C_{2} \times\left(\left(C_{3} \times A_{4}\right) \rtimes C_{2}\right)$ \\
\hline$(5,515)$ & 5 & {$[1,0,4]$} & $G(48,48)$ & $C_{2} \times S_{4}$ & $(5,595)$ & 6 & {$[2,0,4]$} & $G(144,183)$ & $S_{4} \times S_{3}$ \\
\hline$(5,516)$ & 5 & {$[2,0,3]$} & $G(48,48)$ & $C_{2} \times S_{4}$ & $(5,596)$ & 6 & {$[4,0,2]$} & $G(144,183)$ & $S_{4} \times S_{3}$ \\
\hline$(5,517)$ & 5 & {$[1,0,4]$} & $G(96,226)$ & $C_{2}^{2} \times S_{4}$ & $(5,597)$ & 6 & {$[2,0,4]$} & $G(144,183)$ & $S_{4} \times S_{3}$ \\
\hline$(5,518)$ & 12 & {$[2,0,10]$} & $G(192,1537)$ & $C_{2}^{3} \times S_{4}$ & $(5,598)$ & 6 & {$[4,0,2]$} & $G(144,183)$ & $S_{4} \times S_{3}$ \\
\hline$(5,519)$ & 17 & {$[6,0,11]$} & $G(24,13)$ & $C_{2} \times A_{4}$ & $(5,599)$ & 6 & {$[2,0,4]$} & $G(144,183)$ & $S_{4} \times S_{3}$ \\
\hline$(5,520)$ & 17 & {$[10,0,7]$} & $G(24,13)$ & $C_{2} \times A_{4}$ & $(5,600)$ & 6 & {$[2,0,4]$} & $G(144,189)$ & $C_{2} \times\left(\left(C_{3} \times A_{4}\right) \rtimes C_{2}\right)$ \\
\hline$(5,521)$ & 17 & {$[12,0,5]$} & $G(24,12)$ & $S_{4}$ & $(5,601)$ & 3 & {$[2,0,1]$} & $G(144,189)$ & $C_{2} \times\left(\left(C_{3} \times A_{4}\right) \rtimes C_{2}\right)$ \\
\hline$(5,522)$ & 17 & {$[8,0,9]$} & $G(24,12)$ & $S_{4}$ & $(5,602)$ & 3 & {$[1,0,2]$} & $G(144,189)$ & $C_{2} \times\left(\left(C_{3} \times A_{4}\right) \rtimes C_{2}\right)$ \\
\hline$(5,523)$ & 17 & {$[3,0,14]$} & $G(48,49)$ & $C_{2}^{2} \times A_{4}$ & $(5,603)$ & 6 & {$[2,0,4]$} & $G(144,183)$ & $S_{4} \times S_{3}$ \\
\hline$(5,524)$ & 14 & {$[4,0,10]$} & $G(48,49)$ & $C_{2}^{2} \times A_{4}$ & $(5,604)$ & 5 & {$[1,0,4]$} & $G(24,13)$ & $C_{2} \times A_{4}$ \\
\hline$(5,525)$ & 12 & {$[4,0,8]$} & $G(48,49)$ & $C_{2}^{2} \times A_{4}$ & $(5,605)$ & 5 & {$[2,0,3]$} & $G(24,13)$ & $C_{2} \times A_{4}$ \\
\hline$(5,526)$ & 12 & {$[2,0,10]$} & $G(48,49)$ & $C_{2}^{2} \times A_{4}$ & $(5,606)$ & 5 & {$[2,0,3]$} & $G(24,13)$ & $C_{2} \times A_{4}$ \\
\hline$(5,527)$ & 17 & {$[3,0,14]$} & $G(48,48)$ & $C_{2} \times S_{4}$ & $(5,607)$ & 10 & {$[6,0,4]$} & $G(24,12)$ & $S_{4}$ \\
\hline$(5,528)$ & 17 & {$[4,0,13]$} & $G(48,48)$ & $C_{2} \times S_{4}$ & $(5,608)$ & 10 & {$[4,0,6]$} & $G(24,12)$ & $S_{4}$ \\
\hline$(5,529)$ & 17 & {$[6,0,11]$} & $G(48,48)$ & $C_{2} \times S_{4}$ & $(5,609)$ & 3 & {$[1,0,2]$} & $G(288,1033)$ & $C_{2} \times C_{6} \times S_{4}$ \\
\hline$(5,530)$ & 17 & {$[4,0,13]$} & $G(48,48)$ & $C_{2} \times S_{4}$ & $(5,610)$ & 3 & {$[1,0,2]$} & $G(288,1037)$ & $C_{2}^{2} \times A_{4} \times S_{3}$ \\
\hline$(5,531)$ & 17 & {$[10,0,7]$} & $G(48,48)$ & $C_{2} \times S_{4}$ & $(5,611)$ & 3 & {$[1,0,2]$} & $G(288,1034)$ & $C_{2}^{2} \times\left(\left(C_{3} \times A_{4}\right) \rtimes C_{2}\right)$ \\
\hline$(5,532)$ & 12 & {$[4,0,8]$} & $G(48,48)$ & $C_{2} \times S_{4}$ & $(5,612)$ & 6 & {$[2,0,4]$} & $G(288,1028)$ & $C_{2} \times S_{4} \times S_{3}$ \\
\hline$(5,533)$ & 14 & {$[6,0,8]$} & $G(48,48)$ & $C_{2} \times S_{4}$ & $(5,613)$ & 6 & {$[2,0,4]$} & $G(288,1028)$ & $C_{2} \times S_{4} \times S_{3}$ \\
\hline$(5,534)$ & 12 & {$[2,0,10]$} & $G(48,48)$ & $C_{2} \times S_{4}$ & $(5,614)$ & 3 & {$[1,0,2]$} & $G(288,1028)$ & $C_{2} \times S_{4} \times S_{3}$ \\
\hline$(5,535)$ & 14 & {$[5,0,9]$} & $G(48,48)$ & $C_{2} \times S_{4}$ & $(5,615)$ & 3 & {$[2,0,1]$} & $G(288,1028)$ & $C_{2} \times S_{4} \times S_{3}$ \\
\hline$(5,536)$ & 14 & {$[3,0,11]$} & $G(48,48)$ & $C_{2} \times S_{4}$ & $(5,616)$ & 3 & {$[1,0,2]$} & $G(288,1028)$ & $C_{2} \times S_{4} \times S_{3}$ \\
\hline$(5,537)$ & 14 & {$[4,0,10]$} & $G(48,48)$ & $C_{2} \times S_{4}$ & $(5,617)$ & 6 & {$[2,0,4]$} & $G(288,1028)$ & $C_{2} \times S_{4} \times S_{3}$ \\
\hline$(5,538)$ & 14 & {$[4,0,10]$} & $G(48,48)$ & $C_{2} \times S_{4}$ & $(5,618)$ & 6 & {$[2,0,4]$} & $G(288,1028)$ & $C_{2} \times S_{4} \times S_{3}$ \\
\hline$(5,539)$ & 12 & {$[2,0,10]$} & $G(96,228)$ & $C_{2}^{3} \times A_{4}$ & $(5,619)$ & 3 & {$[1,0,2]$} & $G(288,1028)$ & $C_{2} \times S_{4} \times S_{3}$ \\
\hline$(5,540)$ & 17 & {$[3,0,14]$} & $G(96,226)$ & $C_{2}^{2} \times S_{4}$ & $(5,620)$ & 3 & {$[2,0,1]$} & $G(36,11)$ & $C_{3} \times A_{4}$ \\
\hline$(5,541)$ & 14 & {$[4,0,10]$} & $G(96,226)$ & $C_{2}^{2} \times S_{4}$ & $(5,621)$ & 5 & {$[1,0,4]$} & $G(48,49)$ & $C_{2}^{2} \times A_{4}$ \\
\hline$(5,542)$ & 14 & {$[2,0,12]$} & $G(96,226)$ & $C_{2}^{2} \times S_{4}$ & $(5,622)$ & 5 & {$[1,0,4]$} & $G(48,48)$ & $C_{2} \times S_{4}$ \\
\hline$(5,543)$ & 12 & {$[4,0,8]$} & $G(96,226)$ & $C_{2}^{2} \times S_{4}$ & $(5,623)$ & 10 & {$[4,0,6]$} & $G(48,48)$ & $C_{2} \times S_{4}$ \\
\hline$(5,544)$ & 12 & {$[2,0,10]$} & $G(96,226)$ & $C_{2}^{2} \times S_{4}$ & $(5,624)$ & 10 & {$[2,0,8]$} & $G(48,48)$ & $C_{2} \times S_{4}$ \\
\hline$(5,545)$ & 12 & {$[2,0,10]$} & $G(96,226)$ & $C_{2}^{2} \times S_{4}$ & $(5,625)$ & 5 & {$[2,0,3]$} & $G(48,48)$ & $C_{2} \times S_{4}$ \\
\hline$(5,546)$ & 12 & {$[2,0,10]$} & $G(96,226)$ & $C_{2}^{2} \times S_{4}$ & $(5,626)$ & 3 & {$[1,0,2]$} & $G(576,8659)$ & $C_{2}^{2} \times S_{4} \times S_{3}$ \\
\hline$(5,547)$ & 12 & {$[2,0,10]$} & $G(96,226)$ & $C_{2}^{2} \times S_{4}$ & $(5,627)$ & 3 & {$[1,0,2]$} & $G(72,47)$ & $C_{6} \times A_{4}$ \\
\hline$(5,548)$ & 14 & {$[3,0,11]$} & $G(96,226)$ & $C_{2}^{2} \times S_{4}$ & $(5,628)$ & 3 & {$[1,0,2]$} & $G(72,47)$ & $C_{6} \times A_{4}$ \\
\hline$(5,549)$ & 5 & {$[1,0,4]$} & $G(192,1497)$ & $C_{2} \times D_{4} \times A_{4}$ & $(5,629)$ & 3 & {$[2,0,1]$} & $G(72,47)$ & $C_{6} \times A_{4}$ \\
\hline$(5,550)$ & 10 & {$[2,0,8]$} & $G(192,1488)$ & $C_{2} \times\left(\left(C_{2}^{2} \times A_{4}\right) \rtimes C_{2}\right)$ & $(5,630)$ & 3 & {$[1,0,2]$} & $G(72,42)$ & $C_{3} \times S_{4}$ \\
\hline$(5,551)$ & 10 & {$[2,0,8]$} & $G(192,1472)$ & $D_{4} \times S_{4}$ & $(5,631)$ & 3 & {$[1,0,2]$} & $G(72,42)$ & $C_{3} \times S_{4}$ \\
\hline$(5,552)$ & 10 & {$[2,0,8]$} & $G(192,1472)$ & $D_{4} \times S_{4}$ & $(5,632)$ & 3 & {$[2,0,1]$} & $G(72,42)$ & $C_{3} \times S_{4}$ \\
\hline$(5,553)$ & 5 & {$[1,0,4]$} & $G(192,1472)$ & $D_{4} \times S_{4}$ & $(5,633)$ & 3 & {$[2,0,1]$} & $G(72,42)$ & $C_{3} \times S_{4}$ \\
\hline$(5,554)$ & 5 & {$[1,0,4]$} & $G(192,1472)$ & $D_{4} \times S_{4}$ & $(5,634)$ & 6 & {$[4,0,2]$} & $G(72,44)$ & $A_{4} \times S_{3}$ \\
\hline$(5,555)$ & 5 & {$[2,0,3]$} & $G(192,1472)$ & $S_{4} \times D_{4}$ & $(5,635)$ & 6 & {$[2,0,4]$} & $G(72,44)$ & $A_{4} \times S_{3}$ \\
\hline$(5,556)$ & 5 & {$[1,0,4]$} & $G(192,1472)$ & $S_{4} \times D_{4}$ & $(5,636)$ & 6 & {$[2,0,4]$} & $G(72,43)$ & $\left(C_{3} \times A_{4}\right) \rtimes C_{2}$ \\
\hline$(5,557)$ & 5 & {$[1,0,4]$} & $G(192,1470)$ & $C_{2} \times\left(\left(C_{2} \times S_{4}\right) \rtimes C_{2}\right)$ & $(5,637)$ & 6 & {$[4,0,2]$} & $G(72,43)$ & $\left(C_{3} \times A_{4}\right) \rtimes C_{2}$ \\
\hline$(5,558)$ & 5 & {$[1,0,4]$} & $G(192,1469)$ & $C_{2} \times C_{4} \times S_{4}$ & $(5,638)$ & 5 & {$[1,0,4]$} & $G(96,226)$ & $C_{2}^{2} \times S_{4}$ \\
\hline$(5,559)$ & 5 & {$[1,0,4]$} & $G(384,20051)$ & $C_{2} \times D_{4} \times S_{4}$ & $(5,639)$ & 2 & {$[1,0,1]$} & $G(16,5)$ & $C_{8} \times C_{2}$ \\
\hline$(5,560)$ & 5 & {$[2,0,3]$} & $G(48,31)$ & $C_{4} \times A_{4}$ & $(5,640)$ & 2 & {$[2,0,0]$} & $G(16,7)$ & $D_{8}$ \\
\hline
\end{tabular}


Table 16 (continued): stably rational classification of algebraic $k$-tori of dimension 5

\begin{tabular}{|c|c|c|c|c|c|c|c|c|c|}
\hline CARAT & \# & {$[s, r, u]$} & $G(n, i)$ & & CARAT & \# & {$[s, r, u]$} & $G(n, i)$ & \\
\hline$(5,641)$ & 4 & {$[3,0,1]$} & $G(16,7)$ & $D_{8}$ & $(5,721)$ & 4 & {$[1,0,3]$} & $G(32,40)$ & $C_{2} \times Q D_{8}$ \\
\hline$(5,642)$ & 2 & {$[1,0,1]$} & $G(16,7)$ & $D_{8}$ & $(5,722)$ & 1 & {$[0,0,1]$} & $G(384,18101)$ & $C_{2} \times\left(\left(\operatorname{SL}(2,3) \rtimes C_{4}\right) \rtimes C_{2}\right)$ \\
\hline$(5,643)$ & 2 & {$[1,0,1]$} & $G(32,39)$ & $C_{2} \times D_{8}$ & $(5,723)$ & 5 & {$[1,0,4]$} & $G(384,5833)$ & \\
\hline$(5,644)$ & 2 & {$[2,0,0]$} & $G(8,1)$ & $C_{8}$ & $(5,724)$ & 5 & {$[1,0,4]$} & $G(384,5602)$ & \\
\hline$(5,645)$ & 2 & {$[2,0,0]$} & $G(8,1)$ & $C_{8}$ & $(5,725)$ & 5 & {$[1,0,4]$} & $G(384,5602)$ & \\
\hline$(5,646)$ & 2 & {$[0,0,2]$} & $G(1152,157511)$ & & $(5,726)$ & 5 & {$[2,0,3]$} & $G(384,5602)$ & \\
\hline$(5,647)$ & 2 & {$[0,0,2]$} & $G(1152,157478)$ & & $(5,727)$ & 5 & {$[1,0,4]$} & $G(384,20090)$ & \\
\hline$(5,648)$ & 1 & {$[0,0,1]$} & $G(1152,157478)$ & & $(5,728)$ & 5 & {$[1,0,4]$} & $G(384,5602)$ & \\
\hline$(5,649)$ & 1 & {$[0,0,1]$} & $G(1152,157528)$ & & $(5,729)$ & 5 & {$[1,0,4]$} & $G(384,20089)$ & \\
\hline$(5,650)$ & 1 & {$[0,0,1]$} & $G(1152,157478)$ & & $(5,730)$ & 1 & {$[0,0,1]$} & $G(48,32)$ & $C_{2} \times \mathrm{SL}(2,3)$ \\
\hline$(5,651)$ & 8 & {$[2,0,6]$} & $G(128,928)$ & $D_{4}^{2} \rtimes C_{2}$ & $(5,731)$ & 1 & {$[0,0,1]$} & $G(48,33)$ & $\mathrm{SL}(2,3) \rtimes C_{2}$ \\
\hline$(5,652)$ & 4 & {$[1,0,3]$} & $G(128,928)$ & $D_{4}^{2} \rtimes C_{2}$ & $(5,732)$ & 1 & {$[0,0,1]$} & $G(48,33)$ & $\mathrm{SL}(2,3) \rtimes C_{2}$ \\
\hline$(5,653)$ & 8 & {$[2,0,6]$} & $G(128,928)$ & $D_{4}^{2} \rtimes C_{2}$ & $(5,733)$ & 1 & {$[0,0,1]$} & $G(48,29)$ & $\mathrm{GL}(2,3)$ \\
\hline$(5,654)$ & 4 & {$[1,0,3]$} & $G(128,928)$ & $D_{4}^{2} \rtimes C_{2}$ & $(5,734)$ & 1 & {$[0,0,1]$} & $G(48,29)$ & $\mathrm{GL}(2,3)$ \\
\hline$(5,655)$ & 4 & {$[2,0,2]$} & $G(128,928)$ & $D_{4}^{2} \rtimes C_{2}$ & $(5,735)$ & 1 & {$[0,0,1]$} & $G(48,46)$ & $C_{6} \times Q_{8}$ \\
\hline$(5,656)$ & 4 & {$[2,0,2]$} & $G(128,928)$ & $D_{4}^{2} \rtimes C_{2}$ & $(5,736)$ & 1 & {$[0,1,0]$} & $G(48,9)$ & $C_{2} \times\left(C_{3} \rtimes C_{8}\right)$ \\
\hline$(5,657)$ & 8 & {$[2,0,6]$} & $G(128,1755)$ & & $(5,737)$ & 1 & {$[0,0,1]$} & $G(48,17)$ & $\left(C_{3} \times Q_{8}\right) \rtimes C_{2}$ \\
\hline$(5,658)$ & 4 & {$[1,0,3]$} & $G(128,1746)$ & $C_{2} \times\left(\left(C_{4}^{2} \rtimes C_{2}\right) \rtimes C_{2}\right)$ & $(5,738)$ & 1 & {$[0,0,1]$} & $G(48,17)$ & $\left(C_{3} \times Q_{8}\right) \rtimes C_{2}$ \\
\hline$(5,659)$ & 8 & {$[2,0,6]$} & $G(128,850)$ & & $(5,739)$ & 1 & {$[0,0,1]$} & $G(48,17)$ & $\left(C_{3} \times Q_{8}\right) \rtimes C_{2}$ \\
\hline$(5,660)$ & 4 & {$[1,0,3]$} & $G(128,856)$ & & $(5,740)$ & 1 & {$[0,0,1]$} & $G(48,17)$ & $\left(C_{3} \times Q_{8}\right) \rtimes C_{2}$ \\
\hline$(5,661)$ & 1 & {$[0,0,1]$} & $G(144,156)$ & $C_{6} \times \mathrm{SL}(2,3)$ & $(5,741)$ & 5 & {$[1,0,4]$} & $G(48,32)$ & $C_{2} \times \operatorname{SL}(2,3)$ \\
\hline$(5,662)$ & 1 & {$[0,0,1]$} & $G(144,125)$ & $\left(C_{3} \times \mathrm{SL}(2,3)\right) \rtimes C_{2}$ & $(5,742)$ & 5 & {$[2,0,3]$} & $G(48,29)$ & $\mathrm{GL}(2,3)$ \\
\hline$(5,663)$ & 1 & {$[0,0,1]$} & $G(144,125)$ & $\left(C_{3} \times \mathrm{SL}(2,3)\right) \rtimes C_{2}$ & $(5,743)$ & 5 & {$[1,0,4]$} & $G(48,29)$ & $\operatorname{GL}(2,3)$ \\
\hline$(5,664)$ & 4 & {$[1,0,3]$} & $G(16,12)$ & $C_{2} \times Q_{8}$ & $(5,744)$ & 1 & {$[0,0,1]$} & $G(576,8359)$ & \\
\hline$(5,665)$ & 4 & {$[2,0,2]$} & $G(16,6)$ & $C_{8} \rtimes C_{2}$ & $(5,745)$ & 2 & {$[0,0,2]$} & $G(576,8277)$ & \\
\hline$(5,666)$ & 4 & {$[2,0,2]$} & $G(16,6)$ & $C_{8} \rtimes C_{2}$ & $(5,746)$ & 2 & {$[0,0,2]$} & $G(576,8277)$ & \\
\hline$(5,667)$ & 4 & {$[1,0,3]$} & $G(16,6)$ & $C_{8} \rtimes C_{2}$ & $(5,747)$ & 1 & {$[0,0,1]$} & $G(576,8282)$ & \\
\hline$(5,668)$ & 4 & {$[2,0,2]$} & $G(16,13)$ & $\left(C_{4} \times C_{2}\right) \rtimes C_{2}$ & $(5,748)$ & 1 & {$[0,0,1]$} & $G(576,8282)$ & \\
\hline$(5,669)$ & 4 & {$[1,0,3]$} & $G(16,13)$ & $\left(C_{4} \times C_{2}\right) \rtimes C_{2}$ & $(5,749)$ & 4 & {$[1,0,3]$} & $G(64,101)$ & $C_{2} \times\left(C_{4}^{2} \rtimes C_{2}\right)$ \\
\hline$(5,670)$ & 7 & {$[3,0,4]$} & $G(16,13)$ & $\left(C_{4} \times C_{2}\right) \rtimes C_{2}$ & $(5,750)$ & 8 & {$[4,0,4]$} & $G(64,138)$ & \\
\hline$(5,671)$ & 7 & {$[3,0,4]$} & $G(16,13)$ & $\left(C_{4} \times C_{2}\right) \rtimes C_{2}$ & $(5,751)$ & 8 & {$[2,0,6]$} & $G(64,138)$ & \\
\hline$(5,672)$ & 4 & {$[2,0,2]$} & $G(16,8)$ & $Q D_{8}$ & $(5,752)$ & 8 & {$[2,0,6]$} & $G(64,34)$ & \\
\hline$(5,673)$ & 4 & {$[1,0,3]$} & $G(16,8)$ & $Q D_{8}$ & $(5,753)$ & 4 & {$[2,0,2]$} & $G(64,34)$ & \\
\hline$(5,674)$ & 4 & {$[2,0,2]$} & $G(16,8)$ & $Q D_{8}$ & $(5,754)$ & 8 & {$[4,0,4]$} & $G(64,32)$ & $\left(\left(C_{8} \rtimes C_{2}\right) \rtimes C_{2}\right) \rtimes C_{2}$ \\
\hline$(5,675)$ & 4 & {$[1,0,3]$} & $G(16,8)$ & $Q D_{8}$ & $(5,755)$ & 8 & {$[2,0,6]$} & $G(64,32)$ & $\left(\left(C_{8} \rtimes C_{2}\right) \rtimes C_{2}\right) \rtimes C_{2}$ \\
\hline$(5,676)$ & 1 & {$[0,0,1]$} & $G(192,988)$ & $\left(\mathrm{SL}(2,3) \rtimes C_{4}\right) \rtimes C_{2}$ & $(5,756)$ & 4 & {$[2,0,2]$} & $G(64,134)$ & $\left(C_{4}^{2} \rtimes C_{2}\right) \rtimes C_{2}$ \\
\hline$(5,677)$ & 1 & {$[0,0,1]$} & $G(192,988)$ & $\left(\mathrm{SL}(2,3) \rtimes C_{4}\right) \rtimes C_{2}$ & $(5,757)$ & 4 & {$[1,0,3]$} & $G(64,134)$ & $\left(C_{4}^{2} \rtimes C_{2}\right) \rtimes C_{2}$ \\
\hline$(5,678)$ & 1 & {$[0,0,1]$} & $G(192,988)$ & $\left(\mathrm{SL}(2,3) \rtimes C_{4}\right) \rtimes C_{2}$ & $(5,758)$ & 4 & {$[2,0,2]$} & $G(64,134)$ & $\left(C_{4}^{2} \rtimes C_{2}\right) \rtimes C_{2}$ \\
\hline$(5,679)$ & 1 & {$[0,0,1]$} & $G(192,988)$ & $\left(\mathrm{SL}(2,3) \rtimes C_{4}\right) \rtimes C_{2}$ & $(5,759)$ & 4 & {$[1,0,3]$} & $G(64,134)$ & $\left(C_{4}^{2} \rtimes C_{2}\right) \rtimes C_{2}$ \\
\hline$(5,680)$ & 1 & {$[0,0,1]$} & $G(192,981)$ & $C_{2} \times\left(\mathrm{SL}(2,3) \rtimes C_{4}\right)$ & $(5,760)$ & 8 & {$[4,0,4]$} & $G(64,32)$ & $\left(\left(C_{8} \rtimes C_{2}\right) \rtimes C_{2}\right) \rtimes C_{2}$ \\
\hline$(5,681)$ & 1 & {$[0,0,1]$} & $G(192,1502)$ & $C_{2} \times\left(\left(\operatorname{SL}(2,3) \rtimes C_{2}\right) \rtimes C_{2}\right)$ & $(5,761)$ & 8 & {$[2,0,6]$} & $G(64,32)$ & $\left(\left(C_{8} \rtimes C_{2}\right) \rtimes C_{2}\right) \rtimes C_{2}$ \\
\hline$(5,682)$ & 1 & {$[0,0,1]$} & $G(192,1481)$ & $C_{2} \times\left(\mathrm{GL}(2,3) \rtimes C_{2}\right)$ & $(5,762)$ & 4 & {$[2,0,2]$} & $G(64,34)$ & \\
\hline$(5,683)$ & 5 & {$[1,0,4]$} & $G(192,201)$ & & $(5,763)$ & 11 & {$[5,0,6]$} & $G(64,138)$ & \\
\hline$(5,684)$ & 5 & {$[2,0,3]$} & $G(192,201)$ & & $(5,764)$ & 11 & {$[3,0,8]$} & $G(64,138)$ & \\
\hline$(5,685)$ & 5 & {$[1,0,4]$} & $G(192,1508)$ & & $(5,765)$ & 4 & {$[1,0,3]$} & $G(64,134)$ & $\left(C_{4}^{2} \rtimes C_{2}\right) \rtimes C_{2}$ \\
\hline$(5,686)$ & 5 & {$[1,0,4]$} & $G(192,1494)$ & & $(5,766)$ & 4 & {$[1,0,3]$} & $G(64,134)$ & $\left(C_{4}^{2} \rtimes C_{2}\right) \rtimes C_{2}$ \\
\hline$(5,687)$ & 5 & {$[2,0,3]$} & $G(192,1494)$ & & $(5,767)$ & 4 & {$[1,0,3]$} & $G(64,264)$ & $C_{2} \times\left(\left(C_{2} \times D_{4}\right) \rtimes C_{2}\right)$ \\
\hline$(5,688)$ & 5 & {$[1,0,4]$} & $G(192,1493)$ & & $(5,768)$ & 11 & {$[3,0,8]$} & $G(64,90)$ & \\
\hline$(5,689)$ & 5 & {$[2,0,3]$} & $G(192,1493)$ & & $(5,769)$ & 4 & {$[1,0,3]$} & $G(64,92)$ & $C_{2} \times\left(\left(C_{8} \rtimes C_{2}\right) \rtimes C_{2}\right)$ \\
\hline$(5,690)$ & 1 & {$[0,0,1]$} & $G(2304, ?)$ & & $(5,770)$ & 4 & {$[1,0,3]$} & $G(64,254)$ & $C_{2} \times\left(\left(C_{2} \times D_{4}\right) \rtimes C_{2}\right)$ \\
\hline$(5,691)$ & 1 & {$[0,0,1]$} & $G(24,3)$ & $\mathrm{SL}(2,3)$ & $(5,771)$ & 1 & {$[0,0,1]$} & $G(72,25)$ & $C_{3} \times \operatorname{SL}(2,3)$ \\
\hline$(5,692)$ & 1 & {$[0,1,0]$} & $G(24,1)$ & $C_{3} \rtimes C_{8}$ & $(5,772)$ & 5 & {$[1,0,4]$} & $G(768,1088539)$ & \\
\hline$(5,693)$ & 1 & {$[0,1,0]$} & $G(24,1)$ & $C_{3} \rtimes C_{8}$ & $(5,773)$ & 4 & {$[2,0,2]$} & $G(8,4)$ & $Q_{8}$ \\
\hline$(5,694)$ & 1 & {$[0,0,1]$} & $G(24,11)$ & $C_{3} \times Q_{8}$ & $(5,774)$ & 4 & {$[2,0,2]$} & $G(8,4)$ & $Q_{8}$ \\
\hline$(5,695)$ & 1 & {$[0,0,1]$} & $G(24,11)$ & $C_{3} \times Q_{8}$ & $(5,775)$ & 1 & {$[0,0,1]$} & $G(96,200)$ & $C_{2} \times\left(\mathrm{SL}(2,3) \rtimes C_{2}\right)$ \\
\hline$(5,696)$ & 5 & {$[2,0,3]$} & $G(24,3)$ & $\mathrm{SL}(2,3)$ & $(5,776)$ & 1 & {$[0,0,1]$} & $G(96,189)$ & $C_{2} \times \mathrm{GL}(2,3)$ \\
\hline$(5,697)$ & 4 & {$[1,0,3]$} & $G(256,26531)$ & $C_{2} \times\left(D_{4}^{2} \rtimes C_{2}\right)$ & $(5,777)$ & 1 & {$[0,0,1]$} & $G(96,67)$ & $\mathrm{SL}(2,3) \rtimes C_{4}$ \\
\hline$(5,698)$ & 1 & {$[0,0,1]$} & $G(288,860)$ & $\left(\left(\mathrm{SL}(2,3) \rtimes C_{2}\right) \rtimes C_{2}\right) \rtimes C_{3}$ & $(5,778)$ & 1 & {$[0,0,1]$} & $G(96,67)$ & $\mathrm{SL}(2,3) \rtimes C_{4}$ \\
\hline$(5,699)$ & 1 & {$[0,0,1]$} & $G(288,911)$ & $C_{2} \times\left(\left(C_{3} \times \mathrm{SL}(2,3)\right) \rtimes C_{2}\right)$ & $(5,779)$ & 1 & {$[0,0,1]$} & $G(96,201)$ & $\left(\mathrm{SL}(2,3) \rtimes C_{2}\right) \rtimes C_{2}$ \\
\hline$(5,700)$ & 4 & {$[2,0,2]$} & $G(32,11)$ & $C_{4}^{2} \rtimes C_{2}$ & $(5,780)$ & 1 & {$[0,0,1]$} & $G(96,193)$ & $\mathrm{GL}(2,3) \rtimes C_{2}$ \\
\hline$(5,701)$ & 4 & {$[1,0,3]$} & $G(32,11)$ & $C_{4}^{2} \rtimes C_{2}$ & $(5,781)$ & 1 & {$[0,0,1]$} & $G(96,193)$ & $\mathrm{GL}(2,3) \rtimes C_{2}$ \\
\hline$(5,702)$ & 4 & {$[1,0,3]$} & $G(32,11)$ & $C_{4}^{2} \rtimes C_{2}$ & $(5,782)$ & 1 & {$[0,0,1]$} & $G(96,201)$ & $\left(\mathrm{SL}(2,3) \rtimes C_{2}\right) \rtimes C_{2}$ \\
\hline$(5,703)$ & 4 & {$[2,0,2]$} & $G(32,11)$ & $C_{4}^{2} \rtimes C_{2}$ & $(5,783)$ & 1 & {$[0,0,1]$} & $G(96,193)$ & $\operatorname{GL}(2,3) \rtimes C_{2}$ \\
\hline$(5,704)$ & 11 & {$[5,0,6]$} & $G(32,6)$ & $\left(\left(C_{4} \times C_{2}\right) \rtimes C_{2}\right) \rtimes C_{2}$ & $(5,784)$ & 1 & {$[0,0,1]$} & $G(96,148)$ & $C_{2} \times\left(\left(C_{3} \times Q_{8}\right) \rtimes C_{2}\right)$ \\
\hline$(5,705)$ & 11 & {$[6,0,5]$} & $G(32,6)$ & $\left(\left(C_{4} \times C_{2}\right) \rtimes C_{2}\right) \rtimes C_{2}$ & $(5,785)$ & 5 & {$[2,0,3]$} & $G(96,204)$ & $\left(\left(C_{2} \times D_{4}\right) \rtimes C_{2}\right) \rtimes C_{3}$ \\
\hline$(5,706)$ & 14 & {$[6,0,8]$} & $G(32,6)$ & $\left(\left(C_{4} \times C_{2}\right) \rtimes C_{2}\right) \rtimes C_{2}$ & $(5,786)$ & 5 & {$[1,0,4]$} & $G(96,189)$ & $C_{2} \times \mathrm{GL}(2,3)$ \\
\hline$(5,707)$ & 4 & {$[2,0,2]$} & $G(32,49)$ & $\left(C_{2} \times D_{4}\right) \rtimes C_{2}$ & $(5,787)$ & 1 & {$[1,0,0]$} & $G(12,2)$ & $C_{12}$ \\
\hline$(5,708)$ & 4 & {$[1,0,3]$} & $G(32,49)$ & $\left(C_{2} \times D_{4}\right) \rtimes C_{2}$ & $(5,788)$ & 1 & {$[1,0,0]$} & $G(12,2)$ & $C_{12}$ \\
\hline$(5,709)$ & 7 & {$[3,0,4]$} & $G(32,49)$ & $\left(C_{2} \times D_{4}\right) \rtimes C_{2}$ & $(5,789)$ & 1 & {$[1,0,0]$} & $G(24,9)$ & $C_{12} \times C_{2}$ \\
\hline$(5,710)$ & 4 & {$[2,0,2]$} & $G(32,7)$ & $\left(C_{8} \rtimes C_{2}\right) \rtimes C_{2}$ & $(5,790)$ & 1 & {$[1,0,0]$} & $G(24,6)$ & $D_{12}$ \\
\hline$(5,711)$ & 4 & {$[2,0,2]$} & $G(32,7)$ & $\left(C_{8} \rtimes C_{2}\right) \rtimes C_{2}$ & $(5,791)$ & 1 & {$[1,0,0]$} & $G(24,6)$ & $D_{12}$ \\
\hline$(5,712)$ & 4 & {$[1,0,3]$} & $G(32,7)$ & $\left(C_{8} \rtimes C_{2}\right) \rtimes C_{2}$ & $(5,792)$ & 1 & {$[1,0,0]$} & $G(24,6)$ & $D_{12}$ \\
\hline$(5,713)$ & 4 & {$[2,0,2]$} & $G(32,43)$ & $\left(C_{2} \times D_{4}\right) \rtimes C_{2}$ & $(5,793)$ & 1 & {$[1,0,0]$} & $G(48,36)$ & $C_{2} \times D_{12}$ \\
\hline$(5,714)$ & 4 & {$[1,0,3]$} & $G(32,43)$ & $\left(C_{2} \times D_{4}\right) \rtimes C_{2}$ & $(5,794)$ & 1 & {$[1,0,0]$} & $G(12,1)$ & $C_{3} \rtimes C_{4}$ \\
\hline$(5,715)$ & 4 & {$[2,0,2]$} & $G(32,43)$ & $\left(C_{2} \times D_{4}\right) \rtimes C_{2}$ & $(5,795)$ & 1 & {$[1,0,0]$} & $G(12,1)$ & $C_{3} \rtimes C_{4}$ \\
\hline$(5,716)$ & 4 & {$[1,0,3]$} & $G(32,43)$ & $\left(C_{2} \times D_{4}\right) \rtimes C_{2}$ & $(5,796)$ & 1 & {$[1,0,0]$} & $G(144,167)$ & $C_{6} \times\left(\left(C_{6} \times C_{2}\right) \rtimes C_{2}\right)$ \\
\hline$(5,717)$ & 4 & {$[1,0,3]$} & $G(32,43)$ & $\left(C_{2} \times D_{4}\right) \rtimes C_{2}$ & $(5,797)$ & 3 & {$[2,0,1]$} & $G(144,191)$ & $C_{2}^{2} \times\left(C_{3}^{2} \rtimes C_{4}\right)$ \\
\hline$(5,718)$ & 4 & {$[1,0,3]$} & $G(32,43)$ & $\left(C_{2} \times D_{4}\right) \rtimes C_{2}$ & $(5,798)$ & 2 & {$[1,0,1]$} & $G(144,192)$ & $C_{2}^{2} \times S_{3}^{2}$ \\
\hline$(5,719)$ & 4 & {$[1,0,3]$} & $G(32,48)$ & $C_{2} \times\left(\left(C_{4} \times C_{2}\right) \rtimes C_{2}\right)$ & $(5,799)$ & 1 & {$[1,0,0]$} & $G(144,149)$ & \\
\hline$(5,720)$ & 4 & {$[1,0,3]$} & $G(32,37)$ & $C_{2} \times\left(C_{8} \rtimes C_{2}\right)$ & $(5,800)$ & 1 & {$[1,0,0]$} & $G(144,151)$ & $C_{2} \times\left(\left(C_{6} \times S_{3}\right) \rtimes C_{2}\right)$ \\
\hline
\end{tabular}


Table 16 (continued): stably rational classification of algebraic $k$-tori of dimension 5

\begin{tabular}{|c|c|c|c|c|c|c|c|c|c|}
\hline CARAT & $\#$ & {$[s, r, u]$} & $G(n, i)$ & & CARAT & $\#$ & {$[s, r, u]$} & $G(n, i)$ & \\
\hline$(5,801)$ & 6 & {$[3,0,3]$} & $G(144,186)$ & $C_{2} \times\left(S_{3}^{2} \rtimes C_{2}\right)$ & $(5,881)$ & 1 & {$[1,0,0]$} & $G(72,21)$ & $\left(C_{3} \times\left(C_{3} \rtimes C_{4}\right)\right) \rtimes C_{2}$ \\
\hline$(5,802)$ & 2 & {$[2,0,0]$} & $G(144,115)$ & $\left(C_{2} \times\left(C_{3}^{2} \rtimes C_{4}\right)\right) \rtimes C_{2}$ & $(5,882)$ & 2 & {$[1,0,1]$} & $G(72,46)$ & $C_{2} \times S_{3}^{2}$ \\
\hline$(5,803)$ & 2 & {$[2,0,0]$} & $G(144,115)$ & $\left(C_{2} \times\left(C_{3}^{2} \rtimes C_{4}\right)\right) \rtimes C_{2}$ & $(5,883)$ & 1 & {$[1,0,0]$} & $G(72,23)$ & $\left(C_{6} \times S_{3}\right) \rtimes C_{2}$ \\
\hline$(5,804)$ & 2 & {$[2,0,0]$} & $G(144,115)$ & $\left(C_{2} \times\left(C_{3}^{2} \rtimes C_{4}\right)\right) \rtimes C_{2}$ & $(5,884)$ & 1 & {$[1,0,0]$} & $G(72,23)$ & $\left(C_{6} \times S_{3}\right) \rtimes C_{2}$ \\
\hline$(5,805)$ & 3 & {$[2,0,1]$} & $G(144,186)$ & $C_{2} \times\left(S_{3}^{2} \rtimes C_{2}\right)$ & $(5,885)$ & 1 & {$[1,0,0]$} & $G(72,23)$ & $\left(C_{6} \times S_{3}\right) \rtimes C_{2}$ \\
\hline$(5,806)$ & 3 & {$[2,0,1]$} & $G(144,186)$ & $C_{2} \times\left(S_{3}^{2} \rtimes C_{2}\right)$ & $(5,886)$ & 1 & {$[1,0,0]$} & $G(72,23)$ & $\left(C_{6} \times S_{3}\right) \rtimes C_{2}$ \\
\hline$(5,807)$ & 2 & {$[2,0,0]$} & $G(144,115)$ & $\left(C_{2} \times\left(C_{3}^{2} \rtimes C_{4}\right)\right) \rtimes C_{2}$ & $(5,887)$ & 5 & {$[2,0,3]$} & $G(72,46)$ & $C_{2} \times S_{3}^{2}$ \\
\hline$(5,808)$ & 3 & {$[2,0,1]$} & $G(144,186)$ & $C_{2} \times\left(S_{3}^{2} \rtimes C_{2}\right)$ & $(5,888)$ & 4 & {$[2,0,2]$} & $G(72,45)$ & $C_{2} \times\left(C_{3}^{2} \rtimes C_{4}\right)$ \\
\hline$(5,809)$ & 3 & {$[2,0,1]$} & $G(144,186)$ & $C_{2} \times\left(S_{3}^{2} \rtimes C_{2}\right)$ & $(5,889)$ & 6 & {$[3,0,3]$} & $G(72,40)$ & $S_{3}^{2} \rtimes C_{2}$ \\
\hline$(5,810)$ & 4 & {$[2,0,2]$} & $G(144,186)$ & $C_{2} \times\left(S_{3}^{2} \rtimes C_{2}\right)$ & $(5,890)$ & 6 & {$[3,0,3]$} & $G(72,40)$ & $S_{3}^{2} \rtimes C_{2}$ \\
\hline$(5,811)$ & 4 & {$[2,0,2]$} & $G(144,186)$ & $C_{2} \times\left(S_{3}^{2} \rtimes C_{2}\right)$ & $(5,891)$ & 6 & {$[3,0,3]$} & $G(72,40)$ & $S_{3}^{2} \rtimes C_{2}$ \\
\hline$(5,812)$ & 4 & {$[2,0,2]$} & $G(144,186)$ & $C_{2} \times\left(S_{3}^{2} \rtimes C_{2}\right)$ & $(5,892)$ & 6 & {$[3,0,3]$} & $G(72,40)$ & $S_{3}^{2} \rtimes C_{2}$ \\
\hline$(5,813)$ & 1 & {$[1,0,0]$} & $G(144,154)$ & $\left(C_{2} \times S_{3}^{2}\right) \rtimes C_{2}$ & $(5,893)$ & 4 & {$[2,0,2]$} & $G(72,40)$ & $S_{3}^{2} \rtimes C_{2}$ \\
\hline$(5,814)$ & 1 & {$[1,0,0]$} & $G(144,136)$ & $\left(C_{2} \times\left(C_{3}^{2} \rtimes C_{4}\right)\right) \rtimes C_{2}$ & $(5,894)$ & 4 & {$[2,0,2]$} & $G(72,40)$ & $S_{3}^{2} \rtimes C_{2}$ \\
\hline$(5,815)$ & 1 & {$[1,0,0]$} & $G(144,136)$ & $\left(C_{2} \times\left(C_{3}^{2} \rtimes C_{4}\right)\right) \rtimes C_{2}$ & $(5,895)$ & 4 & {$[2,0,2]$} & $G(72,40)$ & $S_{3}^{2} \rtimes C_{2}$ \\
\hline$(5,816)$ & 2 & {$[2,0,0]$} & $G(144,136)$ & $\left(C_{2} \times\left(C_{3}^{2} \rtimes C_{4}\right)\right) \rtimes C_{2}$ & $(5,896)$ & 4 & {$[2,0,2]$} & $G(72,40)$ & $S_{3}^{2} \rtimes C_{2}$ \\
\hline$(5,817)$ & 1 & {$[1,0,0]$} & $G(144,154)$ & $\left(C_{2} \times S_{3}^{2}\right) \rtimes C_{2}$ & $(5,897)$ & 1 & {$[1,0,0]$} & $G(96,209)$ & $C_{2} \times S_{3} \times D_{4}$ \\
\hline$(5,818)$ & 1 & {$[1,0,0]$} & $G(144,154)$ & $\left(C_{2} \times S_{3}^{2}\right) \rtimes C_{2}$ & $(5,898)$ & 1 & {$[1,0,0]$} & $G(10,2)$ & $C_{10}$ \\
\hline$(5,819)$ & 1 & {$[1,0,0]$} & $G(144,154)$ & $\left(C_{2} \times S_{3}^{2}\right) \rtimes C_{2}$ & $(5,899)$ & 1 & {$[1,0,0]$} & $G(10,2)$ & $C_{10}$ \\
\hline$(5,820)$ & 1 & {$[1,0,0]$} & $G(144,154)$ & $\left(C_{2} \times S_{3}^{2}\right) \rtimes C_{2}$ & $(5,900)$ & 3 & {$[3,0,0]$} & $G(10,1)$ & $D_{5}$ \\
\hline$(5,821)$ & 1 & {$[1,0,0]$} & $G(144,154)$ & $\left(C_{2} \times S_{3}^{2}\right) \rtimes C_{2}$ & $(5,901)$ & 3 & {$[3,0,0]$} & $G(10,1)$ & $D_{5}$ \\
\hline$(5,822)$ & 5 & {$[2,0,3]$} & $G(18,3)$ & $C_{3} \times S_{3}$ & $(5,902)$ & 2 & {$[2,0,0]$} & $G(10,2)$ & $C_{10}$ \\
\hline$(5,823)$ & 5 & {$[2,0,3]$} & $G(18,3)$ & $C_{3} \times S_{3}$ & $(5,903)$ & 1 & {$[1,0,0]$} & $G(20,5)$ & $C_{10} \times C_{2}$ \\
\hline$(5,824)$ & 1 & {$[1,0,0]$} & $G(24,7)$ & $C_{2} \times\left(C_{3} \rtimes C_{4}\right)$ & $(5,904)$ & 3 & {$[3,0,0]$} & $G(20,4)$ & $D_{10}$ \\
\hline$(5,825)$ & 1 & {$[1,0,0]$} & $G(24,10)$ & $C_{3} \times D_{4}$ & $(5,905)$ & 1 & {$[1,0,0]$} & $G(20,4)$ & $D_{10}$ \\
\hline$(5,826)$ & 2 & {$[2,0,0]$} & $G(24,10)$ & $C_{3} \times D_{4}$ & $(5,906)$ & 2 & {$[2,0,0]$} & $G(20,4)$ & $D_{10}$ \\
\hline$(5,827)$ & 1 & {$[1,0,0]$} & $G(24,10)$ & $C_{3} \times D_{4}$ & $(5,907)$ & 1 & {$[1,0,0]$} & $G(20,4)$ & $D_{10}$ \\
\hline$(5,828)$ & 2 & {$[2,0,0]$} & $G(24,8)$ & $\left(C_{6} \times C_{2}\right) \rtimes C_{2}$ & $(5,908)$ & 1 & {$[1,0,0]$} & $G(40,13)$ & $C_{2}^{2} \times D_{5}$ \\
\hline$(5,829)$ & 2 & {$[2,0,0]$} & $G(24,8)$ & $\left(C_{6} \times C_{2}\right) \rtimes C_{2}$ & $(5,909)$ & 2 & {$[2,0,0]$} & $G(5,1)$ & $C_{5}$ \\
\hline$(5,830)$ & 2 & {$[2,0,0]$} & $G(24,8)$ & $\left(C_{6} \times C_{2}\right) \rtimes C_{2}$ & $(5,910)$ & 4 & {$[4,0,0]$} & $G(120,35)$ & $C_{2} \times A_{5}$ \\
\hline$(5,831)$ & 2 & {$[2,0,0]$} & $G(24,8)$ & $\left(C_{6} \times C_{2}\right) \rtimes C_{2}$ & $(5,911)$ & 4 & {$[3,1,0]$} & $G(120,34)$ & $S_{5}$ \\
\hline$(5,832)$ & 1 & {$[1,0,0]$} & $G(24,5)$ & $C_{4} \times S_{3}$ & $(5,912)$ & 4 & {$[3,1,0]$} & $G(120,34)$ & $S_{5}$ \\
\hline$(5,833)$ & 1 & {$[1,0,0]$} & $G(24,5)$ & $C_{4} \times S_{3}$ & $(5,913)$ & 2 & {$[2,0,0]$} & $G(120,35)$ & $C_{2} \times A_{5}$ \\
\hline$(5,834)$ & 1 & {$[1,0,0]$} & $G(24,5)$ & $C_{4} \times S_{3}$ & $(5,914)$ & 2 & {$[1,1,0]$} & $G(120,34)$ & $S_{5}$ \\
\hline$(5,835)$ & 1 & {$[1,0,0]$} & $G(24,5)$ & $C_{4} \times S_{3}$ & $(5,915)$ & 2 & {$[2,0,0]$} & $G(120,35)$ & $C_{2} \times A_{5}$ \\
\hline$(5,836)$ & 3 & {$[2,0,1]$} & $G(288,1031)$ & $C_{2}^{2} \times\left(S_{3}^{2} \rtimes C_{2}\right)$ & $(5,916)$ & 2 & {$[1,1,0]$} & $G(120,34)$ & $S_{5}$ \\
\hline$(5,837)$ & 2 & {$[2,0,0]$} & $G(288,880)$ & & $(5,917)$ & 6 & {$[4,2,0]$} & $G(20,3)$ & $C_{5} \rtimes C_{4}$ \\
\hline$(5,838)$ & 2 & {$[2,0,0]$} & $G(288,889)$ & $\left(C_{2}^{2} \times S_{3}^{2}\right) \rtimes C_{2}$ & $(5,918)$ & 6 & {$[4,2,0]$} & $G(20,3)$ & $C_{5} \rtimes C_{4}$ \\
\hline$(5,839)$ & 1 & {$[1,0,0]$} & $G(288,889)$ & $\left(C_{2}^{2} \times S_{3}^{2}\right) \rtimes C_{2}$ & $(5,919)$ & 4 & {$[3,1,0]$} & $G(240,189)$ & $C_{2} \times S_{5}$ \\
\hline$(5,840)$ & 1 & {$[1,0,0]$} & $G(288,889)$ & $\left(C_{2}^{2} \times S_{3}^{2}\right) \rtimes C_{2}$ & $(5,920)$ & 2 & {$[2,0,0]$} & $G(240,190)$ & $C_{2}^{2} \times A_{5}$ \\
\hline$(5,841)$ & 2 & {$[2,0,0]$} & $G(288,889)$ & $\left(C_{2}^{2} \times S_{3}^{2}\right) \rtimes C_{2}$ & $(5,921)$ & 2 & {$[1,1,0]$} & $G(240,189)$ & $C_{2} \times S_{5}$ \\
\hline$(5,842)$ & 1 & {$[1,0,0]$} & $G(288,889)$ & $\left(C_{2}^{2} \times S_{3}^{2}\right) \rtimes C_{2}$ & $(5,922)$ & 2 & {$[1,1,0]$} & $G(240,189)$ & $C_{2} \times S_{5}$ \\
\hline$(5,843)$ & 1 & {$[1,0,0]$} & $G(288,889)$ & $\left(C_{2}^{2} \times S_{3}^{2}\right) \rtimes C_{2}$ & $(5,923)$ & 2 & {$[1,1,0]$} & $G(240,189)$ & $C_{2} \times S_{5}$ \\
\hline$(5,844)$ & 1 & {$[1,0,0]$} & $G(288,941)$ & & $(5,924)$ & 2 & {$[1,1,0]$} & $G(240,189)$ & $C_{2} \times S_{5}$ \\
\hline$(5,845)$ & 1 & {$[1,0,0]$} & $G(288,977)$ & $C_{2} \times\left(\left(C_{2} \times S_{3}^{2}\right) \rtimes C_{2}\right)$ & $(5,925)$ & 2 & {$[1,1,0]$} & $G(240,189)$ & $C_{2} \times S_{5}$ \\
\hline$(5,846)$ & 5 & {$[2,0,3]$} & $G(36,12)$ & $C_{6} \times S_{3}$ & $(5,926)$ & 6 & {$[4,2,0]$} & $G(40,12)$ & $C_{2} \times\left(C_{5} \rtimes C_{4}\right)$ \\
\hline$(5,847)$ & 2 & {$[1,0,1]$} & $G(36,12)$ & $C_{6} \times S_{3}$ & $(5,927)$ & 2 & {$[1,1,0]$} & $G(40,12)$ & $C_{2} \times\left(C_{5} \rtimes C_{4}\right)$ \\
\hline$(5,848)$ & 2 & {$[1,0,1]$} & $G(36,12)$ & $C_{6} \times S_{3}$ & $(5,928)$ & 4 & {$[2,2,0]$} & $G(40,12)$ & $C_{2} \times\left(C_{5} \rtimes C_{4}\right)$ \\
\hline$(5,849)$ & 1 & {$[1,0,0]$} & $G(36,6)$ & $C_{3} \times\left(C_{3} \rtimes C_{4}\right)$ & $(5,929)$ & 2 & {$[1,1,0]$} & $G(40,12)$ & $C_{2} \times\left(C_{5} \rtimes C_{4}\right)$ \\
\hline$(5,850)$ & 1 & {$[1,0,0]$} & $G(36,6)$ & $C_{3} \times\left(C_{3} \rtimes C_{4}\right)$ & $(5,930)$ & 2 & {$[1,1,0]$} & $G(480,1186)$ & $C_{2}^{2} \times S_{5}$ \\
\hline$(5,851)$ & 3 & {$[1,0,2]$} & $G(36,12)$ & $C_{6} \times S_{3}$ & $(5,931)$ & 4 & {$[4,0,0]$} & $G(60,5)$ & $A_{5}$ \\
\hline$(5,852)$ & 6 & {$[3,0,3]$} & $G(36,9)$ & $C_{3}^{2} \rtimes C_{4}$ & $(5,932)$ & 2 & {$[1,1,0]$} & $G(80,50)$ & $C_{2}^{2} \times\left(C_{5} \rtimes C_{4}\right)$ \\
\hline$(5,853)$ & 6 & {$[3,0,3]$} & $G(36,9)$ & $C_{3}^{2} \rtimes C_{4}$ & $(5,933)$ & 3 & {$[1,0,2]$} & $G(160,235)$ & $C_{2} \times\left(C_{2}^{4} \rtimes C_{5}\right)$ \\
\hline$(5,854)$ & 7 & {$[3,0,4]$} & $G(36,10)$ & $S_{3}^{2}$ & $(5,934)$ & 3 & {$[1,0,2]$} & $G(160,234)$ & $\left(C_{2}^{4} \rtimes C_{5}\right) \rtimes C_{2}$ \\
\hline$(5,855)$ & 7 & {$[3,0,4]$} & $G(36,10)$ & $S_{3}^{2}$ & $(5,935)$ & 3 & {$[1,0,2]$} & $G(160,234)$ & $\left(C_{2}^{4} \rtimes C_{5}\right) \rtimes C_{2}$ \\
\hline$(5,856)$ & 8 & {$[3,0,5]$} & $G(36,10)$ & $S_{3}^{2}$ & $(5,936)$ & 3 & {$[1,0,2]$} & $G(1920,240997)$ & $C_{2} \times\left(C_{2}^{4} \rtimes A_{5}\right)$ \\
\hline$(5,857)$ & 2 & {$[2,0,0]$} & $G(48,43)$ & $C_{2} \times\left(\left(C_{6} \times C_{2}\right) \rtimes C_{2}\right)$ & $(5,937)$ & 3 & {$[1,0,2]$} & $G(1920,240996)$ & $\left(C_{2}^{4} \rtimes A_{5}\right) \rtimes C_{2}$ \\
\hline$(5,858)$ & 1 & {$[1,0,0]$} & $G(48,45)$ & $C_{6} \times D_{4}$ & $(5,938)$ & 3 & {$[1,0,2]$} & $G(1920,240996)$ & $\left(C_{2}^{4} \rtimes A_{5}\right) \rtimes C_{2}$ \\
\hline$(5,859)$ & 1 & {$[1,0,0]$} & $G(48,35)$ & $C_{2} \times C_{4} \times S_{3}$ & $(5,939)$ & 3 & {$[1,0,2]$} & $G(320,1635)$ & $\left(C_{2}^{4} \rtimes C_{5}\right) \rtimes C_{4}$ \\
\hline$(5,860)$ & 1 & {$[1,0,0]$} & $G(48,38)$ & $D_{4} \times S_{3}$ & $(5,940)$ & 3 & {$[1,0,2]$} & $G(320,1635)$ & $\left(C_{2}^{4} \rtimes C_{5}\right) \rtimes C_{4}$ \\
\hline$(5,861)$ & 2 & {$[2,0,0]$} & $G(48,38)$ & $D_{4} \times S_{3}$ & $(5,941)$ & 3 & {$[1,0,2]$} & $G(320,1636)$ & $C_{2} \times\left(\left(C_{2}^{4} \rtimes C_{5}\right) \rtimes C_{2}\right)$ \\
\hline$(5,862)$ & 2 & {$[2,0,0]$} & $G(48,38)$ & $D_{4} \times S_{3}$ & $(5,942)$ & 3 & {$[1,0,2]$} & $G(3840, ?)$ & $C_{2} \times\left(\left(C_{2}^{4} \rtimes A_{5}\right) \rtimes C_{2}\right)$ \\
\hline$(5,863)$ & 1 & {$[1,0,0]$} & $G(48,38)$ & $D_{4} \times S_{3}$ & $(5,943)$ & 3 & {$[1,0,2]$} & $G(640,21536)$ & $C_{2} \times\left(\left(C_{2}^{4} \rtimes C_{5}\right) \rtimes C_{4}\right)$ \\
\hline$(5,864)$ & 1 & {$[1,0,0]$} & $G(48,38)$ & $D_{4} \times S_{3}$ & $(5,944)$ & 3 & {$[1,0,2]$} & $G(80,49)$ & $C_{2}^{4} \rtimes C_{5}$ \\
\hline$(5,865)$ & 1 & {$[1,0,0]$} & $G(48,38)$ & $D_{4} \times S_{3}$ & $(5,945)$ & 3 & {$[1,0,2]$} & $G(960,11358)$ & $C_{2}^{4} \rtimes A_{5}$ \\
\hline$(5,866)$ & 1 & {$[1,0,0]$} & $G(576,8418)$ & $C_{2} \times\left(\left(C_{2}^{2} \times S_{3}^{2}\right) \rtimes C_{2}\right)$ & $(5,946)$ & 4 & {$[2,0,2]$} & $G(120,34)$ & $S_{5}$ \\
\hline$(5,867)$ & 2 & {$[1,0,1]$} & $G(72,48)$ & $C_{2} \times C_{6} \times S_{3}$ & $(5,947)$ & 4 & {$[2,0,2]$} & $G(120,34)$ & $S_{5}$ \\
\hline$(5,868)$ & 1 & {$[1,0,0]$} & $G(72,29)$ & $C_{6} \times\left(C_{3} \rtimes C_{4}\right)$ & $(5,948)$ & 4 & {$[0,0,4]$} & $G(120,35)$ & $C_{2} \times A_{5}$ \\
\hline$(5,869)$ & 7 & {$[3,0,4]$} & $G(72,46)$ & $C_{2} \times S_{3}^{2}$ & $(5,949)$ & 4 & {$[0,0,4]$} & $G(1440,5842)$ & $C_{2} \times S_{6}$ \\
\hline$(5,870)$ & 6 & {$[3,0,3]$} & $G(72,45)$ & $C_{2} \times\left(\dot{C}_{3}^{2} \rtimes C_{4}\right)$ & $(5,950)$ & 4 & {$[0,0,4]$} & $G(240,189)$ & $C_{2} \times S_{5}$ \\
\hline$(5,871)$ & 1 & {$[1,0,0]$} & $G(72,30)$ & $C_{3} \times\left(\left(C_{6} \times C_{2}\right) \rtimes C_{2}\right)$ & $(5,951)$ & 4 & {$[1,0,3]$} & $G(360,118)$ & $A_{6}$ \\
\hline$(5,872)$ & 1 & {$[1,0,0]$} & $G(72,30)$ & $C_{3} \times\left(\left(C_{6} \times C_{2}\right) \rtimes C_{2}\right)$ & $(5,952)$ & 4 & {$[2,0,2]$} & $G(60,5)$ & $A_{5}$ \\
\hline$(5,873)$ & 1 & {$[1,0,0]$} & $G(72,30)$ & $C_{3} \times\left(\left(C_{6} \times C_{2}\right) \rtimes C_{2}\right)$ & $(5,953)$ & 4 & {$[1,0,3]$} & $G(720,763)$ & $S_{6}$ \\
\hline$(5,874)$ & 1 & {$[1,0,0]$} & $G(72,30)$ & $C_{3} \times\left(\left(C_{6} \times C_{2}\right) \rtimes C_{2}\right)$ & $(5,954)$ & 4 & {$[0,0,4]$} & $G(720,763)$ & $S_{6}$ \\
\hline$(5,875)$ & 1 & {$[1,0,0]$} & $G(72,21)$ & $\left(C_{3} \times\left(C_{3} \rtimes C_{4}\right)\right) \rtimes C_{2}$ & $(5,955)$ & 4 & {$[0,0,4]$} & $G(720,766)$ & $C_{2} \times A_{6}$ \\
\hline$(5,876)$ & 3 & {$[2,0,1]$} & $G(72,45)$ & $C_{2} \times\left(C_{3}^{2} \rtimes C_{4}\right)$ & & & & & \\
\hline$(5,877)$ & 3 & {$[2,0,1]$} & $G(72,45)$ & $C_{2} \times\left(C_{3}^{2} \rtimes C_{4}\right)$ & & & & & \\
\hline$(5,878)$ & 2 & {$[1,0,1]$} & $G(72,46)$ & $C_{2} \times S_{3}^{2}$ & & & & & \\
\hline$(5,879)$ & 2 & {$[1,0,1]$} & $G(72,46)$ & $C_{2} \times S_{3}^{2}$ & & & & & \\
\hline$(5,880)$ & 1 & {$[1,0,0]$} & $G(72,21)$ & $\left(C_{3} \times\left(C_{3} \rtimes C_{4}\right)\right) \rtimes C_{2}$ & & & & & \\
\hline
\end{tabular}




\section{REFERENCES}

[Arn84] J. E. Arnold, Jr., Groups of permutation projective dimension two, Proc. Amer. Math. Soc. 91 (1984) 505-509.

[Azu50] G. Azumaya. Corrections and supplementaries to my paper concerning Krull-Remak-Schmidt's theorem, Nagoya Math. J. 1 (1950) 117-124.

[Ben91] D. J. Benson, Representations and cohomology. I, Basic representation theory of finite groups and associative algebras. Cambridge Studies in Advanced Mathematics, 30. Cambridge University Press, Cambridge, 1991.

[Bog88] F. A. Bogomolov, The Brauer group of quotient spaces of linear representations, (Russian) Izv. Akad. Nauk SSSR Ser. Mat. 51 (1987) 485-516, 688; translation in Math. USSR-Izv. 30 (1988) 455-485.

[Bog90] F. A. Bogomolov, Brauer groups of the fields of invariants of algebraic groups, (Russian) Mat. Sb. 180 (1989) 279-293; translation in Math. USSR-Sb. 66 (1990) 285-299.

[BMP04] F. A. Bogomolov, J. Maciel, T. Petrov, Unramified Brauer groups of finite simple groups of Lie type $A_{l}$, Amer. J. Math. 126 (2004) 935-949.

[Bou00] S. Bouc, Burnside rings, Handbook of algebra, vol. 2, 739-804, North-Holland, Amsterdam, 2000.

[BBNWZ78] H. Brown, R. Bülow, J. Neubüser, H. Wondratschek, H. Zassenhaus. Crystallographic Groups of Four-Dimensional Space, John Wiley, New York, 1978.

[Bro82] K. S. Brown, Cohomology of Groups, Grad. Texts in Math., vol. 87, Springer-Verlag, 1972.

[BM83] G. Butler, J. McKay, The transitive groups of degree up to eleven, Comm. Algebra 11 (1983) 863-911.

[Carat] J. Opgenorth, W. Plesken, T. Schulz, CARAT, GAP 4 package, version 2.1b1, 2008, available at http://wwwb.math.rwth-aachen.de/carat/

[CE56] H. Cartan, S. Eilenberg, Homological algebra, Princeton University Press, Princeton, N. J., 1956.

[CHKK10] H. Chu, S. Hu, M. Kang, B. E. Kunyavskii, Noether's problem and the unramified Brauer group for groups of order 64, Int. Math. Res. Not. IMRN 2010 2329-2366.

[CHKP08] H. Chu, S. Hu, M. Kang, Y. G. Prokhorov, Noether's problem for groups of order 32, J. Algebra 320 (2008) $3022-3035$.

[CK01] H. Chu, M. Kang, Rationality of p-group actions, J. Algebra 237 (2001) 673-690.

[CK00] A. Cortella, B. Kunyavskii, Rationality problem for generic tori in simple groups, J. Algebra 225 (2000) $771-793$.

[CTS77] J.-L. Colliot-Thélène, J.-J. Sansuc, La R-équivalence sur les tores, Ann. Sci. École Norm. Sup. (4) 10 (1977) $175-229$.

[CTS87] J.-L. Colliot-Thélène, J.-J. Sansuc, Principal homogeneous spaces under flasque tori: Applications, J. Algebra 106 (1987) 148-205.

[CR81] C. W. Curtis, I. Reiner, Methods of representation theory, vol. I, with applications to finite groups and orders, Pure and Applied Mathematics. A Wiley-Interscience Publication. John Wiley \& Sons, Inc., New York, 1981.

[CR87] C. W. Curtis, I. Reiner, Methods of representation theory, vol. II, With applications to finite groups and orders, Pure and Applied Mathematics (New York). A Wiley-Interscience Publication. John Wiley \& Sons, Inc., New York, 1987.

[Dad65] E. C. Dade, The maximal finite groups of $4 \times 4$ integral matrices, Illinois J. Math. 9 (1965) 99-122.

[Dre70] A. Dress, On the Krull-Schmidt theorem for integral group representations of rank 1, Michigan Math. J. 17 (1970) $273-277$.

[Dre73] A. W. M. Dress, Contributions to the theory of induced representations, Algebraic K-theory, II: "Classical" algebraic K-theory and connections with arithmetic (Proc. Conf., Battelle Memorial Inst., Seattle, Wash., 1972), pp.183-240. Lecture Notes in Math., Vol. 342, Springer, Berlin, 1973.

[Dre75] A. W. M. Dress, The permutation class group of a finite group, J. Pure Appl. Algebra 6 (1975) 1-12.

[End11] S. Endo, The rationality problem for norm one tori, Nagoya Math. J. 202 (2011) 83-106.

[End12] S. Endo, private communications, 2012.

[EH79] S. Endo, Y. Hironaka, Finite groups with trivial class groups, J. Math. Soc. Japan 31 (1979) 161-174.

[EM73] S. Endo, T. Miyata, Invariants of finite abelian groups, J. Math. Soc. Japan 25 (1973) 7-26.

[EM74] S. Endo, T. Miyata, On a classification of the function fields of algebraic tori, Nagoya Math. J. 56 (1975) 85-104.

[EM82] S. Endo, T. Miyata, Integral representations with trivial first cohomology groups, Nagoya Math. J. 85 (1982) $231-240$.

[Fac03] A. Facchini, The Krull-Schmidt theorem, Handbook of algebra, Vol. 3, 357-397, North-Holland, Amsterdam, 2003.

[Flo] M. Florence, Non rationality of some norm-one tori, preprint (2006).

[GAP] The GAP Group, GAP - Groups, Algorithms, and Programming, Version 4.4.12; 2008. (http://www.gap-system.org).

[GW93] R. M. Guralnick, A. Weiss, Transitive permutation lattices in the same genus and embeddings of groups, Linear algebraic groups and their representations (Los Angeles, CA, 1992), 21-33, Contemp. Math., 153, Amer. Math. Soc., Providence, RI, 1993.

[Haj83] M. Hajja, A note on monomial automorphisms, J. Algebra 85 (1983) 243-250.

[Haj87]

[HK92]

[HK94]

[HAP]

[Hür84]

[HKO98] P. Hindman, L. Klingler, C. J. Odenthal, On the Krull-Schmidt-Azumaya theorem for integral group rings, Comm. M. Hajja, Rationality of finite groups of monomial automorphisms of $k(x, y)$, J. Algebra 109 (1987) 46-51. M. Hajja, M. Kang, Finite group actions on rational function fields, J. Algebra 149 (1992) 139-154.

M. Hajja, M. Kang, Three-dimensional purely monomial group actions, J. Algebra 170 (1994) 805-860.

G. Ellis, HAP, GAP 4 package, version 1.10.6, 2012, available at http://hamilton.nuigalway.ie/Hap/www/

W. Hürlimann, On algebraic tori of norm type, Comment. Math. Helv. 59 (1984) 539-549.

[HK10] A. Hoshi, M. Kang, Twisted symmetric group actions, Pacific J. Math. 248 (2010) 285-304.

[HKK14] A. Hoshi, M. Kang, H. Kitayama, Quasi-monomial actions and some 4-dimensional rationality problems, J. Algebra 403 (2014) 363-400.

[HKK13] A. Hoshi, M. Kang, B. E. Kunyavskii, Noether's problem and unramified Brauer groups, Asian J. Math. 17 (2013) 689-714. 
[HKY11] A. Hoshi, H. Kitayama, A. Yamasaki, Rationality problem of three-dimensional monomial group actions, J. Algebra 341 (2011) 45-108.

[HR08] A. Hoshi, Y. Rikuna, Rationality problem of three-dimensional purely monomial group actions: the last case, Math. Comp. 77 (2008) 1823-1829.

[Jon65] A. Jones, On representations of finite groups over valuation rings, Illinois J. Math. 9 (1965) 297-303.

[Kan09] M. Kang, Retract rationality and Noether's problem, Int. Math. Res. Not. IMRN 2009 2760-2788.

[Kan12] M. Kang, Retract rational fields, J. Algebra 349 (2012) 22-37.

[KP10] M. Kang, Y. G. Prokhorov, Rationality of three-dimensional quotients by monomial actions, J. Algebra 324 (2010) 2166-2197.

[Knu98] D. E. Knuth, The Art of Computer Programming, Volume 2: Seminumerical Algorithms, Addison-Wesley, third edition, 1998.

[Kun90] B. E. Kunyavskii, Three-dimensional algebraic tori, Selecta Math. Soviet. 9 (1990) 1-21.

[Kun07] B. E. Kunyavskii, Algebraic tori - thirty years after, Vestnik Samara State Univ. (2007) 198-214.

[Kun10] B. E. Kunyavskii, The Bogomolov multiplier of finite simple groups, Cohomological and geometric approaches to rationality problems, 209-217, Progr. Math., 282, Birkhäuser Boston, Inc., Boston, MA, 2010.

[LL00] N. Lemire, M. Lorenz, On certain lattices associated with generic division algebras, J. Group Theory 3 (2000) 385-405.

[LPR06] N. Lemire, V. L. Popov, Z. Reichstein, Cayley groups, J. Amer. Math. Soc. 19 (2006) 921-967.

[LeB95] L. Le Bruyn, Generic norm one tori, Nieuw Arch. Wisk. (4) 13 (1995) 401-407.

[Len74] H. W. Lenstra, Jr., Rational functions invariant under a finite abelian group, Invent. Math. 25 (1974) $299-325$.

[Lor05] M. Lorenz, Multiplicative invariant theory, Encyclopaedia Math. Sci., vol. 135, Springer-Verlag, Berlin, 2005.

[MN98] M. Matsumoto, T. Nishimura, Mersenne Twister: A 623-dimensionally equidistributed uniform pseudorandom number generator, ACM Trans. on Modeling and Computer Simulation 8 (1998) 3-30.

[Mor12] P. Moravec, Unramified Brauer groups of finite and infinite groups, Amer. J. Math. 134 (2012) $1679-1704$.

[OPS98] J. Opgenorth, W. Plesken, T. Schulz, Crystallographic algorithms and tables, Acta Cryst. Sect. A 54 (1998) 517-531.

[Ple78] W. Plesken, On reducible and decomposable representations of orders, J. Reine Angew. Math. 297 (1978) 188-210.

[Ple81] W. Plesken, Bravais groups in low dimensions, Proceedings of the Conference on Kristallographische Gruppen (Univ. Bielefeld, Bielefeld, 1979), Part II. Match No. 10 (1981) 97-119.

[Ple85] W. Plesken, Finite unimodular groups of prime degree and circulants, J. Algebra 97 (1985) $286-312$.

[PH84] W. Plesken, W. Hanrath, The lattices of six-dimensional Euclidean space, Math. Comp. 43 (1984) 573-587.

[PP77] W. Plesken, M. Pohst, On maximal finite irreducible subgroups of $\operatorname{GL}(n, \mathbf{Z})$. I. The five and seven dimensional cases. II. The six dimensional case, Math. Comp. 31 (1977) 536-551, 552-573.

[PP80] W. Plesken, M. Pohst, On maximal finite irreducible subgroups of GL(n, Z). III. The nine dimensional case. IV. Remarks on even dimensions with application to $n=8$. V. The eight dimensional case and a complete description of dimensions less than ten, Math. Comp. 34 (1980) 245-258, 259-275, 277-301.

[PS00] W. Plesken, T. Schulz, Counting crystallographic groups in low dimensions, Exp. Math. 9 (2000) $407-411$.

[Pop98] S. Yu. Popov, Galois lattices and their birational invariants, (Russian) Vestn. Samar. Gos. Univ. Mat. Mekh. Fiz. Khim. Biol. 1998, no. 4, 71-83.

[Rys72a] S. S. Ryshkov, Maximal finite groups of $n \times n$ integral matrices and full integral automorphism groups of positive quadratic forms (Bravais types), (Russian) Collection of articles dedicated to Academician Ivan Matveevič Vinogradov on his eightieth birthday, II. Trudy Mat. Inst. Steklov. 128 (1972) 183-211, 261; translation in Proc. Steklov Inst. Math. 128 (1972) 217-250.

[Rys72b] S. S. Ryshkov, The maximal finite groups of integer $n \times n$ matrices, (Russian) Dokl. Akad. Nauk SSSR 204 (1972) 561-564; translation in Soviet Math. Dokl. 13 (1972) 720-724.

[RL80] S. S. Ryshkov, Z. D. Lomakina, A proof of the theorem on maximal finite groups of integral $5 \times 5$ matrices, (Russian) Geometry of positive quadratic forms. Trudy Mat. Inst. Steklov. 152 (1980) 204-215, 238; translation in Proc. Steklov Inst. Math. 1982, no. 3, 225-236, American Mathematical Society, Providence, R.I., 1982.

[Sal84a] D. J. Saltman, Retract rational fields and cyclic Galois extensions, Israel J. Math. 47 (1984) 165-215.

[Sal84b] D. J. Saltman, Noether's problem over an algebraically closed field, Invent. Math. 77 (1984) 71-84.

[Sal87]

[Sal90] D. J. Saltman, Multiplicative field invariants, J. Algebra 106 (1987) 221-238.

[Sou94] B. Souvignier, Irreducible finite integral matrix groups of degree 8 and 10, Math. Comp. 63 (1994) 335-350.

[Swa60] R. G. Swan, Induced Representations and Projective Modules, Ann. Math. 71 (1960) 552-578.

[Swa83] R. G. Swan, Noether's problem in Galois theory, Emmy Noether in Bryn Mawr (Bryn Mawr, Pa., 1982), 21-40, Springer, New York-Berlin, 1983.

[Swa88] R. G. Swan, Torsion free cancellation over orders, Illinois J. Math. 32 (1988) 329-360.

[Swa10] R. G. Swan, The flabby class group of a finite cyclic group, Fourth International Congress of Chinese Mathematicians, 259-269, AMS/IP Stud. Adv. Math., 48, Amer. Math. Soc., Providence, RI, 2010.

[Vos67] V. E. Voskresenskii, On two-dimensional algebraic tori II, (Russian) Izv. Akad. Nauk SSSR Ser. Mat. 31 (1967) 711-716; translation in Math. USSR-Izv. 1 (1967) 691-696.

[Vos70] V. E. Voskresenskii, Birational properties of linear algebraic groups, (Russian) Izv. Akad. Nauk SSSR Ser. Mat. 34 (1970) 3-19; translation in Math. USSR-Izv. 4 (1970) 1-17.

[Vos74] V. E. Voskresenskii, Stable equivalence of algebraic tori, (Russian) Izv. Akad. Nauk SSSR Ser. Mat. 38 (1974) 3-10; translation in Math. USSR-Izv. 8 (1974) 1-7.

[Vos83] V. E. Voskresenskii, Projective invariant Demazure models, (Russian) Izv. Akad. Nauk SSSR Ser. Mat. 46 (1982) 195-210, 431; translation in Math USSR-Izv. 20 (1983) 189-202. 
[Vos98] V. E. Voskresenskii, Algebraic groups and their birational invariants, Translated from the Russian manuscript by Boris Kunyavskii, Translations of Mathematical Monographs, 179. American Mathematical Society, Providence, RI, 1998.

[Yam12] A. Yamasaki, Negative solutions to three-dimensional monomial Noether problem, J. Algebra 370 (2012) $46-78$.

Department of Mathematics, Nitgata University, Niigata 950-2181, Japan

E-mail address: hoshi@math.sc.niigata-u.ac.jp

Department of Mathematics, Kyoto University, Kyoto 606-8502, Japan

E-mail address: aiichi.yamasaki@gmail.com 